\title{
TENSILE BEHAVIOUR OF ULTRA-HIGH-PERFORMANCE STEEL FIBER REINFORCED CONCRETE
}

\author{
Yuechen Yang \\ A Thesis Submitted to the Faculty of Graduate Studies \\ in Partial Fulfillment of the Requirements \\ for the Degree of Master of Applied Science
}

Graduate Program in Civil Engineering

York University

Toronto, Ontario

December 2019

(C) Yuechen Yang 2019 


\begin{abstract}
Reinforcing bars are provided in reinforced concrete structures on account of conventional concrete's negligible resistance to tension. However, corrosion of steel reinforcement inevitably occurs due to carbonation and chloride ingress, which significantly reduces the service life of structures. An alternative to this predicament is now feasible with the advent in cementitious material technologies, such as ultrahigh-performance, self-consolidating, steel fiber reinforced concrete (UHP-SFRC). The keystone of safe and economically feasible designs with UHP-SFRC is dependant on its characterization in tension. Thus, in the present work, a detailed research study including both experimental and analytical components was conducted to investigate the tensile behaviour of UHP-SFRC: tensile strength was quantified and correlated through direct tension test (DTT), four-point bending test (FPBT), splitting tensile test, nonlinear finite element analysis and a calibrated empirical expression in relation to cylinder compressive strength. In addition, effects of important parameters on flexural strength including casting methodology, volumetric ratio of steel fibers, aspect ratio of bending prism and prism size were assessed. Experimental results indicated that casting methodology had an influence on fiber dispersion and orientation which was directly related to flexural strength. Prisms containing $1 \%$ steel fibers by unit volume failed in a relatively brittle manner and exhibited less flexural strength than those containing $2 \%$ steel fibers. Prisms with an aspect ratio of 1 generally developed greater flexural strength than those with an aspect ratio of 2 . The degree of preferential fiber alignment became more prominent in prisms with a smaller cross-sectional dimension than those with a relatively larger cross-sectional dimension, leading to greater flexural strength results. Moreover, the bilinear stress-strain and stress-crack mouth opening relationships of UHP-SFRC were derived according to the inverse analysis procedures proposed by Annex 8.1 of CSA-S6 (2018) and Annex U of CSA-A23.1 (2019). Furthermore, a nonlinear finite element analysis software, VecTor2, was employed to develop numerical models with the ability to match the response curves obtained from FPBT. Analytical results indicated that cracking strength of UHP-SFRC derived from the inverse analysis method was generally greater than those obtained from direct tension test, splitting tensile test, nonlinear finite element models and the calibrated empirical expression. Additionally, inverse analysis and finite element analysis results indicated that the majority of prisms exhibited tension hardening behaviour with a hardening ratio greater than 1.1 and an ultimate tensile strain greater than $0.1 \%$. Furthermore, it was observed that UHP-SFRC enabled resilient and ductile response in tension by delaying the occurrence of crack localization and sustaining large levels of deformation. In addition to tension tests, a host of nondestructive tests were conducted to assess the physical properties and durability performance of UHP-SFRC.
\end{abstract}




\section{Acknowledgements}

I would like to convey my appreciation to my supervisors, Dr. Pantazopoulou and Dr. Palermo, who guided me along the way, imparted knowledge and provided support unreservedly. Their expertise, understanding and encouragement motivated me to accomplish this research study. What I gained is not only specialized knowledge, but also professional and positive attitudes towards to this area of expertise. It was an honor for me to be their student, and I will cherish this experience for the rest of my life.

I also want to express my most sincere gratitude to my friends who helped me throughout the experimental program: Najmeh Eshghi, Nicolas Joukhadar, Konstantinos Tsiotsias, Tintu Kizhakkethundathil Tomy, Michael Armando Soto and Zoi Ralli. In addition, I would like to acknowledge our laboratory technician, Riad Rajab, who was never tired of my questions, taught me how to use the devices, and provided professional support and advice throughout the experimental program.

Moreover, I want to express my deepest gratitude to my boyfriend, Geer Heng, who shared every single exciting and challenging moments with me. Although he works in a different area, his support is irreplaceable. Without him, I would not be able to attain the achievements so far.

Last but not the least, special thanks to my family, starting with my parents who respected every decision I have made and support me unconditionally. Furthermore, my appreciation is extended to my grandparents who raised me and cared about me even though they live far away from me. 


\section{Table of Contents}

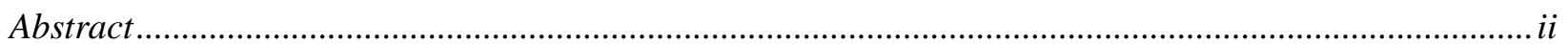

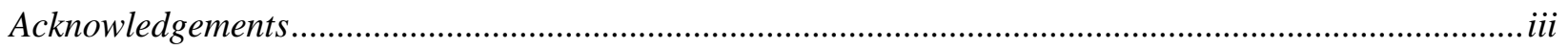

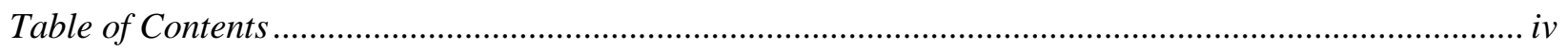

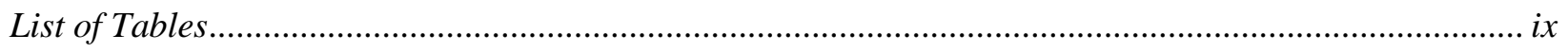

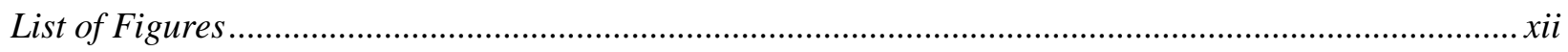

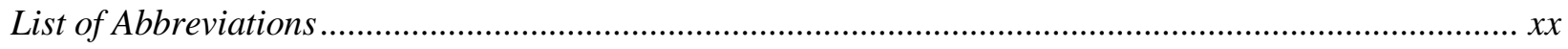

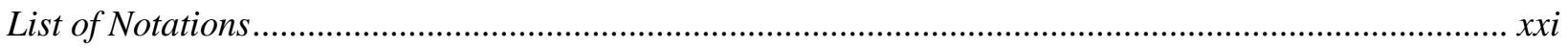

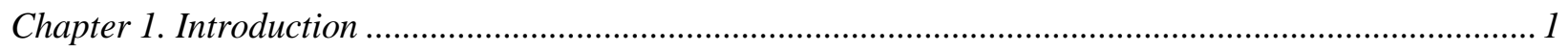

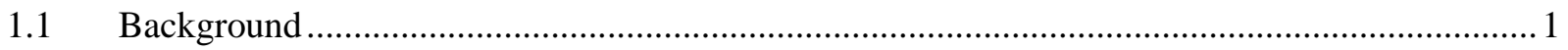

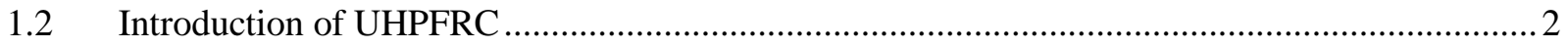

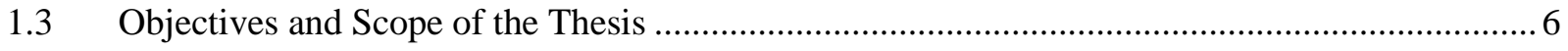

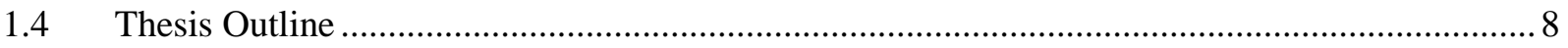

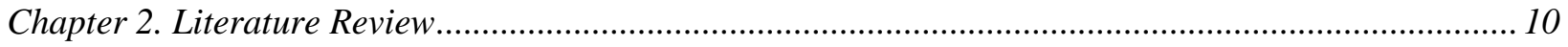

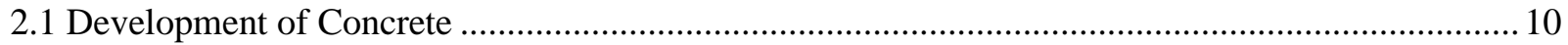

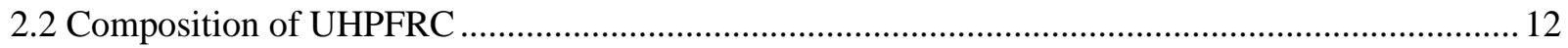

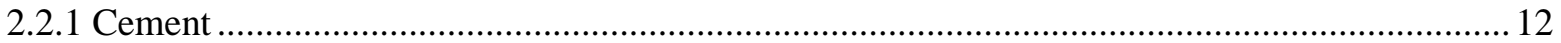

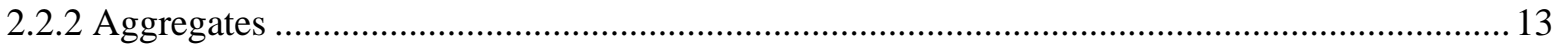

2.2.3 Supplementary Cementitious Materials and Inert Fillers..................................................... 14

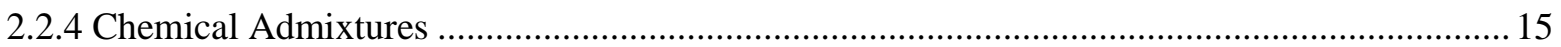

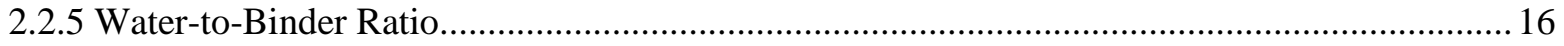

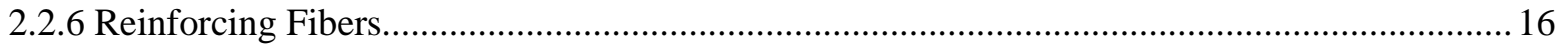

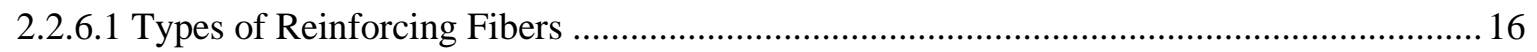

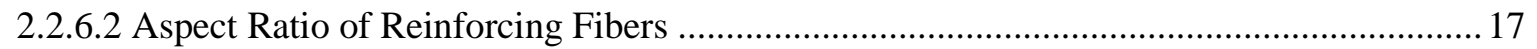

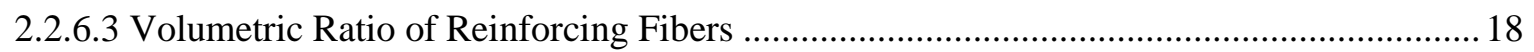

2.2.6.4 Effect of Casting Methodology on Fiber Orientation ...................................................... 18

2.2.6.5 Effect of Brass-Coated Steel Fibers ............................................................................... 19

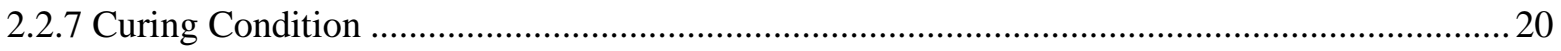

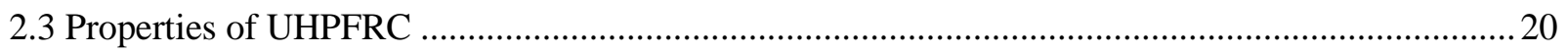

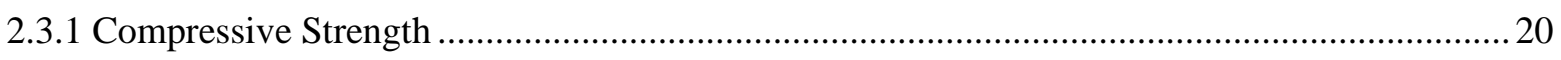

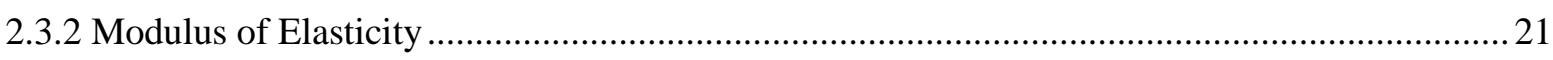

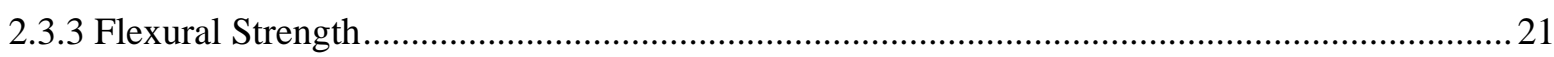

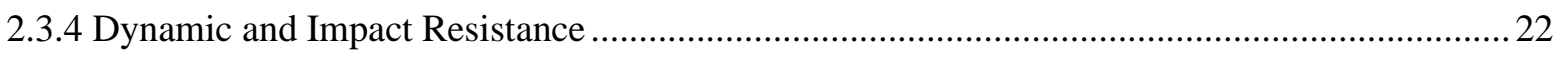




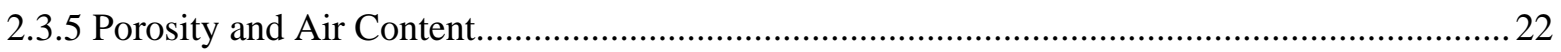

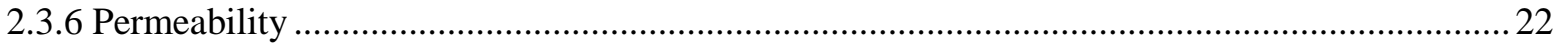

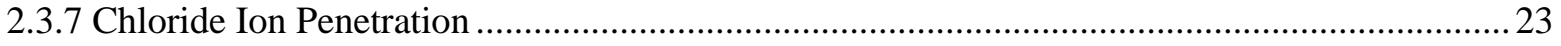

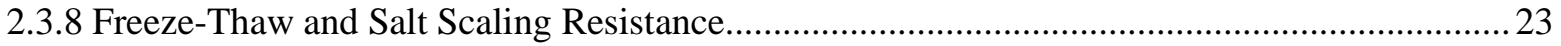

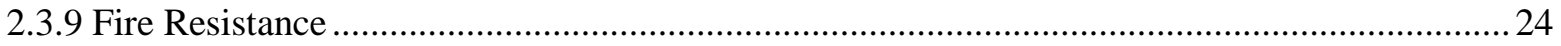

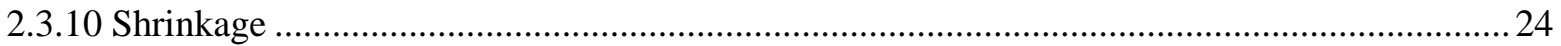

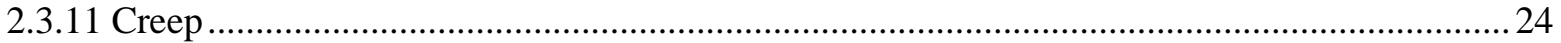

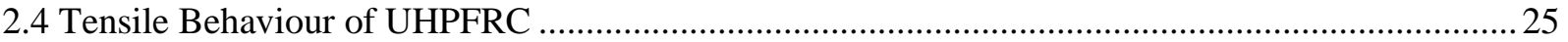

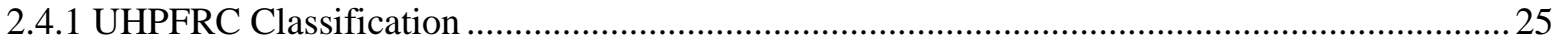

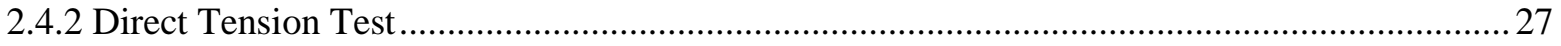

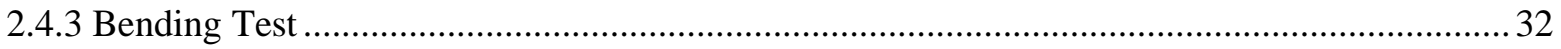

2.4.3.1 Recommended Bending Tests of UHPFRC in Standards .................................................. 33

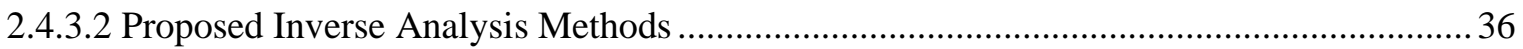

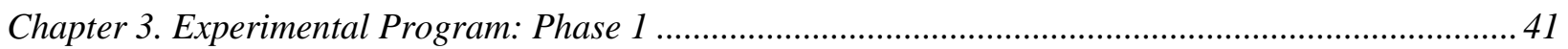

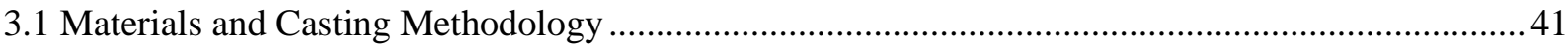

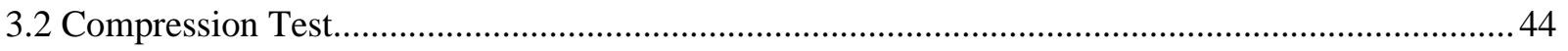

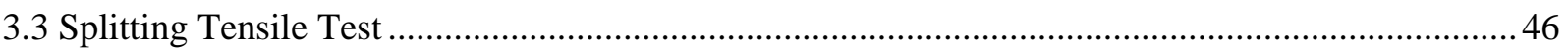

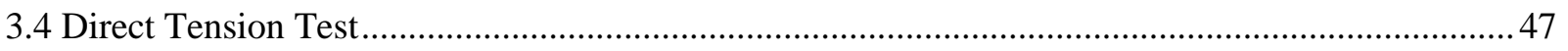

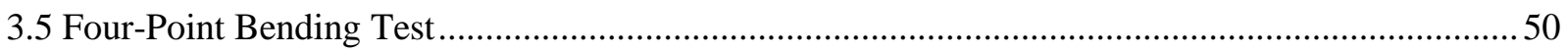

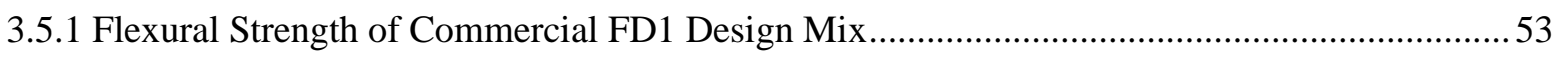

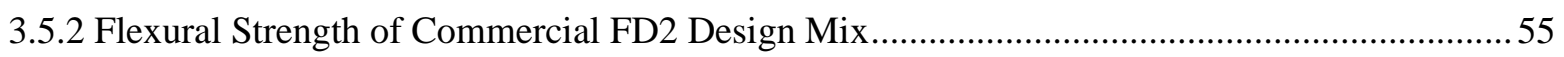

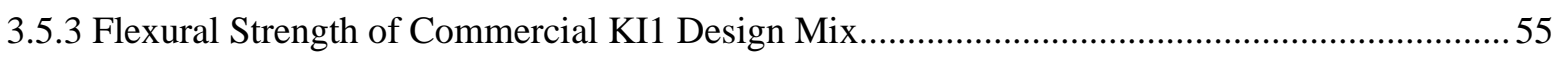

3.5.4 Flexural Strength of Commercial LD1 Design Mix ............................................................56

3.5.5 Flexural Strength of In-House DE1 and DE2 Design Mixes ................................................57

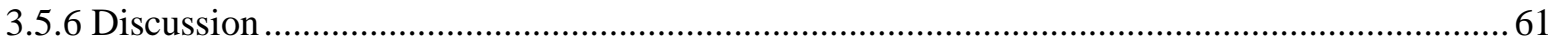

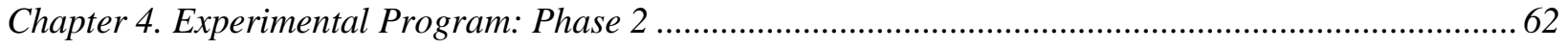

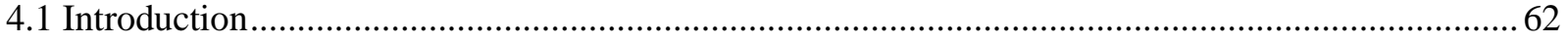

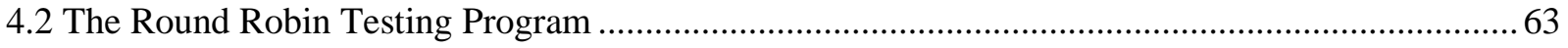

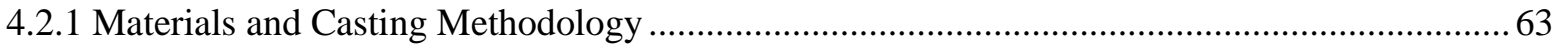

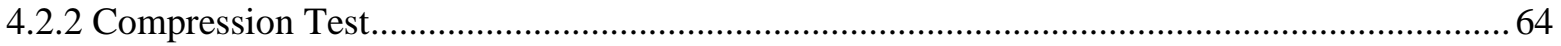

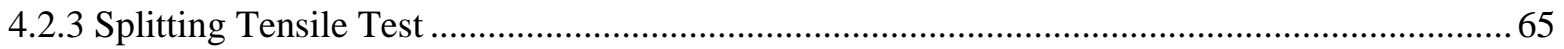

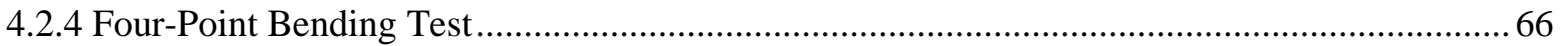

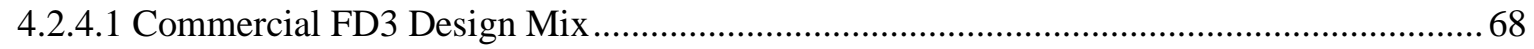

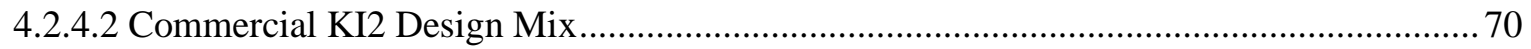




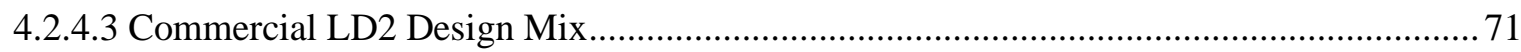

4.2.4.4 Discussion of the Round Robin Testing Program Results ............................................... 71

4.2.4.5 Investigation into the Effect of Support Condition ......................................................... 73

4.3 Experimental Results of Commercial FD4, FD5 and FD6 Design Mixes ....................................... 77

4.3.1 Materials and Casting Methodology .................................................................................... 77

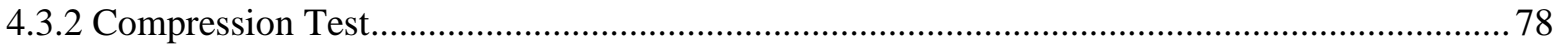

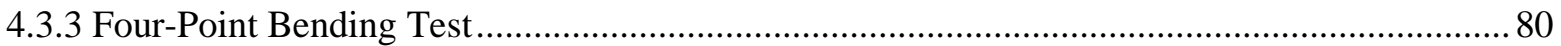

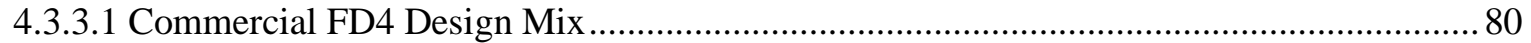

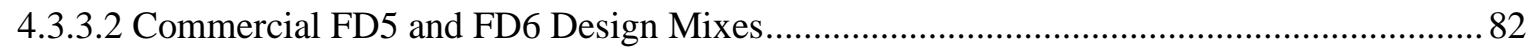

Chapter 5. Non-Destructive Tests and Durability Evaluation ................................................................ 85

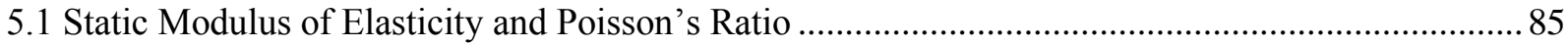

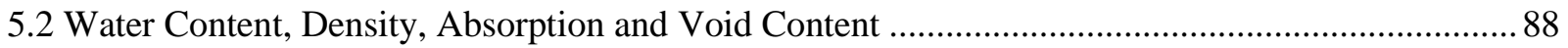

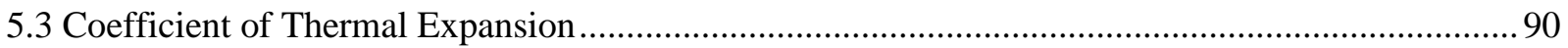

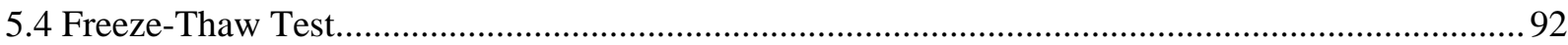

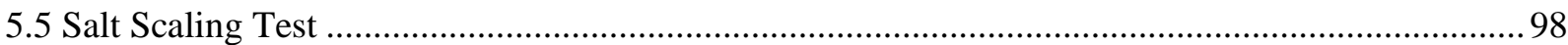

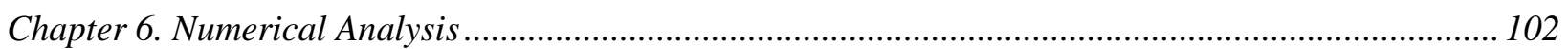

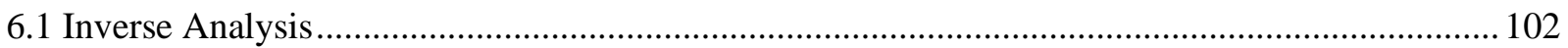

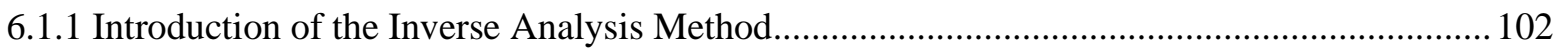

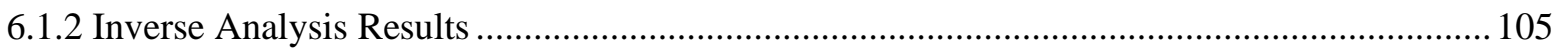

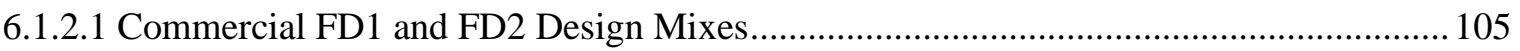

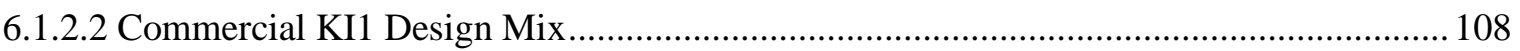

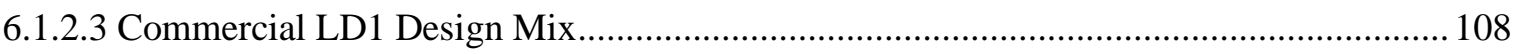

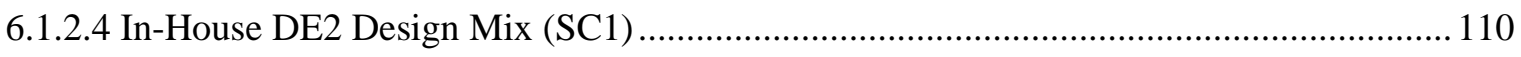

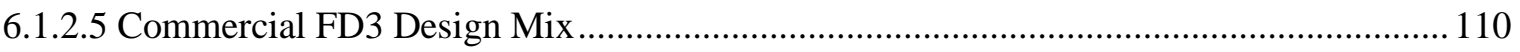

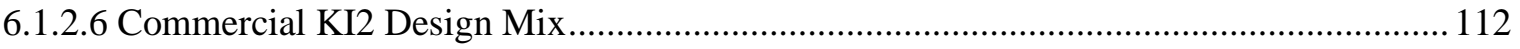

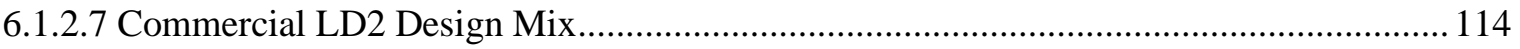

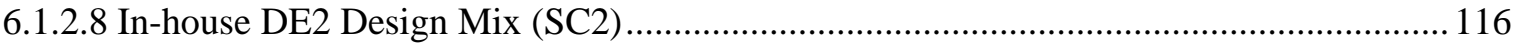

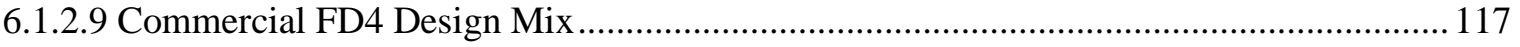

6.1.2.10 Commercial FD5 and FD6 Design Mix ….................................................................. 118

6.1.3 Discussion of the Inverse Analysis Results ......................................................................... 119

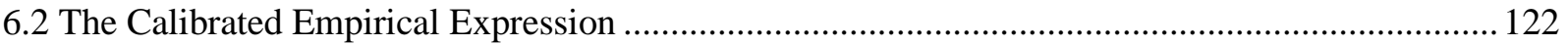

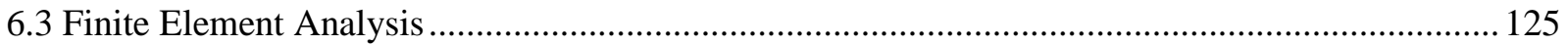

6.3.1 Application of the Finite Element Method in Inverse Analysis ............................................ 125

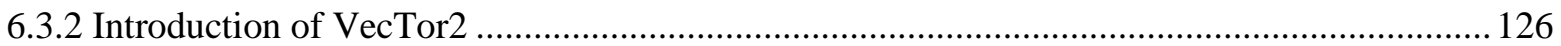




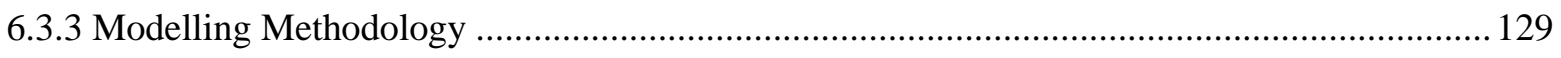

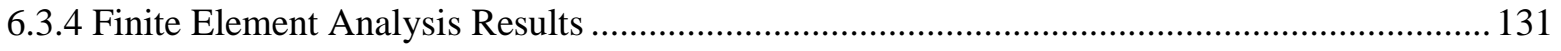

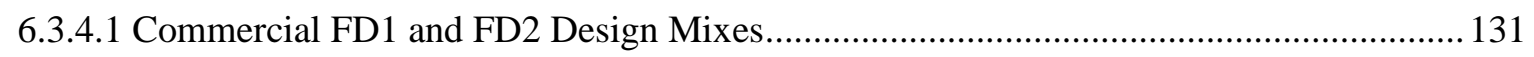

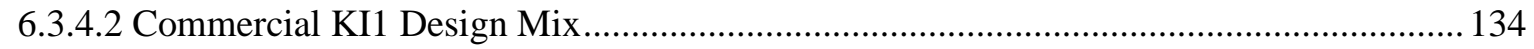

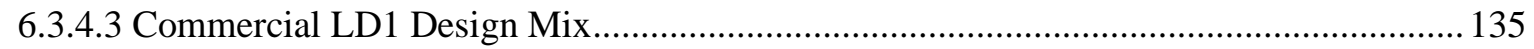

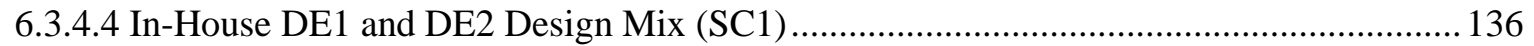

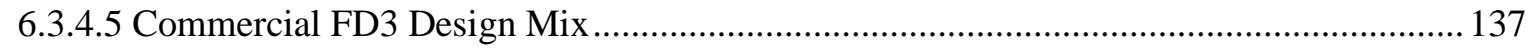

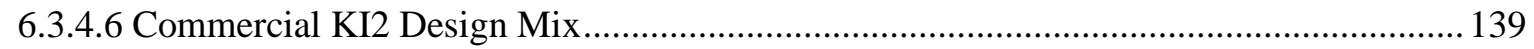

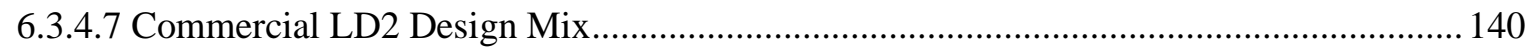

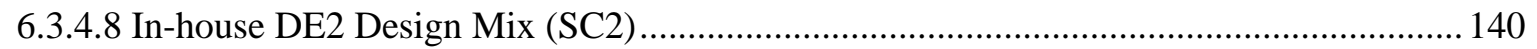

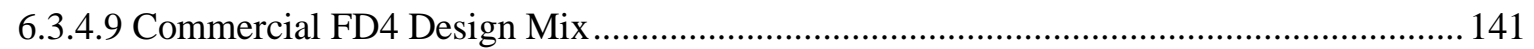

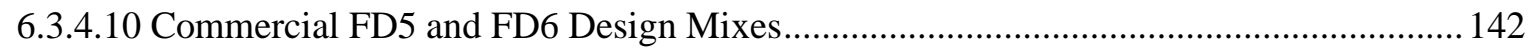

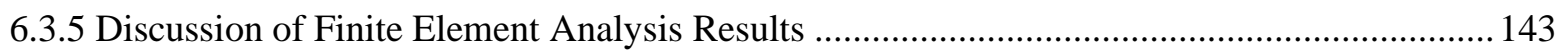

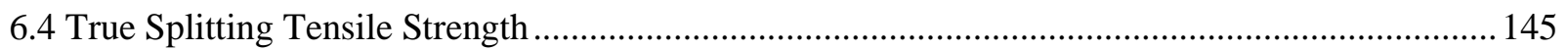

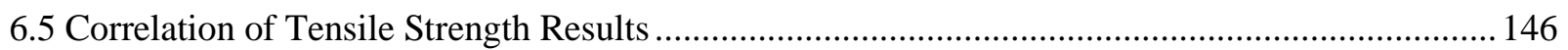

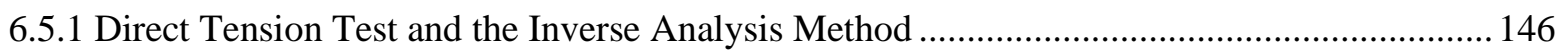

6.5.2 Direct Tension Test and the Calibrated Empirical Expression ............................................ 147

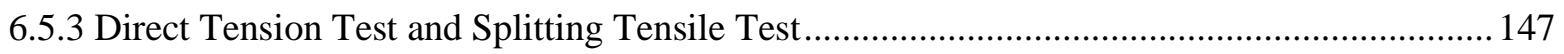

6.5.4 Inverse Analysis Method and the Calibrated Empirical Expression ....................................... 148

6.5.5 Inverse Analysis Method and Splitting Tensile Test ............................................................ 149

6.5.6 Inverse Analysis and Finite Element Analysis................................................................ 149

6.5.7 Finite Element Analysis and the Calibrated Empirical Expression ..................................... 151

6.5.8 Finite Element Analysis and Direct Tension Test.............................................................. 151

6.5.9 Finite Element Analysis and Splitting Tensile Test ............................................................ 152

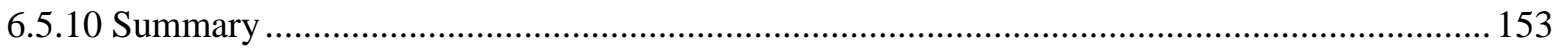

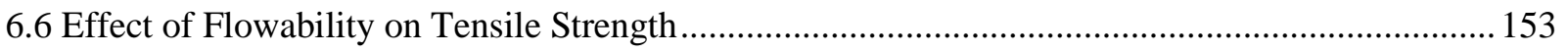

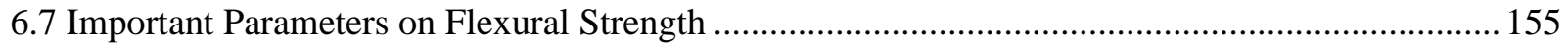

6.7.1 Aspect Ratio of the Bending Prism................................................................................. 155

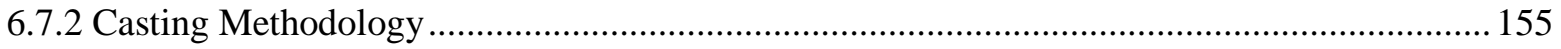

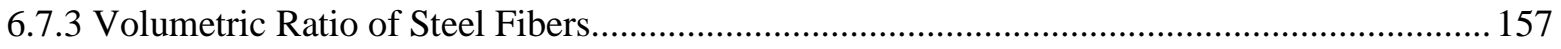

6.7.4 Degree of Preferential Fiber Alignment ............................................................................. 158

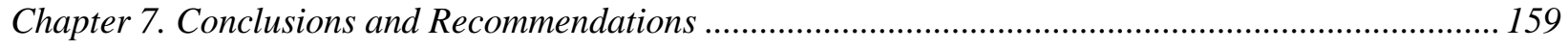

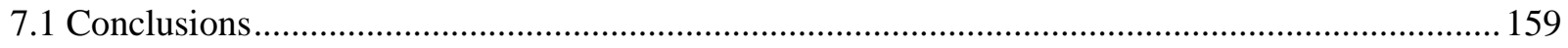

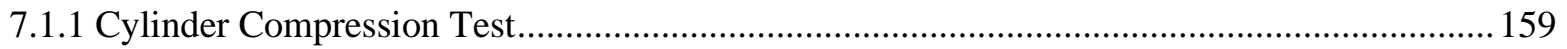


7.1.2 Splitting Tensile Test ………………………………………………………………. 160

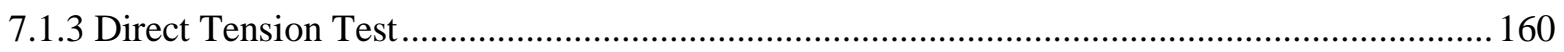

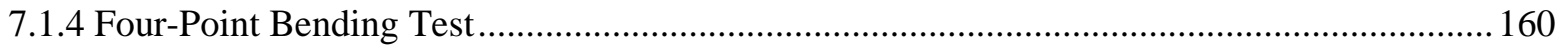

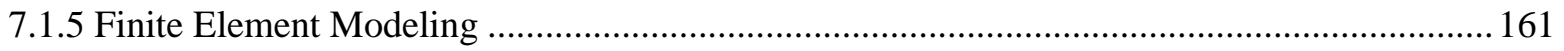

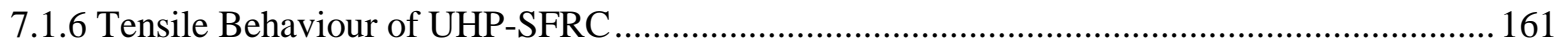

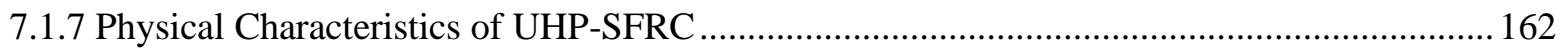

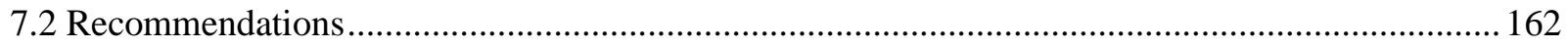

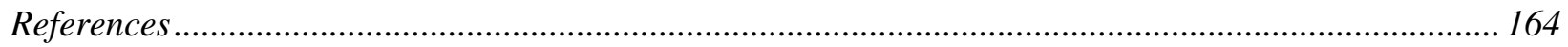

Appendix A. Resistance Curves of Four-Point Bending Tests ............................................................ 176

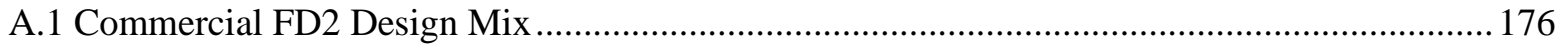

A.2 Commercial KI1 Design Mix ........................................................................................ 177

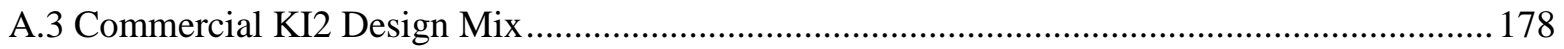

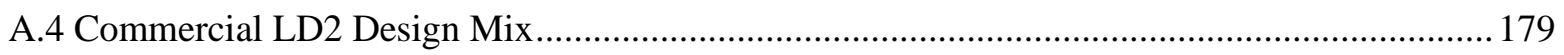

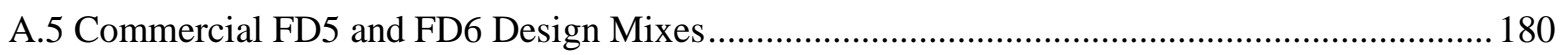

Appendix B. Inverse Analysis (The Four Characteristic Points)........................................................ 181

B.1 Commercial FD1 and FD2 Design Mixes ...................................................................... 181

B.2 Commercial KI1 Design Mix ........................................................................................ 181

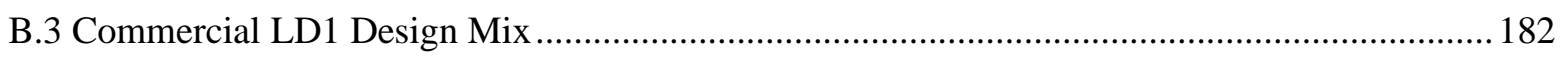

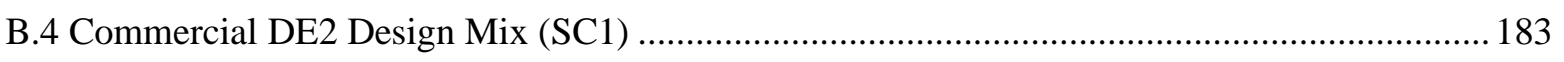

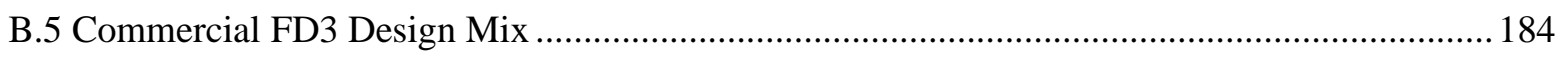

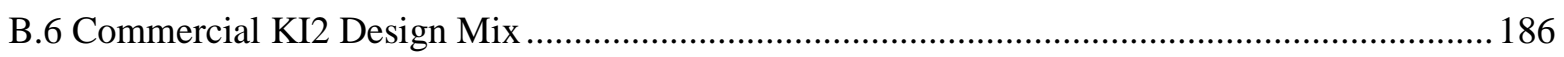

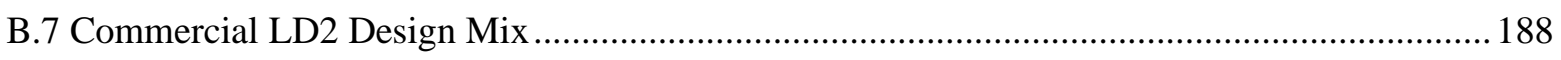

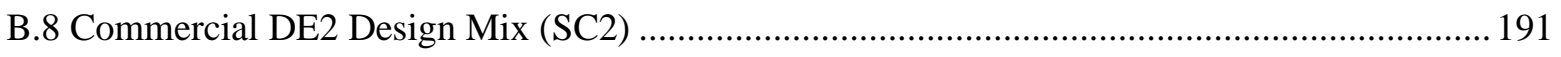

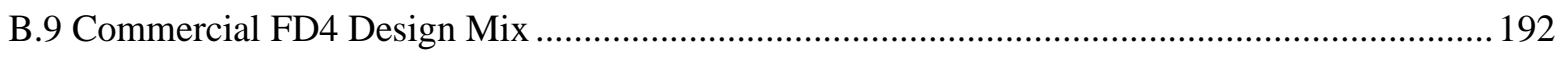

B.10 Commercial FD5 and FD6 Design Mixes ....................................................................... 192

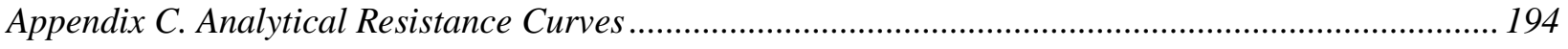

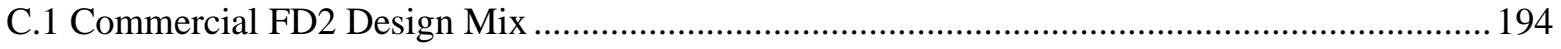

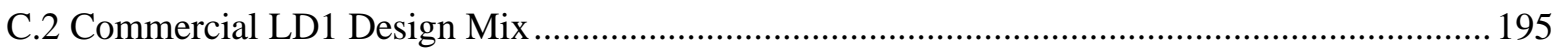

C.3 Commercial DE2 Design Mix (SC1) ……………………………………………….... 195

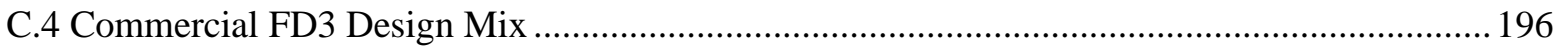

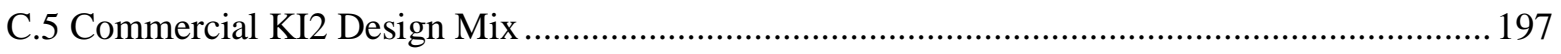

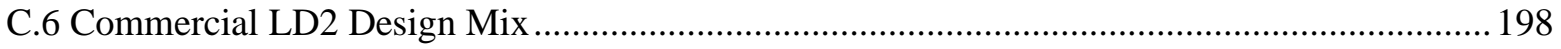

C.7 Commercial FD1 and FD2 Design Mixes ............................................................................ 199 


\section{List of Tables}

Table 2- 1: Proposed fiber length and prism size of four-point bending test (Annex U of CSA-A23.1 2019)

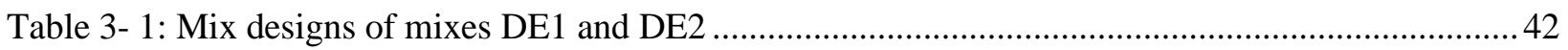

Table 3- 2: Compressive strength results (Phase 1 of the experimental program) ...................................45

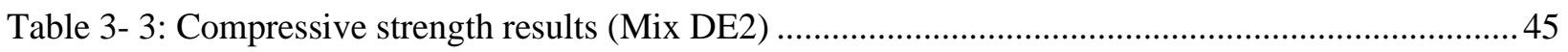

Table 3- 4: Splitting tensile strength results (Phase 1 of the experimental program) ............................... 46

Table 3- 5: Splitting tensile strength results (Mix DE2) ….................................................................... 47

Table 3- 6: Tensile strength results obtained from direct tension tests ................................................. 49

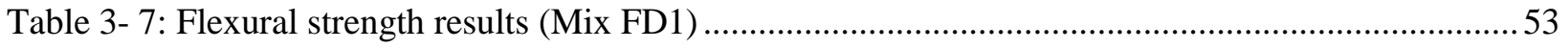

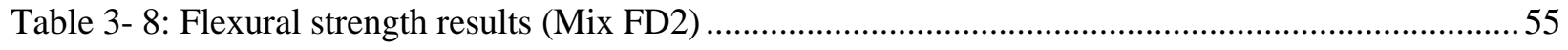

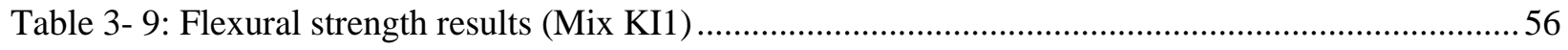

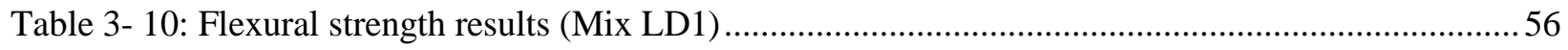

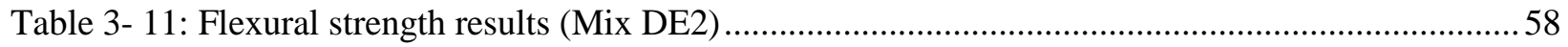

Table 3- 12: Flexural strength results of the prisms from mix DE1 .....................................................58

Table 3- 13: Flexural strength results of the thin prisms from mix DE2 ................................................60

Table 4- 1: Specified prism size in terms of fiber length (ASTM C1856 2017).....................................64

Table 4- 2: Compressive strength results (The Round Robin Testing Program) ......................................65

Table 4- 3: Splitting tensile strength results (Mixes FD3, KI2 and LD2) ..............................................65

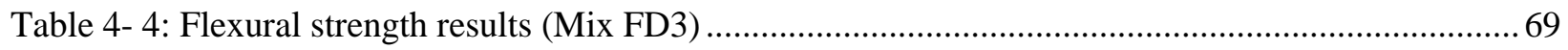

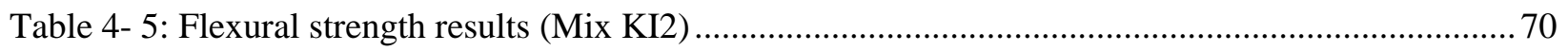

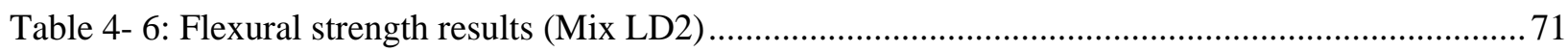

Table 4- 7: Flexural strength results of the prisms from DE2 tested with SC1 and SC2 respectively ....... 74

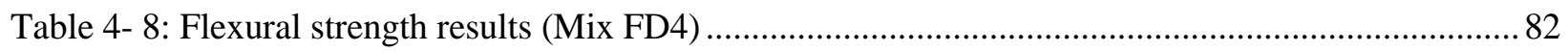

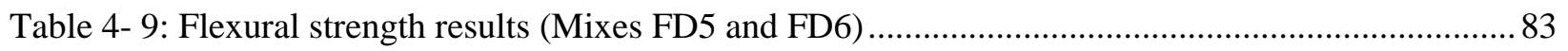

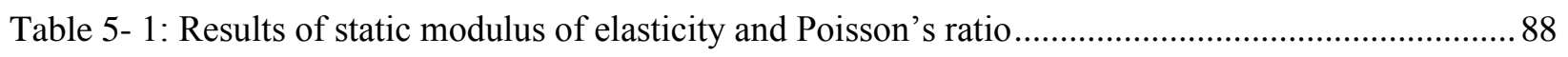

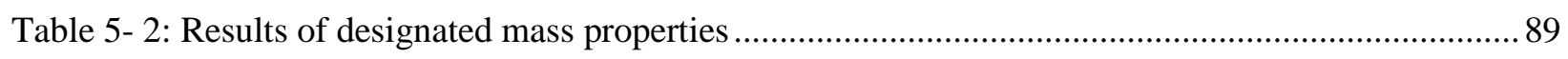

Table 5- 3: Results of water content, density, absorption and void content ...........................................90

Table 5- 4:Coefficient of thermal expansion results obtained by using DEMEC mechanical strain gauge92

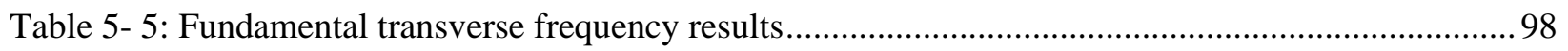


Table 5- 6: Results of salt scaling test.. 101

Table 6- 1: Derived expressions of the inverse analysis method (Annex 8.1 of CSA-S6 2018) .............. 103

Table 6- 2: Inverse analysis results of the short prisms from mix FD1 ................................................. 106

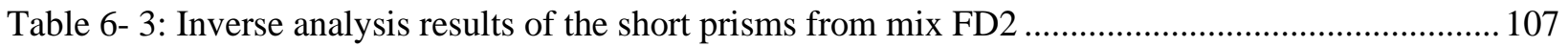

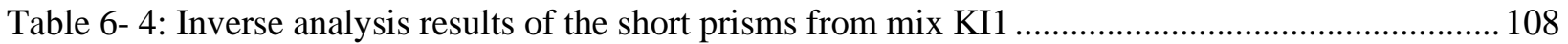

Table 6- 5: Inverse analysis results of the short prisms from mix LD1 ............................................... 109

Table 6- 6: Inverse analysis results of the short prisms from mix DE2 tested with SC1 ....................... 110

Table 6- 7: Inverse analysis results of the prisms from mix FD3 ….................................................... 111

Table 6- 8: Inverse analysis results of the prisms from mix KI2 ….................................................... 113

Table 6- 9: Inverse analysis results of the prisms from mix LD2 ...................................................... 115

Table 6- 10: Inverse analysis results of the prisms from mix DE2 tested with SC2 ….......................... 116

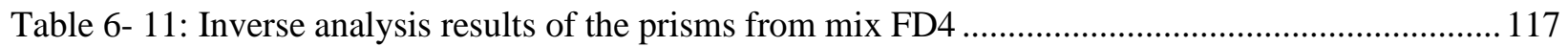

Table 6- 12: Inverse analysis results of the prisms from mixes FD5 and FD6 ..................................... 119

Table 6- 13: Cracking strength results calculated with the calibrated empirical expression .................... 124

Table 6- 14: Selected models in VecTor2 used in the present simulations............................................ 131

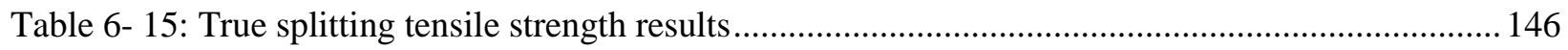

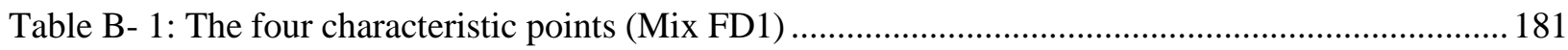

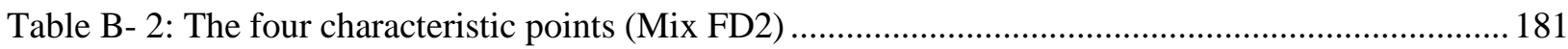

Table B- 3: The four characteristic points utilized for the inverse analysis of the short prisms from mix

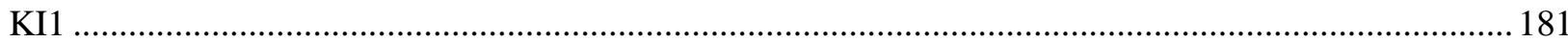

Table B- 4: The four characteristic points utilized for the inverse analysis of the short prisms from mix

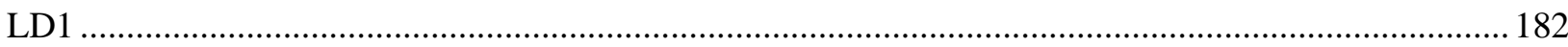

Table B- 5: The four characteristic points utilized for the inverse analysis of the short prisms from mix

DE2

Table B- 6: The four characteristic points utilized for the inverse analysis of the prisms from mix FD3 tested by York University

Table B- 7: The four characteristic points utilized for the inverse analysis of the prisms from mix FD3 tested by École Polytechnique-Montréal

Table B- 8: The four characteristic points utilized for the inverse analysis of the prisms from mix FD3 tested by Queen's University 184

Table B- 9: The four characteristic points utilized for the inverse analysis of the prisms from mix KI2 tested by York University 186 
Table B- 10: The four characteristic points utilized for the inverse analysis of the prisms from mix KI2 tested by École Polytechnique-Montréal

Table B- 11: The four characteristic points utilized for the inverse analysis of the prisms from mix KI2

tested by Queen's University.

Table B- 12: The four characteristic points utilized for the inverse analysis of the prisms from mix LD2

tested by York University

Table B- 13: The four characteristic points utilized for the inverse analysis of the prisms from mix LD2

tested by École Polytechnique-Montréal

Table B- 14: The four characteristic points utilized for the inverse analysis of the prisms from mix LD2

tested by Queen's University.

Table B- 15: The four characteristic points utilized for the inverse analysis of the OW prisms from mix

DE2 tested with SC2...

Table B- 16: The four characteristic points utilized for the inverse analysis of the prisms from mix FD4

Table B- 17: The four characteristic points utilized for the inverse analysis of the prisms from mix FD5 and FD6. 


\section{List of Figures}

Figure 1- 1: The cumulative number of bridges constructed with UHPFRC since 2006 (FHWA-HRT-18$0362018)$

Figure 1- 2: Field-cast UHPFRC connections: (a) The casting of UHPFRC connections; and (b) The Pulaski Skyway Bridge constructed with UHPFRC connections (Rahman and McQuaker 2016) ..............5

Figure 1- 3: MuCEM in Marseille (Ricciotti 2013) ............................................................................... 5

Figure 2- 1: Relationship between water-to-binder ratio and compressive strength (Sohail et al. 2018) ... 16

Figure 2- 2: Types of steel fibers: (a) Straight smooth; (b) Hooked; and (c) Twisted (Tai et al. 2016) ..... 17

Figure 2- 3: Brass-coated steel fibers: (a) Non-abraded fiber; (b) Partially abraded fiber; and (c). The

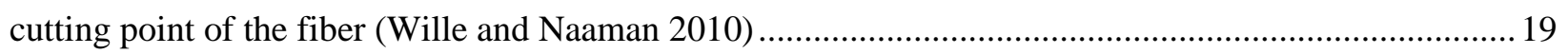

Figure 2- 4: General classification of FRC (Naaman and Reinhardt 2006) ............................................25

Figure 2- 5: UHPFRC classification proposed by Wille et al. (2014): (a) Level 3; and (b) Level 4 ..........26

Figure 2- 6: Idealized uniaxial tensile mechanical response of UHP-SFRC proposed by Russel and

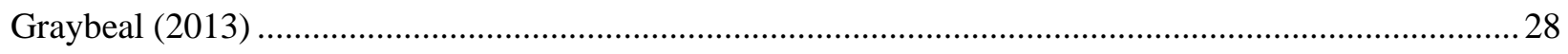

Figure 2- 7: Typical uniaxial tensile response of UHPFRC proposed by Wille et al. (2014)...................29

Figure 2- 8: Dog-bone shaped specimen proposed in the Swiss standard for direct tension test (SIA2052

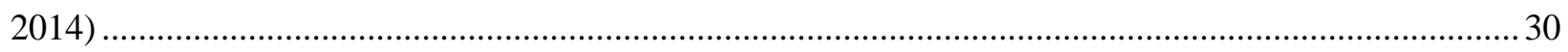

Figure 2- 9: Unnotched prism proposed by Graybeal and Baby (2013) for direct tension test ................. 30

Figure 2- 10: Direct tension test setup proposed by Chasioti and Vecchio (2017): (a) Specimen shape; and

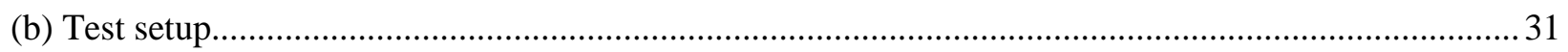

Figure 2- 11: Direct tension test setup: (a) Dog-bone shaped specimen proposed in AASHTO T132-87

(2018); and (b) The notched prism (Kusumawardaningsih et al. 2015) ..................................................31

Figure 2- 12: Multiple micro-cracks of a UHPFRC specimen observed from a notched three-point

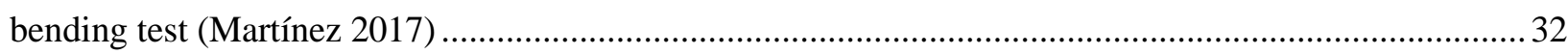

Figure 2- 13: Bending test setup of UHPFRC proposed in the French standard (NF P18-470 2016): (a)

Strain-softening material; and (b) Strain-hardening material ............................................................. 34

Figure 2- 14: Four-point bending test setup of UHPFRC proposed in the Swiss standard (SIA2052 2014)

Figure 2- 15: Four-point bending test setup of UHPFRC proposed in the Canadian standard (Annex U of

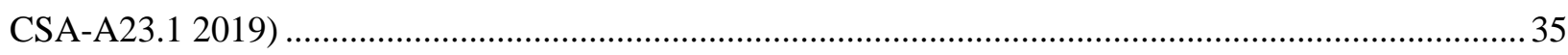

Figure 2- 16: Experimental test setup of the unnotched four-point bending test proposed by Kanakubo (2006). 
Figure 2-17: Stress distribution considered for the inverse analysis method proposed by Kanakubo (2006)

Figure 2-18: Simplified inverse analysis method proposed in the French standard (NF P18-470 2016): (a)

Bilinear stress-strain relationship of strain-hardening UHPFRC; and (b) Linearity limit and elastic

modulus determined from the moment-deflection curve .38

Figure 2- 19: Stress distribution considered for the inverse analysis method proposed in the French standard (NF P18-470 2016) 39

Figure 2- 20: Stress distribution proposed in the Swiss standard (SIA2052 2014) .39

Figure 3- 1: Steel fibers utilized in mixes FD1 and FD2: (a) Straight steel fibers; and (b) Hooked-end steel fibers

Figure 3- 2: Concrete mixing of mixes DE1 and DE2: (a) Mixing station (b) Dry mix (c) Mix state after adding all the water; and (d) Addition of steel fibers .42

Figure 3- 3: Flow test 42

Figure 3- 4: Molds prepared for prisms, cylinders and direct-tension specimens .43

Figure 3- 5: Experimental test setup of cylinder compression test .44

Figure 3- 6: Failure mode of cylinder compression test .45

Figure 3- 7: Splitting tensile test: (a) Experimental test setup; and (b) Failure mode .46

Figure 3- 8: Nominal dimensions of direct- tension specimens: from left to right: Type G specimen (FHWA 2013); Type A specimen (Georgiou and Pantazopoulou 2016); and Type S specimen (SIA2052 2014)

Figure 3- 9: Experimental test setup of direct tension test: (a) Type G and Type S specimens (sideview); and (b) Type A specimens .48

Figure 3-10: Failure planes of direct-tension specimens: (a) Desired failure planes, top to bottom: Type S, Type $\mathrm{G}$ and Type A specimens; and (b) Challenges with direct tension test, left to right: crack near CFRP strips, bending phenomenon, stress concentration due to the tolerance in geometry …............................50

Figure 3- 11: Schematic of the experimental test setup of four-point bending test .................................51

Figure 3-12: Experimental test setup of four-point bending test: (a) Support rollers, (b) Loading rollers,

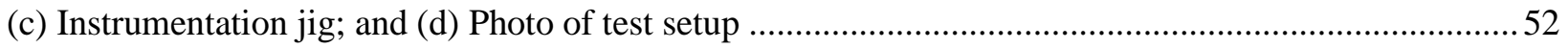

Figure 3- 13: Resistance curves of the prisms from mix FD1 ..........................................................5

Figure 3- 14: Failure mode of four-point bending test: (a) Short prisms; and (b) Long prisms .................54

Figure 3- 15: Resistance curves of the prisms from mix LD1 ..............................................................57

Figure 3- 16: Resistance curves: (a) Prisms from mix DE2; and (b) Prisms from mix DE1 .....................59 
Figure 3- 17: Critical failure planes of four-point bending tests: (a) Prisms containing 1\% steel fibers; and

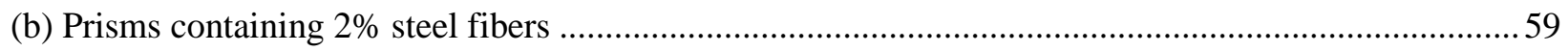

Figure 3- 18: Resistance curves of the thin prisms from mix DE2 ..................................................... 60

Figure 3-19: Failure modes of the thin prisms subjected to four-point loading: (a) Flexural failure; and (b)

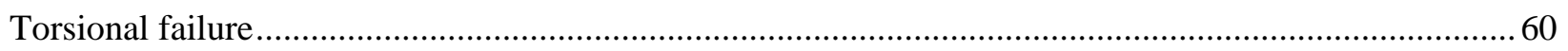

Figure 3- 20: Average flexural strength results (Phase 1 of the experimental program) ..........................61 61

Figure 4- 1: Sealed pails containing prisms and cylinders for each university........................................63

Figure 4- 2: Prepared molds for the prismatic and cylindrical specimens for the Round Robin Testing

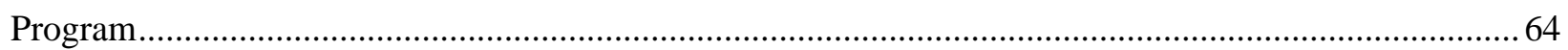

Figure 4- 3: Concrete grinding for compression test ...................................................................... 65

Figure 4- 4: Stepwise loading rate proposed by École Polytechnique-Montréal (ASTM C1609 2012).....67

Figure 4- 5: Support and loading rollers of four-point bending test: (a) York University's support rollers; (b) York University's loading rollers; (c) Queen's University's support and loading rollers; and (d) École Polytechnique-Montréal's support and loading rollers (Annex U of CSA-A23.1 2019).

Figure 4- 6: Failure Modes of four-point bending tests: (a) Flexural failure; (b) Torsional failure; and (c) Shear failure. .68

Figure 4- 7: Resistance curves of the prisms from mix FD3 (York University)...................................... 69

Figure 4- 8: Resistance curves of the prisms from mix FD3 (École Polytechnique-Montréal)..................69

Figure 4- 9: Resistance curves of the prisms from mix FD3 (Queen's University) ...................................70

Figure 4- 10: Average flexural strength results (the Round Robin Testing Program) ............................. 72

Figure 4- 11: Support conditions of four-point bending tests: (a) SC1; and (b) SC2 ….........................73

Figure 4- 12: Resistance curves of the prisms from mix DE2 tested with SC1 and SC2 respectively ....... 74

Figure 4- 13: Comparison of the horizontal force: (a) SC1; and (b) SC2 ............................................. 75

Figure 4- 14: Comparison of the frictional force: (a) SC1; and (b) SC2 .............................................76

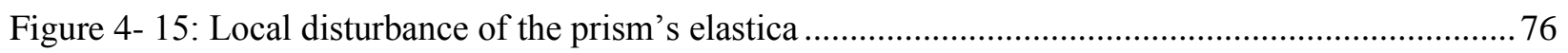

Figure 4- 16: Prepared molds for prismatic and cylindrical specimens (Mixes FD4, FD5 and FD6) ........78

Figure 4- 17: Imperfections of the cylinders from mix FD4: (a) Bubbles on the top surface; (b) Peeling

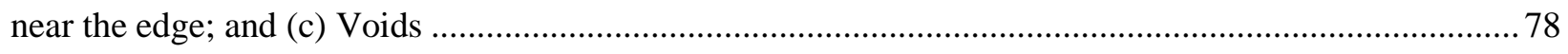

Figure 4- 18: Cylinders from mix FD4 capped with mortar and epoxy paste, and wrapped with CFRP

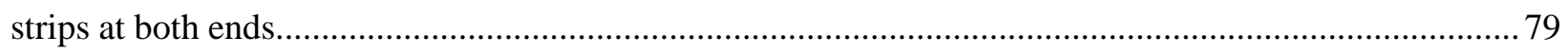

Figure 4- 19: Local failure near the junction of the two ends of CFRP strip ........................................ 79

Figure 4- 20: Critical failure planes of the prisms from mix FD4: (a) $100 \mathrm{~mm}$ by $100 \mathrm{~mm}$ prisms; and (b) $150 \mathrm{~mm}$ by $150 \mathrm{~mm}$ prisms 80 
Figure 4- 21: Fiber segregation observed from the prisms from mix FD4 ........................................... 81

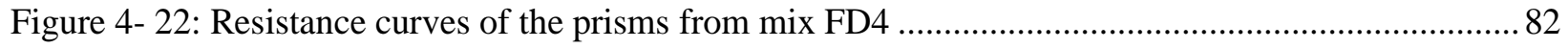

Figure 4- 23: Average flexural strength results of mixes FD4, FD5 and FD6........................................ 84

Figure 5- 1: Experimental test setup to determine static modulus of elasticity and Poisson's ratio: (a) Foil

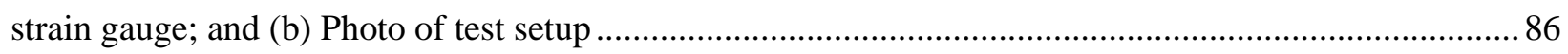

Figure 5- 2: Response curve of static modulus of elasticity and Poisson's ratio test ................................ 86

Figure 5- 3: DEMEC strain gauge and accessories: (a) Reference bar and gauge points; and (b) Strain

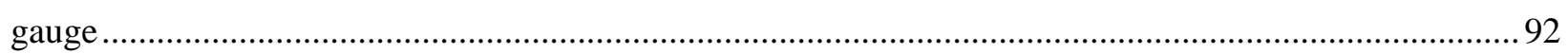

Figure 5- 4: Experimental test setup of freeze-thaw test: (a) ThermoStream System; and (b) Modified

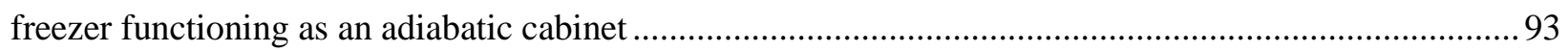

Figure 5- 5: Temperature variations of a freeze-thaw cycle ................................................................ 94

Figure 5- 6: (a) Containers; and (b) The control specimen .................................................................94

Figure 5- 7: Experimental test setup of fundamental transverse frequency test: (a) Impactor, sponge supports, accelerator and double-sided adhesive tape; (b) Data amplifier and CatmanEasy data acquisition software; and (c) Locations of the impact point, accelerator, and support points.....................................95

Figure 5- 8: Response curve of a fundamental transverse frequency test ..............................................96

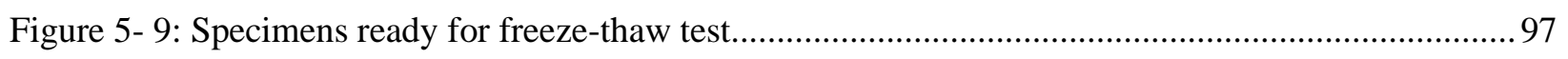

Figure 5- 10: The textured roller and the slightly textured test surface .................................................99

Figure 5- 11: The dyke of a salt-scaling specimen: (a) Schematic of the dyke (sideview); and (b) Photo of

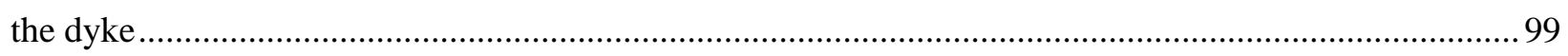

Figure 5- 12: Temperature probes and temperature data recorder ..................................................... 100

Figure 5-13:Test surfaces at the beginning of the thawing period ....................................................... 101

Figure 5-14: (a) The sieve utilized for collecting scaling residue; and (b) Water loss measurement to

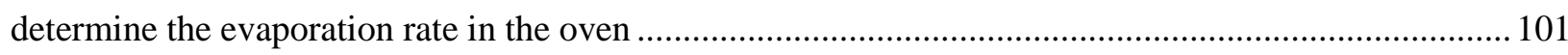

Figure 6- 1: Characteristic points on the resistance curve of four-point bending test (Annex 8.1 of CSA-S6 2018)

Figure 6- 2: Horizontal distance between the location of crack tip to the midspan of the prism (from both front and back faces of the prism). 104

Figure 6- 3: Linearized tensile stress-strain-crack mouth opening relationships (Annex 8.1 of CSA-S6 2018) 104

Figure 6- 4: Linearized tensile stress-strain-crack mouth opening relationships of the short prisms from mix FD1 106 
Figure 6- 5: Linearized tensile stress-strain-crack mouth opening relationships of the short prisms from mix FD2

Figure 6- 6: Linearized tensile stress-strain-crack mouth opening relationships of the short prisms from $\operatorname{mix} \mathrm{LD} 1$

Figure 6- 7: Linearized tensile stress-strain-crack mouth opening relationships of the prisms from mix

FD3 tested by York University

Figure 6- 8: Linearized tensile stress-strain-crack mouth opening relationships of the prisms from mix KI2 tested by York University

Figure 6- 9: Linearized tensile stress-strain-crack mouth opening relationships of the prisms from mix DE2 tested with SC2.

Figure 6-10: Linearized tensile stress-strain-crack mouth opening relationships of the prisms from mix FD4

Figure 6- 11: Average ultimate tensile strength and ultimate tensile strain results obtained from the inverse analysis (Mixes FD1, FD2, KI1 and LD1)

Figure 6-12:Average cracking strength and ultimate tensile strength results obtained from the inverse analysis (Mixes FD3, KI2 and LD2).

Figure 6-13:Average cracking strength and ultimate tensile strength results obtained from the inverse analysis (Mix DE2).

Figure 6-14: Average cracking strength and ultimate tensile strength results obtained from the inverse analysis (Mixes FD4, FD5 and FD6).

Figure 6- 15: Criteria of THFRC classification based on the inverse analysis results: (a) Average cracking strength; (b) Average ultimate tensile strain; and (c) Hardening ratio.

Figure 6- 16: Average tensile strength obtained from direct tension test and cracking strength results calculated with the calibrated empirical expression.

Figure 6- 17: Reinforced concrete element subjects to in-plane stresses (Vecchio and Collins 1986)..... 127 Figure 6- 18: Tensile stress distribution of the cracked reinforced concrete (Lin 2010): (a) Uniaxial tensile loading; (b) Stress distribution of the reinforcing bar; and (c) Stress distribution of concrete. 127

Figure 6- 19: Longitudinal stress distribution along the span of the prism (Lin 2010) 128

Figure 6- 20: (a) Average strain in concrete and reinforcement; and (b) Deformation due to crack shear slip (Vecchio et al. 2013)..... 128

Figure 6- 21: Finite element models: (a) $75 \mathrm{~mm}$ by $75 \mathrm{~mm}$ by $280 \mathrm{~mm}$ prisms; (b) $100 \mathrm{~mm}$ by $100 \mathrm{~mm}$ by $370 \mathrm{~mm}$ prisms; (c) $75 \mathrm{~mm}$ by $75 \mathrm{~mm}$ by $500 \mathrm{~mm}$ prisms; and (d) $150 \mathrm{~mm}$ by $150 \mathrm{~mm}$ by $500 \mathrm{~mm}$ prisms 130

Figure 6- 22: Finite element analysis results of one-way prisms from mix FD1 132 
Figure 6- 23: Finite element analysis results of random prisms from mix FD1 133

Figure 6- 24: Finite element analysis results of the prisms from mix FD2. 133

Figure 6- 25: Finite element analysis results of one-way prisms from mix KI1 134

Figure 6- 26: Finite element analysis results of random prisms from mix KI1 135

Figure 6- 27: Finite element analysis results of the prisms from mix LD1 ... 136

Figure 6- 28: Finite element analysis results of the prisms from mixes DE1 and DE2 137

Figure 6- 29: Finite element analysis results: (a) Resistance curves (YU); and (b) Tensile stress-strain relationship. 138

Figure 6- 30: Finite element analysis results of the prisms from mix KI2. 139

Figure 6- 31: Finite element analysis results of the prisms from mix LD2 140

Figure 6- 32: Finite element analysis results of the prisms from mix DE2 141

Figure 6- 33: Analytical response curves of the prisms from mix FD4. 141

Figure 6- 34: Tensile stress-strain relationship of the prisms from mix FD4 142

Figure 6- 35: Tensile stress-strain relationship of the prisms from mixes FD5 and FD6..... 142

Figure 6- 36: Classification criteria of THFRC based on finite element analysis results: (a) Cracking strength; (b) Ultimate tensile strain; and (c) Hardening ratio 144

Figure 6- 37: Ultimate tensile strength results obtained from finite element analysis 145

Figure 6- 38: Biaxial stress state developed in the cylinder under diametrically compressive loading (Carmona and Aguado 2012) 145

Figure 6- 39: Relationship between direct tension test and inverse analysis 146

Figure 6- 40: Relationship between direct tension test and the calibrated empirical expression...... 147

Figure 6- 41: Relationship between direct tension test and splitting tensile test 148

Figure 6- 42: Relationship between the inverse analysis method and the calibrated empirical expression 148

Figure 6- 43: Relationship between the inverse analysis method and splitting tensile test 149

Figure 6- 44: Relationship between inverse analysis and finite element analysis: (a) Cracking strength; and (b) Ultimate tensile strength. 150

Figure 6- 45: Relationship between finite element analysis and the calibrated empirical expression ....... 151

Figure 6- 46: Relationship between finite element analysis and direct tension test............................... 152

Figure 6- 47: Relationship between finite element analysis and splitting tensile test. 152

Figure 6- 48: Correlation of tensile strength results through direct tension test, the inverse analysis method, splitting tensile test, finite element analysis and the calibrated empirical expression 153 
Figure 6- 49: Effect of flowability on tensile strength: (a) Cracking strength results of inverse analysis against spread values; and (b) Ultimate tensile strength results of inverse analysis against spread values

Figure 6- 50: Effect of aspect ratio of the bending prism on flexural strength ..... 155

Figure 6- 51: Effect of casting methodology on flexural strength 156

Figure 6- 52: The number of steel fibers present near the tension face of the prism: (a) Cutting plane and the area of interest; and (b) Relationship between the number of steel fibers present near the tension face of the prism and average flexural strength 157

Figure 6- 53: Effect of volumetric ratio of steel fibers on flexural strength .... 158

Figure 6- 54: Effect of degree of preferential fiber alignment on flexural strength. 158

Figure A- 1: Resistance curves of the prisms from mix FD2 176

Figure A-2: Resistance curves of the prisms from mix KI1 177

Figure A- 3: Resistance curves of the prisms from mix KI2 (York University) 178

Figure A- 4: Resistance curves of the prisms from mix KI2 (École Polytechnique-Montréal)................ 178

Figure A- 5: Resistance curves of the prisms from mix KI2 (Queen's University) ................................. 178

Figure A- 6: Resistance curves of the prisms from mix LD2 (York University).................................... 179

Figure A- 7: Resistance curves of the prisms from mix LD2 (École Polytechnique-Montréal)............... 179

Figure A- 8: Resistance curves of the prisms from mix LD2 (Queen's University) ............................... 179

Figure A- 9: Resistance curves of the prisms from mixes FD5 and FD6 tested at the age of 21 days ..... 180

Figure A- 10: Resistance curves of the prisms from mixes FD5 and FD6 tested at the age of 28 days ... 180

Figure B- 1: Linearized tensile stress-strain-crack mouth opening relationships of the short prisms from mix KI1

Figure B- 2: Linearized tensile stress-strain-crack mouth opening relationships of the short prisms from mix DE2 tested with SC1.....

Figure B- 3: Linearized tensile stress-strain-crack mouth opening relationships of the prisms from mix

FD3 tested by École Polytechnique-Montréal .

Figure B- 4: Linearized tensile stress-strain-crack mouth opening relationships of the prisms from mix FD3 tested by Queen's University...... 186

Figure B- 5: Linearized tensile stress-strain-crack mouth opening relationships of the prisms from mix KI2 tested by École Polytechnique-Montréal 187

Figure B- 6: Linearized tensile stress-strain-crack mouth opening relationships of the prisms from mix KI2 tested by Queen's University 188 
Figure B- 7: Linearized tensile stress-strain-crack mouth opening relationships of the prisms from mix LD2 tested by York University.

Figure B- 8: Linearized tensile stress-strain-crack mouth opening relationships of the prisms from mix LD2 tested by École Polytechnique-Montréal.

Figure B- 9: Linearized tensile stress-strain-crack mouth opening relationships of the prisms from mix LD2 tested by Queen's University 191

Figure B- 10: Linearized tensile stress-strain-crack mouth opening relationships of the prisms from mixes FD5 and FD6 193

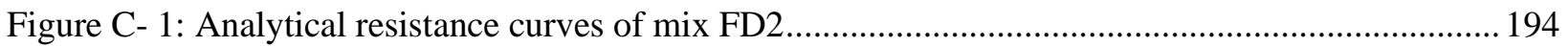

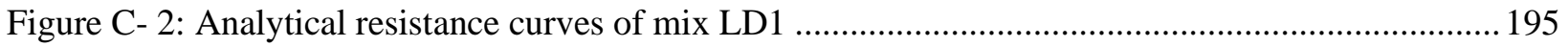

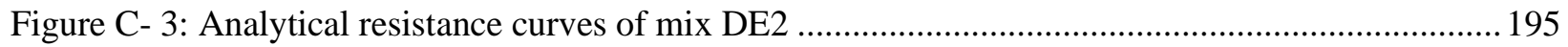

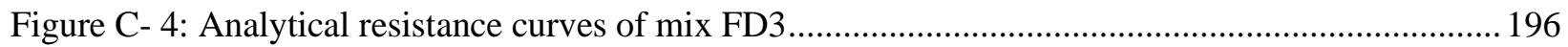

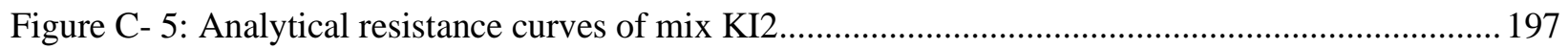

Figure C- 6: Analytical resistance curves of mix LD2 _.................................................................... 198

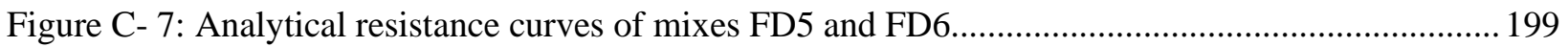




\section{List of Abbreviations}

\begin{tabular}{|c|c|}
\hline ASR & Alkali-Silica Reaction \\
\hline CFRP & Carbon Fiber Reinforced Polymer \\
\hline CTE & Coefficient of Thermal Expansion \\
\hline DSFM & Disturbed Stress Field Model \\
\hline DSP & Densified Small Particles-Based Concrete \\
\hline DTT & Direct Tension Test \\
\hline ECC & Engineered Cementitious Composites \\
\hline FA & Fly Ash \\
\hline FPBT & Four-Point Bending Test \\
\hline FRC & Fiber-Reinforced Concrete \\
\hline GGBS & Ground Granulated Blast Furnace Slag \\
\hline HPC & High-Performance Concrete \\
\hline ITZ & Interfacial Transition Zone \\
\hline MCFT & Modified Compression Field Theory \\
\hline MDF & Macro-Defect-Free Cement \\
\hline $\mathrm{OPC}$ & Ordinary Portland Cement \\
\hline OW Prism & One-Way Prism \\
\hline PP & Polypropylene \\
\hline PVA & Polyvinyl Alcohol \\
\hline QS & Quartz Sand \\
\hline R Prism & Random Prism \\
\hline RHA & Rice Husk Ash \\
\hline $\mathrm{RPC}$ & Reactive Powder Concrete \\
\hline $\mathrm{SCC}$ & Self-Compacting Concrete \\
\hline SCMs & Supplementary Cementitious Materials \\
\hline $\mathrm{SF}$ & Silica Fume \\
\hline SFL & Silica Flour \\
\hline SIFCON & Slurry Infiltrated Concrete \\
\hline SP & Superplasticizer \\
\hline THFRC & Tension-Hardening Fiber Reinforced Concrete \\
\hline UHPFRC & Ultra-High-Performance Fiber Reinforced Concrete \\
\hline UHP-SFRC & Ultra-High-Performance Steel Fiber Reinforced Concrete \\
\hline
\end{tabular}




\section{List of Notations}

A mass of the cylinder after reaching its mass equilibrium after drying relative distance between gauge points

$B \quad$ mass of surface-dried cylinder in air after immersion relative distance between gauge points

C boiled, surface-dried mass of the cylinder relative distance between gauge points

$d_{o} \quad$ horizontal distance between crack tip to midspan

D mass of the cylinder while suspending in water diameter of the support roller

b cross-sectional width

$d \quad$ cylinder diameter

\{d\} nodal displacement matrix

$E, E_{c o}, E_{c} \quad$ concrete static modulus of elasticity

$E_{S} \quad$ steel static modulus of elasticity

$E^{*} \quad$ unloading modulus

$f \quad$ frictional force

$f_{c}^{\prime} \quad$ compressive strength at the age of 28 days or at a mature age

$f_{c r}, f_{t}, f_{t}^{\prime} \quad$ cracking strength

$f_{t, u}, f_{c t f^{*},} f_{F u} \quad$ ultimate tensile strength

$f_{t, d} \quad$ stress at which bilinear tensile stress-crack opening relationship changes slope

$f_{y} \quad$ yielding strength of reinforcement

$F \quad$ force transferred from the prism to the support rollers

\{F\} load matrix

$G \quad$ mass of the cylinder after reaching its mass equilibrium after curing

h cylinder height

cross-sectional depth

$H \quad$ horizontal component force

I mass of the cylinder immediately after demolding

$K \quad$ normalized parameters

[k] local stiffness matrix

$[K] \quad$ global stiffness matrix 


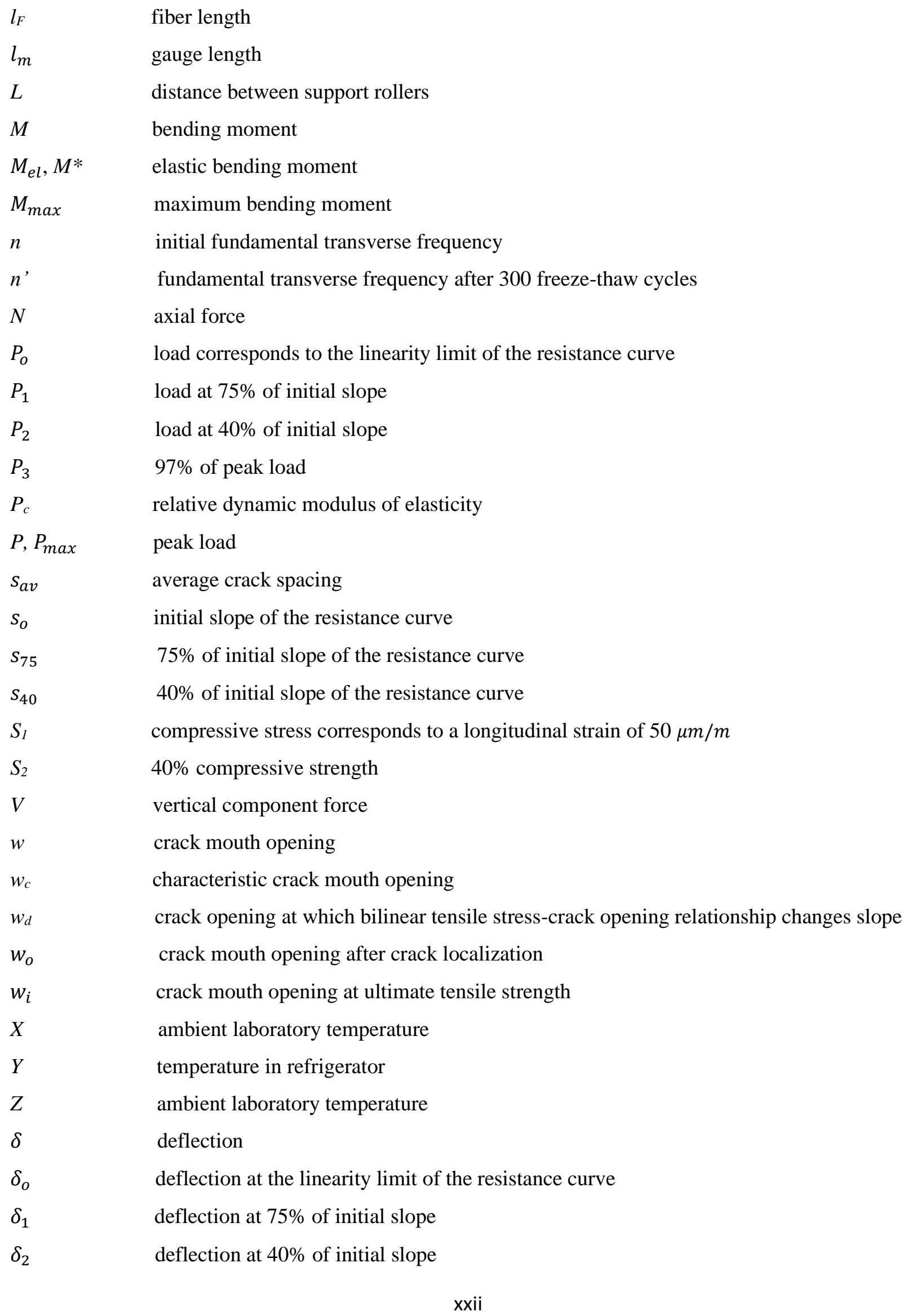




\begin{tabular}{ll}
$\delta_{3}$ & midspan deflection at $97 \%$ of peak load \\
$\delta_{4}^{*}$ & corrected deflection of four-point bending test \\
$\delta_{e l}$ & deflection at cracking strength \\
$\delta_{c r}$ & deflection at ultimate tensile strength \\
$\delta_{s}$ & crack-shear-slip deformation \\
$\sigma$ & axial stress \\
$\sigma_{s}$ & splitting tensile strength \\
$\sigma_{f}$ & equivalent flexural strength \\
$\varepsilon_{1}$ & compressive longitudinal strain of $50 \mu m / m$ \\
$\varepsilon_{2}$ & compressive longitudinal strain at $40 \%$ of compressive strength \\
$\varepsilon_{t o}$ & calculated effective strain value \\
$\varepsilon_{t 1}$ & transverse strain corresponds to a longitudinal strain of $50 \mu m / m$ \\
$\varepsilon_{t 2}$ & transverse strain at $40 \%$ of compressive strength \\
$\varepsilon_{c o}, \varepsilon_{o}$ & longitudinal strain at compressive strength \\
$\varepsilon_{t}, \varepsilon_{e l}, \varepsilon_{c r}$ & cracking strain \\
$\varepsilon_{t, u}, \varepsilon_{l i m}, \varepsilon_{t u}$ & ultimate tensile strain \\
$\phi_{e l}$ & elastic curvature \\
$\phi_{m a x}, \chi$ & maximum curvature \\
$\theta$ & rotation of the support roller about its centroid of four-point bending test \\
$\mu$ & average net shear strain \\
$v$ & shear stress \\
$\gamma$ & coinforcement ratio \\
\hline &
\end{tabular}




\section{Chapter 1. Introduction}

\subsection{Background}

Conventional concrete has been the material of choice in the construction industry throughout the $20^{\text {th }}$ century due to its inexpensive constituents, easy preparation on site from local source materials, favourable compressive strength and ability to be placed in multifarious forms. On account of concrete's negligible resistance to tension, reinforcing bars are provided in concrete structures to develop strength and ductility. However, when conventional reinforced concrete structures are exposed to aggressive environments, corrosion of steel reinforcement inevitably occurs due to carbonation and chloride ingress, which leads to premature deterioration and a significant reduction in the service life (Mindess et al. 2003; Kumar and Burrows 2001).

The corrosion process of steel reinforcement can be divided into two stages: the initiation phase and the propagation phase. Two mechanisms, namely carbonation and chloride ingress, initiate the corrosion process. Carbonation results from acidic atmospheric carbon dioxide ingress, whereas additives containing chloride ions utilized during concrete mixing (i.e., cast-in chlorides) and de-icing agents (i.e., external environment induced chlorides) are the roots of chloride ingress. In addition, cyclic wetting and drying can accelerate carbonation and chloride ingress (fib Bulletin 62 2012). Nevertheless, corrosion of rebars is not evident during the initiation phase owing to the protection provided by concrete cover and the passive oxide film around the rebars. During the propagation phase, corrosion of rebars becomes severe and structural degradation becomes noticeable over time. The ongoing corrosion process can be explained by anodic and cathodic reactions, so-called Half-Cell reactions. Anodic reaction is an oxidation process which leads to the loss of metal, whereas cathodic reaction is a reduction process which reduces the amount of dissolved oxygen as hydroxyl ions form. Along with time, rebars become depassivated. In addition, rebars expand as corrosion propagates, and this expansion generates stress which is one of the primary causes of concrete cracking and spalling (Bhalla and Bhattacharjee 2014). Moreover, concrete cracking, scaling and crumbling may occur after exposure to successive freeze-thaw cycles. Under freezing conditions, the water in concrete freezes and expands approximately $9 \%$ in volume, which imposes pressure to the surrounding concrete, and results in concrete dilation and rupture when the pressure exceeds concrete's tensile strength (Portland Cement Association 2002). 
In view of the issues mentioned above, maintenance, rehabilitation and retrofitting of existing structures are necessary. However, the cost and time required to implement these measures are considerable: the amount of money spent on maintaining existing structures exceeds that spent on building new structures (Yau 1998). Therefore, novel approaches, such as air-entrained concrete, glass fiber-reinforced polymer bars and ultra-high-performance fiber reinforced concrete (UHPFRC), have been proposed to address current challenges and to develop high-strength and high-performance materials. The entrained air voids can mitigate the pressure in concrete by providing empty chambers which allow water penetration and water expansion under freezing environment (Portland Cement Association 2002), and glass fiber-reinforced polymer bars are a competitive alternative to steel reinforcement owing to its non-corrosive nature (Carmo and Júlio 2017). Among all the countermeasures, UHPFRC is distinguished by its exclusive mechanical properties and durability performance.

\subsection{Introduction of UHPFRC}

An increasing number of documents have been issued by U.S. and Canadian public authorities, drawing attention to the needs of infrastructure investment (Mowat Center 2014; Canadian Chamber of Commerce 2013; Canadian Center for Policy Alternatives 2013; Canada West Foundation 2013; Federation of Canadian Municipalities 2012). The Ontario government alone will invest several billion dollars to repair and to expand provincial highways and bridges in the next few years in order to meet the future transportation needs (Ministry of Transportation of Ontario 2015). With the harsh climate in Canada and the poor durability performance of conventional concrete, existing infrastructures are ageing quickly and are in urgent need of immediate replacement or retrofit. In consideration of the tremendous cost of repair, public authorities have given priority to research on sustainable designs of infrastructures with enhanced resistance and extended service life, aiming to reduce long-term financial losses. In this context, the emergence of UHPFRC technology developed in the last 20 years opens up opportunities for designing much longer and dependable service lives of infrastructures (Larsen et al. 2017).

UHPFRC is a new class of materials which exhibits superior workability, mechanical properties and durability performance. According to the Annex U of CSA-A23.1 (2019), UHPFRC is defined as a cementitious-based composite material with a compressive strength greater than 120MPa, a cracking strength greater than $4 \mathrm{MPa}$ and exclusive durability performance. The desired mechanical and durability properties of UHPFRC are mainly attributed to its novel mix design. In general, UHPFRC comprises Portland cement, supplementary cementitious materials (SCMs), inert fillers, water, superplasticizer (SP), fine aggregates and reinforcing fibers. In addition, UHPFRC typically has a low water-to-cement ratio less 
than 0.25 and a high cementitious material content in excess of $1000 \mathrm{~kg} / \mathrm{m}^{3}$ (Russell and Graybeal 2013; Rangaraju et al. 2013; Yu et al. 2014). Studies indicate that SCMs could be used to replace partial cement content without compromising mechanical and durability performance of UHPFRC (Mindess et al. 2003). Widely used SCMs are silica fume (SF), fly ash (FA), ground granulated blast furnace slag (GGBS) and metakaolin (Wille and Cotulio 2013). Tricalcium silicate $\left(C_{3} S\right)$ and dicalcium silicate $\left(C_{2} S\right)$ are the wellknown constituents in Portland cement which produce C-S-H gel and calcium hydroxide $(\mathrm{CH})$ through the hydration process, and C-S-H gel is the primary contributor to material strength. With the use of SCMs, additional C-S-H gel can be produced through the reaction between SCMs and $\mathrm{CH}$, so-called pozzolanic reaction, which promotes the less porous microstructure and higher compressive strength of UHPFRC. Inert fillers are fine particles which do not present pozzolanic reactivity at ambient temperature. However, they can physically optimize the grading and packing of fine aggregates and thereby improving the material's density, strength and permeability resistance (Lawrence et al. 2003). Widely used inert fillers are silica flour (SFL), quartz sand (QS) and rice husk ash (RHA) (Wille et al. 2011). Superplasticizer is an essential chemical admixture for UHPFRC. It enables flowable cementitious materials with low water-to-cement ratio and thereby improving concrete workability and strength. The addition of reinforcing fibers improves tensile strength and ductility, and the mechanism between fibers and the matrix is as follows. At the level of micro-cracks, the bridging effect of fibers provides a stress transfer media which can delay the formation of localized crack. At the level of macro-cracks (i.e., the localized crack), the fibers cross crack opening and restrain crack growth (Banthia 2012). However, the effectiveness of fibers depends on the fiber-matrix bond strength and fiber tensile strength. Moreover, owing to its mix design, UHPFRC has high density and limited connected capillary pores which can delay the ingress of corrosive agents and thereby enabling excellent durability performance.

To date, many projects have incorporated UHPFRC, comprising either repairs or new designs (Doiron 2017). The market size of UHPFRC is expected to grow 7\% annually between 2017 and 2023 due to distinct advantages of UHPFRC in applications where narrow formwork and dense reinforcement are inevitable, high-strength material is required and the surrounding environment is aggressive (Darling 2018). UHPFRC, in its present form, became commercially available in North America in the late 1990s, and the first North American bridge constructed with UHPFRC is located in Sherbrooke, Canada (Blais and Couture 1999). Figure 1-1 depicts the cumulative number of bridges constructed with UHPFRC since 2006. 


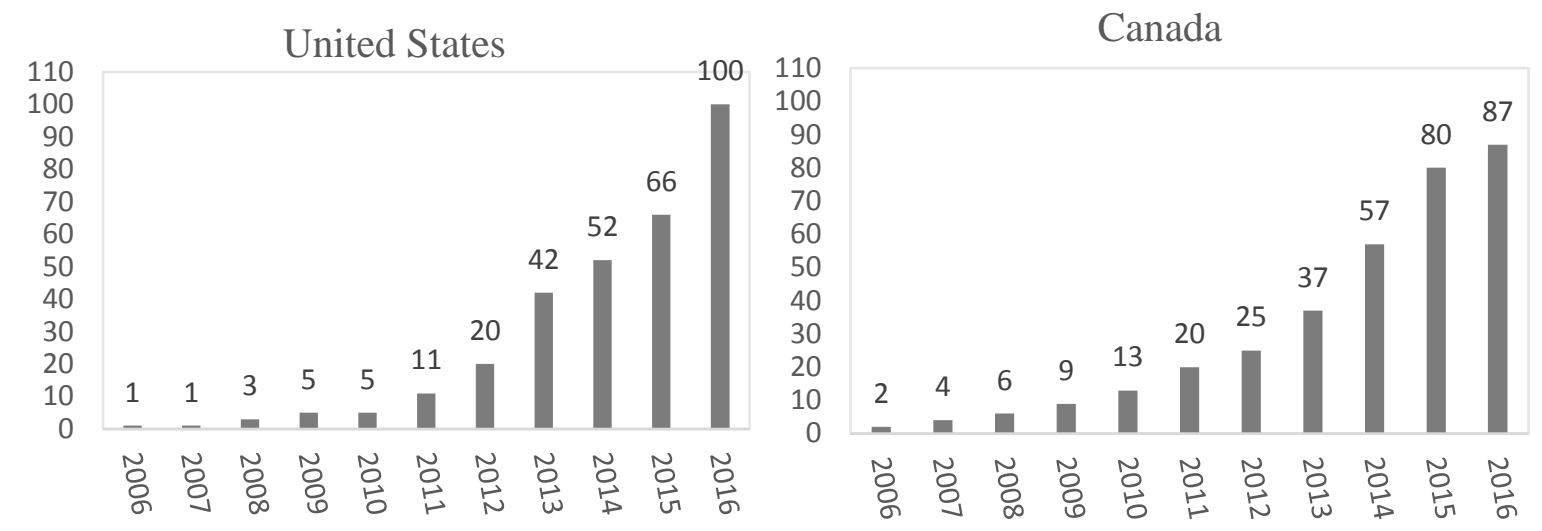

Figure 1- 1: The cumulative number of bridges constructed with UHPFRC since 2006 (FHWA-HRT-18-036 2018)

Field-cast connections is one of the primary applications of UHPFRC. Until 2016, UHPFRC connections have been constructed in more than 130 bridges in the United States and Canada (Rahman and McQuaker 2016). UHPFRC connections require less volume of field-cast concrete and do not need post-tensioning, which accelerates bridge construction. Figure 1-2 depicts the casting of UHPFRC connections, and the Pulaski Skyway Bridge constructed with UHPFRC connections in the deck. With regard to deteriorated bridge decks, UHPFRC can be used as an overlay material for rehabilitation, which not only strengthens the existing bridges but also limits further contaminant ingress owing to the material's low permeability and discontinuous capillary pores. In addition, UHPFRC overlays are typically $25 \mathrm{~mm}$ to $51 \mathrm{~mm}$ thick, which are thinner than conventional overlays ( $51 \mathrm{~mm}$ to $152 \mathrm{~mm}$ thick) and thereby reducing the additional dead load. However, it is worth noting that UHPFRC overlays are different from usual UHPFRC in terms of rheological property: usual UHPFRC is self-consolidating (i.e., it flows under gravity); whereas UHPFRC utilized for overlays are thixotropic (i.e., it is a non-Newtonian fluid), meaning it only flows under agitation. The thixotropic characteristic is ideal for deck overlay construction by preventing the overlay material from flowing along ramps (FHWA-HRT-17-097 2017). Other ongoing and potential applications of UHPFRC include: 1) tall wind turbine towers which can generate an increasing output and more renewable energy; 2) tunnels; and 3) as illustrated in Figure 1-3, architectural designs with much thinner and more complex shapes owing to its self-consolidation characteristic (Darling 2018). 

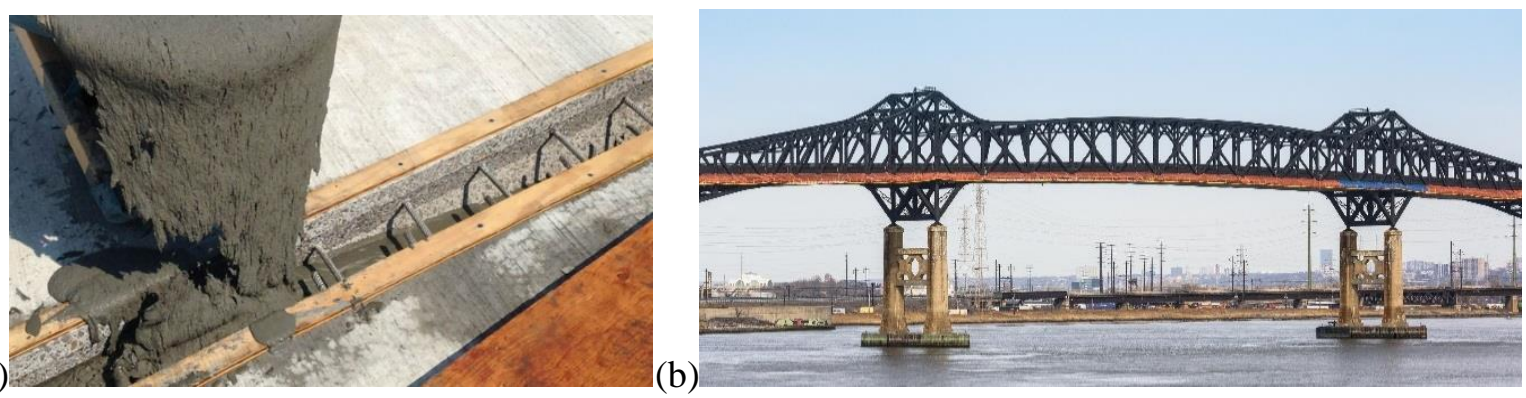

Figure 1- 2: Field-cast UHPFRC connections: (a) The casting of UHPFRC connections; and (b) The Pulaski Skyway Bridge constructed with UHPFRC connections (Rahman and McQuaker 2016)

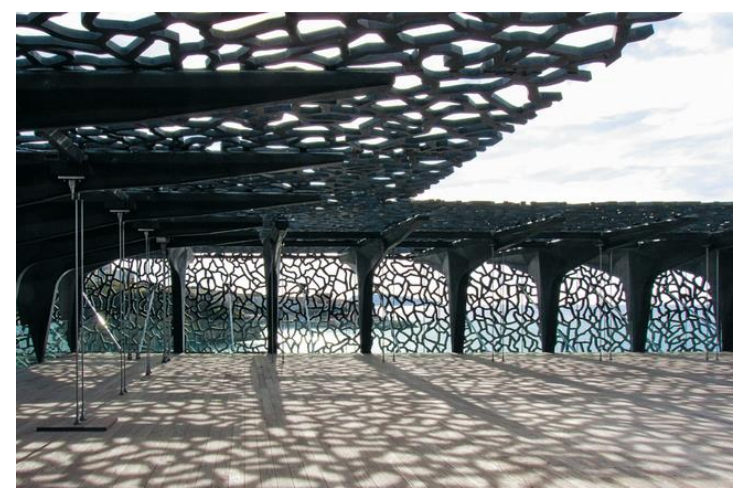

Figure 1- 3: MuCEM in Marseille (Ricciotti 2013)

From an environmental perspective, 2.2 billion tons of cement was produced in 2005 due to the fact that Portland cement is one of the most widely used raw material in the concrete industry. However, cement is not eco-friendly: considerable amount of carbon dioxide, which is a major contributor of the green house effect and global warming, is emitted during the cement production process (Srinivasa and Venkatesh 2014). Tuan et al. (2011) indicated that 690 million metric tons of rice paddy waste is generated annually, and 20\% of it was processed into RHA. In addition, the American Coal Ash Association reported that 71 million tons of FA was produced in 2005, and $41 \%$ of it was used in the concrete industry (ACAA 2015), indicating that there is growing awareness about extensive environmental damages due to waste materials. As mentioned previously, SCMs and filler materials can be used to replace partial cement and fine aggregate contents in UHPFRC, which directly reduces green house gas emission. Therefore, the reuse of industrial and agricultural wastes such as SCMs and fillers in UHPFRC has a positive environmental impact.

From a cost-effective perspective, the unit price of UHPFRC is much higher compared with conventional concrete. However, the dimensions of structural elements can be reduced due to UHPFRC's superior strength, which leads to substantial cost saving due to the reduction in dead load and steel reinforcement 
(Swar et al. 2015). In addition, cement and SF replacement with limestone, calcined clay, FA and GGBS is economical (Yu et al. 2015; Huang et al. 2017; Afroughsabet et al. 2016). Moreover, SCMs can reduce the heat of hydration and improve a structure's long-term durability, indicating lower maintenance and repair costs (Reddy et al. 2012). Furthermore, according to life cycle cost analysis of the Eder Bridge in Felsberg, the cost of precast UHPFRC box girders filled with lightweight concrete is lower than that of conventional prestressed concrete members (Piotrowski and Schmidt 2012).

\subsection{Objectives and Scope of the Thesis}

The Canadian Standards Association has introduced UHPFRC in two standards: Annex 8.1 of CSA-S6 (2018) focuses on structural design and Annex U of CSA-A23.1 (2019) focuses on the material. Both standards bring attention to a number of practical and theoretical issues regarding the characterization of UHPFRC in tension. In both standards, the FPBT and an associated inverse analysis method were adopted on account of the fact that the DTT is challenging to conduct. However, test setup, specimen size and the manner of casting can significantly affect the FPBT results. In addition, the relationship between FPBT and DTT is still unclear, and this is critical when both tests are considered for UHPFRC characterization. Thus, it is necessary to standardize a clear and repeatable testing procedure by which tensile properties of UHPFRC can be assessed. Moreover, the inverse analysis method proposed to determine the tensile properties of UHPFRC is based on the load-deflection response curve obtained from FPBT, and it is an empirical procedure which has not been proof-tested with UHPFRC materials utilized in Canada. In this context, extensive investigation is necessary to ensure that the inverse analysis method possesses sufficient repeatability, consistency and conservatism.

This research study includes both experimental and analytical components. The experimental program considered several proprietary and non-proprietary ultra-high-performance steel fiber reinforced concrete (UHP-SFRC) materials in order to support generalized conclusions from the investigation. With regard to DTT, three types of specimens were tested to compare the experimental results with respect to specimen shape and test setup. In addition, splitting tensile tests were conducted in accordance with CSA A23.2-13C (2014). Four-point bending tests were conducted according to Annex 8.1 of CSA-S6 (2018), combined with parameter variations to evaluate the effects of casting methodology, volumetric ratio of steel fibers, aspect ratio (i.e., prism shear span-to-depth ratio) of the bending prism and prism size on flexural strength. Moreover, a Round Robin Testing Program in collaboration with Queen's University and École Polytechnique-Montréal was carried out to assess the replicability of experimental results by testing identical prisms in different laboratories. Furthermore, freeze-thaw tests and salt-scaling tests were 
conducted to evaluate the long-term performance of UHP-SFRC. Additionally, the coefficient of thermal expansion, static modulus of elasticity, Poisson's ratio and absorption properties were determined to complement the experimental program. The experimental work was divided into three parts: the first part comprised the testing of four proprietary and two non-proprietary UHP-SFRC materials; the second part comprised the Round Robin Testing Program and the testing of three proprietary UHP-SFRC materials; and the third part comprised non-destructive tests to evaluate the physical properties and durability performance of UHP-SFRC. The analytical work was also divided into three parts: the first part focused on obtaining the linearized tensile stress-strain and stress-crack mouth opening relationships of UHP-SFRC by using the inverse analysis procedures adopted in Annex 8.1 of CSA-S6 (2018); the second part focused on developing numerical models with the ability to match the response curves obtained from FPBT by using a nonlinear finite element analysis software (VecTor2), and deriving the tensile properties of UHP-SFRC from the numerical models; and the third part focused on correlating the tensile strength results of UHPSFRC obtained from DTT, inverse analysis of FPBT, splitting tensile test, nonlinear finite element analysis and the calibrated empirical expression in relation to compressive strength.

The main objectives of this research study are defined as follows:

1. To validate the proposed FPBT setup, to investigate the effects of casting methodology, volumetric ratio of steel fibers, aspect ratio of the bending prism and prism size on flexural strength, and to provide recommendations in order to improve the repeatability of test results.

2. To validate the inverse analysis method, and to obtain the linearized tensile stress-strain and stresscrack mouth opening relationships of UHP-SFRC.

3. To develop or validate direct tension test setups and direct-tension specimens.

4. To develop numerical models, and to derive analytical tensile strength values from the nonlinear finite element models.

5. To classify the UHP-SFRC materials considered in this research study based on their tensile properties.

6. To correlate the tensile strength results obtained from different tension tests, numerical models and the calibrated empirical expression proposed in the Canadian standards.

7. To determine the physical properties and durability performance of UHP-SFRC. 


\subsection{Thesis Outline}

This report comprises seven chapters, and the details of each chapter are as follows:

Chapter 1-Introduction: This chapter starts with the introduction of the issues observed from conventional reinforced concrete structures, followed by a brief introduction of the composition, applications and practicability of UHPFRC in view of environmental and cost-effective considerations. In addition, the objectives and scope of this research study was presented in this chapter.

Chapter 2-Literature Review: This chapter focuses on reviewing the historical background of concrete and the raw materials commonly utilized in UHPFRC. In addition, experimental results relating to the physical properties, mechanical properties and durability performance of UHPFRC assessed by other researchers are presented. Moreover, this chapter includes a detailed review on the classification of UHPFRC, various tension test setups and inverse analysis methods proposed in different standards.

Chapter 3-Phase 1 of the Experimental Program: This chapter presents the experimental results of both proprietary and non-proprietary UHP-SFRC materials considered in the Phase 1 of this research study, including cylinder compressive strength, splitting tensile strength, cracking strength obtained from DTT, and flexural strength. Four parameters of the FPBT were evaluated in this chapter, which includes the casting methodology, volumetric ratio of steel fibers, aspect ratio of the bending prism and the degree of fiber alignment.

Chapter 4-Phase 2 of the Experimental Program: This chapter presents the flexural strength results obtained from the Round Robin Testing Program. An investigation was carried out to address the reasons which led to discrepancies in results among the three universities. In addition, flexural strength results of three proprietary UHP-SFRC mixes were also presented in this chapter, and the effect of the degree of fiber alignment on flexural strength was evaluated.

Chapter 5-Non-Destructive Tests: This chapter presents the experimental test setups and results regarding the physical properties and durability performance of UHP-SFRC, which includes the determination of static modulus of elasticity, Poisson's ratio, absorption properties, coefficient of thermal expansion, and resistance to freeze-thaw cycles and salt scaling. 
Chapter 6-Analytical Studies: This chapter comprises three parts. The first part introduces the inverse analysis method proposed by the Canadian standards (Annex 8.1 of CSA-S6 2018; Annex U of CSA-A23.1 2019), and presents the linearized tensile stress-strain and stress-crack mouth opening relationships of UHPSFRC obtained from the inverse analysis. The second part presents the modelling methodology considered for finite element analysis and the numerical results relating to tensile properties of UHP-SFRC derived from the finite element models. The third part presents the classification of the UHP-SFRC materials considered in this research study according to the Canadian standards (Annex 8.1 of CSA-S6 2018; Annex U of CSA-A23.1 2019) and correlates the tensile strength results obtained from DTT, FPBT, splitting tensile test, finite element analysis and the empirical calibrated expression.

Chapter 7-Conclusions and Recommendations: This chapter summarizes the main findings of this research program. In addition, several recommendations are provided with respect to experimental test setup and specimen size. 


\section{Chapter 2. Literature Review}

This literature review starts with an introduction of the concrete development history and the typical constituents of UHPFRC, followed by experimental results and observations regarding the physical properties, mechanical properties, durability performance and fire resistance of UHPFRC assessed by other researchers. In addition, this chapter reviews the tensile behaviour of UHPFRC by presenting various classification criteria, tension tests and inverse analysis methods proposed by several standards and researchers.

\subsection{Development of Concrete}

Concrete is known as a biphasic composite material with a matrix (i.e., binder paste) incorporating a filling material (i.e., granular skeleton). Addis and Bussell (2002) indicated that concrete-type materials can be traced back to approximately 5,000 years ago. For instance, the Roman concrete, also referred as hydraulic concrete which hardens under water, is similar to modern concrete. Another type of concrete, a nonhydraulic concrete (i.e., also known as lime concrete) was used in medieval times. Lime concrete hardens through a carbonation process during which quicklime reacts with atmospheric carbon dioxide (fib Bulletin 62 2012).

In 1824, Portland cement was invented by Joseph Aspdin, which was a landmark for concrete development history due to the fact that Portland cement is still one of the most commonly used materials to date. Aspdin produced this binder by burning powdered limestone with clay and grinding the obtained clinker to fine powders (Tejuosho et al. 2016). Portland cement comprises four main chemical compounds which are tricalcium silicate $\left(\mathrm{C}_{3} \mathrm{~S}\right)$, dicalcium silicate $\left(\mathrm{C}_{2} \mathrm{~S}\right)$, tricalcium aluminate $\left(\mathrm{C}_{3} \mathrm{~A}\right)$ and tetracalcium aluminoferrite $\left(\mathrm{C}_{4} \mathrm{AF}\right)$. The American Society for Testing and Materials classifies Portland cement into six groups based on its chemical contents, fineness and color. For instance, Type I Portland cement, also known as ordinary Portland cement (OPC), contains a relatively high $\mathrm{C}_{3} \mathrm{~S}$ content, and Type II and Type V Portland cements which are generally used to construct structures exposed to sulfate ions contain low $\mathrm{C}_{3} \mathrm{~A}$ contents less than $8 \%$ and 5\%, respectively (Dunuweera and Rajapakse 2018).

Addis and Bussell (2002) found that there was a steady improvement in compressive strength with time: concrete had a compressive strength of $11 \mathrm{MPa}$ to $15 \mathrm{MPa}$ prior to $1915,15 \mathrm{MPa}$ to $20 \mathrm{MPa}$ by the $1930 \mathrm{~s}$, and $20 \mathrm{MPa}$ to $30 \mathrm{MPa}$ in the 1960s. Mindess and Young (1981) indicated that concrete strength was related 
to its porosity, and Brownyard (1948) brought to light that low water-to-cement ratio could effectively reduce the porosity and increase the strength of concrete. With the development of superplasticizers (SP) and pozzolanic admixtures, macro-defect-free cement (MDF) and densified small particles-based concrete (DSP) arose in the 1980s. A Research group led by Birchall manufactured MDF with a low water-to-cement ratio between 0.08 and 0.2. MDF relies on high-shear mixing and mechano-chemical reaction among calcium aluminate cement, water-soluble polymers and glycerol plasticizer, and it exhibits exclusive mechanical properties compared with OPC. MDF can achieve a flexural strength of more than $150 \mathrm{MPa}$ compared with that of $5 \mathrm{MPa}$ to $10 \mathrm{MPa}$ for OPC (Donatello et al. 2009). However, MDF has poor moisture resistance: its flexural strength decreases up to $55 \%$ after immersing in water for 24 hours (Donatello et al. 2009). DSP is a mixture of Portland cement and silica fume, and it leans upon the use of SP (Bache 1987). In general, DSP has a micro-silica to cement ratio between 0.15 and 0.25 and a water-to-binder ratio between 0.15 and 0.2 (Verma and Sharma 2019). However, brittle failure of DSP was observed, thus the addition of fibers in cementitious materials was introduced to improve ductility.

Slurry infiltrated concrete (SIFCON) was first reported in 1984, and it comprises fibers and cement-based slurry. The slurry consists of cement or a combination of cement and sand, or cement and SCMs. In general, $6 \%$ to $20 \%$ of glass, steel, polypropylene (PP), polyester or carbon fibers are used in SIFCON. For casting SIFCON, fibers are placed in the mold prior to mixing so that the cementitious material can infiltrate through the fiber layer (Thamilselvi et al. 2017). Engineered cementitious composites (ECC) is another type of fiber-reinforced material which comprises less than $2 \%$ of PP or polyvinyl alcohol (PVA) fibers. ECC is a ductile material by having a tensile strain capacity in the range of $3 \%$ to $5 \%$, which is several hundred times that of conventional concrete (i.e., the tensile strain capacity of conventional concrete is about $0.01 \%$ ) (Srinivasa and Venkatesh 2014). Both SIFCON and ECC fall into the category of highperformance concrete (HPC) because their compressive strengths are greater than $55 \mathrm{MPa}$ but less than 120 MPa (ACI C363.2R 2011 and ASTM C1856 2017). Self-compacting concrete (SCC) was first proposed in Japan in the late 1980s to ease poor compaction issues. SCC has a low water-to-cement ratio and possesses exclusive rheological property which allows it to fully encapsulate reinforcement under its own weight without any external vibration or compaction. In addition, coarse and fine aggregates replacement with finer particles can improve the homogeneity of SCC and lower its segregation potential (Painuly and Uniyal 2016).

The concept of reactive powder concrete (RPC) was first introduced by Richard and Cheyrezy, and it was first produced in the early 1990s in France (fib Bulletin 62 2012). RPC characterizes with high density, low porosity and the addition of fibers. RPC can attain a compressive strength of $200 \mathrm{MPa}$, a tensile strength of 
$8 \mathrm{MPa}$ and a flexural strength of $40 \mathrm{MPa}$ to $50 \mathrm{MPa}$. Differing from conventional concrete, coarse aggregates are not used in RPC, but fine particles whose sizes are between $0.02 \mu \mathrm{m}$ to $300 \mu \mathrm{m}$ are employed to enhance homogeneity. SP is applied to RPC to reduce water-to-binder ratio to around 0.2 , to reduce porosity, and to improve strength. In addition, the addition of metallic or synthetic fibers introduces new reinforcement systems and improves ductility (Anson et al. 2002). Moreover, properties of RPC depend on the chosen raw materials (i.e., chemical admixtures, mineral additives, and fiber content) and curing condition. Mansour and Alkafaji (2014) found that heat treatment can increase the compressive strength, indirect tensile strength, modulus of elasticity and flexural strength of RPC; and the studies carried out by Hassani et al. (2014) indicated that by increasing silica fume content from $0 \%$ to $30 \%$, RPC's compressive strength improved significantly, whereas its tensile strength improved slightly. It is worth noting that main differences between RPC and SIFCON are fiber content and mixing methodology. RPC generally contains $1 \%$ to $3 \%$ of fibers by volume which are mixed with other raw materials during concrete mixing, whereas SIFCON typically contains $6 \%$ to $20 \%$ of fibers which are preplaced in molds and allow the cementitious materials to infiltrate (Thamilselvi et al. 2017).

\subsection{Composition of UHPFRC}

UHPFRC generally consists of Portland cement, fine sand, SCMs, reinforcing fibers, SP and chemical admixtures and water. However, the properties and dosage of each type of constituent and curing methodology can result in diversities in UHPFRC's properties. The following sections introduce the commonly used raw materials in UHPFRC, and explain their effects in terms of workability, physical properties, mechanical properties and durability performance.

\subsubsection{Cement}

It is well known that the exothermic chemical reaction, so-called hydration process, promotes the hardening of concrete when compounds in cement form chemical bonds with water molecules and produce C-S-H gel (Mindess et al. 2003). $\mathrm{C}_{3} \mathrm{~S}, \mathrm{C}_{2} \mathrm{~S}, \mathrm{C}_{3} \mathrm{~A}$ and $\mathrm{C}_{4} \mathrm{AF}$ are the major chemical compounds in Portland cement, and they affect the properties of cement differently. $\mathrm{C}_{3} \mathrm{~A}$ releases considerable amount of heat during the early stage of hydration, but its contribution to concrete strength is negligible. In addition, cement with a low $\mathrm{C}_{3} \mathrm{~A}$ content possesses more sulfate resistance. Furthermore, $\mathrm{C}_{3} \mathrm{~A}$ reacts with SP and causes insufficient SP left to guarantee the workability of fresh concrete (Mindess et al. 2003). $\mathrm{C}_{3} \mathrm{~S}$ hydrates and hardens rapidly, and it is responsible for cement's early strength; whereas $\mathrm{C}_{2} \mathrm{~S}$ hydrates much slower, and it is responsible for the strength gain after one week. $\mathrm{C}_{4} \mathrm{AF}$ hydrates fast, but it only benefits the manufacture process of 
cement by lowering raw materials' melting temperature rather than contributing to concrete strength (Kosmatka and Panarese 1988). Moreover, the amount of heat generated, and the reaction rate of hydration can cause thermal cracking at early ages. Another concern is attributed to the alkali content in cement (known as $\mathrm{Na}_{2} \mathrm{O}_{\text {eq }}$ ): alkali-silica reaction (ASR) takes place when highly alkaline cement reacts with reactive amorphous silica contained in common aggregates, leading to additional shrinkage under drying condition (Burrows 1998). Additionally, cement containing a high alkali content produces alkali-containing C-S-H gel, resulting in a porous microstructure and low compressive strength of the hardened concrete. Furthermore, Mindess et al. (2003) indicated that high-fineness cement enables fast hydration and high early strength. However, high-fineness cement may cause workability issues. With regard to UHPFRC, the particle size of cement is typically between $10 \mu \mathrm{m}$ to $45 \mu \mathrm{m}$, and CEM 152.5 , ASTM Types I, II, IV and V cements are recommended by researchers (Deeb et al. 2012; EL-Dieb 2009; Huo and Wong 2000; Thomason 2009; Willey 2013). Type II Portland cement has a $\mathrm{C}_{3} \mathrm{~A}$ content less than $8 \%$; Type IV Portland cement reduces the heat of hydration; and Type V Portland cement has a $\mathrm{C}_{3} \mathrm{~A}$ content less than $5 \%$ and a lower cost (EI-Tawil 2016).

\subsubsection{Aggregates}

Coarse aggregates are usually excluded in UHPFRC to reduce the damage potential at interfacial transition zone (ITZ) and porosity (Metha and Monteiro 2006). Russell and Graybeal (2013) recommended the maximum grain size utilized in UHPFRC should be less than $0.8 \mathrm{~mm}$. However, Ma and Orgass (2004) reported that UHPFRC containing $2 \mathrm{~mm}$ to $5 \mathrm{~mm}$ crushed basalt aggregates exhibited identical compressive strength as that containing finer particles, and coarse basalt aggregates can reduce the autogenous shrinkage.

Aggregates type, size distribution and aggregate-to-cement ratio can significantly affect the workability, strength and durability of UHPFRC (Mindess et al. 2003; Cortes et al. 2008). Chemically stable aggregates are preferred to reduce ASR potential. In addition, Wille and Cotulio (2013) indicated that quartz aggregate provided the greatest compressive strength, followed by basalt aggregate, volcanic rock and limestone. With regard to fine aggregate (i.e., sand), a study showed that the resistance to chloride ion penetration of UHPFRC improved with sand content up to a certain amount, but the resistance started to decrease beyond this amount because permeability increases when sand content drastically increases due to the formation of more connected capillary pores resulted from the adjacent ITZs (Mindess et al. 2003; Winslow et al. 1994). Moreover, sand-to-cement ratio between 1 to 1.4 was found to be optimal for UHPFRC (Park et al. 2008; Wille et al. 2011; Li et al. 2014). 


\subsubsection{Supplementary Cementitious Materials and Inert Fillers}

Silica fume (SF) between $0.1 \mu \mathrm{m}$ to $1 \mu \mathrm{m}$, fly ash (FA) between $13 \mu \mathrm{m}$ to $40 \mu \mathrm{m}$, quartz powder between $5 \mu \mathrm{m}$ to $45 \mu \mathrm{m}$, ground granulated blast furnace slag (GGBS) between $6.5 \mu \mathrm{m}$ to $45 \mu \mathrm{m}$, rice husk ash (RHA) between $3.8 \mu \mathrm{m}$ to $10 \mu \mathrm{m}$ and lime powder are commonly-used SCMs and inert fillers in UHPFRC. Through pozzolanic reactions, SCMs react with calcium hydroxide $(\mathrm{CH})$ (i.e., a product of cement hydration process) and produce additional C-S-H gel, which promotes a less-porous microstructure and better bond between fibers and the matrix (Sohail et al. 2018). Studies showed that up to $40 \%$ cement content by volume could be replaced by SCMs without affecting the compressive strength of UHPFRC (Ma and Schneider 2002; Soutsos et al. 2005; Yazici 2006). In addition, because SCMs and inert fillers are fine particles, they can fill the voids and release the trapped water, which directly improves material's density and mechanical properties.

SF is one of the most commonly used SCMs in cementitious materials because it can improve material's compressive strength through pozzolanic reaction. In addition, on account of the fact that SF are fine particles with large surface areas, it can reduce bleeding potential and increase cohesiveness of fresh concrete (Nehdi et al. 1998), and the recommended dosage of SF for UHPFRC is $20 \%$ to $30 \%$ of the total binder material (Ma and Schneider 2002; Matte and Moranville 1999; Chan and Chu 2004). Moreover, SF can improve material's resistance to freeze-thaw cycles, scaling and chloride ion penetration owing to its ability to densify the microstructure (Hooton 1993; Sabir 1997; Thomas et al. 1999).

The spherical shape of FA allows it to function as ball bearings in cementitious materials, which can improve the workability of fresh concrete (Mindess et al. 2003). In addition, loss of ignition (LOI) is a measurement of the carbon content in FA, and it affects concrete workability and properties since FA with a high LOI value has higher water requirement; and FA with a low LOI value can reduce the heat of hydration (Nagataki et al. 1984; Dhir et al. 1988). The American Society for Testing and Materials (2015) requires the LOI of FA to be less than 4\%, and it classifies FA into two types based on their chemical composition (i.e., Type F and Type C). Type F FA is preferred to be used in concrete. Similar to SF, the aluminosilicate components in FA undergo pozzolanic reaction and produce additional cementitious compounds which can reduce the pore interconnectivity in concrete and improve concrete's mechanical and long-term performance. Regarding compressive strength, the pozzolanic effect of FA is inappreciable at early ages (<7 days) but prominent at later ages (Dhir et al. 1988). Furthermore, FA consumes alkali content of cement under pozzolanic reaction, which indirectly reduces ASR and sulfate attack potential (Lopes-Flores 1981). 
GGBS is a type of mineral admixture to replace partial amount of cement, and it is characterized by the resulting high compressive strength, low heat of hydration, better workability and cost-effectiveness. Regarding workability, GGBS results in an extended setting time, which enables fresh concrete to remain workable for longer periods and promotes quality control. With regard to mechanical properties, unlike Portland cement concrete which can attain $75 \%$ compressive strength at 28 days, concrete containing high amount of GGBS only develops $45 \%$ to $55 \%$ compressive strength at 28 days, followed by a continued strength gain until 90 days (Suresh and Ngaraju 2015).

Particles that do not present pozzolanic reactivity at ambient temperature are referred as inert fillers which include silica flour (SFL), QP, QS, RHA and lime powder. Due to their fineness, inert fillers can improve concrete's density and strength (Wille et al. 2011). In addition, studies showed that inert fillers accelerated hydration process because they provided more surface areas for portlandite to crystallize and thereby promoted early strength gain (Lawrence et al. 2003; Soroka and Stern 1976).

\subsubsection{Chemical Admixtures}

SP is an essential chemical admixture for UHPFRC in order to obtain flowable cementitious materials with low water-to-cement ratio and thereby improving concrete workability and strength. Widely used SPs are lignosulphonates, sulphonated melamine formaldehyde (SMF), sulphonated naphthalene formaldehyde (SNF) and polycarboxylate-ether-based (PCE). Lignosulphonates, SMF and SNF-based SPs rely on electrostatic charges, whereas PCE-based SPs require stirring to deflocculate powder particles (Sohail et al. 2018). It was reported that PCE-based SPs provided more flowable materials compared with the others (Deeb et al. 2012; Golaszewski and Szwabowski 2004). In addition, Mindess et al. (2003), and Jiang and Kim (1999) indicated that SP with higher molecular weight has better performance. Moreover, the dosage of SP depends on the properties of aggregates and SCMs, and the excessive use of SP can delay concrete setting and compressive strength development (Mindess et al. 2003). Furthermore, Tue et al. (2008) found that the stepwise addition of SP was more effectively than adding SP at once.

Shrinkage is one of the critical concerns of UHPFRC owing to its high cement content and thereby shrinkage reducing admixture is sometimes applied. However, studies showed that shrinkage reducing admixture reduced the compressive strength of UHPFRC at early ages (Rangaraju et al. 2013; Graybeal 2006; Soliman 2011). Another commonly used chemical admixture is an accelerator which can accelerate concrete setting and strength development. 


\subsubsection{Water-to-Binder Ratio}

Richard and Cheyrezy (1995); Larrard and Sedran 1994; and Gao et al. 2006 recommended a water-tocement ratio of 0.13 to 0.2 for UHPFRC. Figure 2-1 illustrates the relationship between water-to-binder ratio and the compressive strength of HPC and UHPFRC (i.e., UHPC) based on the studies conducted by several researchers. It is evident that low water-to-binder ratio leads to the increase in compressive strength. The data are scattered owing to different mix designs. For instance, some researchers did not apply SP, resulting in less workable materials and lower strengths (Sohail et al. 2018).

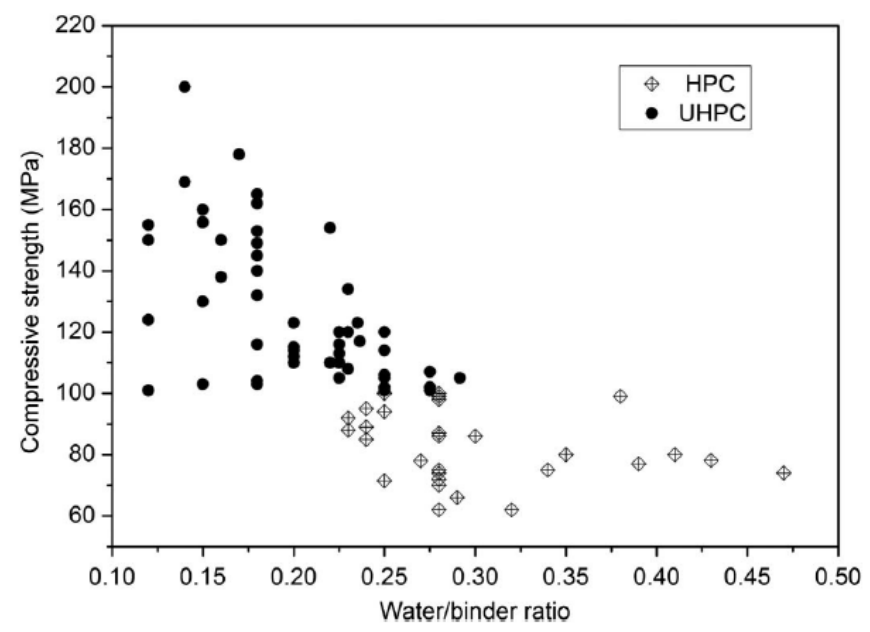

Figure 2- 1: Relationship between water-to-binder ratio and compressive strength (Sohail et al. 2018)

\subsubsection{Reinforcing Fibers}

On account of the brittle nature of cementitious materials, fibers are utilized in UHPFRC to improve its ductility and energy absorption capacity. The following sections focus on introducing various types of fibers available in the market and the effects of aspect ratio, volumetric ratio, shape, coating and distribution of fibers on UHPFRC properties.

\subsubsection{Types of Reinforcing Fibers}

Steel fibers and polyvinyl alcohol fibers (PVA) are commonly used in UHPFRC, although carbon, polypropylene, polyethylene (PP), nylon, polyester and glass fibers are commercially available as well (Sohail et al. 2018). Fibers can restrain crack propagation, resulting in the exclusive tensile strength and ductile response of UHPFRC. 
PVA fibers are made of PVOH resin, and they are the critical constituents in ECC technology. In addition, PVA fibers are hydrophilic and tend to form strong bonds with the surrounding matrix. To moderate the interfacial strength, PVA fibers were treated with surfactants in some studies (Li 2002, Georgiou and Pantazopoulou 2016). PVA fibers used in UHPFRC are usually $8 \mathrm{~mm}$ or $12 \mathrm{~mm}$ in length and $0.039 \mathrm{~mm}$ or $0.1 \mathrm{~mm}$ in diameter, and they generally have a density of $1100 \mathrm{~kg} / \mathrm{m}^{3}$, a tensile strength of $1235 \mathrm{MPa}$ and an elastic modulus of $29 \mathrm{GPa}$.

As depicted in Figure 2-2, a variety of steel fibers are commercially available. Due to the fact that the specific gravity of steel fibers is greater than the matrix, segregation is critical for UHP-SFRC (Li 2015). In addition, the shape of fibers has an impact on the mechanical properties of UHPFRC since hooked and twisted fibers develop stronger bonds with the matrix than straight smooth fibers owing to the improved mechanical anchorage (Wille et al. 2011). A study showed that the tensile strength and strain of specimens containing twisted fibers were $60 \%$ and $200 \%$ higher than those containing straight smooth fibers (Wille et al. 2012). However, straight smooth fibers usually provide better workability (Wille et al. 2011). Moreover, fibers with different shapes and aspect ratios can be introduced in UHPFRC in a hybrid form, and studies showed that the hybrid fiber reinforcement led to exclusive strain hardening results which were much higher than that obtained with a single fiber type (Sohail et al. 2018).

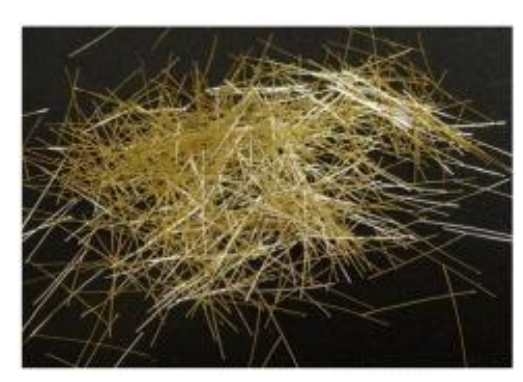

(a)

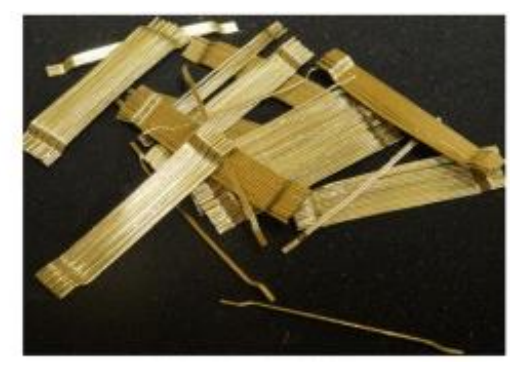

(b)

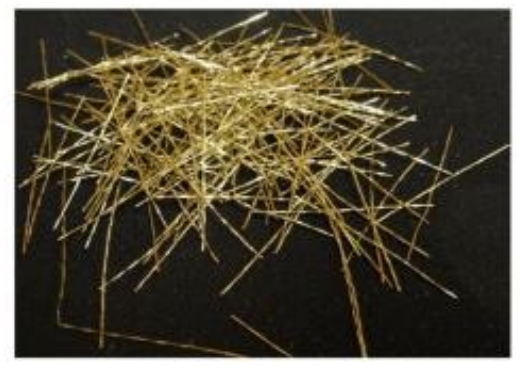

(c)

Figure 2- 2: Types of steel fibers: (a) Straight smooth; (b) Hooked; and (c) Twisted (Tai et al. 2016)

\subsubsection{Aspect Ratio of Reinforcing Fibers}

Steel fibers are generally $6 \mathrm{~mm}$ to $60 \mathrm{~mm}$ in length and $0.15 \mathrm{~mm}$ to $0.75 \mathrm{~mm}$ in diameter (Sohail et al. 2018). The most commonly used steel fibers are $13 \mathrm{~mm}$ in length and $0.2 \mathrm{~mm}$ in diameter, corresponding to an aspect ratio of 65 (Schmidt et al. 2004). Eldin et al. (2014) tested UHP-SFRC specimens containing steel fibers with an aspect ratio of 30 and 50 respectively, and they found that fibers with an aspect ratio of 50 provided greater results in terms of compressive strength, splitting tensile strength and flexural strength. 
The relationship between the aspect ratio of fibers and flexural strength can be explained by the degree of fiber bridging effect: there is an increased number of fibers present in concrete when fibers with smaller diameters (i.e., higher aspect ratio) are utilized, which promotes fiber bridging effect and flexural strength (Ye et al. 2012). However, previous studies indicated that fibers with low aspect ratio improved workability (Abbas et al. 2016). Wille et al. (2011) reported that steel fibers with an aspect ratio of 40 were used up to $10 \%$ by unit volume without causing workability issue, whereas the maximum volumetric ratio of fibers with an aspect ratio of 80 without causing workability issue was $3 \%$.

\subsubsection{Volumetric Ratio of Reinforcing Fibers}

Based on a total of 24 UHP-SFRC mixtures, Maca et al. (2012) recommended that $2 \%$ to $3 \%$ volume fraction of steel fibers was optimal in terms of mechanical properties and workability. In addition, Eldin $e t$ al. (2014) reported that regardless of the aspect ratio of fibers, specimens containing 3\% steel fibers by unit volume exhibited higher strengths under splitting tensile and flexural loading conditions than those containing lower volume fraction of fibers. Moreover, many researchers indicated that the compressive strength of UHPFRC did not increase with the volumetric ratio of steel fibers. To the contrary, specimens with higher steel fiber content are prone to fiber clotting which develops weak spots, reduces fiber efficiency and decreases compressive strength (Reda et al. 1999; Schmidt et al. 2003). Furthermore, the presence of fibers in UHPFRC not only improves mechanical properties but also impacts the failure mode of concrete. Graybeal (2006) reported that fiber reinforced specimens subjected to compression exhibited a ductile failure mode (i.e., the specimen held its integrity beyond failure) rather than failing explosively as observed from conventional concrete. In addition, Kazemi and Lubell (2012) observed that owing to the bridging effect of fibers, flexural prisms exhibited multiple micro-cracks prior to the formation of a localized vertical macro-crack, which resulted in a steady drop in load capacity rather than a sudden drop after the formation of the first crack.

\subsubsection{Effect of Casting Methodology on Fiber Orientation}

Lappa et al. (2004) reported that pouring concrete from one end of the mold provided a flexural strength which was up to $56 \%$ greater than that of pouring concrete at different locations because the degree of fiber alignment became more prominent along the flow direction. Furthermore, it was observed that prisms cast at the middle of the mold exhibited 16\% lower flexural strength than those cast from the mold end (Yang et al. 2010). In addition, owing to fiber orientation, prisms cast with lower chute speed $(0.13 \mathrm{~m} / \mathrm{s})$ exhibited lower flexural strength than those cast with higher speed $(0.5 \mathrm{~m} / \mathrm{s})$ : high chute speed enabled the formation 
of thin layers and stronger fiber alignment along the prism axis, whereas low chute speed led to thick layers and vertical fiber alignment (Wille and Parra-Montesinos 2012). With regard to compressive strength, the effect of casting methodology is not obvious. Steil et al. (2004) reported that the difference in compressive strength results of cube specimens subjected to load perpendicular and parallel to the casting direction was less than $2 \%$.

\subsubsection{Effect of Brass-Coated Steel Fibers}

Brass-coated steel fibers with a modulus of elasticity of $200 \mathrm{GPa}$ and a tensile strength of $2000 \mathrm{MPa}$ are most commonly used in UHP-SFRC. The coating was introduced to facilitate cutting the steel wire into fibers. In addition, experimental results indicated that brass-coated steel fibers had better fiber-matrix adhesion than regular steel fibers (Chan and Li 1997). According to pullout tests conducted by Wille and Naaman (2013) of brass-coated straight steel fibers which were $13 \mathrm{~mm}$ in length and $0.2 \mathrm{~mm}$ in diameter, the obtained response curves provided a very ductile behavior with a developed fiber stress exceeding 1200 $\mathrm{MPa}$, which was equivalent to a peak bond stress of 9.2 $\mathrm{MPa}$ for an anchorage length of $6.5 \mathrm{~mm}$. As illustrated in Figure 2-3, the pullout behavior was detected by the abrasion on the lateral surfaces of coated steel fibers. Moreover, local deformations at the cutting points might also contribute to capacity (Wille and Naaman 2010).

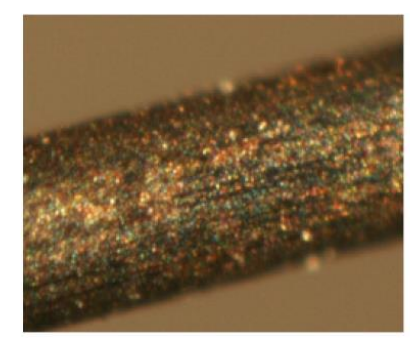

(a)

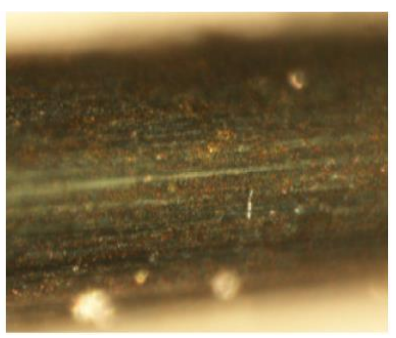

(b)

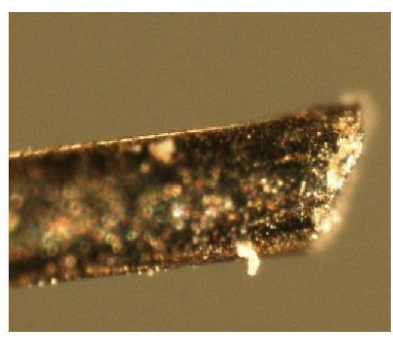

(c)

Figure 2- 3: Brass-coated steel fibers: (a) Non-abraded fiber; (b) Partially abraded fiber; and (c) The cutting point of the fiber (Wille and Naaman 2010) 


\subsubsection{Curing Condition}

In accordance with ASTM C192 (2002) and ASTM C1856 (2017), UHPFRC specimens should be cured in water storage tanks at a temperature of $23 \pm 2^{\circ} \mathrm{C}$. However, other curing conditions are also permitted.

Heat treatment (HT) (i.e., steam curing at elevated temperatures) is usually employed to gain high strength at an early age ( $<7$ days). It was observed that specimens steam-cured at $90^{\circ} \mathrm{C}$ for 48 hours developed their full compressive strength at the age of four days (FHWA-HRT-13-060 2013). Kang et al. (2017) cured UHPFRC specimens under various conditions: HT temperature ranged from $20^{\circ} \mathrm{C}$ to $90^{\circ} \mathrm{C}$, and $\mathrm{HT}$ duration ranged from 12 hours to 96 hours. They reported that the increase in both HT temperature and duration resulted in accelerated cement hydration and pozzolanic reaction, and reduced pore size less than $100 \mathrm{~nm}$. In addition, $\mathrm{HT}$ temperature of $90^{\circ} \mathrm{C}$ led to no increase in compressive strength between 7 days and 28 days, whereas HT temperature of $60^{\circ} \mathrm{C}$ allowed a steady increase in strength until 28 days.

Pressure application is another way to improve the properties of UHPFRC. Justs et al. (2011) applied a pressure between 0 to $50 \mathrm{MPa}$ to UHPFRC cylinders during the initial hardening phase, and they found that $10 \mathrm{MPa}$ led to $31.5 \%$ increase in compressive strength, and $50 \mathrm{MPa}$ led to $48 \%$ increase. In addition, the porosity of cylinders decreased up to $7.8 \%$, indicating pressure application can effectively eliminate pores, remove excess capillary water, and improve density and the compressive strength of UHPFRC (Justs et al. 2011).

\subsection{Properties of UHPFRC}

\subsubsection{Compressive Strength}

The high density and less porous microstructure of UHPFRC enable the superior compressive strength, and the addition of fibers improves its lateral strain tolerance and thereby also contributing to compressive strength (Kazemi and Lubell 2012; Hassan et al. 2012; Orgass and Klug 2004; Magureanu et al. 2012; Ye et al. 2012-UHPC). In addition, Skazlic et al. (2008) reported on a specimen size effect on the compressive strength of UHPFRC: $70 \mathrm{~mm}$ by $140 \mathrm{~mm}$ cylinders exhibited a compressive strength which was $21 \%$ higher than $100 \mathrm{~mm}$ by $200 \mathrm{~mm}$ cylinders. On account of the high compressive strength, AFGC-SETRA (2002) allows UHPFRC specimens to be tested under compression with a loading rate ranging between $0.24 \mathrm{MPa} / \mathrm{s}$ to 1.7 MPa/s. Furthermore, Kazemi and Lubll (2012), and Graybeal and Hartmann (2003) reported that a loading rate up to $1 \mathrm{MPa} / \mathrm{s}$ can be utilized without significantly affecting the compressive results of 
UHPFRC. Moreover, the minimum compressive strength requirement of UHPFRC is 130MPa in AFNOR NF EN1370/CN (2013) and 120MPa in Annex 8.1 of CSA-S6 (2018) and Annex U of CSA-A23.1 (2019).

\subsubsection{Modulus of Elasticity}

Shehata and Thomas (2000) concluded that the elastic modulus (E) of UHPFRC was generally around 50 GPa. In addition, Yoo et al. (2013) reported that the elastic modulus in compression increased with steel fiber content up to $3 \%$, whereas it showed a downtrend for specimens containing more than $3 \%$ steel fibers. In addition, Bonneau et al. (1996) reported that the modulus of elasticity increased $7 \%$ by adding $2 \%$ steel fibers by unit volume. Moreover, the use of SF and shrinkage reducing admixture do not significantly affect the elastic modulus values of UHPFRC (Rangaraju et al. 2013; Li et al. 2014). Furthermore, Equation 2-1 to Equation 2-4 present the empirical relationships between the compressive strength and modulus of elasticity of UHPFRC proposed by different researchers (Ma and Schneider 2002; Sritharan et al. 2003; Ma et al. 2004; Graybeal 2007).

$E=16364 \ln \left(f_{c}^{\prime}\right)-34828$

$E=4150 \sqrt{f_{c}^{\prime}}$

$E=19000 \sqrt[3]{f_{c}^{\prime} / 10}$

$E=3840 \sqrt{f_{c}^{\prime}}$

\subsubsection{Flexural Strength}

Kazemi and Lubell (2012), Magureanu et al. (2012), and Wille and Parra-Montesinos (2012) reported that prism size affected fiber orientation and flexural capacity, known as wall effect. Fiber orientation near the mold surfaces shows two-dimensional patterns, whereas it shows three-dimensional patterns away from the mold surfaces (Reineck and Greiner 2004). The two-dimensional pattern is preferred because it promotes fiber orientation (i.e., an increased number of fibers are perpendicular to the failure plane under bending tests), and it is more prominent in relatively small prisms and thereby resulting in higher flexural strengths. Results obtained by Wille and Parra-Montesinos (2012) indicated that prisms with $100 \mathrm{~mm}$ by $100 \mathrm{~mm}$ cross-sectional dimensions exhibited 6\% higher flexural strength than those with $150 \mathrm{~mm}$ by $150 \mathrm{~mm}$ crosssectional dimensions. In addition, flexural capacity is dependent on support conditions. Wille and ParraMontesinos (2012) reported that flexural strength results obtained by using high-frictional supports were not comparable with those obtained by using low-frictional supports because frictional force introduced additional internal moment. 


\subsubsection{Dynamic and Impact Resistance}

UHPFRC enables higher energy dissipation under impact loads compared with conventional concrete. Sun and Jiao (2011) indicated that the impact axial tensile strength of UHP-SFRC was 1.5 times greater than that of conventional concrete. In addition, Astarlioglu and Krauthammer (2014) reported that columns constructed with UHPFRC exhibited 30\% smaller displacement and four times higher loading capacity compared with those constructed with conventional concrete under blast loading according to a single degree of freedom analysis. Moreover, UHPFRC structural elements subjected to blast loading did not present fragmentation or spalling issues and were able to mitigate global structural damage (Millon et al. 2012).

\subsubsection{Porosity and Air Content}

Depending on the mix design, Wille et al. (2011) reported that the air content of UHPFRC ranged from $0.3 \%$ to $5.4 \%$, and Graybeal (2006) reported that Ductal ${ }^{\circledR}$ proprietary UHPFRC had an air content of $5.7 \%$ to $7.3 \%$. In addition, Ingo et al. (2004) pointed that the ring-type mixer with high shear forces resulted in lower air content compared with the laboratory mixer with high mixing speed. Moreover, the pore size of UHPFRC was reported to be less than $5 \mathrm{~nm}$ (Vernet 2004; Heinz and Ludwig 2004; Teichmann and Schmidt 2004; Herold and Muller 2004), and it was observed that heat and pressure treatment could significantly reduce the porosity of UHPFRC: porosity decreased from $8.4 \%$ to $1.5 \%$ due to heat treatment, and reduced $50 \%$ due to the applied pressure (Cwirzen 2007; Herold and Muller 2004; Cheyrezy et al. 1995).

\subsubsection{Permeability}

UHPFRC has a water absorption capacity 60 times less than conventional concrete (Schmidt and Fehling 2005; Ghafari et al. 2012; Roux et al. 1996; Pierard and Cauberg 2009), and its water permeability coefficient and water sorptivity coefficient are much lower compared with HPC and conventional concrete, which are around 0.0005 and $0.044 \mathrm{~kg} / \mathrm{m}^{2} / \mathrm{h}^{0.5}$, respectively (Wang et al. 2015; Ghafari et al. 2012). In addition, UHPFRC possesses limited oxygen permeability which is about $10^{-9} \mathrm{~m}^{2}$ (Vernet 2004 and Wang et al. 2015), and no carbon dioxide penetration was observed after 90 days of exposure (Roux et al. 1996). After six months of exposure, the carbonation depth of UHPFRC was found to be $0.5 \mathrm{~mm}$, and it increased to $1.5 \mathrm{~mm}$ after three years of exposure, which was still 4.5 times less than conventional concrete (Schmidt et al. 2003; Perry and Zakariasen 2004). 


\subsubsection{Chloride Ion Penetration}

As reported by Roux et al. (1996), the chloride diffusion coefficient of UHPFRC was around $2 \times 10^{-14} \mathrm{~m}^{2} / \mathrm{s}$ which was much lower than HPC $\left(6 \times 10^{-13} \mathrm{~m}^{2} / \mathrm{s}\right)$ and conventional concrete $\left(10^{-12} \mathrm{~m}^{2} / \mathrm{s}\right)$. ASTM C1202 (2012) allows to evaluate chloride ion penetration in terms of the number of coulombs passing through the specimen, referred to as rapid chloride ion penetrability test. Graybeal (2006) and Alkaysi et al. (2016) reported that this test methodology can be adopted for UHP-SFRC because steel fibers were short and distributed discontinuously, and electric short circuiting was not observed. In addition, Alkaysi et al. (2016) reported that UHPFRC had negligible chloride ion penetration, indicated by a coulomb value less than 100 coulombs.

\subsubsection{Freeze-Thaw and Salt Scaling Resistance}

Graybeal (2006) and Bonneau et al. (2000) concluded that UHPFRC possesses exclusive freeze-thaw and salt scaling resistance owing to its limited porosity and interconnected pores. The research carried out by Alkaysi et al. (2016) reported that no deterioration of UHPFRC was observed after exposing to freezingthawing conditions for 300 cycles. In addition, Alkaysi et al. (2016) studied the effect of cement type and silica powder content on the resistance of UHPFRC to freeze-thaw cycles. In this study, three types of cement were utilized, which were Portland Type I, Portland Type V and a 50:50 mixture of Portland Type I with GGBS; and silica-to-cement ratio by weight ranged between $0 \%$ to $25 \%$. They found that all mixtures exhibited exceptional freeze-thaw resistance, indicating cement type and silica powder content did not affect the long-term performance of UHPFRC. Moreover, Vernet (2004) indicated that UHPFRC did not experience significant deterioration after exposing to 500 freeze-thaw cycles along with 4500 wettingdrying cycles. Additionally, Juanhong et al. (2009), and Shaheen and Shrive (2006) indicated that there was no significant length or weight change of UHPFRC specimens after exposing to 300 freeze-thaw cycles. In terms of salt scaling resistance, Bonneau et al. (1997), and Perry and Zakariasen (2004) reported that surface scaling (i.e., mass loss) due to de-icing salts after exposing to 50 freeze-thaw cycles was $8 \mathrm{~g} / \mathrm{m}^{2}$ to $60 \mathrm{~g} / \mathrm{m}^{2}$. 


\subsubsection{Fire Resistance}

Owing to UHPFRC's less porous microstructure, vapor pressure accumulates within the material at elevated temperatures, and may result in physical damages, such as concrete spalling (Way and Wille 2012). Therefore, many researchers engaged in mitigating this issue, and observed that polypropylene (PP) fibers melted at high temperatures and left space for internal pressure release (Schmidt et al. 2004; Heinz and Ludwig 2004). In addition, Annex U of CSA-A23.1 (2019) classifies UHPFRC into three groups depending on its PP fiber content: Class FN is non-fire-exposed UHPFRC containing no PP fiber; Class F1 is fireexposed UHPFRC containing at least $0.2 \%$ PP fibers by unit volume; and Class F2 requires the UHPFRC which is expected to withstand a hydrocarbon fire contains at least $0.6 \%$ PP fibers with an aspect ratio greater than 65 .

\subsubsection{Shrinkage}

ASTM C596 (2018) and ASTM C157 (2017) are the mostly referred standards to measure drying shrinkage owing to the loss of moisture from concrete. Previous studies indicated that the addition of steel fibers and shrinkage reducing admixture significantly reduced the drying shrinkage of UHPFRC (Li et al. 2014). In addition, Russell and Graybeal (2013) reported that $75 \mathrm{~mm}$ by $75 \mathrm{~mm}$ by $285 \mathrm{~mm}$ UHPFRC specimens cured under ambient temperature had a drying shrinkage of 555 micro-strains. Another type of shrinkage is autogenous shrinkage resulting from the loss of water during the hydration process. A study in accordance with ASTM C1698 (2014) showed that the use of SF, steel fibers and shrinkage reducing admixture can effectively reduce the autogenous shrinkage of UHPFRC, and the ultimate autogenous shrinkage was 290 micro-strains two days after casting under ambient temperature curing condition (Russell and Graybeal 2013).

\subsubsection{Creep}

Depending on curing method, Graybeal (2006) indicated that the specific creep of $102 \mathrm{~mm}$ by $203 \mathrm{~mm}$ UHPFRC cylinders was between 0.04 millionth/psi to 0.15 millionth/psi after one-year loading, which was much lower than that of conventional concrete ( 0.25 millionth/psi to 1 millionths/psi). In addition, studies showed that creep coefficients decreased with the age of loading and increased with specimen size, which was similar to what had been observed from conventional concrete (Russell and Graybeal 2013). 


\subsection{Tensile Behaviour of UHPFRC}

In structural design, tensile strength is always assumed to be zero for conventional concrete's flexural design. However, due to the addition of fibers, UHPFRC possesses much higher tensile strength even after the formation of the first crack. Thus, it is necessary to consider the tensile strength of UHPFRC in designs. The following sections focus on introducing the classification of UHPFRC based on its tensile properties and presenting various types of tension tests standardized in codes or proposed by other researchers.

\subsubsection{UHPFRC Classification}

As depicted in Figure 2-4, Naaman and Reinhardt (2006) proposed the general classification of fiberreinforced concrete (FRC) based on its tensile stress-strain and load-deflection responses. FRC can be generally divided into two groups according to its stress-strain response in tension, which are strainhardening and strain-softening materials. In addition, strain-softening material comprises two subclassifications according to its load-deflection response in bending, which are deflection-hardening and deflection-softening materials. It is worth noting that deflection-hardening material can fall into either strain-hardening or strain-softening category, and strain-softening material may exhibit deflectionsoftening response, whereas strain-hardening material exhibits deflection-hardening response in bending.

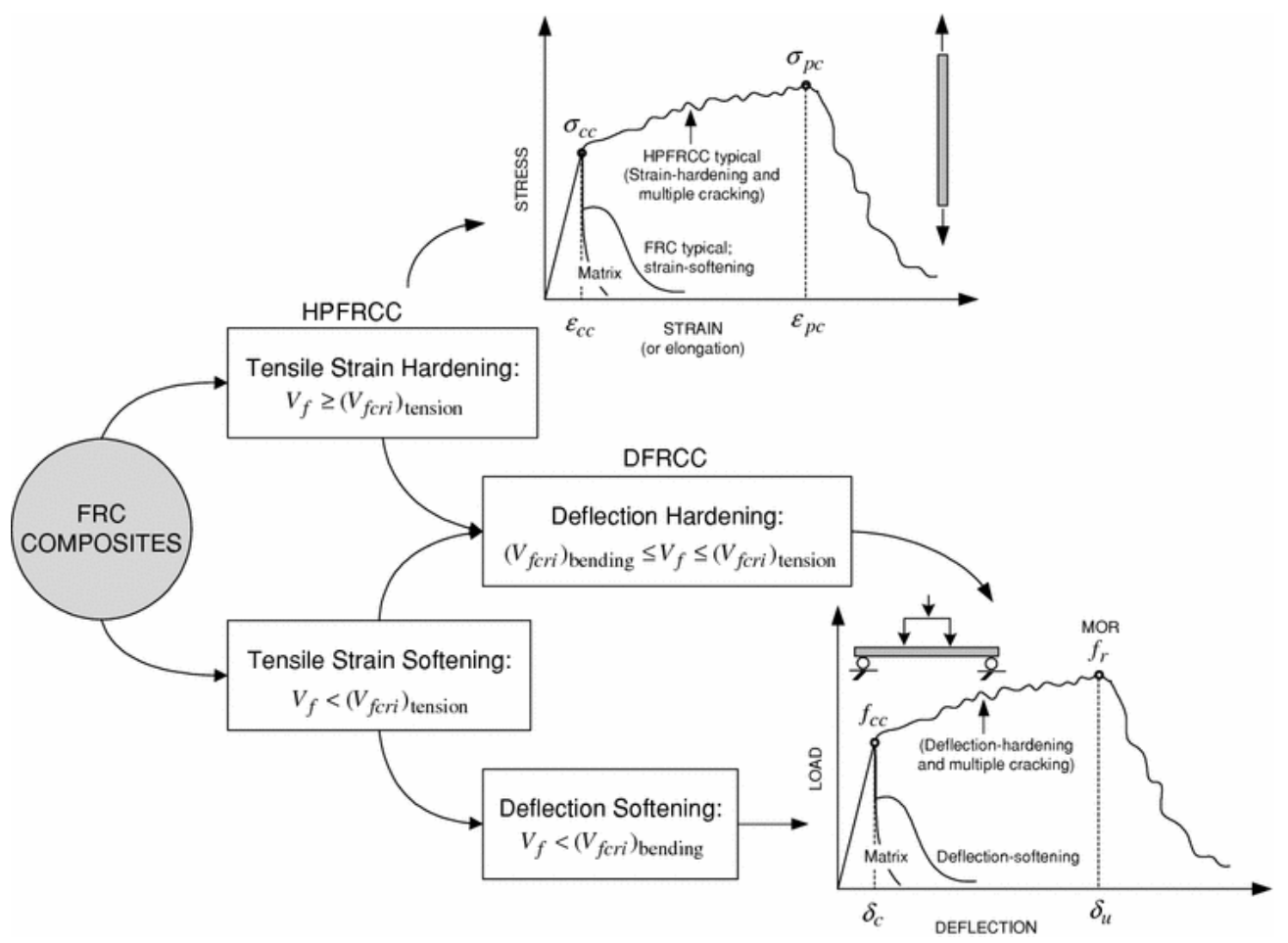

Figure 2- 4: General classification of FRC (Naaman and Reinhardt 2006) 
In light of the FRC classification, there are several classification criteria proposed specifically for UHPFRC. Naaman and Reinhardt (2006) classified the UHPFRC exhibiting strain-hardening behaviour into five groups based on its ultimate tensile strength, which are T-2.5, T-5, T-10, T-15 and T-20 (T represents tension, followed by the value of ultimate tensile strength). In addition, Naaman and Reinhardt (2006) proposed two criteria of strain-hardening material: 1) The modulus of elasticity should be greater than 10.5 $\mathrm{GPa}$; and 2) The ultimate tensile strain upon crack localization should be greater than $0.5 \%$. The second criterion is controversial due to the fact that $0.5 \%$ appears to be on high side.

As illustrated in Figure 2-5, Wille et al. (2014) classified the UHPFRC exhibiting strain-hardening behaviour into four levels based on its volumetric energy absorption capacity prior to tension softening. Level 1corresponds to deflection-softening material; Level 2 corresponds to deflection-hardening material; and Level 3 and Level 4 correspond to strain-hardening material: if the material has an energy absorption capacity greater than $50 \mathrm{~kJ} / \mathrm{m}^{3}$, it falls into the Level 4 category.

(a)
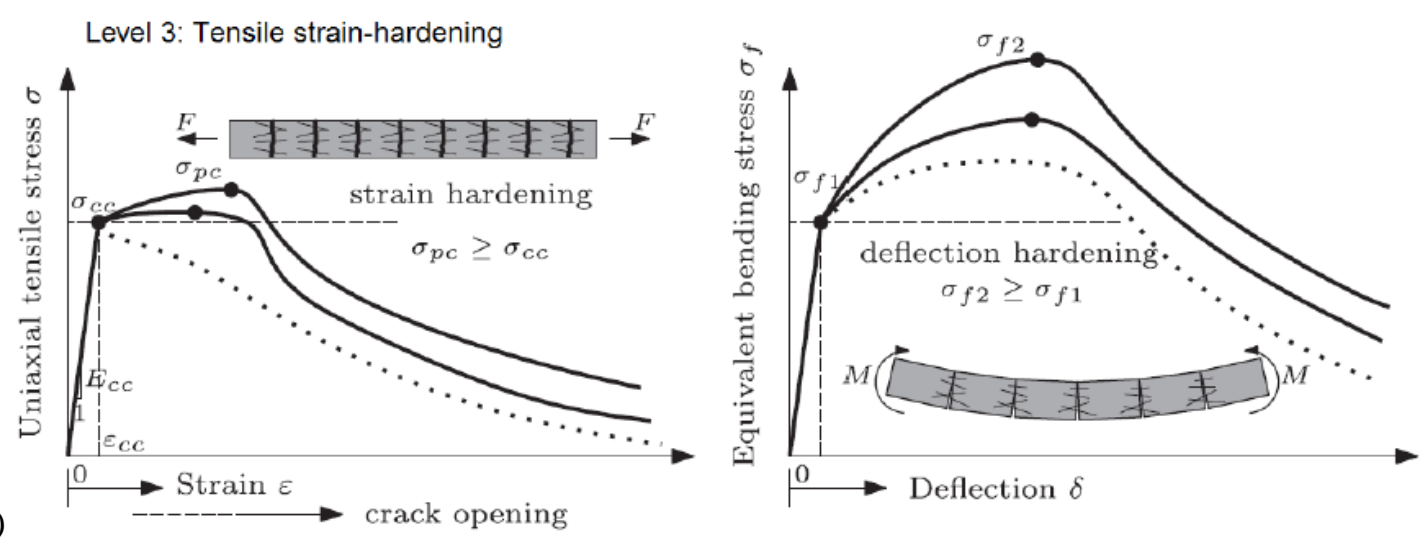

Level 4: High energy absorbing
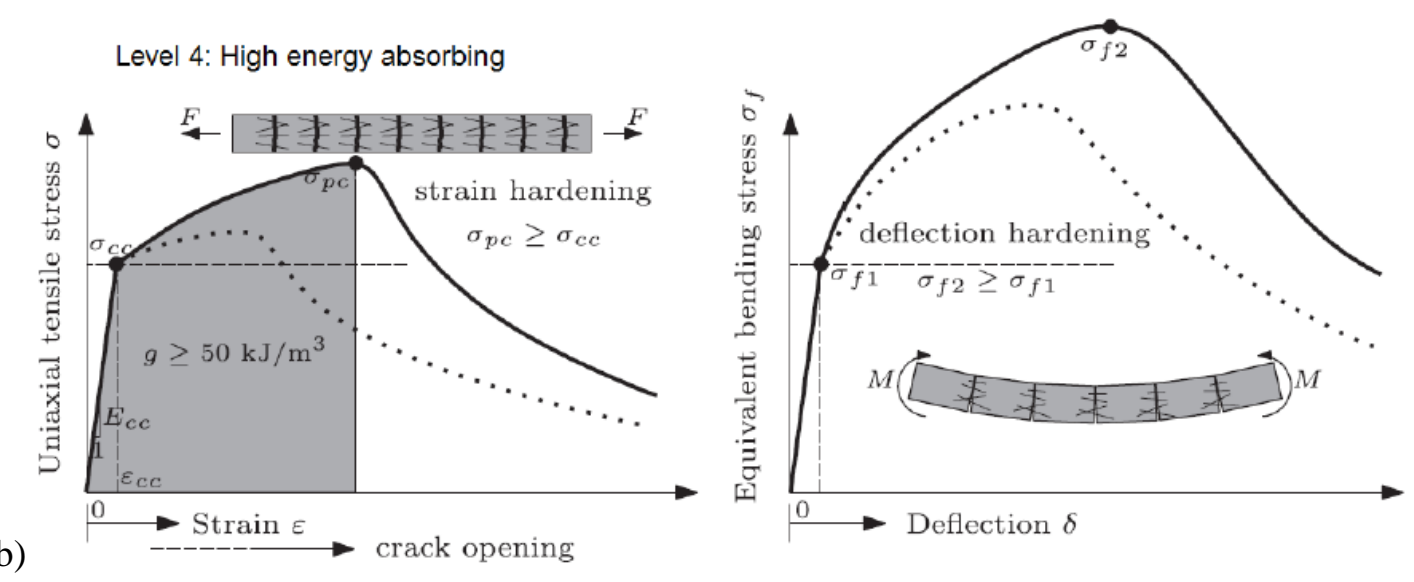

Figure 2- 5: UHPFRC classification proposed by Wille et al. (2014): (a) Level 3; and (b) Level 4 
The Canadian standards (Annex 8.1 of CSA-S6 2018; Annex U of CSA-A23.1 2019) classifies UHPFRC into two groups which are tension-softening fiber reinforced concrete (TSFRC) and tension-hardening fiber reinforced concrete (THFRC). Three criteria are proposed: 1) The cracking strength upon the formation of micro-cracks should be at least $5 \mathrm{MPa}$ for THFRC and at least $4 \mathrm{MPa}$ for TSFRC; 2) The ratio between ultimate tensile strength upon crack localization and cracking strength (i.e., hardening ratio) should be at least 1.1 for THFRC; and 3) The ultimate tensile strain should be at least $0.1 \%$ for THFRC. In addition, as presented in Equation 2-5, Annex 8.1 of CSA-S6 (2018) proposes an empirical relationship between cracking strength $\left(f_{c r}\right)$ and compressive strength $\left(f_{c}^{\prime}\right)$.

$f_{c r}=0.6 \sqrt{f_{c}^{\prime}} \leq 7.35 \mathrm{MPa}$

for TSFRC (2-5a)

$f_{c r}=$ the greater of $\left\{\begin{array}{c}\text { cracking strength obtained from direct tension test } \\ 0.6 \sqrt{f_{c}^{\prime}}\end{array}\right.$ for THFRC (2-5b)

The French standard (NF P18-470 2016) classifies UHPFRC based on the hardening ratio: if the material has a hardening ratio greater than 1.25, it falls into the strain-hardening category; otherwise, it is defined as strain-softening material.

The Swiss standard (SIA2052 2014) classifies UHPFRC into three groups (i.e., UO, UA and UB) based on its cracking strength, hardening ratio and ultimate tensile strain. The strain-softening material with at least $7 \mathrm{MPa}$ cracking strength falls into category UO; the material with at least $7 \mathrm{MPa}$ cracking strength, $0.15 \%$ ultimate tensile strain and 1.1 hardening ratio falls into category $\mathrm{UA}$; and the material with at least $8.5 \mathrm{MPa}$ cracking strength, $0.25 \%$ ultimate tensile strain and 1.2 hardening ratio falls into category UB.

\subsubsection{Direct Tension Test}

Direct tension test (DTT) is the most straightforward testing method to obtain the tensile behaviour of UHPFRC. As reported by Russell and Graybeal (2013), the cracking strength of $102 \mathrm{~mm}$ by $203 \mathrm{~mm}$ cylinders subjected to uniaxial tensile loading was 5.5 MPa to 6.9 MPa. Figure 2-6 depicts the idealized uniaxial tensile mechanical response of UHP-SFRC exhibiting strain-hardening behaviour (Russell and Graybeal 2013). The response is characterized by four phases: Phase I depicts the elastic behaviour; Phase II corresponds to cracking strength at which the formation of multiple micro-cracks takes place; Phase III corresponds to ultimate tensile strength owing to the strain-hardening behaviour of UHP-SFRC, indicating cracks widen as stress increases due to fiber bridging effect; and Phase IV depicts strain-softening behaviour, indicating macro-crack localizes and widens as stress decreases due to fiber pullout or rupture. It is evident 
that for THFRC, the limit of fiber bridging strength (i.e., ultimate tensile strength) upon crack localization is greater than the cracking strength upon the initial formation of micro-cracks (Russell and Graybeal 2013). In addition, as presented in Equation 2-6 (in psi), Russell and Graybeal (2013) proposed an empirical relationship between cracking strength $\left(f_{c r}\right)$ and compressive strength $\left(f_{c}^{\prime}\right)$.

$f_{c r}=7.8 \sqrt{f_{c}^{\prime}}$ or $8.3 \sqrt{f_{c}^{\prime}}$ depending on the stream curing method

$f_{c r}=6.7 \sqrt{f_{c}^{\prime}} \quad$ for the specimens cured in ambient laboratory temperature $(2-6 \mathrm{~b})$

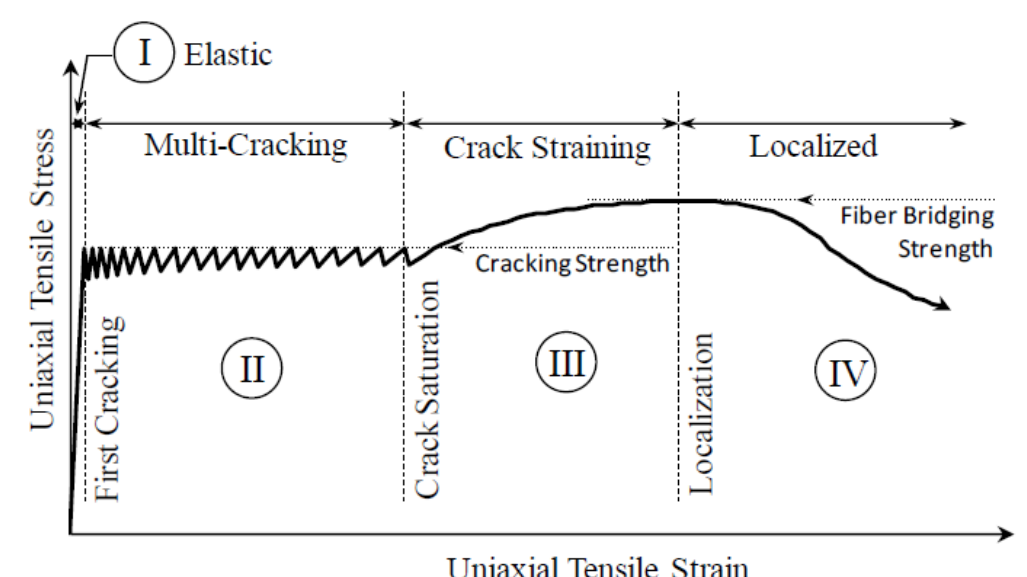

Figure 2- 6: Idealized uniaxial tensile mechanical response of UHP-SFRC proposed by Russel and Graybeal (2013)

Wille et al. (2014) indicated that for simplicity, bilinear relationships should be considered to describe the tensile behaviour of UHPFRC in accordance with the stress-strain and stress-cracking mouth opening relationships standardized in RILEM TC 162-TDF (2002; 2003c). As depicted in Figure 2-7 (the blue line), eight tensile parameters constitute the bilinear stress-strain and stress-crack mouth opening relationships, which are: 1$)$. the modulus of elasticity $(E) ; 2)$. the cracking strength $\left.\left(f_{t}\right) ; 3\right)$. the ultimate tensile strength $\left.\left(f_{t, u}\right) ; 4\right)$. the ultimate tensile strain $\left.\left(\varepsilon_{t, u}\right) ; 5\right)$. the unloading modulus $\left(E^{*}\right)$ beyond the peak load (i.e., tension softening portion); 6). the stress at which bilinear stress-crack mouth opening relationship changes slope $\left.\left(f_{t, d}\right) ; 7\right)$. the crack mouth opening at which bilinear stress-crack mouth opening relationship changes slope $\left(w_{d}\right)$; and 8$)$. the characteristic crack mouth opening when stress drops to zero $\left(w_{c}\right)$. However, according to considerable experimental results, certain assumptions were proposed to reduce the number of parameters: 1). $E$ is the same in tension and compression; 2$). w_{c}$ is equal to half of the fiber length $\left(l_{f}\right)$ (Wille et al. 2014); 3). $f_{t, d}$ is equal to $1 / 3 f_{t, u}$ (Wille et al. 2014; Nguyen et al. 2014; Graybeal et al. 2013; Mahmud et al. 2013; Tailhan et al. 2012; Kim et al. 2008; Kanakubo 2006); and 4). $E^{*}$ is equal to $10 \%$ to $20 \%$ of $E$ (Wille et al. 2014). Based on the above assumptions, there are only four parameters (i.e., $f_{t}, f_{t, u}, \varepsilon_{t, u}$ and $w_{d}$ ) to be determined from the experimental uniaxial tensile response curve. 


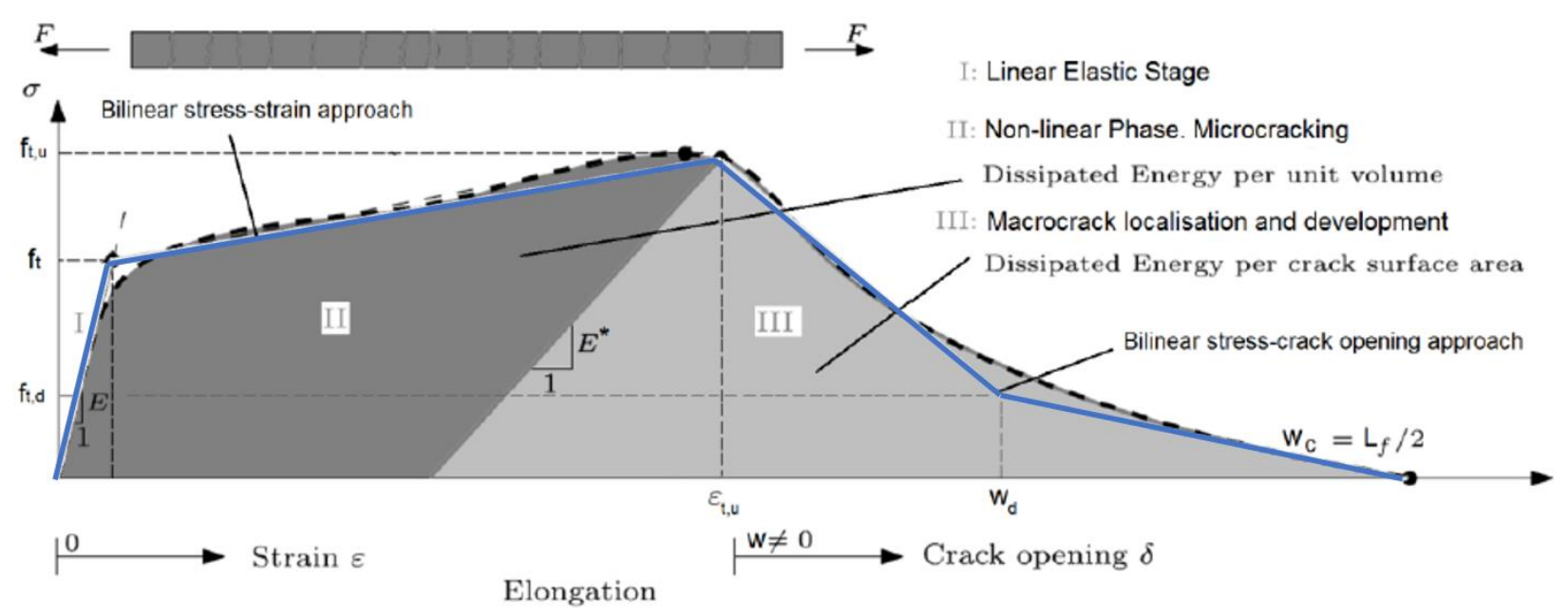

Figure 2- 7: Typical uniaxial tensile response of UHPFRC proposed by Wille et al. (2014)

Depending on the boundary conditions of the test setup, DTT can be classified into two groups. The setup allowing rotation at the ends of the specimen (i.e., pinned end condition) may only provide cracking strength because local inconsistencies in stiffness at the planes of micro-cracks can cause rotation and fiber pullout before attaining the ultimate tensile strength. The second type of boundary condition restrains rotation at cracks (i.e., fixed end condition), and allows the attainment of both cracking strength and ultimate tensile strength. However, direct tension tests are challenging to conduct with either boundary condition as the alignment between gripping devices and the specimen in the loading frame are not easy to control, and misalignment can introduce the secondary bending moments to the specimen (Russel and Graybeal 2013; Amin et al. 2019). In addition, direct tension test is sensitive to specimen imperfections and non-uniformity of the material itself (Kanakubo 2006; Ostergaard et al. 2005; Qian and Li 2007).

Three typical types of direct-tension specimens are unnotched prisms or cylinders, notched prisms or cylinders, and unnotched dog-bone shaped specimens. In addition, high-strength adhesive bonding is always applied between gripping devices and the specimen to promote the critical failure plane to occur within the gauge length. However, Graybeal and Baby (2013), and Abrishambaf et al. (2017) reported that the failure plane may localize near the adhered boundary due to stress concentration. Moreover, Graybeal and Baby (2013), Marzouk and Chen (1995), and Li and Ansari (2000) indicated that notched specimens resulted in inaccurate strength results because the notched cross section might not be the weakest plane. Furthermore, many researchers are engaging in developing various types of dog-bone shaped specimens to proof-test how specimen shape and gripping devices affect test results (Graybeal and Baby 2013; Reineck and Frettlor 2010; Denarié and Brühwiler 2015). It was observed that unnotched dog-bone shaped 
specimens with larger cross-sectional areas at the supports and a smooth geometry transition were helpful to avoid support failures and stress concentrations (Martínez 2017). Figure 2-8 depicts the dog-bone shaped specimen proposed in the Swiss standard (SIA2052 2014). The dimensions labelled in Figure 2-8 are fixed (i.e., they do not depend on fiber length). In addition, the standard requires to employ fixed end boundary condition, and aluminum transfer plates need to be bonded at the specimen ends. Figure 2-9 depicts the experimental test setup of a $50.8 \mathrm{~mm}$ by $50.8 \mathrm{~mm}$ by $431.8 \mathrm{~mm}$ unnotched prism with a gauge length of $102 \mathrm{~mm}$ subjected to uniaxial tensile loading, where aluminum gripping plates were bonded to the specimen ends by using high-strength structural epoxy, and two extensometers were installed on each side of the prism to measure displacement. Additionally, the loading rate was $0.00254 \mathrm{~mm} / \mathrm{s}$. (Graybeal and Baby 2013).

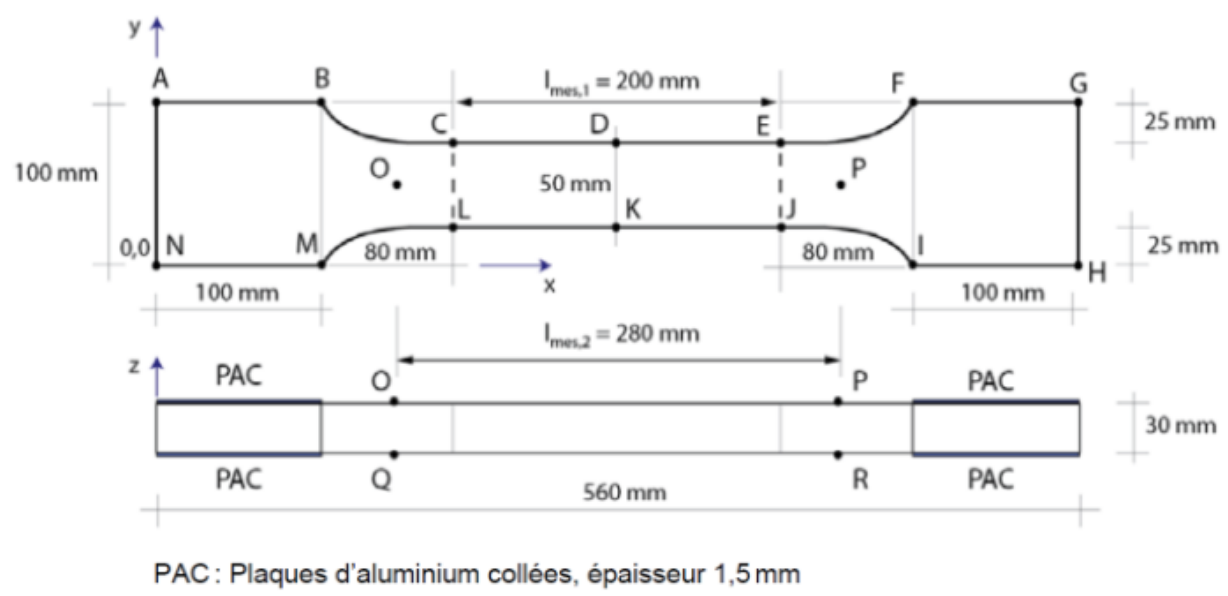

Figure 2- 8: Dog-bone shaped specimen proposed in the Swiss standard for direct tension test (SIA2052 2014)
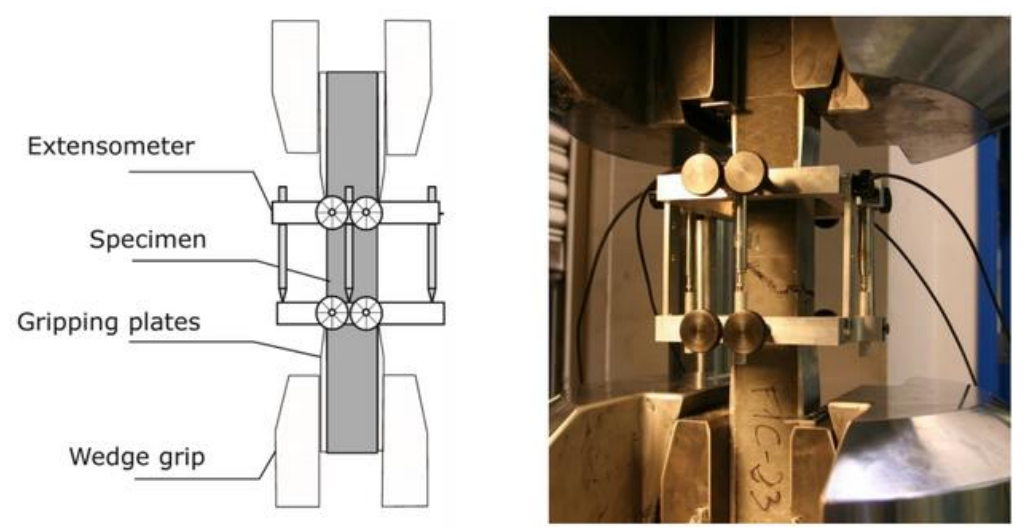

Figure 2- 9: Unnotched prism proposed by Graybeal and Baby (2013) for direct tension test

Figure 2-10 depicts the dog-bone shaped specimen and its associated test setup proposed by Chasioti and Vecchio (2017). There were two high-strength threaded steel rods embedded for a length of $225 \mathrm{~mm}$ at the ends of the specimen, and a wooden rod was placed within the gauge length. The wooden rod and partial 
threaded steel rods were connected by plastic tape to align the end rods and thereby reduce the secondary moment. In addition, two layers of steel wire were placed in the end regions to avoid failure near the end rods. Figure 2-11a depicts the dog-bone shaped specimen proposed in AASHTO T132-87 (2018). The specimen is $76 \mathrm{~mm}$ long, $25 \mathrm{~mm}$ thick, and its cross-sectional area at mid-length is $645 \mathrm{~mm}^{2}$. Moreover, special gripping devices were utilized to promote uniform loading (Plank et al. 2009). Furthermore, Figure $2-11$ b depicts a $40 \mathrm{~mm}$ by $40 \mathrm{~mm}$ prism with $5 \mathrm{~mm}$ by $5 \mathrm{~mm}$ notches subjected to uniaxial tensile loading (Kusumawardaningsih et al. 2015).

(a)

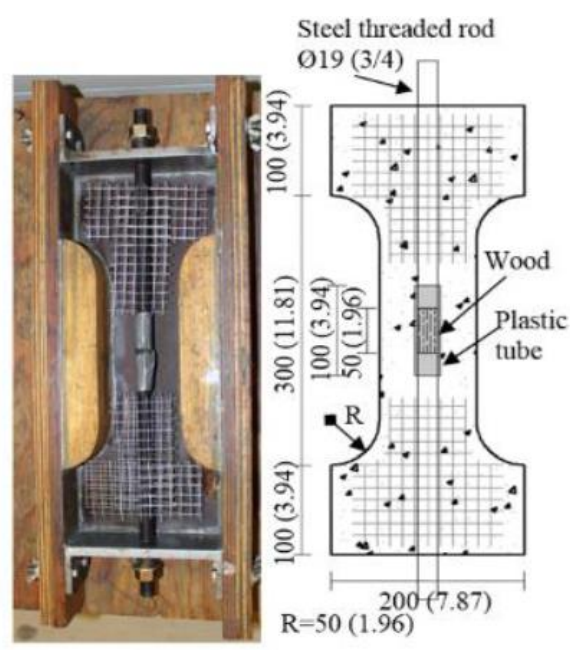

(b)

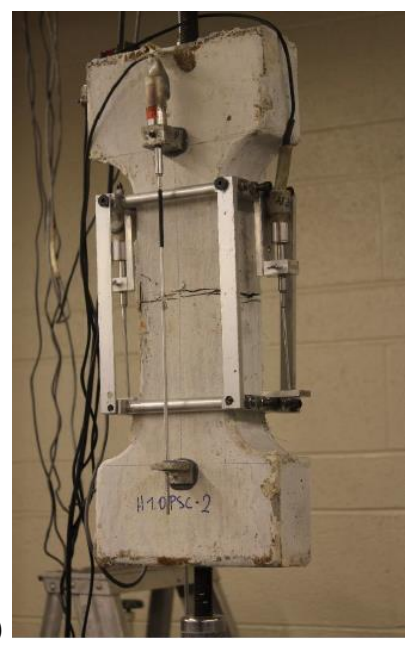

Figure 2- 10: Direct tension test setup proposed by Chasioti and Vecchio (2017): (a) Specimen shape; and (b) Test setup
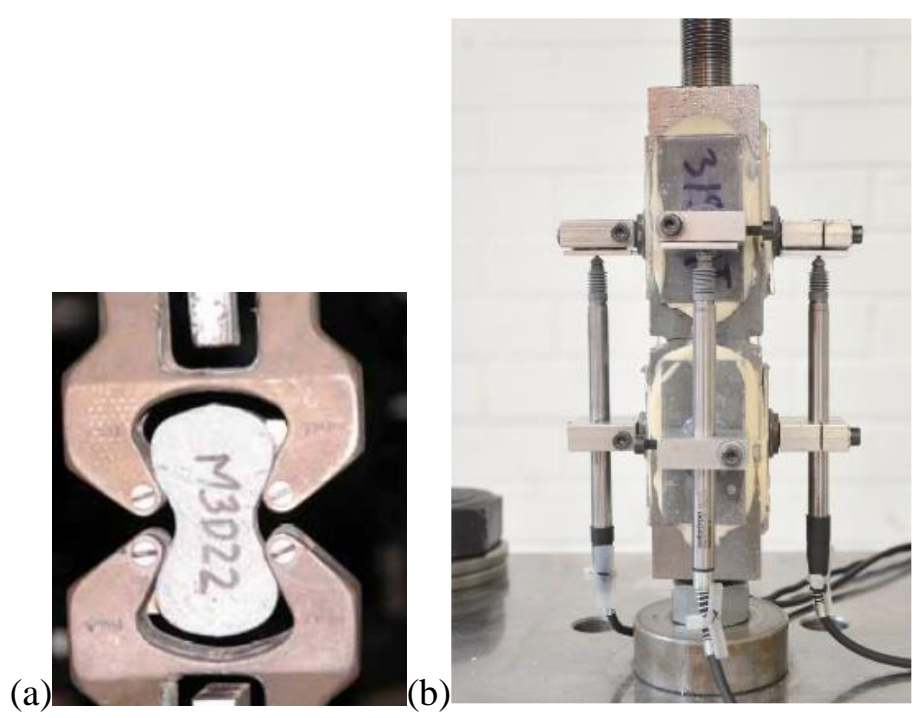

Figure 2- 11: Direct tension test setup: (a) Dog-bone shaped specimen proposed in AASHTO T132-87 (2018); and (b) The notched prism (Kusumawardaningsih et al. 2015) 


\subsubsection{Bending Test}

Bending test is relatively easy to conduct compared with DTT, but it requires particular interpretations of experimental results and usually relies on inverse analysis procedures to obtain the tensile properties of the material (Gopalaratnam and Gettu 1995). Two widely adopted bending tests are notched three-point bending test and unnotched four-point bending test (FPBT). For conventional FRC, notched three-point bending test was conducted to determine the stress-crack mouth opening relationship, and FPBT was conducted to determine the stress-strain relationship (Casanova and Rossi 1996; Chanvillard 2000).

Regarding the notched three-point bending test, a single crack is expected to form at the notch and propagates along the specimen depth after attaining material's cracking strength. In addition, as the crack propagates at notch, the rest of the specimen can be assumed to unload elastically, and the fracture energy indicated as the area below the response curve is solely attributed to the fracture along the notch. However, this assumption does not apply to UHPFRC because UHPFRC not only exhibits a macro-crack at the notch but also multiple micro-cracks near the notch. As illustrated in Figure 2-12, the multiple micro-cracks indicate plastic deformations near the notch. Moreover, the inverse analysis method proposed by RILEM TC 162-TDF (2002) does not incorporate the multiple micro-cracks phenomenon but assumes that displacement was only attributed to the single crack at the notch. In the case of UHPFRC, displacement is attributed to the combined effect of the single macro-crack at notch and the smeared micro-cracks near notch and thereby the assumption of the inverse analysis method in RILEM TC 162-TDF (2002) may overestimate the tensile behaviour of UHPFRC. In this context, notched three-point bending test may not be appropriate for testing UHPFRC.

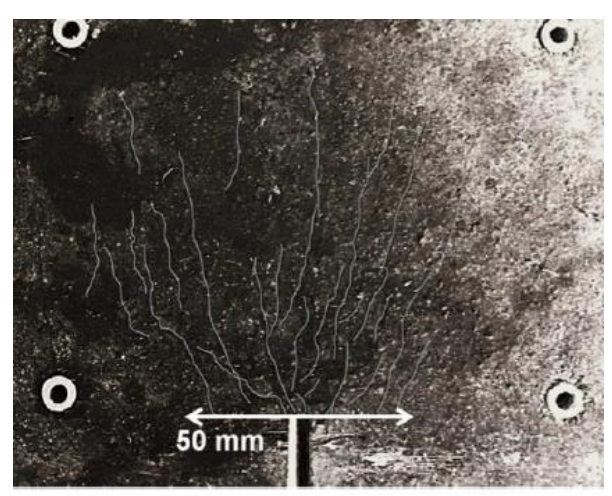

Figure 2- 12: Multiple micro-cracks of a UHPFRC specimen observed from a notched three-point bending test (Martínez 2017) 
Unnotched FPBT provides a constant bending moment region between two loading points. The point loads cause changes in stress field near the loading points (i.e., shear-moment interaction), and their effect reduces as the aspect ratio of the bending prism increases. The most widely used unnotched prism subjected to fourpoint bending has an aspect ratio of 1 , and the distance between loading rollers is equal to prism depth as well. Discrepancies in flexural strength results were observed between unnotched FPBT and notched threepoint test because the failure plane of an unnotched prism is the weakest cross section within the constant moment region, whereas the failure plane at the notch may not be the weakest one (Amin et al. 2015). In this context, unnotched FPBT provides more conservative strength results. In addition, due to the fact that cross sections within the constant moment region only subjected to axial force, the empirical relationships (as presented in Equation 2-7) describing the uniaxial tensile properties can be modified and applied to FPBT (Baby et al. 2013). The stress-crack mouth opening relationship of UHPFRC subjected to uniaxial tensile loading is calculated with Equation 2-7, where $\delta_{e l}$ is the deflection at cracking strength, $\delta_{c r}$ is the deflection at ultimate tensile strength, $\delta$ and $w$ are any deflection or crack mouth opening value within the tension softening portion, $w_{i}$ is the crack mouth opening at ultimate tensile strength, $E^{*}$ is the modulus of the tension softening portion, $\Delta \sigma$ is the tensile stress difference between any stress within the tension softening portion and ultimate tensile stress, $l_{m}$ is gauge length and $s_{a v}$ is the average crack spacing (Wille et al. 2014).

$$
\begin{aligned}
& w=w_{i}+\left(\delta-\delta_{c r}\right)-\frac{\Delta \sigma}{E^{*}} l_{m}, \text { where } \Delta \sigma<0 \\
& w_{i}=\frac{\left(\delta_{c r}-\delta_{e l}\right)}{l_{m}} S_{a v}
\end{aligned}
$$

\subsubsection{Recommended Bending Tests of UHPFRC in Standards}

As mentioned in Section 2.4.1, the French standard (NF P18-470 2016) classifies UHPFRC into two groups (i.e., strain-hardening material and strain-softening material). In addition, it proposes two different test setups for each type of material by assuming the material classification is known prior to testing. As depicted in Figure 2-13a, for the strain-softening material, an unnotched prism with an aspect ratio of 1 (the

prism depth is 5 to 7 times of fiber length) is subjected to four-point bending in order to determine the cracking strength which is assumed to be equal to the linearity limit value of the response curve; in addition, a notched prism is subjected to three-point bending in order to determine crack mouth opening values which need to be correlated with the cracking strength result obtained from FPBT; and the experimental results obtained from both tests are analyzed by using a point-by-point inverse analysis method in order to develop the bilinear stress-strain and stress-crack mouth opening relationships. For the strain-hardening material, 
the French standard only considers the elastic and strain-hardening portions. The experimental test setup of a strain-hardening prism (the prism depth is 3 times of fiber length) subjected to four-point bending is illustrated in Figure 2-13b, and the bilinear stress-strain relationship can be obtained through a point-bypoint method or a simplified inverse analysis method.

(a)
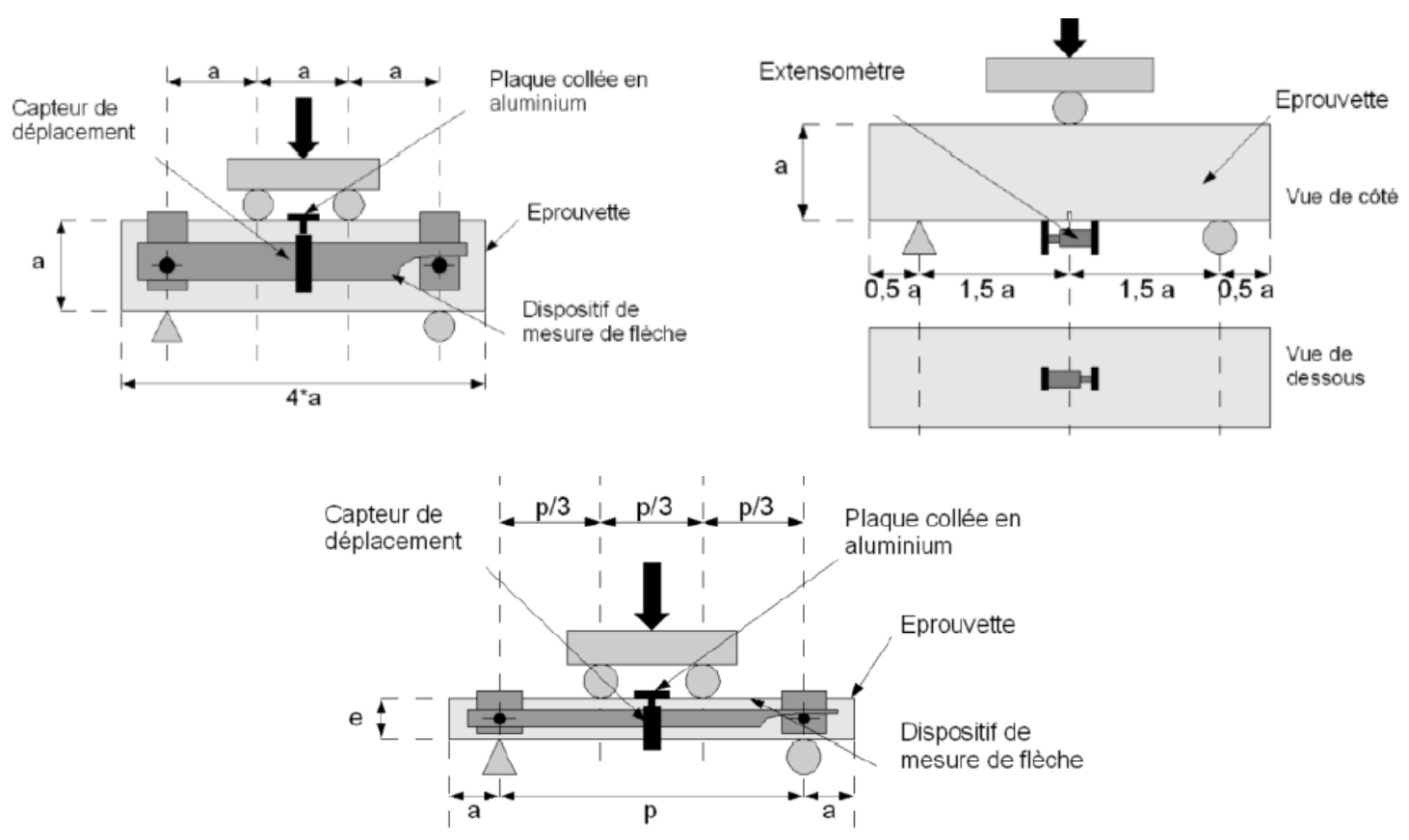

(b)

$a=\max (e / 2 ; 3 \mathrm{~cm}) ; p=L p-2 a$

Figure 2- 13: Bending test setup of UHPFRC proposed in the French standard (NF P18-470 2016): (a) Strain-

softening material; and (b) Strain-hardening material

Similar to the French standard, the Swiss Standard (SIA2052 2014) also only considers the stress-strain relationship prior to tension softening. Despite the dog-bone shaped specimen proposed for DTT (refer to Section 2.4.2), the Swiss standard also recommends obtaining the tensile behaviour of UHPFRC through FPBT. The experimental test setup is depicted in Figure 2-14, which is similar to that proposed in the French standard for strain-hardening material except for the prism size. It is worth noting that the dimensions (the prism width is $100 \mathrm{~mm}$ ) shown in Figure 2-14 are fixed and are independent on fiber length. In addition, to determine the bilinear stress-stain relationship of UHPFRC, a simplified inverse analysis method is proposed in the Swiss standard. 


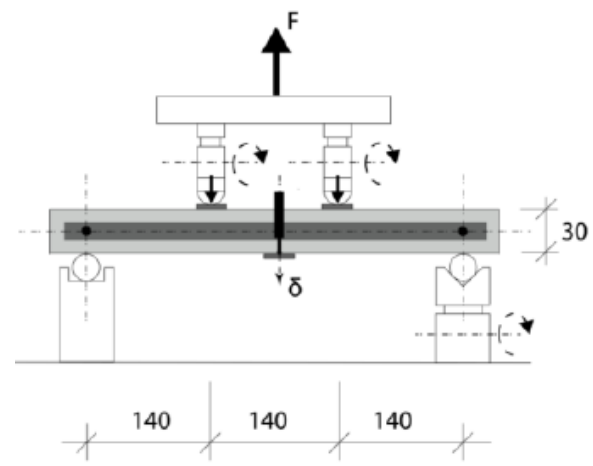

Figure 2- 14: Four-point bending test setup of UHPFRC proposed in the Swiss standard (SIA2052 2014)

Figure 2-15 illustrates the experimental test setup of FPBT of UHPFRC proposed in the Canadian standard (Annex U of CSA-A23.1 2019). The prism has an aspect ratio of 1, and the distance between loading points is equal to prism depth as well. In addition, as mentioned in Section 2.3.3, the wall effect promotes fiber alignment and results in higher flexural strength. As presented in Table 2-1, to accommodate the wall effect, Annex U of CSA-A23.1 (2019) correlates fiber length with prism size. Moreover, similar to the French and Swiss standards, the Canadian standard proposes a simplified inverse analysis method in order to obtain the bilinear stress-strain and stress-crack mouth opening relationships of UHPFRC based on the flexural response curve.

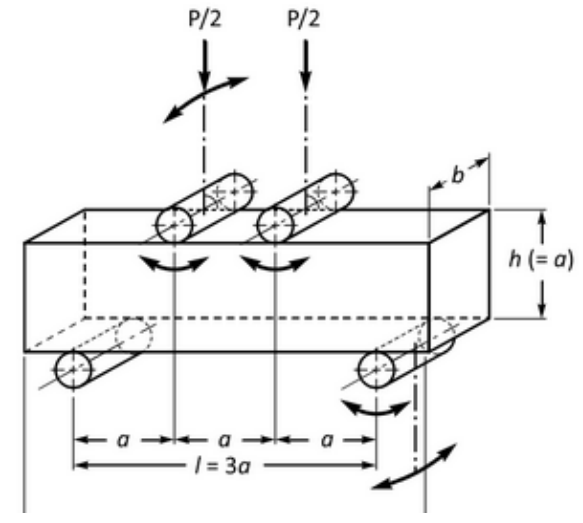

Figure 2- 15: Four-point bending test setup of UHPFRC proposed in the Canadian standard (Annex U of CSAA23.1 2019)

Table 2- 1: Proposed fiber length and prism size of four-point bending test (Annex U of CSA-A23.1 2019)

\begin{tabular}{|c|c|}
\hline Fiber Length & Nominal Prism Size \\
\hline$\leq 15 \mathrm{~mm}$ & $75 \mathrm{~mm}$ by $75 \mathrm{~mm}$ by $280 \mathrm{~mm}$ \\
\hline$>15 \mathrm{~mm}$ to $20 \mathrm{~mm}$ & $100 \mathrm{~mm}$ by $100 \mathrm{~mm}$ by $370 \mathrm{~mm}$ \\
\hline$>20 \mathrm{~mm}$ to $25 \mathrm{~mm}$ & $150 \mathrm{~mm}$ by $150 \mathrm{~mm}$ by $550 \mathrm{~mm}$ \\
\hline$>25 \mathrm{~mm}$ to $60 \mathrm{~mm}$ & $200 \mathrm{~mm}$ by $200 \mathrm{~mm}$ by $720 \mathrm{~mm}$ \\
\hline
\end{tabular}




\subsubsection{Proposed Inverse Analysis Methods}

There are several inverse analysis methods standardized in codes or proposed by researchers in order to determine the tensile behaviour of UHPFRC based on the response curve obtained from bending tests. The inverse analysis methods can be divided into two groups: the methods relying on the key points of the experimental response curve are referred as simplified methods; and the others relying on the complete experimental response curve is referred as iterative methods or point-by-point methods. The iterative methods require computational analysis effort, which is time-consuming and sophisticated. Therefore, simplified methods are preferred. However, all the inverse analysis methods have a point in common: fiber orientation is not taken into consideration. The following section focuses on introducing different simplified inverse analysis methods.

Figure 2-16 depicts the experimental test setup of an unnotched FPBT proposed by Kanakubo (2006), where three linear displacement transducers were utilized to measure the midspan deflection, and another two linear displacement transducers were utilized to determine the average curvature. In addition, as presented in Figure 2-17, the stress distribution along prism depth is divided into two stages: the first stage associates with cracking strength $\left(f_{t}\right)$, elastic curvature $\left(\phi_{e l}\right)$, cracking strain $\left(\varepsilon_{t}\right)$, and the bending moment $\left(M_{e l}\right)$ upon reaching the cracking strength; and the second stage associates with ultimate tensile strength $\left(f_{t, u}\right)$, maximum curvature $\left(\phi_{\max }\right)$, ultimate tensile strain $\left(\varepsilon_{t, u}\right)$, and the maximum bending moment $\left(M_{\max }\right)$. Moreover, a linear elastic stress distribution in compression is assumed for both stages. Furthermore, Kanakubo (2006) proposed two cases for the second stage: Case 1 assumed the tensile stress linearly increased from neutral axis to tension face, whereas Case 2 assumed uniform stress distribution in tension. Regarding Case 1, $f_{t}$ and $\varepsilon_{t}$ can be determined with Equation 2-8. Moreover, $f_{t, u}$ and crack depth $(h-x)$ can be determined with Equation 2-9. Additionally, $\varepsilon_{t, u}$ can be obtained from Equation 2-10. With regard to Case 2, $f_{t, u}$ and crack depth (h-x) should be calculated with Equation 2-11, whereas the calculations of $f_{t}, \varepsilon_{t}$ and $\varepsilon_{t, u}$ remain the same as Case 1. It is evident that this inverse analysis method only provides stress-strain relationship of UHPFRC, but not the stress-crack mouth opening relationship.

$\begin{aligned} \varepsilon_{t} & =\frac{h \phi_{e l}}{2} \\ f_{t} & =\frac{6 M_{e l}}{b h^{2}}\end{aligned}$

$\sum N=0 \rightarrow \frac{E b \phi_{\max }}{2} x^{2}=\frac{b}{2}\left(f_{t}+f_{t, u}\right)(h-x)$

$M_{\max }=\frac{E b \phi_{\max }}{3} x^{3}+\frac{b}{6}\left(f_{t}+2 f_{t, u}\right)(h-x)^{2}$ 
$\varepsilon_{t, u}=\phi_{\max }(h-x)$

$\sum N=0 \rightarrow \frac{E b \phi_{\max }}{2} x^{2}=b f_{t, u}(h-x)$

$$
M_{\max }=\frac{E b \phi_{\max }}{3} x^{3}+\frac{b f_{t, u}}{2}(h-x)^{2}
$$

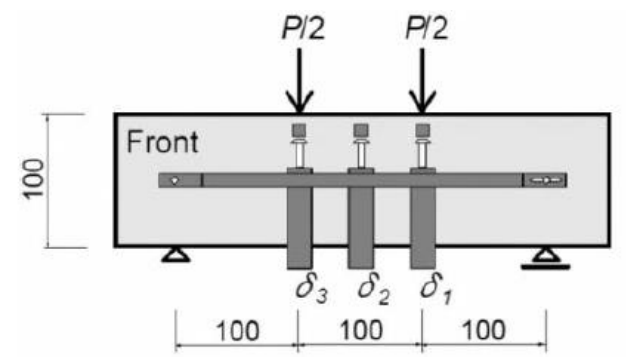

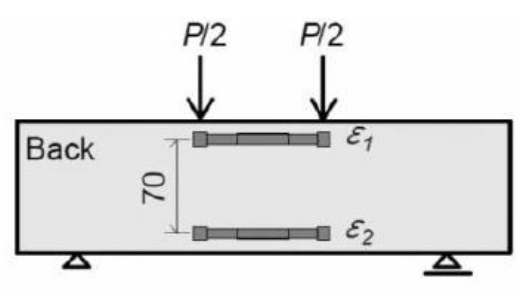

Figure 2- 16: Experimental test setup of the unnotched four-point bending test proposed by Kanakubo (2006)

Stage 1

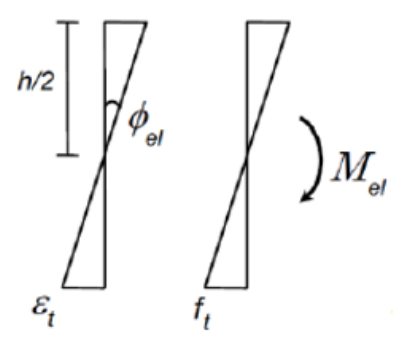

Stage 2-Case 1

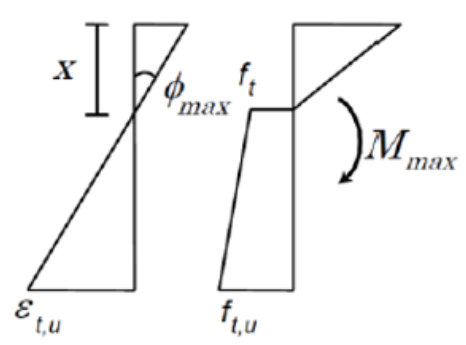

Stage 2-Case 2

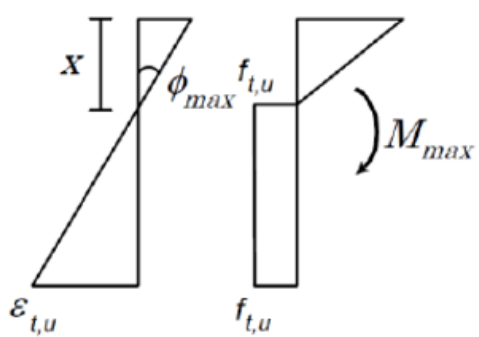

Figure 2- 17: Stress distribution considered for the inverse analysis method proposed by Kanakubo (2006)

Figure 2-18a depicts the bilinear tensile stress-strain relationship of the strain-hardening UHPFRC obtained from the simplified inverse analysis method proposed in the French standard (NF P18-470 2016), where cracking strength is assumed to be equal to the ultimate tensile strength $\left(f_{\text {ctf }}\right), \varepsilon_{\text {lim }}$ is the ultimate tensile strain, $\varepsilon_{e l}$ is the elastic tensile strain (i.e., cracking strain), and $E$ is the elastic modulus in tension which is assumed to be equal to the elastic modulus in bending. As illustrated in Figure 2-18b, $E$ can be determined based on the moment $(M)$-deflection $(f)$ curve obtained experimentally. $M^{*}$ is the bending moment corresponding to linearity limit of the response curve, and it is equal to $3 \Delta M$. $E$ is equal to the slope of the central third part multiplied by a factor of $\left[\left(23 p^{2}\right) /\left(216 b e^{3} / 12\right)\right]$, where $p, b$ and $e$ are prism span, width and depth, respectively. In addition, the maximum curvature $(\chi)$ can be calculated with Equation 2-12 based on an elastic relationship. Moreover, Figure 2-19 depicts the stress distribution along prism depth considered for this inverse analysis method. Based on the stress distribution diagram, axial force $(N)$ and bending 
moment $(M)$ can be expressed with Equation 2-13. For $N$ set equal to zero, $f_{c t} *$ can be solved in terms of $b$, $e, \alpha, E$ and $\chi$. Equation 2-14 can be derived by substituting $f_{c t f^{*}}$ into Equation 2-13b; and $\alpha$ can be calculated with Equation 2-14. Additionally, $f_{c t f^{*}}$ and $\varepsilon_{\text {lim }}$ can be calculated with Equation 2-15, and $\varepsilon_{e l}$ can be determined by knowing $E$ and $f_{c t \text { ** }}$.

$\delta=\frac{23}{216} \chi p^{2}$

$N=b e f_{c t f}^{*}+\frac{1}{2} b(1-\alpha)^{2} e^{2} \chi E$

$M=\frac{b e^{2}}{3} f_{c t f}^{*}+b\left(\frac{1}{3}-\frac{\alpha}{2}+\frac{\alpha^{3}}{6}\right) e^{3} \chi E$

$M=\left(2 \alpha^{3}-3 \alpha^{2}+1\right) \frac{b e^{3} \chi E}{12}$

$f_{c t f}^{*}=-\frac{1}{2}(1-\alpha)^{2}$ e $\chi E$

$\varepsilon_{\text {lim }}=-\chi \alpha e+\frac{f_{c t f}^{*}}{E}$

(a)

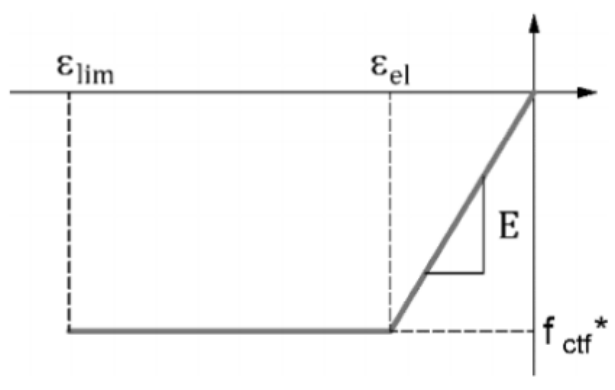

(b)

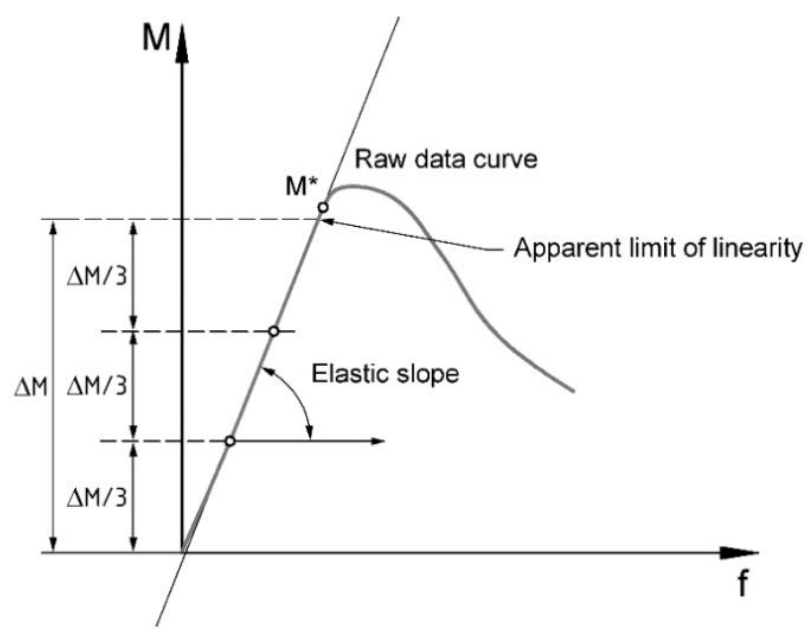

Figure 2- 18: Simplified inverse analysis method proposed in the French standard (NF P18-470 2016): (a) Bilinear stress-strain relationship of strain-hardening UHPFRC; and (b) Linearity limit and elastic modulus determined from the moment-deflection curve 


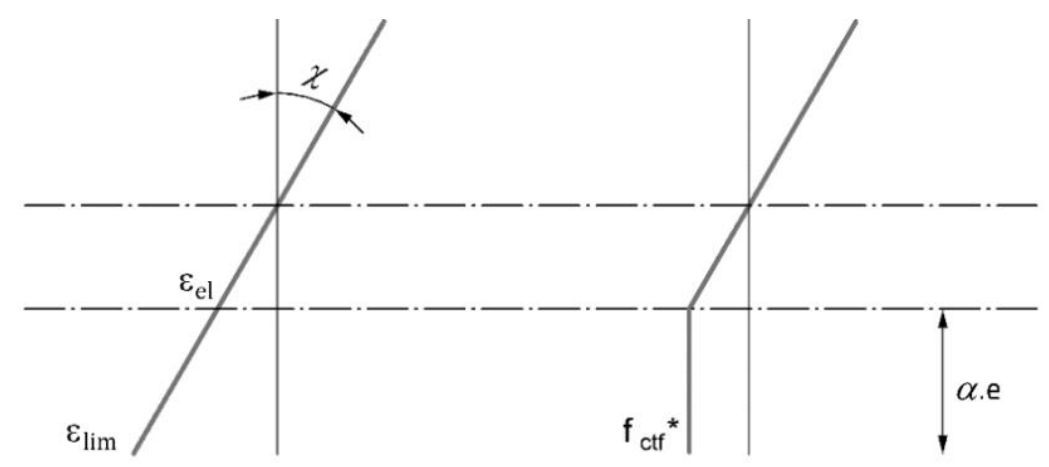

Figure 2- 19: Stress distribution considered for the inverse analysis method proposed in the French standard (NF

P18-470 2016)

In the Swiss standard (SIA2052 2014), cracking strength is defined as the elastic stress at which stiffness reduces $1 \%$ compared with the initial stiffness. However, owing to the strain-hardening behaviour of UHPFRC, the loss of stiffness is challenging to be identified from experimental response curve. Therefore, the Swiss standard requires to conduct elastic loading-unloading cycle for three times so that the estimation of elastic modulus is accurate. In addition, linear elastic theory which overlooks shear effect is utilized to determine elastic modulus. For the prism proposed by the Swiss standard (refer to Figure 2-14) whose span to depth ratio is 14 , linear elastic theory seems applicable. However, for the prisms proposed by the French standard (refer to Figure 2-13) whose span to depth ratio is small, linear elastic theory may cause noticeable difference in elastic modulus results (Graybeal 2006). Figure 2-20 depicts the stress distribution proposed in the Swiss standard, where neutral axis is assumed to be located at a distance of $0.82 \mathrm{hm}$ ( $\mathrm{hm}$ is prism depth) from the tension face. Moreover, ultimate tensile strength $\left(f_{t, u}\right)$ is assumed to be equal to the equivalent flexural strength multiplied by 0.383 . This assumption is based on the analytical simulation of more than one million UHPFRC specimens (López et al. 2016). The drawback of this inverse analysis method is that tensile strain properties can only be determined through an iterative process.

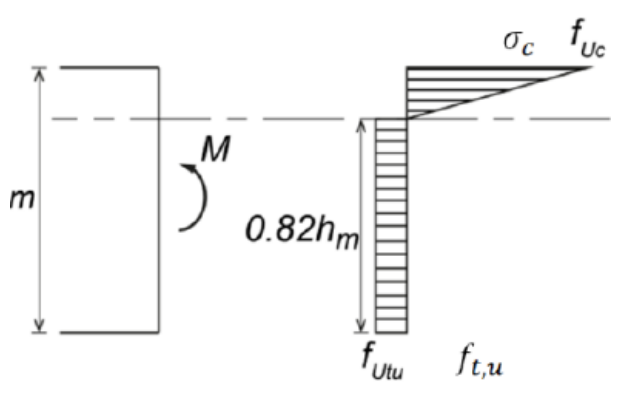

Figure 2- 20: Stress distribution proposed in the Swiss standard (SIA2052 2014) 
The Canadian standards (Annex 8.1 of CSA-S6 2018; Annex U of CSA-A23.1 2019) proposes an inverse analysis method relying on four characteristic points of the load-midspan deflection response curve obtained from unnotched FPBT. This inverse analysis method was employed for the research study, and its analysis procedures is described in Section 6.1.1. 


\section{Chapter 3. Experimental Program: Phase 1}

\subsection{Materials and Casting Methodology}

Six mixes of steel fiber reinforced concrete were prepared for the Phase 1 of the experimental program. Of those, four were commercial mixes, referred herein as mixes FD1, FD2, KI1 and LD1; and two were inhouse mixes, referred herein as mixes DE1 and DE2. With regard to commercial design mixes, the commercial companies provided all the necessary materials, and concrete mixing and casting were performed in collaboration with expert representatives from the respective commercial providers. Mixes FD1 and FD2 were cast on February $28^{\text {th }}$ and March $14^{\text {th }}, 2018$, respectively. Mix KI1 was cast on February $14^{\text {th }}, 2018$, and Mix LD1 was cast on December $15^{\text {th }}, 2017$. Mixes DE1 and DE2 were cast on April $16^{\text {th }}$, 2019. In addition, all commercial design mixes contained $2 \%$ steel fibers by unit volume. Mixes KI1 and LD1 contained $13 \mathrm{~mm}$ long, straight steel fibers with a diameter of $0.2 \mathrm{~mm}$, whereas mixes FD1 and FD2 contained $1 \%$ straight steel fibers $(20 \mathrm{~mm}$ in length and $0.2 \mathrm{~mm}$ in diameter) and $1 \%$ hooked-end steel fibers (20 $\mathrm{mm}$ in length and $0.3 \mathrm{~mm}$ in diameter). The straight and hooked-end steel fibers utilized in mixes FD1 and FD2 are depicted in Figure 3-1. With regard to in-house mixes, DE1 and DE2 were prepared by using Densit ${ }^{\circledR}$ premixed dry powders and the $13 \mathrm{~mm}$ long, straight steel fibers with a diameter of $0.2 \mathrm{~mm}$ from Nycon. Mix DE1 contained 1\% steel fibers, and mix DE2 contained 2\% steel fibers. The mix designs of mixes DE1 and DE2 are presented in Table 3-1. Moreover, Figure 3-2 illustrates the mixing procedures of mixes DE1 and DE2. Initially, premixed dry powders were mixed for two minutes. Then half the amount of water was added while the mixer was still rotating. After five minutes, the remainder of water was added for mixing for another five minutes. Lastly, steel fibers were added and mixed for three minutes. It is worth noting that fiber clotting was observed during the mixing of the DE2 design mix.
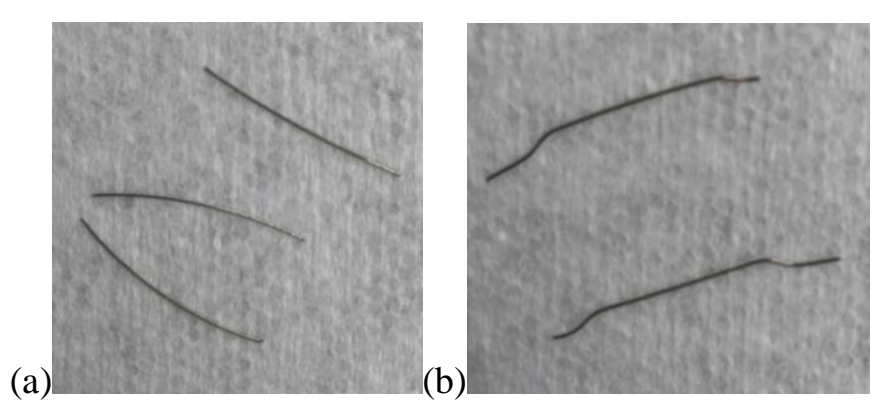

Figure 3- 1: Steel fibers utilized in mixes FD1 and FD2: (a) Straight steel fibers; and (b) Hooked-end steel fibers 
Table 3- 1: Mix designs of mixes DE1 and DE2

\begin{tabular}{|c|c|c|c|c|c|c|c|}
\hline \multicolumn{3}{|c|}{ DE1 } & \multicolumn{4}{c|}{ DE2 } \\
\hline $\begin{array}{c}\text { Densit } \\
\text { Dry Mix }\end{array}$ & Water & $\begin{array}{c}\text { Steel } \\
\text { Fibers }\end{array}$ & $\begin{array}{c}\text { Total } \\
\text { Volume }\end{array}$ & $\begin{array}{c}\text { Densit } \\
\text { Dry Mix }\end{array}$ & Water & $\begin{array}{c}\text { Steel } \\
\text { Fibers }\end{array}$ & $\begin{array}{c}\text { Total } \\
\text { Volume }\end{array}$ \\
\hline $\mathrm{kg}$ & $\mathrm{kg}$ & $\mathrm{kg}$ & $\mathrm{L}$ & $\mathrm{kg}$ & $\mathrm{kg}$ & $\mathrm{kg}$ & $\mathrm{L}$ \\
\hline 34.77 & 4.17 & 1.10 & 14 & 193.13 & 23.18 & 12.50 & 80 \\
\hline
\end{tabular}
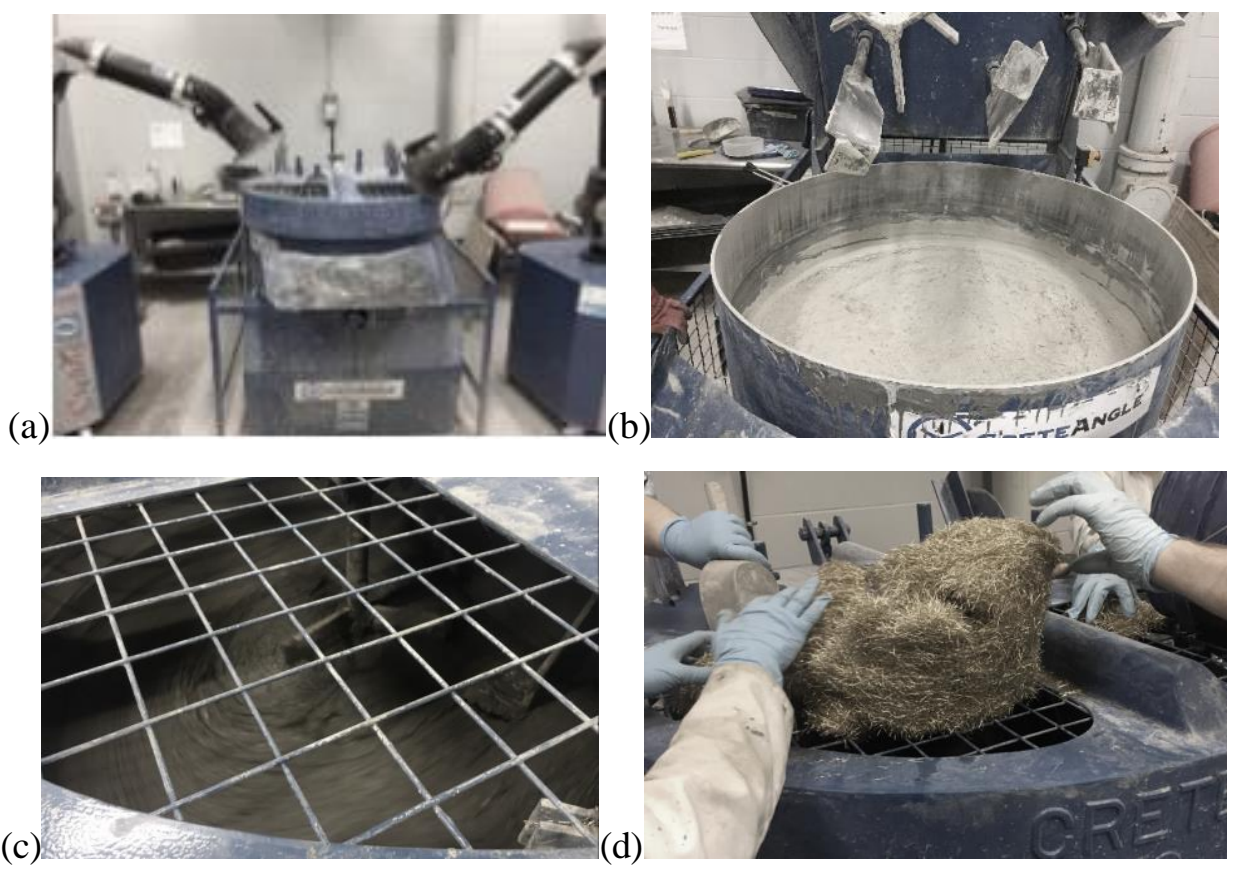

Figure 3- 2: Concrete mixing of mixes DE1 and DE2: (a) Mixing station (b) Dry mix (c) Mix state after adding all the water; and (d) Addition of steel fibers

In accordance with ASTM C1856 (2017), flowability of each mix was determined immediately after concrete mixing. As depicted in Figure 3-3, the mold of the flow table was filled with a single layer of fresh cementitious composites, and the average of minimum and maximum diameters of the material spread measured two minutes after lifting the mold is the reported flow value. The flow value was found to be 210 $\mathrm{mm}$ for mixes FD1 and KI1, $218 \mathrm{~mm}$ for mix FD2, $228 \mathrm{~mm}$ for mix LD1, $183 \mathrm{~mm}$ for mix DE1 and 233 mm for mix DE2.

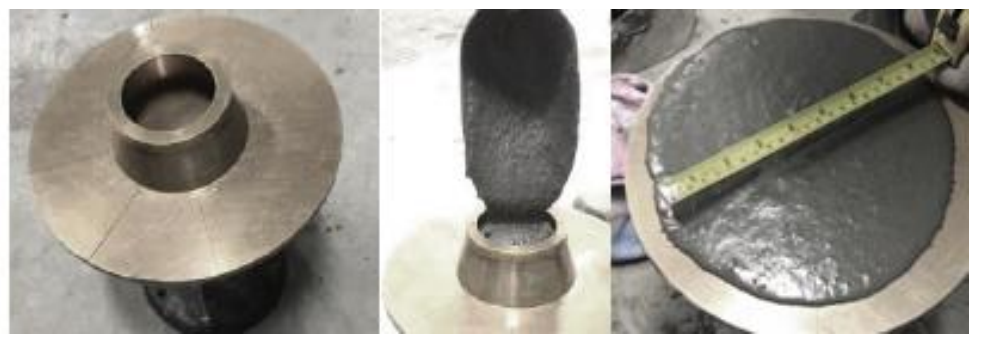

Figure 3- 3: Flow test 
As depicted in Figure 3-4, wood and acrylic molds were prepared for prisms and direct-tension specimens, and standard plastic molds were prepared for cylinder specimens. Prior to concrete casting, a release agent was applied to all the molds. Prisms with an aspect ratio of 1 (i.e., $75 \mathrm{~mm}$ by $75 \mathrm{~mm}$ by $280 \mathrm{~mm}$ prisms, referred herein as short prisms), prisms with an aspect ratio of 2 (i.e., $75 \mathrm{~mm}$ by $75 \mathrm{~mm}$ by $500 \mathrm{~mm}$ prisms, referred herein as long prisms), and prisms proposed by AFNOR NF EN 13670 (2013) which are $50 \mathrm{~mm}$ (depth) by $100 \mathrm{~mm}$ (width) by $400 \mathrm{~mm}$ (span) were prepared for conducting four-point bending test (FPBT). ASTM C1856 (2017) states that for UHP-SFRC, prisms should be cast by pouring fresh concrete from one end of the mold, and the mold should be filled with a single layer of material. However, to study the effect of casting methodology on flexural strength, prisms were cast with two approaches: 1). the prisms which were cast by pouring fresh material from one end with a single layer are referred herein as one-way prisms (OW Prisms); and 2). the prisms which were cast by pouring fresh material at different locations along the span of the prism with multiple layers are referred herein as random prisms (R Prisms). In addition, $75 \mathrm{~mm}$ by $150 \mathrm{~mm}$ cylinders were prepared for conducting cylinder compression test and splitting tensile test. When casting cylinder specimens, molds were filled in one layer. Moreover, three types of specimens were prepared for conducting direct tension test (DTT). Similar to OW prisms, direct-tension specimens were cast by filling the mold from one end with a single layer. In accordance with ASTM C1856 (2017), all the specimens were consolidated by tapping the sides of the mold 30 times with a mallet (i.e., external vibration only). Furthermore, the specimens containing commercial mixes were demolded one day after casting, and the specimens containing in-house mixes were demolded two days after casting. Thereafter, the specimens were placed in water tanks in ambient laboratory temperature for curing until testing.
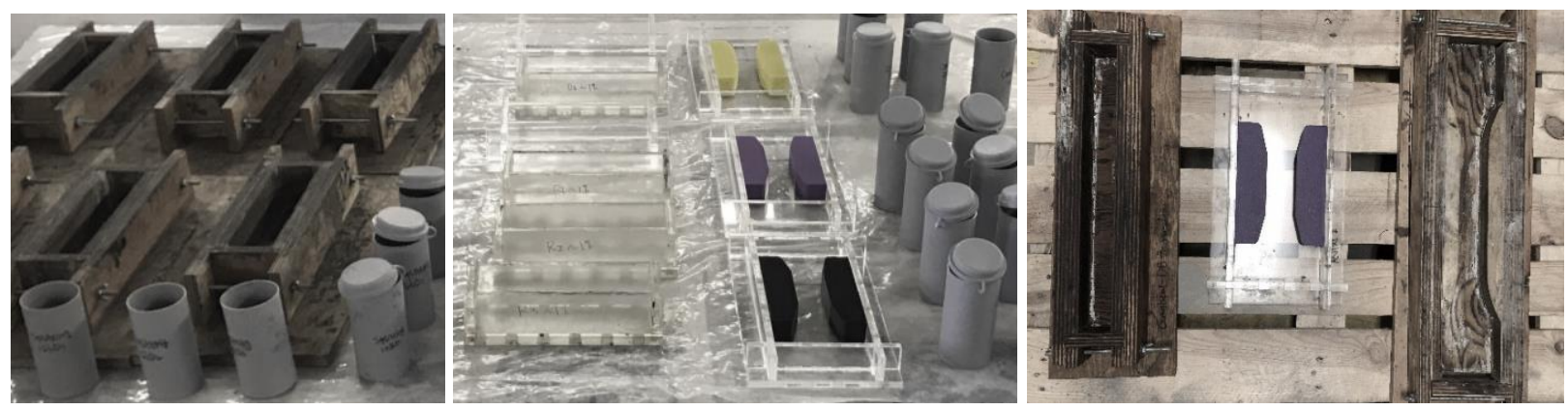

Figure 3- 4: Molds prepared for prisms, cylinders and direct-tension specimens 


\subsection{Compression Test}

A load-controlled compression-testing machine (Controls Pilot) was used to test $75 \mathrm{~mm}$ by $150 \mathrm{~mm}$ cylinders under compression at a loading rate of $0.259 \mathrm{MPa} / \mathrm{s}$ in accordance with ASTM C39 (2005). Cylinders from mixes FD1, FD2, KI1 and LD1 were tested at a mature age (i.e., >56 days); those from mix DE1 were tested at the age of 28 days; and those from mix DE2 were tested at the age of 28 days, 56 days, 108 days and 156 days respectively to study the age effect on compressive strength. Prior to testing, top and bottom surfaces of each cylinder were grinded. Figure 3-5 depicts the experimental test setup of cylinder compression test, where two steel blocks with high stiffness were placed under the cylinder to avoid premature failure.

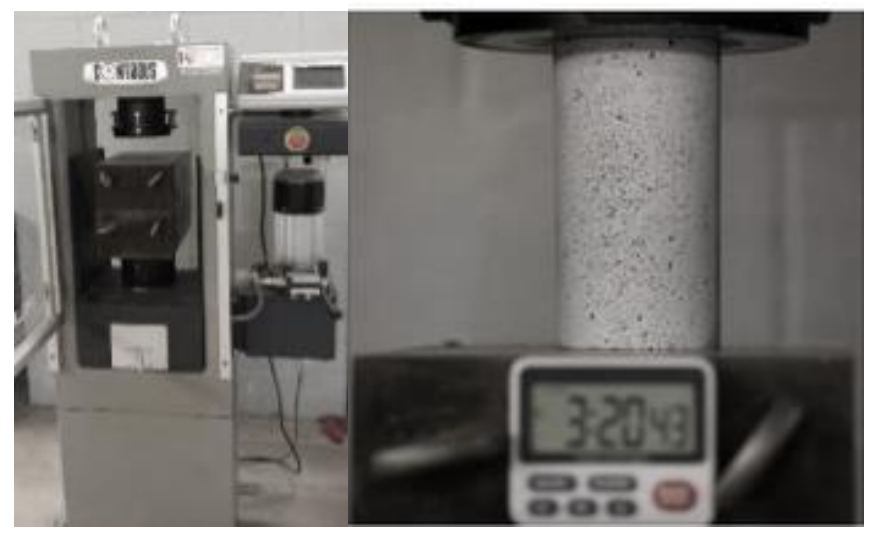

Figure 3- 5: Experimental test setup of cylinder compression test

The average compressive strength was $126 \mathrm{MPa}$ for mix FD1, $115 \mathrm{MPa}$ for mix FD2, $123 \mathrm{MPa}$ for mix KI1, $204 \mathrm{MPa}$ for mix LD1, $164 \mathrm{MPa}$ for DE1 and $155 \mathrm{MPa}$ for mix DE2 (at the age of 28 days). The compressive strength $\left(f_{c}^{\prime}\right)$ was calculated with Equation 3-1, where $P$ is the peak load and $d$ is the measured diameter ( $d$ was determined by averaging two diameters measured at right angles to each other at midheight of the cylinder). The compressive strength result of each cylinder is presented in Table 3-2.

As illustrated in Figure 3-6, cylinders generally held their integrity (i.e., they did not collapse into fragments) beyond attainment of the peak load. In addition, cracks were parallel to the loading direction, revealing the bridging effect of steel fibers crossing the cracks. Moreover, it is evident that the average compressive strength result of the cylinders containing 1\% steel fibers (from mix DE1) is greater than those containing 2\% steel fibers (from mix DE2). Georgiou and Pantazopoulou (2016) indicated that the internal confinement (i.e., compressive pressure) provided by the steel fibers was equal in magnitude to the intrinsic tensile strength of the material. Furthermore, given that the confining pressure is passive, its effect is only 
noticeable in the deformation capacity of the crushed concrete cylinder, whereas the effect of confining pressure on cylinder compressive strength is negligible. In this regard, compressive strength seems insensitive to the volumetric ratio of steel fibers, and the compressive strength of the material with a high fiber content appears slightly compromised due to fiber clotting in some cases, which corresponds to what was observed from the average compressive strength results of mixes DE1 and DE2. Furthermore, compressive strength results of the cylinders containing mix DE2 tested at the age of 56 days, 108 days and 156 days are presented in Table 3-3. It is evident that the average compressive strength of mix DE2 increased from $155 \mathrm{MPa}$ (at the age of 28 days) to $209 \mathrm{MPa}$ (at the age of 156 days), revealing the age effect on compressive strength.

$f_{c}^{\prime}=\frac{P}{0.25 \pi d^{2}}$

Table 3-2: Compressive strength results (Phase 1 of the experimental program)

\begin{tabular}{|c|c|c|c|c|c|c|c|c|c|c|c|c|c|c|}
\hline Mix & FD1 & FD2 & \multicolumn{4}{|c|}{ KI1 } & \multicolumn{2}{|c|}{ LD1 } & \multicolumn{3}{|c|}{ DE1 } & \multicolumn{3}{|c|}{ DE2 (28 days) } \\
\hline Cylinder & $\mathrm{C} 1$ & $\mathrm{C} 1$ & $\mathrm{C} 1$ & $\mathrm{C} 2$ & C3 & $\mathrm{C} 4$ & $\mathrm{C} 1$ & $\mathrm{C} 2$ & $\mathrm{C} 1$ & $\mathrm{C} 2$ & $\mathrm{C} 3$ & $\mathrm{C} 1$ & $\mathrm{C} 2$ & C3 \\
\hline Peak Load $[\mathrm{kN}]$ & 572 & 508 & 706 & 530 & 498 & 450 & 936 & 868 & 766 & 773 & 735 & 681 & 759 & 702 \\
\hline $\begin{array}{c}\text { Compressive } \\
\text { Strength [MPa] }\end{array}$ & 126 & 115 & 156 & 120 & 113 & 105 & 212 & 197 & 166 & 167 & 159 & 148 & 164 & 152 \\
\hline $\begin{array}{c}\text { Average } \\
\text { Compressive } \\
\text { Strength }[\mathrm{MPa}]\end{array}$ & 126 & 115 & \multicolumn{4}{|c|}{123} & \multicolumn{2}{|c|}{204} & \multicolumn{3}{|c|}{164} & \multicolumn{3}{|c|}{155} \\
\hline
\end{tabular}

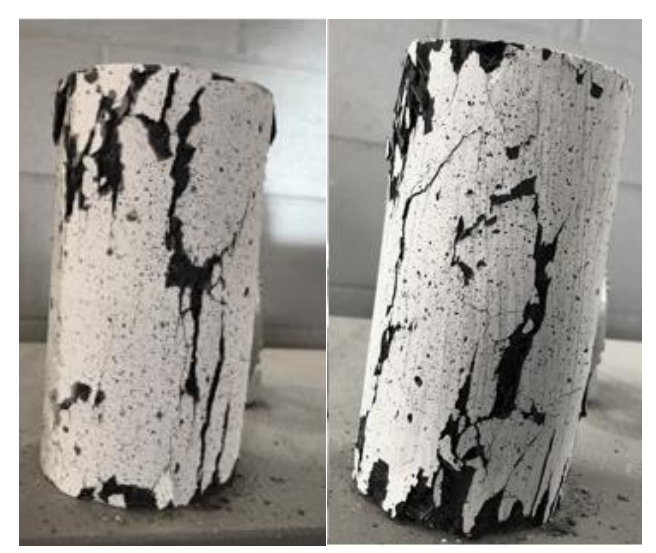

Figure 3- 6: Failure mode of cylinder compression test

Table 3- 3: Compressive strength results (Mix DE2)

\begin{tabular}{|c|c|c|c|c|c|c|c|c|c|}
\hline Mix & \multicolumn{3}{|c|}{ DE2 (56 days) } & \multicolumn{3}{c|}{ DE2 (108 days) } & \multicolumn{3}{c|}{ DE2 (156 days) } \\
\hline Cylinder & C1 & C2 & C3 & C1 & C2 & C3 & C1 & C2 & C3 \\
\hline Peak Load $[\mathrm{kN}]$ & 752 & 824 & 864 & 851 & 846 & 848 & 927 & 941 & 902 \\
\hline Compressive Strength $[\mathrm{MPa}]$ & 163 & 178 & 188 & 193 & 191 & 192 & 210 & 213 & 204 \\
\hline Average Compressive Strength $[\mathrm{MPa}]$ & \multicolumn{4}{|c|}{176} & \multicolumn{3}{c|}{192} & \multicolumn{5}{c|}{209} \\
\hline
\end{tabular}




\subsection{Splitting Tensile Test}

The load-controlled compression-testing machine (Controls Pilot) was used to obtain the splitting tensile strength of $75 \mathrm{~mm}$ by $150 \mathrm{~mm}$ cylinders at a loading rate of $0.016 \mathrm{MPa} / \mathrm{s}$ (CSA-A23.2-13C 2014). As illustrated in Figure 3-7a, wood shims were placed above and below the cylinder to improve load distribution, and to promote a longitudinal crack to form upon the attainment of the material's splitting tensile strength. In addition, as depicted in Figure 3-7b, cylinders maintained their integrity beyond failure (i.e., the two halves defined by the diametric crack were not separated). The cylinders containing mixes FD1, FD2, KI1 and LD1 were tested at a mature age (>56 days), and those from mix DE2 were tested at the age of 28 days, 56 days, 108 days and 156 days respectively to study the age effect on splitting tensile strength. Splitting tensile strength $\left(\sigma_{s}\right)$ was calculated with Equation 3-2, where $P$ is the peak load, $d$ is the average diameter, and $h$ is the height of the cylinder. The average splitting tensile strength was $25.1 \mathrm{MPa}$ for mixes FD1 and DE2 (at the age of 28 days), 25.8 MPa for mix FD2, 20.5 MPa for mix KI1 and 25.5 MPa for mix LD1. Table 3-4 presents the splitting tensile strength of each cylinder. In addition, the splitting tensile strength results of the cylinders containing mix DE2 tested at the age of 56 days, 108 days and 156 days are presented in Table 3-5. It is evident that the average splitting tensile strength of mix DE2 increased from $25.1 \mathrm{MPa}$ (at the age of 28 days) to $27.3 \mathrm{MPa}$ (at the age of 156 days), revealing the age effect on splitting tensile strength.

$\sigma_{s}=2 P / \pi d h$
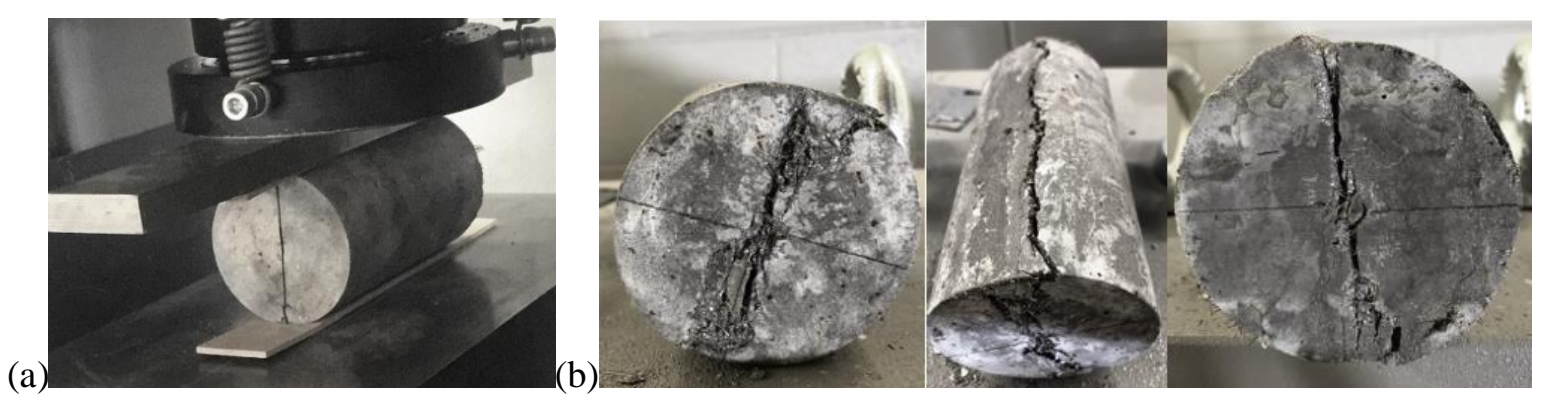

Figure 3- 7: Splitting tensile test: (a) Experimental test setup; and (b) Failure mode

Table 3-4: Splitting tensile strength results (Phase 1 of the experimental program)

\begin{tabular}{|c|c|c|c|c|c|c|c|}
\hline & FD1 & FD2 & KI1 & LD1 & \multicolumn{3}{|c|}{ DE2 (28 days) } \\
\hline Mix & C1 & C1 & C1 & C1 & C1 & C2 & C3 \\
\hline Peak Load [kN] & 443 & 456 & 364 & 438 & 463 & 509 & 394 \\
\hline $\begin{array}{c}\text { Splitting Tensile Strength [MPa] } \\
\text { Average Splitting Tensile Strength } \\
{[\mathrm{MPa}]}\end{array}$ & 25.1 & 25.8 & 20.5 & 25.5 & 25.8 & 28.1 & 21.6 \\
\hline & 25.1 & 25.8 & 20.5 & 25.5 & \multicolumn{4}{|c|}{25.1} \\
\hline
\end{tabular}


Table 3- 5: Splitting tensile strength results (Mix DE2)

\begin{tabular}{|c|c|c|c|c|c|c|c|c|c|}
\hline Mix & \multicolumn{3}{|c|}{ DE2 (56 days) } & \multicolumn{3}{|c|}{ DE2 (108 days) } & \multicolumn{3}{|c|}{ DE2 (156 days) } \\
\hline Cylinder & $\mathrm{C} 1$ & $\mathrm{C} 2$ & $\mathrm{C} 3$ & $\mathrm{C} 1$ & $\mathrm{C} 2$ & $\mathrm{C} 3$ & $\mathrm{C} 1$ & $\mathrm{C} 2$ & $\mathrm{C} 3$ \\
\hline Peak Load $[\mathrm{kN}]$ & 463 & 470 & 479 & 467 & 471 & 468 & 493 & 477 & 477 \\
\hline Splitting Tensile Strength $[\mathrm{MPa}]$ & 25.3 & 26.0 & 26.2 & 26.4 & 26.7 & 26.5 & 27.9 & 27.0 & 27.0 \\
\hline $\begin{array}{l}\text { Average Splitting Tensile Strength } \\
{[\mathrm{MPa}]}\end{array}$ & & 25.8 & & & 26.5 & & & 27.3 & \\
\hline
\end{tabular}

\subsection{Direct Tension Test}

Three types of direct-tension specimens (referred herein as Type A, Type $\mathrm{G}$ and Type $\mathrm{S}$ specimens) were tested by using a servo-controlled, closed loop universal testing machine at a loading rate of $0.00254 \mathrm{~mm} / \mathrm{s}$ (Graybeal an Baby 2013). The nominal dimensions of the specimens are illustrated in Figure 3-8. Thixotropic epoxy was utilized to wrap the ends of the specimen with carbon fiber reinforced polymer (CFRP) strips to eliminate support failure and to promote the formation of the critical cracking plane within the gauge length. The experimental test setup is presented in Figure 3-9. Type $G$ and Type $S$ specimens were gripped at the ends; therefore, the load was transferred through shear action between the gripping plates, CFRP layer, epoxy layer and the specimen. However, as depicted in Figure 3-9b, Type A specimens were supported through bearing action at four points of contact with the supporting brackets. Thus, the load transfer mechanism was different for the three types of direct-tension specimens. In all cases, a relevant instrumentation jig was attached to measure strains and elongations in the central $100 \mathrm{~mm}$ region.
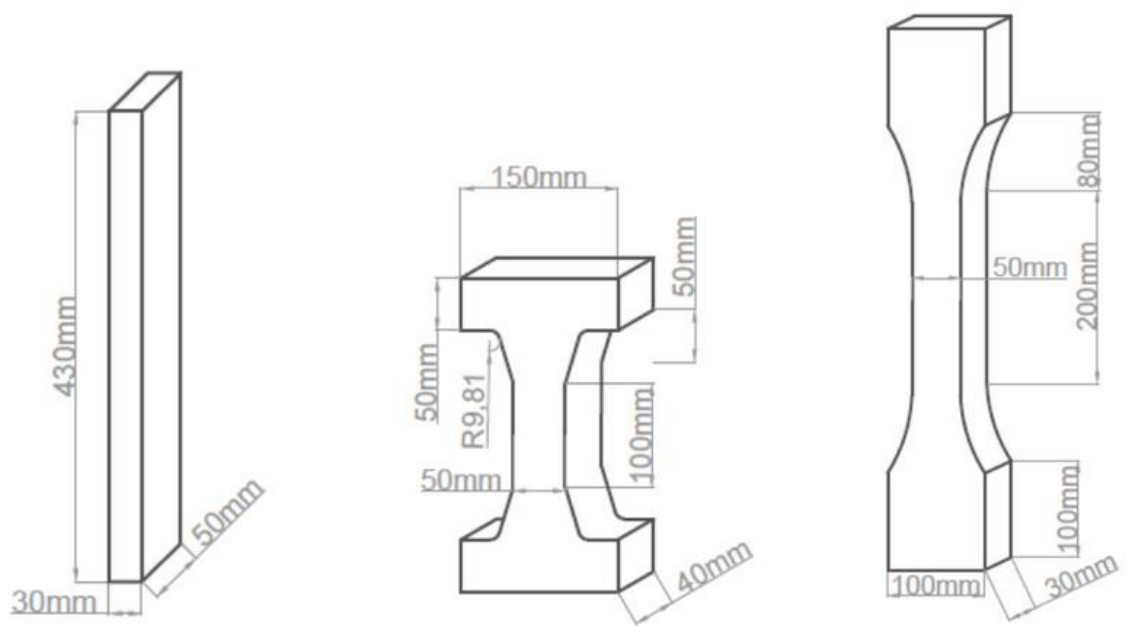

Figure 3- 8: Nominal dimensions of direct-tension specimens: from left to right: Type G specimen (FHWA 2013);

Type A specimen (Georgiou and Pantazopoulou 2016); and Type S specimen (SIA2052 2014) 

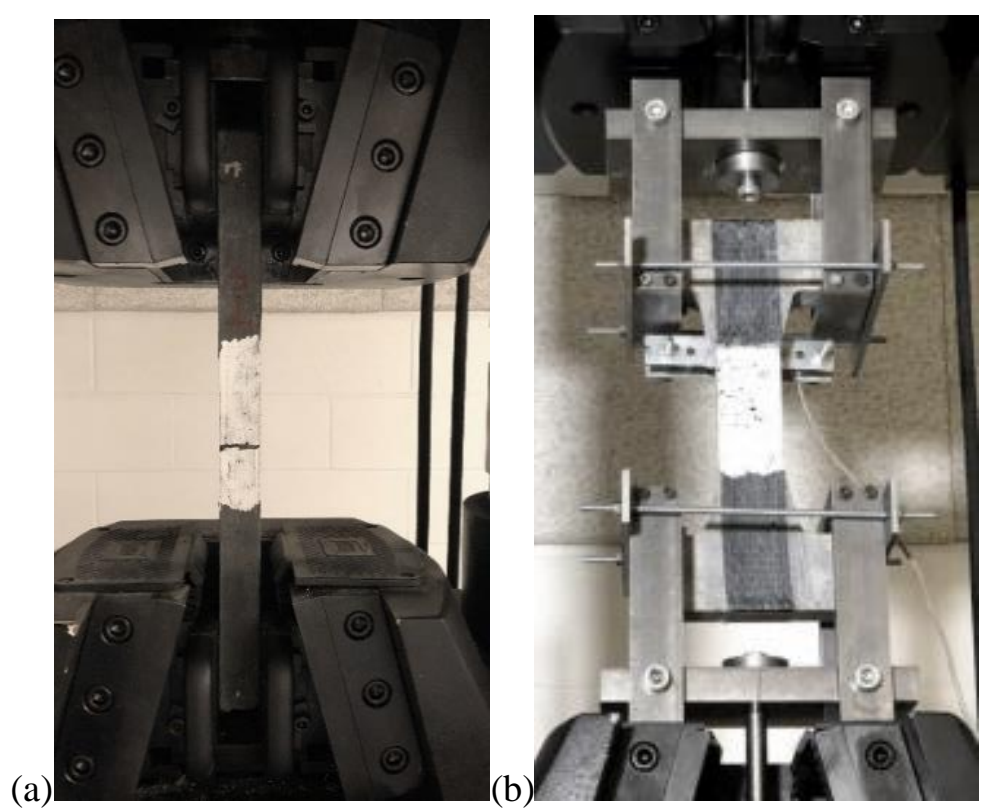

Figure 3- 9: Experimental test setup of direct tension test: (a) Type G and Type S specimens (sideview); and (b) Type A specimens

It's worth noting that some researchers proposed to glue aluminum transfer plates to the two parallel faces at each end of the direct-tension specimen (Graybeal and Baby 2013). However, as illustrated in Figure 39, the gripping devices of the Universal Testing Machine restricts the specimen's dimensions. In this context, to fit the specimen in the grips, it is advisable to glue CFRP strips to the ends of the specimen, which functions equivalently as the aluminum transfer plates.

The direct-tension specimens containing mixes FD1, FD2, KI1 and LD1 were tested at a mature age (>56 days), whereas the specimens from mix DE2 were tested at the age of 36 days and 38 days, respectively. Tensile strength (i.e., cracking strength) was calculated from the ratio of the peak load divided by the crosssectional area of the specimen. The average tensile strength of Type A specimens is $10.09 \mathrm{MPa}$ for mix FD1, 8.13 MPa for mix FD2, 5.28 MPa for mix KI1, 7.72 MPa for mix LD1 and 10.04 MPa for mix DE2; the average tensile strength of Type $\mathrm{G}$ specimens is 8.02 MPa for mix FD1, 8.39 MPa for mix FD2, 5.59 MPa for mix KI1 and 8.15 MPa for mix LD1; and the average tensile strength of Type S specimens is 7.96 MPa for mix FD1, 11.91 MPa for mix FD2, 6.57 MPa for mix KI1, 9.82 MPa for mix LD1 and 10.6 MPa for mix DE2. Table 3-6 summarizes the tensile strength results of each direct-tension specimen, and the tensile strength results are relatively consistent for the three types of specimens considered. 
Table 3- 6: Tensile strength results obtained from direct tension tests

\begin{tabular}{|c|c|c|}
\hline \multicolumn{3}{|c|}{ Mix FD1 } \\
\hline Specimen & Tensile Strength [MPa] & Average Tensile Strength [MPa] \\
\hline \multirow[b]{2}{*}{ Type G } & 6.68 & \multirow{2}{*}{ 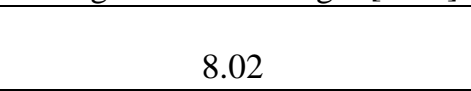 } \\
\hline & 9.35 & \\
\hline \multirow[b]{2}{*}{ Type $S$} & 9.84 & \multirow[b]{2}{*}{7.96} \\
\hline & 6.09 & \\
\hline \multirow[b]{2}{*}{ Type A } & 9.91 & \multirow[b]{2}{*}{10.09} \\
\hline & 10.27 & \\
\hline \multicolumn{3}{|c|}{ Mix FD2 } \\
\hline Specimen & Tensile Strength [MPa] & Average Tensile Strength [MPa] \\
\hline \multirow{3}{*}{ Type G } & 7.46 & \multirow{3}{*}{ ( } \\
\hline & 7.74 & \\
\hline & 9.96 & \\
\hline \multirow{3}{*}{ Type S } & 14.01 & \multirow{3}{*}{11.91} \\
\hline & 11.33 & \\
\hline & 10.36 & \\
\hline \multirow[b]{2}{*}{ Type A } & 8.42 & \multirow[b]{2}{*}{8.13} \\
\hline & 7.84 & \\
\hline \multicolumn{3}{|c|}{ Mix KI1 } \\
\hline Specimen & Tensile Strength [MPa] & Average Tensile Strength [MPa] \\
\hline \multirow{3}{*}{ Type G } & 6.22 & \multirow[t]{3}{*}{ 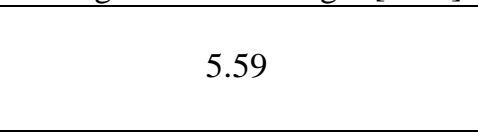 } \\
\hline & 6.00 & \\
\hline & 4.54 & \\
\hline \multirow{3}{*}{ Type S } & 7.09 & \multirow{3}{*}{6.57} \\
\hline & 6.94 & \\
\hline & 5.70 & \\
\hline \multirow[b]{2}{*}{ Type A } & 5.25 & \multirow[b]{2}{*}{5.28} \\
\hline & 5.31 & \\
\hline \multicolumn{3}{|c|}{ Mix LD1 } \\
\hline Specimen & Tensile Strength [MPa] & Average Tensile Strength [MPa] \\
\hline \multirow[b]{2}{*}{ Type G } & 8.47 & \multirow[t]{2}{*}{ ( } \\
\hline & 7.83 & \\
\hline \multirow{3}{*}{ Type S } & 8.94 & \\
\hline & 9.05 & 9.82 \\
\hline & 11.46 & \\
\hline & 7.56 & \\
\hline Type A & 9.00 & 7.72 \\
\hline & 6.60 & \\
\hline & Mix DE2 & \\
\hline Specimen & Tensile Strength [MPa] & Average Tensile Strength [MPa] \\
\hline & 11.7 & \\
\hline Type S & 9.47 & 10.60 \\
\hline & 10.67 & \\
\hline & 10.12 & \\
\hline Type A & 9.96 & 10.04 \\
\hline $\begin{array}{l}\text { Note: Tensile } \\
\text { outside the gal } \\
\text { the average re }\end{array}$ & $\begin{array}{l}\text { th results of the specin } \\
\text { igths were considered as o }\end{array}$ & $\begin{array}{l}\text { I whose failure planes occurred } \\
\text { iers and were not used to calculate }\end{array}$ \\
\hline
\end{tabular}

Some specimens developed failure planes outside the gauge lengths or experienced bending owing to the non-symmetry created after cracking. Setup conditions and tolerances in the specimens' dimensions could 
have contributed to the development of initial eccentricities, whereas the most significant challenge was the bending moments that developed inevitably when a crack initiated from one side of the specimen. In addition, owing to stress concentration, the failure planes of some Type A specimens were observed to occur near the region where cross section changed geometry. Desired as well as improper failure planes of the direct-tension specimens are illustrated in Figure 3-10.

(a)

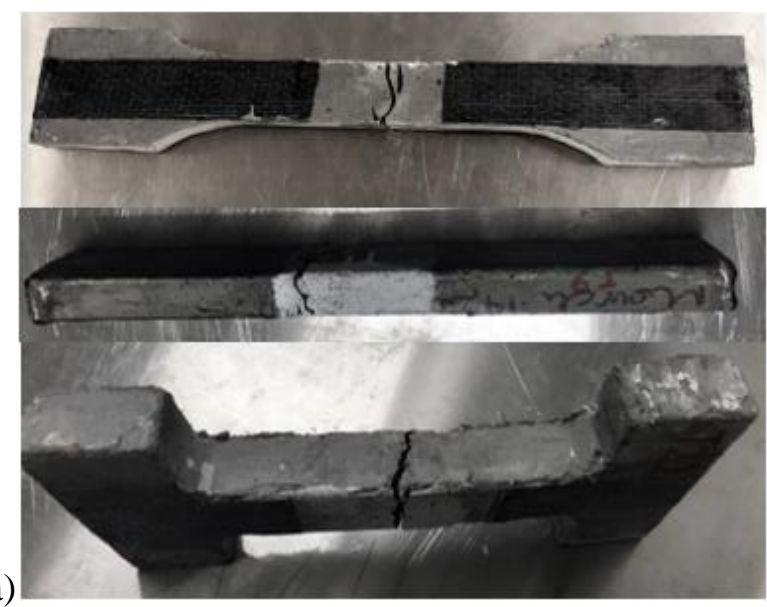

(b)

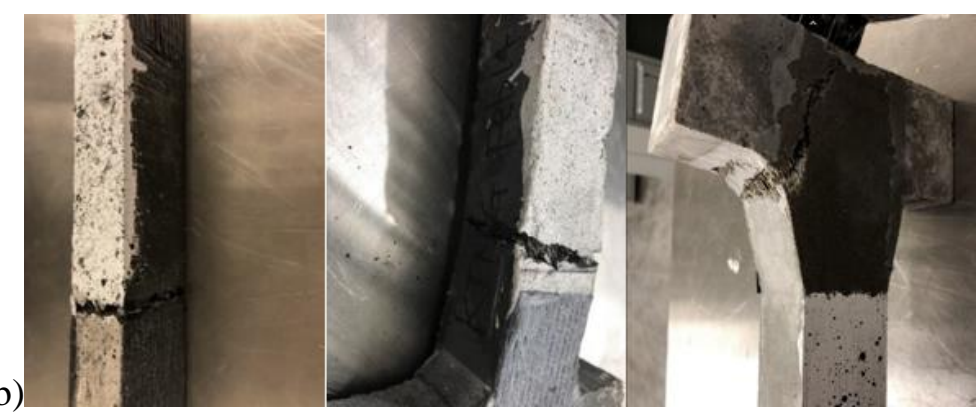

Figure 3-10: Failure planes of direct-tension specimens: (a) Desired failure planes, top to bottom: Type S, Type G and Type A specimens; and (b) Challenges with direct tension test, left to right: crack near CFRP strips, bending phenomenon, stress concentration due to the tolerance in geometry

\subsection{Four-Point Bending Test}

Prisms containing mixes FD1, FD2, KI1 and LD1 subjected to four-point loading were tested by using the universal testing machine at a constant displacement-controlled loading rate of $0.3 \mathrm{~mm} / \mathrm{min}$, and the prisms containing mixes DE1 and DE2 were tested at a stepwise loading rate in accordance with ASTM C1609 (2012). With regard to the stepwise loading rate, a loading rate of $0.15 \mathrm{~mm} / \mathrm{min}$ was applied to the prisms before reaching $70 \%$ post-peak load, and the loading rate was increased by $0.05 \mathrm{~mm} / \mathrm{min}$ for the post-peak softening branch after the load dropped below $70 \%$ of the peak load. In addition, regardless of the loading 
rate, all the prisms were tested until the post-peak residual strength had degraded to $20 \%$ of the peak load. Moreover, the prisms from commercial mixes were tested at a mature age ( $>56$ days), and the prisms from in-house mixes were tested at the age of 32 days, 40 days and 41 days, respectively. Figure 3-11 depicts the schematic of the experimental test setup, where ' $\mathrm{B}$ ' is shear span (i.e., the distance between a loading roller to the nearest support roller), ' $\mathrm{A}$ ' is the distance between loading rollers, and ' $\mathrm{C}$ ' is the depth of the prism. With regard to the short prisms with an aspect ratio of 1 (i.e., $75 \mathrm{~mm}$ by $75 \mathrm{~mm}$ by $280 \mathrm{~mm}$ prisms), the shear span from each side and the distance between loading rollers are $75 \mathrm{~mm}$, and the distance between support rollers is $225 \mathrm{~mm}$. With regard to the long prisms with an aspect ratio of 2 (i.e., $75 \mathrm{~mm}$ by $75 \mathrm{~mm}$ by $500 \mathrm{~mm}$ prisms), the shear span from each side is $150 \mathrm{~mm}$, the distance between loading rollers is 100 $\mathrm{mm}$, and the distance between support rollers is $400 \mathrm{~mm}$. With regard to the thin prisms (i.e., $50 \mathrm{~mm}$ by $100 \mathrm{~mm}$ by $400 \mathrm{~mm}$ prisms), the shear span from each side and the distance between loading points are 100 $\mathrm{mm}$, and the distance between support rollers is $300 \mathrm{~mm}$. As illustrated in Figure 3-12c and d, to measure the net vertical deflection at midspan, a linear displacement transducer was placed at midspan and mounted at the mid-height of the prism on the specimen chord, which was defined by the support points (i.e., Point 1 and Point 2 as depicted in Figure 3-11) using a fabricated instrumentation jig; the transducer measured the downwards relative displacement of the top compression face of the specimen. Moreover, as illustrated in Figure 3-12a, the support rollers are free to rotate about their own axis and are supported on their axles through bearings in order to eliminate friction. Furthermore, horizontal translations are free to occur at the points of contact between the prism and support rollers, but the distance between the axles of rollers is restricted and the span of the prism is not allowed to change during the test. Figure 3-12b depicts the loading rollers which are also free to rotate and are supported on their axle through bearings. Additionally, the loading rollers can adjust rotation about the longitudinal axis of the prism.

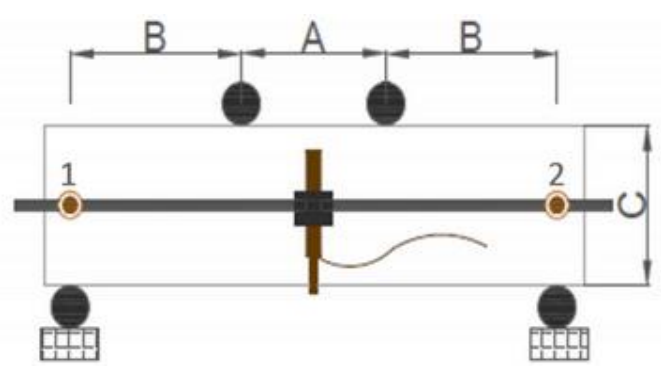

Figure 3-11: Schematic of the experimental test setup of four-point bending test 
(a)

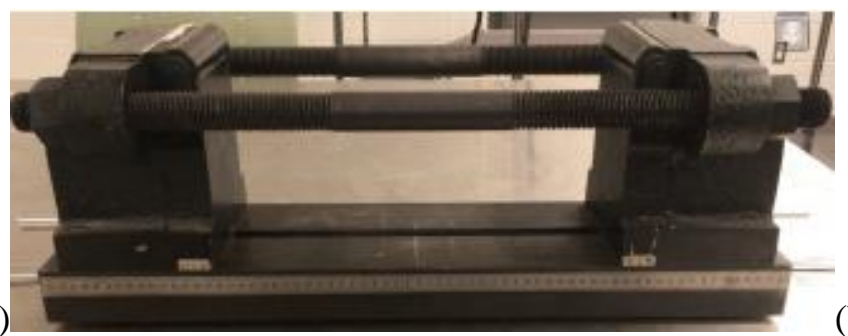

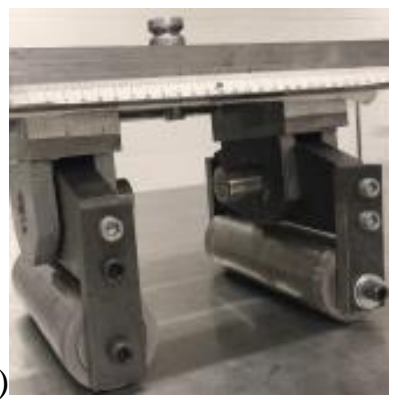

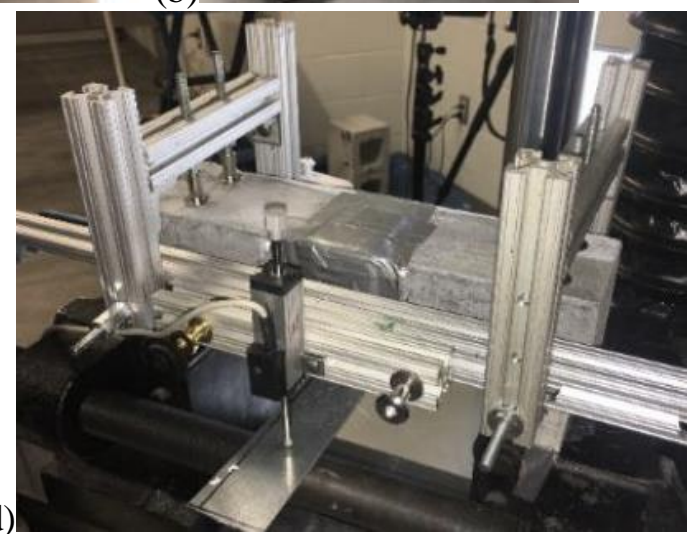

(c)

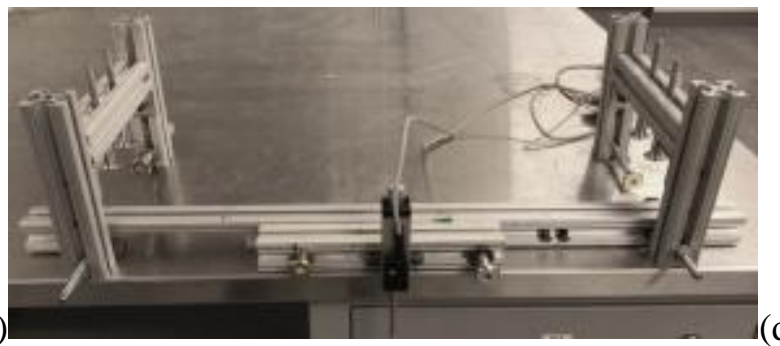

Figure 3- 12: Experimental test setup of four-point bending test: (a) Support rollers, (b) Loading rollers, (c) Instrumentation jig; and (d) Photo of test setup

Long prisms ( $75 \mathrm{~mm}$ by $75 \mathrm{~mm}$ by $500 \mathrm{~mm}$ prisms) and short prisms ( $75 \mathrm{~mm}$ by $75 \mathrm{~mm}$ by $280 \mathrm{~mm}$ ) were tested to study the effect of aspect ratio of the bending prism on flexural strength. OW prisms and R prisms were tested to study the effect of casting methodology. Short prisms containing $1 \%$ and $2 \%$ steel fibers from mixes DE1 and DE2 respectively were tested to study the effect of volumetric ratio of steel fibers. Thin prisms (50 mm by $100 \mathrm{~mm}$ by $400 \mathrm{~mm}$ prisms) from mix DE2 were tested to assess whether this proposed prism size (AFNOR NF EN 13670 2013) is adequate by comparing their flexural strength results with those of long prisms from mix DE2. In addition, the prisms containing $20 \mathrm{~mm}$ long, steel fibers (prims from mixes FD1 and FD2) were tested to study the effect of the degree of preferential fiber alignment on flexural strength by comparing their results with those obtained from the prisms containing $13 \mathrm{~mm}$ long, steel fibers. The equivalent flexural strength $\left(\sigma_{f}\right)$ was calculated with Equation 3-3 for short prisms and was calculated with Equation 3-4 for long prisms. In the equations, $P$ is the experimental peak load, $L$ is the total distance between supports, and $b$ and $h$ are the cross-sectional width and depth near the failure plane measured after each test. The flexural strength results of each design mix are presented in the following sections.

$$
\begin{aligned}
& \sigma_{f}=P L / b h^{2} \\
& \sigma_{f}=9 P L / 8 b h^{2}
\end{aligned}
$$




\subsubsection{Flexural Strength of Commercial FD1 Design Mix}

With regard to the long prisms ( $75 \mathrm{~mm}$ by $75 \mathrm{~mm}$ by $500 \mathrm{~mm}$ prisms), the average flexural strength is 39.81 $\mathrm{MPa}$ for OW prisms and $30.97 \mathrm{MPa}$ for R prisms. With regard to the short prisms $(75 \mathrm{~mm}$ by $75 \mathrm{~mm}$ by $280 \mathrm{~mm}$ prisms), the average flexural strength is $35.28 \mathrm{MPa}$ for OW prisms and $39.57 \mathrm{MPa}$ for R prisms. Figure 3-13 and Table 3-7 present the resistance curve and flexural strength result of each prism. Note that the resistance curves were adjusted to relatively smooth curves by selecting 15 to 30 points from the experimental response curve, and thereby the shape of the resistance curve remains the same after adjustment. In addition, in some cases, the linear displacement transducer slipped and did not measure the vertical deflection at midspan properly, so the corresponding resistance curves of these prisms are not illustrated in Figure 3-13. Moreover, as depicted in Figure 3-14, the critical failure planes of both short and long prisms formed within the central constant moment region (i.e., between the loading rollers).

Table 3- 7: Flexural strength results (Mix FD1)

\begin{tabular}{|c|c|c|c|}
\hline \multicolumn{3}{|c|}{ Long Prisms } \\
\hline \multirow{2}{*}{ Prism } & Peak Load & Flexural Strength & Average Flexural Strength \\
\cline { 2 - 3 } & kN & MPa & MPa \\
\hline OW1 & 32.57 & 34.29 & \multirow{2}{*}{39.81} \\
\hline OW2 & 38.70 & 40.21 & \multirow{2}{*}{30.97} \\
\hline OW3 & 42.13 & 44.94 & \\
\hline R1 & 36.87 & 38.30 & \multirow{2}{*}{} \\
\hline R2 & 26.20 & 27.58 & \multirow{2}{*}{ MPa } \\
\hline R3 & 26.72 & 27.03 & \multirow{2}{*}{35.28} \\
\hline \multicolumn{3}{|c|}{ Short Prisms } \\
\hline \multirow{2}{*}{ Prism } & Peak Load & Flexural Strength & Average Flexural Strength \\
\cline { 2 - 3 } & kN & MPa & \multirow{2}{*}{39.57} \\
\hline OW1 & 73.47 & 37.68 & \\
\hline OW2 & 64.10 & 32.87 & \\
\hline R1 & 79.72 & 43.10 & \\
\hline R2 & 65.93 & 34.70 & \\
\hline R3 & 80.13 & 40.57 & \multirow{2}{*}{} \\
\hline R4 & 76.82 & 39.91 & \\
\hline
\end{tabular}


FD1: One-Way Short Prisms

—One-Way Prism 1 One-Way Prism 2

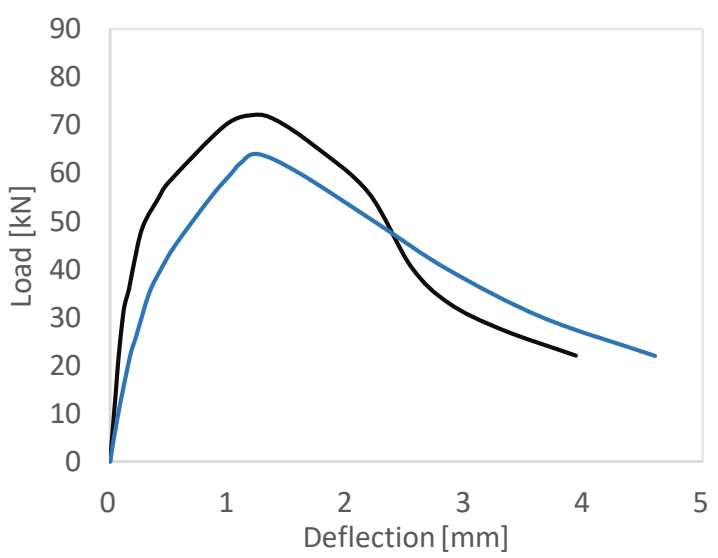

FD 1: Random Short Prisms

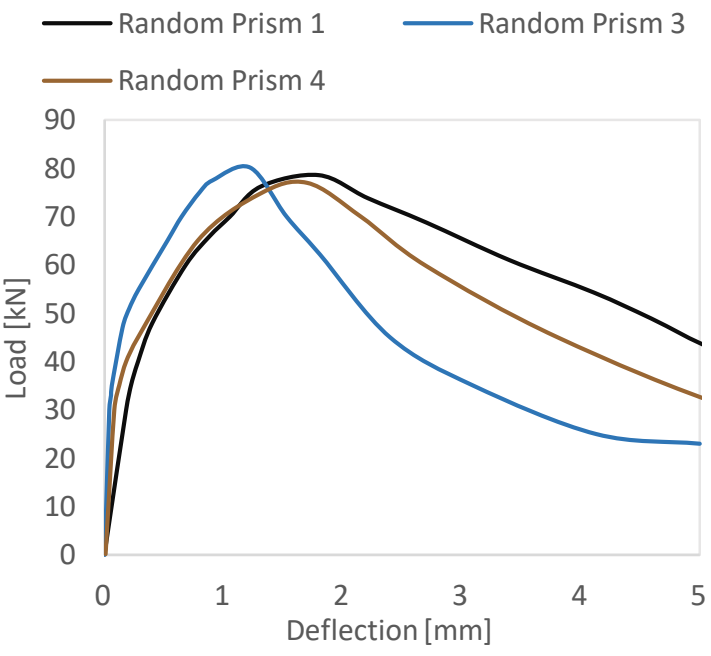

FD 1: One-Way Long Prisms

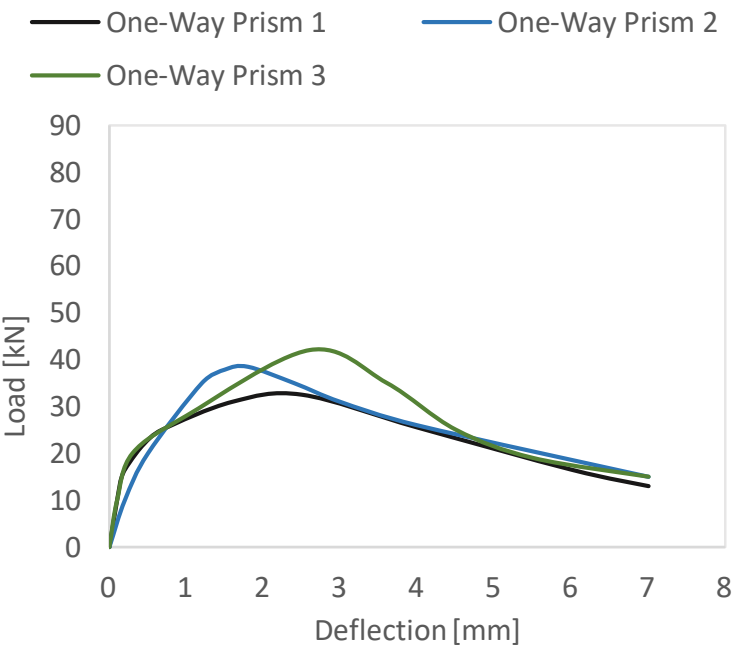

FD 1: Random Long Prisms
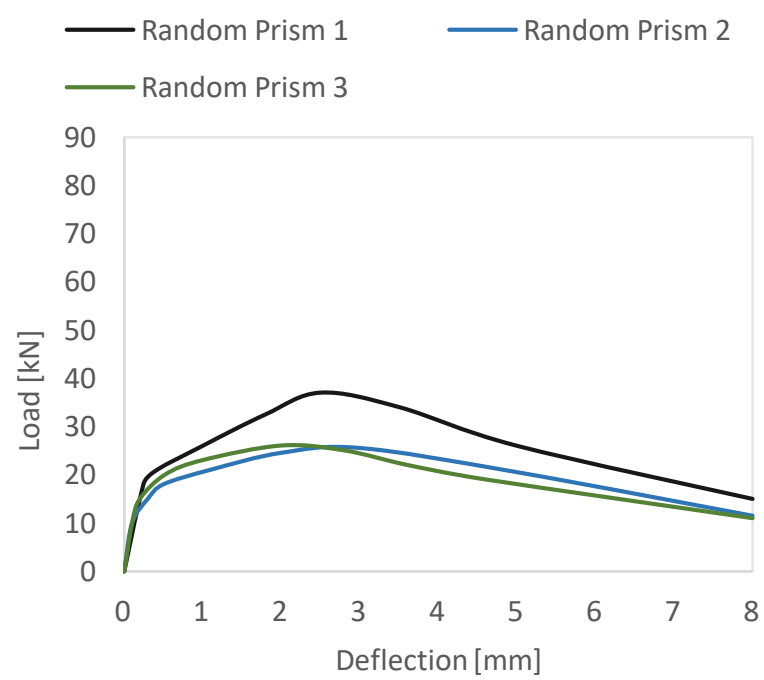

Figure 3-13: Resistance curves of the prisms from mix FD1

(a)
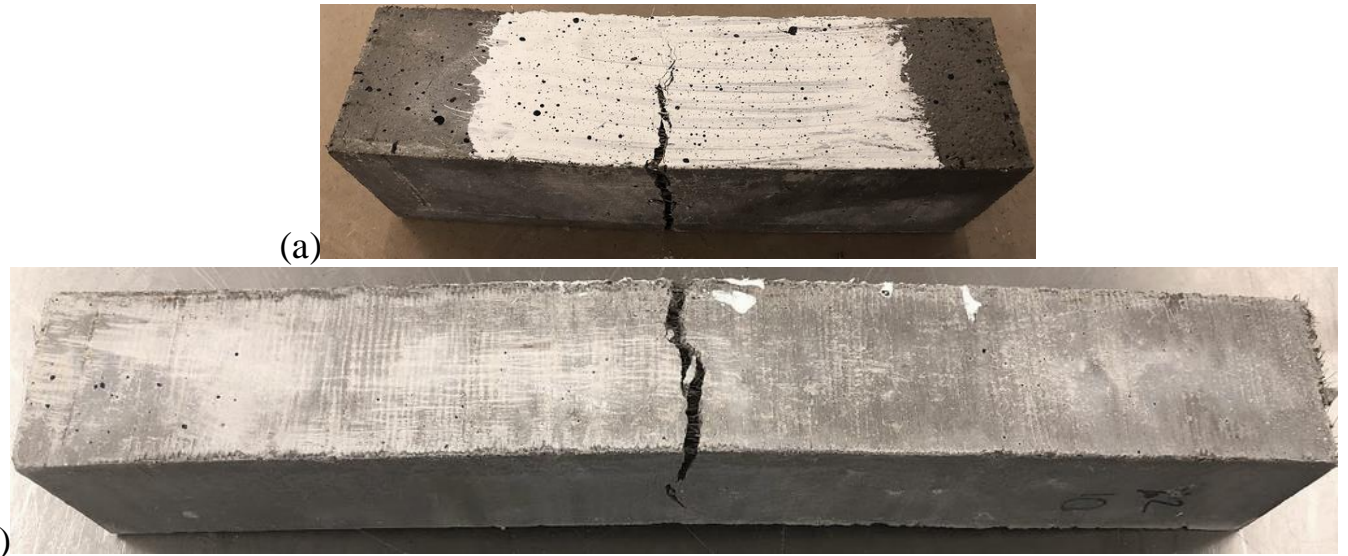

Figure 3-14: Failure mode of four-point bending test: (a) Short prisms; and (b) Long prisms 


\subsubsection{Flexural Strength of Commercial FD2 Design Mix}

With regard to the long prisms, the average flexural strength is $36.97 \mathrm{MPa}$ for OW prisms, while the $\mathrm{R}$ prism was not prepared for this mix. With regard to the short prisms, the average flexural strength is 38.78 $\mathrm{MPa}$ for OW prisms and $40.42 \mathrm{MPa}$ for $\mathrm{R}$ prisms. Table 3-8 presents the flexural strength result of each prism, and the resistance curves can be found in Appendix A.1.1.

Table 3- 8: Flexural strength results (Mix FD2)

\begin{tabular}{|c|c|c|c|}
\hline \multicolumn{4}{|c|}{ Long Prisms } \\
\hline & Peak Load & Flexural Strength & Average Flexural Strength \\
\hline Prism & $\mathrm{kN}$ & $\mathrm{MPa}$ & $\mathrm{MPa}$ \\
\hline OW1 & 32.33 & 34.49 & \multirow{6}{*}{36.97} \\
\hline OW2 & 34.37 & 37.16 & \\
\hline OW3 & 34.57 & 36.39 & \\
\hline OW4 & 34.72 & 37.03 & \\
\hline OW5 & 39.61 & 41.69 & \\
\hline OW6 & 32.45 & 35.09 & \\
\hline \multicolumn{4}{|c|}{ Short Prisms } \\
\hline & Peak Load & Flexural Strength & Average Flexural Strength \\
\hline Prism & $\mathrm{kN}$ & $\mathrm{MPa}$ & $\mathrm{MPa}$ \\
\hline OW1 & 74.60 & 40.87 & \multirow{3}{*}{38.78} \\
\hline OW2 & 80.09 & 42.71 & \\
\hline OW3 & 61.43 & 32.76 & \\
\hline $\mathrm{R} 1$ & 73.43 & 38.15 & \multirow{3}{*}{40.42} \\
\hline R2 & 76.36 & 40.73 & \\
\hline R3 & 79.50 & 42.40 & \\
\hline
\end{tabular}

\subsubsection{Flexural Strength of Commercial KI1 Design Mix}

With regard to the long prisms, the average flexural strength is $26.29 \mathrm{MPa}$ for OW prisms and 25.25 $\mathrm{MPa}$ for R prisms. With regard to the short prisms, the average flexural strength is $30.08 \mathrm{MPa}$ for OW prisms and 22.98 MPa for R prisms. Table 3-9 presents the flexural strength result of each prism, and the resistance curves can be found in Appendix A.1.2. 
Table 3- 9: Flexural strength results (Mix KII)

\begin{tabular}{|c|c|c|c|}
\hline \multicolumn{4}{|c|}{ Long Prisms } \\
\hline & Peak Load & Flexural Strength & Average Flexural Strength \\
\hline Prism & $\mathrm{kN}$ & $\mathrm{MPa}$ & $\mathrm{MPa}$ \\
\hline OW1 & 26.66 & 28.07 & \multirow{3}{*}{26.29} \\
\hline OW2 & 23.53 & 25.44 & \\
\hline OW3 & 24.11 & 25.38 & \\
\hline R1 & 27.29 & 29.11 & \multirow{3}{*}{25.25} \\
\hline R2 & 20.22 & 21.57 & \\
\hline R3 & 23.80 & 25.06 & \\
\hline \multicolumn{4}{|c|}{ Short Prisms } \\
\hline & Peak Load & Flexural Strength & Average Flexural Strength \\
\hline Prism & $\mathrm{kN}$ & $\mathrm{MPa}$ & $\mathrm{MPa}$ \\
\hline OW1 & 55.45 & 29.19 & \multirow{3}{*}{30.08} \\
\hline OW2 & 62.94 & 33.57 & \\
\hline OW3 & 52.20 & 27.48 & \\
\hline R1 & 40.87 & 21.51 & \multirow{3}{*}{22.98} \\
\hline R2 & 50.92 & 27.16 & \\
\hline R3 & 38.01 & 20.27 & \\
\hline
\end{tabular}

\subsubsection{Flexural Strength of Commercial LD1 Design Mix}

With regard to the long prisms, the average flexural strength is $31.96 \mathrm{MPa}$ for OW prisms and $19.41 \mathrm{MPa}$ for R prisms. With regard to the short prisms, the average flexural strength is $29.74 \mathrm{MPa}$ for OW prisms and 29.55 MPa for R prisms. Figure 3-15 and Table 3-10 present the resistance curve and flexural strength result of each prism.

Table 3- 10: Flexural strength results (Mix LD1)

\begin{tabular}{|c|c|c|c|}
\hline \multicolumn{4}{|c|}{ Long Prisms } \\
\hline & Peak Load & Flexural Strength & Average Flexural Strength \\
\hline Prism & $\mathrm{kN}$ & $\mathrm{MPa}$ & $\mathrm{MPa}$ \\
\hline OW1 & 30.71 & 30.68 & \multirow{3}{*}{31.96} \\
\hline OW2 & 30.74 & 31.53 & \\
\hline OW3 & 33.29 & 33.69 & \\
\hline R1 & 25.14 & 26.82 & \multirow{3}{*}{19.41} \\
\hline $\mathrm{R} 2$ & 17.91 & 19.10 & \\
\hline R3 & 11.24 & 12.31 & \\
\hline \multicolumn{4}{|c|}{ Short Prisms } \\
\hline & Peak Load & Flexural Strength & Average Flexural Strength \\
\hline Prism & $\mathrm{kN}$ & $\mathrm{MPa}$ & $\mathrm{MPa}$ \\
\hline OW1 & 72.44 & 37.63 & \multirow{3}{*}{29.74} \\
\hline OW2 & 50.12 & 26.38 & \\
\hline OW3 & 47.29 & 25.22 & \\
\hline R1 & 63.28 & 33.75 & \multirow{3}{*}{29.55} \\
\hline $\mathrm{R} 2$ & 50.60 & 26.63 & \\
\hline R3 & 54.41 & 28.26 & \\
\hline
\end{tabular}



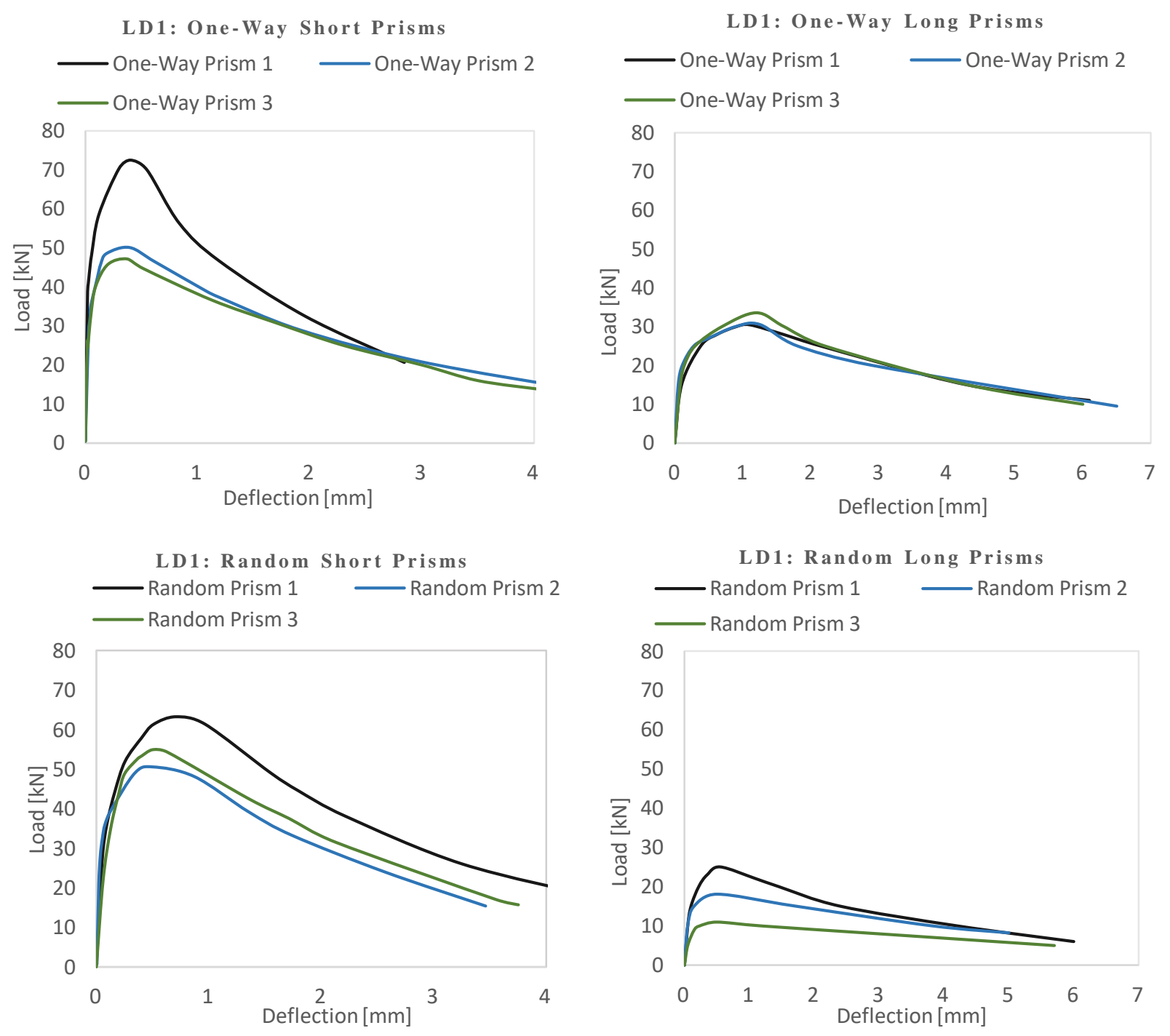

Figure 3-15: Resistance curves of the prisms from mix LDI

\subsubsection{Flexural Strength of In-House DE1 and DE2 Design Mixes}

With regard to the long prisms from mix DE2, the average flexural strength is $22.55 \mathrm{MPa}$ for OW prisms and 16.69 MPa for R prisms. With regard to the short prisms from mix DE2, the average flexural strength is 26.23 MPa for OW prisms and 21.83 MPa for R prisms. Figure 3-16a and Table 3-11 present the resistance curves and flexural strength results of the prisms containing mix DE2.

With regard to the short prisms from mix DE1, the average flexural strength is $23.36 \mathrm{MPa}$ for OW prisms and 18.49 MPa for R prisms. The resistance curve and flexural strength result of each prism from mix DE1 are presented in Figure 3-16b and Table 3-12. 
Table 3- 11: Flexural strength results (Mix DE2)

\begin{tabular}{|c|c|c|c|}
\hline \multicolumn{4}{|c|}{ Long Prisms } \\
\hline \multirow{2}{*}{ Prism } & Peak Load & Flexural Strength & Average Flexural Strength \\
\hline & $\mathrm{kN}$ & $\mathrm{MPa}$ & $\mathrm{MPa}$ \\
\hline OW1 & 22.43 & 24.08 & \multirow{3}{*}{22.55} \\
\hline OW2 & 19.64 & 21.09 & \\
\hline OW3 & 20.64 & 22.47 & \\
\hline $\mathrm{R} 1$ & 14.64 & 15.62 & \multirow{3}{*}{16.69} \\
\hline $\mathrm{R} 2$ & 19.90 & 21.37 & \\
\hline $\mathrm{R} 3$ & 12.27 & 13.09 & \\
\hline \multicolumn{4}{|c|}{ Short Prisms } \\
\hline \multirow{2}{*}{ Prism } & Peak Load & Flexural Strength & Average Flexural Strength \\
\hline & $\mathrm{kN}$ & $\mathrm{MPa}$ & $\mathrm{MPa}$ \\
\hline OW1 & 47.00 & 25.58 & \multirow{3}{*}{26.23} \\
\hline OW2 & 50.32 & 27.20 & \\
\hline OW3 & 48.25 & 25.90 & \\
\hline $\mathrm{R} 1$ & 32.86 & 17.41 & \multirow{3}{*}{21.83} \\
\hline $\mathrm{R} 2$ & 42.78 & 22.81 & \\
\hline $\mathrm{R} 3$ & 47.04 & 25.26 & \\
\hline
\end{tabular}

Table 3-12: Flexural strength results of the prisms from mix DE1

\begin{tabular}{|c|c|c|c|}
\hline \multirow{2}{*}{ Prism } & Peak Load & Flexural Strength & Average Flexural Strength \\
\cline { 2 - 3 } & kN & MPa & MPa \\
\hline OW1 & 45.10 & 24.38 & \multirow{2}{*}{23.36} \\
\hline OW2 & 44.20 & 23.42 & \multirow{2}{*}{1} \\
\hline OW3 & 41.78 & 22.28 & \multirow{2}{*}{18.49} \\
\hline R1 & 29.12 & 15.64 & \\
\hline R2 & 37.27 & 19.88 & \\
\hline R3 & 37.44 & 19.97 & \\
\hline
\end{tabular}
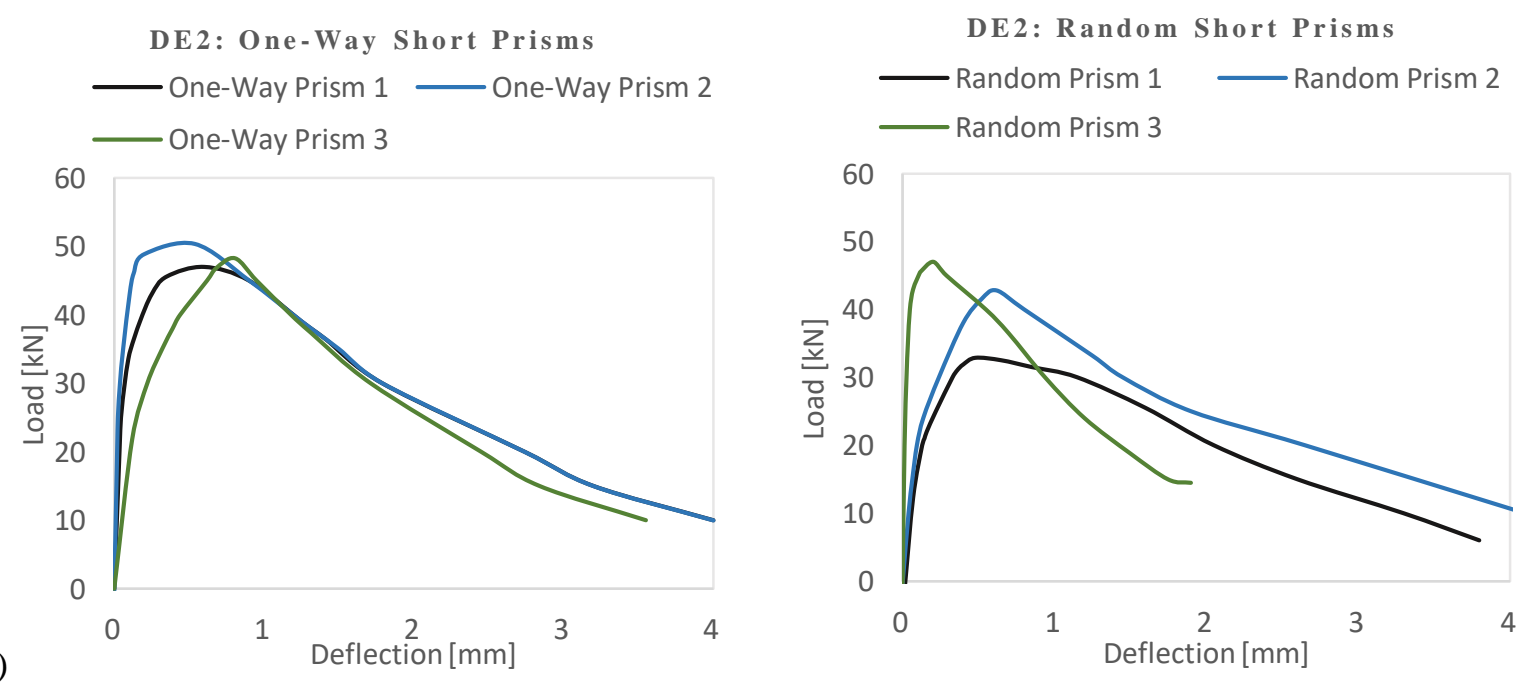
DE1: One-Way Short Prisms

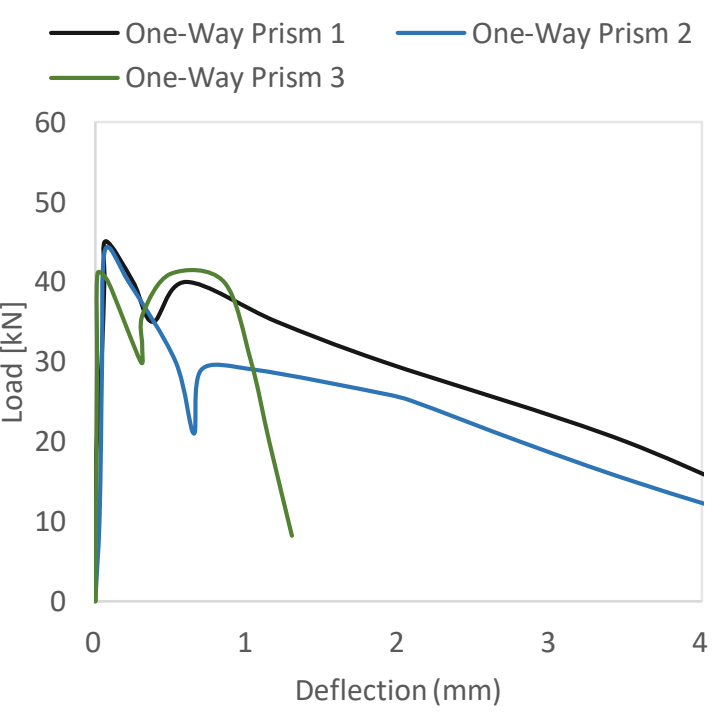

DE 1: Random Short Prisms

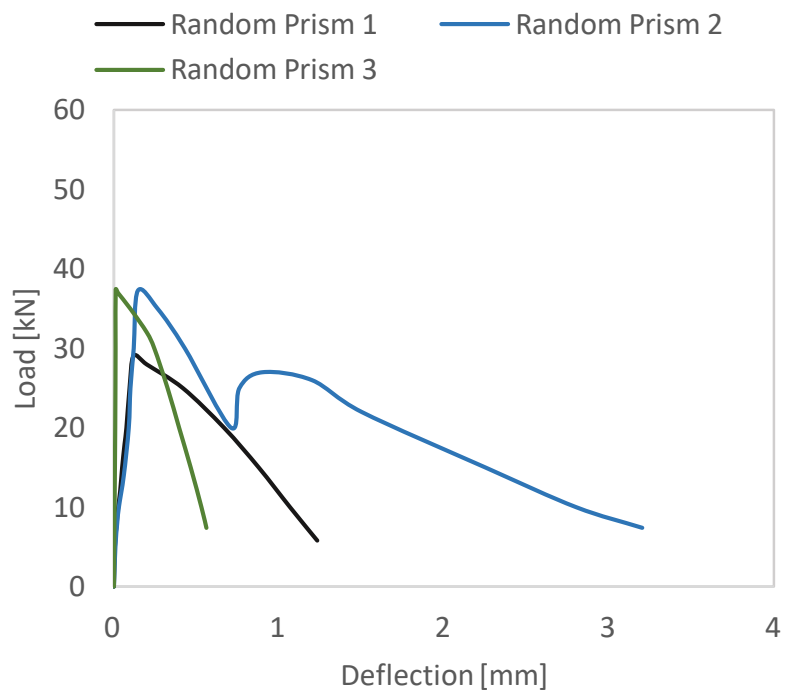

Figure 3- 16: Resistance curves: (a) Prisms from mix DE2; and (b) Prisms from mix DE1

It is evident that the average flexural strength of the short prisms containing $1 \%$ steel fibers from mix DE1 is lower than those containing $2 \%$ steel fibers from mix DE2. As illustrated in Figure 3-16b, prisms from mix DE1 failed in a brittle manner, indicated in resistance curves by the sudden drop of load-carrying capacity. However, due to the presence of $1 \%$ steel fibers, OW prism 1, OW prism 2 and R prism 2 from mix DE1 still had the capacity to sustain load while deflecting considerably. In addition, as depicted in Figure 3-17, the critical failure planes of the prisms from mixes DE1 and DE2 formed within the central constant moment region, whereas the cracking mouth opening of the prisms from mix DE2 is much wider than those from mix DE1, indicating the fiber bridging effect is more prominent in mix DE2.

(a)

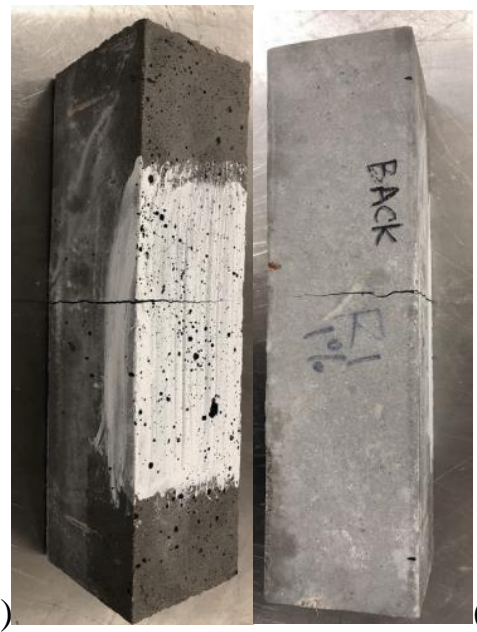

(b)

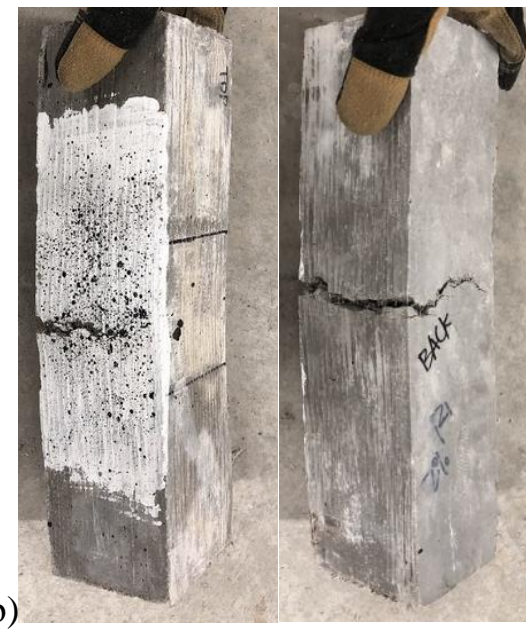

Figure 3-17: Critical failure planes of four-point bending tests: (a) Prisms containing 1\% steel fibers; and (b) Prisms containing $2 \%$ steel fibers 
The average flexural strength of one-way thin prisms ( $50 \mathrm{~mm}$ by $100 \mathrm{~mm}$ by $400 \mathrm{~mm}$ prisms) is $25.57 \mathrm{MPa}$. Figure 3-18 and Table 3-13 present the resistance curve and flexural strength result of each prism. As depicted in Figure 3-19b, OW prism 1 failed under torsion, so its result was discarded. The average flexural strength result of thin prisms $(25.57 \mathrm{MPa})$ is compared with the result of OW long prisms (22.55 MPa) from mix DE2 because both sets of prisms had an aspect ratio of 2. The difference in average flexural strength is $13.4 \%$, indicating the thin prism proposed by AFNOR NF EN 13670 (2013) is adequate.

Table 3- 13: Flexural strength results of the thin prisms from mix DE2

\begin{tabular}{|c|c|c|c|}
\hline \multirow{2}{*}{ Prism } & Peak Load & Flexural Strength & Average Flexural Strength \\
\cline { 2 - 3 } & $\mathrm{kN}$ & $\mathrm{MPa}$ & $\mathrm{MPa}$ \\
\hline OW2 & 22.22 & 26.66 & \multirow{2}{*}{25.57} \\
\hline OW3 & 20.40 & 24.48 & \\
\hline
\end{tabular}

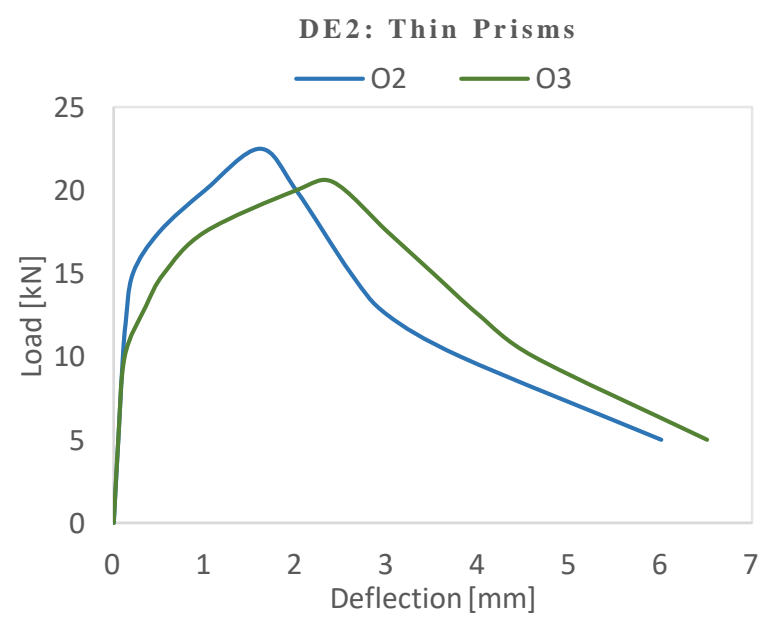

Figure 3-18: Resistance curves of the thin prisms from mix DE2

(a)

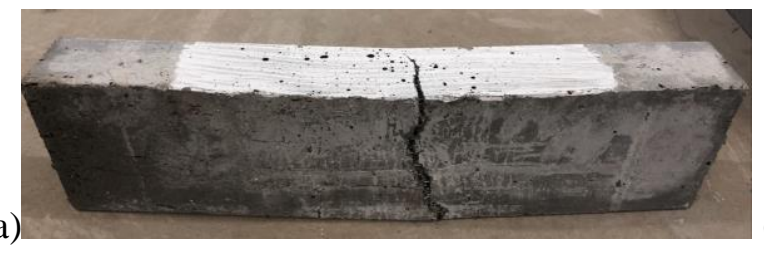

(b)

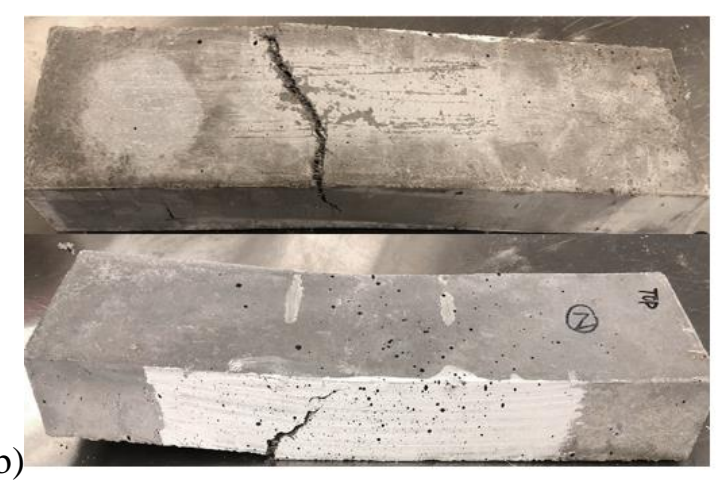

Figure 3-19: Failure modes of the thin prisms subjected to four-point loading: (a) Flexural failure; and (b) Torsional failure 


\subsubsection{Discussion}

As a summary, Figure 3-20 plots the average flexural strength result of each design mix. With regard to casting methodology, it is evident that the average equivalent flexural strength of $\mathrm{R}$ prisms is lower than that of OW prisms for the majority of the tests regardless of aspect ratio of the bending prism and volumetric ratio of steel fibers. In addition, the difference in average flexural strength between $\mathrm{OW}$ and $\mathrm{R}$ prisms is not consistent, but ranges from $0.6 \%$ (short prisms from LD1) to $64.7 \%$ (long prisms from LD1). Fresh concrete was poured at different locations along the prism span with multiple layers in casting the $\mathrm{R}$ prisms and thereby caused the fiber distribution and orientation to vary significantly in different prisms. With regard to the aspect ratio of the bending prism, long prisms generally exhibited a lower average flexural strength than short prisms. Moreover, for most prisms tested, the resistance curves presented in previous sections highlight that the midspan deflection of long prisms at peak load is approximately twofold the midspan deflection of short prisms at peak load regardless of casting methodology. Furthermore, it is evident that average flexural strength of the prisms containing mixes FD1 and FD2 is much higher than the others on account of the $20 \mathrm{~mm}$ long, steel fibers used in the mixes (other design mixes comprise $13 \mathrm{~mm}$ long, steel fibers): the degree of preferential fiber alignment is more prominent for the prisms from mixes FD1 and FD2 because they contain the fibers which are relatively long compared with their cross-sectional dimensions, leading to higher flexural strengths. Furthermore, prisms containing $1 \%$ steel fibers exhibited lower strengths than those containing $2 \%$ steel fibers, and the difference in average flexural strength was $12.3 \%$.

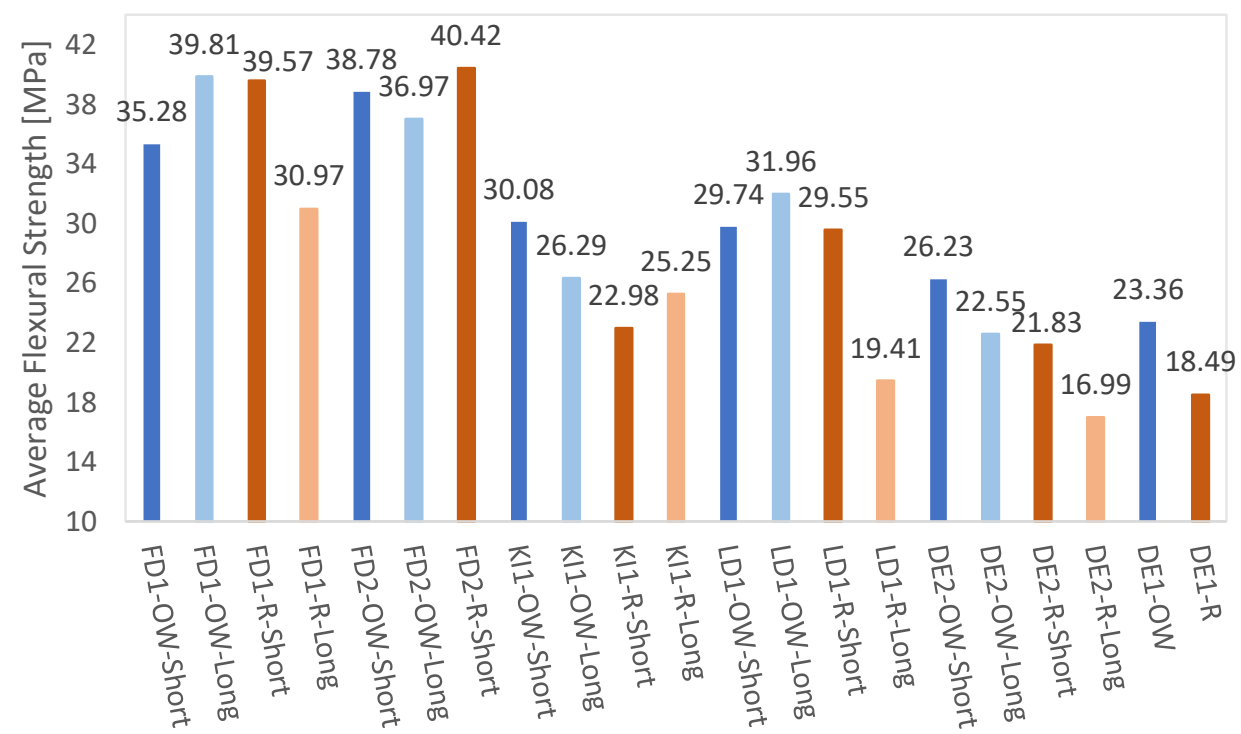

Figure 3-20: Average flexural strength results (Phase 1 of the experimental program) 


\section{Chapter 4. Experimental Program: Phase 2}

\subsection{Introduction}

During Phase 2 of the experimental program, a Round Robin Testing Program was conducted in collaboration with École Polytechnique-Montréal and Queen's University by testing specimens casted from three commercial design mixes, referred herein as mixes FD3, KI2 and LD2. The purpose of this testing program was to assess the replicability of results by testing identical specimens in different laboratories on account of the variability in the custom details of four-point bending test (FPBT) setups and the uncertainties introduced by the operator. In this context, specimens were cast at York University or in the facilities of the commercial companies. After curing for two weeks in uniform conditions at York University, the specimens were shipped to the other two universities. As illustrated in Figure 4-1, specimens were wrapped with wet burlaps, sealed in plastic bags and placed vertically in plastic pails. In addition, the void space between specimens and the pail was filled with foam and burlap to prevent the specimens from damage. A total of 54 prisms with an aspect ratio of 1cast by pouring fresh material from one end of the mold with a single layer or along the span of the prism with multiple layers (i.e., OW prisms and R prisms) and 36 cylinders $(75 \mathrm{~mm}$ by $150 \mathrm{~mm}$ ) were prepared for the Round Robin Testing Program (i.e., 18 prisms were prepared for each university to conduct FPBT, six cylinders were prepared for York University to conduct splitting tensile test, and the other cylinders were prepared for each university to conduct cylinder compression testing). Moreover, each university tested the specimens after the 28-day curing period to eliminate age effect on experimental results. However, significant differences were observed from the flexural strength results among the three universities, and it became evident that the supports and loading rollers utilized by each university were different in some degree. Therefore, two sets of OW prisms from mix DE2 were tested at York University under two different support conditions to investigate whether the support conditions caused the discrepancies in results. Furthermore, to further study the effects of the degree of preferential fiber alignment and prism size on flexural strength, another three commercial design mixes, referred herein as mixes FD4, FD5 and FD6, were prepared by the commercial provider and tested at York University. The prisms from mixes FD4, FD5 and FD6 had an aspect ratio of 1 and were cast by pouring the fresh material from one end of the mold in a single layer (i.e., OW prisms). Six $100 \mathrm{~mm}$ by $100 \mathrm{~mm}$ by $370 \mathrm{~mm}$ prisms and six $150 \mathrm{~mm}$ by $150 \mathrm{~mm}$ by $500 \mathrm{~mm}$ prisms containing $13 \mathrm{~mm}$ long, straight steel fibers were cast with the FD4 design mix. 24 prisms ( $150 \mathrm{~mm}$ by $150 \mathrm{~mm}$ by $500 \mathrm{~mm}$ prisms) containing $20 \mathrm{~mm}$ long, steel fibers were cast with FD5 and FD6 design mixes; of those, 12 prisms were tested at the age of 21 days, and the other 12 prisms were tested at the age of 28 days. 


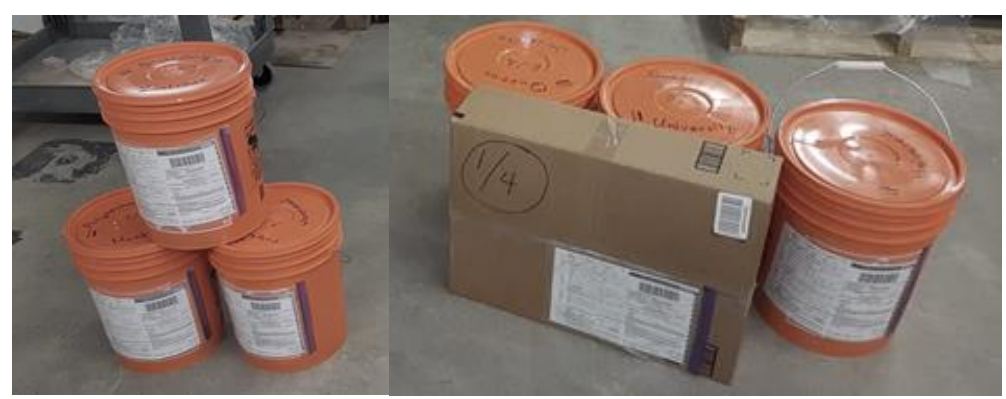

Figure 4- 1: Sealed pails containing prisms and cylinders for each university

\subsection{The Round Robin Testing Program}

\subsubsection{Materials and Casting Methodology}

The commercial companies provided all the necessary materials for the three design mixes (i.e., mixes FD3, KI2 and LD2), and they also appointed technicians to supervise and assist in concrete mixing and casting. Mix FD3 was cast on November $14^{\text {th }}, 2018$; mix KI2 was cast on November $16^{\text {th }}$, 2018; and mix LD2 was cast on November $19^{\text {th }}, 2018$. All three design mixes contained $2 \%$ steel fibers by unit volume. Mixes KI2 and LD2 comprised $13 \mathrm{~mm}$ long, straight steel fibers with a diameter of $0.2 \mathrm{~mm}$, whereas mix FD3 comprised $1 \% 20 \mathrm{~mm}$ long, straight steel fibers with a dimeter of $0.2 \mathrm{~mm}$ and $1 \% 20 \mathrm{~mm}$ long, hooked-end steel fibers with a diameter of $0.3 \mathrm{~mm}$. In addition, to determine the flowability of each design mix, the mold of the flow table was filled with a single layer of fresh cementitious composites, and the average of minimum and maximum diameters of the spread of the fluid material on the flow table measured two minutes after lifting the mold constituted the flow value. Flowability was found to be $228 \mathrm{~mm}$ for mix FD3, $225 \mathrm{~mm}$ for mix KI2 and $235 \mathrm{~mm}$ for mix LD2. As depicted in Figure 4-2, wood and acrylic molds were prepared for prisms and standard plastic molds were prepared for cylinders. A release agent was applied to the molds prior to casting. With regard to the cylinder specimens, molds were filled in a single layer. With regard to the prisms, similar to the prisms considered for the Phase 1 of the experimental program, two casting methodologies were applied, and both OW prisms and R prisms were cast for the Round Robin Testing Program. Six prisms from each commercial design mix were prepared for each university including three OW prisms and three R prisms to study the effect of casting methodology on flexural strength. In addition, all the prisms had an aspect ratio of 1, and Table 4-1 presents the prism size in terms of fiber length specified in ASTM C1856 (2017). Therefore, dimensions of the prisms from mixes KI2 and LD2 are $75 \mathrm{~mm}$ by $75 \mathrm{~mm}$ by $280 \mathrm{~mm}$ owing to the use of $13 \mathrm{~mm}$ long steel fibers, whereas dimensions of the prisms from mix FD3 are $100 \mathrm{~mm}$ by $100 \mathrm{~mm}$ by $370 \mathrm{~mm}$ owing to the use of $20 \mathrm{~mm}$ long steel fibers. Furthermore, specimens were consolidated by tapping the sides of the mold 30 times with a mallet. 
Thereafter, specimens were demolded one day after casting and stored in water tanks in ambient laboratory temperature.

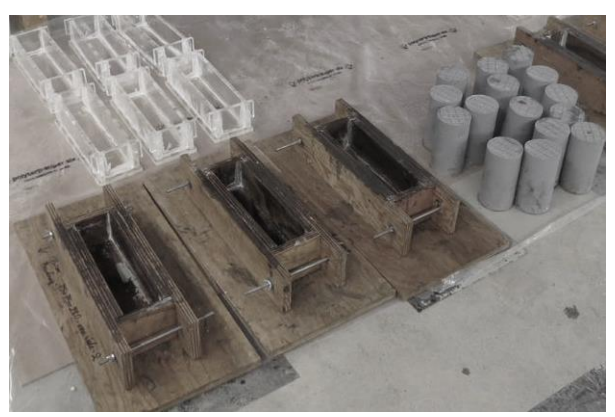

Figure 4- 2: Prepared molds for the prismatic and cylindrical specimens for the Round Robin Testing Program

Table 4- 1: Specified prism size in terms of fiber length (ASTM C1856 2017)

\begin{tabular}{|c|c|}
\hline Fiber Length & Nominal Prism Cross Section \\
\hline$<15 \mathrm{~mm}$ & $75 \mathrm{~mm} \times 75 \mathrm{~mm}$ \\
\hline $15 \mathrm{~mm}$ to $20 \mathrm{~mm}$ & $100 \mathrm{~mm} \times 100 \mathrm{~mm}$ \\
\hline $20 \mathrm{~mm}$ to $25 \mathrm{~mm}$ & $150 \mathrm{~mm} \times 150 \mathrm{~mm}$ \\
\hline$>25 \mathrm{~mm}$ & $200 \mathrm{~mm} \times 200 \mathrm{~mm}$ \\
\hline
\end{tabular}

\subsubsection{Compression Test}

A load-controlled compression-testing machine (Controls Pilot) was used to test $75 \mathrm{~mm}$ by $150 \mathrm{~mm}$ cylinders under compression at a loading rate of $0.259 \mathrm{MPa} / \mathrm{s}$ (ASTM C39 2005). Each university utilized the same loading rate and tested the cylinders at the age of 28 days to eliminate loading rate effect and age effect on compressive strength results. As depicted in Figure 4-3, top and bottom surfaces of the cylinders were grinded prior to testing, and the test setup considered at York University was the same as the one utilized for the Phase 1 of the experimental program (refer to Figure 3-5). In addition, similar to what was observed from Phase 1 of the experimental program, the cylinders generally held their integrity beyond attainment of the peak load, and the cracks formed parallel to the loading direction. For mix FD3, the average compressive strength was found to be 111.1 MPa by York University and 123.7 MPa by Queen's University. For mix KI2, the average compressive strength was found to be $129 \mathrm{MPa}$ by York University and 122.2 MPa by Queen's University. For mix LD2, the average compressive strength was to be $166 \mathrm{MPa}$ by York University and 121.1 MPa by Queen's University. The compressive strength result of each cylinder is presented in Table 4-2, where ' $\mathrm{S}$ ' represents the cylinders with substandard quality (i.e., large voids formed at top surfaces, requiring significant reduction of cylinder height to obtain dependable results). 


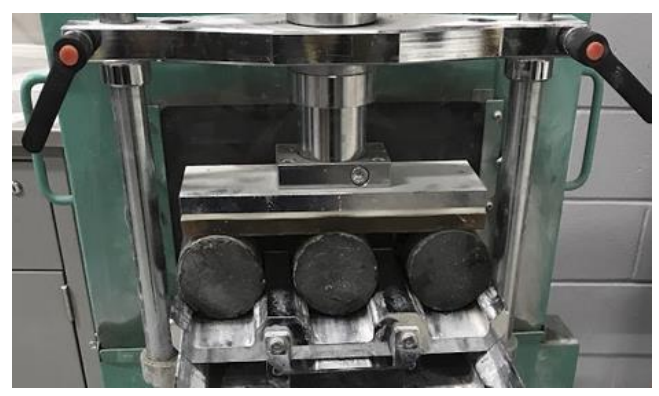

Figure 4- 3: Concrete grinding for compression test

Table 4- 2: Compressive strength results (The Round Robin Testing Program)

\begin{tabular}{|c|c|c|c|c|c|c|c|c|c|c|c|c|}
\hline \multicolumn{13}{|c|}{ York University } \\
\hline Mix & \multicolumn{6}{|c|}{ FD3 } & \multicolumn{3}{|c|}{$\mathrm{KI} 2$} & \multicolumn{3}{|c|}{ LD2 } \\
\hline Cylinder & \multicolumn{2}{|c|}{$\mathrm{C} 1$} & \multirow{2}{*}{\multicolumn{2}{|c|}{$\begin{array}{c}\mathrm{C} 2 \\
\mathrm{~S}\end{array}$}} & \multicolumn{2}{|c|}{$\mathrm{C} 3$} & $\mathrm{C} 1$ & $\mathrm{C} 2$ & $\mathrm{C} 3$ & $\mathrm{C} 1$ & $\mathrm{C} 2$ & $\mathrm{C} 3$ \\
\hline Compressive Strength $[\mathrm{MPa}]$ & \multicolumn{2}{|c|}{$\mathrm{S}$} & & & \multicolumn{2}{|c|}{111} & 120 & 137 & 130 & 178 & 156 & 164 \\
\hline $\begin{array}{l}\text { Average Compressive } \\
\text { Strength [MPa] }\end{array}$ & \multicolumn{6}{|c|}{111} & \multicolumn{3}{|c|}{129} & \multicolumn{3}{|c|}{166} \\
\hline \multicolumn{13}{|c|}{ Queen's University } \\
\hline Mix & \multicolumn{6}{|c|}{ FD3 } & \multicolumn{3}{|c|}{ KI2 } & \multicolumn{3}{|c|}{ LD2 } \\
\hline Cylinder & $\mathrm{C} 1$ & $\mathrm{C} 2$ & $\mathrm{C} 3$ & $\mathrm{C} 4$ & $\mathrm{C} 5$ & C6 & $\mathrm{C} 1$ & $\mathrm{C} 2$ & $\mathrm{C} 3$ & $\mathrm{C} 1$ & $\mathrm{C} 2$ & $\mathrm{C} 3$ \\
\hline Compressive Strength [MPa] & 134 & 117 & 115 & 127 & 117 & 131 & 121 & 120 & 126 & 129 & 123 & 11 \\
\hline $\begin{array}{l}\text { Average Compressive } \\
\text { Strength }[\mathrm{MPa}]\end{array}$ & \multicolumn{6}{|c|}{124} & \multicolumn{3}{|c|}{122} & \multicolumn{3}{|c|}{121} \\
\hline
\end{tabular}

\subsubsection{Splitting Tensile Test}

Two $75 \mathrm{~mm}$ by $150 \mathrm{~mm}$ cylinders from each design mix were subjected to diametrically compressive loading at a loading rate of $0.016 \mathrm{MPa} / \mathrm{s}$ (CSA-A23.2-13C 2014) after 30 days from casting. The experimental test setup is the same as the one utilized for the Phase 1 of the experimental program (refer to Figure 3-7a). The average splitting tensile strength is $25 \mathrm{MPa}$ for mix FD3, 23.6 MPa for KI2 and 23.4 MPa for mix LD2. Table 4-3 presents the splitting tensile strength result of each cylinder.

Table 4- 3: Splitting tensile strength results (Mixes FD3, KI2 and LD2)

\begin{tabular}{|c|c|c|c|c|c|c|}
\hline Mix & \multicolumn{2}{|c|}{ FD3 } & \multicolumn{2}{c|}{ KI2 } & \multicolumn{2}{c|}{ LD2 } \\
\hline Cylinder & C1 & C2 & C1 & C2 & C1 & C2 \\
\hline Peak Load [kN] & 431 & 443 & 411 & 434 & 371 & 448 \\
\hline Splitting Tensile Strength [MPa] & 25.1 & 24.9 & 23.0 & 24.2 & 21.3 & 25.5 \\
\hline Average Splitting Tensile Strength [MPa] & \multicolumn{2}{|c|}{25.0} & \multicolumn{2}{|c|}{23.6} & \multicolumn{2}{|c|}{23.4} \\
\hline
\end{tabular}




\subsubsection{Four-Point Bending Test}

The prisms subjected to four-point loading were tested at the age of 28 days by each university. As illustrated in Figure 4-4, École Polytechnique-Montréal proposed a stepwise loading rate in light of ASTM C1609 (2012), and all three universities tested the prims with the same loading rate to exclude the potential inconsistency in results caused by the loading rate effect. The stepwise loading rate comprised two stages: a loading rate of $0.15 \mathrm{~mm} / \mathrm{min}$ was applied to prisms before reaching $70 \%$ post-peak load, then the loading rate was increased by $0.05 \mathrm{~mm} / \mathrm{min}$ for the post-peak softening branch after the load dropped below $70 \%$ of the peak load. In addition, the prisms were tested until the post-peak residual strength had degraded to $20 \%$ of the peak load. As depicted in Figure 3-11, the experimental test setup was generally the same among the three universities. With regard to the $75 \mathrm{~mm}$ by $75 \mathrm{~mm}$ by $280 \mathrm{~mm}$ prisms from mixes KI2 and LD2, the shear span and the distance between loading points is $75 \mathrm{~mm}$. With regard to the $100 \mathrm{~mm}$ by $100 \mathrm{~mm}$ by $370 \mathrm{~mm}$ prisms from mix FD3, the shear span and the distance between loading points is $100 \mathrm{~mm}$. However, as depicted in Figure 4-5, there were some discrepancies in support and loading rollers among the three university test setups. The support rollers employed at York University are 1.5 inch in diameter which are free to rotate about their own axes and are supported on their axles through bearings in order to eliminate friction (as depicted in Figure 4-5a). Furthermore, horizontal translations are free to manifest at the points of contact between the prism and support rollers, but the distance between the axles of support rollers is restrained and the span of the prism does not change during testing. Moreover, York University's loading rollers are 2.5 inch in diameter which are supported on their axles through bearings and free to rotate (as depicted in Figure 4-5b). Additionally, the loading rollers can adjust rotation about the longitudinal axis of the prism. The test setups at Queen's University and École Polytechnique-Montréal incorporated support rollers of 2 inch in diameter and 1 inch in diameter, respectively, where one roller is fixed against any rotation and translation, whereas the other is free to translate in the longitudinal direction of the setup (as depicted in Figure 4-5c and Figure 4-5d). The support conditions utilized by Queen's University and École Polytechnique-Montréal are in accordance with Annex U of CSA-A23.1 (2019), while the support condition utilized by York University is in accordance with ASTM C1609 (2012). Furthermore, the loading rollers at Queen's University and École Polytechnique-Montréal are 1 inch in diameter. In addition to the support and loading rollers, the position of the prisms in the test setup is another discrepancy among the three universities. The prisms generally had some roughness on the free surface created through casting operation. In this regard, York University and Queen's University rotated the prisms at 90 degrees with respect to the position at casting such that the surfaces in contact with the support and loading rollers would be smooth. However, École Polytechnique-Montréal applied a layer of plaster between the prism and loading rollers rather than rotating the prisms. The equivalent flexural strength of each prism was 
calculated with Equation 3-3, and the results obtained by each university are presented in the following sections.

\begin{tabular}{|c|c|}
\hline \multicolumn{2}{|c|}{ Polytechnique Montréal } \\
\hline Criteria & Procedure \\
\hline $\begin{array}{l}\text { Up to } 70 \% \text { of peak load } \\
\text { in the post-peak region }\end{array}$ & $\begin{array}{l}\text { - Rate of increase of net deflection } \\
\text { shall be of } 0.15 \mathrm{~mm} / \mathrm{min} \\
(0.0025 \mathrm{~mm} / \mathrm{s})\end{array}$ \\
\hline \multirow[t]{3}{*}{$\begin{array}{l}\text { Beyond } 70 \% \text { of peak } \\
\text { load in the post-peak } \\
\text { region }\end{array}$} & $\begin{array}{l}\text { - When increasing the loading rate, } \\
\text { the rate of increase of net deflection } \\
\text { shall be increased in increment of } \\
\text { speed of } \\
\Delta V=(0.05 \mathrm{~mm} / \mathrm{min}) / 60 \mathrm{~s} \\
\Delta V=0.000833 \mathrm{~mm} / \mathrm{min} / \mathrm{s}\end{array}$ \\
\hline & $\begin{array}{l}\text { - Subsequent increases of the rate } \\
\text { of net deflection shall be at } 1 \mathrm{~s} \text { apart. }\end{array}$ \\
\hline & $\begin{array}{l}\text { - The maximum rate of increase of } \\
\text { net deflection shall not exceed } 1 \\
\mathrm{~mm} / \mathrm{min} \text {. }\end{array}$ \\
\hline End of test & $\begin{array}{l}\text { - Test shall be stopped when post- } \\
\text { peak load reached } 3 \mathrm{kN}\end{array}$ \\
\hline
\end{tabular}

\begin{tabular}{ll} 
& \multicolumn{1}{c}{ ASTM C1609 } \\
\hline Criteria & Procedure \\
\hline $\begin{array}{l}\text { Up to net deflection } \\
\text { of L/900 }\end{array}$ & $\begin{array}{l}\text { - Rate of increase of net deflection shall be of } \\
0.025 \text { to } 0.075 \mathrm{~mm} / \mathrm{min}\end{array}$ \\
& $\begin{array}{l}(0.00042 \mathrm{~mm} / \mathrm{s} \text { to } 0.0013 \mathrm{~mm} / \mathrm{s}) \\
\text { - The initial loading rate up to deflection of } \mathrm{L} / 900 \\
\text { [...] shall be based on reaching the first-peak } \\
\text { deflection } 40 \text { to } 100 \mathrm{~s} \text { after the start of the test }\end{array}$ \\
\hline $\begin{array}{l}\text { Beyond to net } \\
\text { deflection of L/900 }\end{array}$ & $\begin{array}{l}\text { - When increasing the loading rate, the rate of } \\
\text { increase of net deflection shall be increased in } \\
\text { increments not exceeding } 0.05 \mathrm{~mm} / \mathrm{min} .\end{array}$
\end{tabular}

- Subsequent increases of the rate of net deflection shall be at least $30 \mathrm{~s}$ apart.

- The rate of increase of net deflection shall not exceed 8 times the initial rate until the specified end-point deflection is reached.

\begin{tabular}{ll}
\hline End of test & - Unless otherwise required by the specifier of \\
& tests, terminate the test at a net deflection of \\
1150 of the span
\end{tabular}

Figure 4- 4: Stepwise loading rate proposed by École Polytechnique-Montréal (ASTM C1609 2012)

(a)

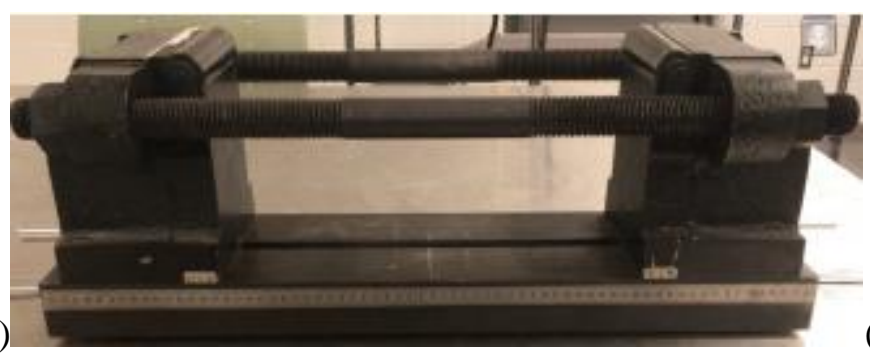

(b)

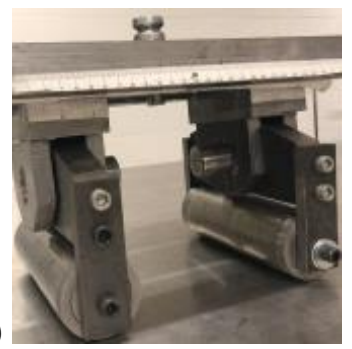

(c)

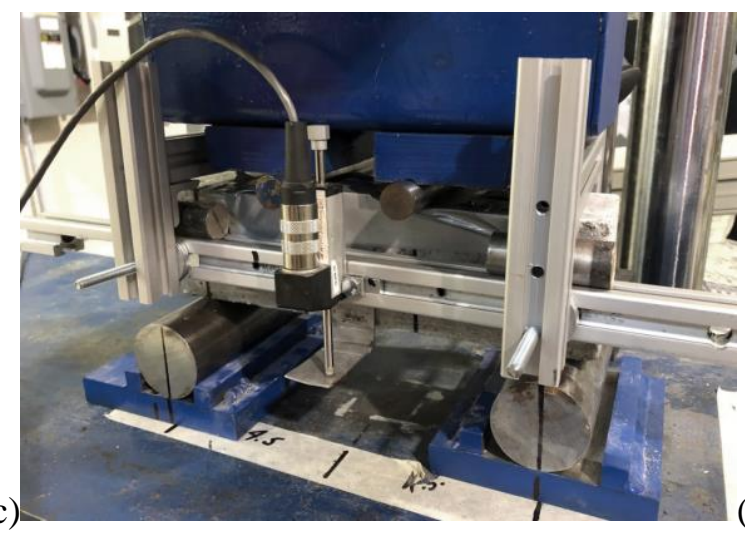

(d)

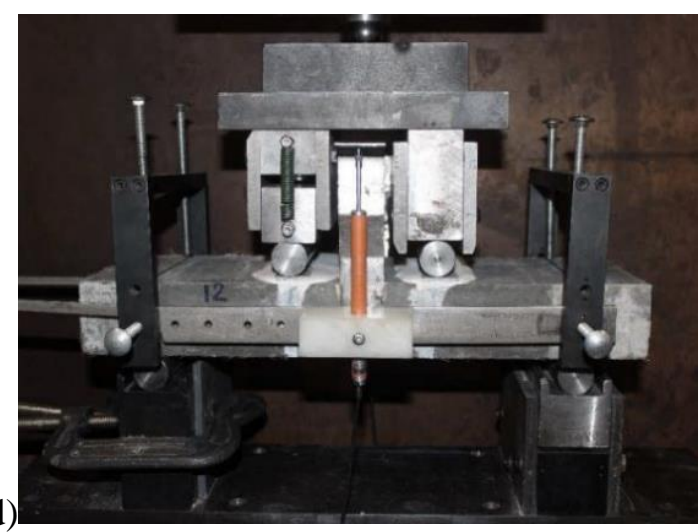

Figure 4- 5: Support and loading rollers of four-point bending test: (a) York University's support rollers; (b) York University's loading rollers; (c) Queen's University's support and loading rollers; and (d) École PolytechniqueMontréal's support and loading rollers (Annex U of CSA-A23.1 2019) 


\subsubsection{Commercial FD3 Design Mix}

The average flexural strength results for the commercial design mix FD3 obtained by York University is 40.22 MPa for OW prisms and 31.73 MPa for R prisms; the average flexural strength results obtained by École Polytechnique-Montréal is 32.61 MPa for OW prisms and 26.86 MPa for R prisms; and the average flexural strength results obtained by Queen's University is $30.71 \mathrm{MPa}$ for OW prisms and 24.68 MPa for $\mathrm{R}$ prisms. It is evident that York University's results were consistently higher than École PolytechniqueMontréal while Queen's University reported the lowest results regardless of casting methodology. In addition, the average flexural strength result of $\mathrm{R}$ prisms obtained by each university was lower than that of OW prisms. As depicted in Figure 4-6a, most prisms failed due to flexure. However, as depicted in Figure 4-6b, several prisms failed in torsion owing to the ability of support and loading rollers to adjust rotation about the longitudinal axis of the prism. Torsional failure was reflected by the critical crack propagating across the width of the tension face with an inclination relative to the transverse axis of the prism. In addition, as depicted in Figure 4-6c, the critical failure planes of some prisms formed outside the constant moment region (i.e., shear failure), and the results of these prisms were considered as outliers. Moreover, due to the poor performance of the instrumentation in some tests, the linear displacement transducer slipped and did not measure the vertical deflection at midspan properly. Figure 4-7 to Figure 49 illustrate the resistance curve of each prism (with the exception of the prisms that experienced poor performance of the instrumentation, torsional failure or shear failure). The flexural strength result of each prism is presented in Table 4-4 (with the exception of the prisms that experienced torsional failure or shear failure).
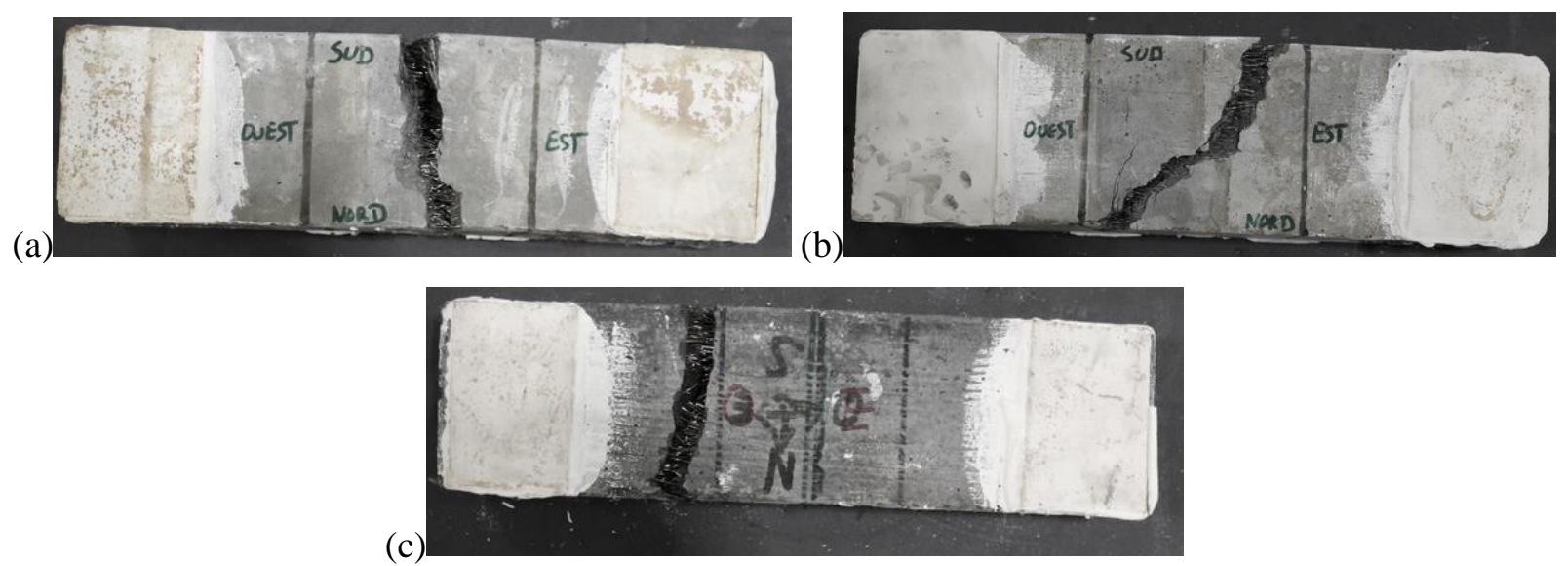

Figure 4- 6: Failure Modes of four-point bending tests: (a) Flexural failure; (b) Torsional failure; and (c) Shear failure 
Table 4- 4: Flexural strength results (Mix FD3)

\begin{tabular}{|c|c|c|c|}
\hline \multicolumn{4}{|c|}{ York University } \\
\hline & Peak Load & Flexural Strength & Average Flexural Strength \\
\hline Prism & $\mathrm{kN}$ & $\mathrm{MPa}$ & $\mathrm{MPa}$ \\
\hline OW1 & 138.62 & 40.77 & \multirow{3}{*}{40.22} \\
\hline OW2 & 145.81 & 43.31 & \\
\hline OW3 & 121.95 & 36.59 & \\
\hline $\mathrm{R} 1$ & 97.24 & 29.17 & \multirow{3}{*}{31.73} \\
\hline $\mathrm{R} 2$ & 111.47 & 32.15 & \\
\hline R3 & 116.31 & 33.88 & \\
\hline \multicolumn{4}{|c|}{ Ecole Polytechnique-Montréal } \\
\hline & Peak Load & Flexural Strength & Average Flexural Strength \\
\hline Prism & $\mathrm{kN}$ & $\mathrm{MPa}$ & $\mathrm{MPa}$ \\
\hline OW1 & 100.20 & 30.06 & \multirow[b]{2}{*}{32.61} \\
\hline OW2 & 117.20 & 35.16 & \\
\hline $\mathrm{R} 1$ & 103.30 & 30.99 & \multirow{3}{*}{26.86} \\
\hline R2 & 87.00 & 26.10 & \\
\hline R3 & 78.30 & 23.49 & \\
\hline \multicolumn{4}{|c|}{ Queen's University } \\
\hline & Peak Load & Flexural Strength & Average Flexural Strength \\
\hline Prism & $\mathrm{kN}$ & $\mathrm{MPa}$ & $\mathrm{MPa}$ \\
\hline OW2 & 108.50 & 32.55 & \multirow{2}{*}{30.71} \\
\hline OW3 & 96.20 & 28.86 & \\
\hline R2 & 74.10 & 22.23 & \multirow{2}{*}{24.68} \\
\hline R3 & 90.40 & 27.12 & \\
\hline
\end{tabular}

FD 3-YorkU: One-Way Prisms

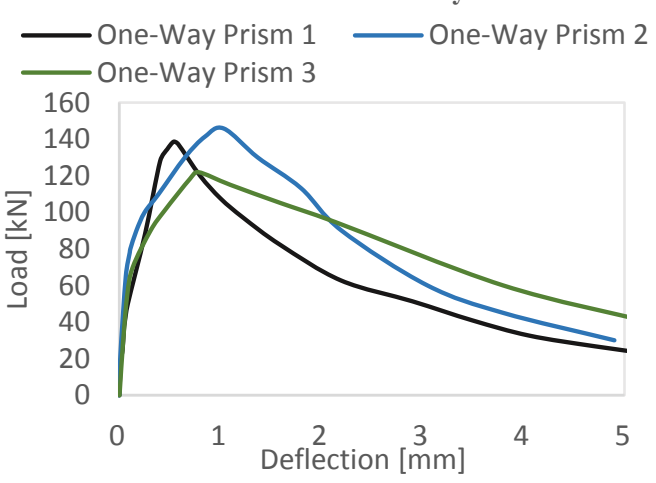

FD3 - YorkU: Random Prisms

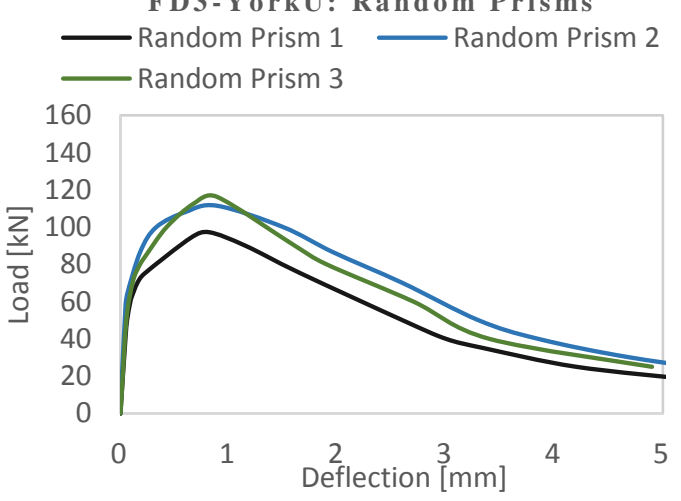

Figure 4- 7: Resistance curves of the prisms from mix FD3 (York University)

FD3-EP: One-Way Prisms

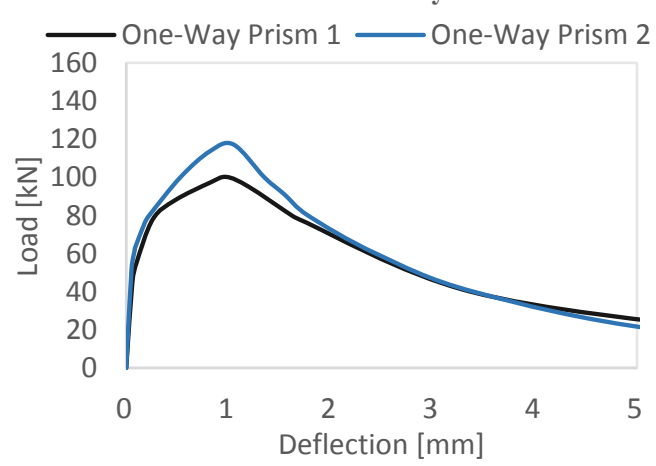

FD 3 - EP : Random Prisms

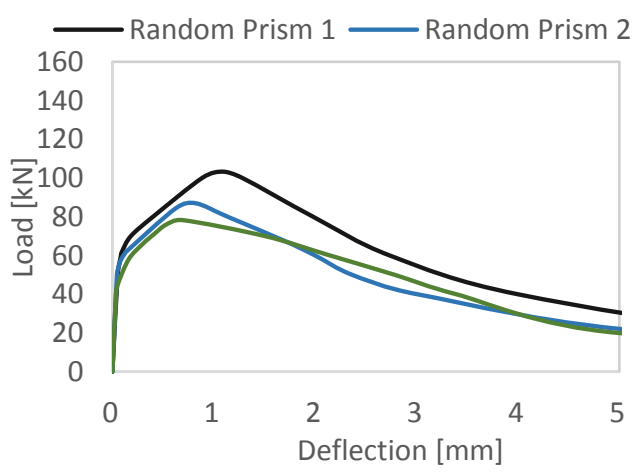

Figure 4- 8: Resistance curves of the prisms from mix FD3 (École Polytechnique-Montréal) 

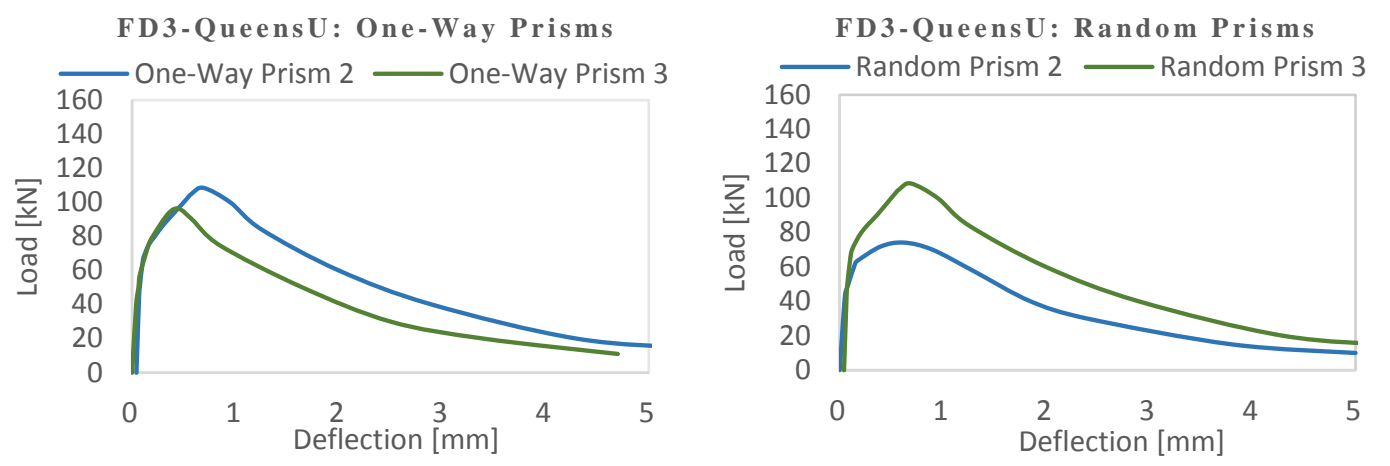

Figure 4- 9: Resistance curves of the prisms from mix FD3 (Queen's University)

\subsubsection{Commercial KI2 Design Mix}

The average flexural strength results for the commercial design mix KI2 obtained by York University is 27.19 MPa for OW prisms and 30.47 MPa for R prisms; the average flexural strength results obtained by École Polytechnique-Montréal is $22 \mathrm{MPa}$ for OW prisms and 24.37 MPa for R prisms; and the average flexural strength results obtained by Queen's University is $20.53 \mathrm{MPa}$ for OW prisms and $22.34 \mathrm{MPa}$ for $\mathrm{R}$ prisms. As with the previous mix, York University's results were consistently higher than École Polytechnique-Montréal's while Queen's University recorded the lowest results. The flexural strength result of each prism is presented in Table 4-5 (with the exception of the prisms that experienced torsional failure or shear failure), and the resistance curves can be found in Appendix A.1.3.

Table 4- 5: Flexural strength results (Mix KI2)

\begin{tabular}{|c|c|c|c|}
\hline \multicolumn{4}{|c|}{ York University } \\
\hline \multirow{2}{*}{ Prism } & Peak Load & Flexural Strength & Average Flexural Strength \\
\hline & $\mathrm{kN}$ & $\mathrm{MPa}$ & $\mathrm{MPa}$ \\
\hline OW1 & 55.08 & 28.25 & \multirow{2}{*}{27.19} \\
\hline OW3 & 50.98 & 26.13 & \\
\hline R1 & 49.38 & 26.34 & \multirow{3}{*}{30.47} \\
\hline $\mathrm{R} 2$ & 57.20 & 30.51 & \\
\hline R3 & 68.31 & 34.56 & \\
\hline \multicolumn{4}{|c|}{ École Polytechnique-Montréal } \\
\hline \multirow{2}{*}{ Prism } & Peak Load & Flexural Strength & Average Flexural Strength \\
\hline & $\mathrm{kN}$ & $\mathrm{MPa}$ & $\mathrm{MPa}$ \\
\hline OW1 & 44.10 & 23.52 & \multirow{2}{*}{22.00} \\
\hline OW2 & 38.40 & 20.48 & \\
\hline R3 & 45.70 & 24.37 & 24.37 \\
\hline \multicolumn{4}{|c|}{ Queen's University } \\
\hline \multirow{2}{*}{ Prism } & Peak Load & Flexural Strength & Average Flexural Strength \\
\hline & $\mathrm{kN}$ & $\mathrm{MPa}$ & $\mathrm{MPa}$ \\
\hline OW1 & 37.74 & 20.13 & \multirow{2}{*}{20.53} \\
\hline OW3 & 39.24 & 20.93 & \\
\hline R2 & 33.37 & 17.80 & \multirow{2}{*}{22.34} \\
\hline R3 & 50.38 & 26.87 & \\
\hline
\end{tabular}




\subsubsection{Commercial LD2 Design Mix}

The average flexural strength results for the commercial design mix LD2 obtained by York University is 26.54 MPa for OW prisms and 25.05 MPa for R prisms; the average flexural strength results obtained by École Polytechnique-Montréal is 20.35 MPa for OW prisms and 21.36 MPa for R prisms; and the average flexural strength results obtained by Queen's University is 20.94 MPa for OW prisms and 17.51 MPa for $\mathrm{R}$ prisms. The flexural strength result of each prism is presented in Table 4-6 (with the exception of the prisms that experienced torsional failure or shear failure), and the resistance curves can be found in Appendix A.1.4.

Table 4- 6: Flexural strength results (Mix LD2)

\begin{tabular}{|c|c|c|c|}
\hline \multicolumn{4}{|c|}{ York University } \\
\hline \multirow{2}{*}{ Prism } & Peak Load & Flexural Strength & Average Flexural Strength \\
\hline & $\mathrm{kN}$ & $\mathrm{MPa}$ & $\mathrm{MPa}$ \\
\hline OW1 & 56.03 & 29.49 & \multirow{3}{*}{26.54} \\
\hline OW2 & 41.39 & 21.50 & \\
\hline OW3 & 53.69 & 28.63 & \\
\hline $\mathrm{R} 1$ & 40.11 & 20.31 & \multirow{2}{*}{25.05} \\
\hline R3 & 55.86 & 29.79 & \\
\hline \multicolumn{4}{|c|}{ École Polytechnique-Montréal } \\
\hline \multirow{2}{*}{ Prism } & Peak Load & Flexural Strength & Average Flexural Strength \\
\hline & $\mathrm{kN}$ & MPa & $\mathrm{MPa}$ \\
\hline OW1 & 43.30 & 23.09 & \multirow{3}{*}{20.35} \\
\hline OW2 & 39.90 & 21.28 & \\
\hline OW3 & 31.30 & 16.69 & \\
\hline $\mathrm{R} 1$ & 43.50 & 23.20 & \multirow{2}{*}{21.36} \\
\hline R3 & 36.60 & 19.52 & \\
\hline \multicolumn{4}{|c|}{ Queens University } \\
\hline \multirow{2}{*}{ Prism } & Peak Load & Flexural Strength & Average Flexural Strength \\
\hline & $\mathrm{kN}$ & $\mathrm{MPa}$ & $\mathrm{MPa}$ \\
\hline OW1 & 38.59 & 20.58 & \multirow{2}{*}{20.94} \\
\hline OW3 & 39.91 & 21.29 & \\
\hline R1 & 32.22 & 17.19 & \multirow{3}{*}{17.51} \\
\hline $\mathrm{R} 2$ & 35.72 & 19.05 & \\
\hline R3 & 30.56 & 16.30 & \\
\hline
\end{tabular}

\subsubsection{Discussion of the Round Robin Testing Program Results}

As a summary, Figure 4-10 illustrates the average flexural strength results of the prisms containing mixes FD3, KI2 and LD2. It is evident that regardless of casting methodology, the results obtained by York University were consistently higher than those obtained by École Polytechnique-Montréal, and Queen's University reported the lowest results in general. The difference in average flexural strength result is 
between 22.2\% (based on the results of random prisms from mix FD3 obtained by York University and Queen's University) and 30.3\% (based on the results of random prisms from mix LD2 obtained by York University and Queen's University). The difference is attributed to the following reasons: 1). Different testing equipment available in each lab; 2). The position of the prism in the test setup (York University and Queen's University rotated the prisms at 90 degrees with respect to the position at casting); 3). The use of a softer material layer between the prism and rollers (École Polytechnique-Montréal did not rotate the prism at 90 degrees but placed a layer of plaster at the points of contact between the prism and rollers); and 4). The ability of the support and loading rollers to adjust rotation about the longitudinal axis of the prism to eliminate torsional effects. Note that prisms that experienced torsional effects failed prematurely at lower loads.

Theoretically, École Polytechnique-Montréal was expected to obtain higher strength results than York University and Queen's University as a result of the way the prisms were placed in the test setup. Due to effects of gravity, it is highly probable that an increased number of steel fibers were present along the bottom surface of the prism at casting. For York University and Queen's University, the bottom surface at casting was not the tension face during testing due to the rotation of the prism by 90 degrees, whereas for École Polytechnique-Montréal, the bottom surface at casting was the tension face during testing. This suggests that there was a higher concentration of steel fibers present near the tension face of the prisms tested by École Polytechnique-Montréal compared with the prisms tested by the other two universities, thus École Polytechnique-Montréal's results were expected to be the highest. However, the results reported by École Polytechnique-Montréal were consistently lower than York University. One of the reasons is attributed to the application of the plaster layer which led to premature softening response. The other probable reason is the difference in support and loading rollers.

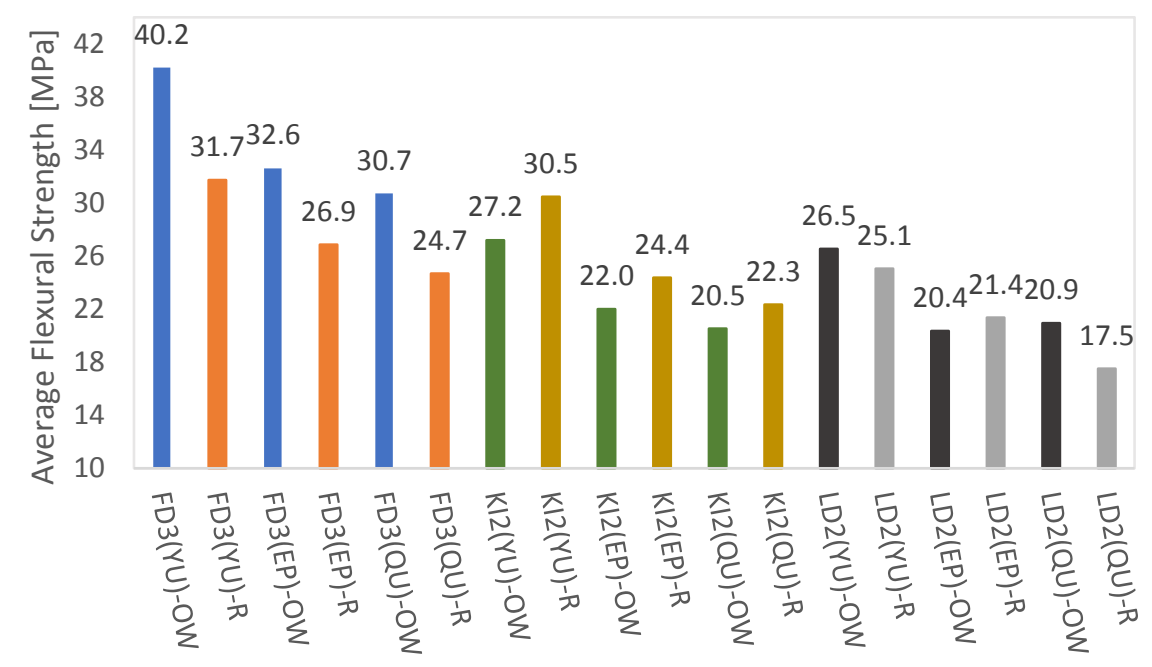

Figure 4- 10: Average flexural strength results (the Round Robin Testing Program) 


\subsubsection{Investigation into the Effect of Support Condition}

To assess whether the difference in support rollers among the three universities contributed to the discrepancies in flexural strength results, two sets of OW prisms with an aspect ratio of 1 (75 $\mathrm{mm}$ by 75 $\mathrm{mm}$ by $280 \mathrm{~mm}$ prisms) from mix DE2 were subjected to four-point loading under two different support conditions. As illustrated in Figure 4-11a, support rollers which restrain horizontal translation, and utilized by York University during the Round Robin Testing Program are referred herein as support condition 1 (SC1); and the support condition depicted in Figure 4-11b comprising a fixed roller (a shim was placed between the C-channel and roller to restrict the right roller from translation and rotation) and a roller with the ability to translate laterally (there is some space between the C-channel and left roller) is referred herein as support condition 2 (SC2). SC2 is identical to that utilized by Queen's University and École Polytechnique-Montréal during the Round Robin Testing Program. In addition, the diameter of the support rollers of SC2 is 2 inch. The loading rollers used to test these two sets of prisms are those utilized for the Round Robin Testing Program. Moreover, the stepwise loading rate utilized during the Round Robin Testing Program was applied to these two sets of prisms.
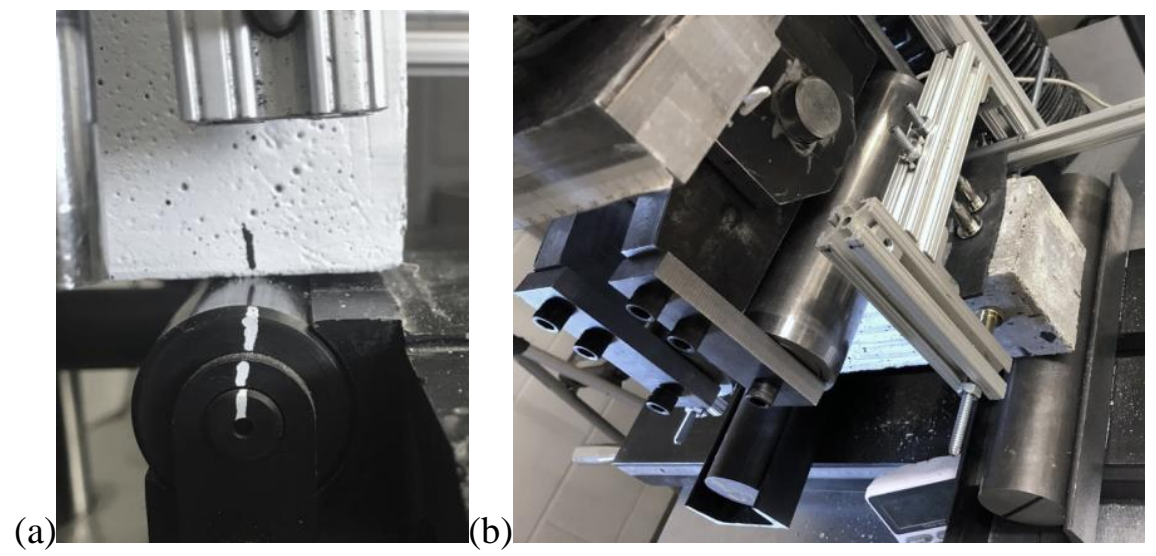

Figure 4- 11: Support conditions of four-point bending tests: (a) SC1; and (b) SC2

The average flexural strength is $26.23 \mathrm{MPa}$ for the prisms tested with SC1 and 19.6 MPa for those tested with SC2. The difference in average flexural strength is significant (i.e., 25.3\% difference), which indicates the effect of support condition on flexural strength is critical. The resistance curve and flexural strength result of each prism tested under SC1 or SC2 are presented in Figure 4-12 and Table 4-7. 
Table 4- 7: Flexural strength results of the prisms from DE2 tested with SC1 and SC2 respectively

\begin{tabular}{|c|c|c|c|}
\hline \multicolumn{3}{|c|}{ SC1 } \\
\hline \multirow{2}{*}{ Prism } & Peak Load & Flexural Strength & Average Flexural Strength \\
\cline { 2 - 3 } & kN & MPa & MPa \\
\hline OW1 & 47.00 & 25.58 & \multirow{2}{*}{26.23} \\
\hline OW2 & 50.32 & 27.20 & \\
\hline OW3 & 48.25 & 25.90 & \\
\hline \multicolumn{3}{|c|}{ SC2 } \\
\hline \multirow{2}{*}{ Prism } & Peak Load & Flexural Strength & Average Flexural Strength \\
\cline { 2 - 3 } & kN & MPa & MPa \\
\hline OW1 & 36.44 & 19.43 & \multirow{2}{*}{19.60} \\
\hline OW2 & 33.50 & 18.11 & \\
\hline OW3 & 39.62 & 21.27 & \\
\hline
\end{tabular}
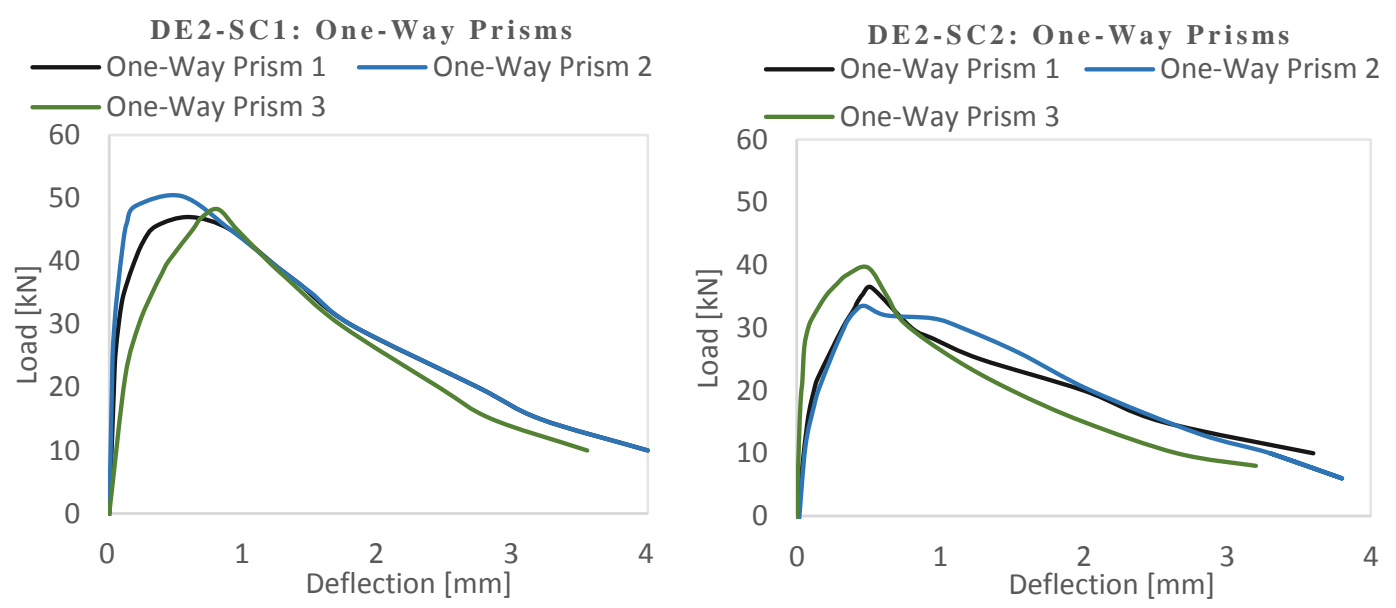

Figure 4- 12: Resistance curves of the prisms from mix DE2 tested with SC1 and SC2 respectively

The following section focuses on explaining three phenomena relating to support conditions which affect the experimental results of FPBT. Figure 4-13 illustrates one of the rollers of SC1 and the roller which allows rotation and horizontal translation of SC2, where $H$ and $V$ are the horizontal and vertical component forces of the force $(F)$ transferred from the prism to the roller at the point of contact, and $\theta$ is the rotation of the roller about its centroid. The roller of $\mathrm{SC} 1$ rotates about its own axle (point $\mathrm{O}_{1}$ ), whereas the roller of $\mathrm{SC} 2$ rotates about the contact point with the base (point $\mathrm{O}_{2}$ ). For the roller of SC1, moment equilibrium about point $\mathrm{O}_{1}$ can be described with Equation 4-1, where $D_{l}$ is the diameter of the roller. For the roller of $\mathrm{SC} 2$, the moment equilibrium about point $\mathrm{O}_{2}$ can be described with Equation 4-2, where $D_{2}$ is the diameter of the roller. As depicted in Figure 4-13, the prism exerts a counterclockwise moment of $M$ into the roller (shown as the black arrow), whereas $H_{l}$ exerts a clockwise moment of $\frac{H_{1} D_{1}}{2}$ (shown as the green arrow) into the roller, indicating this portion of moment is counteracted by the effect of $H_{l}$ and the resultant moment becomes $\left(M-\frac{H_{1} D_{1}}{2}\right)$. With regard to the roller of SC2, $H_{2}$ exerts a clockwise moment of $H_{2} \cdot D_{2}$ which does 
not counteract the moment resulting from the applied load but converts into kinetic energy since the roller of SC2 can translate laterally. Therefore, the resultant moment under SC2 remains $M$, which is greater than that under SC1 and results in lower flexural strength results. In addition, the larger the diameter of the roller of SC1, the smaller the resultant moment, leading to a higher flexural strength result.

(a)

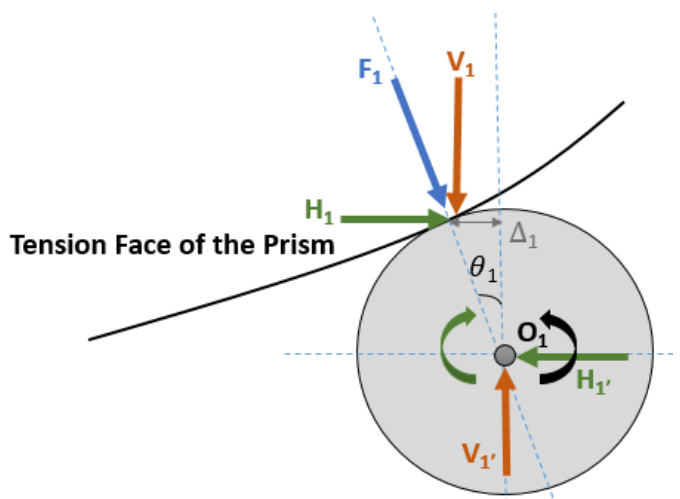

(b)

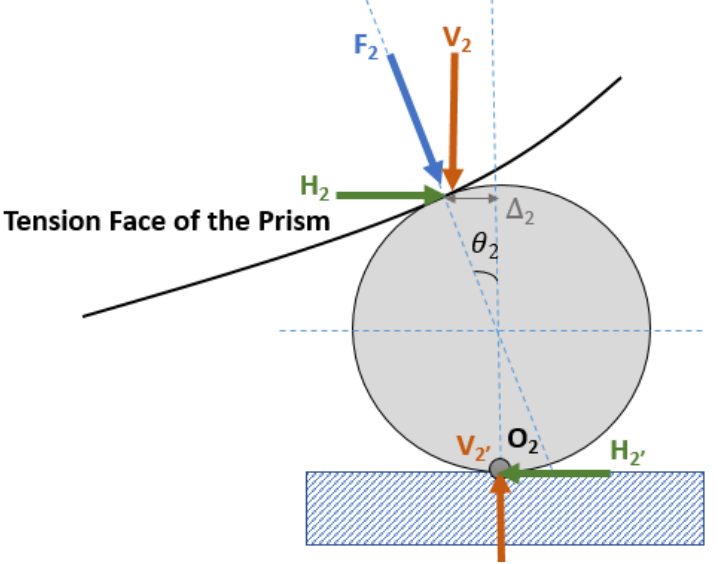

Figure 4- 13: Comparison of the horizontal force: (a) SC1; and (b) SC2

$H_{1} \cdot \frac{D_{1}}{2}=V_{1} \cdot \Delta_{1}$

$H_{2} \cdot D_{2}=V_{2} \cdot \Delta_{2}$

Figure 4-14 depicts the rollers of SC1 and SC2 at their points of contact with the prism, where $V$ is the nominal component force and $f$ is the frictional force generated owing to the contact between the prism and the roller. Again, the roller of SC1 rotates about point $\mathrm{O}_{1}$, whereas the roller of SC2 rotates about point $\mathrm{O}_{2}$. With regard to the roller of $\mathrm{SC} 1$, the moment resulting from $f_{1}$ about point $\mathrm{O}_{1}$ can be described with Equation 4-3, where $\mu$ is the coefficient of static friction. With regard to the roller of SC2, the moment resulting from $f_{2}$ about point $\mathrm{O}_{2}$ can be described with Equation 4-4. As illustrated in Figure 4-14, the prism exerts a counterclockwise moment of $M$ into the roller (shown as the black arrow), and $f_{2}$ also exerts a counterclockwise moment (shown as the green arrow) into the roller. For the roller of SC1, the moment resulting from $f_{l}$ is negligible due to the fact that the roller can rotate about its own axis and is supported on its axle through bearings. Therefore, the resultant moment under SC1 remains $M$. For the roller of SC2, the resultant moment becomes $\left(f_{2} \cdot D_{2}+M\right)$, which is greater than that under SC1 and results in lower flexural strength results. In addition, the larger the diameter of the roller of SC2, the larger the resultant moment, leading to a lower flexural strength result. 
(a)

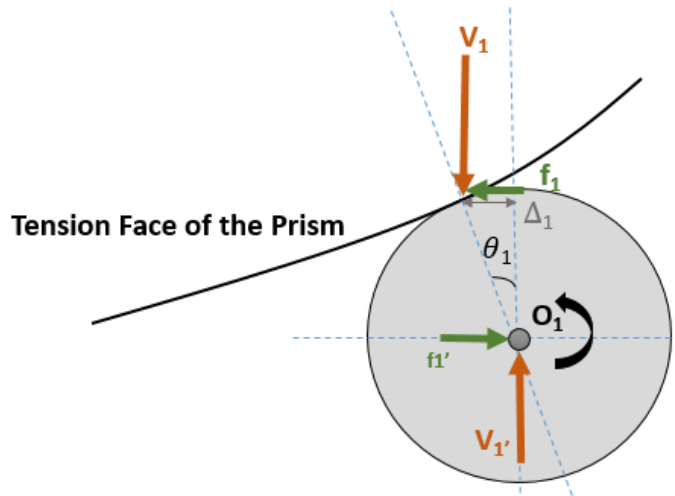

(b)

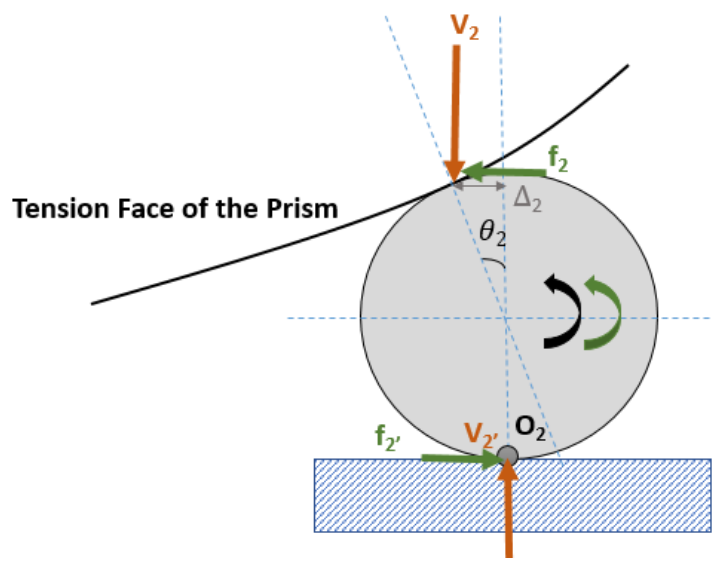

Figure 4- 14: Comparison of the frictional force: (a) SC1; and (b) SC2

$f_{1} \cdot \frac{D_{1}}{2}=\mu \cdot V_{1} \cdot \frac{D_{1}}{2}$

$f_{2} \cdot D_{2}=\mu \cdot V_{2} \cdot D_{2}$

The third phenomenon is the local bending about support rollers. As illustrated in Figure 4-15, the ideal support condition assumes support rollers are points (i.e., rollers with infinitely small diameters). However, support rollers have finite dimensions, which enforces local deformation constraints and causes the actual deformation curve of the prism to deviate from the natural elastica. The deformation constraints impose a clockwise moment which counteracts a portion of the counterclockwise moment resulting from the applied load, leading to higher flexural strength results. In addition, the amount of moment imposed by the support roller is a function of the diameter of the roller: the smaller the roller, the lower the moment. Moreover, the deformation constraints can be eliminated if the roller is free to translate, meaning one of the rollers of SC2 does not correspond to this phenomenon, whereas the other roller of SC2 and both rollers of SC1 affect experimental results in this way.

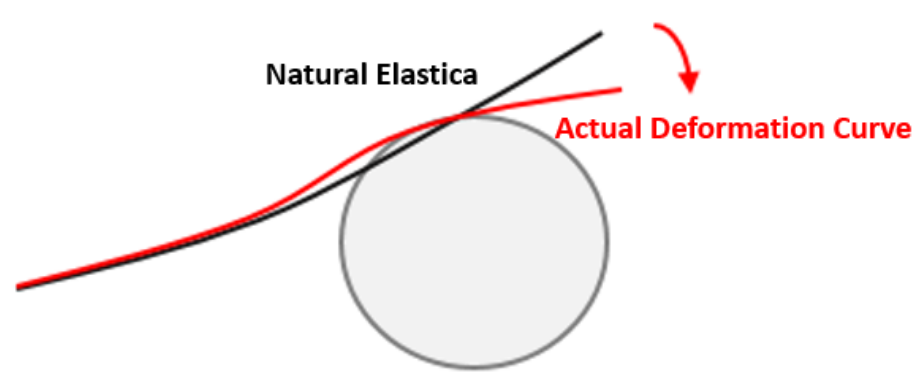

Figure 4- 15: Local disturbance of the prism's elastica 
In this context, all three universities experienced some degree of challenges in terms of FPBT setup. York University's support rollers minimized the frictional force but added equivalent fixed-end moment which counteracted a portion of moment imposed by the applied load and resulted in greater apparent flexural strength results. École Polytechnique-Montréal's and Queen's University's support rollers avoided the horizontal force effect but imposed additional moment to the prism due to frictional force, leading to lower apparent flexural strength results. In addition, the fixed roller enforced deformation constraints, whereas the free roller did not, leading to bias and nonsymmetry in the response. In conclusion, it is recommended that both support rollers are free to translate laterally and to be greased to minimize friction. With regard to loading rollers, they should be capable of adjusting rotation about the longitudinal axis of the prism in order to eliminate torsional effect. Moreover, it is recommended to rotate the prism at 90 degrees prior to testing rather than placing a plaster layer since the plaster layer may cause premature softening response. Alternatively, a glass layer can be placed on top of the free surface of the prism after casting to reduce surface roughness.

\subsection{Experimental Results of Commercial FD4, FD5 and FD6 Design Mixes}

\subsubsection{Materials and Casting Methodology}

The commercial company provided all the necessary materials for FD4, FD5 and FD6 design mixes. In addition, concrete mixing and casting took place in the facilities of the commercial company and were conducted by the technicians of the commercial company. Mix FD4 was cast on May $2^{\text {nd }}, 2019$, and mixes FD5 and FD6 were cast on June $17^{\text {th }}, 2019$. All three design mixes contain $2 \%$ steel fibers by unit volume. Mix FD4 utilized $13 \mathrm{~mm}$ long, straight steel fibers with a diameter of $0.2 \mathrm{~mm}$, whereas mixes FD5 and FD6 utilized $20 \mathrm{~mm}$ long, straight steel fibers with a diameter of $0.2 \mathrm{~mm}$. A total of $33 \mathrm{OW}$ prisms with an aspect ratio of 1 were prepared for conducting FPBT. Of those, the dimensions of three prisms from mix FD4 are $100 \mathrm{~mm}$ by $100 \mathrm{~mm}$ by $370 \mathrm{~mm}$; the dimensions of six prisms from mix FD4 are $150 \mathrm{~mm}$ by $150 \mathrm{~mm}$ by $500 \mathrm{~mm}$; and the dimensions of 24 prisms from mixes FD5 and FD6 are $150 \mathrm{~mm}$ by $150 \mathrm{~mm}$ by $500 \mathrm{~mm}$. In addition, $75 \mathrm{~mm}$ by $150 \mathrm{~mm}$ cylinders were cast with FD4 design mix by filling the molds in a single layer for conducting compression test. Moreover, flowability was found to be $247.5 \mathrm{~mm}$ for mix FD4 and $230 \mathrm{~mm}$ for mixes FD5 and FD6. As illustrated in Figure 4-16, wood molds were prepared for prisms and standard plastic molds were prepared for cylinders. A release agent was applied to the molds prior to casting, and specimens were consolidated by tapping the sides of the mold 30 times with a mallet. Thereafter, the specimens were shipped to York University and demolded one day after casting and stored in water tanks for curing in ambient laboratory temperature. 


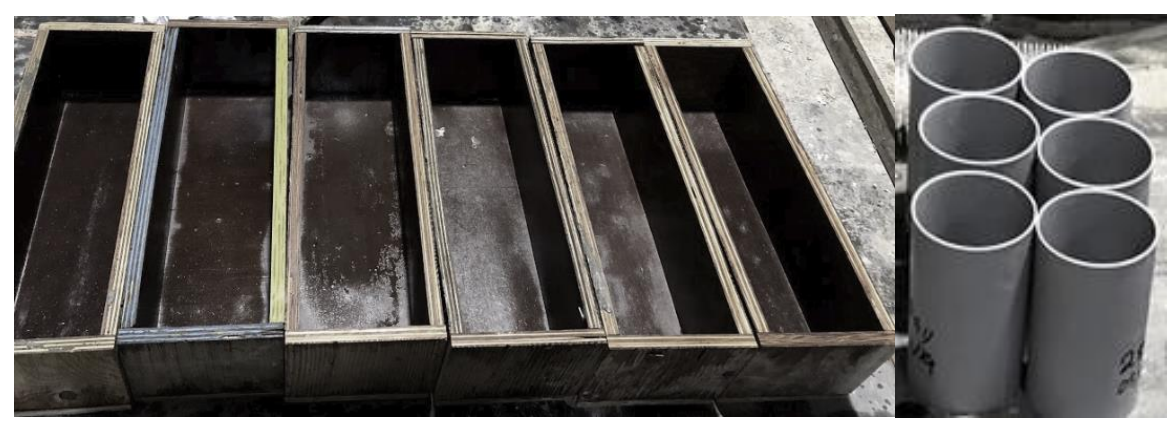

Figure 4- 16: Prepared molds for prismatic and cylindrical specimens (Mixes FD4, FD5 and FD6)

\subsubsection{Compression Test}

Regarding mix FD4, a load-controlled compression-testing machine (Controls Pilot) was used to test the cylinders under compression at a loading rate of $1 \mathrm{MPa} / \mathrm{s}$ in accordance with ASTM C1856 (2017). As depicted in Figure 4-17a and Figure 4-17b, bubbles appeared on the cylinder's top surface, and concrete peeling was observed near the edges as a result of excess release agent applied to the molds. In addition, as illustrated in Figure 4-17c, large and deep voids appeared after grinding approximately $1 \mathrm{~mm}$ from the surface of the cylinder. The cylinders were further grinded to $145 \mathrm{~mm}$ in height, but further voids appeared, which indicates that the cylinders were poorly compacted. As depicted in Figure 4-18, to test these cylinders, the voids were filled with Densit ${ }^{\circledR}$ mortar and epoxy paste, and the ends of the cylinders were wrapped with CFRP strips to avoid local failure.

(a)

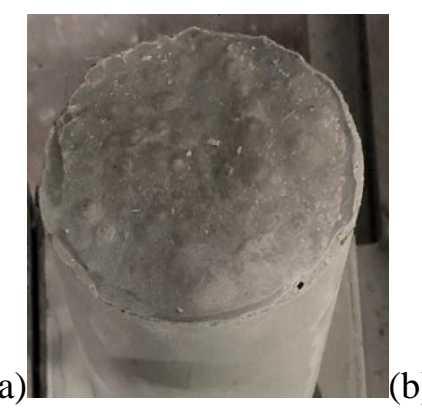

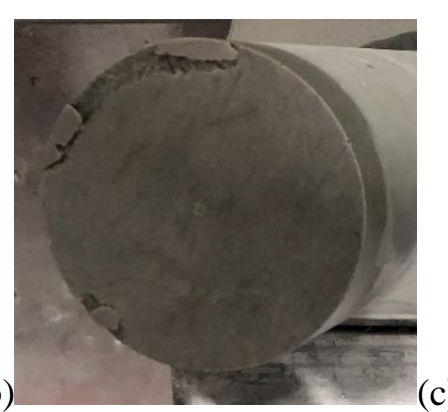

(c)

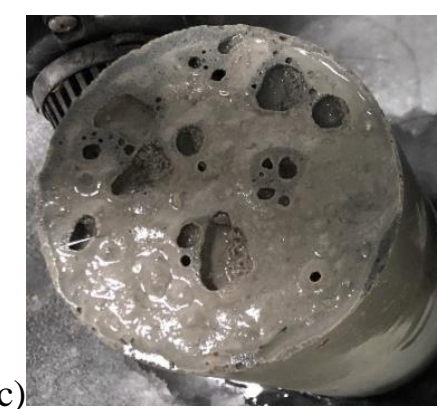

Figure 4- 17: Imperfections of the cylinders from mix FD4: (a) Bubbles on the top surface; (b) Peeling near the edge; and (c) Voids 


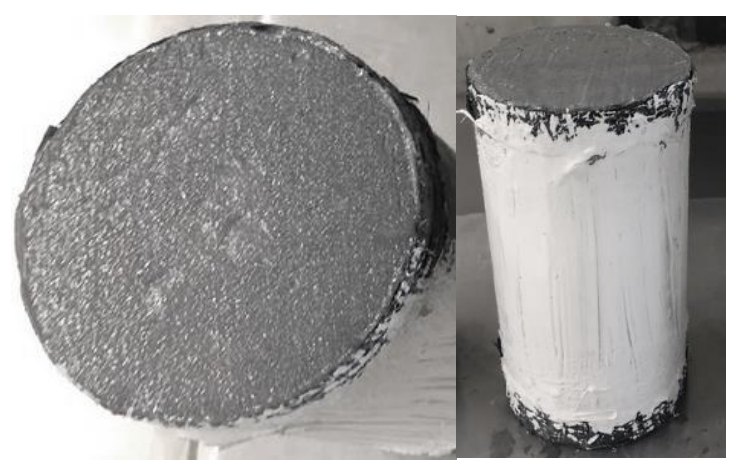

Figure 4- 18: Cylinders from mix FD4 capped with mortar and epoxy paste, and wrapped with CFRP strips at both ends

After the mortar and epoxy paste dried, the cylinders were tested at the age of 31 days, and the compressive strength result of each cylinder is $78.2 \mathrm{MPa}, 95 \mathrm{MPa}$ and 107.6 MPa, which is much lower than those obtained from the other design mixes. Other than the voids and peeling issues, improper use of the CFRP stips is also responsible for the unexpected low strength results. As illustrated in Figure 4-19, the two ends of CFRP strip did not overlap at the junction, which not only caused the CFRP strips to lost their efficacy but also created a weak region near the junction, leading to local failure. Therefore, these compressive strength results were discarded, and three additional cylinders ( $75 \mathrm{~mm}$ by $150 \mathrm{~mm}$ ) from mix FD4 were used to determine the compressive strength. To eliminate the voids, these cylinders were grinded down to $139 \mathrm{~mm}, 140 \mathrm{~mm}$ and $142 \mathrm{~mm}$, corresponding to a cylinder height-to-diameter ratio of 1.81, 1.84 and 1.85, respectivley. According to CSA A23.2-9C (2014), correction factors do not need to apply to compressive strength results if the cylinder's heigh-to-diamter ratio is greater than 1.8. The average compressive stength is $130.8 \mathrm{MPa}$ (the compressive strength result of each cylinder is $112.4 \mathrm{MPa}, 137.8 \mathrm{MPa}$ and $142.3 \mathrm{MPa}$, respectively). It is worth noting that prior to compression test, these three cylinders were loaded to $222 \mathrm{kN}$ (i.e., about $50 \mathrm{MPa}$ ) under compression to determine the modulus of elasticity and Poisson's ratio of mix FD4.

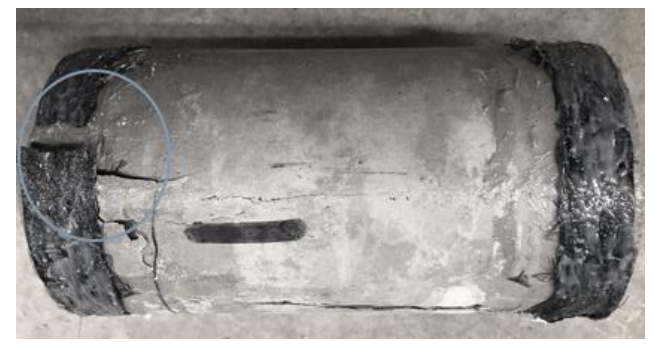

Figure 4- 19: Local failure near the junction of the two ends of CFRP strip

With respect to mixes FD5 and FD6, the cylinders were tested by the commercial company. The average compressive strength after the 21-day curing period is 131 MPa for mix FD5 and 131.2 MPa for mix FD6, 
and the average compressive strength after the 28-day curing period is $136.2 \mathrm{MPa}$ for mix FD5 and 133.3 MPa for mix FD6.

\subsubsection{Four-Point Bending Test}

The prisms from mix FD4 were tested at the age of 28 days and the prisms from mixes FD5 and FD6 were tested at the age of 21 days and 28 days, respectively. The universal testing machine was utilized to apply load at the stepwise rate. In addition, the experimental test setup utilized by York University during the Round Robin Testing Program was used to test the prisms from mixes FD4, FD5 and FD6. Regarding the $100 \mathrm{~mm}$ by $100 \mathrm{~mm}$ by $370 \mathrm{~mm}$ prisms from mix FD4, the shear span and distance between loading rollers are $100 \mathrm{~mm}$. Regarding the $150 \mathrm{~mm}$ by $150 \mathrm{~mm}$ by $500 \mathrm{~mm}$ prisms from mixes FD4, FD5 and FD6, the shear span and distance between loading rollers are $150 \mathrm{~mm}$. The equivalent flexural strength results of each design mix are presented in following sections.

\subsubsection{Commercial FD4 Design Mix}

The average flexural strength result of the prisms from mix FD4 is $18.24 \mathrm{MPa}$ for $100 \mathrm{~mm}$ by $100 \mathrm{~mm}$ by $370 \mathrm{~mm}$ prisms and $15.44 \mathrm{MPa}$ for $150 \mathrm{~mm}$ by $150 \mathrm{~mm}$ by $500 \mathrm{~mm}$ prisms. As depicted in Figure 4-20, critical failure planes of both $100 \mathrm{~mm}$ by $100 \mathrm{~mm}$ prisms and $150 \mathrm{~mm}$ by $150 \mathrm{~mm}$ prisms formed within the central constant moment region. However, as depicted in Figure 4-21, fiber segregation was observed through the critical failure plane of the prism: more steel fibers present near side surface 1 compared with side surface 2 (note that when conducting FPBT, prisms were rotated at 90 degrees with respect to the position at casting, so side surface 1 is the bottom surface of the prism at casting and side surface 2 is the free surface at casting). Mix FD4 was very flowable with a flow value of $247.5 \mathrm{~mm}$, which may have led to fiber segregation.
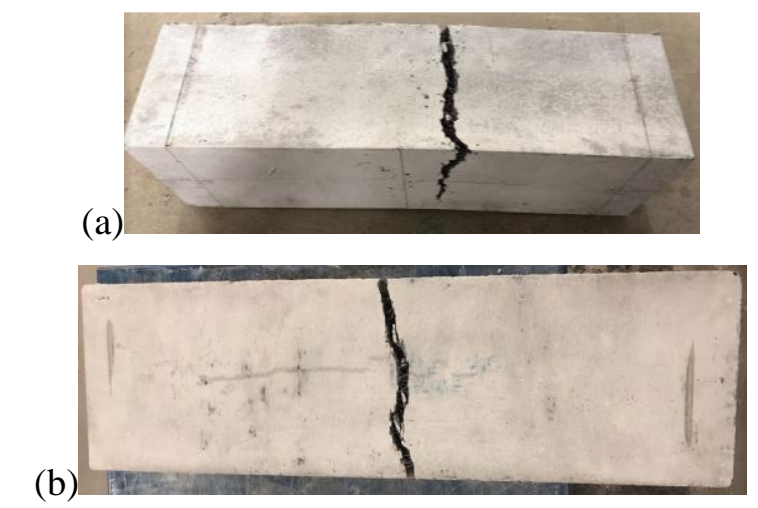

Figure 4- 20: Critical failure planes of the prisms from mix FD4: (a) $100 \mathrm{~mm}$ by $100 \mathrm{~mm}$ prisms; and (b) $150 \mathrm{~mm}$ by $150 \mathrm{~mm}$ prisms 


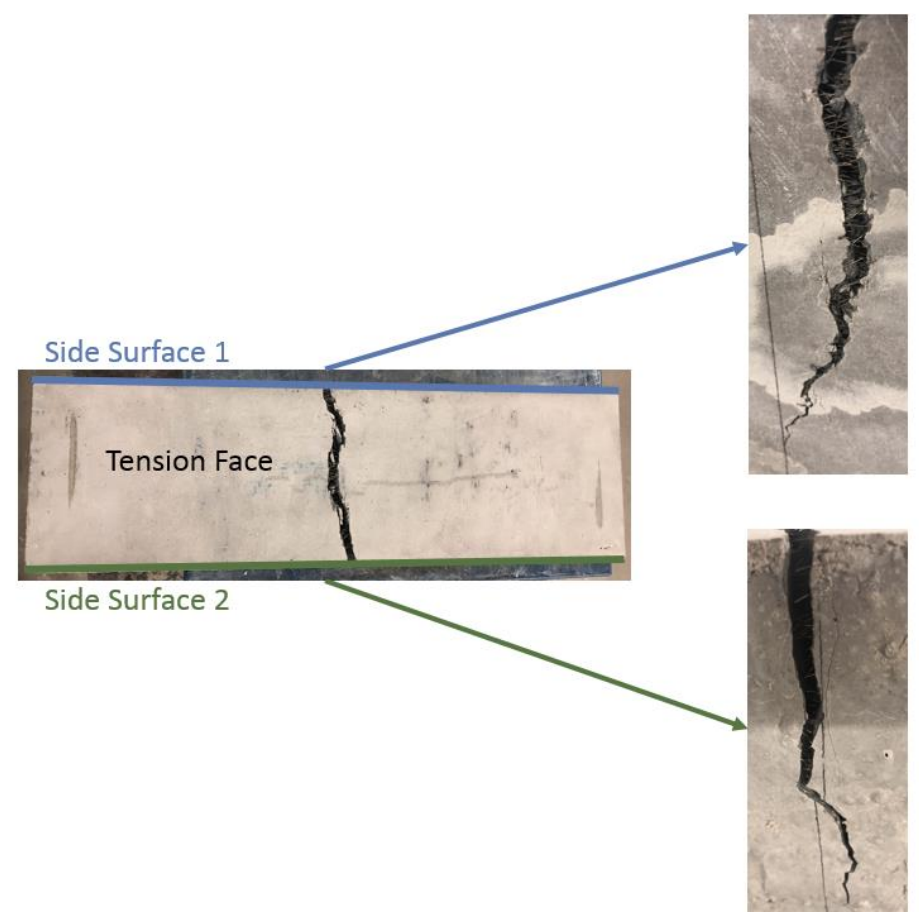

Figure 4- 21: Fiber segregation observed from the prisms from mix FD4

Figure 4-22 illustrates the resistance curve of each prism and the flexural strength results calculated with Equation 3-3 are presented in Table 4-8. It is evident that the flexural strength results of $100 \mathrm{~mm}$ by 100 $\mathrm{mm}$ prisms are greater than those of $150 \mathrm{~mm}$ by $150 \mathrm{~mm}$ prisms. According to ASTM C1856 (2017) (refer to Table 4-1), the steel fibers which are $15 \mathrm{~mm}$ to $20 \mathrm{~mm}$ in length should be utilized in $100 \mathrm{~mm}$ by 100 $\mathrm{mm}$ prisms, and fibers which are $20 \mathrm{~mm}$ to $25 \mathrm{~mm}$ in length should be utilized in $150 \mathrm{~mm}$ by $150 \mathrm{~mm}$ prisms. Thus, prisms from mix FD4 with $13 \mathrm{~mm}$ long fibers did not meet this criterion. In addition, the degree of preferential fiber alignment is more prominent in the prisms containing fibers that are relatively long compared with the prism's cross-sectional dimensions (ASTM C1609 2012), therefore the degree of preferential fiber alignment is more remarkable in $100 \mathrm{~mm}$ by $100 \mathrm{~mm}$ prisms than in $150 \mathrm{~mm}$ by $150 \mathrm{~mm}$ prisms, leading to greater flexural strength results. 
Table 4- 8: Flexural strength results (Mix FD4)

\begin{tabular}{|c|c|c|c|}
\hline \multicolumn{4}{|c|}{$100 \mathrm{~mm}$ by $100 \mathrm{~mm}$ Prisms } \\
\hline Prism & Peak Load $[\mathrm{kN}]$ & Flexural Strength $[\mathrm{MPa}]$ & Average Flexural Strength [MPa \\
\hline Prism 1 & 54.21 & 15.40 & \multirow{3}{*}{ 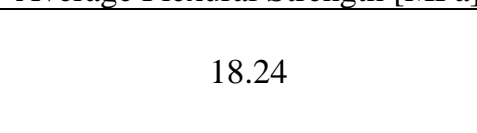 } \\
\hline Prism 2 & 69.97 & 20.37 & \\
\hline Prism 3 & 63.77 & 18.94 & \\
\hline \multicolumn{4}{|c|}{$150 \mathrm{~mm}$ by $150 \mathrm{~mm}$ Prisms } \\
\hline Prism & Peak Load $[\mathrm{kN}]$ & Flexural Strength $[\mathrm{MPa}]$ & Average Flexural Strength [MPa] \\
\hline Prism 1 & 124.07 & 16.49 & \multirow{6}{*}{ 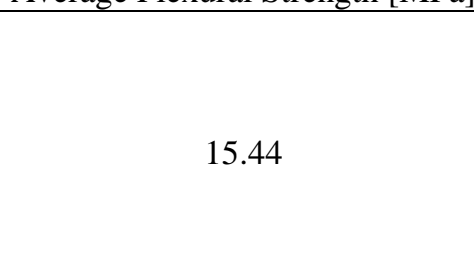 } \\
\hline Prism 2 & 122.20 & 15.81 & \\
\hline Prism 3 & 109.28 & 14.05 & \\
\hline Prism 4 & 135.06 & 17.19 & \\
\hline Prism 5 & 105.30 & 13.67 & \\
\hline Prism 6 & 101.39 & 12.78 & \\
\hline
\end{tabular}
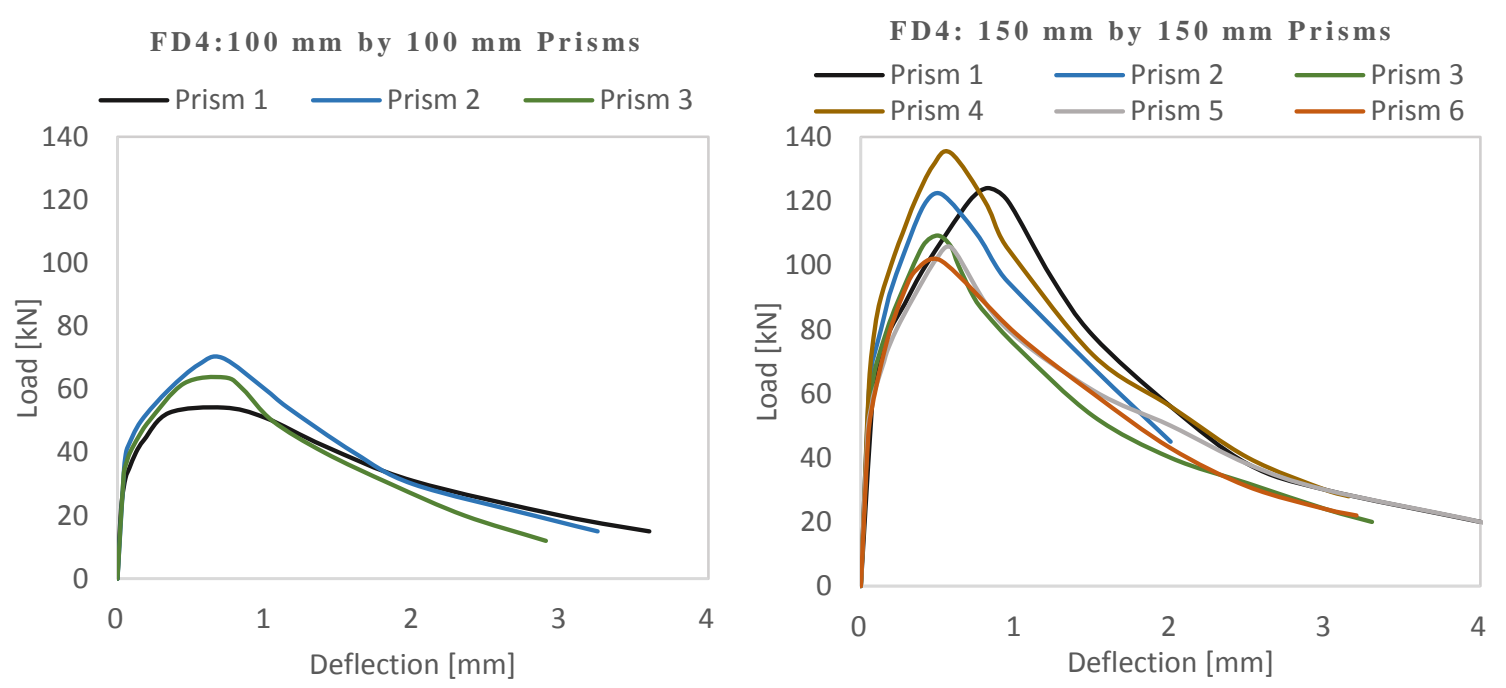

Figure 4- 22: Resistance curves of the prisms from mix FD4

\subsubsection{Commercial FD5 and FD6 Design Mixes}

The average flexural strength results of the prisms ( $150 \mathrm{~mm}$ by $150 \mathrm{~mm}$ by $500 \mathrm{~mm}$ prisms) from mix FD5 is $22.01 \mathrm{MPa}$ at the age of 21 days and $21.75 \mathrm{MPa}$ at the age of 28 days, and the average flexural strength results of the prisms ( $150 \mathrm{~mm}$ by $150 \mathrm{~mm}$ by $500 \mathrm{~mm}$ prisms) from mix FD6 is $22.24 \mathrm{MPa}$ at the age of 21 days and $20.54 \mathrm{MPa}$ at the age of 28 days. It is evident that the average flexural strength at the age of 21 days is higher than that at the age of 28 days for both design mixes, which could be explained by the bond strength development. Regarding the prisms tested at the age of 21 days, the bond strength between the matrix and steel fibers was low in general, so multiple micro-cracks formed prior to crack localization; whereas for the prisms tested at the age of 28 days, the bond between certain steel fibers and the matrix developed, thus the region with comparatively low bond strength would be the weakest path and crack 
would localize faster than those tested at the age of 21 days, resulting in lower flexural strength results. Table 4-9 presents the flexural strength result of each prism, and the resistance curves can be found in Appendix A.1.5.

Table 4- 9: Flexural strength results (Mixes FD5 and FD6)

\begin{tabular}{|c|c|c|c|}
\hline \multicolumn{4}{|c|}{ Prisms from mix FD5 (21 Days) } \\
\hline Prism & Peak Load $[\mathrm{kN}]$ & Flexural Strength [MPa] & Average Flexural Strength [MPa] \\
\hline Prism 1 & 156.81 & 20.91 & \multirow{6}{*}{22.01} \\
\hline Prism 2 & 193.36 & 25.78 & \\
\hline Prism 3 & 161.81 & 21.29 & \\
\hline Prism 4 & 185.08 & 24.51 & \\
\hline Prism 5 & 146.73 & 19.56 & \\
\hline Prism 6 & 148.87 & 19.98 & \\
\hline \multicolumn{4}{|c|}{ Prisms from mix FD5 (28 Days) } \\
\hline Prism & Peak Load $[\mathrm{kN}]$ & Flexural Strength [MPa] & Average Flexural Strength [MPa] \\
\hline Prism 1 & 165.30 & 22.04 & \multirow{6}{*}{21.75} \\
\hline Prism 2 & 186.14 & 24.82 & \\
\hline Prism 3 & 141.43 & 18.86 & \\
\hline Prism 4 & 147.23 & 19.63 & \\
\hline Prism 5 & 160.54 & 21.41 & \\
\hline Prism 6 & 177.94 & 23.73 & \\
\hline \multicolumn{4}{|c|}{ Prisms from mix FD6 (21 Days) } \\
\hline Prism & Peak Load [kN] & Flexural Strength [MPa] & Average Flexural Strength [MPa] \\
\hline Prism 1 & 150.98 & 20.13 & \multirow{6}{*}{22.24} \\
\hline Prism 2 & 169.86 & 22.35 & \\
\hline Prism 3 & 183.26 & 24.43 & \\
\hline Prism 4 & 159.86 & 21.31 & \\
\hline Prism 5 & 171.39 & 22.85 & \\
\hline Prism 6 & 164.26 & 22.35 & \\
\hline \multicolumn{4}{|c|}{ Prisms from mix FD6 (28 Days) } \\
\hline Prism & Peak Load $[\mathrm{kN}]$ & Flexural Strength [MPa] & Average Flexural Strength [MPa] \\
\hline Prism 1 & 147.21 & 19.63 & \multirow{6}{*}{20.54} \\
\hline Prism 2 & 140.59 & 18.75 & \\
\hline Prism 3 & 143.78 & 19.17 & \\
\hline Prism 4 & 177.34 & 23.65 & \\
\hline Prism 5 & 154.63 & 20.62 & \\
\hline Prism 6 & 160.53 & 21.40 & \\
\hline
\end{tabular}

Figure 4-23 plots the average flexural strength results of the $150 \mathrm{~mm}$ by $150 \mathrm{~mm}$ prisms from mix FD4 and the prisms from mixes FD5 and FD6 tested at the age of 28 days. As mentioned previously, mix FD4 comprises $13 \mathrm{~mm}$ long, steel fibers, whereas mixes FD5 and FD6 comprises $20 \mathrm{~mm}$ long, steel fibers. It is evident that the average flexural strength of the prisms from mixes FD5 and FD6 is greater than those from mix FD4. Fiber segregation might contribute to the low strength results of mix FD4. In addition, the degree of preferential fiber alignment was more prominent in the prisms from mixes FD5 and FD6 due to the use of $20 \mathrm{~mm}$ long, steel fibers, leading to greater flexural strength results. 


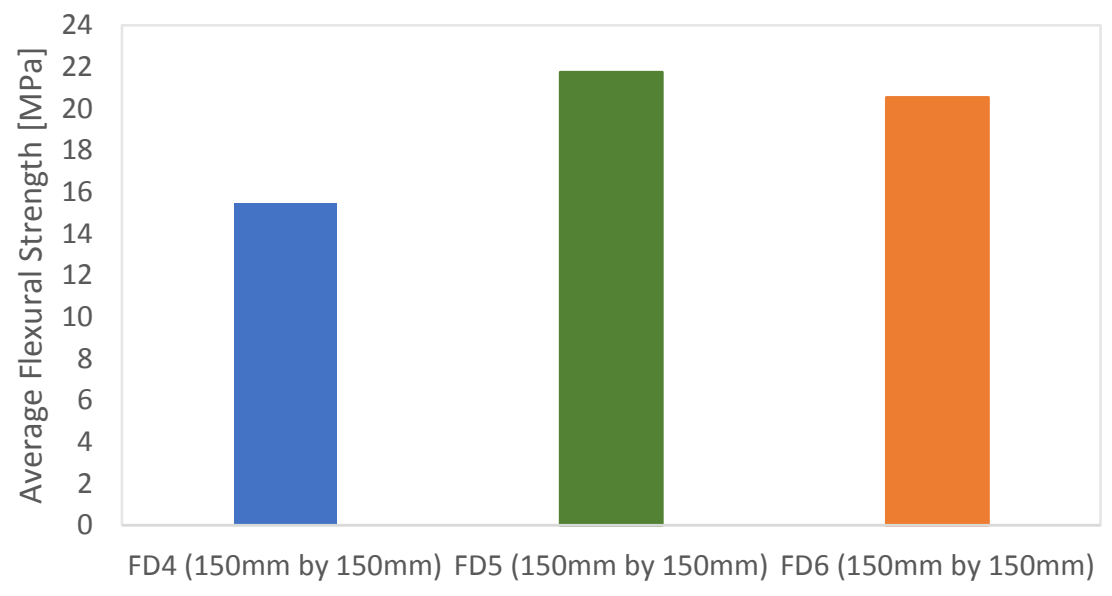

Figure 4- 23: Average flexural strength results of mixes FD4, FD5 and FD6 


\section{Chapter 5. Non-Destructive Tests and Durability Evaluation}

The experimental program also explores the physical properties and durability performance of UHP-SFRC. With regard to physical properties, static modulus of elasticity, Poisson's ratio, coefficient of thermal expansion and absorption properties of UHP-SFRC were determined. With regard to durability performance, freeze-thaw tests and salt-scaling tests were conducted to assess the long-term performance of this class of materials. The following sections present the experimental test setups, testing procedures and experimental results of each test.

\subsection{Static Modulus of Elasticity and Poisson's Ratio}

Three $75 \mathrm{~mm}$ by $150 \mathrm{~mm}$ cylinders from mix FD4 were subjected to compressive loading at the age of 32 days to determine the static modulus of elasticity and Poisson's ratio. The test was conducted in accordance with ASTM C469 (2010). The cylinders were demolded one day after casting and stored in a water tank in ambient laboratory temperature for curing until testing. Prior to testing, both top and bottom surfaces of the cylinder were grinded smooth. As mentioned previously, large and deep voids appeared on top surface of the cylinder due to poor compaction. Therefore, to avoid local failure, the cylinders were grinded down by $139 \mathrm{~mm}, 140 \mathrm{~mm}$ and $142 \mathrm{~mm}$ heightwise, respectively. In addition, the diameter of each cylinder was measured to be $76.7 \mathrm{~mm}, 76.3 \mathrm{~mm}$ and $76.6 \mathrm{~mm}$, respectively. As depicted in Figure 5-1, four foil strain gauges which had laminated surfaces and integral leads were glued along the two diametrically opposite lines near the mid-height of the cylinder: two strain gauges were placed vertically to measure longitudinal strains, and the other two strain gauges were placed horizontally to measure transverse strains. The reported strain values in Table 5-1 were calculated by averaging the readings of the strain gauges attached on the diametrically opposite sides of the cylinder. The wire leads of strain gauges were soldered with electric wires, and the electric wires were connected with the universal testing machine so that strain readings synchronized with the applied load values. Figure 5-2 illustrates the response curve of a test, where the horizontal axis represents the applied load values and the vertical axis represents strain values for the four strain gauges. 
(a)

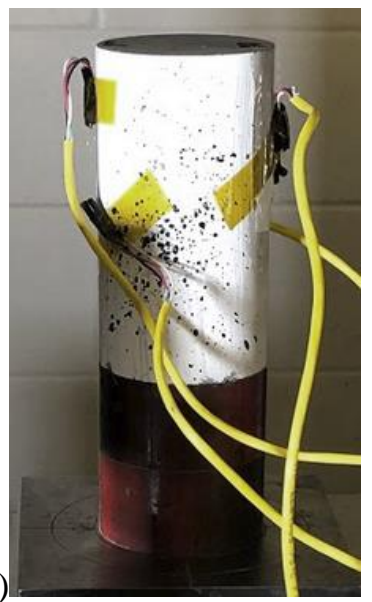

Figure 5- 1: Experimental test setup to determine static modulus of elasticity and Poisson's ratio: (a) Foil strain gauge; and (b) Photo of test setup

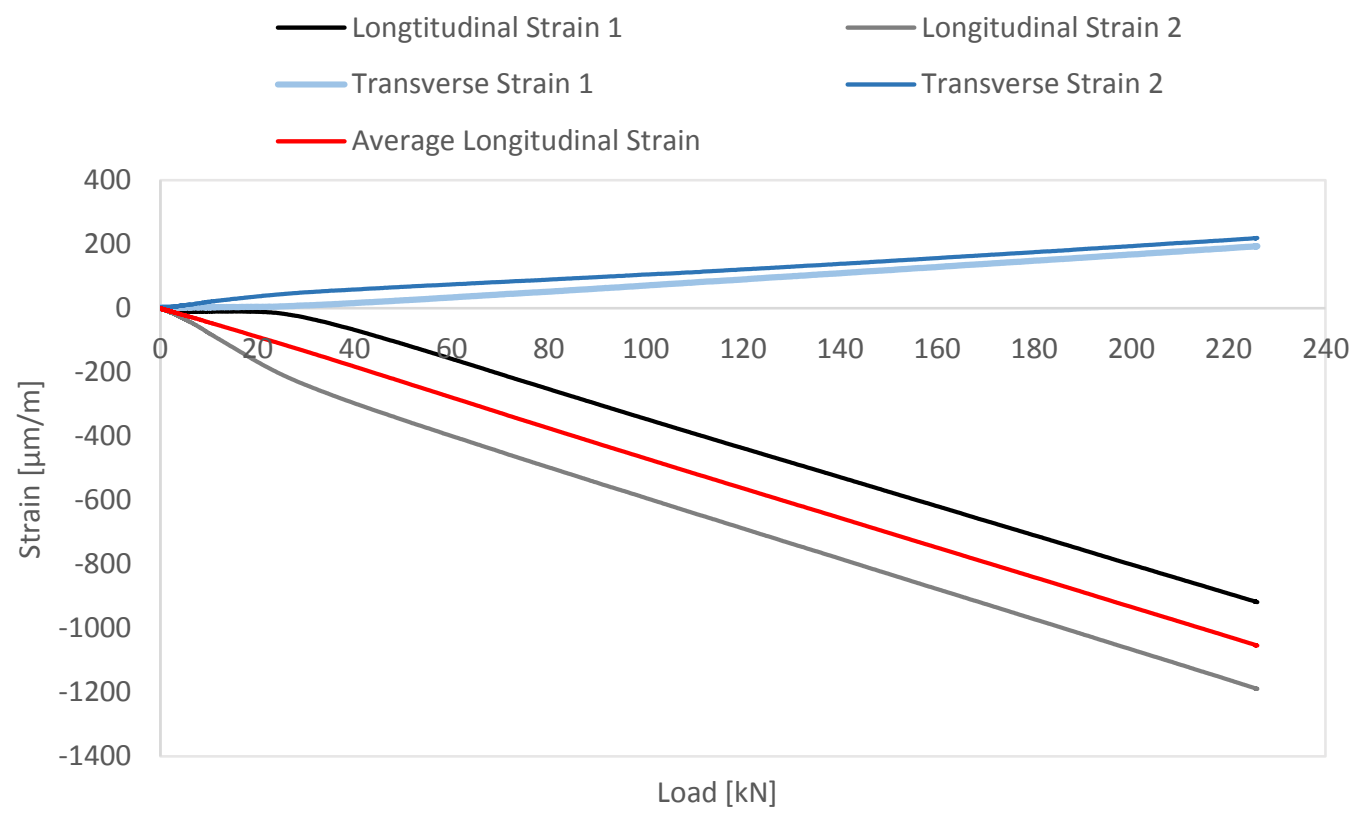

Figure 5- 2: Response curve of static modulus of elasticity and Poisson's ratio test

ASTM C469 (2010) states that concrete modulus of elasticity and Poisson's ratio should be determined by loading the cylinders in compression with a stress less than $40 \%$ of the compressive strength. Due to the fact that the compressive tests of mix FD4 were not acceptable on account of the voids at the top resulting from excessive flowability, (see Section 4.3.2), a compressive strength of $120 \mathrm{MPa}$ was assumed, and the cylinders were loaded up to around $48 \mathrm{MPa}$. In addition, according to ASTM 1856 (2017), the loading rate of compression test is $1 \mathrm{MPa} / \mathrm{s}$ for UHP-FRC, and a loading rate of $0.0023 \mathrm{~mm} / \mathrm{s}$ which is equivalent to 1 $\mathrm{MPa} / \mathrm{s}$ was utilized for modulus of elasticity and Poisson's ratio test under displacement control. The 
resulting loading rate was calculated with Equation 5-1 by assuming the linearity limit of concrete compressive stress-strain curve is at $70 \%$ of the compressive strength and the longitudinal strain at attainment of compressive strength is $0.003 \mathrm{~mm} / \mathrm{mm}$. In Equation $5-1, \varepsilon_{c o}$ is the longitudinal strain corresponding to peak stress, and $(\sigma, \varepsilon)$ is any point on the compressive stress-strain curve. As presented in Equation 5-1, the longitudinal strain at $70 \%$ of the compressive strength (i.e., $84 \mathrm{MPa}$ ) is $0.00136 \mathrm{~mm} / \mathrm{mm}$. For a loading rate of $1 \mathrm{MPa} / \mathrm{s}$, it will take $84 \mathrm{~s}$ to attain $84 \mathrm{MPa}$, meaning the strain rate is equal to $1.615 \mathrm{E}-$ $5 \mathrm{~mm} / \mathrm{mm} / \mathrm{s}$ (i.e., $\frac{0.00136 \mathrm{~mm} / \mathrm{mm}}{84 \mathrm{~s}}$ ). The average height of the three cylinders was approximately $140 \mathrm{~mm}$, so the displacement-controlled loading rate was $0.0023 \mathrm{~mm} / \mathrm{s}$ (i.e., $1.615 \mathrm{E}-5 \mathrm{~mm} / \mathrm{mm} / \mathrm{s} \cdot 140 \mathrm{~mm}$ ).

$\sigma=f_{c}^{\prime}\left[2 \frac{\varepsilon}{\varepsilon_{c o}}-\left(\frac{\varepsilon}{\varepsilon_{c o}}\right)^{2}\right]$

$0.7 f_{c}^{\prime}=f_{c}^{\prime}\left[2 \frac{\varepsilon}{\varepsilon_{c o}}-\left(\frac{\varepsilon}{\varepsilon_{c o}}\right)^{2}\right]$

$0.7=2 \frac{\varepsilon}{\varepsilon_{c o}}-\left(\frac{\varepsilon}{\varepsilon_{c o}}\right)^{2}$

$0.7=2 \frac{\varepsilon}{0.003}-\left(\frac{\varepsilon}{0.003}\right)^{2}$

$\varepsilon=0.00136 \mathrm{~mm} / \mathrm{mm}$

In accordance with ASTM C469 (2010), each cylinder was tested three times. The average static modulus of elasticity $(E)$ was $46.58 \mathrm{GPa}$ and the average Poisson's ratio $(v)$ is 0.204 . Table $5-1$ presents the experimental results of each test, where $S_{1}$ and $S_{2}$ are the compressive stresses corresponding to a longitudinal strain of $50 \mu \mathrm{m} / \mathrm{m}$ and $40 \%$ of the assumed compressive strength, respectively; $\varepsilon_{1}$ represents a longitudinal strain of $50 \mu \mathrm{m} / \mathrm{m}$, and $\varepsilon_{2}$ is the longitudinal strain corresponding to $S_{2} ; \varepsilon_{t 1}$ and $\varepsilon_{t 2}$ are the transverse strains corresponding to $S_{1}$ and $S_{2}$, respectively; $E$ was calculated with Equation 5-2, and $v$ was calculated with Equation 5-3.

$\mathrm{E}=\frac{S_{2}-S_{1}}{\varepsilon_{2}-\varepsilon_{1}}$

$v=\frac{\varepsilon_{t 2}-\varepsilon_{t 1}}{\varepsilon_{2}-\varepsilon_{1}}$ 
Table 5- 1: Results of static modulus of elasticity and Poisson's ratio

\begin{tabular}{|c|c|c|c|c|c|c|c|c|c|}
\hline Cylinder & $\begin{array}{c}\varepsilon_{1} \\
{[\mu \mathrm{m} / \mathrm{m}]}\end{array}$ & $\begin{array}{c}\varepsilon_{t 1} \\
{[\mu \mathrm{m} / \mathrm{m}]}\end{array}$ & $\begin{array}{c}S_{1} \\
{[\mathrm{MPa}]}\end{array}$ & $\begin{array}{c}\varepsilon_{2} \\
{[\mu \mathrm{m} / \mathrm{m}]}\end{array}$ & $\begin{array}{c}\varepsilon_{t 2} \\
{[\mu \mathrm{m} / \mathrm{m}]}\end{array}$ & $\begin{array}{c}S_{2} \\
{[\mathrm{MPa}]}\end{array}$ & $\begin{array}{c}E \\
{[\mathrm{GPa}]}\end{array}$ & $v$ & $\begin{array}{c}\text { Average } E \\
\text { [GPa] }\end{array}$ \\
\hline \multirow{3}{*}{$\mathrm{C} 1$} & 50.16 & 11.54 & 2.24 & 1040.40 & 215.83 & 48.05 & 46.27 & 0.21 & \multirow{4}{*}{46.58} \\
\hline & 50.14 & 11.85 & 2.26 & 1036.37 & 215.93 & 48.08 & 46.46 & 0.21 & \\
\hline & 50.07 & 11.46 & 2.31 & 1036.54 & 215.06 & 48.05 & 46.37 & 0.21 & \\
\hline \multirow{3}{*}{$\mathrm{C} 2$} & 50.05 & 12.76 & 2.56 & 1039.11 & 194.51 & 48.63 & 46.58 & 0.18 & \\
\hline & 50.19 & 12.89 & 2.49 & 1035.36 & 202.52 & 48.62 & 46.83 & 0.19 & Average $\gamma$ \\
\hline & 50.10 & 12.83 & 2.47 & 1034.39 & 202.82 & 48.62 & 46.88 & 0.19 & \multirow{4}{*}{0.204} \\
\hline \multirow{3}{*}{$\mathrm{C} 3$} & 50.09 & 10.42 & 2.01 & 1113.19 & 228.42 & 48.25 & 43.50 & 0.21 & \\
\hline & 50.16 & 9.50 & 1.57 & 1017.55 & 222.19 & 48.25 & 48.25 & 0.22 & \\
\hline & 50.12 & 9.35 & 1.62 & 1019.86 & 222.29 & 48.24 & 48.08 & 0.22 & \\
\hline
\end{tabular}

\subsection{Water Content, Density, Water Absorption Capacity and Void Content}

Three $75 \mathrm{~mm}$ by $150 \mathrm{~mm}$ cylinders were tested to determine the physical properties of mix FD4 in terms of water content, density, absorption and void content. Similar to other cylinder specimens, these cylinders were cast by filling the molds with a single layer of fresh cementitious composites. However, as per CSA A23.2 -11C (2014), no release agent was applied to the molds of these cylinders prior to casting. The cylinders were demolded one day after casting and were weighed immediately after demolding, designating the result as mass $I$. The value of $I$ of each cylinder was found to be $1675.4 \mathrm{~g}, 1680.65 \mathrm{~g}$ and $1681.1 \mathrm{~g}$, respectively. In addition, differing from other specimens which were stored in water tanks in ambient laboratory temperature, these cylinders were placed in a freezer for curing. The freezer was not connected with power source, so it simply functioned as an insulated chamber. CSA A23.2 -11C (2014) states that the specimens should be cured under the condition of $50 \% \pm 5 \%$ relative humidity and $23^{\circ} \mathrm{C} \pm 2{ }^{\circ} \mathrm{C}$ temperature. Since the relative humidity in lab ranges between $36 \%$ to $42 \%$, an automatic humidifier was placed in the chamber and was set to maintain the relative humidity inside the freezer at $50 \%$ to meet the curing requirement. Besides, a digital thermometer was placed near the freezer to monitor the ambient temperature in lab, which was around $22{ }^{\circ} \mathrm{C}$, satisfying the curing requirement. Moreover, the cylinders were weighed after a 28-day curing period, and the mass of each cylinder was $1668.5 \mathrm{~g}, 1673.4 \mathrm{~g}$ and $1673.9 \mathrm{~g}$, respectively. Subsequently, the cylinders were returned to the chamber for curing for another seven days. After seven days, the cylinders were weighed again, and the mass of each cylinder was $1668.1 \mathrm{~g}, 1673.2 \mathrm{~g}$ and $1673.8 \mathrm{~g}$ respectively which had less than $0.5 \%$ difference compared with the weight values measured after the 28-day curing period, indicating the cylinders have reached their mass equilibrium. The mass values obtained after 35-day curing period were designated as mass $G$. After that point, the cylinders were dried in an oven at $110^{\circ} \mathrm{C}$ for three days, and then returned to the chamber for one day so that the cylinders 
cooled down to room temperature. After the cooling period, the mass of each cylinder was found to be $1634.8 \mathrm{~g}, 1639.9 \mathrm{~g}$ and $1640.4 \mathrm{~g}$, respectively. Then the cylinders were placed in an oven for drying and returned to the chamber for cooling for another cycle, and the mass of each cylinder after completing the second cycle was $1630.6 \mathrm{~g}, 1635.2 \mathrm{~g}$ and $1636.2 \mathrm{~g}$ respectively. These values had less than $0.5 \%$ difference compared with the weight values measured after completing the first cycle, and were designated as mass $A$. Furthermore, to determine the mass of surface-dried cylinder in air after immersion (referred herein as mass $B)$, the cylinders were placed in a water tank in ambient laboratory temperature for one day; then the cylinders were taken out and wiped with a damp cloth to remove surface water and weighed; the mass of each cylinder was $1656.4 \mathrm{~g}, 1661.3 \mathrm{~g}$ and $1661.9 \mathrm{~g}$, respectively. Afterwards, the cylinders were returned to the water tank for another cycle, and the mass of each cylinder after completing the second cycle was $1659.1 \mathrm{~g}, 1663.4 \mathrm{~g}$ and $1664 \mathrm{~g}$ respectively which had less than $0.5 \%$ difference compared with the weight values after completing the first cycle and were considered as the results of mass $B$. To determine the boiled, surface-dried mass $C$, the cylinders were put in a container filled with tap water and placed in an oven at $110^{\circ} \mathrm{C}$ for five hours. Therewith, the cylinders were returned to the chamber for one day to cool down to room temperature. After the cooling period, the mass (mass $C$ ) of each cylinder was found to be $1663.7 \mathrm{~g}$, $1663.5 \mathrm{~g}$ and $1658.9 \mathrm{~g}$, respectively. Lastly, the cylinder was suspended in water by using a wire, and a hanging scale was used to weigh the cylinder in water; the mass of each cylinder was $1015.1 \mathrm{~g}, 1010.3 \mathrm{~g}$ and $1005 \mathrm{~g}$ respectively, designating as mass $D$. For quick reference, the results of designated mass properties are summarized in Table 5-2.

Table 5- 2: Results of designated mass properties

\begin{tabular}{|c|c|c|c|c|c|c|}
\hline Cylinder & Mass $I[\mathrm{~g}]$ & Mass $G[\mathrm{~g}]$ & Mass $A[\mathrm{~g}]$ & Mass $B[\mathrm{~g}]$ & Mass $C[\mathrm{~g}]$ & Mass $D[\mathrm{~g}]$ \\
\hline $\mathrm{C} 1$ & 1675.40 & 1668.10 & 1630.55 & 1659.05 & 1663.65 & 1015.10 \\
\hline $\mathrm{C} 2$ & 1680.65 & 1673.15 & 1635.15 & 1663.35 & 1663.45 & 1010.30 \\
\hline $\mathrm{C} 3$ & 1681.10 & 1673.75 & 1636.15 & 1663.95 & 1658.90 & 1005.00 \\
\hline
\end{tabular}

Based on the results of designated mass properties, water content, density, absorption and void content of mix FD4 were calculated using Equations 5-4 to 5-11. On average, the water content was 2.76\%, absorption after immersion was $1.72 \%$, absorption after immersion and boiling was $1.72 \%$, air-dry density was 2564.5 $\mathrm{kg} / \mathrm{m}^{3}$, oven-dried density was $2506.6 \mathrm{~kg} / \mathrm{m}^{3}$, density after immersion was $2549.8 \mathrm{~kg} / \mathrm{m}^{3}$, density after immersion and boiling was $2549.6 \mathrm{~kg} / \mathrm{m}^{3}$, and the void content was $4.31 \%$. The results for each cylinder are presented in Table 5-3.

Water content $=\frac{I-A}{A}$ 
Absorption after immersion $=\frac{B-A}{A}$

Absorption after immersion and boiling $=\frac{C-A}{A}$

Air - dry density $=\frac{G}{C-D}$

Oven - dried density $=\frac{A}{C-D}$

Density after immersion $=\frac{B}{C-D}$

Density after immersion and boiling $=\frac{C}{C-D}$

Void content $=\frac{C-A}{C-D}$

Table 5- 3: Results of water content, density, absorption and void content

\begin{tabular}{|c|c|c|c|c|}
\hline Cylinder & $\begin{array}{c}\text { Water Content } \\
{[\%]}\end{array}$ & $\begin{array}{c}\text { Absorption after } \\
\text { Immersion [\%] }\end{array}$ & $\begin{array}{c}\text { Absorption after Immersion } \\
\text { and Boiling [\%] }\end{array}$ & $\begin{array}{c}\text { Air-dry Density } \\
{\left[\mathrm{kg} / \mathrm{m}^{3}\right]}\end{array}$ \\
\hline $\mathrm{C} 1$ & 2.75 & 1.75 & 2.03 & 2572.05 \\
\hline $\mathrm{C} 2$ & 2.78 & 1.72 & 1.73 & 2561.66 \\
\hline C3 & 2.75 & 1.70 & 1.39 & 2559.64 \\
\hline Average & 2.76 & 1.72 & 1.72 & 2564.45 \\
\hline Cylinder & $\begin{array}{c}\text { Oven-dried } \\
\text { Density }\left[\mathrm{kg} / \mathrm{m}^{3}\right]\end{array}$ & $\begin{array}{c}\text { Density after } \\
\text { Immersion }\left[\mathrm{kg} / \mathrm{m}^{3}\right]\end{array}$ & $\begin{array}{c}\text { Density after Immersion and } \\
\text { Boiling }\left[\mathrm{kg} / \mathrm{m}^{3}\right]\end{array}$ & Void [\%] \\
\hline C1 & 2514.15 & 2558.09 & 2565.18 & 5.10 \\
\hline C2 & 2503.48 & 2546.66 & 2546.81 & 4.33 \\
\hline C3 & 2502.14 & 2544.66 & 2536.93 & 3.48 \\
\hline Average & 2506.59 & 2549.80 & 2549.64 & 4.31 \\
\hline
\end{tabular}

\subsection{Coefficient of Thermal Expansion}

Three $100 \mathrm{~mm}$ by $200 \mathrm{~mm}$ cylinders were tested to determine the coefficient of thermal expansion (CTE) of mix FD4. These cylinders were placed in a water tank filled with saturated limewater for curing. After a 44-day curing period, the cylinders were taken out of the water tank, wiped with a towel to remove surface moisture and weighed. The mass of each cylinder was $4006.25 \mathrm{~g}, 4013.15 \mathrm{~g}$ and $4003.45 \mathrm{~g}$, respectively. Subsequently, the cylinders were returned to the water tank for curing for another two days. At the age of 46 days, the cylinders were weighed again after removing the surface moisture with a towel, and the mass of each cylinder was $4006.45 \mathrm{~g}, 4013.4 \mathrm{~g}$ and $4003.65 \mathrm{~g}$, respectively. It is evident that the difference between the two successive weighings measured at the age of 44 days and 46 days was less than $0.5 \%$, indicating the cylinders were saturated and reached mass equilibrium. It is known that the degree of saturation of concrete affects CTE results, so the verification of cylinders' saturated condition is critical prior to testing (AASHTO T336 2015). 
Four DEMEC gauge points were glued along two diametrically opposite lines of the cylinder, and the distance between the gauge points was set to be $100 \mathrm{~mm}$ by using a reference bar as illustrated in Figure 53a. As depicted in Figure 5-3b, after the gauge points were fixed, the DEMEC strain gauge was utilized to measure the relative distance between the gauge points on each side of the cylinder, and the readings were designated as $A$. The strain gauge has two locating points, one is fixed and the other one is moveable. In addition, room temperature was monitored by a thermometer, designating as $X$. Therewith, the cylinders were placed in a refrigerator for three hours, allowing the cylinders to reach thermal equilibrium. A thermometer was also put in the refrigerator to monitor the temperature, and this temperature was designated as $Y$. Then, the cylinders were taken out of the refrigerator after three hours and the relative distance between the gauge points on each side of the cylinder was measured by the strain gauge again, and this distance was designated as $B$. Subsequently, the cylinders were placed on a table in ambient laboratory temperature for three hours, and the relative distance between the gauge points were measured after, designating as $C$; in the meantime, room temperature was recorded, designated as $Z$. CTE was calculated with Equation 5-12, where Equation 5-12a corresponds to the temperature transition from room temperature to refrigerator temperature, and Equation 5-12b corresponds to the temperature transition from refrigerator temperature to room temperature. As presented in Equation 5-12, the above procedures provided two CTE results. Since CTE results are small and sensitive to each reading obtained throughout the test, the above procedures were repeated three times for each cylinder. After testing each cylinder for three times (i.e., after obtaining six CTE results of each cylinder), the average CTE result of each cylinder was found to be 9.936E$6 \mathrm{~mm} / \mathrm{mm} /{ }^{\circ} \mathrm{C}, 9.79 \mathrm{E}-6 \mathrm{~mm} / \mathrm{mm} /{ }^{\circ} \mathrm{C}$ and $9.89 \mathrm{E}-6 \mathrm{~mm} / \mathrm{mm} /{ }^{\circ} \mathrm{C}$ respectively, and CTE of mix FD4 is $9.87 \mathrm{E}-06$ $\mathrm{mm} / \mathrm{mm} /{ }^{\circ} \mathrm{C}$ by averaging the CTE result of each cylinder. Table 5-4 presents the relative distance readings between gauge points and CTE result of each test.

$$
\begin{aligned}
\text { CTE1 } & =\frac{B-A}{100 \mathrm{~mm}} \\
Y-X & \\
\text { CTE2 } & =\frac{C-B}{100 \mathrm{~mm}} \\
Z-Y &
\end{aligned}
$$



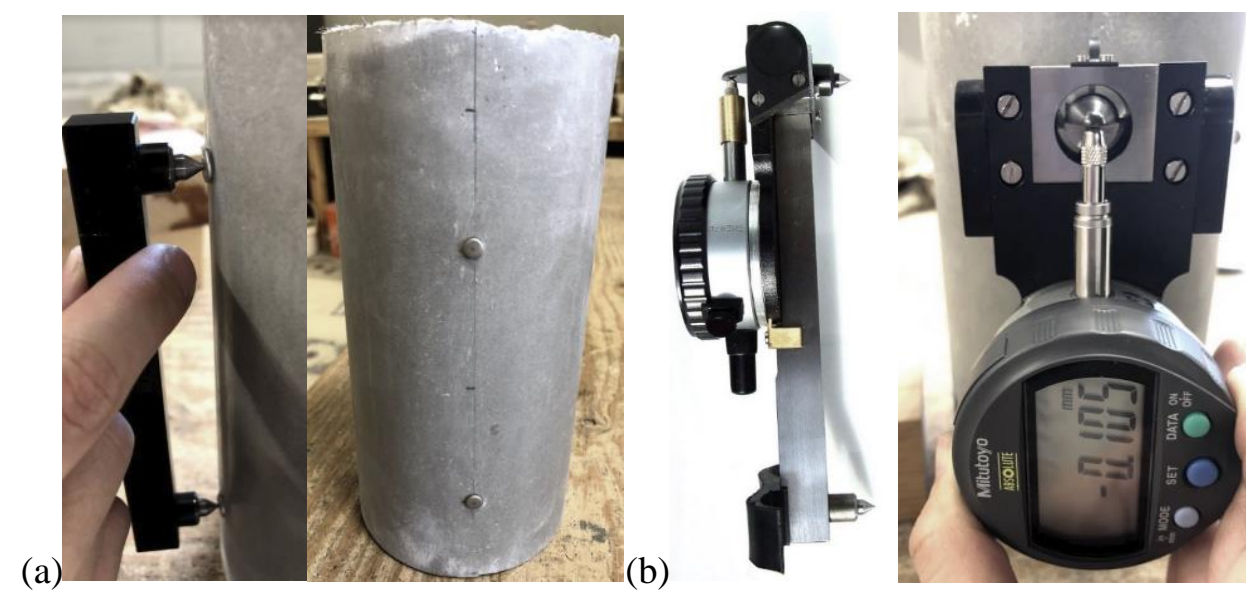

Figure 5- 3: DEMEC strain gauge and accessories: (a) Reference bar and gauge points; and (b) Strain gauge

Table 5- 4:Coefficient of thermal expansion results obtained by using DEMEC mechanical strain gauge

\begin{tabular}{|c|c|c|c|c|c|c|c|c|c|}
\hline Cylinder & \multicolumn{3}{|c|}{ C1 } & \multicolumn{3}{|c|}{$\mathrm{C} 2$} & \multicolumn{3}{|c|}{$\mathrm{C3}$} \\
\hline Test & 1 & 2 & 3 & 1 & 2 & 3 & 1 & 2 & 3 \\
\hline$X\left[{ }^{\circ} \mathrm{C}\right]$ & 22.3 & 22.3 & 23.2 & 22.3 & 22.3 & 23.2 & 22.3 & 22.3 & 23.2 \\
\hline$A$ (Left) $[\mathrm{mm}]$ & -0.005 & -0.005 & -0.004 & 0.021 & 0.020 & 0.021 & -0.108 & -0.112 & -0.110 \\
\hline$A$ (Right) $[\mathrm{mm}]$ & -0.003 & -0.004 & -0.003 & -0.029 & -0.029 & -0.028 & -0.001 & -0.002 & -0.006 \\
\hline$Y\left[{ }^{\circ} \mathrm{C}\right]$ & 5.9 & 5.9 & 5.9 & 5.9 & 5.9 & 5.9 & 5.9 & 5.9 & 5.9 \\
\hline$B$ (Left) $[\mathrm{mm}]$ & -0.021 & -0.021 & -0.021 & 0.005 & 0.004 & 0.004 & -0.124 & -0.128 & -0.127 \\
\hline$B$ (Right) $[\mathrm{mm}]$ & -0.019 & -0.020 & -0.021 & -0.045 & -0.045 & -0.045 & -0.017 & -0.018 & -0.023 \\
\hline CTE1 (Left) & 9.756 & 9.756 & 9.827 & 9.756 & 9.756 & 9.827 & 9.756 & 9.756 & 9.827 \\
\hline$\left[\mathrm{mm} / \mathrm{mm} /{ }^{\circ} \mathrm{C}\right]$ & E-06 & E-06 & E-06 & E-06 & E-06 & E-06 & E-06 & E-06 & E-06 \\
\hline CTE1 (Right) & 9.756 & 9.756 & 1.040 & 9.756 & 9.756 & 9.827 & 9.756 & 9.756 & 9.827 \\
\hline$\left[\mathrm{mm} / \mathrm{mm} /{ }^{\circ} \mathrm{C}\right]$ & E-06 & E-06 & E-05 & E-06 & E-06 & E-06 & E-06 & E-06 & E-06 \\
\hline Average CTE1 & 9.756 & 9.756 & 1.012 & 9.756 & 9.756 & 9.827 & 9.756 & 9.756 & 9.827 \\
\hline$\left[\mathrm{mm} / \mathrm{mm} /{ }^{\circ} \mathrm{C}\right]$ & E-06 & E-06 & E-05 & E-06 & E-06 & E-06 & E-06 & E-06 & E-06 \\
\hline$Z\left[{ }^{\circ} \mathrm{C}\right]$ & 22.3 & 23.2 & 23.2 & 22.3 & 23.2 & 23.2 & 22.3 & 23.2 & 23.2 \\
\hline$C$ (Left) $[\mathrm{mm}]$ & -0.005 & -0.003 & -0.004 & 0.021 & 0.021 & 0.021 & -0.107 & -0.110 & -0.110 \\
\hline$C$ (Right) $[\mathrm{mm}]$ & -0.003 & -0.004 & -0.002 & -0.029 & -0.028 & -0.028 & -0.001 & -0.001 & -0.006 \\
\hline CTE2 (Left) & 9.756 & 9.827 & 9.827 & 9.756 & 9.827 & 9.827 & 9.756 & 9.827 & 9.827 \\
\hline$\left[\mathrm{mm} / \mathrm{mm} /{ }^{\circ} \mathrm{C}\right]$ & E-06 & E-06 & E-06 & E-06 & E-06 & E-06 & E-06 & E-06 & E-06 \\
\hline CTE2 (Right) & 9.756 & 9.827 & 1.098 & 9.756 & 9.827 & 9.827 & 1.037 & 1.040 & 9.827 \\
\hline$\left[\mathrm{mm} / \mathrm{mm} /{ }^{\circ} \mathrm{C}\right]$ & E-06 & E-06 & E-05 & E-06 & E-06 & E-06 & E-05 & E-05 & E-06 \\
\hline Average CTE2 & 9.756 & 9.827 & 1.040 & 9.756 & 9.827 & 9.827 & 1.006 & 1.011 & 9.827 \\
\hline$\left[\mathrm{mm} / \mathrm{mm} /{ }^{\circ} \mathrm{C}\right]$ & E-06 & E-06 & E-05 & E-06 & E-06 & E-06 & E-05 & E-05 & E-06 \\
\hline $\begin{array}{l}\text { Average CTE } \\
{\left[\mathrm{mm} / \mathrm{mm} /{ }^{\circ} \mathrm{C}\right]}\end{array}$ & & 0265 & & & $.790 \mathrm{E}-0$ & & & $.890 \mathrm{E}-0$ & \\
\hline
\end{tabular}

\subsection{Freeze-Thaw Test}

Three $75 \mathrm{~mm}$ by $75 \mathrm{~mm}$ by $285 \mathrm{~mm}$ prisms from mix FD 4 were tested to determine the effects of variations in the properties of UHP-SFRC as subjecting to freezing-and-thawing conditions in accordance with ASTM C1856 (2017), ASTM C666 (2008) and ASTM C215 (2002). The specimens were cast by filling the mold 
from one end with a single layer of fresh concrete and were demolded one day after casting. Subsequently, the specimens were cured in saturated limewater in ambient laboratory temperature and the test was started at the age of 41 days. Figure 5-4 depicts the devices utilized for the freeze-thaw test. The ThermoStream which is a programmable temperature forcing system allowing rapid thermal cycling and long saturation at a precise temperature was utilized, and the specimens were placed in a freezer which was connected with the ThermoStream system so that the ThermoStream system controlled the temperature in the freezer. Note that the freezer was not connected to power source and functioned as an airtight and adiabatic cabinet by placing a lid made of thermal insulating materials. In addition, a temperature sensor was placed in the freezer to monitor temperature.
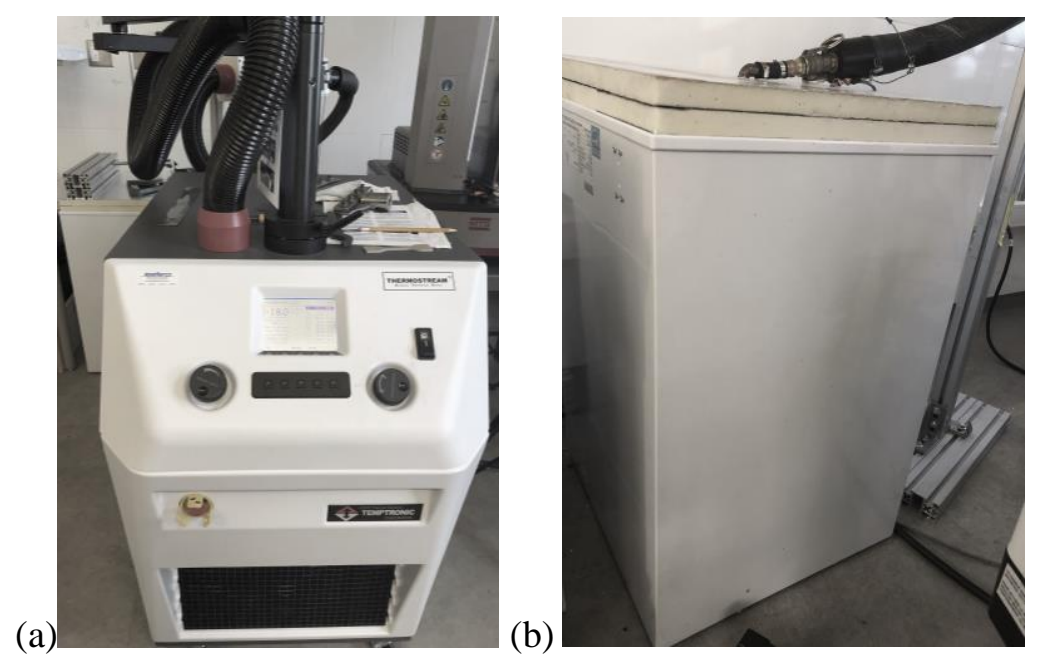

Figure 5- 4: Experimental test setup of freeze-thaw test: (a) ThermoStream System; and (b) Modified freezer functioning as an adiabatic cabinet

ASTM C666 (2008) requires to alternately lower the temperature of the cabinet from $4{ }^{\circ} \mathrm{C}$ to $-18{ }^{\circ} \mathrm{C}$ and raise it from $-18{ }^{\circ} \mathrm{C}$ to $4{ }^{\circ} \mathrm{C}$ in not less than 2 hours nor more than 5 hours. Besides, at least $25 \%$ of the time of each freeze-thaw cycle should be used for thawing. Figure 5-5 depicts the temperature variations in the freezer for a freeze-thaw cycle. It is evident that the transition time from $4{ }^{\circ} \mathrm{C}$ to $-18^{\circ} \mathrm{C}$ was about 12 minutes, the freezing period (i.e., the period of time during which temperature in the freezer maintained at $-18{ }^{\circ} \mathrm{C}$ ) was approximately 110 minutes, the transition time from $-18^{\circ} \mathrm{C}$ to $4^{\circ} \mathrm{C}$ was around 12 minutes, and the thawing period (i.e., the period of time during which temperature in the freezer maintained at $4{ }^{\circ} \mathrm{C}$ ) was approximately 50 minutes. Thus, the time required to complete one freeze-thaw cycle was around 3 hours, and about $27 \%$ of the time was used for thawing, which satisfied the temperature requirements set by the standard. 


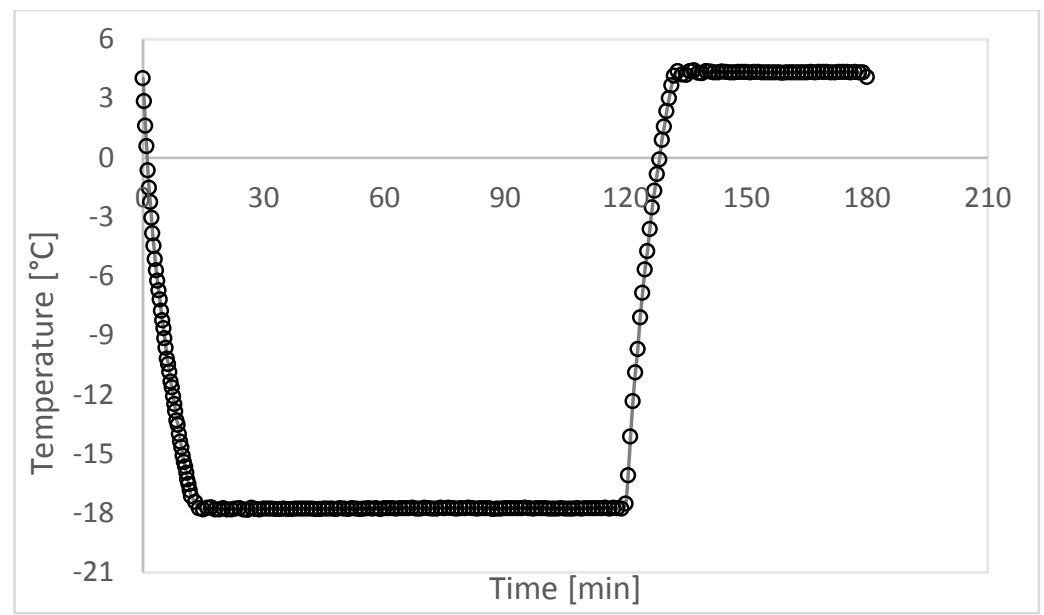

Figure 5- 5: Temperature variations of a freeze-thaw cycle

According to ASTM C666 (2008) and ASTM C1856 (2017), the specimens should be completely surrounded by no less than $1 \mathrm{~mm}$ nor more than $3 \mathrm{~mm}$ of water at all times while they are subjected to freezing-and-thawing conditions. As depicted in Figure 5-6a, to satisfy this requirement, thin aluminum sheets were used to make containers. In addition, paper clips were folded and placed at the bottom of the container as supports so that there was $1 \mathrm{~mm}$ to $3 \mathrm{~mm}$ water below the bottom surface of the specimen. Note that rigid metal was not selected for making the containers because during the freezing period, ice or water pressure had the potential to damage metal containers if they were rigid, and possibly the specimens therein. Moreover, as depicted in Figure 5-6b, a control specimen was cast, and a thermometer was imbedded in it. The thermometer tip was located near the center of the control specimen.

(a)

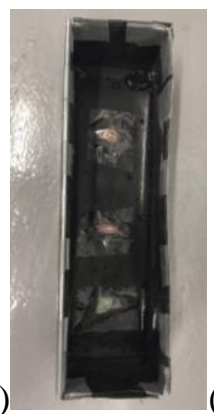

Figure 5- 6: (a) Containers; and (b) The control specimen

Prior to subjecting the specimens to freeze-thaw cycles, the prisms (without the containers) were placed in the freezer, and the ThermoStream system was programmed to bring the temperature of the prisms to $4{ }^{\circ} \mathrm{C}$ which was the target thaw temperature. Subsequently, the fundamental transverse frequency test was conducted in accordance with ASTM C215 (2002). Figure 5-7 depicts the experimental test setup of 
fundamental transverse frequency test, where $L$ and $b$ were the length and width of the prism, respectively. The impact point was located at the center of the prism (i.e., at Point $\mathrm{O}$ ), whereas the support points (i.e., nodal points) were located 0.224 of the length of the prism measured from each end. Additionally, as required by ASTM C215 (2002), the prism was placed on soft sponge supports, and the accelerometer was attached to the prism by using a double-sided adhesive tape and a rubber band. The accelerometer was connected with a data amplifier which was connected to a laptop running the CatmanEasy data acquisition software. Thus, the accelerometer signal could trigger data acquisition, and FFT (Fast Fourier Transform) analysis was performed by using the CatmanEasy software in order to identify through resonance the frequency of the fundamental mode of vibration. When testing the prism, an impactor was used to strike the prism normal to the surface at the center of the prism, and each prism was tested for three times. Figure 5-8 presents the response curve of a test, where the horizontal axis provides transverse frequency values and vertical axis provides root-mean-square of acceleration amplitude. The fundamental transverse frequency was the frequency with the highest peak in amplitude on the response curve, and the initial fundamental transverse frequency result of each prism determined prior to subjecting the specimens to freeze-thaw cycles was $3555 \mathrm{~Hz}, 3516 \mathrm{~Hz}$ and $3555 \mathrm{~Hz}$, respectively.

(a)

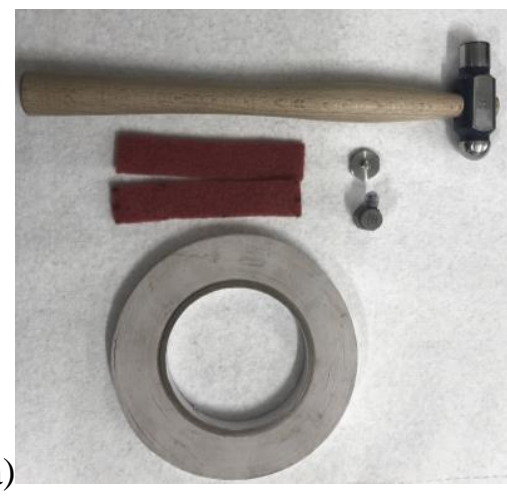

(b)
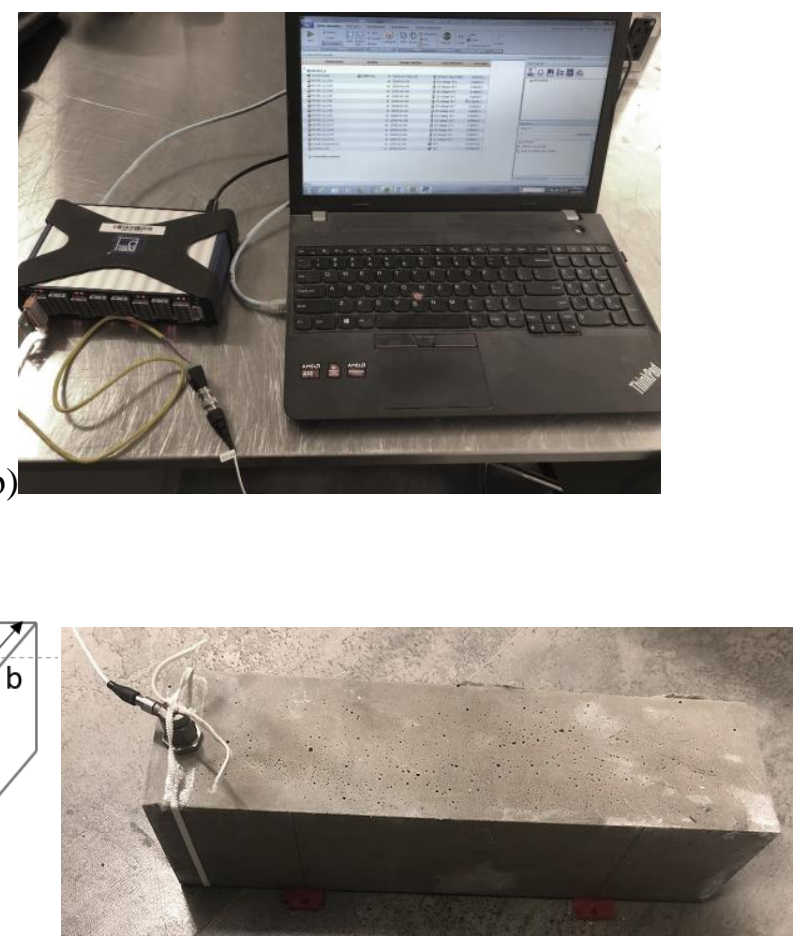

(c)

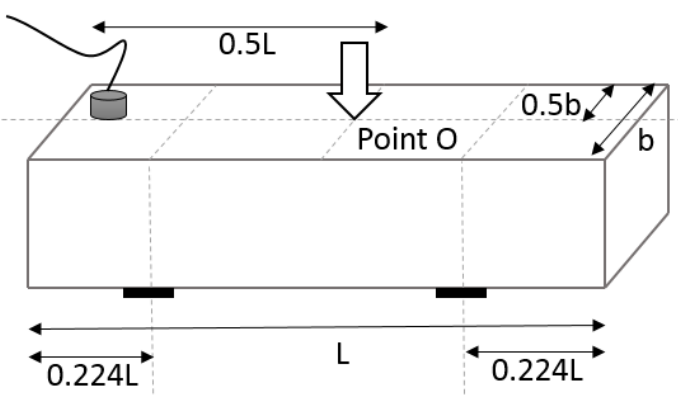

Figure 5- 7: Experimental test setup of fundamental transverse frequency test: (a) Impactor, sponge supports, accelerator and double-sided adhesive tape; (b) Data amplifier and CatmanEasy data acquisition software; and (c) Locations of the impact point, accelerator, and support points 


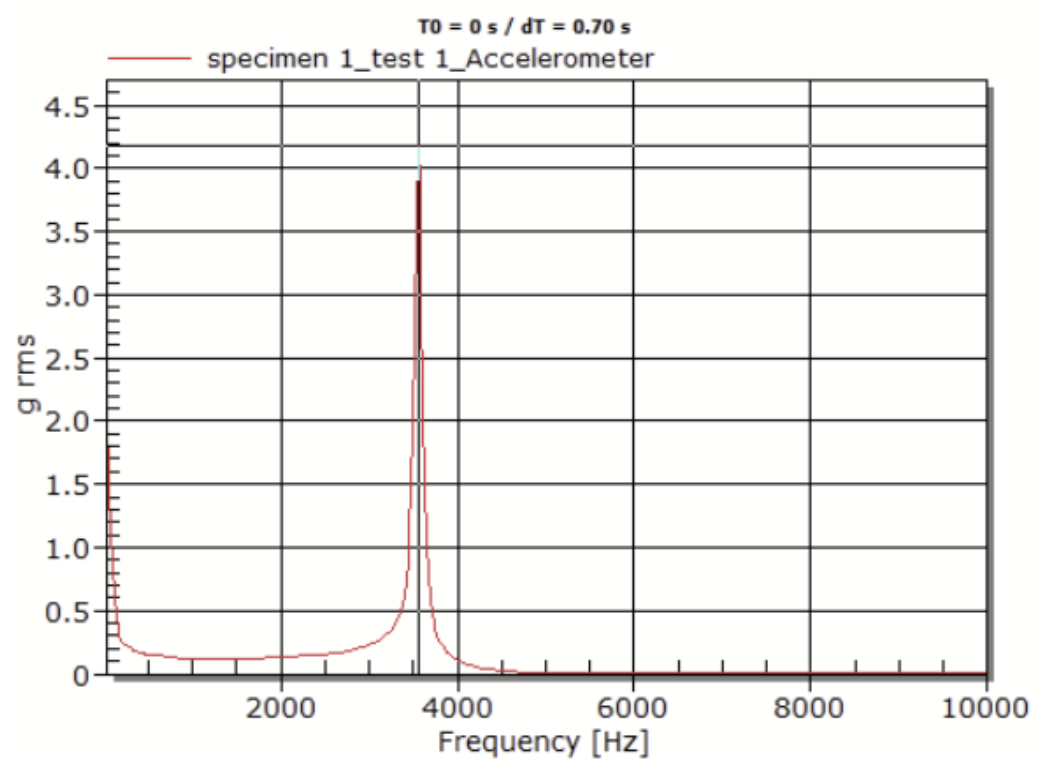

Figure 5- 8: Response curve of a fundamental transverse frequency test

As illustrated in Figure 5-9, the freeze-thaw test was started immediately after obtaining the initial fundamental transverse frequency results by placing the prisms in the containers filled with water in the freezer which was connected with the ThermoStream system. In addition, the prisms were taken out of the freezer under a thawed condition after completing every 36 cycles of exposure to the freezing-and thawing conditions in order to determine the fundamental transverse frequency during the test progress. Before removing the prisms from the freezer, it was essential to ensure the prisms were completely thawed. This was accomplished by holding the prism at the end of the thawing period in the freezer for a sufficient time and by checking the temperature of the control specimen. As mentioned previously, the thermometer tip was located near the center of the control specimen, so the thermometer measured the temperature inside the control specimen and could be used to evaluate if the prisms were fully thawed. After obtaining the fundamental transverse frequency results, the containers were rinsed out and clean water was added, and the prisms were returned to the freezer for exposing to more freeze-thaw cycles. ASTM C666 (2008) and ASTM C1856 (2017) state that each specimen should be tested until subjecting to 300 freeze-thaw cycles or until its relative dynamic modulus of elasticity reaches $90 \%$ of the initial modulus, whichever occurs first. For the three prims considered, all prisms were subjected to 300 freeze-thaw cycles and concrete deterioration was not observed. It is worth noting that the 300 freeze-thaw cycles were not completed continuously because the ThermoStream system was used by other researchers sometimes; whenever the cycles were interrupted, the specimens were stored in an operational freezer at $-18^{\circ} \mathrm{C}$, which was permitted according with ASTM C666 (2008). 


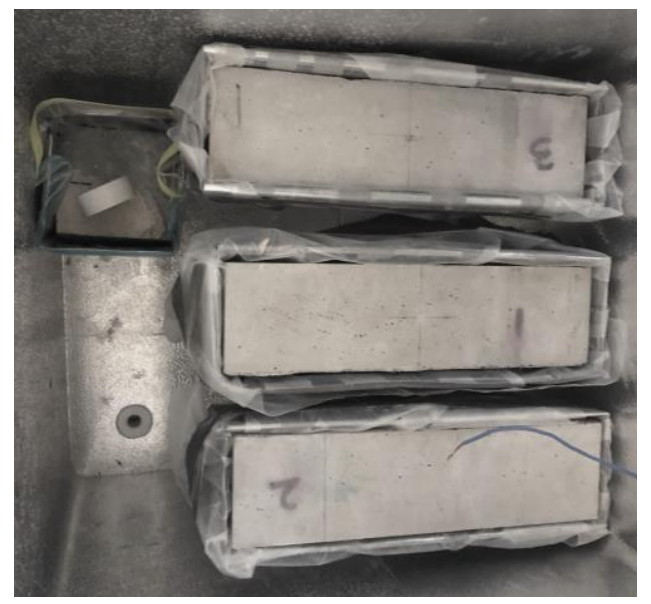

Figure 5- 9: Specimens ready for freeze-thaw test

Table 5-5 presents the fundamental transverse frequency results obtained throughout the freeze-thaw test. It is evident that compared with the initial fundamental transverse frequency results, the frequency values slightly decreased after exposing to 300 freeze-thaw cycles. In addition, the fundamental transverse frequency results increased after subjecting the specimens to a certain number of freeze-thaw cycles. It is most likely that the UHP-FRC material of the prisms might have contained unhydrated Portland cement, and the surrounding water absorption and further hydration could have caused the increase in results (ASTM C1856 2017). Moreover, the relative dynamic modulus of elasticity $\left(P_{c}\right)$ was calculated with Equation 5-13a, where $n$ is the initial fundamental transverse frequency at 0 cycles of freezing and thawing, and $n$ 'is the fundamental transverse frequency after 300 cycles. The relative dynamic modulus of elasticity of each prism was found to be $97.7 \%, 99.2 \%$ and $98.3 \%$ respectively, and the average relative dynamic modulus of elasticity of mix FD4 is $98.3 \%$. The durability factor $(D F)$ was determined with Equation 513b (ASTM C666 2008), where $N$ is the number of freeze-thaw cycles at which the relative dynamic modulus of elasticity would reach the specified minimum value (i.e., $90 \%$ of the initial modulus) for discontinuing the test (in other words, this is the number of cycles at which the exposure would have to be terminated), and $M$ is the specified maximum number of cycles at which the exposure would be terminated according with the test. Regarding the three prisms tested in the present study, $M$ is equal to $N$ (i.e., $M=N$ $=300$ cycles), so the durability factor is equal to the relative dynamic modulus of elasticity values.

$P_{c}=\left(n^{2} / n^{2}\right) \times 100 \%$

$D F=P_{c} N / M$ 
Table 5- 5: Fundamental transverse frequency results

\begin{tabular}{|c|c|c|c|c|c|c|c|}
\hline & Prism 1 & Prism 2 & Prism 3 & & Prism 1 & Prism 2 & Prism 3 \\
\hline \multirow{3}{*}{0 Cycle } & 3555 & 3516 & 3555 & \multirow{3}{*}{180 Cycles } & 3555 & 3516 & 3613 \\
\hline & 3555 & 3516 & 3555 & & 3555 & 3516 & 3555 \\
\hline & 3555 & 3516 & 3555 & & 3555 & 3516 & 3613 \\
\hline Average $[\mathrm{Hz}]$ & 3555 & 3516 & 3555 & Average $[\mathrm{Hz}]$ & 3555 & 3516 & 3594 \\
\hline \multirow{3}{*}{36 Cycles } & 3574 & 3535 & 3574 & \multirow{3}{*}{216 Cycles } & 3551 & 3508 & 3549 \\
\hline & 3574 & 3535 & 3574 & & 3551 & 3508 & 3549 \\
\hline & 3574 & 3535 & 3574 & & 3551 & 3508 & 3549 \\
\hline Average $[\mathrm{Hz}]$ & 3574 & 3535 & 3574 & Average [Hz] & 3551 & 3508 & 3549 \\
\hline \multirow{3}{*}{72 Cycles } & 3555 & 3516 & 3574 & \multirow{3}{*}{252 Cycles } & 3514 & 3508 & 3549 \\
\hline & 3555 & 3516 & 3574 & & 3514 & 3508 & 3549 \\
\hline & 3555 & 3516 & 3555 & & 3514 & 3508 & 3549 \\
\hline Average $[\mathrm{Hz}]$ & 3555 & 3516 & 3568 & Average [Hz] & 3514 & 3508 & 3549 \\
\hline \multirow{3}{*}{108 Cycles } & 3574 & 3516 & 3574 & \multirow{3}{*}{288 Cycles } & 3514 & 3502 & 3536 \\
\hline & 3574 & 3516 & 3574 & & 3514 & 3502 & 3536 \\
\hline & 3574 & 3516 & 3574 & & 3514 & 3502 & 3536 \\
\hline Average $[\mathrm{Hz}]$ & 3574 & 3516 & 3574 & Average $[\mathrm{Hz}]$ & 3514 & 3502 & 3536 \\
\hline \multirow{3}{*}{144 Cycles } & 3574 & 3516 & 3555 & \multirow{3}{*}{300 Cycles } & 3514 & 3502 & 3521 \\
\hline & 3574 & 3516 & 3555 & & 3514 & 3502 & 3521 \\
\hline & 3574 & 3516 & 3555 & & 3514 & 3502 & 3521 \\
\hline Average $[\mathrm{Hz}]$ & 3574 & 3516 & 3555 & Average [Hz] & 3514 & 3502 & 3521 \\
\hline
\end{tabular}

\subsection{Salt Scaling Test}

Two $300 \mathrm{~mm}$ by $300 \mathrm{~mm}$ by $75 \mathrm{~mm}$ specimens were prepared for conducting the salt scaling test so as to determine the resistance to scaling of a horizontal UHP-SFRC surface exposed to freeze-thaw cycles in the presence of de-icing chemicals. During concrete casting, the specimens were cast by filling the mold with a single layer of fresh concrete and consolidated by tapping each side of the mold for 30 times. Besides, excess concrete was screened with a wood strike-off board and the surface was finished with two passes of a wood float, one in each direction. Additionally, as depicted in Figure 5-10, top surface of the specimen was gently brushed by a textured roller to provide the desired texture of the test surface. In addition, the specimens were demolded one day after casting and stored in water tanks in ambient laboratory temperature for curing for 14 days. At the age of 14 days, the specimens were removed from the water tanks and placed in the lab which had a temperature of approximately $23^{\circ} \mathrm{C}$ and a relative humidity of $40 \%$. The specimens were placed in lab for curing until testing. During the dry period (i.e., curing in lab), a dyke was applied to each specimen. As illustrated in Figure 5-11, the dyke was made of $25 \mathrm{~mm}$-thick plastic boards which were non-absorptive and waterproof. Each piece of the dyke was fixed to the specimens by using highly adhesive gel. Additionally, rubber sealant was applied around the dyke perimeter so that the dyke was leakproof. On account of the thickness of the plastic boards, the effective test surface area which was exposed to salt solution became $0.0625 \mathrm{~m}^{2}$, reduced from $0.09 \mathrm{~m}^{2}$. 


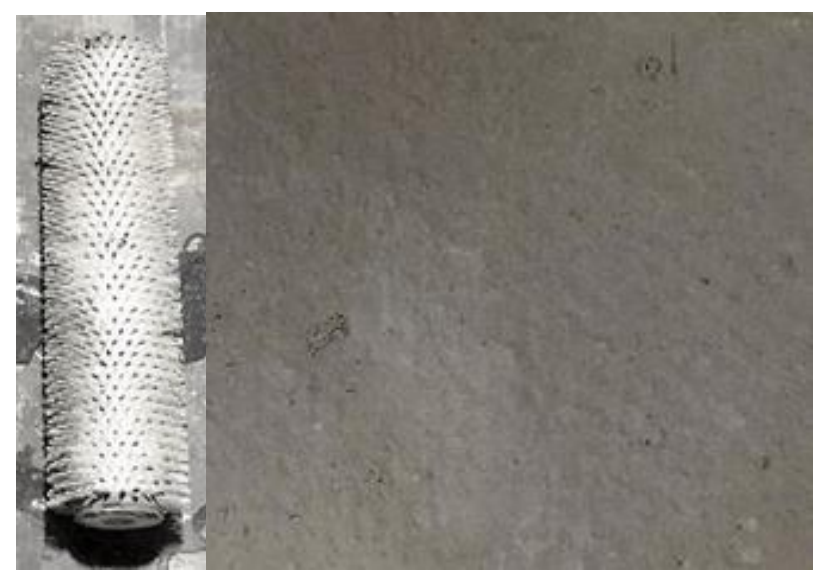

Figure 5-10: The textured roller and the slightly textured test surface

(a)

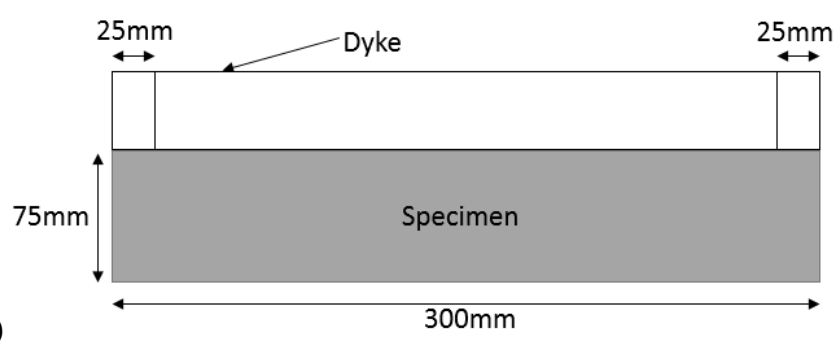

(b)

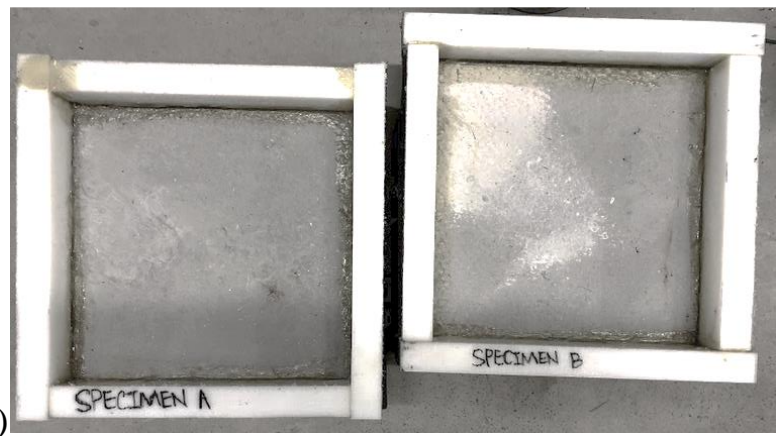

Figure 5-11: The dyke of a salt-scaling specimen: (a) Schematic of the dyke (sideview); and (b) Photo of the dyke

The salt scaling test was started at a mature age ( $>56$ days). The test surfaces were covered with approximately $6 \mathrm{~mm}$ of 3\% sodium chloride solution and then they were exposed to freeze-thaw cycles. Each freeze-thaw cycle took 24 hours to complete, was divided into a freezing period of 17 hours and a thawing period of 7 hours. The freezing condition was accomplished by placing the specimens in a freezer at approximately $-16^{\circ} \mathrm{C}$, and the thawing condition was accomplished by placing the specimens in ambient laboratory environment at approximately $23{ }^{\circ} \mathrm{C}$. As depicted in Figure 5-12, temperature probes and a temperature data recorder were utilized to monitor the temperature at the interface between concrete test surface and salt solution during the first three freeze-thaw cycles to ensure temperature requirements were satisfied. Regarding the freeze-thaw cycles, each specimen was supported by two wood strips to allow free 
air circulation under, around and over the specimen. In addition, the specimens were covered by a plastic sheet whenever they were placed in the lab to keep the test surfaces clean.

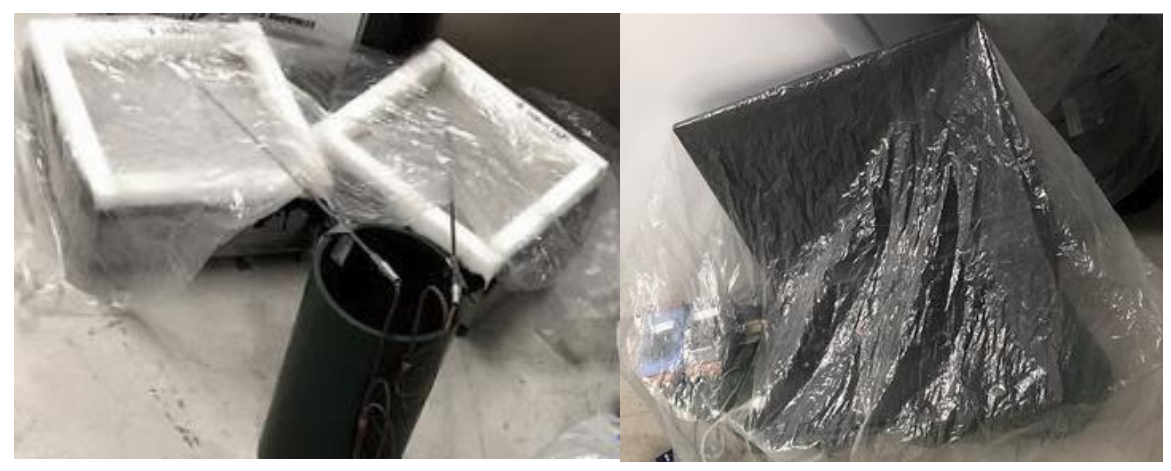

Figure 5-12: Temperature probes and temperature data recorder

Figure 5-13 depicts the test surfaces at the beginning of the thawing period. In accordance with CSA A23.222C (2014) and LS-412 (2017), the mass of the scaling residue from the test surfaces was measured after $5,15,30$ and 50 freeze-thaw cycles. When measuring the mass loss, the salt solution was removed with the flaked off concrete from the test surfaces by tilting the specimen into a funnel and washing the test surfaces with fresh salt solution until all loose particles were collected. Subsequently, salt solution and scaling residue was strained through a pre-weighed $80 \mu \mathrm{m}$ sieve (as depicted in Figure 5-14a), then the sieve and scaling residue were dried in an oven at $110^{\circ} \mathrm{C}$ for six hours. It is worth noting that the sieve was dried in the oven at $110^{\circ} \mathrm{C}$ for three hours then weighed prior to collecting the scaling residue so that moisture content of the sieve did not affect mass loss results. Moreover, CSA A23.2-22C (2014) requires the evaporation rate in the oven to be greater than 25 grams per hour. As depicted in Figure 5-14b, the evaporation rate in the oven was determined by placing beakers at a corner and the center of the oven and measuring the water loss after drying at $110^{\circ} \mathrm{C}$ for four hours. The average evaporation rate was found to be 34.52 grams per hour, which satisfied the requirement. Furthermore, new salt solution was applied to the test surfaces after each measuring operation, and test continued until the specimens were exposed to 50 freeze- thaw cycles. 

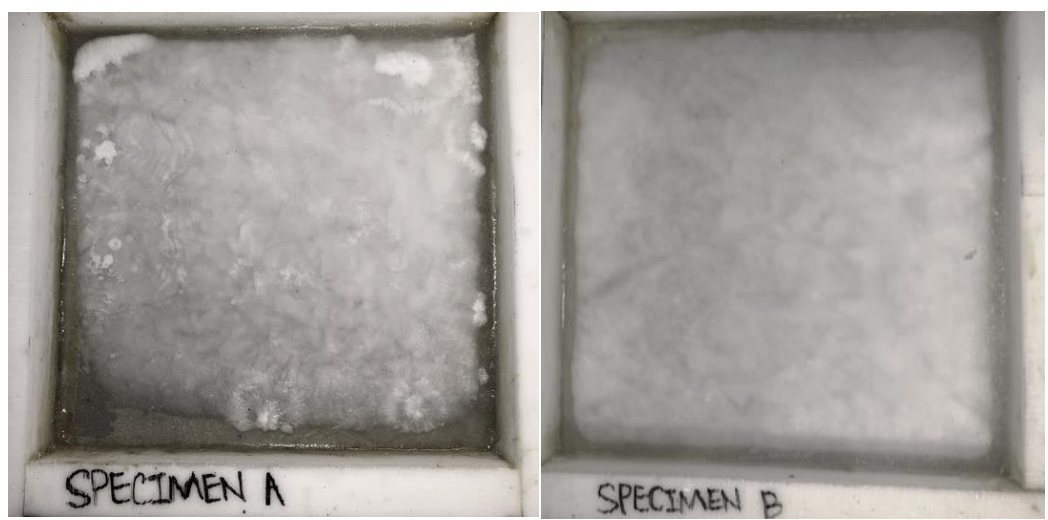

Figure 5-13:Test surfaces at the beginning of the thawing period

(a)

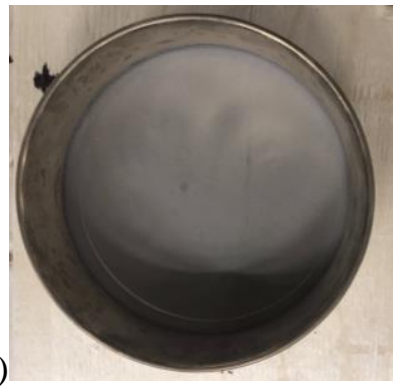

(b)

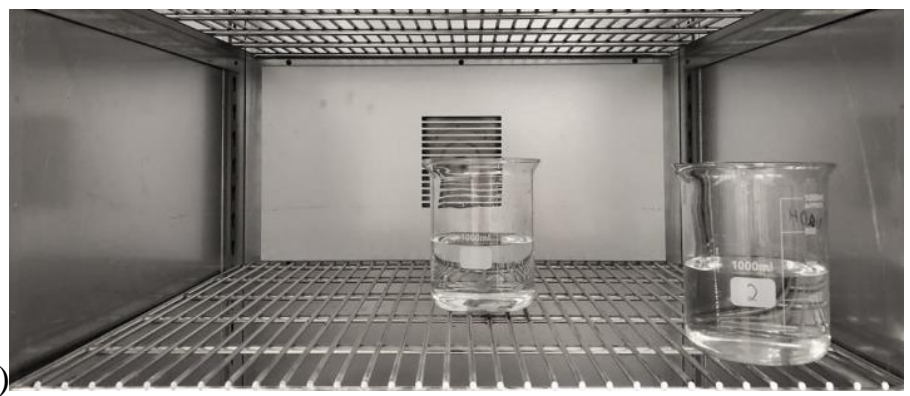

Figure 5-14: (a) The sieve utilized for collecting scaling residue; and (b) Water loss measurement to determine the evaporation rate in the oven

The cumulative mass loss of the test surface of each specimen after exposing to 50 freeze-thaw cycles with the presence of de-icing chemicals was $1.93 \mathrm{~g}$ and $1.48 \mathrm{~g}$ respectively, which was equivalent to $30.88 \mathrm{~g} / \mathrm{m}^{2}$ and $23.68 \mathrm{~g} / \mathrm{m}^{2}$, found by diving the cumulative mass loss results with the effective test surface area (i.e., $0.0625 \mathrm{~m}^{2}$ ). Table 5-6 presents the measured mass loss results after 5,15, 30 and 50 freeze-thaw cycles.

Table 5- 6: Results of salt scaling test

\begin{tabular}{|c|c|c|}
\hline Cycle & Specimen A- Mass Loss [g] & Specimen B- Mass Loss [g] \\
\hline 5 & 0.11 & 0.13 \\
\hline 15 & 0.23 & 0.19 \\
\hline 30 & 0.62 & 0.39 \\
\hline 50 & 0.97 & 0.77 \\
\hline Total Mass Loss [g] & 1.93 & 1.48 \\
\hline
\end{tabular}




\section{Chapter 6. Numerical Analysis}

This chapter starts with the introduction of the inverse analysis procedures of four-point bending test (FPBT) prescribed in Annex 8.1 of CSA-S6 (2018) and Annex U of CSA-A23.1 (2019), followed by the introduction of a calibrated empirical expression which links the tensile strength of UHP-SFRC to cylinder compressive strength. In addition, the modelling methodology utilized to obtain the tensile properties of UHP-SFRC from a nonlinear finite element analysis platform (VecTor2) is explained. The estimated tensile properties obtained from the inverse analysis and finite element analysis are correlated in this chapter, and a correction factor is introduced to calibrate the experimental results of splitting tensile test (i.e., apparent splitting tensile strength) so that the true splitting tensile strength results are comparable with the tensile strength values obtained from other tests. Thus, the tensile strength results of UHP-SFRC are evaluated through direct tension test (DTT), FPBT, splitting tensile test, nonlinear finite element analysis and the calibrated empirical expression. The implications of excessive flowability on tensile behaviour of UHPSFRC is explored through the experimental trends. With the available database assembled from all the experiments conducted in the course of the present thesis, it was possible to study the effects of important parameters on flexural strength including casting methodology, volumetric ratio of steel fibers, aspect ratio of the bending prism, and the degree of preferential fiber alignment.

\subsection{Inverse Analysis}

\subsubsection{Introduction of the Inverse Analysis Method}

As mentioned in the previous chapters, a load-deflection resistance curve was obtained from each FPBT by placing a linear displacement transducer at midspan of the prism. The inverse analysis method prescribed in Annex 8.1 of CSA-S6 (2018) and Annex U of CSA-A23.1 (2019) evaluates the characteristic points of the resistance curve of the bending test at distinct levels of deflection to failure under load applied at the third points of the span. As illustrated in Figure 6-1, $P$ is the total applied force at the third points of the prism, $\delta$ is the vertical deflection at midspan of the prism, point $\left(P_{o}, \delta_{o}\right)$ determines the linearity limit and initial slope $\left(s_{o}\right)$ of the resistance curve. In total, a set of four characteristic points on the resistance curve of the FPBT test were utilized for the inverse analysis method. These are defined as follows: Points $\left(P_{1}, \delta_{1}\right)$ and $\left(P_{2}, \delta_{2}\right)$ are defined by the intersection of the resistance curve with a straight line from the origin with a slope of $s_{75}$ and $s_{40}$ corresponding to $75 \%$ and $40 \%$ of $s_{o}$, respectively. Point $\left(P_{3}, \delta_{3}\right)$ corresponds to $97 \%$ of peak load $\left(P_{\max }\right)$, and point $\left(P_{4}, \delta_{4}\right)$ is in the post-peak range at $80 \%$ of $P_{3}$. 


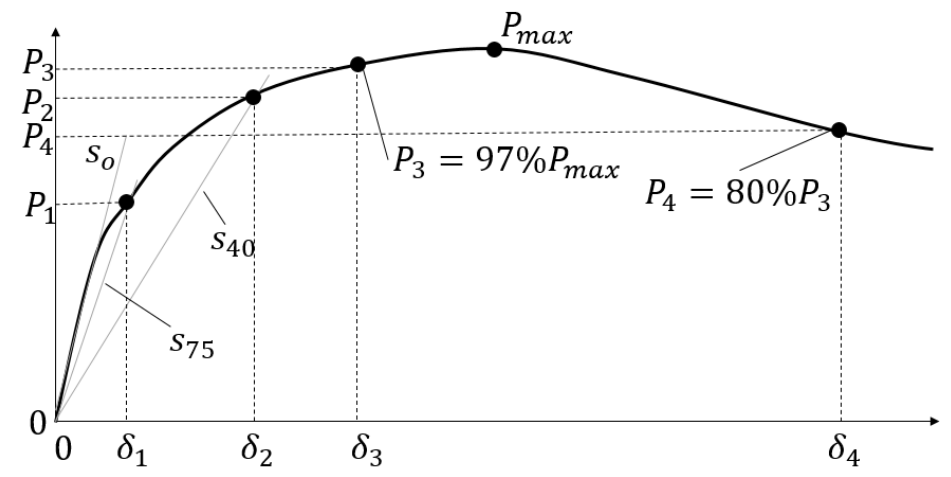

Figure 6- 1: Characteristic points on the resistance curve of four-point bending test used for the hand-calculated inverse analysis (Annex 8.1 of CSA-S6 2018)

Table 6-1 summarizes the expressions derived for the inverse analysis method, where $E_{c o}$ is the modulus of elasticity in tension, $L$ is the span between the axles of support rollers, $b$ and $h$ are the cross-sectional width and depth respectively near the failure plane, $\varepsilon_{t o}$ is a calculated effective strain value, $\delta_{4}^{*}$ is the corrected deflection of the fourth characteristic point by taking the crack location into consideration (on account of the fact that the crack rarely localizes exactly at midspan of the prism), $d_{o}$ is the average horizontal distance between the location of crack tip to midspan measured from both front and back faces of the prism (as depicted in Figure 6-2), and $K_{1}$ to $K_{5}$ are normalized parameters. The expressions shown in Table 6-1 are only applicable to the prisms subjected to loading at the third points of the span. However, the expressions were derived based on the Euler-Bernoulli Beam Theory, so the concept can be generalized to other bending test setups with proper modifications (Georgiou and Pantazopoulou 2016).

Table 6- 1: Derived expressions of the inverse analysis method (Annex 8.1 of CSA-S6 2018)

\begin{tabular}{|c|c|}
\hline$E_{c o}=\frac{7.2}{b} s_{o}$ & $K_{1}=\frac{\left(\frac{P_{1}}{P_{2}}\right)^{0.19}}{1.63}$ \\
\hline$f_{c r}=K_{1} \frac{P_{1} L}{b h^{2}}$ & \\
\hline$\varepsilon_{c r}=\frac{f_{c r}}{E_{c o}}$ & $K_{2}=7.65 \frac{\delta_{3}}{\delta_{1}}-10.53$ \\
\hline$\varepsilon_{t u}=K_{2} \varepsilon_{c r}$ & $K_{3}=K_{2}^{-0.18}\left(2.46 \frac{P_{3}}{P_{1}}-1.76\right)$ \\
\hline$f_{F u}=K_{3} f_{c r}$ & $K_{5}=1+\frac{0.6}{L} d_{0}$ \\
\hline$\delta_{4}^{*}=K_{5} \delta_{4}$ & $K_{4}=K_{3}^{-0.37} K_{2}^{0.88}\left(3 \frac{\delta_{4}^{*}}{\delta_{3}}-1.8\right)$ \\
\hline$\varepsilon_{t o}=K_{4} \varepsilon_{c r}$ & \\
\hline$w_{o}=\left(\varepsilon_{t o}-\varepsilon_{t u}+\frac{10 f_{F u}}{3 E_{c o}}\right) 1.5 h$ & \\
\hline
\end{tabular}




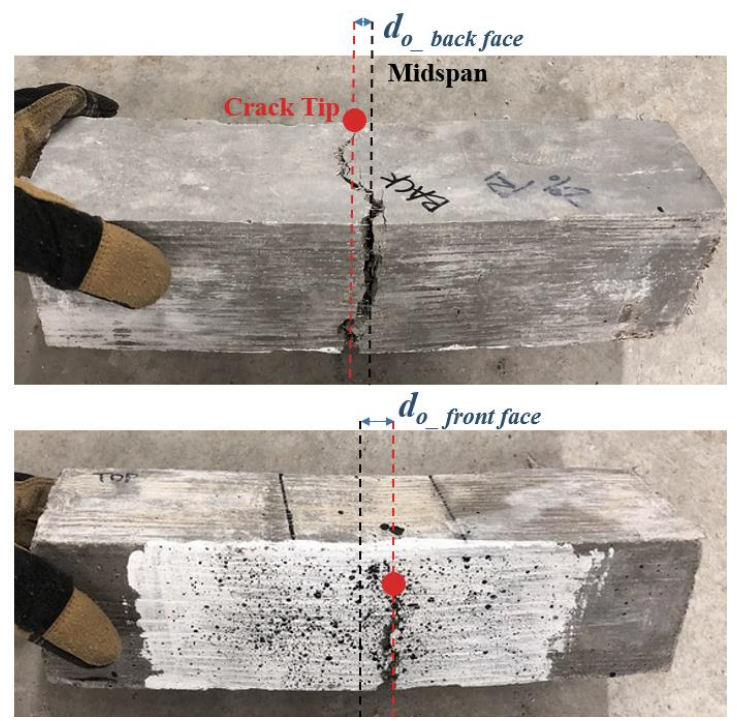

Figure 6- 2: Horizontal distance between the location of crack tip to the midspan of the prism (from both front and back faces of the prism)

The main objective of this inverse analysis method is to obtain tensile properties of UHP-SFRC. As illustrated in Figure 6-3, by using the closed-form expressions provided in Table 6-1, a simplified tensile behaviour of UHP-SFRC can be developed, where $f_{c r}$ is the cracking strength corresponding to the onset of crack formation, $f_{F u}$ is the ultimate tensile strength corresponding to crack localization, $\varepsilon_{c r}$ and $\varepsilon_{t u}$ are the strains corresponding to $f_{c r}$ and $f_{F u}$ respectively, $w_{o}$ is crack mouth opening in post-peak stage of the response (i.e., after crack localization), and $l_{F}$ is fiber length. Regarding the simplified tensile behaviour of UHP-SFRC depicted in Figure 6-3, the first part represents a bilinear stress-strain relationship upon crack localization, and the second part represents a bilinear stress-crack mouth opening relationship beyond crack localization. In addition, tensile strength is taken to be equal to zero when crack mouth opening reaches half of the fiber length. It is worth noting that the stress-crack mouth opening relationship depicted in Figure 6-3 is given here for completeness and should not be used in design models. Furthermore, the inverse analysis method discussed in this section is only applicable when $P_{3}$ is greater than $P_{2}$ on the resistance curve.

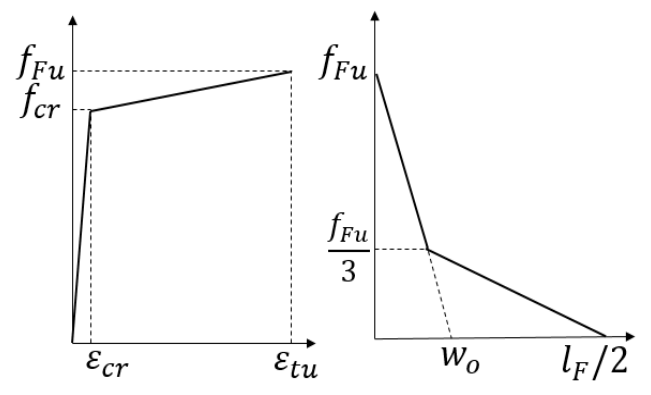

Figure 6- 3: Linearized tensile stress-strain-crack mouth opening relationships (Annex 8.1 of CSA-S6 2018) 


\subsubsection{Inverse Analysis Results}

The following sections provide the inverse analysis results of each design mix. However, only the prisms satisfying the two prerequisites of the inverse analysis procedures were analyzed. The two prerequisites are: 1). the prisms were subjected to loading at the third points of the span; and 2). $P_{3}$ is greater than $P_{2}$ on the resistance curve as depicted in Figure 6-1. Thus, the long prisms with an aspect ratio of 2 tested during Phase 1 of the experimental program can not be analyzed with this inverse analysis method. Besides, the prisms from mix DE1 containing $1 \%$ steel fibers by unit volume were not analyzed because it was challenging to select the four characteristic points from resistance curves (as depicted in Figure 3-16b). In addition, the prisms that had failed in shear or torsion (as observed from the trajectory of the critical crack path) were not analyzed. It is also noted that the resistance curves of a number of specimens could not be used to conduct the inverse analysis due to poor performance of the instrumentation (e.g., the linear displacement transducers slipped during the test and did not measure the vertical deflection at midspan of the prism properly).

\subsubsection{Commercial FD1 and FD2 Design Mixes}

The short prisms ( $75 \mathrm{~mm}$ by $75 \mathrm{~mm}$ by $280 \mathrm{~mm}$ prisms) obtained from mixes FD1 and FD2 and tested during Phase 1 of the experimental program were analyzed with the inverse analysis procedures. The linearized tensile stress-strain-crack mouth opening relationships and corresponding results of tensile properties of each mix are presented in Figure 6-4 to Figure 6-5 and Table 6-2 to Table 6-3, respectively, in which the 'Average' values and curves were obtained by averaging the analysis results of the prisms from the same set. In addition, the four characteristic points on each resistance curve utilized to perform the inverse analysis can be found in Appendix B.1.

With reference to mix FD1, the average flexural strength of OW prisms was $35.28 \mathrm{MPa}$, corresponding to a cracking strength of $11 \mathrm{MPa}$ and an ultimate tensile strength of $16.06 \mathrm{MPa}$; the average flexural strength of $\mathrm{R}$ prisms was $39.57 \mathrm{MPa}$, corresponding to a cracking strength of $11.91 \mathrm{MPa}$ and an ultimate tensile strength of 17.76 MPa. Regarding mix FD2, the average flexural strength of OW prisms was $38.78 \mathrm{MPa}$, corresponding to a cracking strength of $10.87 \mathrm{MPa}$ and an ultimate tensile strength of $16.72 \mathrm{MPa}$; the average flexural strength of R prisms was $40.42 \mathrm{MPa}$, corresponding to a cracking strength of $13.07 \mathrm{MPa}$ and an ultimate tensile strength of 15.99 MPa. Moreover, since mixes FD1 and FD2 comprised 1\% of 20 
$\mathrm{mm}$ long, straight steel fibers and $1 \%$ of $20 \mathrm{~mm}$ long, hooked-end steel fibers, their tensile strengths were assumed to be equal to zero when crack mouth opening reached $10 \mathrm{~mm}$.

Table 6- 2: Inverse analysis results of the short prisms from mix FD1

\begin{tabular}{|c|c|c|c|c|c|c|}
\hline \multicolumn{7}{|c|}{ Mix FD1 } \\
\hline \multirow{2}{*}{ Prism } & $\mathbf{f}_{\text {cr }}$ & $\varepsilon_{\mathrm{cr}}$ & $\mathbf{f}_{\mathrm{Fu}}$ & $\varepsilon_{\mathrm{tu}}$ & $\mathbf{w}_{\mathbf{0}}$ & $\mathbf{f}_{\mathrm{Fu}} / \mathbf{3}$ \\
\hline & [MPa] & {$[\mathrm{mm} / \mathbf{m m}]$} & [MPa] & {$[\mathrm{mm} / \mathrm{mm}]$} & {$[\mathrm{mm}]$} & [MPa] \\
\hline OW1 & 11.73 & 0.00045 & 16.31 & 0.01296 & 3.21 & 5.44 \\
\hline OW2 & 10.28 & 0.00077 & 15.81 & 0.01107 & 2.60 & 5.27 \\
\hline Average & 11.00 & 0.00061 & 16.06 & 0.01202 & 2.90 & 5.35 \\
\hline R1 & 14.82 & 0.00095 & 19.45 & 0.01474 & 5.84 & 6.48 \\
\hline R3 & 10.28 & 0.00014 & 16.62 & 0.01432 & 1.90 & 5.54 \\
\hline $\mathrm{R} 4$ & 10.63 & 0.00028 & 17.72 & 0.02000 & 3.23 & 5.73 \\
\hline Average & 11.91 & 0.00046 & 17.76 & 0.01635 & 3.66 & 5.92 \\
\hline
\end{tabular}

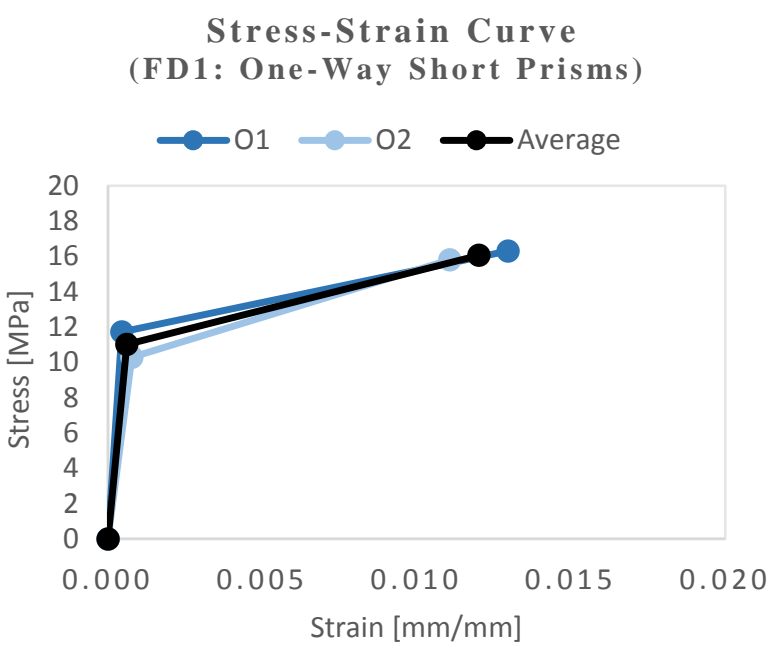

Stress-Strain Curve

(FD1: Random Short Prisms)

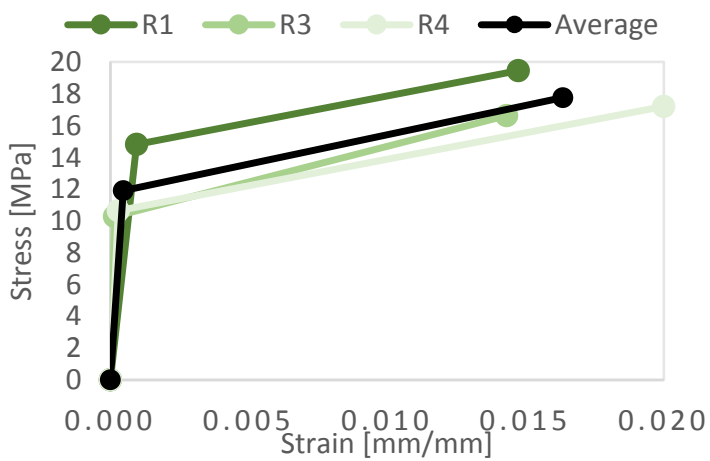

Stress-Crack Width Curve (FD1: One-Way Short Prisms)

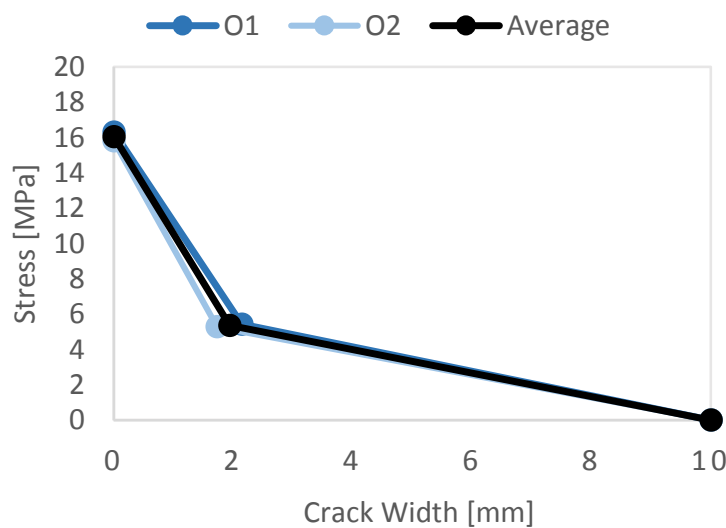

Stress-Crack Width Curve

(FD1: Random Short Prisms)

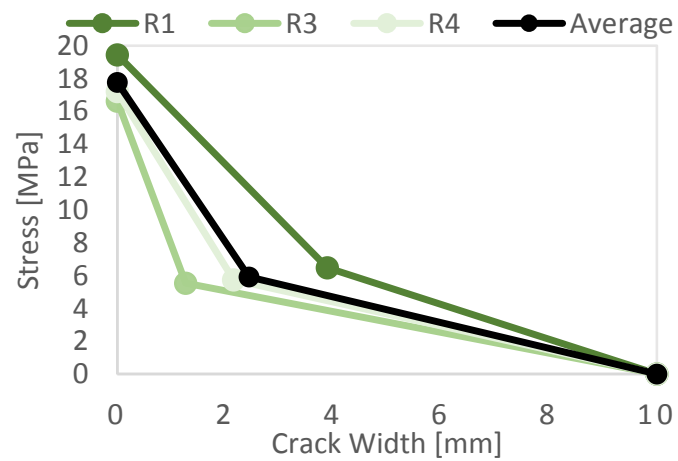

Figure 6- 4: Linearized tensile stress-strain-crack mouth opening relationships of the short prisms from mix FD1 
Table 6- 3: Inverse analysis results of the short prisms from mix FD2

\begin{tabular}{|c|c|c|c|c|c|c|}
\hline \multicolumn{7}{|c|}{ Mix FD2 } \\
\hline \multirow{2}{*}{ Prism } & $\mathbf{f}_{\mathrm{cr}}$ & $\varepsilon_{\mathrm{cr}}$ & $\mathbf{f}_{\mathrm{Fu}}$ & $\varepsilon_{\text {tu }}$ & $\mathbf{w}_{\mathbf{o}}$ & $\mathbf{f}_{\mathrm{Fu}} / \mathbf{3}$ \\
\hline & {$[\mathrm{MPa}]$} & {$[\mathrm{mm} / \mathrm{mm}]$} & {$[\mathrm{MPa}]$} & {$[\mathrm{mm} / \mathrm{mm}]$} & {$[\mathrm{mm}]$} & {$[\mathbf{M P a}]$} \\
\hline OW1 & 10.67 & 0.00010 & 16.61 & 0.01111 & 1.35 & 5.54 \\
\hline OW2 & 11.59 & 0.00033 & 19.35 & 0.01361 & 0.89 & 6.45 \\
\hline OW3 & 10.34 & 0.00027 & 14.21 & 0.00853 & 3.60 & 4.74 \\
\hline Average & 10.87 & 0.00023 & 16.72 & 0.01109 & 1.95 & 5.57 \\
\hline R1 & 11.07 & 0.00009 & 13.82 & 0.01185 & 3.17 & 4.61 \\
\hline R2 & 12.14 & 0.00009 & 15.02 & 0.01336 & 2.81 & 5.01 \\
\hline R3 & 15.99 & 0.00063 & 19.13 & 0.00787 & 2.44 & 6.38 \\
\hline Average & 13.07 & 0.00027 & 15.99 & 0.01103 & 2.81 & 5.33 \\
\hline
\end{tabular}

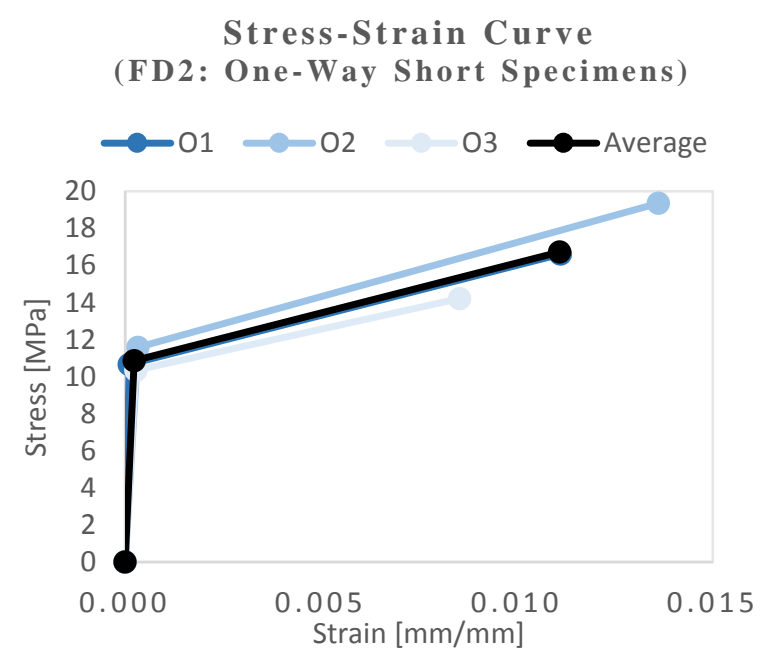

Stress-Strain Curve

(FD2: Random Short Prisms)

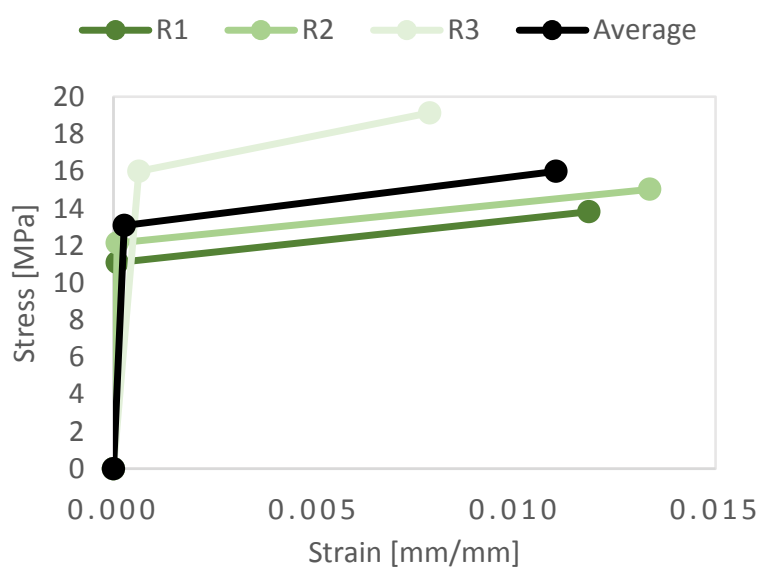

Stress-Crack Width Curve

(FD2: One-Way Short Specimens)

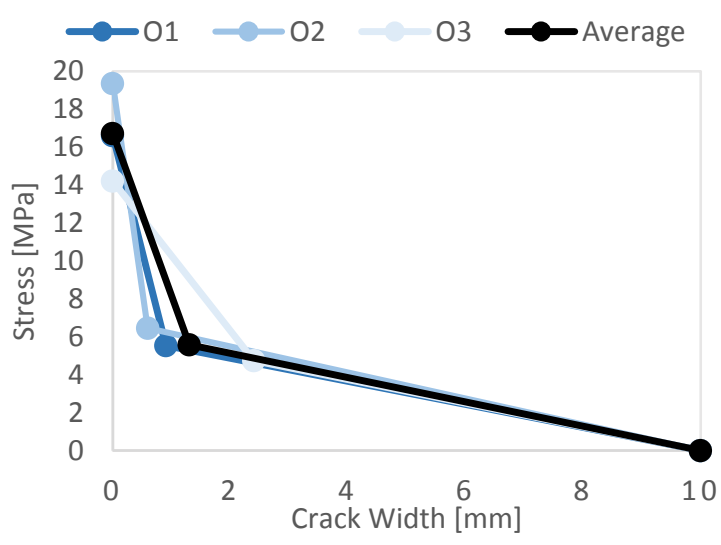

Stress-Crack Width Curve (FD2: Random Short Prisms)

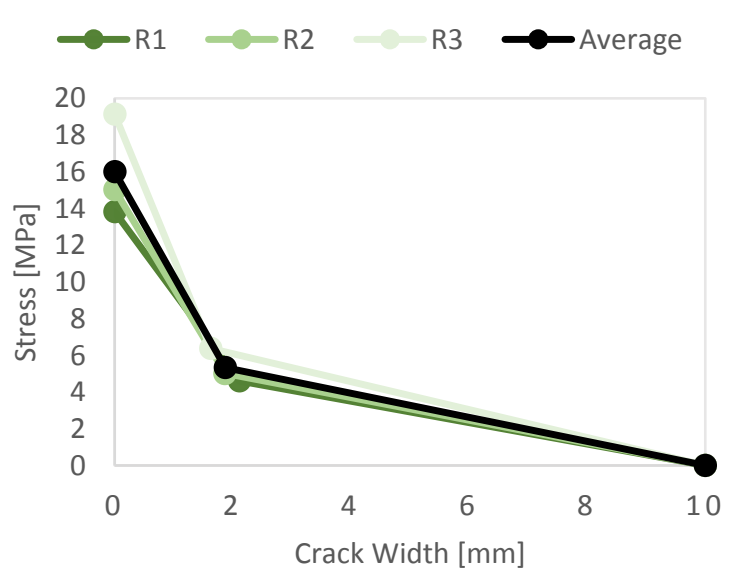

Figure 6- 5: Linearized tensile stress-strain-crack mouth opening relationships of the short prisms from mix FD2 


\subsubsection{Commercial KII Design Mix}

In this section, results obtained from the Phase I - short prism tests ( $75 \mathrm{~mm}$ by $75 \mathrm{~mm}$ by $280 \mathrm{~mm}$ prisms) of mix KI1 are presented. The average flexural strengths of OW short prisms and R short prisms were 30.08 $\mathrm{MPa}$ and 22.98 MPa respectively, corresponding to a cracking strength of $10.62 \mathrm{MPa}$ and an ultimate tensile strength of $13.50 \mathrm{MPa}$ for $\mathrm{OW}$ prisms; and a cracking strength of $10.42 \mathrm{MPa}$ and an ultimate tensile strength of $12.03 \mathrm{MPa}$ for $\mathrm{R}$ prisms. The results of tensile properties are presented in Table $6-4$, and the linearized tensile stress-strain-crack mouth opening relationships and the four characteristic points on each resistance curve utilized to perform the inverse analysis can be found in Appendix B.2. In addition, since mix KI1 comprised $13 \mathrm{~mm}$ long, straight steel fibers, its tensile strength was assumed to be equal to zero when crack mouth opening reached $6.5 \mathrm{~mm}$.

Table 6- 4: Inverse analysis results of the short prisms from mix KII

\begin{tabular}{|c|c|c|c|c|c|c|}
\hline \multicolumn{7}{|c|}{ Mix KI1 } \\
\hline \multirow[b]{2}{*}{ Prism } & $f_{\text {cr }}$ & Ecr & fFu & $\varepsilon_{t u}$ & Wo $_{0}$ & $\mathbf{f F u}_{\mathrm{Fu}} / \mathbf{3}$ \\
\hline & [MPa] & {$[\mathrm{mm} / \mathrm{mm}]$} & [MPa] & {$[\mathrm{mm} / \mathrm{mm}]$} & [mm] & [MPa] \\
\hline OW1 & 10.16 & 0.00023 & 13.08 & 0.00387 & 1.03 & 4.36 \\
\hline OW2 & 12.88 & 0.00058 & 14.61 & 0.00560 & 1.69 & 4.87 \\
\hline OW3 & 8.82 & 0.00030 & 12.82 & 0.00476 & 1.32 & 4.27 \\
\hline Average & 10.62 & 0.00037 & 13.50 & 0.00474 & 1.35 & 4.50 \\
\hline R2 & 10.42 & 0.00043 & 12.03 & 0.00528 & 1.70 & 4.01 \\
\hline
\end{tabular}

\subsubsection{Commercial LD1 Design Mix}

The short prisms from mix LD1 tested during Phase 1 of the experimental program were analyzed in this section. The average flexural strength of OW short prisms and R short prisms was $29.74 \mathrm{MPa}$ and 29.55 $\mathrm{MPa}$ respectively, corresponding to a cracking strength of $10.23 \mathrm{MPa}$ and an ultimate tensile strength of 11.67 MPa for OW prisms; and a cracking strength of 9.98 MPa and an ultimate tensile strength of 12.44 $\mathrm{MPa}$ for R prisms. The linearized tensile stress-strain-crack mouth opening relationships and corresponding results of tensile properties are provided in Figure 6-6 and Table 6-5, and the four characteristic points on each resistance curve utilized to perform the inverse analysis can be found in Appendix B.3. In addition, the tensile strength of mix LD1 was assumed to be equal to zero when crack mouth opening reached $6.5 \mathrm{~mm}$ owing to the use of $13 \mathrm{~mm}$ long, straight steel fibers. 
Table 6- 5: Inverse analysis results of the short prisms from mix LD1

\begin{tabular}{|c|c|c|c|c|c|c|}
\hline \multicolumn{7}{|c|}{ Mix LD1 } \\
\hline \multirow{2}{*}{ Prism } & $\mathbf{f}_{\text {cr }}$ & $\boldsymbol{\varepsilon}_{\text {cr }}$ & $\mathbf{f}_{\text {Fu }}$ & $\boldsymbol{\varepsilon}_{\text {tu }}$ & $\mathbf{w}_{\mathbf{o}}$ & $\mathbf{f}_{\mathrm{Fu}} / \mathbf{3}$ \\
\cline { 2 - 8 } & {$[\mathbf{M P a}]$} & {$[\mathbf{m m} / \mathbf{m m}]$} & {$[\mathbf{M P a}]$} & {$[\mathbf{m m} / \mathbf{m m}]$} & {$[\mathbf{m m}]$} & {$[\mathbf{M P a}]$} \\
\hline OW1 & 12.61 & 0.00006 & 14.29 & 0.00495 & 1.57 & 4.76 \\
\hline OW2 & 9.81 & 0.00010 & 10.91 & 0.00253 & 2.72 & 3.64 \\
\hline OW3 & 8.28 & 0.00005 & 9.80 & 0.00335 & 2.93 & 3.27 \\
\hline Average & 10.23 & 0.00007 & 11.67 & 0.00361 & 2.41 & 3.89 \\
\hline R1 & 10.98 & 0.00023 & 14.36 & 0.00715 & 3.01 & 4.79 \\
\hline R2 & 8.62 & 0.00007 & 10.10 & 0.00551 & 2.96 & 3.37 \\
\hline R3 & 10.32 & 0.00030 & 12.84 & 0.00410 & 2.29 & 4.28 \\
\hline Average & 9.98 & 0.00020 & 12.44 & 0.00559 & 2.75 & 4.15 \\
\hline
\end{tabular}
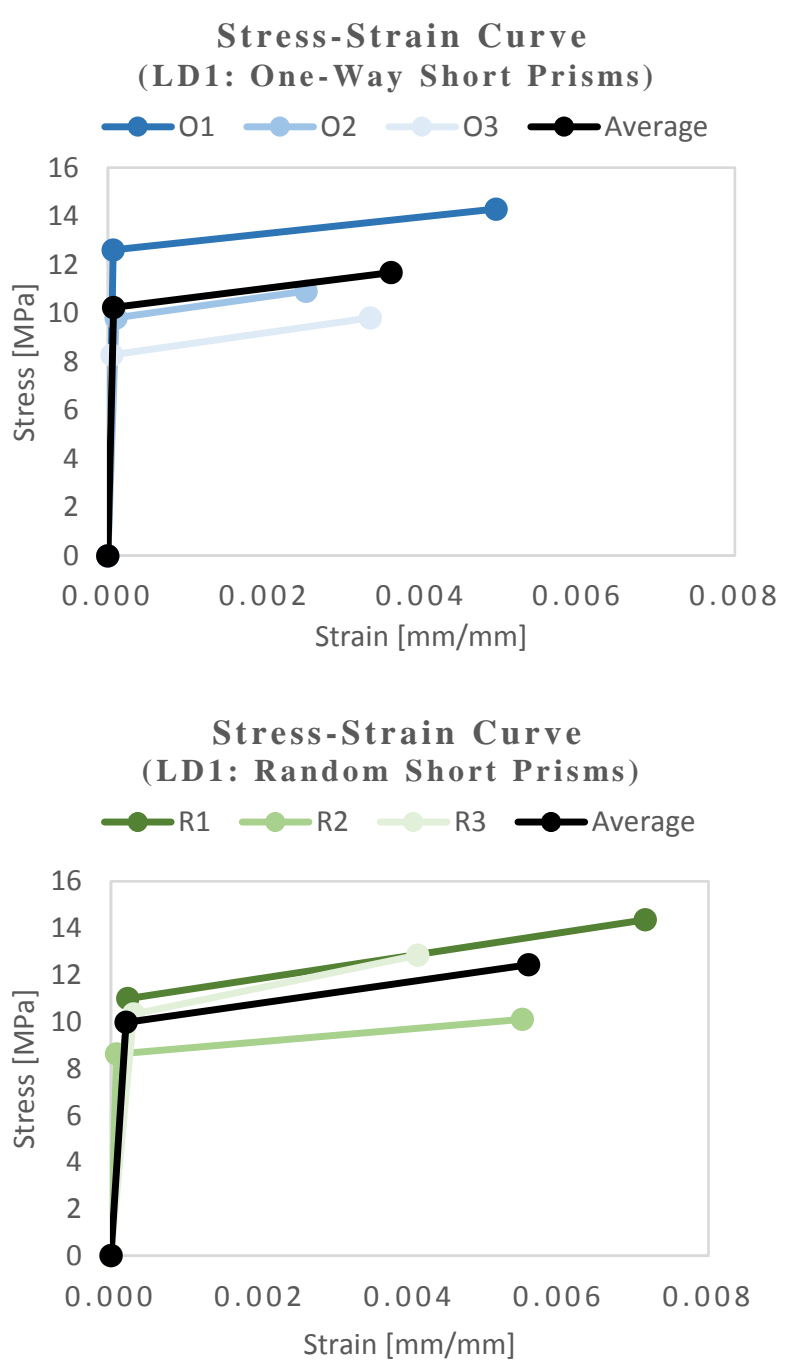
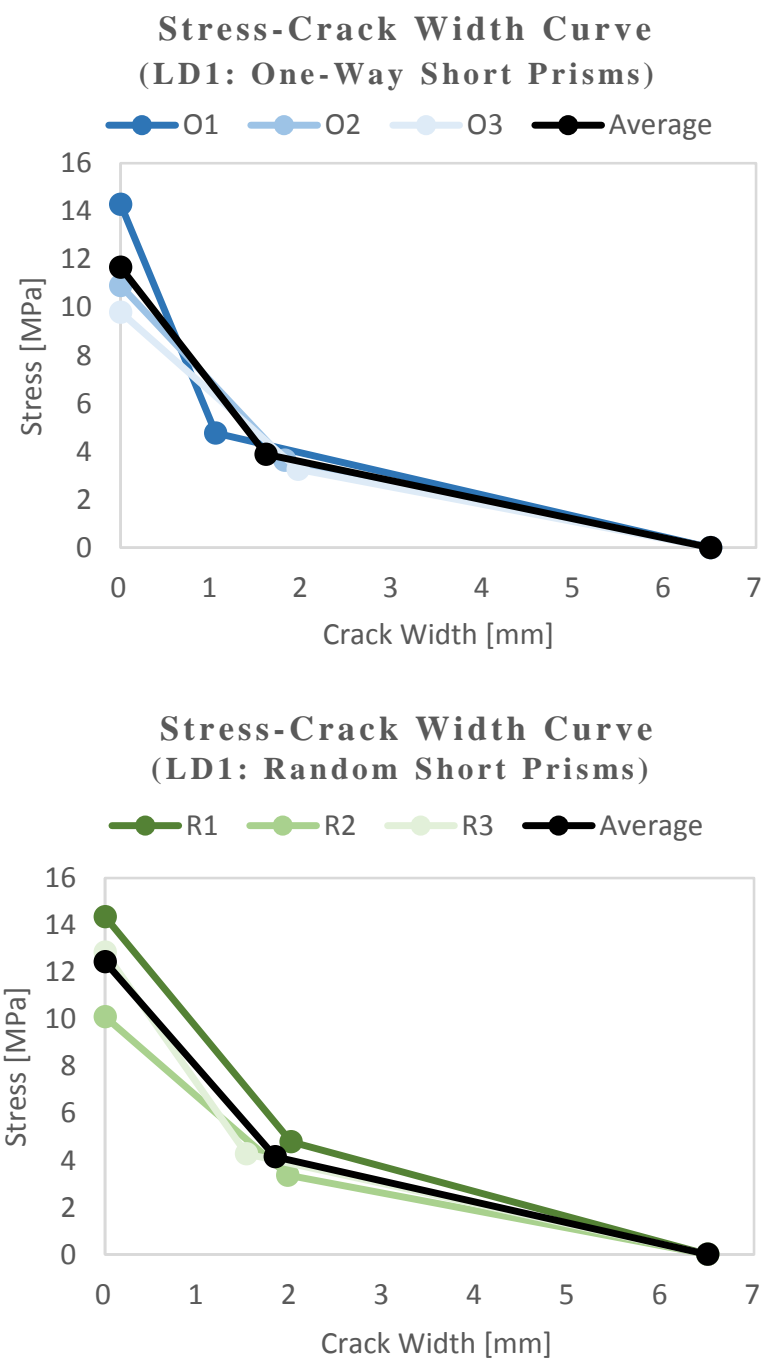

Figure 6- 6: Linearized tensile stress-strain-crack mouth opening relationships of the short prisms from mix LD1 


\subsubsection{In-House DE2 Design Mix (SC1)}

The short prisms from mix DE2 tested with SC1 (as depicted in Figure 4-11a) during Phase 1 of the experimental program were analyzed. The average flexural strength of OW short prisms and R short prisms were 26.23 MPa and 21.83 MPa respectively, corresponding to a cracking strength of 9.32 $\mathrm{MPa}$ and an ultimate tensile strength of 11.29 MPa for OW prisms; and a cracking strength of 7.47 MPa and an ultimate tensile strength of $10.06 \mathrm{MPa}$ for R prisms. The results of tensile properties were presented in Table 6-6, and the linearized tensile stress-strain-crack mouth opening relationships and the four characteristic points on each resistance curve utilized to perform the inverse analysis can be found in Appendix B.4. In addition, the tensile strength of mix DE2 was assumed to be equal to zero when crack mouth opening reached 6.5 $\mathrm{mm}$ owing to the use of $13 \mathrm{~mm}$ long, straight steel fibers.

Table 6- 6: Inverse analysis results of the short prisms from mix DE2 tested with SC1

\begin{tabular}{|c|c|c|c|c|c|c|}
\hline \multirow{2}{*}{ Prism } & $\mathbf{f}_{\text {cr }}$ & $\boldsymbol{\varepsilon}_{\text {cr }}$ & $\mathbf{f}_{\text {Fu }}$ & $\boldsymbol{\varepsilon}_{\text {tu }}$ & $\mathbf{w}_{\mathbf{o}}$ & $\mathbf{f}_{\text {Fu }} / \mathbf{3}$ \\
\cline { 2 - 7 } & {$[\mathbf{M P a}]$} & {$[\mathbf{m m} / \mathbf{m m}]$} & {$[\mathbf{M P a}]$} & {$[\mathbf{m m} / \mathbf{m m}]$} & {$[\mathbf{m m}]$} & {$[\mathbf{M P a}]$} \\
\hline OW1 & 9.53 & 0.00017 & 10.52 & 0.00435 & 3.15 & 3.51 \\
\hline OW2 & 9.29 & 0.00009 & 11.38 & 0.00260 & 2.90 & 3.79 \\
\hline OW3 & 9.15 & 0.00052 & 11.98 & 0.00676 & 1.59 & 3.99 \\
\hline Average & 9.32 & 0.00026 & 11.29 & 0.00457 & 2.54 & 3.76 \\
\hline R1 & 5.98 & 0.00026 & 8.40 & 0.00284 & 2.39 & 2.80 \\
\hline R2 & 7.20 & 0.00031 & 10.61 & 0.00580 & 1.74 & 3.54 \\
\hline R3 & 9.23 & 0.00006 & 11.17 & 0.00095 & 1.53 & 3.72 \\
\hline Average & 7.47 & 0.00021 & 10.06 & 0.00320 & 1.89 & 3.35 \\
\hline
\end{tabular}

\subsubsection{Commercial FD3 Design Mix}

The prisms (100 mm by $100 \mathrm{~mm}$ by $370 \mathrm{~mm}$ prisms) from mix FD3 tested during the Round Robin Testing Program were analyzed. The average flexural strength of OW prisms was 40.22 MPa for York University, 32.61 MPa for École Polytechnique-Montréal, and 30.71 MPa for Queen's University; and the corresponding cracking strength and ultimate tensile strength were 12.95 $\mathrm{MPa}$ and 16.99 $\mathrm{MPa}$ for York University, 10.08 MPa and 12.86 MPa for École Polytechnique-Montréal and 9.94 MPa and 11.94 MPa for Queen's University. The average flexural strength of R prisms was 31.73 MPa for York University, 26.86 MPa for École Polytechnique-Montréal and 24.68 MPa for Queen's University; and the corresponding cracking strength and ultimate tensile strength were 11.59 MPa and 12.97 MPa for York University, 7.74 
$\mathrm{MPa}$ and 8.69 MPa for École Polytechnique-Montréal, and 9.99 MPa and 11.24 MPa for Queen's University.

The linearized tensile stress-strain-crack mouth opening relationships of the prisms tested by York University and corresponding results of tensile properties are presented in Figure 6-7 and Table 6-7, and the four characteristic points on each resistance curve utilized to perform the inverse analysis, and the linearize tensile relationships of the prisms tested by École Polytechnique-Montréal and Queen's University can be found in Appendix B.5. In addition, the tensile strength of mix FD3 was assumed to be equal to zero when crack mouth opening reached $10 \mathrm{~mm}$ owing to the use of $1 \%$ of $20 \mathrm{~mm}$ long, straight steel fibers and $1 \%$ of $20 \mathrm{~mm}$ long, hooked-end steel fibers.

Table 6- 7: Inverse analysis results of the prisms from mix FD3

\begin{tabular}{|c|c|c|c|c|c|c|}
\hline \multicolumn{7}{|c|}{ York University } \\
\hline \multirow{2}{*}{ Prism } & $\mathbf{f}_{\text {cr }}$ & Ecr & $\mathbf{f}_{\mathrm{Fu}}$ & $\varepsilon_{t u}$ & $\mathbf{w}_{\mathbf{0}}$ & $\mathbf{f}_{\mathrm{Fu}} / \mathbf{3}$ \\
\hline & [MPa] & {$[\mathrm{mm} / \mathbf{m m}]$} & {$[\mathrm{MPa}]$} & {$[\mathrm{mm} / \mathbf{m m}]$} & {$[\mathrm{mm}]$} & [MPa] \\
\hline OW2 & 13.56 & 0.00019 & 17.90 & 0.01022 & 2.69 & 5.97 \\
\hline OW3 & 12.34 & 0.00024 & 16.09 & 0.00707 & 4.37 & 5.36 \\
\hline Average & 12.95 & 0.00022 & 16.99 & 0.00864 & 3.53 & 5.66 \\
\hline R2 & 10.88 & 0.00014 & 12.38 & 0.00729 & 4.19 & 4.13 \\
\hline R3 & 12.29 & 0.00019 & 13.55 & 0.00765 & 2.83 & 4.52 \\
\hline Average & 11.59 & 0.00017 & 12.97 & 0.00747 & 3.51 & 4.33 \\
\hline \multicolumn{7}{|c|}{ École Polytechnique-Montréal } \\
\hline \multirow{2}{*}{ Prism } & $\mathbf{f}_{\text {cr }}$ & $\varepsilon_{\text {cr }}$ & $\mathbf{f}_{\mathrm{Fu}}$ & $\varepsilon_{\text {tu }}$ & $\mathbf{w}_{\mathbf{0}}$ & $\mathbf{f}_{\mathrm{Fu}} / \mathbf{3}$ \\
\hline & [MPa] & {$[\mathrm{mm} / \mathbf{m m}]$} & [MPa] & {$[\mathrm{mm} / \mathbf{m m}]$} & {$[\mathrm{mm}]$} & [MPa] \\
\hline OW1 & 9.29 & 0.00018 & 11.79 & 0.00916 & 2.54 & 3.93 \\
\hline OW2 & 10.87 & 0.00015 & 13.93 & 0.00996 & 2.14 & 4.64 \\
\hline Average & 10.08 & 0.00016 & 12.86 & 0.00956 & 2.34 & 4.29 \\
\hline R3 & 7.74 & 0.00010 & 8.69 & 0.00640 & 5.16 & 2.90 \\
\hline \multicolumn{7}{|c|}{ Queen's University } \\
\hline \multirow{2}{*}{ Prism } & $\mathbf{f}_{\text {cr }}$ & $\varepsilon \mathrm{er}$ & $\mathbf{f F u}_{\mathrm{Fu}}$ & $\varepsilon_{\text {tu }}$ & $\mathbf{w}_{0}$ & $\mathbf{f}_{\mathrm{Fu}} / \mathbf{3}$ \\
\hline & [MPa] & {$[\mathrm{mm} / \mathbf{m m}]$} & [MPa] & {$[\mathrm{mm} / \mathbf{m m}]$} & {$[\mathrm{mm}]$} & [MPa] \\
\hline OW3 & 9.94 & 0.00025 & 11.94 & 0.00404 & 1.33 & 3.98 \\
\hline R3 & 9.14 & 0.00019 & 11.24 & 0.00659 & 2.17 & 3.75 \\
\hline
\end{tabular}



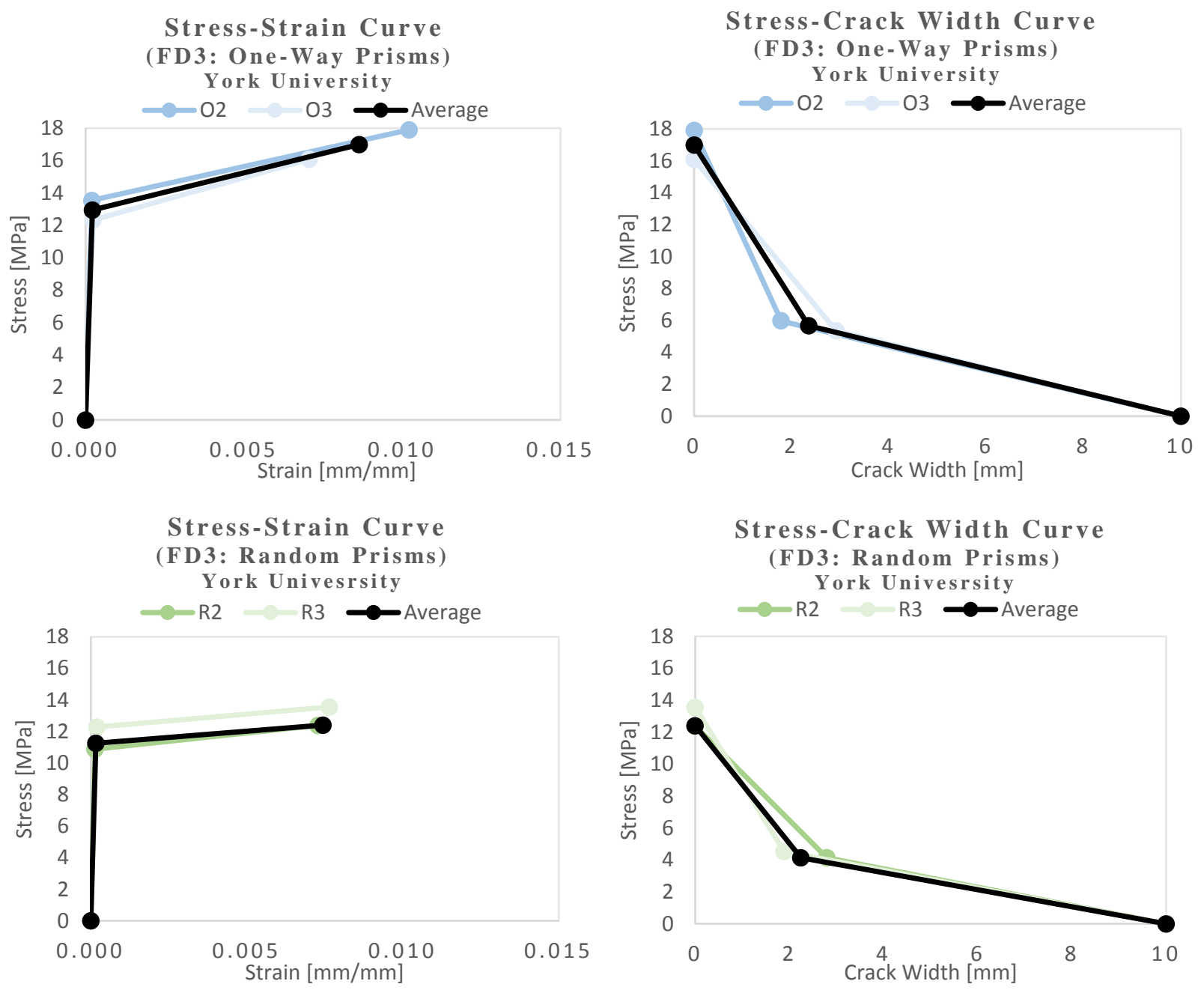

Figure 6- 7: Linearized tensile stress-strain-crack mouth opening relationships of the prisms from mix FD3 tested by York University

\subsubsection{Commercial KI2 Design Mix}

The prisms ( $75 \mathrm{~mm}$ by $75 \mathrm{~mm}$ by $280 \mathrm{~mm}$ prisms) from mix KI2 tested during the Round Robin Testing Program were also analyzed. The average flexural strength of OW prisms was 27.19 MPa for York University, 22 MPa for École Polytechnique-Montréal and 20.53 MPa for Queen's University; and the corresponding cracking strength and ultimate tensile strength were 8.41 $\mathrm{MPa}$ and 10.59 $\mathrm{MPa}$ for York University, 7.19 MPa and 8.81 MPa for École Polytechnique-Montréal, and 7.07 MPa and 7.89 MPa for Queen's University. The average flexural strength of R prisms was 30.47 MPa for York University, 24.37 MPa for École Polytechnique-Montréal and 22.34 MPa for Queen's University; and the corresponding 
cracking strength and ultimate tensile strength were 9.92 $\mathrm{MPa}$ and 13.09 $\mathrm{MPa}$ for York University, 7.34 $\mathrm{MPa}$ and 9.68 MPa for École Polytechnique-Montréal, and 6.51 MPa and 8.60 MPa for Queen's University.

The linearized tensile stress-strain-crack mouth opening relationships of the prisms tested by York University and corresponding results of tensile properties are presented in Figure 6-8 and Table 6-8, and the four characteristic points on each resistance curve utilized to perform the inverse analysis can be found in Appendix B.6. In addition, the tensile strength of mix KI2 was assumed to be equal to zero when crack mouth opening reached $6.5 \mathrm{~mm}$ owing to the use of $13 \mathrm{~mm}$ long, straight steel fibers.

Table 6- 8: Inverse analysis results of the prisms from mix KI2

\begin{tabular}{|c|c|c|c|c|c|c|}
\hline \multicolumn{7}{|c|}{ York University } \\
\hline \multirow{2}{*}{ Prism } & $\mathbf{f}_{\text {cr }}$ & $\varepsilon_{\text {cr }}$ & $\mathbf{f}_{\mathrm{Fu}}$ & $\varepsilon_{\text {tu }}$ & $\mathbf{w}_{\mathbf{0}}$ & $\mathbf{f}_{\mathrm{Fu}} / \mathbf{3}$ \\
\hline & [MPa] & {$[\mathrm{mm} / \mathbf{m m}]$} & [MPa] & {$[\mathbf{m m} / \mathbf{m m}]$} & {$[\mathrm{mm}]$} & [MPa] \\
\hline OW1 & 8.00 & 0.00004 & 9.99 & 0.00658 & 2.42 & 3.33 \\
\hline OW3 & 8.81 & 0.00014 & 11.19 & 0.00377 & 2.79 & 3.73 \\
\hline Average & 8.14 & 0.00009 & 10.59 & 0.00518 & 2.61 & 3.53 \\
\hline $\mathrm{R} 1$ & 8.52 & 0.00014 & 11.09 & 0.00462 & 3.46 & 3.70 \\
\hline $\mathrm{R} 2$ & 9.66 & 0.00020 & 13.50 & 0.00522 & 1.75 & 4.50 \\
\hline R3 & 11.59 & 0.00016 & 14.68 & 0.00571 & 2.71 & 4.89 \\
\hline Average & 9.92 & 0.00017 & 13.09 & 0.00518 & 2.64 & 4.36 \\
\hline \multicolumn{7}{|c|}{ École Polytechnique-Montréal } \\
\hline \multirow{2}{*}{ Prism } & $\mathbf{f}_{\text {cr }}$ & $\varepsilon_{\text {cr }}$ & $\mathbf{f}_{\mathrm{Fu}}$ & $\varepsilon_{\text {tu }}$ & $\mathbf{w}_{\mathbf{0}}$ & $\mathbf{f}_{\mathrm{Fu}} / \mathbf{3}$ \\
\hline & [MPa] & {$[\mathbf{m m} / \mathbf{m m}]$} & [MPa] & {$[\mathbf{m m} / \mathbf{m m}]$} & {$[\mathrm{mm}]$} & [MPa] \\
\hline OW1 & 7.30 & 0.00021 & 9.65 & 0.00648 & 0.91 & 3.22 \\
\hline OW2 & 7.09 & 0.00015 & 7.96 & 0.00444 & 1.41 & 2.65 \\
\hline Average & 7.19 & 0.00018 & 8.81 & 0.00546 & 1.16 & 2.94 \\
\hline R3 & 7.34 & 0.00015 & 9.68 & 0.00674 & 1.41 & 3.23 \\
\hline \multicolumn{7}{|c|}{ Queen's University } \\
\hline \multirow{2}{*}{ Prism } & $\mathbf{f}_{\text {cr }}$ & $\varepsilon_{\mathrm{cr}}$ & $\mathbf{f}_{\mathrm{Fu}}$ & $\varepsilon$ tu & Wo & $\mathbf{f}_{\mathrm{Fu}} / \mathbf{3}$ \\
\hline & [MPa] & {$[\mathrm{mm} / \mathbf{m m}]$} & [MPa] & {$[\mathrm{mm} / \mathbf{m m}]$} & [mm] & [MPa] \\
\hline OW3 & 7.07 & 0.00010 & 7.89 & 0.00357 & 2.23 & 2.63 \\
\hline $\mathrm{R} 2$ & 5.94 & 0.00010 & 6.88 & 0.00360 & 3.73 & 2.29 \\
\hline R3 & 7.07 & 0.00011 & 10.32 & 0.00977 & 5.35 & 3.44 \\
\hline Average & 6.51 & 0.00011 & 8.60 & 0.00669 & 4.54 & 2.87 \\
\hline
\end{tabular}



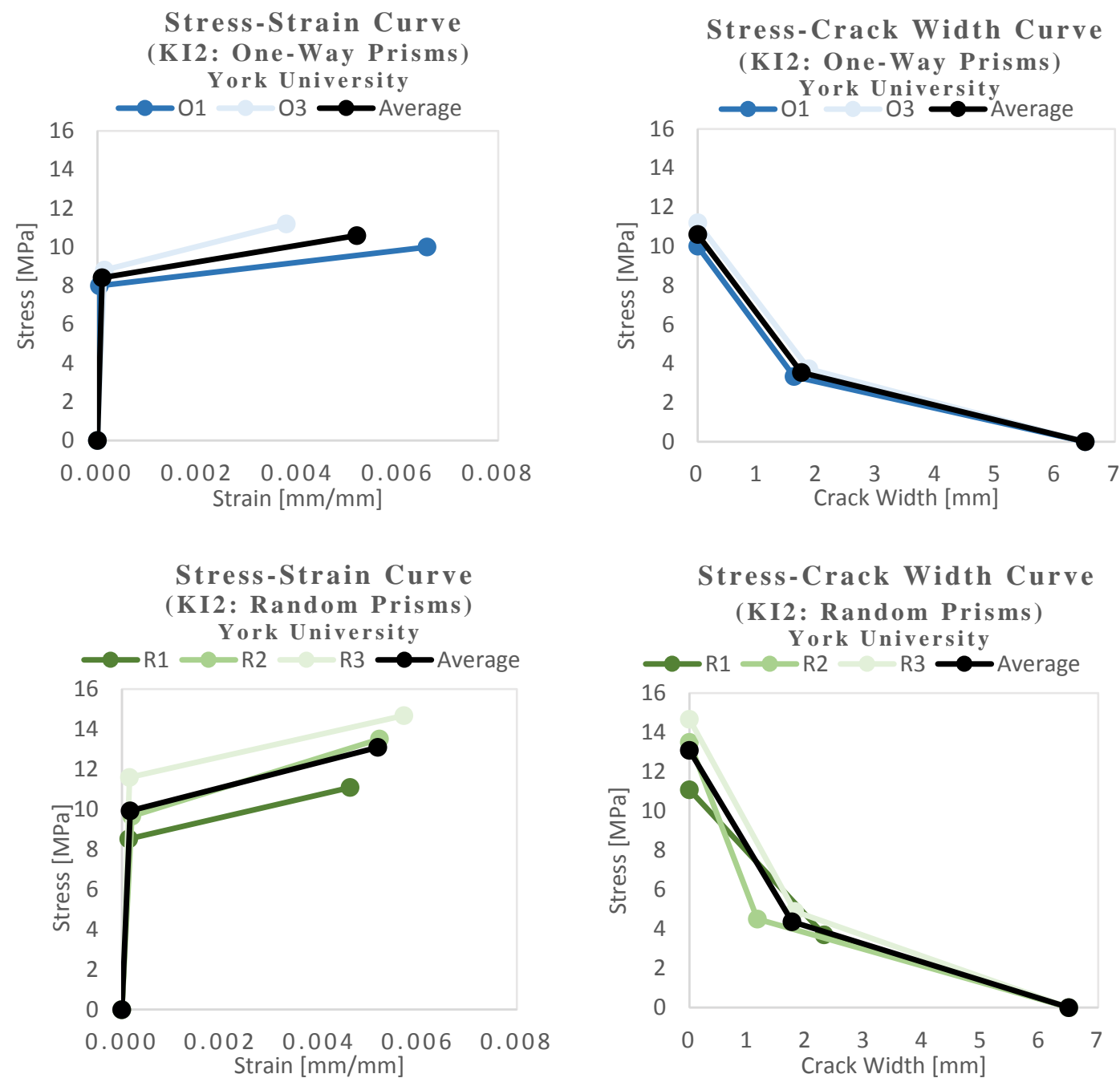

Figure 6- 8: Linearized tensile stress-strain-crack mouth opening relationships of the prisms from mix KI2 tested by York University

\subsubsection{Commercial LD2 Design Mix}

This section presents the inverse analysis results of the prisms tested during the Round Robin Testing Program from mix LD2. The average flexural strength of OW prisms was $26.54 \mathrm{MPa}$ for York University, 20.35 MPa for École Polytechnique-Montréal and 20.94 MPa for Queen's University; and the corresponding cracking strength and ultimate tensile strength were 9.76 MPa and $11.64 \mathrm{MPa}$ for York University, 6.72 MPa and 8.18 MPa for École Polytechnique-Montréal, and 7.16 MPa and 9.02 MPa for Queen's University. The average flexural strength of R prisms was 25.05 MPa for York University, 21.36 MPa for École Polytechnique-Montréal and 17.51 MPa for Queen's University; and the corresponding 
cracking strength and ultimate tensile strength were $8.80 \mathrm{MPa}$ and 11.33 MPa for York University, 7.60 $\mathrm{MPa}$ and 9.89 MPa for École Polytechnique-Montréal, and 6.02 MPa and 7.26 MPa for Queen's University.

The results of tensile properties are presented in Table 6-9, and the linearized tensile stress-strain-crack mouth opening relationships and the four characteristic points on each resistance curve utilized to perform the inverse analysis can be found in Appendix B.7. In addition, the tensile strength of mix LD2 was assumed to be equal to zero when crack mouth opening reached $6.5 \mathrm{~mm}$ owing to the use of $13 \mathrm{~mm}$ long, straight steel fibers.

Table 6- 9: Inverse analysis results of the prisms from mix LD2

\begin{tabular}{|c|c|c|c|c|c|c|}
\hline \multicolumn{7}{|c|}{ York University } \\
\hline \multirow{2}{*}{ Prism } & $\mathbf{f}_{\text {cr }}$ & $\varepsilon_{\text {cr }}$ & $\mathbf{f}_{\mathrm{Fu}}$ & $\varepsilon_{\text {tu }}$ & $\mathbf{w}_{\mathbf{0}}$ & $\mathbf{f}_{\mathrm{Fu}} / \mathbf{3}$ \\
\hline & [MPa] & {$[\mathrm{mm} / \mathbf{m m}]$} & [MPa] & {$[\mathbf{m m} / \mathbf{m m}]$} & {$[\mathrm{mm}]$} & [MPa] \\
\hline OW1 & 11.50 & 0.00025 & 12.95 & 0.00291 & 1.81 & 4.32 \\
\hline OW2 & 7.01 & 0.00017 & 10.02 & 0.00266 & 2.17 & 3.34 \\
\hline OW3 & 10.76 & 0.00008 & 11.96 & 0.00169 & 1.53 & 3.99 \\
\hline Average & 9.76 & 0.00017 & 11.64 & 0.00242 & 1.84 & 3.88 \\
\hline $\mathrm{R} 1$ & 7.55 & 0.00013 & 9.40 & 0.00140 & 0.91 & 3.13 \\
\hline R3 & 10.06 & 0.00016 & 13.25 & 0.00329 & 3.17 & 4.42 \\
\hline Average & 8.80 & 0.00014 & 11.33 & 0.00234 & 2.04 & 4.78 \\
\hline \multicolumn{7}{|c|}{ École Polytechnique-Montréal } \\
\hline \multirow{2}{*}{ Prism } & $\mathbf{f}_{\text {cr }}$ & $\varepsilon_{\mathrm{cr}}$ & $\mathbf{f}_{\mathbf{F u}}$ & $\varepsilon_{\text {tu }}$ & $\mathbf{w}_{\mathbf{0}}$ & $\mathbf{f}_{\mathrm{Fu}} / \mathbf{3}$ \\
\hline & [MPa] & {$[\mathbf{m m} / \mathbf{m m}]$} & [MPa] & {$[\mathbf{m m} / \mathbf{m m}]$} & {$[\mathrm{mm}]$} & [MPa] \\
\hline OW1 & 7.20 & 0.00013 & 9.27 & 0.00594 & 2.56 & 3.09 \\
\hline OW2 & 7.47 & 0.00014 & 8.44 & 0.00383 & 1.53 & 2.81 \\
\hline OW3 & 5.48 & 0.00008 & 6.82 & 0.00274 & 2.30 & 2.27 \\
\hline Average & 6.72 & 0.00012 & 8.18 & 0.00417 & 2.13 & 2.73 \\
\hline $\mathrm{R} 1$ & 7.60 & 0.00022 & 9.89 & 0.00450 & 1.81 & 3.30 \\
\hline \multicolumn{7}{|c|}{ Queen's University } \\
\hline \multirow{2}{*}{ Prism } & $\mathbf{f}_{\text {cr }}$ & $\varepsilon \mathrm{er}$ & $\mathbf{f}_{\mathrm{Fu}}$ & 8 tu & Wo & $\mathbf{f}_{\mathrm{Fu}} / \mathbf{3}$ \\
\hline & [MPa] & {$[\mathrm{mm} / \mathbf{m m}]$} & [MPa] & {$[\mathrm{mm} / \mathbf{m m}]$} & {$[\mathrm{mm}]$} & [MPa] \\
\hline OW1 & 7.16 & 0.00012 & 9.02 & 0.00185 & 1.97 & 3.01 \\
\hline $\mathrm{R} 1$ & 6.66 & 0.00009 & 7.49 & 0.00103 & 1.42 & 2.50 \\
\hline $\mathrm{R} 3$ & 5.38 & 0.00007 & 7.03 & 0.00161 & 1.39 & 2.34 \\
\hline Average & 6.02 & 0.00008 & 7.26 & 0.00132 & 1.41 & 2.42 \\
\hline
\end{tabular}




\subsubsection{In-house DE2 Design Mix (SC2)}

The OW prisms from mix DE2 tested with SC2 (as depicted in Figure 4-11b) during Phase 2 of the experimental program are analyzed in this section. The average flexural strength of prisms was 19.6 MPa, corresponding to a cracking strength of $6.11 \mathrm{MPa}$ and an ultimate tensile strength of $8.8 \mathrm{MPa}$. The linearized tensile stress-strain-crack mouth opening relationships and corresponding results of tensile properties are presented in Figure 6-9 and Table 6-10, and the four characteristic points on each resistance curve utilized to perform the inverse analysis can be found in Appendix B.8.

Table 6- 10: Inverse analysis results of the prisms from mix DE2 tested with SC2

\begin{tabular}{|c|c|c|c|c|c|c|}
\hline \multicolumn{2}{|c|}{ Mix DE2 } \\
\hline \multirow{2}{*}{ Prism } & $\mathbf{f}_{\text {cr }}$ & $\boldsymbol{\varepsilon}_{\text {cr }}$ & $\mathbf{f}_{\mathbf{F u}}$ & $\boldsymbol{\varepsilon}_{\text {tu }}$ & $\mathbf{w}_{\mathbf{o}}$ & $\mathbf{f}_{\mathbf{F u}} / \mathbf{3}$ \\
\cline { 2 - 7 } & {$[\mathbf{M P a}]$} & {$[\mathbf{m m} / \mathbf{m m}]$} & {$[\mathbf{M P a}]$} & {$[\mathbf{m m} / \mathbf{m m}]$} & {$[\mathbf{m m}]$} & {$[\mathbf{M P a}]$} \\
\hline OW1 & 6.63 & 0.00034 & 9.10 & 0.00457 & 1.17 & 3.03 \\
\hline OW2 & 5.31 & 0.00021 & 8.90 & 0.00360 & 2.23 & 2.97 \\
\hline OW3 & 6.40 & 0.00009 & 8.39 & 0.00543 & 1.07 & 2.80 \\
\hline Average & 6.11 & 0.00021 & 8.80 & 0.00453 & 1.49 & 2.93 \\
\hline
\end{tabular}
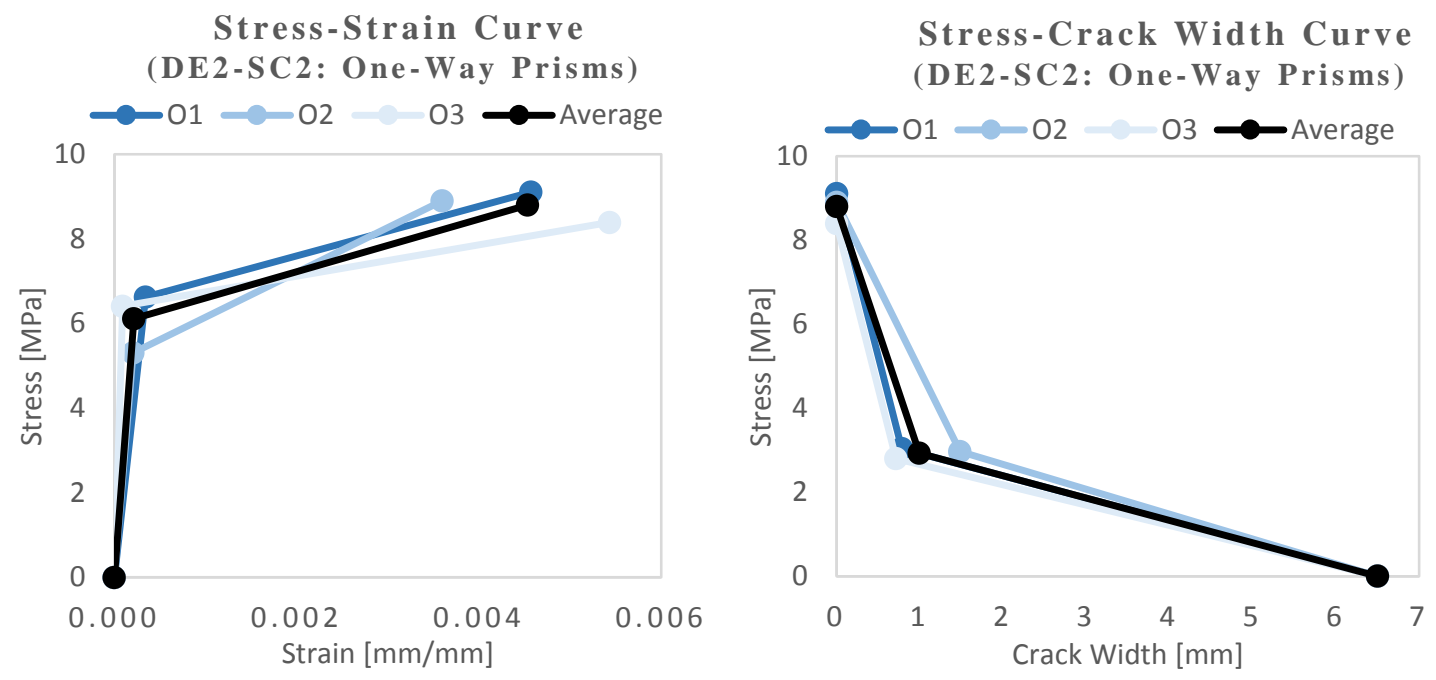

Figure 6- 9: Linearized tensile stress-strain-crack mouth opening relationships of the prisms from mix DE2 tested with $S C 2$ 


\subsubsection{Commercial FD4 Design Mix}

This section presents inverse analysis results of OW prisms $(100 \mathrm{~mm}$ by $100 \mathrm{~mm}$ by $370 \mathrm{~mm}$ prisms and $150 \mathrm{~mm}$ by $150 \mathrm{~mm}$ by $500 \mathrm{~mm}$ prisms) from mix FD4 tested during Phase 2 of the experimental program. The average flexural strength of $100 \mathrm{~mm}$ by $100 \mathrm{~mm}$ prisms was $18.24 \mathrm{MPa}$, corresponding to a cracking strength of $6.6 \mathrm{MPa}$ and an ultimate tensile strength of $7.47 \mathrm{MPa}$; and the average flexural strength $150 \mathrm{~mm}$ by $150 \mathrm{~mm}$ was $15.44 \mathrm{MPa}$, corresponding to a cracking strength of $5.2 \mathrm{MPa}$ and an ultimate tensile strength of $6.72 \mathrm{MPa}$.

The linearized tensile stress-strain-crack mouth opening relationships and corresponding results of tensile properties are presented in Figure 6-10 and Table 6-11, and the four characteristic points on each resistance curve utilized to perform the inverse analysis can be found in Appendix B.9. In addition, the tensile strength of mix FD4 was assumed to be equal to zero when crack mouth opening reached $6.5 \mathrm{~mm}$ owing to the use of $13 \mathrm{~mm}$ long, straight steel fibers.

Table 6- 11: Inverse analysis results of the prisms from mix FD4

\begin{tabular}{|c|c|c|c|c|c|c|}
\hline \multicolumn{7}{|c|}{100 mm by 100 mm Prisms } \\
\hline \multirow{2}{*}{ Prism } & $\mathbf{f}_{\text {cr }}$ & $\varepsilon_{\mathrm{cr}}$ & $\mathbf{f}_{\mathrm{Fu}}$ & $\varepsilon_{\text {tu }}$ & $\mathbf{w}_{\mathbf{0}}$ & $\mathbf{f}_{\mathrm{Fu}} / \mathbf{3}$ \\
\hline & [MPa] & {$[\mathrm{mm} / \mathbf{m m}]$} & [MPa] & {$[\mathrm{mm} / \mathbf{m m}]$} & {$[\mathrm{mm}]$} & [MPa] \\
\hline Prism 1 & 6.05 & 0.00015 & 6.66 & 0.00338 & 3.29 & 2.22 \\
\hline Prism 2 & 7.33 & 0.00013 & 8.09 & 0.00640 & 1.98 & 2.70 \\
\hline Prism 3 & 6.43 & 0.00012 & 7.65 & 0.00515 & 1.92 & 2.55 \\
\hline Average & 6.60 & 0.00013 & 7.47 & 0.00498 & 2.40 & 2.49 \\
\hline \multicolumn{7}{|c|}{$150 \mathrm{~mm}$ by $150 \mathrm{~mm}$ Prisms } \\
\hline \multirow{2}{*}{ Prism } & for & $\varepsilon$ cr & $\mathbf{f F u}_{\mathrm{Fu}}$ & 8 tu & Wo & $\mathbf{f}_{\mathrm{Fu}} / \mathbf{3}$ \\
\hline & [MPa] & {$[\mathbf{m m} / \mathbf{m m}]$} & [MPa] & {$[\mathrm{mm} / \mathbf{m m}]$} & {$[\mathrm{mm}]$} & [MPa] \\
\hline Prism 1 & 5.66 & 0.00015 & 7.12 & 0.00498 & 1.55 & 2.37 \\
\hline Prism 2 & 5.53 & 0.00010 & 7.12 & 0.00274 & 1.58 & 2.37 \\
\hline Prism 3 & 5.06 & 0.00010 & 6.31 & 0.00267 & 1.20 & 2.10 \\
\hline Prism 4 & 6.24 & 0.00011 & 7.71 & 0.00318 & 1.44 & 2.57 \\
\hline Prism 5 & 4.36 & 0.00008 & 6.01 & 0.00368 & 1.28 & 2.00 \\
\hline Prism 6 & 4.34 & 0.00009 & 6.07 & 0.00225 & 1.76 & 2.02 \\
\hline Average & 5.20 & 0.00011 & 6.72 & 0.00325 & 1.47 & 2.24 \\
\hline
\end{tabular}


Stress-Strain Curve

(FD 4: $100 \mathrm{~mm}$ By $100 \mathrm{~mm}$ Prisms)

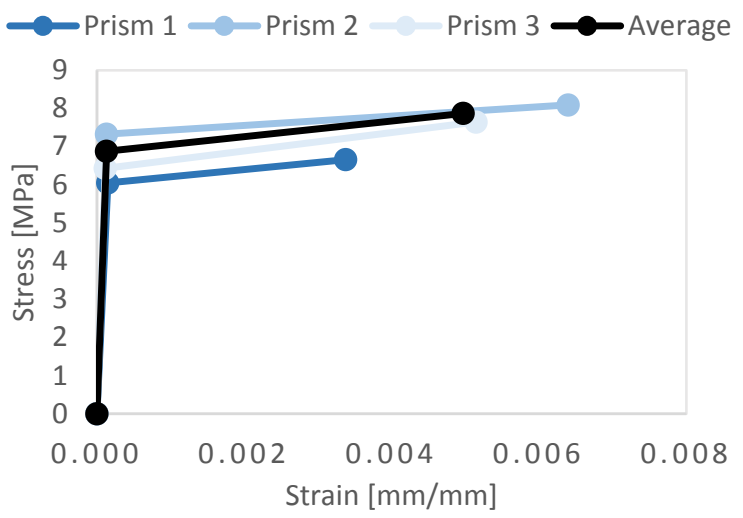

Stress-Strain Curve (FD 4: $150 \mathrm{~mm}$ by $150 \mathrm{~mm}$ Prisms)

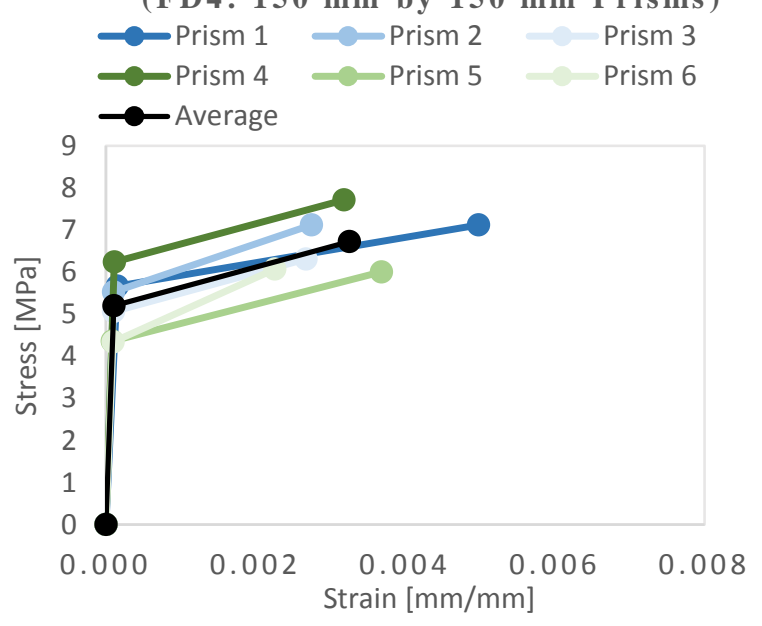

Stress-Crack Width Curve (FD 4: $100 \mathrm{~mm}$ by $100 \mathrm{~mm}$ Prisms)

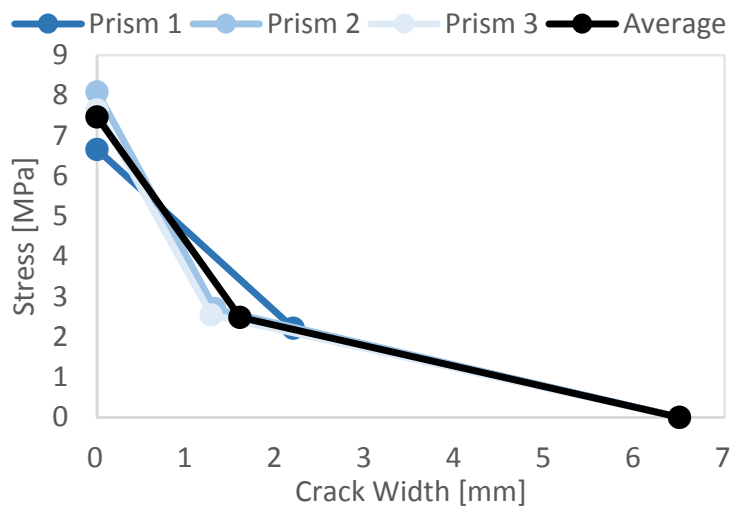

Stress-Crack Width Curve (FD 4: $150 \mathrm{~mm}$ by $150 \mathrm{~mm}$ Prisms)

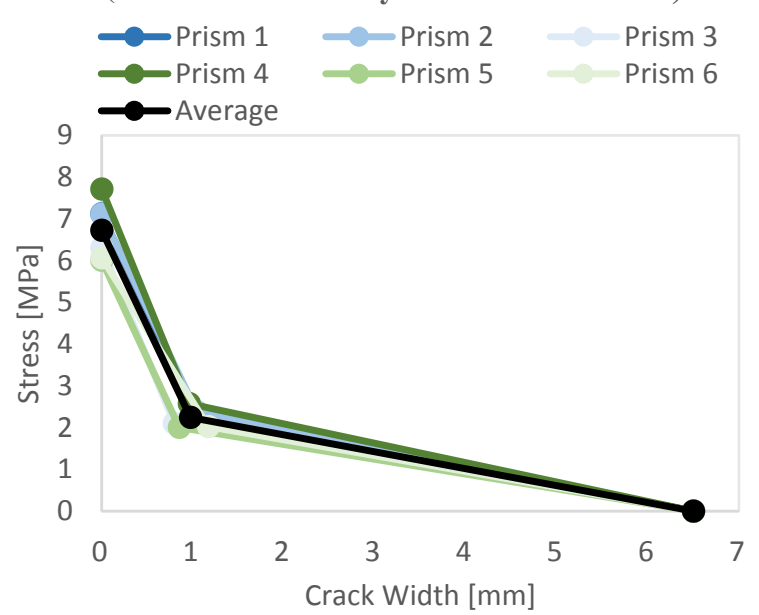

Figure 6- 10: Linearized tensile stress-strain-crack mouth opening relationships of the prisms from mix FD4

\subsubsection{Commercial FD5 and FD6 Design Mixes}

The OW prisms ( $150 \mathrm{~mm}$ by $150 \mathrm{~mm}$ by $500 \mathrm{~mm}$ prisms) from mixes FD5 and FD6 tested during Phase 2 of the experimental program are analyzed here. The average flexural strength of mix FD5 after 28-day curing period was $21.75 \mathrm{MPa}$, corresponding to a cracking strength of $7.18 \mathrm{MPa}$ and an ultimate tensile strength of 8.09 MPa; and the average flexural strength of mix FD6 after 28-day curing period was 20.54 $\mathrm{MPa}$, corresponding to a cracking strength of $7.34 \mathrm{MPa}$ and an ultimate tensile strength of 8.75 $\mathrm{MPa}$. The results of tensile properties are presented in Table 6-12, and the linearized tensile stress-strain-crack mouth opening relationships, and the four characteristic points on each resistance curve utilized to perform the inverse analysis can be found in Appendix B.10. In addition, the tensile strengths of mixes FD5 and FD6 
were assumed to be equal to zero when crack mouth opening reached $10 \mathrm{~mm}$ owing to the use of $20 \mathrm{~mm}$ long, straight steel fibers.

Table 6- 12: Inverse analysis results of the prisms from mixes FD5 and FD6

\begin{tabular}{|c|c|c|c|c|c|c|}
\hline \multicolumn{7}{|c|}{ Mix FD5 } \\
\hline \multirow{2}{*}{ Prism } & $\mathbf{f}_{\text {cr }}$ & Ecr & $\mathbf{f}_{\mathrm{Fu}}$ & 8 tu & Wo & $\mathbf{f}_{\mathrm{Fu}} / \mathbf{3}$ \\
\hline & [MPa] & {$[\mathrm{mm} / \mathbf{m m}]$} & [MPa] & {$[\mathrm{mm} / \mathbf{m m}]$} & {$[\mathrm{mm}]$} & [MPa] \\
\hline Prism 1 & 7.47 & 0.00009 & 8.58 & 0.00410 & 3.65 & 2.86 \\
\hline Prism 3 & 6.21 & 0.00008 & 7.15 & 0.00242 & 2.31 & 2.38 \\
\hline Prism 4 & 7.15 & 0.00011 & 7.95 & 0.00345 & 3.68 & 2.65 \\
\hline Prism 5 & 7.35 & 0.00009 & 8.15 & 0.00318 & 2.85 & 2.72 \\
\hline Prism 6 & 7.72 & 0.00006 & 8.60 & 0.00516 & 3.54 & 2.87 \\
\hline Average & 7.18 & 0.00009 & 8.09 & 0.00366 & 3.20 & 2.70 \\
\hline \multicolumn{7}{|c|}{ Mix FD6 } \\
\hline \multirow{2}{*}{ Prism } & $f_{\text {cr }}$ & Ecr & $\mathbf{f}_{\mathrm{Fu}}$ & $\varepsilon_{\text {tu }}$ & Wo & $\mathbf{f}_{\mathrm{Fu}} / \mathbf{3}$ \\
\hline & [MPa] & {$[\mathrm{mm} / \mathbf{m m}]$} & [MPa] & {$[\mathrm{mm} / \mathrm{mm}]$} & {$[\mathrm{mm}]$} & [MPa] \\
\hline Prism 1 & 7.02 & 0.00011 & 8.46 & 0.00210 & 1.86 & 2.82 \\
\hline Prism 2 & 6.68 & 0.00011 & 8.33 & 0.00169 & 2.21 & 2.78 \\
\hline Prism 3 & 6.73 & 0.00011 & 7.80 & 0.00339 & 2.57 & 2.60 \\
\hline Prism 4 & 8.41 & 0.00017 & 9.94 & 0.00398 & 3.24 & 3.31 \\
\hline Prism 5 & 7.86 & 0.00015 & 9.20 & 0.00178 & 3.54 & 2.00 \\
\hline Average & 7.34 & 0.00013 & 8.75 & 0.00259 & 2.68 & 2.70 \\
\hline
\end{tabular}

\subsubsection{Discussion of the Inverse Analysis Results}

As depicted in Figure 3-20, the average flexural strengths of mixes FD1 and FD2 are much higher than those of other mixes regardless of casting methodology. Figure 6-11 illustrates the inverse analysis results in terms of average ultimate tensile strength $\left(f_{F u}\right)$ and average ultimate tensile strain $\left(\varepsilon_{t u}\right)$ of each set of prisms tested during Phase 1 of the experimental program, and it is evident that $f_{F u}$ and $\varepsilon_{t u}$ results of mixes FD1 and FD2 are much greater than those of other mixes due to the addition of $20 \mathrm{~mm}$ long steel fibers, whereas mixes KI1 and LD1 comprised $13 \mathrm{~mm}$ long steel fibers. Since the cross-sectional dimensions of the prisms from the four mixes are the same $(75 \mathrm{~mm}$ by $75 \mathrm{~mm})$, the degree of preferential fiber alignment was more prominent in the prisms from mixes FD1 and FD2 containing fibers that were relatively long compared with the cross-sectional dimensions, resulting in higher tensile strengths. Besides, owing to the addition of longer fibers, fiber bridging effect was more prominent in the prisms from mixes FD1 and FD2, which also contributed to the greater tensile strength and strain results. 
Average Ultimate Tensile Strength
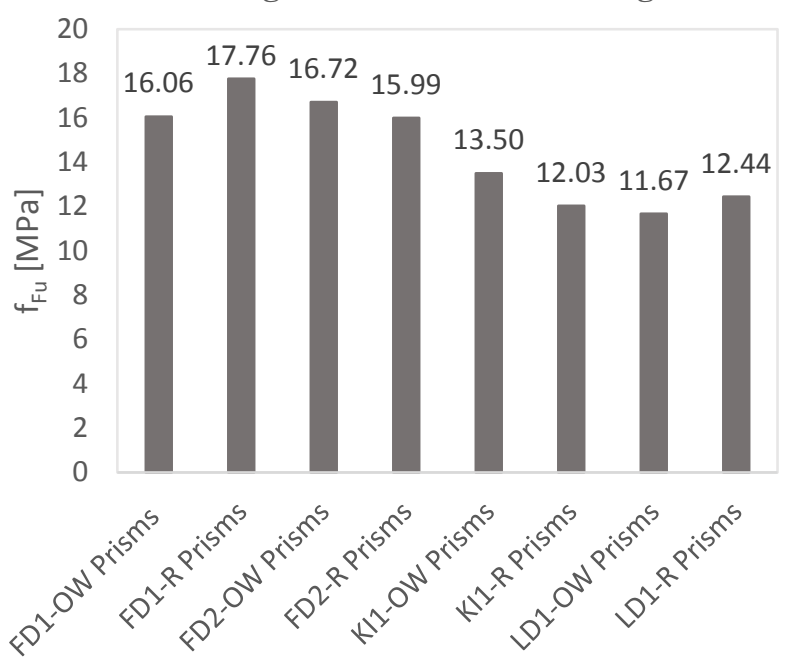

Average Ultimate Tensile Strain

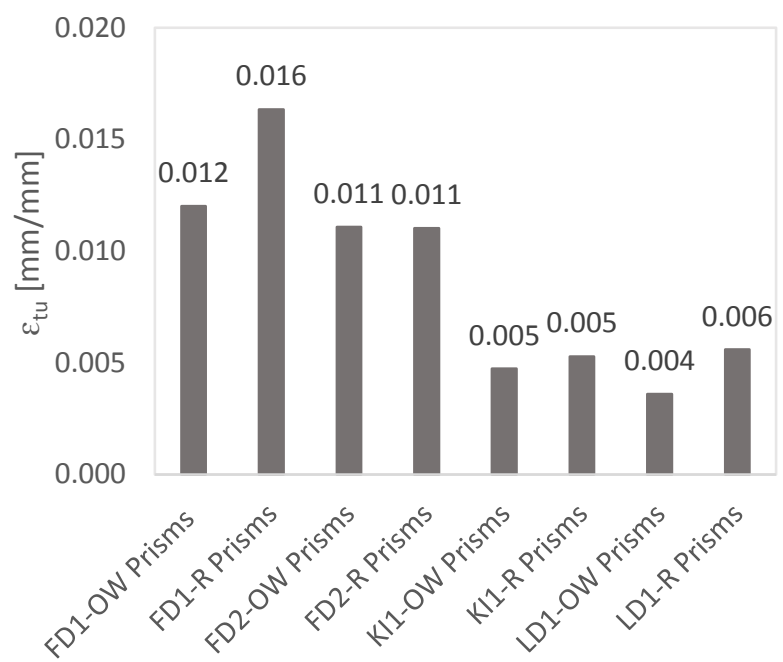

Figure 6- 11: Average ultimate tensile strength and ultimate tensile strain results obtained from the inverse analysis (Mixes FD1, FD2, KI1 and LD1 - each bar represents the average of a triplicate of identical specimens)

As mentioned in Chapter 4, with reference to the Round Robin Testing Program, York University's (YU) flexural strength results are consistently higher than École Polytechnique-Montréal's (EP) while Queen's University (QU) reported the lowest results. Figure 6-12 plots the inverse analysis results in terms of average cracking strength $\left(f_{c r}\right)$ and ultimate tensile strength $\left(f_{F u}\right)$ of the prisms from mixes FD3, KI2 and LD2 from where it is clear that York University's results are the greatest, and École PolytechniqueMontréal's results are generally greater than Queen's University's.

Average Cracking Strength

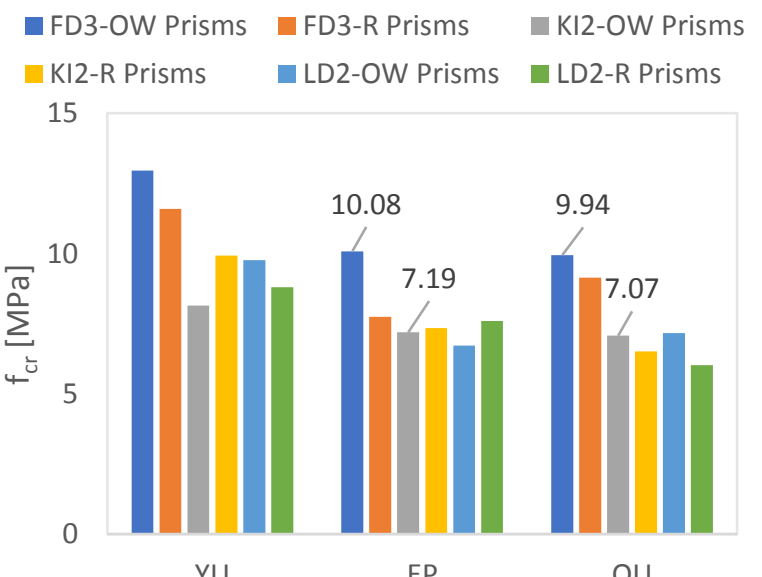

Average Ultimate Tensile Strength

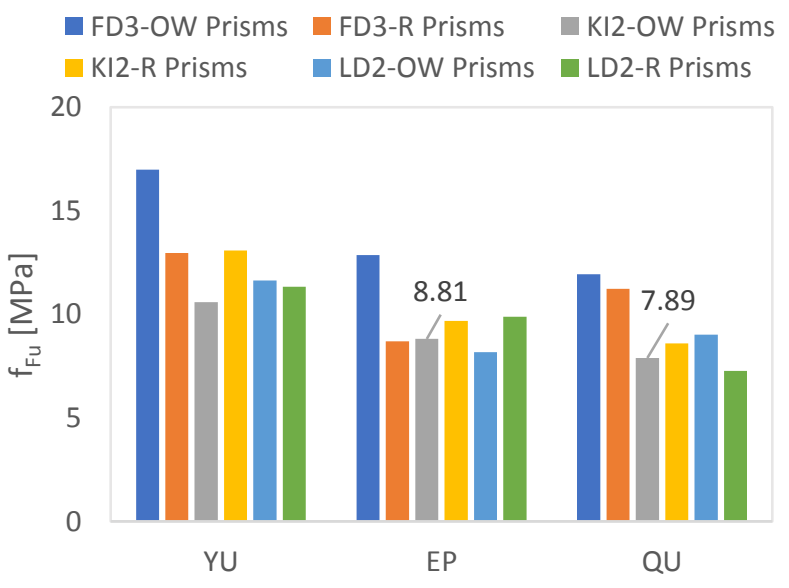

Figure 6- 12:Average cracking strength and ultimate tensile strength results obtained from the inverse analysis (Mixes FD3, KI2 and LD2-each bar represents the average of a triplicate of identical tests) 
As depicted in Table 4-7, the average flexural strength result of OW short prisms ( $75 \mathrm{~mm}$ by $75 \mathrm{~mm}$ by 280 $\mathrm{mm}$ prisms) from mix DE2 tested with $\mathrm{SC} 1$ is greater than those tested with $\mathrm{SC} 2$. Figure 6-13 depicts the inverse analysis results in terms of average cracking strength $\left(f_{c r}\right)$ and ultimate tensile strength $\left(f_{F u}\right)$ of the prisms from mix DE2 tested under different support conditions, and it is evident that SC1 provides greater tensile strength results than SC2.

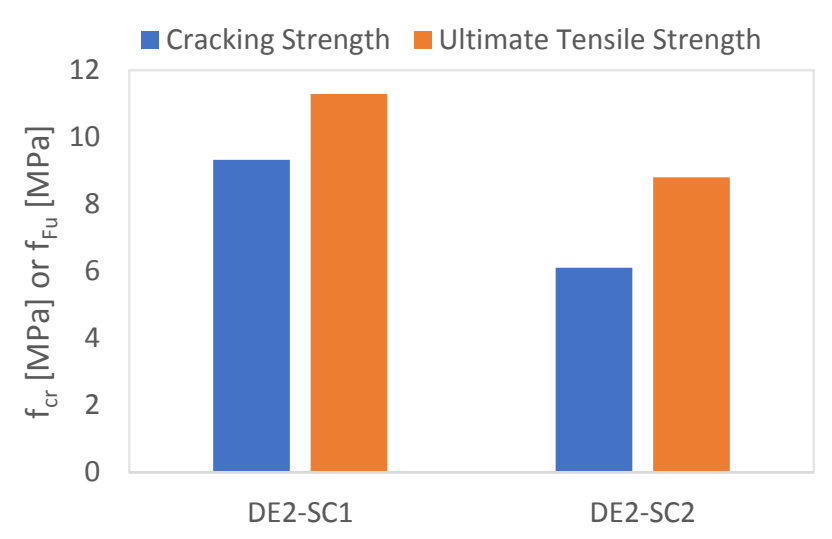

Figure 6- 13:Average cracking strength and ultimate tensile strength results obtained from the inverse analysis (Mix DE2 - each bar represents the average of a triplicate of identical tests)

As mentioned in Sections 4.3.3.1 and 4.3.3.2, $100 \mathrm{~mm}$ by $100 \mathrm{~mm}$ by $370 \mathrm{~mm}$ prisms exhibited greater flexural strength than $150 \mathrm{~mm}$ by $150 \mathrm{~mm}$ by $500 \mathrm{~mm}$ prisms although they comprised the same cementitious materials and fiber contents, and the $150 \mathrm{~mm}$ by $150 \mathrm{~mm}$ by $500 \mathrm{~mm}$ prisms from mix FD4 comprising $13 \mathrm{~mm}$ long steel fibers exhibited lower flexural strength than those from mixes FD5 and FD6 comprising $20 \mathrm{~mm}$ long steel fibers. Figure 6-14 plots the average cracking strength $\left(f_{c r}\right)$ and ultimate tensile strength $\left(f_{F u}\right)$ results of the prisms from mixes FD4, FD5 and FD6; and it is evident that the inverse analysis results in terms of $f_{c r}$ and $f_{F u}$ of $100 \mathrm{~mm}$ by $100 \mathrm{~mm}$ prisms are greater than those of $150 \mathrm{~mm}$ by $150 \mathrm{~mm}$ prisms from mix FD4, and $f_{c r}$ and $f_{F u}$ results of the $150 \mathrm{~mm}$ by $150 \mathrm{~mm}$ prisms from mix FD4 are lower than those from mixes FD5 and FD6. Again, this is can be explained by the degree of preferential fiber alignment: fiber length is relatively longer for $100 \mathrm{~mm}$ by $100 \mathrm{~mm}$ cross sections compared with $150 \mathrm{~mm}$ by $150 \mathrm{~mm}$ cross sections, and mixes FD5 and FD6 comprised longer steel fibers than mix FD4, leading to higher tensile strengths. 


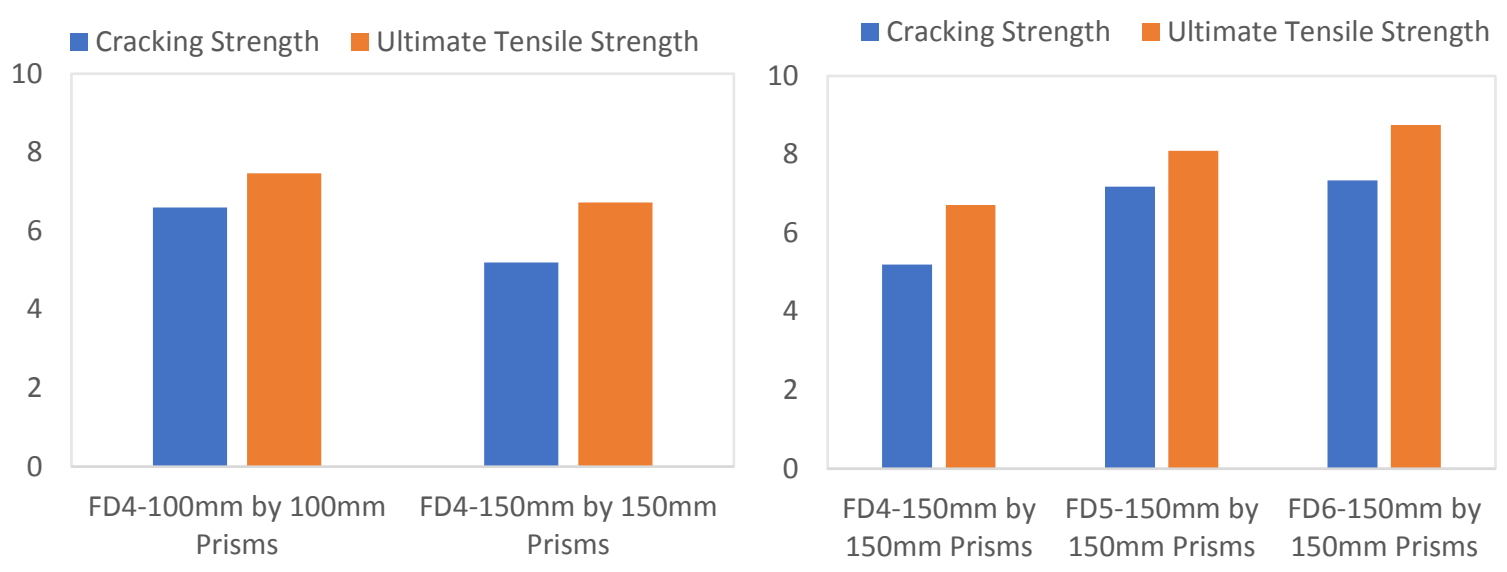

Figure 6- 14: Average cracking strength and ultimate tensile strength results obtained from the inverse analysis (Mixes FD4, FD5 and FD6 - each bar represents the average of a triplicate of identical tests)

\subsection{The Calibrated Empirical Expression}

UHP-SFRC can be classified into two groups: tension-hardening fiber reinforced concrete (THFRC) and tension-softening fiber reinforced concrete (TSFRC). Both Annex 8.1 of CSA-S6 (2018) and Annex U of CSA-A23.1 (2019) provide criteria to distinguish between THFRC and TSFRC, and these criteria rely on the inverse analysis results of FPBT or the experimental results of DTT. According to Clause A8.1.4.3.3 prescribed in Annex 8.1 of CSA-S6 (2018), UHP-SFRC should satisfy the following to be qualified as THFRC: 1$)$, the ratio between ultimate tensile strength $\left(f_{F u}\right)$ and cracking strength $\left(f_{c r}\right)$ should be greater than 1.1; and 2). the ultimate tensile strain $\left(\varepsilon_{t u}\right)$ should be greater than $0.1 \%$. In addition, Annex U of CSAA23.1 (2019) proposes an additional requirement on the basis of Clause A8.1.4.3.3, which is that THFRC should have a cracking strength $\left(f_{c r}\right)$ greater than $5 \mathrm{MPa}$, whereas $f_{c \mathrm{r}}$ should be greater than $4 \mathrm{MPa}$ for TSFRC. Figure 6-15 presents the inverse anlysis results in terms of $f_{c r}$, hardening ratio $\left(f_{F u} / f_{c r}\right)$ and $\varepsilon_{t u}$ of each design mix considered in the experimental program in order to evaluate their classification. The required $f_{c r}, \varepsilon_{t u}$ and $f_{F u} / f_{c r}$ values of THFRC are represented by the black horizontal lines in plots, and it is evident that all design mixes satisfy the criteria and thereby should be classified as THFRC. 


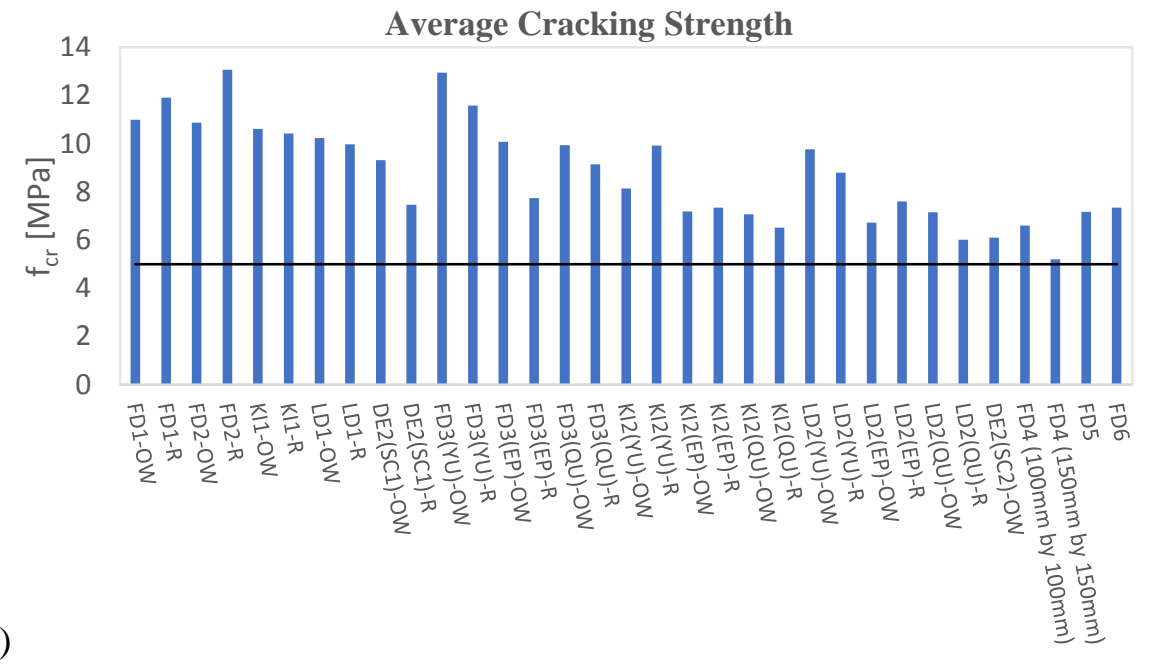

(a)
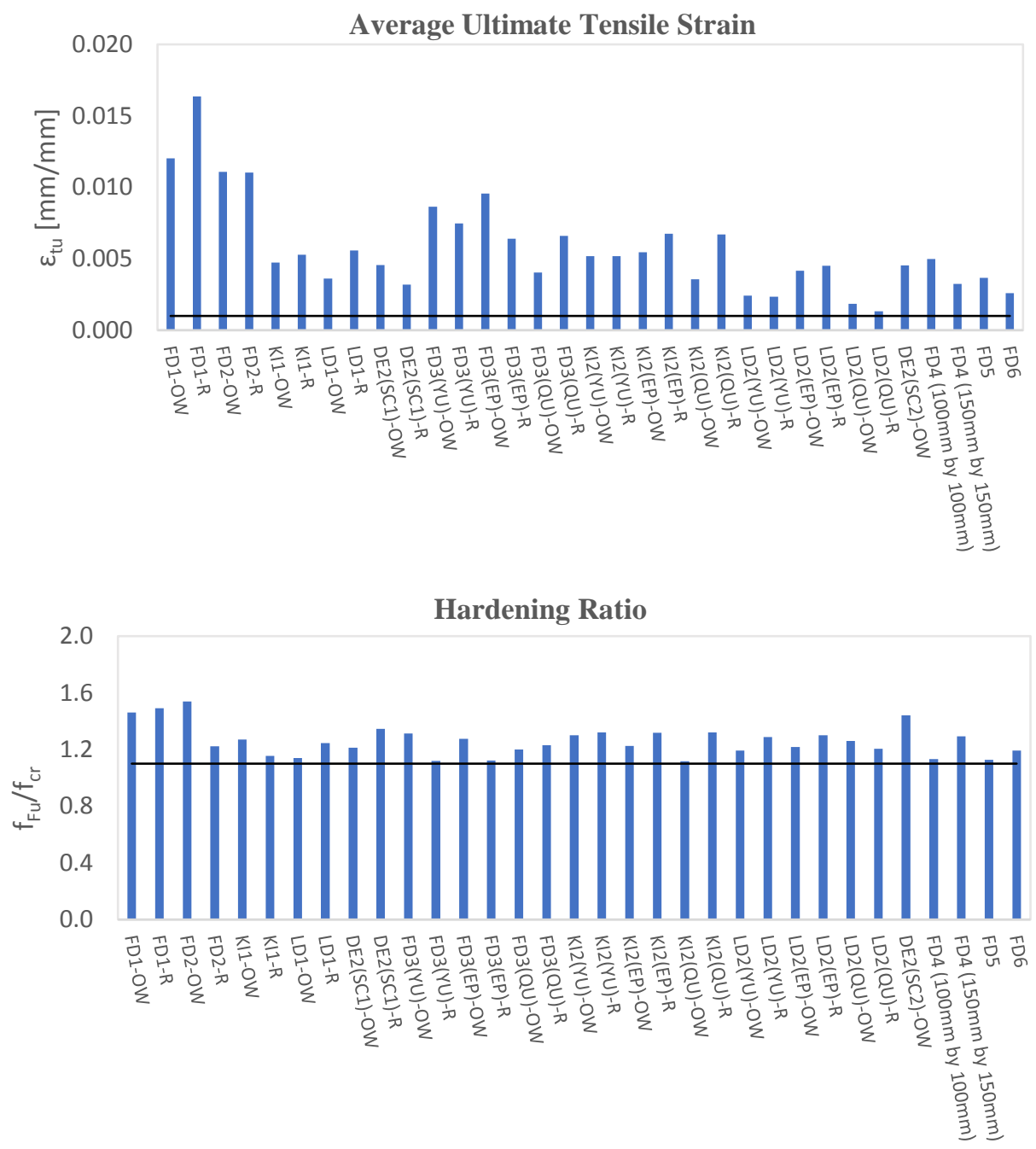

(c)

Figure 6- 15: Criteria of THFRC classification based on the inverse analysis results: (a) Average cracking strength;

(b) Average ultimate tensile strain; and (c) Hardening ratio 
Regarding THFRC, Annex 8.1 of CSA-S6 (2018) also proposes that its cracking strength $\left(f_{c r}\right)$ shall be taken as the greatest of the following: 1). the average tensile strength obtained from DTTs; and 2). the value calculated with the calibrated empirical expression provided in Equation 6-1, which links the cracking strength of UHP-SFRC to its cylinder compressive strength $\left(f_{c}^{\prime}\right)$. The calibrated empirical expression is applicable to the design mixtures considered in the experimental program because the materials were verified to be THFRC based on the inverse analysis results, and Table 6-13 presents the cracking strength result of each design mixture calculated with the calibrated empirical expression. It is worth noting that the compressive strength results obtained by York University were used to estimate the cracking strength of the prisms tested by École Polytechnique-Montréal since the compressive test results of École Polytechnique-Montréal were not available.

$f_{c r}=0.6 \sqrt{f_{c}^{\prime}}$

Table 6-13: Cracking strength results calculated with the calibrated empirical expression

\begin{tabular}{|c|c|c|c|c|c|}
\hline Mix & $\mathbf{f}_{\text {cr }}[\mathbf{M P a}]$ & Mix & $\mathbf{f}_{\text {cr }}[\mathbf{M P a}]$ & Mix & $\mathbf{f}_{\text {cr }}[\mathbf{M P a}]$ \\
\hline FD1 & 6.73 & FD3(EP) & 6.39 & LD2(EP) & 7.73 \\
\hline FD2 & 6.43 & FD3(QU) & 6.67 & LD2(QU) & 6.61 \\
\hline KI1 & 6.67 & KI2(YU) & 6.81 & FD4 & 6.86 \\
\hline LD1 & 8.58 & KI2(EP) & 6.81 & FD5 & 7.00 \\
\hline DE2 & 7.46 & KI2(QU) & 6.64 & FD6 & 6.93 \\
\hline FD3(YU) & 6.39 & LD2(YU) & 7.73 & & \\
\hline
\end{tabular}

Figure 6-16 depicts the average tensile strength results of mixes FD1, FD2, KI1, LD1 and DE2 obtained from each type of direct-tension specimens and the cracking strength results calculated with the calibrated empirical expression. As mentioned previously, the final cracking strength result of THFRC should be the greater value between DTT results and the values provided by the empirical expression. As illustrated in Figure 6-16, tensile strength obtained from DTT governs in general with the exception of mixes KI1 and LD1. 


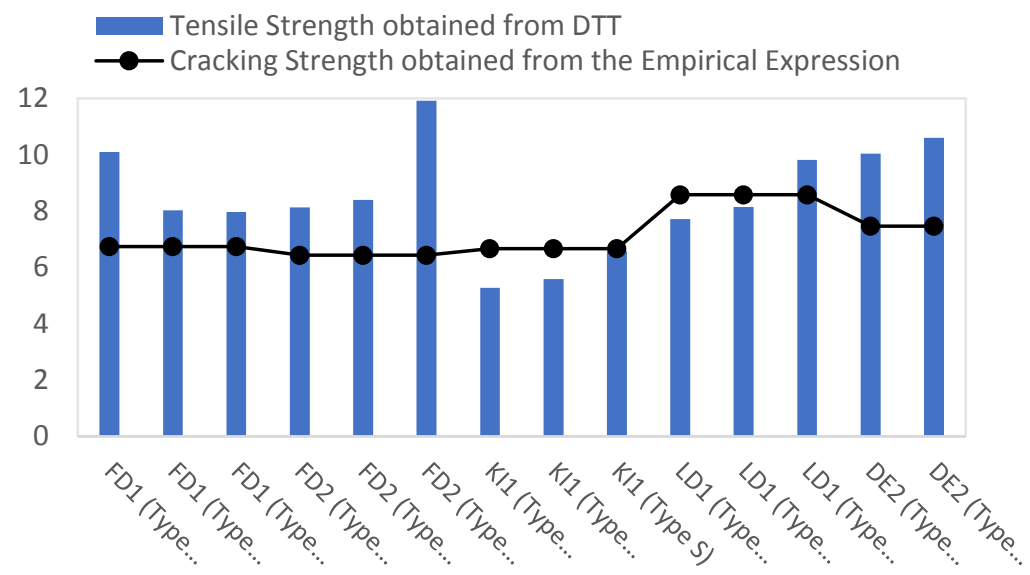

Figure 6-16: Average tensile strength obtained from direct tension test and cracking strength results calculated with the calibrated empirical expression (each bar represents the average of a triplicate of identical tests)

\subsection{Finite Element Analysis}

\subsubsection{Application of the Finite Element Method in Inverse Analysis}

Nowadays, finite element analysis becomes one of the most popular tools to conduct structural analysis, or to solve heat transfer, fluid flow, mass transport and electromagnetic potential problems. The major advantage of finite element analysis reflects in settling problems with complicated geometries, loading conditions and material properties. For those problems, it is usually challenging to get mathematical solutions through ordinary or partial differential equations. Therefore, the finite element method is employed to get approximate but reasonable solutions through a system of simultaneous algebraic equations. Finite element analysis starts with discretization during which the structure is divided into a number of smaller units. These smaller units are called elements which interconnect with one another through nodes, lines and/or surfaces. Unlike using differential equations to solve the entire structure in one operation, an algebraic equation can be developed for each element, and the solution of the entire structure can be obtained after formulating a system of simultaneous algebraic equations.

Even though the finite element method provides convenience compared with solving differential equations, solving the group of simultaneous algebraic equations could be time-consuming and erroneous, so finite element analysis is generally conducted through software platforms. The key steps for developing finite element models are summarized as follows: 1). use the preprocessor platform to define geometry, loading conditions and boundary conditions; 2). define element type and material properties in the preprocessor 
platform; 3). run the finite element model, and the postprocessor will compute the local stiffness matrix $([k])$ for each element and assemble them into a global stiffness matrix $([K])$ for the entire structure; and 4). the postprocessor will provide the results of nodal displacement matrix $(\{d\})$ by using Equation 6-2, where $\{F\}$ is the applied forces defined in step 1. It is worth noting that the finite element model will not run successfully unless equilibrium and compatibility conditions are satisfied. In addition, there are two types of finite element analysis: linear analysis and nonlinear analysis. The linear analysis is used when linear relation holds between the applied forces and displacements. Besides, as presented in Equation 6-2a, the stiffness matrix remains constant in linear analysis. In contrast, as presented in Equation 6-2b, the stiffness matrix is not constant but a function of displacement in nonlinear analysis (Kythe et al. 2003).

$$
\begin{aligned}
& {[K]\{d\}=\{F\}} \\
& {[K(d)]\{d\}=\{F\}}
\end{aligned}
$$

\subsubsection{Introduction of VecTor2}

To verify and correlate the inverse analysis results with finite element analysis, the two-dimensional nonlinear finite element analysis software VecTor2 was used to model the prisms subjected to four-point loading and to identify the stress-strain relationship in tension by matching the analytical resistance curve obtained from VecTor2 with that obtained experimentally.

VecTor 2 combines the Modified Compression Field Theory (MCFT) and the Disturbed Stress Field Model (DSFM) in a single formulation. As depicted in Figure 6-17, MCFT can estimate the load-deflection response of reinforced concrete elements subjecting to shear and normal stresses by determining the average and local strains and stresses of concrete and reinforcement, and the widths and orientation of cracks. In addition, to understand the mechanisms behind MCFT, it is essential to distinguish between discrete crack model and smeared crack model. To separate the cracked and uncracked concrete elements, discrete crack model simulates the cracked reinforced concrete by imposing displacement discontinuity to the cross section where crack localizes. To the contrary, regarding the smeared crack model, compatibility of nodal displacements still holds between the cracked and uncracked elements. However, both models have drawbacks. The discontinuity concept adopted by discrete crack model is not consistent with the compatibility characteristics of continuum mechanics that underlies finite element analysis. Besides, the crack path is predefined and constrained by the discrete crack model, which may not correspond to experimental observation. Conversely, the smeared crack model is incompatible with the discontinuous characteristics of localized cracks. Nevertheless, the smeared crack model does not restrict the orientation 
of cracks by allowing crack planes to concurrently rotate with the axis of principal strain. Moreover, smeared crack model simulates reinforced concrete as an orthotropic material. MCFT utilizes the smeared crack model; and it assumes the axes of principal stress and principal strain overlap, and perfect bond is assumed between concrete and reinforcement (Vecchio et al. 2013).

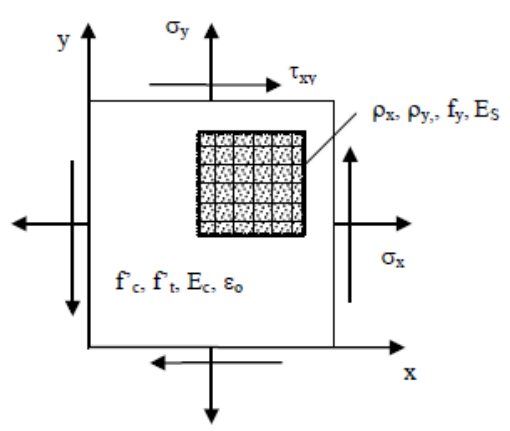

Figure 6- 17: Reinforced concrete element subjects to in-plane stresses (Vecchio and Collins 1986)

The MCFT also encompasses the tension stiffening behaviour. Cracks form upon the attainment of concrete's tensile strength, and the tensile strength of concrete is usually assumed to drop to zero immediately after cracking. However, this is not true for conventional reinforced concrete which loses its stiffness in tension at a slower rate compared with plain concrete, a phenomenon known as tension stiffening in the literature. Tension stiffening is the macroscopic result of partial bond loss between concrete and reinforcement. Figure 6-18 depicts a prism subjecting to uniaxial tensile loading $(P)$, and the stress distribution of concrete and the reinforcing bar along the span of the prism. As illustrated in Figure 6-18b and c, internal tensile stress is carried by the reinforcement alone at individual cracks, whereas the stress is carried by both concrete and reinforcement between the cracks because stress is transferred from steel to concrete through the bond mechanism. In addition, Figure 6-19 depicts the longitudinal stress distribution along the span of the prism, and it is evident that stress becomes uniform for the sections away from the individual crack, and stress concentrates near the reinforcing bar for the section near the individual crack.

(a)

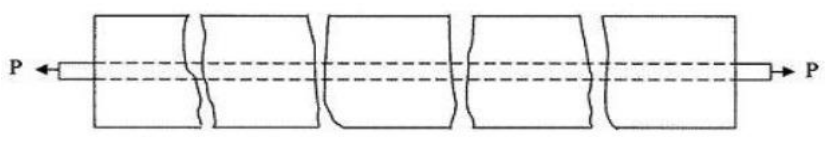

(b)

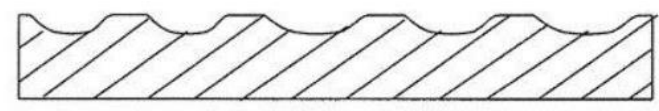

(c)

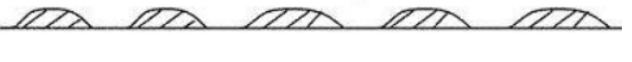

Figure 6- 18: Tensile stress distribution of the cracked reinforced concrete (Lin 2010): (a) Uniaxial tensile loading;

(b) Stress distribution of the reinforcing bar; and (c) Stress distribution of concrete 


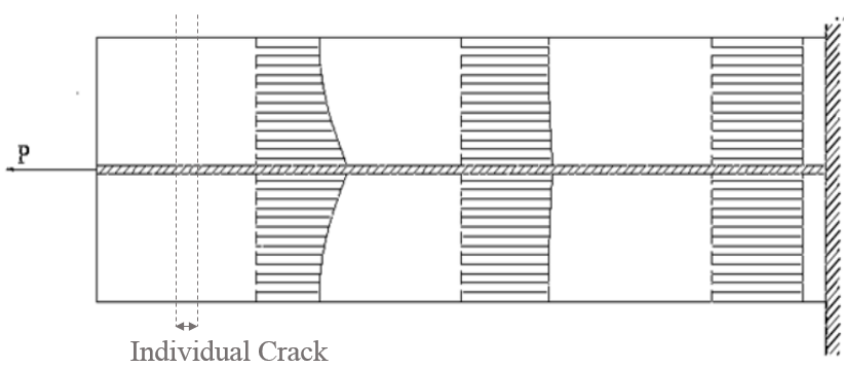

Figure 6- 19: Longitudinal stress distribution along the span of the prism (Lin 2010)

As mentioned above, the MCFT assumes the principal strain and principal stress are coaxial. However, this assumption is not always valid after cracking. For instance, crack shear slip is critical for lightly reinforced elements, and the principal strain field rotates at a faster rate than the principal stress field. As illustrated in Figure 6-20 (where $s$ is average crack spacing, $w$ is average crack width, $\delta_{s}$ is crack-shear-slip deformation, $\varepsilon$ is average net concrete axial strain, and $\gamma$ is average net shear strain), the total strain comprises two components: the response of reinforced concrete to the applied loads (i.e., the average strains in concrete and reinforcement as depicted in Figure 6-20a), and the deformation caused by crack shear slip (as depicted in Figure 6-20b). However, stress development is only attributed to the applied loads, which leads to a differential lag between the orientations of the principal strain field and principal stress field. Therefore, the Disturbed Stress Field Model (DSFM) is utilized to complement MCFT. DSFM is a smeared delayed rotating-crack model which allows the orientation of principal stress field to differ from the orientation of principal strain field by distinguishing the strains caused by crack-shear-slip deformations from the strains caused by the applied loads (Vecchio et al. 2013). In addition, VecTor2 incorporates a variety of models to simulate compression softening, tension softening, creep and relaxation, hysteretic response, dynamic loading and bond behaviour. Moreover, VecTor 2 incorporates constant strain triangle, plane stress rectangle and quadrilateral elements to model reinforced concrete; and truss bar, link, and four-noded contact elements to model reinforcement. Furthermore, FormWorks and Augustus are the preprocessor and postprocessor employed by VecTor2.

(a)

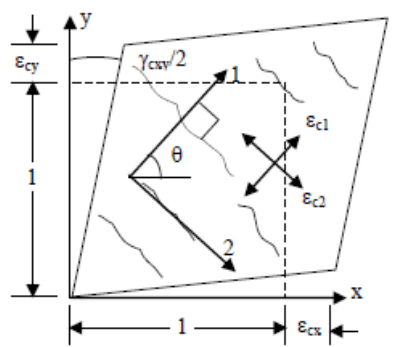

(b)

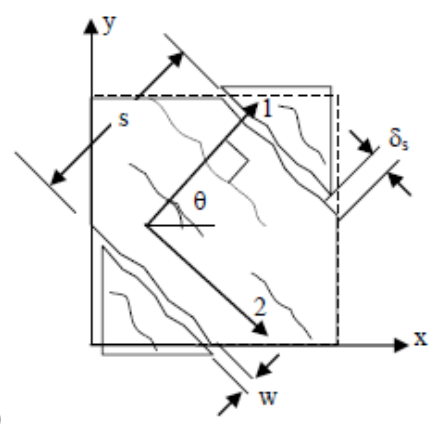

Figure 6- 20: (a)Average strain in concrete and reinforcement; and (b) Deformation due to crack shear slip (Vecchio et al. 2013) 


\subsubsection{Modelling Methodology}

On account of the fact that UHP-SFRC is reinforced with the distributed reinforcement in form of steel fibers, MCFT and DSFM are considered applicable for modelling the prisms subjected to four-point loading. However, constitutive models of UHP-SFRC are not available in most finite element software platforms because this class of materials is relatively new. In this direction, pertinent models were chosen from VecTor2 to simulate the various aspects of behaviour of UHP-SFRC. Table 6-14 presents the selected models from VecTor2. In VecTor2, Hognestad Parabola Model is the default model of concrete's pre-peak behaviour in compression. However, the Hognestad Parabola Model is only applicable to concrete having a compressive strength less than $40 \mathrm{MPa}$. Thus, in the present study the model by Popovics which is intended for High-Strength Concrete was utilized. With regards to tensile behaviour, four points were input to define the tensile stress-strain curve as follows: the first point corresponded to the onset of crack formation (i.e., it corresponded to cracking strength, $f_{c r}$ ); the second point corresponded to crack localization when the prism reached its peak load (i.e., it corresponded to ultimate tensile strength, $f_{F u}$ ); and the last two points were used to describe tension softening behaviour (i.e., the post-peak stage of the response). The tensile stress-strain curve was iteratively modified until the analytical resistance curve obtained from VecTor2 matched with the average resistance curve of each set of prisms obtained experimentally from FPBT. Regarding the material's compressive properties, the cylinder compressive strength obtained experimentally, the elastic modulus $(E)$ calculated with Equation 6-3, and the compressive strain at peak stress $\left(\varepsilon_{c}\right)$ calculated with Equation 6-4 were input to the finite element models (Vecchio et al. 2013). In addition, it was assumed that Poisson's ratio equaled 0.21 , the density of concrete was 2400 $\mathrm{kg} / \mathrm{m}^{3}$, and the maximum aggregate size was $0.5 \mathrm{~mm}$. Moreover, the volumetric ratio and dimensions of steel fibers were input according to each design mix, and the tensile strength of steel fibers was assumed to be $1900 \mathrm{MPa}$. As illustrated in Figure 6-21, finite element models were developed for all the prisms subjected to four-point loading. A fine mesh comprising plane stress rectangular elements $(5 \mathrm{~mm}$ by $5 \mathrm{~mm}$ element) was used throughout the study. Besides, loading rate controlled with $0.05 \mathrm{~mm}$ displacement increment per load step was used to displace the symmetric loading points at midspan in the vertical direction. For mixes FD1, FD2, KI1 and LD1, after matching the analytical and experimental responses for the short prisms ( $75 \mathrm{~mm}$ by $75 \mathrm{~mm}$ by $280 \mathrm{~mm}$ prisms) by fine tuning the stress-strain relationship of the material in tension, the defined models and the tensile stress-strain curve were used as input data and were verified by independently calculating the response of long prisms ( $75 \mathrm{~mm}$ by $75 \mathrm{~mm}$ by $500 \mathrm{~mm}$ ) without further refinement.

$E=5000 \sqrt{f^{\prime} c}$ 
(b)

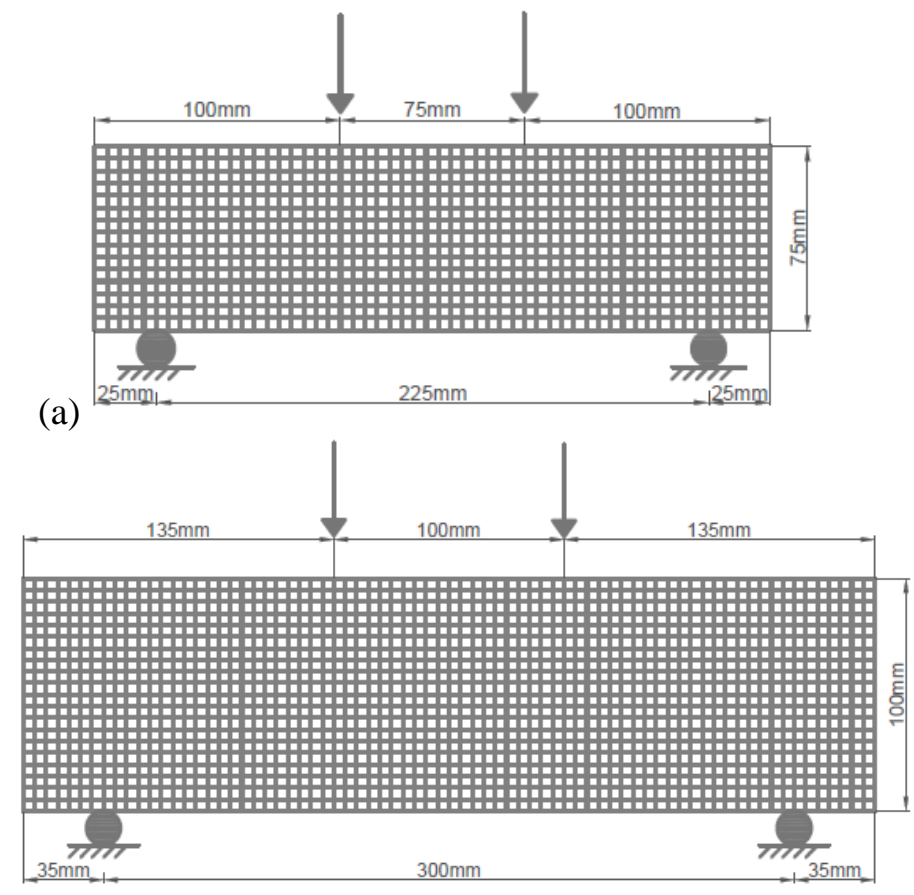

(c)

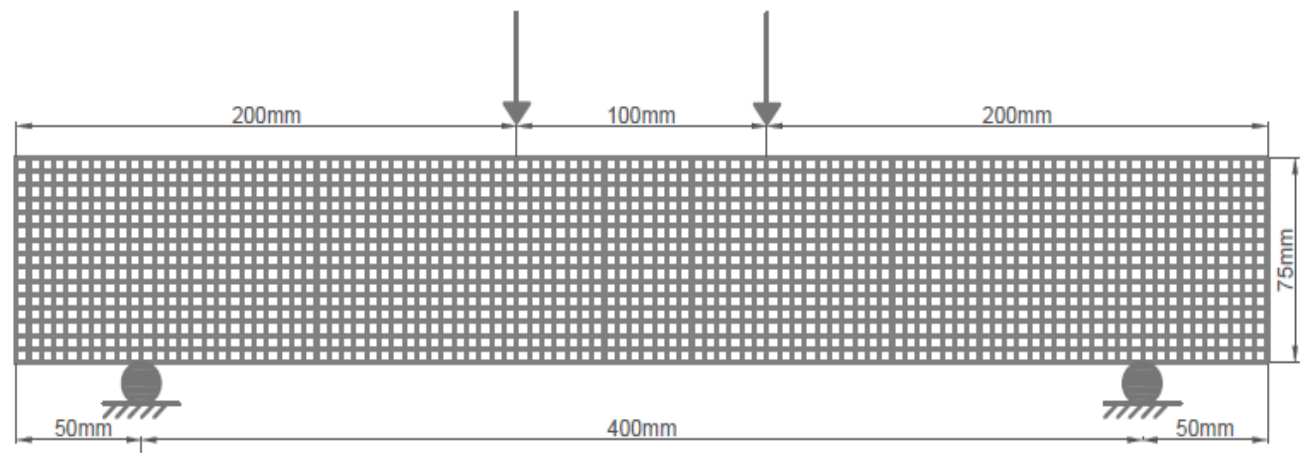

(d)

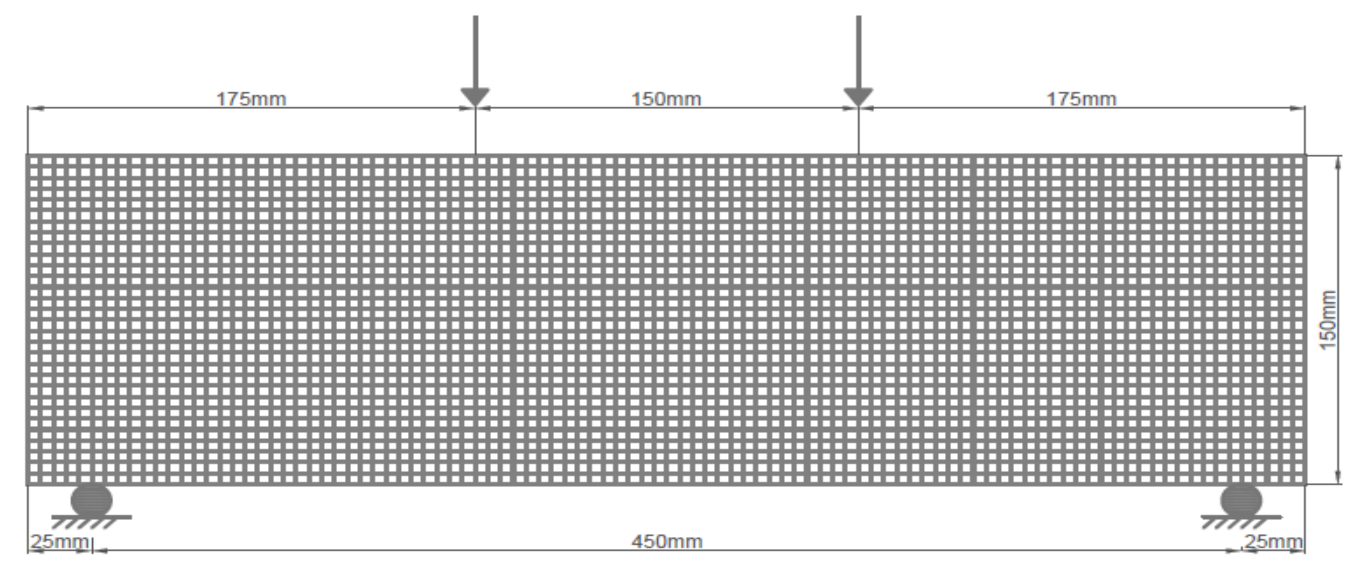

Figure 6- 21: Finite element models: (a) $75 \mathrm{~mm}$ by $75 \mathrm{~mm}$ by $280 \mathrm{~mm}$ prisms; (b) $100 \mathrm{~mm}$ by $100 \mathrm{~mm}$ by $370 \mathrm{~mm}$ prisms; (c) $75 \mathrm{~mm}$ by $75 \mathrm{~mm}$ by $500 \mathrm{~mm}$ prisms; and (d) $150 \mathrm{~mm}$ by $150 \mathrm{~mm}$ by $500 \mathrm{~mm}$ prisms 
Table 6- 14: Selected models in VecTor2 used in the present simulations

\begin{tabular}{|l|l|}
\hline \multicolumn{1}{|c|}{ Concrete Behaviour } & \multicolumn{1}{c|}{ Selected Model } \\
\hline Concrete Compression Pre-Peak & Popovic (HSC) \\
\hline Concrete Compression Post-Peak & Modified Park-Kent \\
\hline Concrete Compression Softening & Vecchio 1992-A (e1/e2-Form) \\
\hline Concrete Tension Stiffening & Modified Bentz 2003 \\
\hline Concrete Tension Softening & Custom Input (Strain Based) \\
\hline Concrete FRC Tension & SDEM-Monotonic \\
\hline Concrete Confined Strength & Kupfer/Richart \\
\hline Concrete Cracking Criterion & Mohr-Coulomb (Stress) \\
\hline Concrete Dilation & Variable - Isotropic \\
\hline Concrete Stress Calculation & Basic (DSFM/MCFT) \\
\hline Concrete Crack Width Check & Aggregate/2.5 Max Crack Width \\
\hline Concrete Slip Calculation & Walraven \\
\hline Concrete Creep and Relaxation & Not Considered \\
\hline Concrete Hysteretic Response & Nonlinear w/Plastic Offsets \\
\hline Concrete Bond & Eligehausen \\
\hline Note: The underlined models are non-default models. \\
\hline
\end{tabular}

\subsubsection{Finite Element Analysis Results}

Owing to limitations in the instrumentation, the resistance curves of some prisms were problematic and were not considered to correlate with the analytical curves obtained from finite element models. In addition, those prisms that had failed under shear or torsion were not considered for finite element analysis.

\subsubsection{Commercial FD1 and FD2 Design Mixes}

The cylinder compressive strength of $126 \mathrm{MPa}$, an elastic modulus of $56.125 \mathrm{GPa}$ calculated with Equation 6-3, and a compressive strain at peak stress of $2.745 \mathrm{~mm} / \mathrm{m}$ calculated with Equation 6-4 were input to the finite element models of the prisms from mix FD1. Regarding mix FD2, a cylinder compressive strength of $115 \mathrm{MPa}$, an elastic modulus of $53.619 \mathrm{GPa}$, and a compressive strain at peak stress of $2.663 \mathrm{~mm} / \mathrm{m}$ were input to the corresponding finite element models. In addition, since only one type of steel fibers was allowed to be defined in VecTor2, $1.5 \%$ of $20 \mathrm{~mm}$ long, hooked-end steel fibers with a diameter of $0.3 \mathrm{~mm}$ were input in the finite element models although mixes FD1 and FD2 actually contained 1\% of $20 \mathrm{~mm}$ long, straight steel fibers with a diameter of $0.2 \mathrm{~mm}$ and $1 \%$ of $20 \mathrm{~mm}$ long, hooked-end steel fibers with a diameter of $0.3 \mathrm{~mm}$.

Regarding mix FD1, the cracking strength and ultimate tensile strength of OW prisms (for both short and long prisms) was $10.5 \mathrm{MPa}$ and $16 \mathrm{MPa}$, respectively; the cracking strength and ultimate tensile strength 
of $\mathrm{R}$ short prisms was $15 \mathrm{MPa}$ and $17 \mathrm{MPa}$, respectively; and the cracking strength and ultimate tensile strength of R long prisms was $11 \mathrm{MPa}$ and $13 \mathrm{MPa}$, respectively. It is worth noting that the defined tensile stress-strain curve utilized for modelling $\mathrm{R}$ short prisms can not be verified by the response of $\mathrm{R}$ long prisms due to the considerable difference in flexural strength results: the average flexural strength was $39.57 \mathrm{MPa}$ for short prisms and $30.97 \mathrm{MPa}$ for long prisms. Regarding mix FD2, the cracking strength and ultimate tensile strength of OW prisms was $13 \mathrm{MPa}$ and $15 \mathrm{MPa}$, respectively; and the cracking strength and ultimate tensile strength of R short prisms ( $\mathrm{R}$ long prism was not cast with FD2 design mixture) was $15 \mathrm{MPa}$ and 18 $\mathrm{MPa}$, respectively.

The analytical and experimental resistance curves of mix FD1, and the first two points defined for the tensile stress-strain relationship are illustrated in Figure 6-22 to Figure 6-24, where the 'Average' curve was obtained by averaging the experimental resistance curves of each set of prisms. The analytical resistance curves of mix FD2 can be found in Appendix C.1.
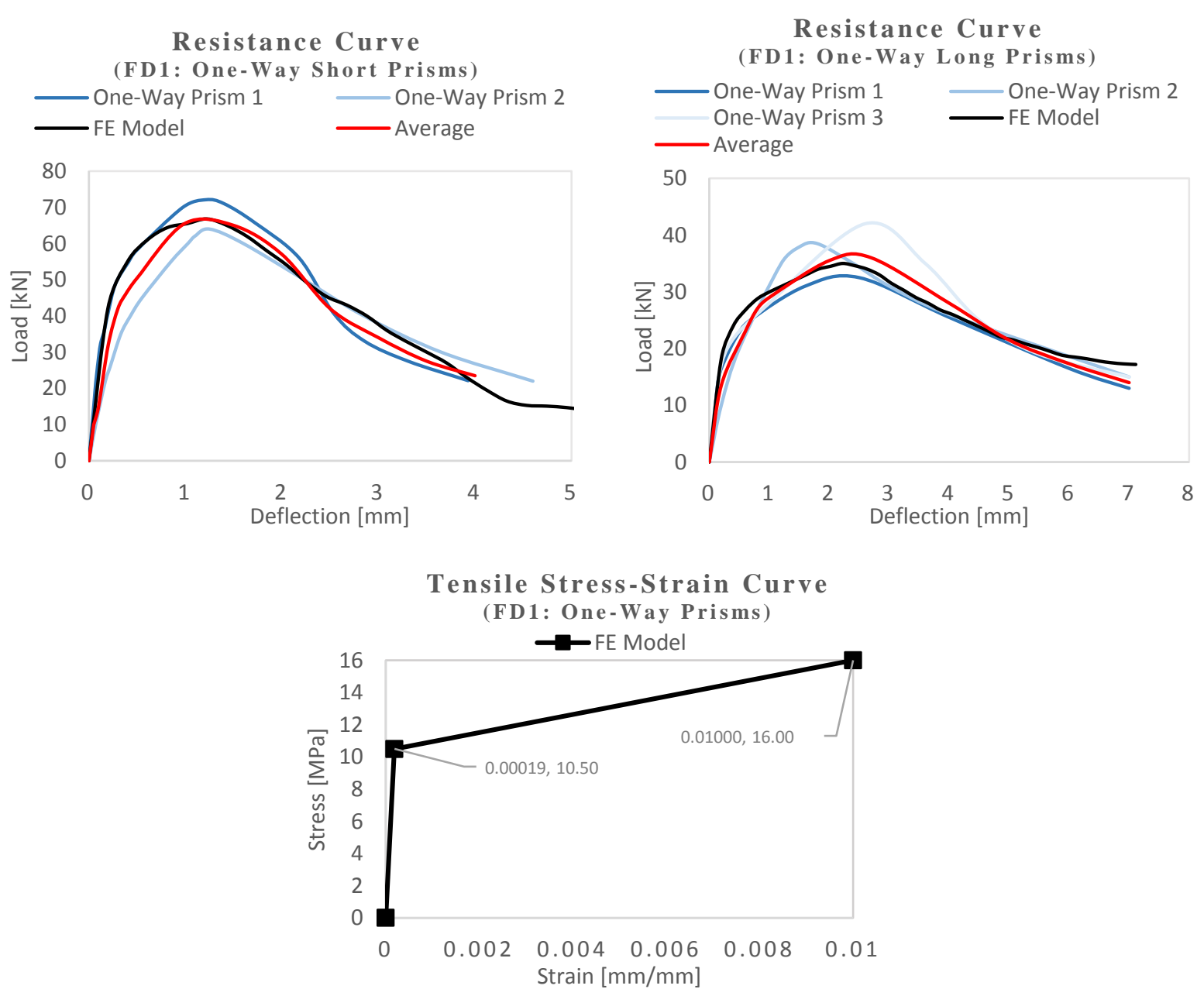

Figure 6- 22: Finite element analysis results of one-way prisms from mix FD1 

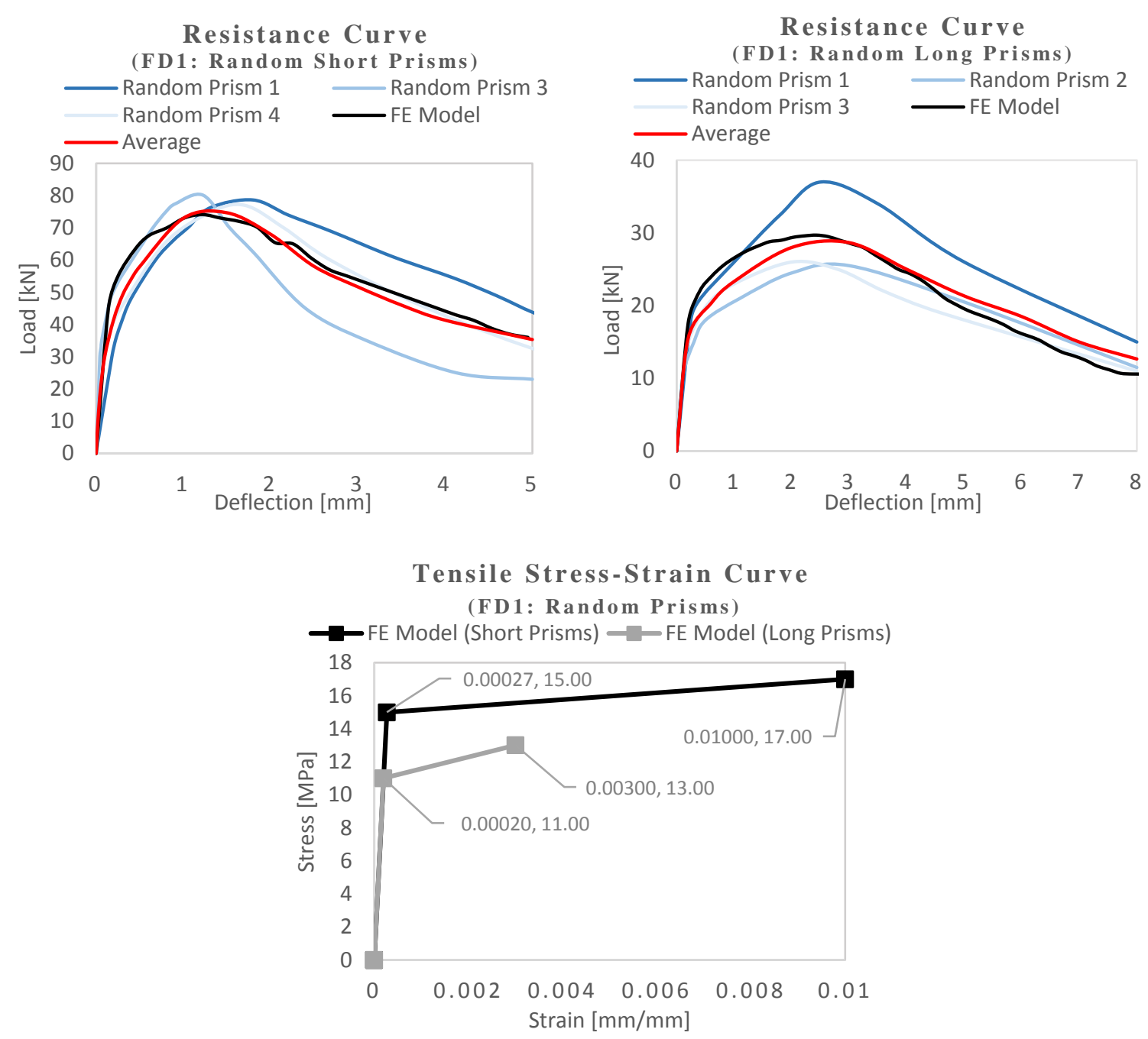

Figure 6- 23: Finite element analysis results of random prisms from mix FD1
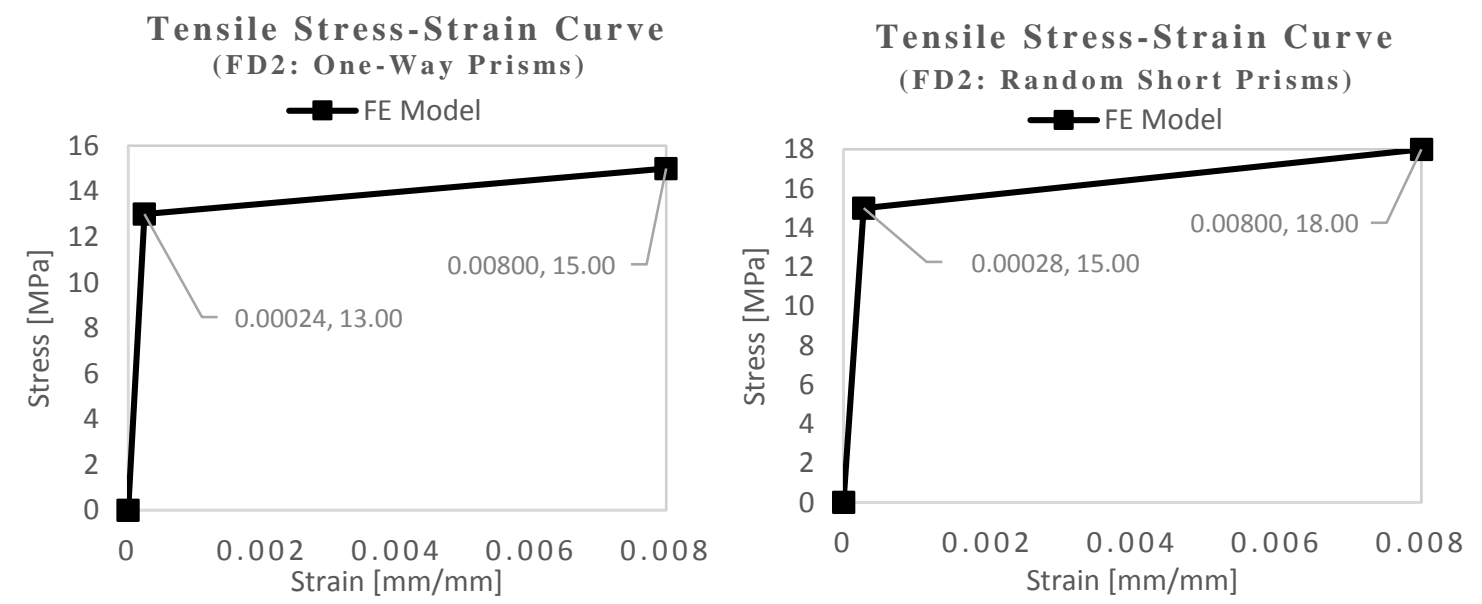

Figure 6- 24: Finite element analysis results of the prisms from mix FD2 


\subsubsection{Commercial KII Design Mix}

The cylinder compressive strength of $123.5 \mathrm{MPa}$, an elastic modulus of $56.565 \mathrm{GPa}$, and a compressive strain at peak stress of $2.726 \mathrm{~mm} / \mathrm{m}$ were input to the finite element models. The cracking strength and ultimate tensile strength of OW prisms was $9 \mathrm{MPa}$ and $12 \mathrm{MPa}$, respectively; and the cracking strength and ultimate tensile strength of $\mathrm{R}$ prisms was $8 \mathrm{MPa}$ and $9.5 \mathrm{MPa}$, respectively. The analytical and experimental resistance curves, and the first two points defined for the tensile stress-strain relationship are illustrated in Figure 6-25 and Figure 6-26.
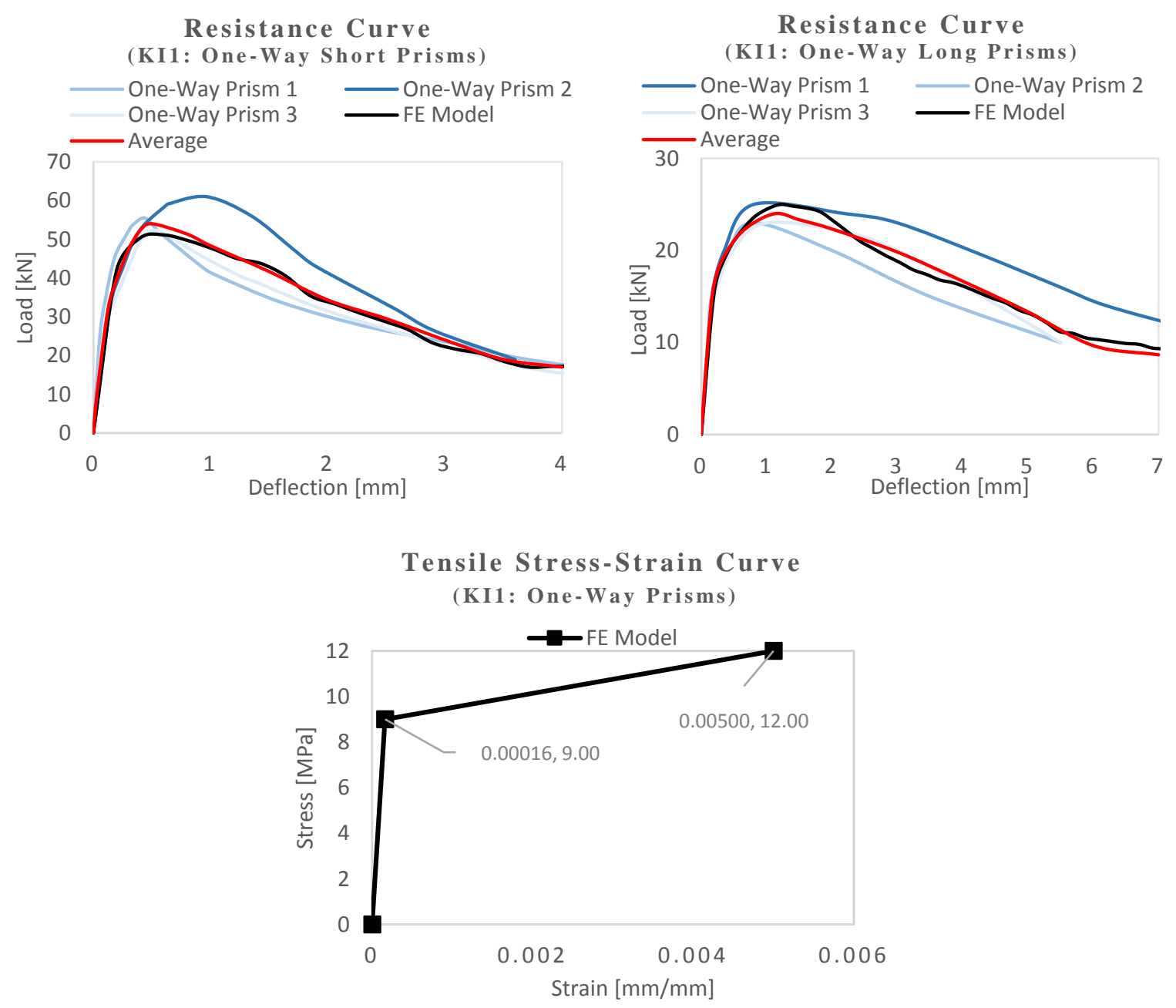

Figure 6- 25: Finite element analysis results of one-way prisms from mix KII 

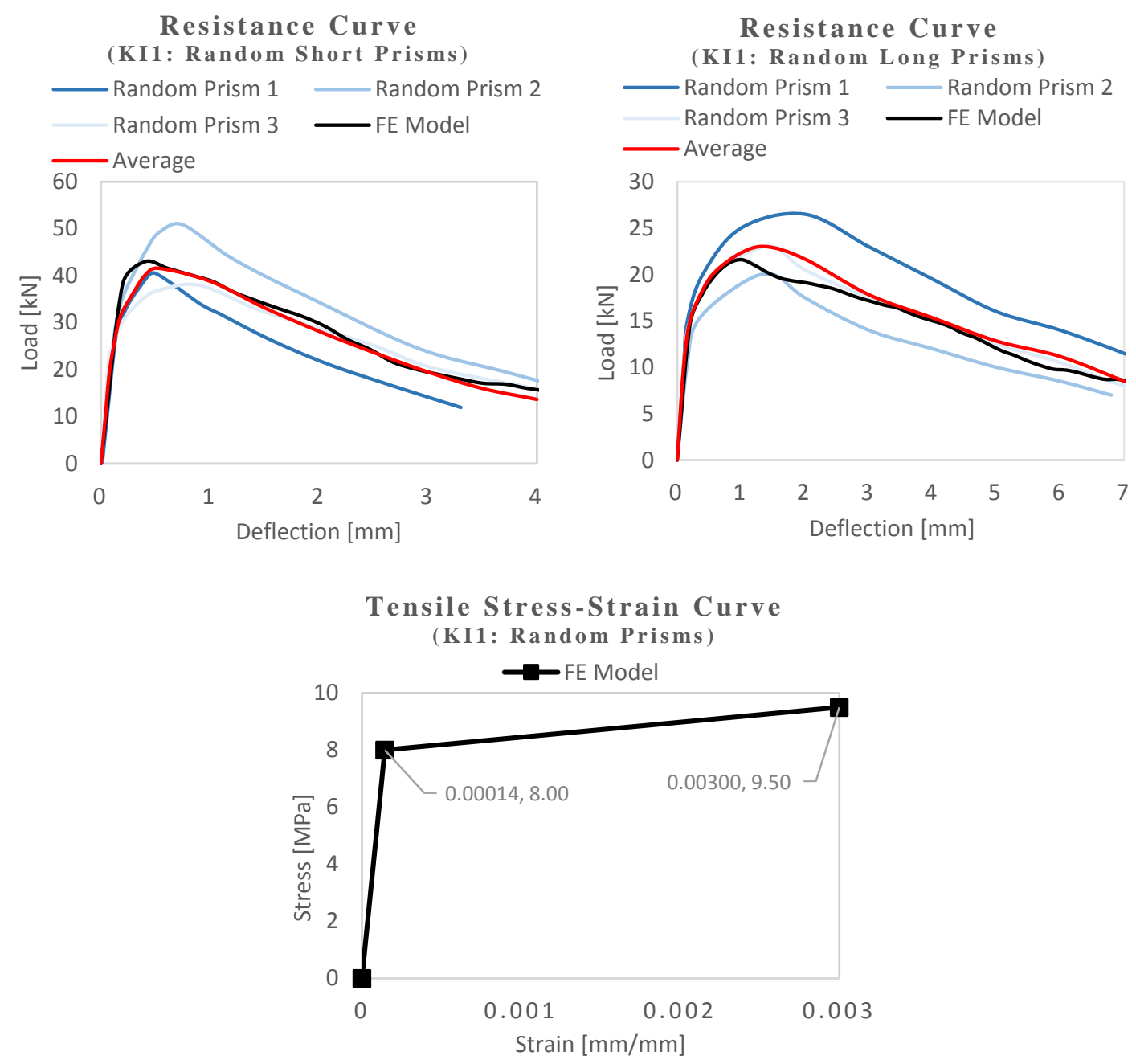

Figure 6- 26: Finite element analysis results of random prisms from mix KII

\subsubsection{Commercial LD1 Design Mix}

The cylinder compressive strength of $204.5 \mathrm{MPa}$, an elastic modulus of $71.414 \mathrm{GPa}$, and a compressive strain at peak stress of $3.33 \mathrm{~mm} / \mathrm{m}$ were input to the finite element models. The cracking strength and ultimate tensile strength of OW prisms was $9 \mathrm{MPa}$ and $12 \mathrm{MPa}$, respectively; the cracking strength and ultimate tensile strength of $\mathrm{R}$ short prisms was $10 \mathrm{MPa}$ and $12 \mathrm{MPa}$, respectively; and the cracking strength and ultimate tensile strength of R long prisms was $6 \mathrm{MPa}$ and $8 \mathrm{MPa}$, respectively. The defined tensile stress-strain curve utilized for modelling $\mathrm{R}$ short prisms can not be verified by the response of $\mathrm{R}$ long prisms owing to the considerable difference in flexural strength results: the average flexural strength was 29.55 MPa for short prisms and 19.41 MPa for long prisms. The first two points defined for the tensile stressstrain relationship are illustrated in Figure 6-27, and the analytical and experimental resistance curves can be found in Appendix C.2. 

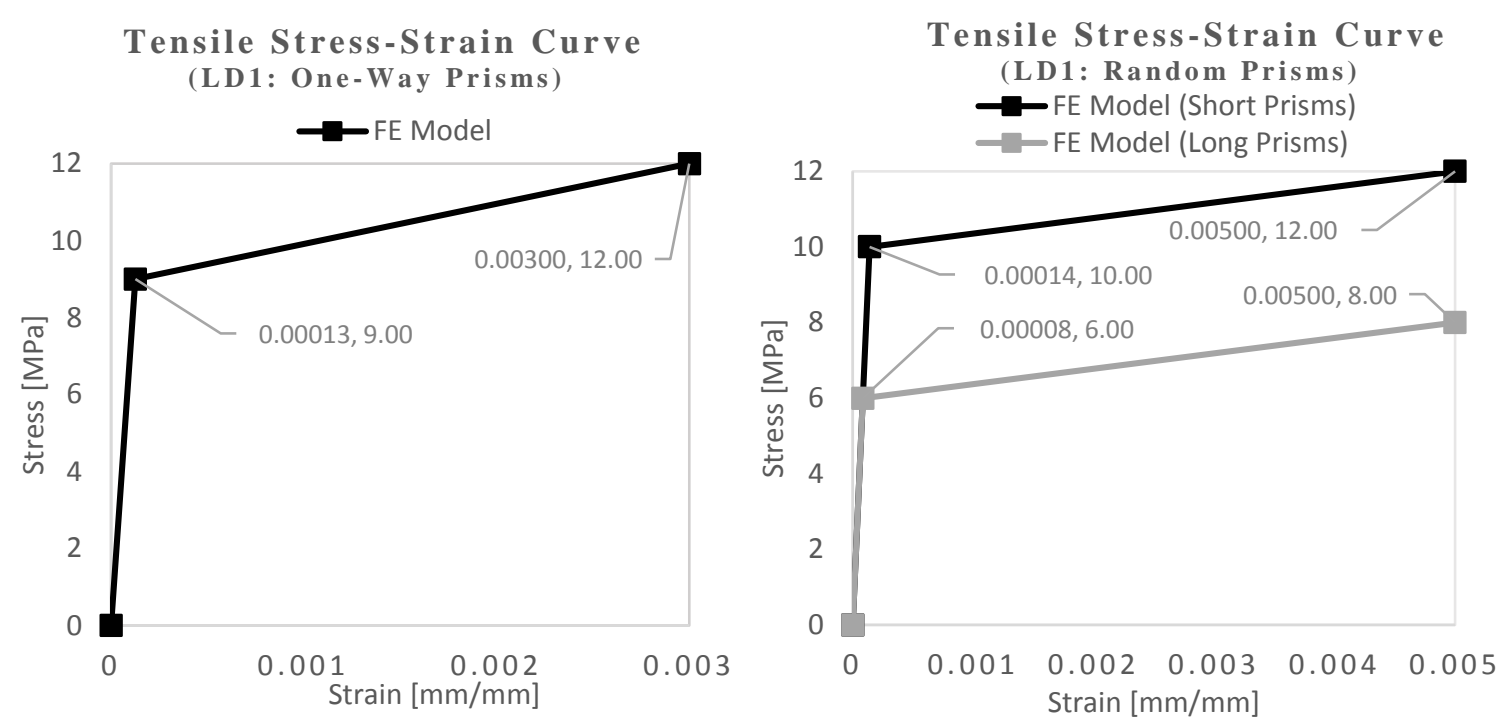

Figure 6- 27: Finite element analysis results of the prisms from mix LD1

\subsubsection{In-House DE1 and DE2 Design Mix (SC1)}

Regarding to mix DE1, a cylinder compressive strength of $164 \mathrm{MPa}$, an elastic modulus of $64.031 \mathrm{GPa}$, and a compressive strain at peak stress of $3.03 \mathrm{~mm} / \mathrm{m}$ were input to the finite element models; the cracking strength of OW prisms was $9 \mathrm{MPa}$, whereas the cracking strength of $\mathrm{R}$ prisms was $7 \mathrm{MPa}$. As depicted in Figure 6-28, it was challenging to match the analytical resistance curves of mix DE1 with those obtained experimentally, and it was assumed that mix DE1 did not exhibit tension-hardening behaviour. Regarding mix DE2, a cylinder compressive strength of $155 \mathrm{MPa}$, an elastic modulus of $62.249 \mathrm{GPa}$, and a compressive strain at peak stress of $2.963 \mathrm{~mm} / \mathrm{m}$ were input to the finite element models; the cracking strength and ultimate tensile strength of OW prisms was $9 \mathrm{MPa}$ and $10.5 \mathrm{MPa}$, respectively, and the cracking strength and ultimate tensile strength of $\mathrm{R}$ prisms was $7 \mathrm{MPa}$ and $8 \mathrm{MPa}$, respectively. The analytical and experimental resistance curves of mix DE1and the first two points defined for the tensile stress-strain relationship are illustrated in Figure 6-28, and the analytical resistance curves of mix DE2 can be found in Appendix C.3. 

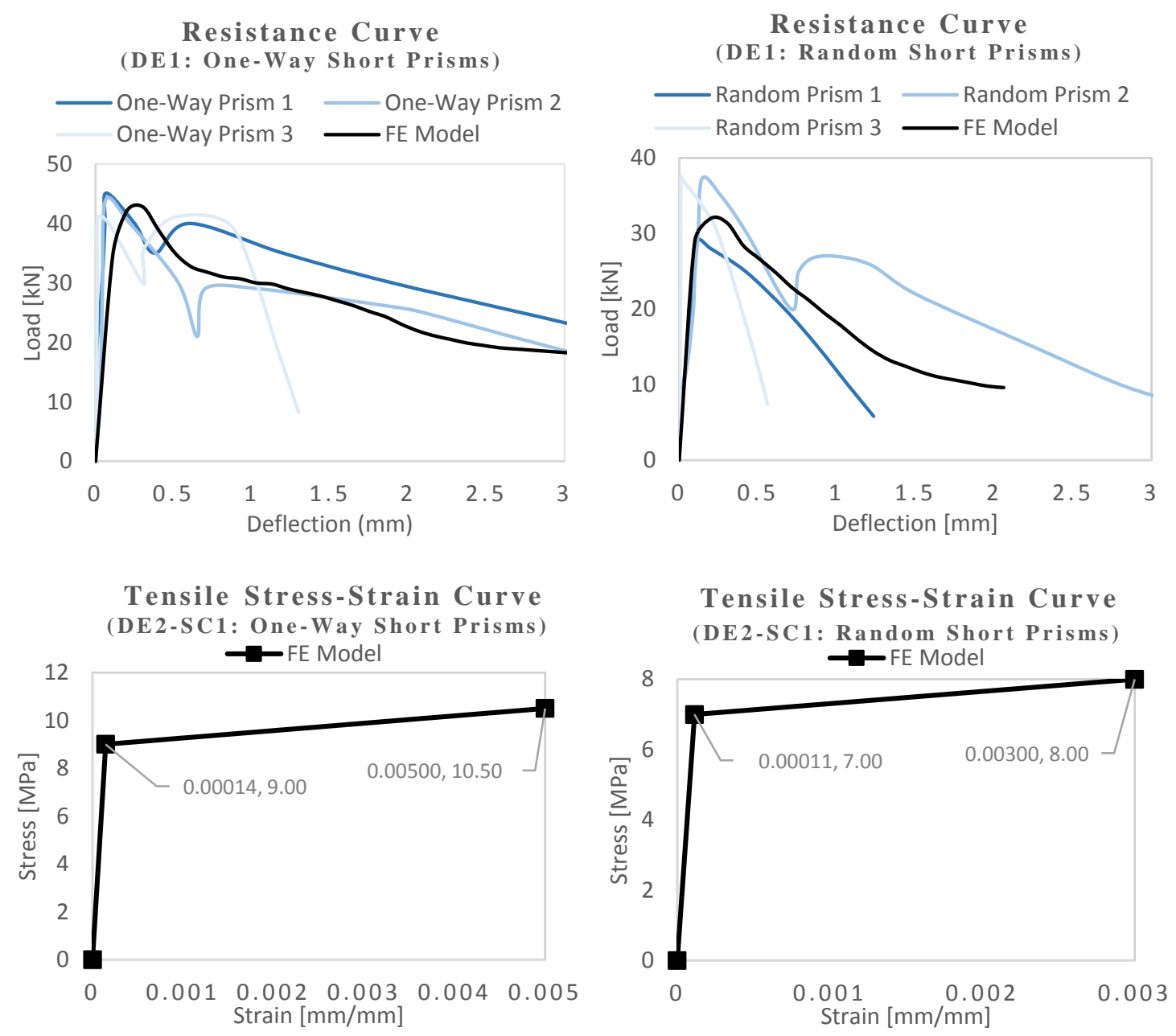

Figure 6- 28: Finite element analysis results of the prisms from mixes DE1 and DE2

\subsubsection{Commercial FD3 Design Mix}

The prisms (100 mm by $100 \mathrm{~mm}$ by $370 \mathrm{~mm}$ prisms) tested by York University, École PolytechniqueMontréal and Queen's University were also analyzed by the finite element models. However, to analyze the differences in the test results obtained by different labs on identical prism specimens, compressive strength, modulus of elasticity and strain at peak stress were taken the same for all three partners on account of the fact that all specimens were cast from the same batch. The cylinder compressive strength of $111 \mathrm{MPa}$, an elastic modulus of $52.678 \mathrm{GPa}$, and a compressive strain at peak stress of $2.434 \mathrm{~mm} / \mathrm{m}$ were input to the finite element models of the prisms. The differences in flexural strength results obtained by each university are most likely traced to the different boundary conditions. In each case, the average experimental curve was matched with the analytical one. In this context, regrading the prisms tested by York University, the cracking strength and ultimate tensile strength of $\mathrm{OW}$ prisms was $12.5 \mathrm{MPa}$ and 17.5 $\mathrm{MPa}$, respectively; 
and the cracking strength and ultimate tensile strength of $\mathrm{R}$ prisms was $11.5 \mathrm{MPa}$ and $13.75 \mathrm{MPa}$, respectively. Regarding the prisms tested by École Polytechnique-Montréal, the cracking strength and ultimate tensile strength of OW prisms was $12 \mathrm{MPa}$ and 14.5 MPa, respectively; and the cracking strength and ultimate tensile strength of $\mathrm{R}$ prisms was $9.5 \mathrm{MPa}$ and $11 \mathrm{MPa}$, respectively. The prisms tested by Queen's University were matched using the cracking strength and ultimate tensile strength of OW prisms equal to $10.5 \mathrm{MPa}$ and $12.5 \mathrm{MPa}$, respectively; and the cracking strength and ultimate tensile strength of $\mathrm{R}$ prisms was equal to $9.5 \mathrm{MPa}$ and $10.75 \mathrm{MPa}$, respectively. The analytical and experimental resistance curves of the prisms tested by York University, and the first two points defined for the tensile stress-strain relationship are illustrated in Figure 6-29, whereas the analytical resistance curves of the prisms tested by École Polytechnique-Montréal and Queen's University can be found in Appendix C.4.

(a)
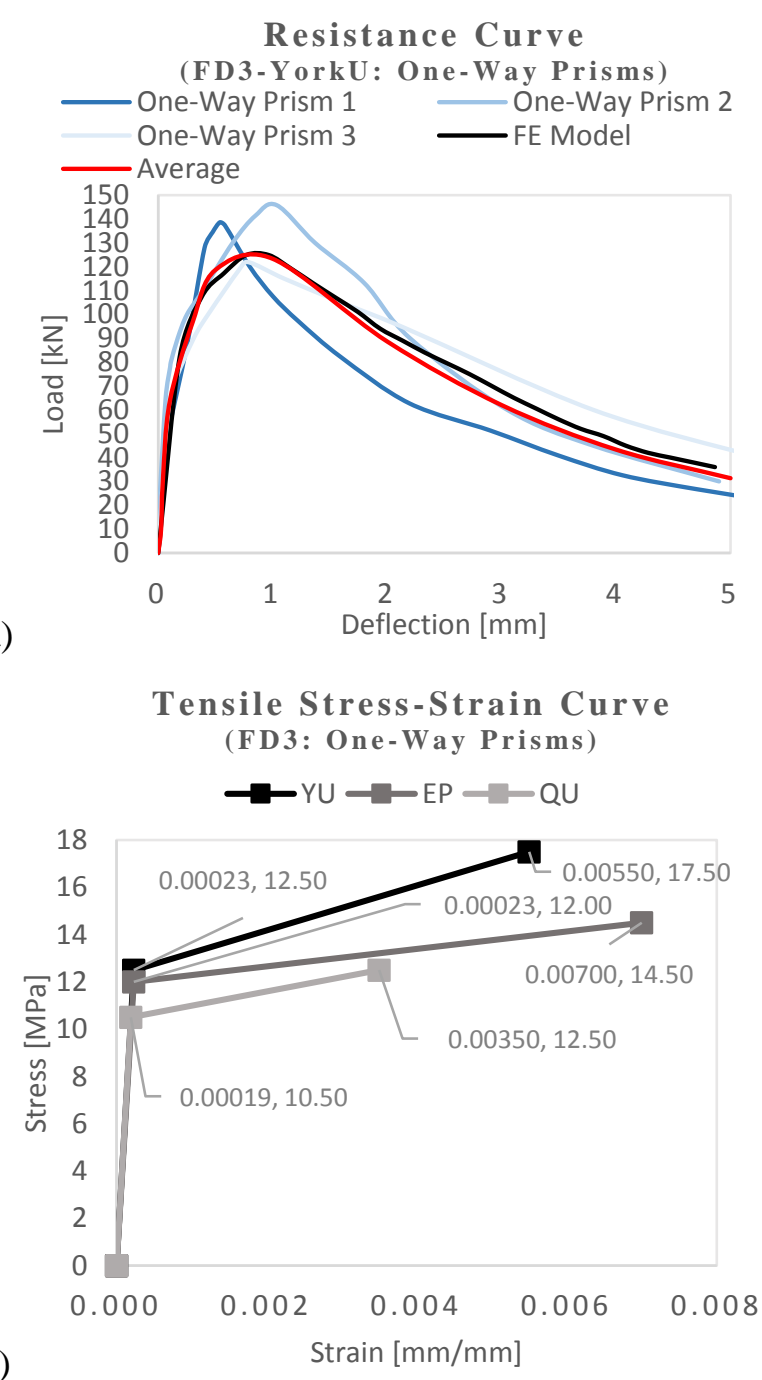

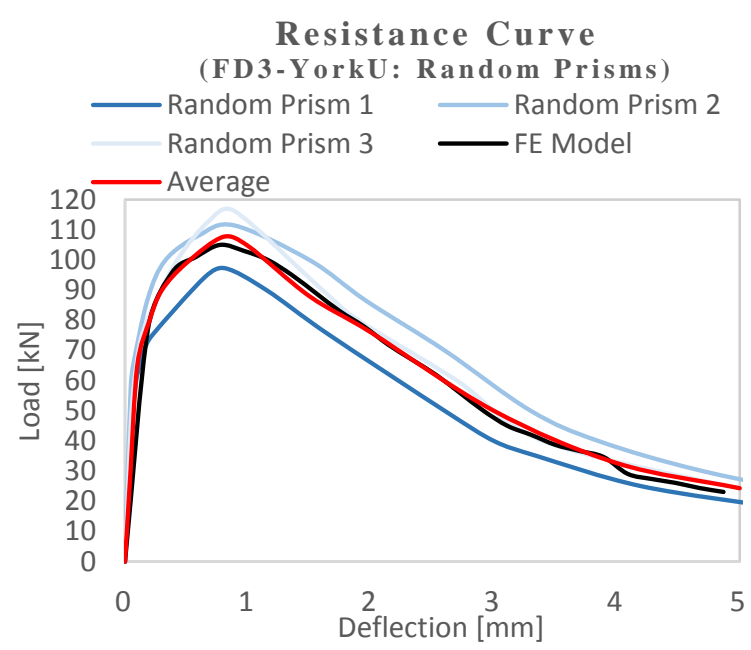

Tensile Stress-Strain Curve (FD3: Random Prisms)

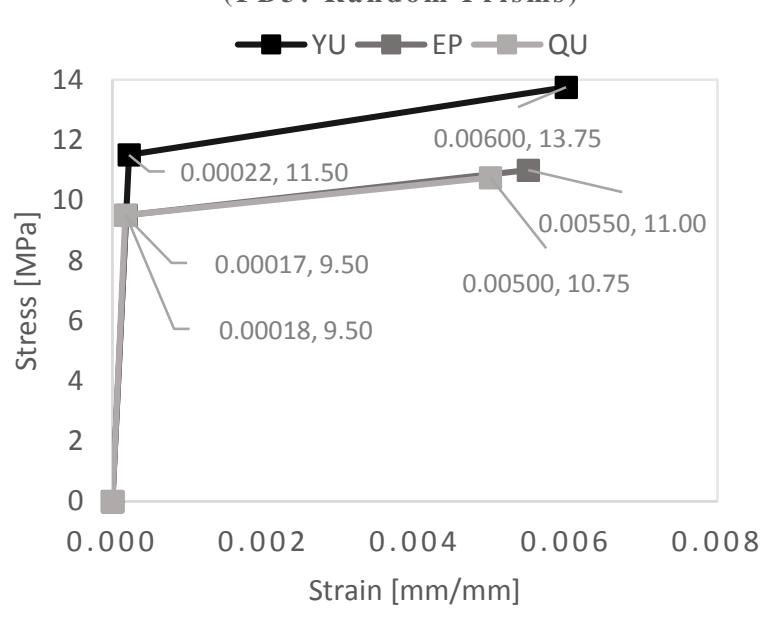

Figure 6- 29: Finite element analysis results: (a) Resistance curves (YU); and (b) Tensile stress-strain relationship 


\subsubsection{Commercial KI2 Design Mix}

This section presents the finite element analysis results of the KI2 prisms $(75 \mathrm{~mm}$ by $75 \mathrm{~mm}$ by $280 \mathrm{~mm}$ prisms) tested during the Round Robin Testing Program. A cylinder compressive strength value of 129 $\mathrm{MPa}$, an elastic modulus of $56.789 \mathrm{GPa}$ and a compressive strain at peak stress of $2.768 \mathrm{~mm} / \mathrm{m}$ were input to the finite element models of the prisms tested by all three partners. Regarding the prisms tested by York University, the cracking strength and ultimate tensile strength of OW prisms was $9 \mathrm{MPa}$ and $11.5 \mathrm{MPa}$, respectively; and the cracking strength and ultimate tensile strength of $\mathrm{R}$ prisms was $9 \mathrm{MPa}$ and $12.5 \mathrm{MPa}$, respectively. Regarding the prisms tested by École Polytechnique-Montréal, the cracking strength and ultimate tensile strength of OW prisms was $7 \mathrm{MPa}$ and 8.5 MPa, respectively; and the cracking strength and ultimate tensile strength of $\mathrm{R}$ prisms was $7 \mathrm{MPa}$ and $9.5 \mathrm{MPa}$, respectively. Regarding the prisms tested by Queen's University, the cracking strength and ultimate tensile strength of OW prisms was $6.5 \mathrm{MPa}$ and 7.75 MPa, respectively; and the cracking strength and ultimate tensile strength of R prisms was $6.5 \mathrm{MPa}$ and 8.5 $\mathrm{MPa}$, respectively. The first two points defined for the tensile stress-strain relationship are illustrated in Figure 6-30, and the analytical resistance curves can be found in Appendix C.5.
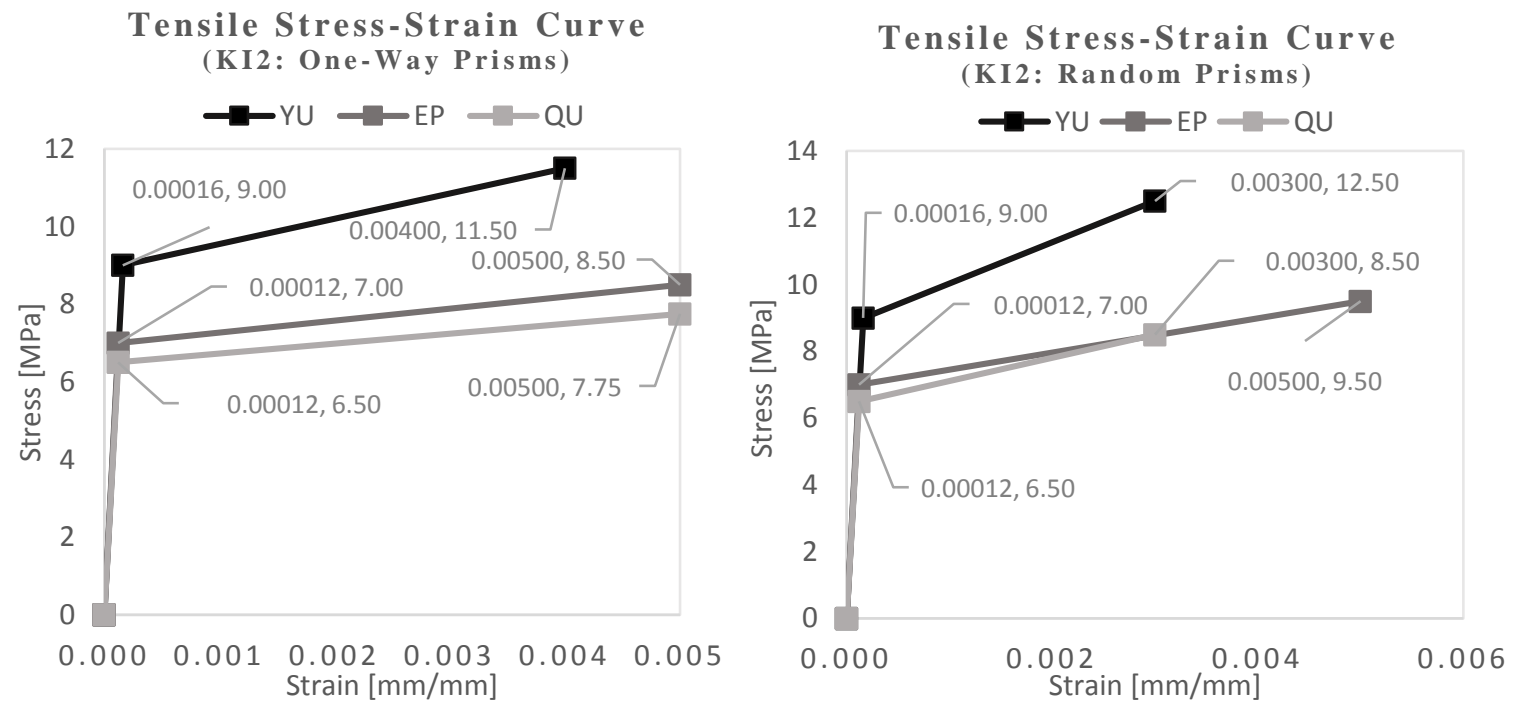

Figure 6- 30: Finite element analysis results of the prisms from mix KI2 


\subsubsection{Commercial LD2 Design Mix}

The cylinder compressive strength of $166 \mathrm{MPa}$, an elastic modulus of $64.42 \mathrm{GPa}$ and a compressive strain at peak stress of $3.045 \mathrm{~mm} / \mathrm{m}$ were input to the finite element models of the prisms tested by all three partners. Regarding the prisms tested by York University, the cracking strength and ultimate tensile strength of OW prisms was $8 \mathrm{MPa}$ and $10.75 \mathrm{MPa}$, respectively; and the cracking strength and ultimate tensile strength of R prisms was 7.5 $\mathrm{MPa}$ and $10 \mathrm{MPa}$, respectively. Regarding the prisms tested by École Polytechnique-Montréal, the cracking strength and ultimate tensile strength of OW prisms was 6.5 MPa and $8 \mathrm{MPa}$, respectively; and the cracking strength and ultimate tensile strength of $\mathrm{R}$ prisms was $7 \mathrm{MPa}$ and 8.5 MPa, respectively. Regarding the prisms tested by Queen's University, the cracking strength and ultimate tensile strength of $\mathrm{OW}$ prisms was $6.5 \mathrm{MPa}$ and $8.5 \mathrm{MPa}$, respectively; and the cracking strength and ultimate tensile strength of $\mathrm{R}$ prisms was $5.5 \mathrm{MPa}$ and $6.5 \mathrm{MPa}$, respectively. The first two points defined for the tensile stress-strain relationship are illustrated in Figure 6-31, and the analytical resistance curves can be found in Appendix C.6.
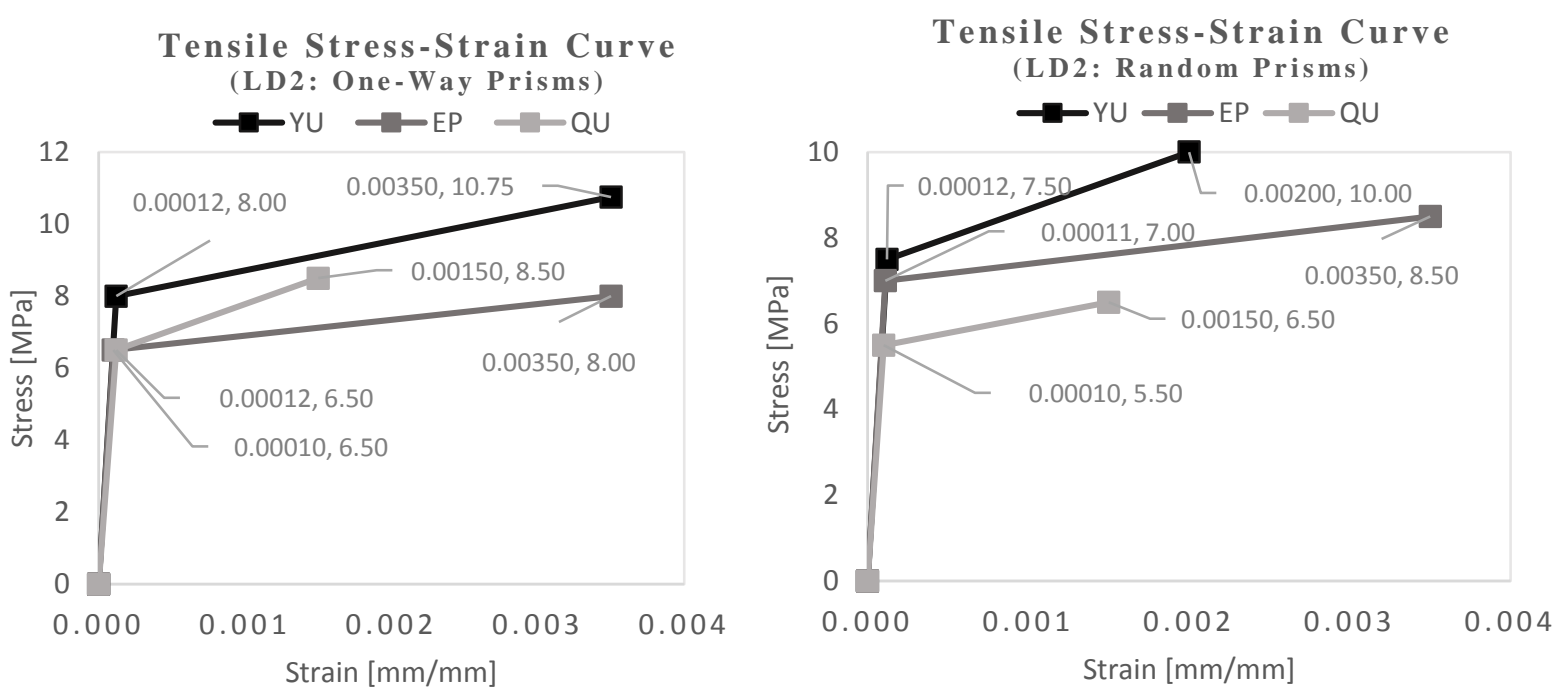

Figure 6- 31: Finite element analysis results of the prisms from mix LD2

\subsubsection{In-house DE2 Design Mix (SC2)}

The cylinder compressive strength of $155 \mathrm{MPa}$, an elastic modulus of $62.249 \mathrm{GPa}$ and a compressive strain at peak stress of $2.963 \mathrm{~mm} / \mathrm{m}$ were input to the finite element models, and the cracking strength and ultimate tensile strength was $6 \mathrm{MPa}$ and $8.75 \mathrm{MPa}$, respectively. The analytical and experimental resistance curves, and the first two points defined for the tensile stress-strain relationship are illustrated in Figure 6-32. 

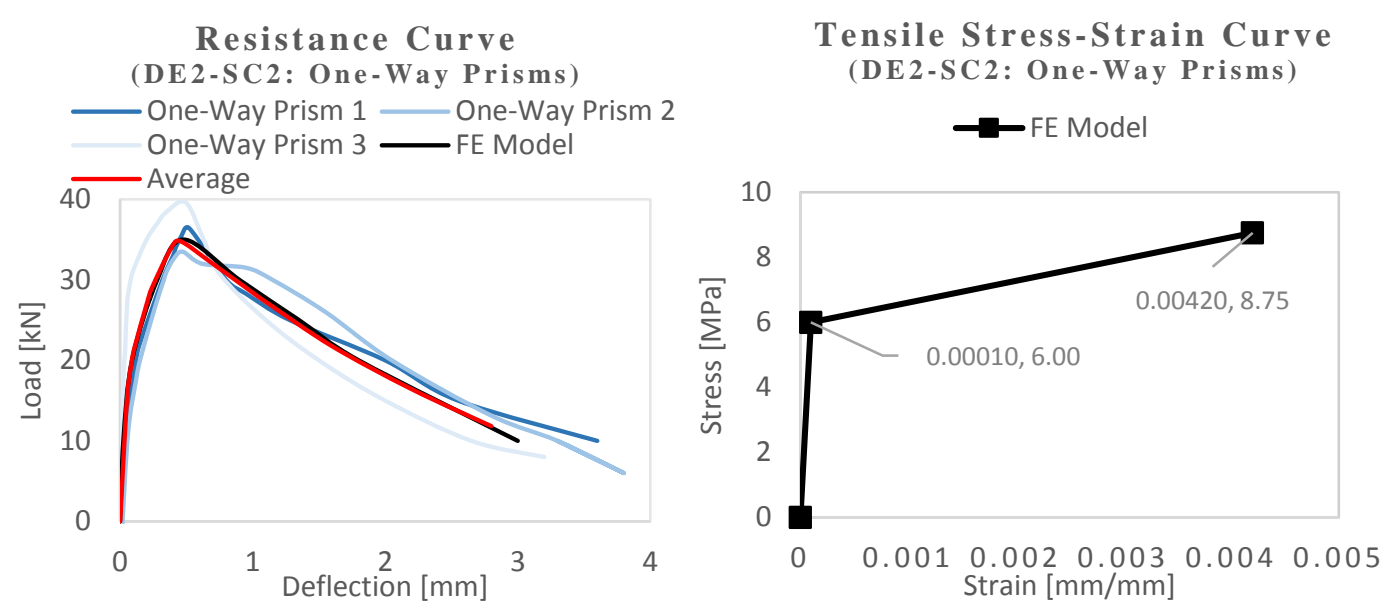

Figure 6- 32: Finite element analysis results of the prisms from mix DE2

\subsubsection{Commercial FD4 Design Mix}

The elastic modulus and Poisson's ratio of mix FD4 were measured experimentally (refer to Section 5.1), and were found to be $46.58 \mathrm{GPa}$ and 0.204 , respectively; and the cylinder compressive strength was 130.7 MPa corresponding to a compressive strain at peak stress of $2.781 \mathrm{~mm} / \mathrm{m}$. With regards to the $100 \mathrm{~mm}$ by $100 \mathrm{~mm}$ by $370 \mathrm{~mm}$ prisms, the cracking strength and ultimate tensile strength were $6 \mathrm{MPa}$ and $7 \mathrm{MPa}$, respectively. Similarly, for the $150 \mathrm{~mm}$ by $150 \mathrm{~mm}$ by $500 \mathrm{~mm}$ prisms, the cracking strength and ultimate tensile strength were 4.5 $\mathrm{MPa}$ and $6.25 \mathrm{MPa}$, respectively. The analytical and experimental resistance curves, and the first two points defined for the tensile stress-strain relationship are illustrated in Figure 633 and Figure 6-34.
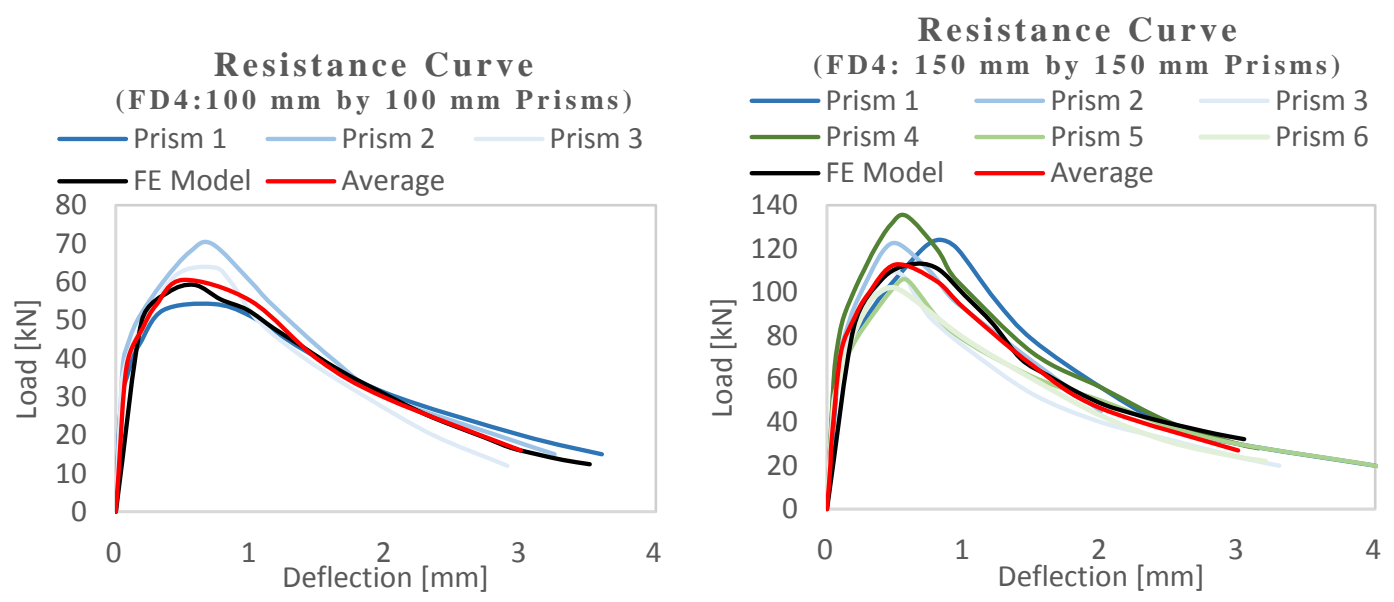

Figure 6- 33: Analytical response curves of the prisms from mix FD4 

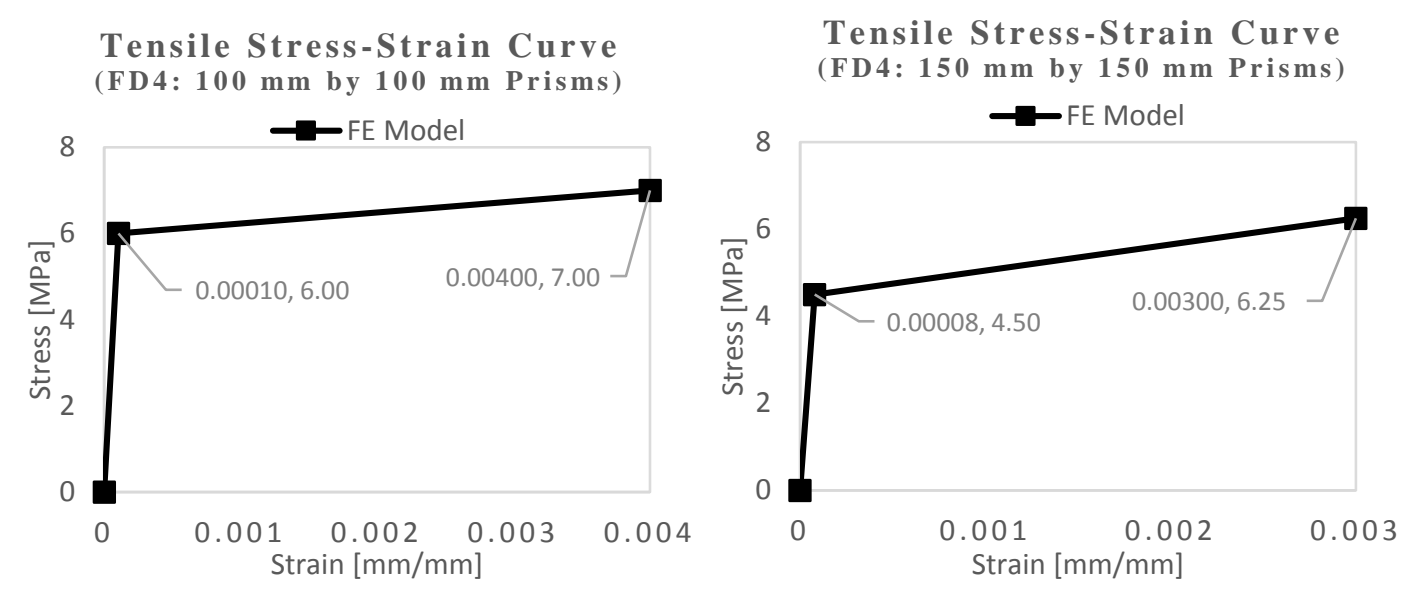

Figure 6- 34: Tensile stress-strain relationship of the prisms from mix FD4

\subsubsection{Commercial FD5 and FD6 Design Mixes}

The cylinder compressive strength of $136.2 \mathrm{MPa}$ (at the age of 28 days), an elastic modulus of $58.352 \mathrm{GPa}$, and a compressive strain at peak stress of $2.822 \mathrm{~mm} / \mathrm{m}$ were input to the finite element models of the prisms from mix FD5; the cylinder compressive strength of $133.3 \mathrm{MPa}$ (at the age of 28 days), an elastic modulus of $57.728 \mathrm{GPa}$, and a compressive strain at peak stress of $2.8 \mathrm{~mm} / \mathrm{m}$ were input to the finite element models of the prisms from mix FD6. With regards to mix FD5, the cracking strength and ultimate tensile strength were 7.25 MPa and 8.35 MPa, respectively. Similarly, for mix FD6, the cracking strength and ultimate tensile strength were 7.25 $\mathrm{MPa}$ and $8.6 \mathrm{MPa}$, respectively. The first two points defined for the tensile stressstrain relationship are illustrated in Figure 6-35, and the analytical response curves can be found in Appendix C.7.
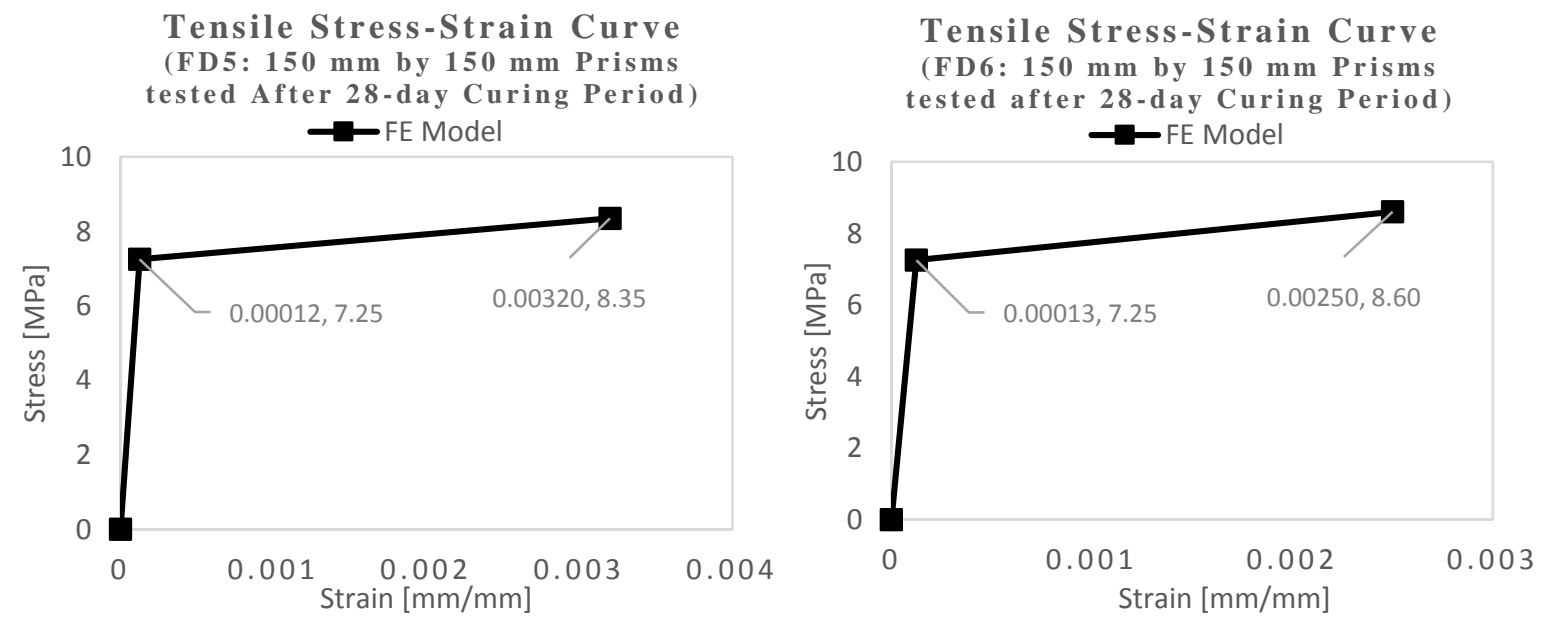

Figure 6- 35: Tensile stress-strain relationship of the prisms from mixes FD5 and FD6 


\subsubsection{Discussion of Finite Element Analysis Results}

According to the classification criteria of THFRC introduced in Section 6.2, Figure 6-36 plots the results of cracking strength $\left(f_{c r}\right)$, ultimate tensile strain $\left(\varepsilon_{t u}\right)$ and hardening ratio $\left(f_{F u} / f_{c r}\right)$ obtained from finite element analysis. It is evident that all sets of prisms have a cracking strength greater than $5 \mathrm{MPa}$ except for the 150 $\mathrm{mm}$ by $150 \mathrm{~mm}$ by $500 \mathrm{~mm}$ prisms from mix FD4. However, the $100 \mathrm{~mm}$ by $100 \mathrm{~mm}$ by $370 \mathrm{~mm}$ prisms containing the same cementitious materials and fiber contents satisfy the cracking strength criterion. As required by ASTM C1856 (2017), the fibers should be $15 \mathrm{~mm}$ to $20 \mathrm{~mm}$ long for $100 \mathrm{~mm}$ by $100 \mathrm{~mm}$ prisms, and $20 \mathrm{~mm}$ to $25 \mathrm{~mm}$ long for $150 \mathrm{~mm}$ by $150 \mathrm{~mm}$ prisms (refer to Table 4-1). Thus, owing to the $13 \mathrm{~mm}$ long fibers in mix FD4, the degree of fiber alignment recedes in $150 \mathrm{~mm}$ by $150 \mathrm{~mm}$ prisms, leading to low flexural and tensile strength results. Other scale effects might be in action, however, that would need to be explored in the future. As depicted in Figure 6-36b and c, all sets of prisms have an ultimate tensile strain greater than $0.001 \mathrm{~mm} / \mathrm{mm}$ and a hardening ratio greater than 1.1 . Therefore, all design mixes considered in the experimental progran can be classified as THFRC also based on the finite element analysis results.

Figure 6-37 plots the ultimate tensile strength results $\left(f_{F u}\right)$ obtained from finite element analysis. It is evident that the strengths of mixes FD1 and FD2 are greater than those of mixes KI1 and LD1 regardless of casting methodology. Besides, with regards to the prisms tested during the Round Robin Testing Program (i.e., prisms from mixes FD3, KI2 and LD2), York University's results are the greatest while Queen's University's results are the lowest in general. Additionally, support condition 1 (SC1) results in greater ultimate tensile strength results than SC2. Moreover, the $150 \mathrm{~mm}$ by $150 \mathrm{~mm}$ by $500 \mathrm{~mm}$ prisms from mixes FD5 and FD6 comprising $20 \mathrm{~mm}$ long fibers exhibit higher strengths than those from mix FD4 comprising $13 \mathrm{~mm}$ long fibers. 


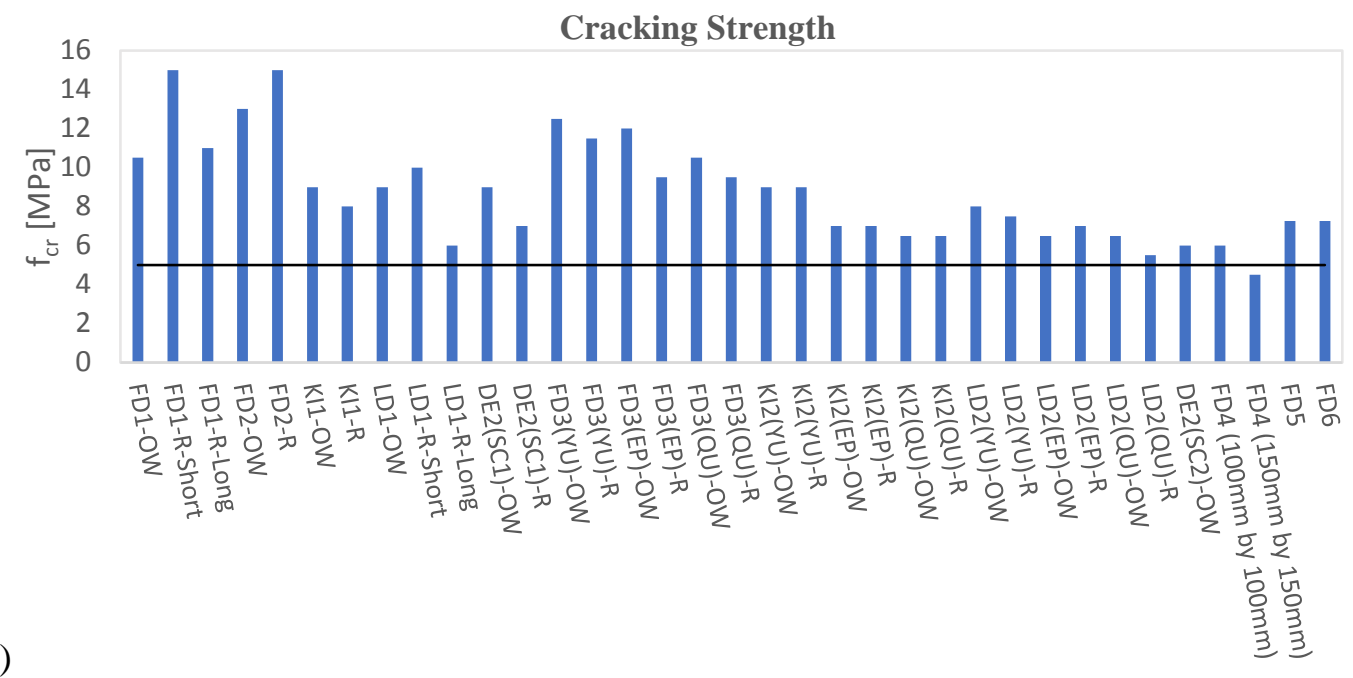

(a)

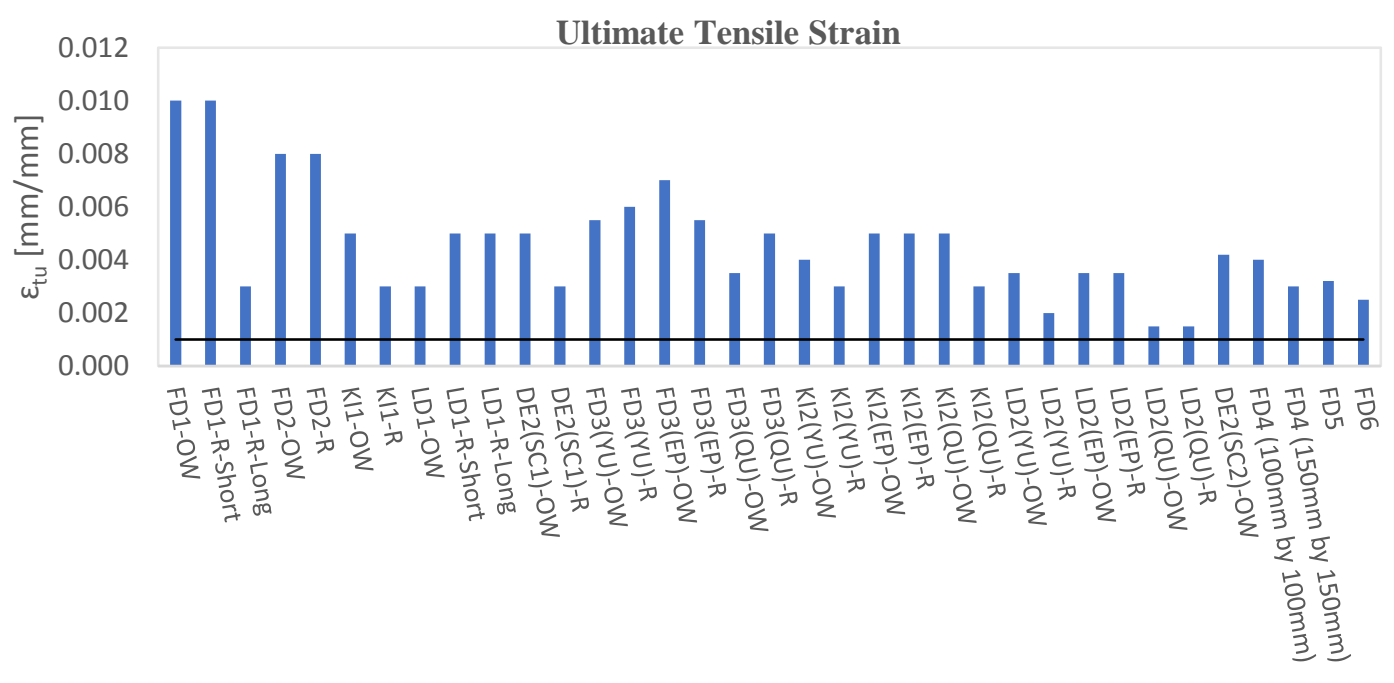

(b)

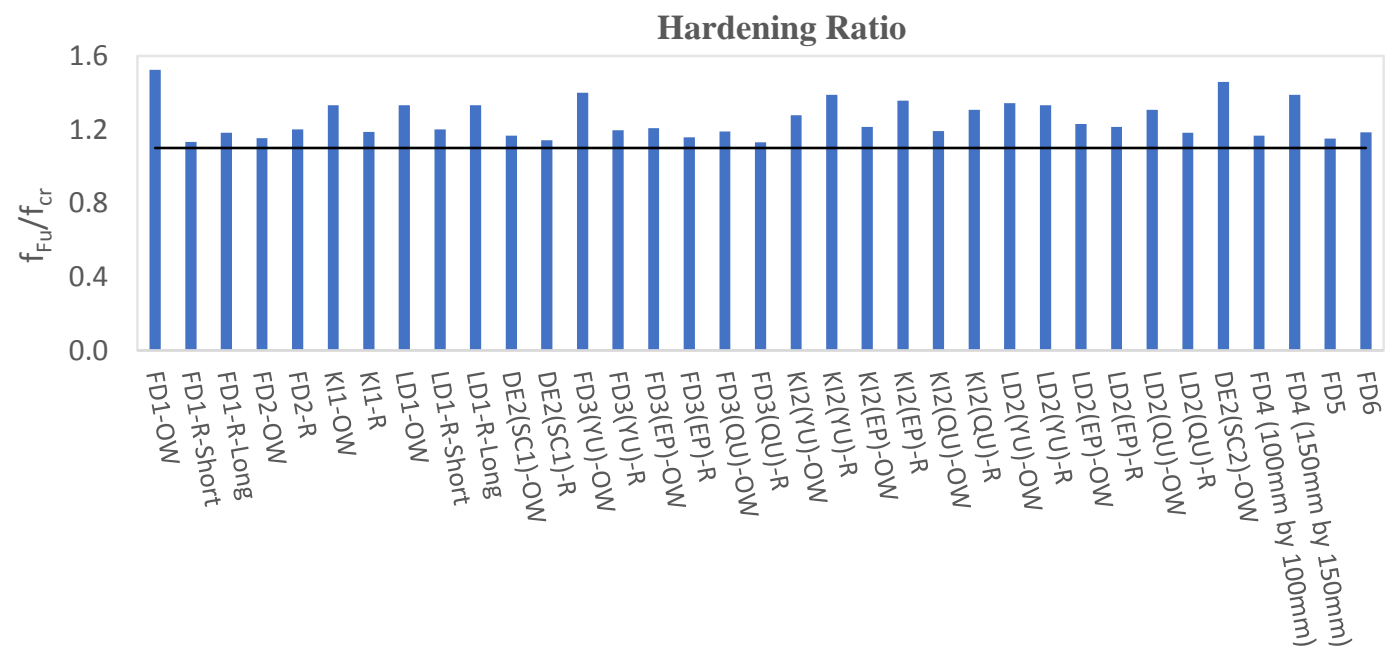

Figure 6- 36: Classification criteria of THFRC based on finite element analysis results: (a) Cracking strength; (b) Ultimate tensile strain; and (c) Hardening ratio (each bar represents the average of a triplicate set of identical 


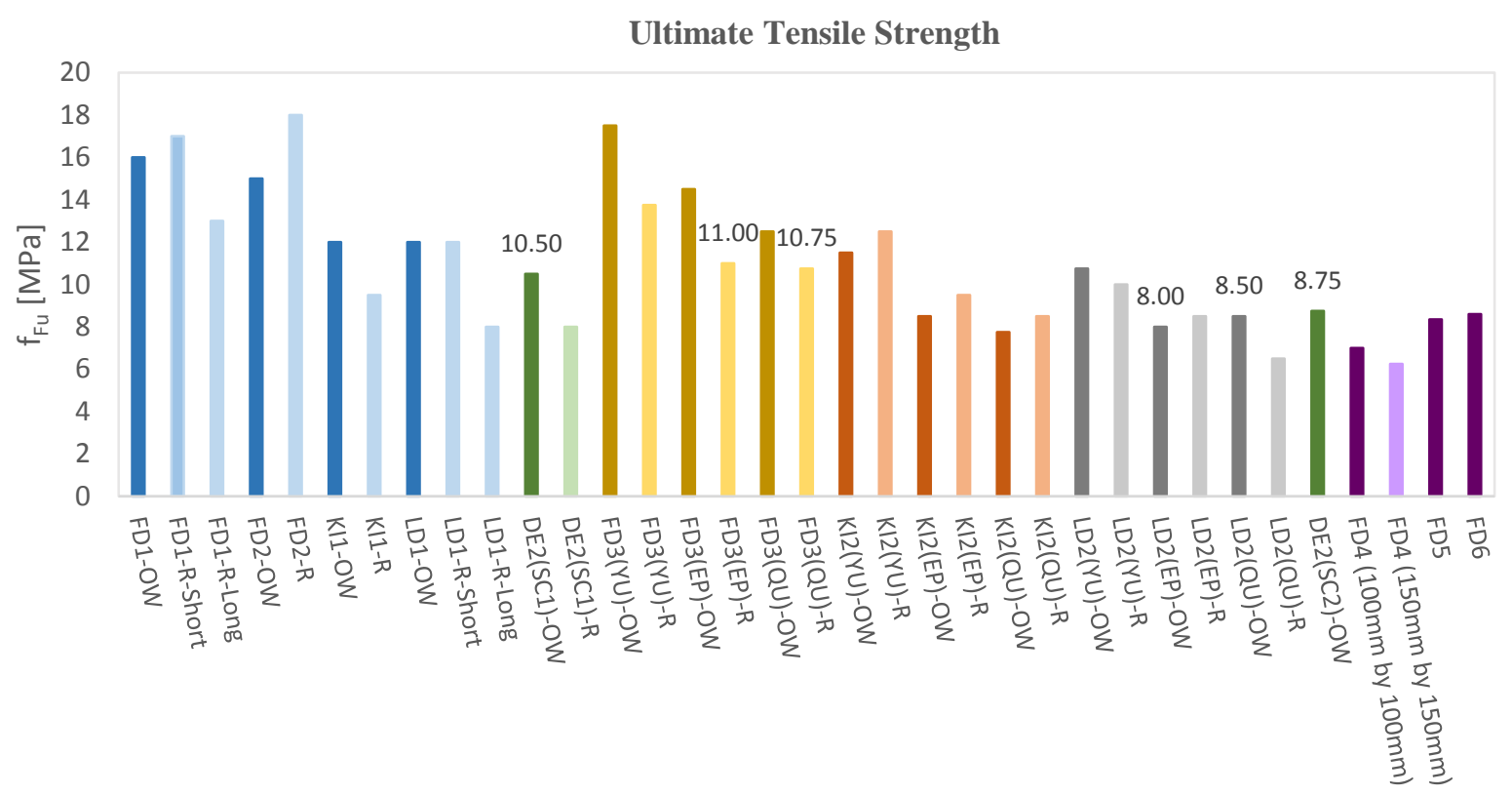

Figure 6- 37: Ultimate tensile strength results obtained from finite element analysis (each bar represents the average of a triplicate set of identical tests)

\subsection{True Splitting Tensile Strength}

Splitting tensile test was conducted by applying diametrically compressive load along the two opposite sides of the cylinder specimen, and as depicted in Figure 6-38, the applied distributed load resulted in a biaxial stress state within the cylinder. Thus, the splitting tensile strength obtained experimentally can not be compared with the uniaxial tensile strength obtained from DTTs. In this context, true splitting tensile strength was calculated by multiplying apparent splitting tensile strength results with a correction factor which was assumed to be equal to the ratio of longitudinal loading plane area to the circumference area of the cylinder (i.e., $1 / \pi)$. The resulting true splitting tensile strength results $\left(\sigma_{s}\right)$ are summarized in Table 615.

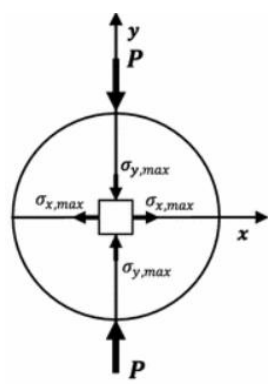

Figure 6- 38: Biaxial stress state developed in the cylinder under diametrically compressive loading (Carmona and Aguado 2012) 
Table 6- 15: True splitting tensile strength results

\begin{tabular}{|c|c|c|c|}
\hline Mix & $\boldsymbol{\sigma}_{\boldsymbol{s}}[\mathbf{M P a}]$ & $\mathbf{M i x}$ & $\boldsymbol{\sigma}_{\boldsymbol{s}}[\mathbf{M P a}]$ \\
\hline FD1 & 7.99 & FD2 & 8.21 \\
\hline FD3 & 7.96 & KI1 & 6.53 \\
\hline KI2 & 7.51 & LD1 & 8.12 \\
\hline LD2 & 7.45 & DE2 (at 28 days) & 7.99 \\
\hline
\end{tabular}

\subsection{Correlation of Tensile Strength Results}

\subsubsection{Direct Tension Test and the Inverse Analysis Method}

Figure 6-39 depicts the tensile strength results obtained from DTT and the inverse analysis of the flexural tests: the vertical axis provides the average tensile strength results of each type of direct-tension specimen from mixes FD1, FD2, KI1, LD1 and DE2, and the horizontal axis provides the average cracking strength of each set of OW prisms with an aspect ratio of 1 from mixes FD1, FD2, KI1, LD1 and DE2. The average cracking strength of each set of R prisms were not used to compare with DTT results because all directtension specimens were cast by pouring fresh concrete from one end of the mold in a single layer. In addition, the cracking strength result of the prisms from mix DE2 tested with SC1 was plotted instead of those from the same mix but tested with SC2 because prisms from mixes FD1, FD2, KI1 and LD1 were also tested with SC1. As illustrated in Figure 6-39, it is evident that the cracking strength results derived from the inverse analysis are generally greater than the tensile strengths obtained from DTTs. The difference in strength is expected, considering the different stress states developed in FPBT and DTT. With regards to DTT, a state of uniaxial tensile stress is developed (i.e., all the fibers of the specimen are subjected to approximately the same amount of tension stresses). In the case of FPBT, a biaxial state of stress is developed due to deflection and curvature, and only a portion of the critical cross-sectional plane is subjected to normal tension stresses. Therefore, DTT provides lower strength values which are believed to be closer to the true tensile strength of the material.

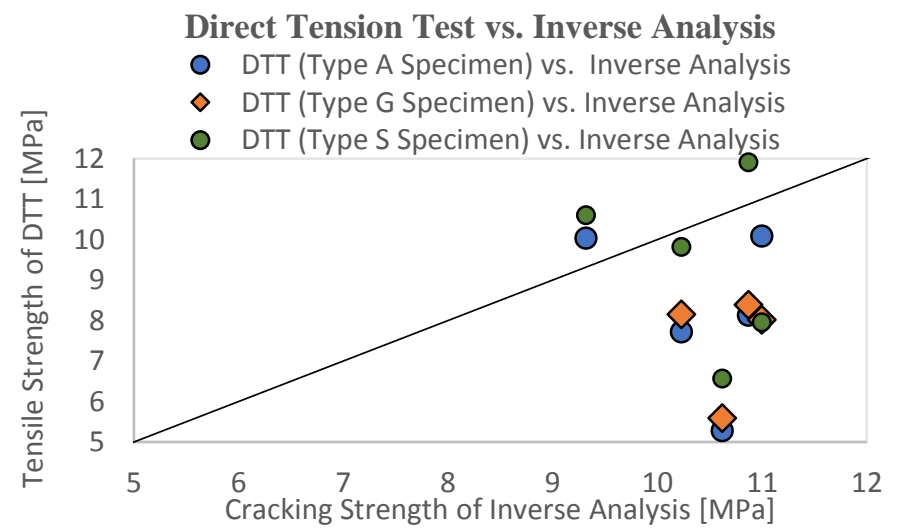

Figure 6- 39: Relationship between direct tension test and inverse analysis (each point represents the average of a triplicate set of identical tests) 


\subsubsection{Direct Tension Test and the Calibrated Empirical Expression}

Figure 6-40 depicts the tensile strength results obtained from DTT and the calibrated empirical expression: the vertical axis provides the average tensile strength results of the direct-tension specimens from mixes FD1, FD2, KI1, LD1 and DE2, and the horizontal axis provides the cracking strength calculated based on the average cylinder compressive strength of the corresponding design mixture. It is evident that DTT provides greater strength values than the calibrated empirical expression in general.

Direct Tension Test vs. Calibrated Empirical Expression

- DTT (Type A Specimen) vs. Empirical Expression

$\diamond$ DTT (Type G Specimen) vs. Empirical Expression

- DTT (Type S Specimen) vs. Empirical Expression

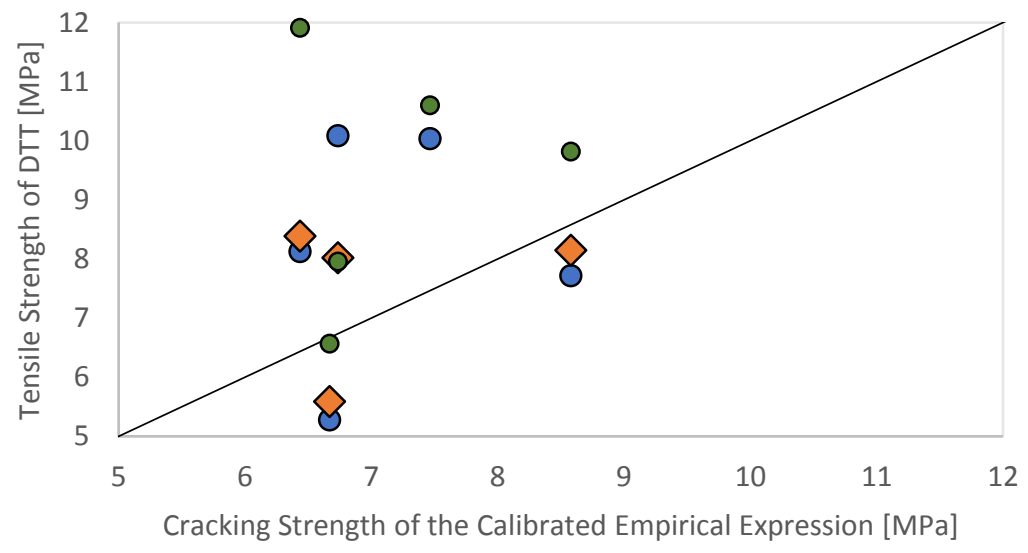

Figure 6- 40: Relationship between direct tension test and the calibrated empirical expression (each point represents the average of a triplicate set of identical tests)

\subsubsection{Direct Tension Test and Splitting Tensile Test}

Figure 6-41 illustrates the tensile strength results obtained from DTT and splitting tensile test: the vertical axis provides the average tensile strength results of each type of direct-tension specimens from mixes FD1, FD2, KI1, LD1 and DE2, and the horizontal axis provides the true splitting tensile strength results. The plot indicates that in general, the true splitting tensile strength values are close to the tensile strength values of DTTs: seven out of 14 points plotted in Figure 6-41 are located near the 45-degree line; five points are above the line; and two points are below the line. The difference is attributed to the deviation between the actual splitting failure plane developed of each test and the diametrical loading plane assumed for the correction factor (i.e., the diametrical loading plane was assumed to cross the cylinder's center of gravity). 


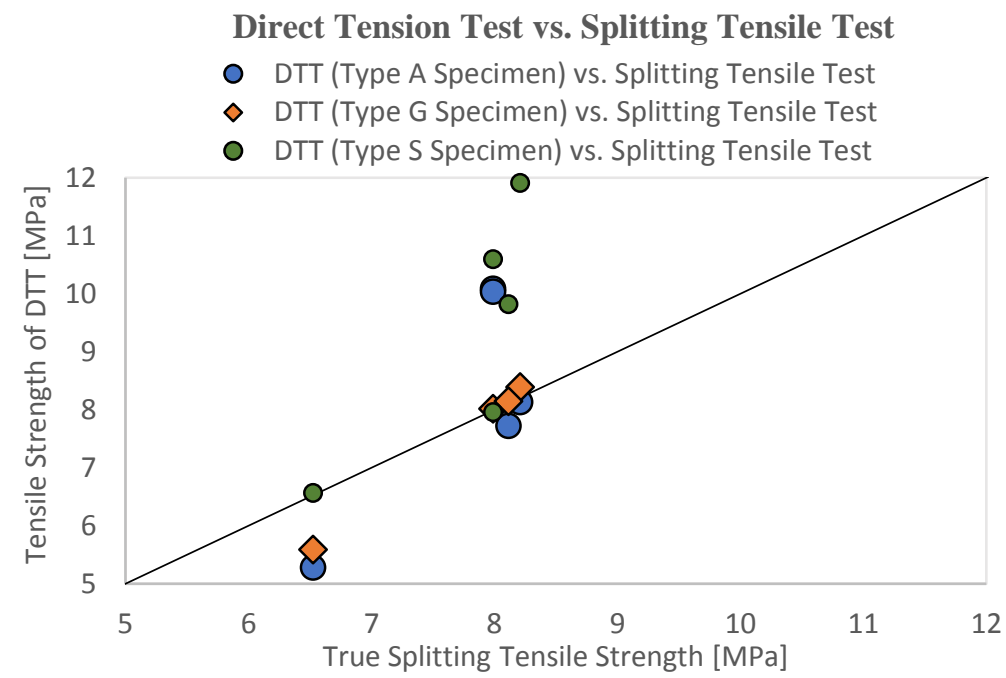

Figure 6- 41: Relationship between direct tension test and splitting tensile test (each point represents the average of a triplicate set of identical tests)

\subsubsection{Inverse Analysis Method and the Calibrated Empirical Expression}

Figure 6-42 illustrates the tensile strength results obtained from the inverse analysis method and the calibrated empirical expression: the vertical axis provides the average cracking strength results of each set of prisms with an aspect ratio of 1 (both OW and R prisms) obtained from the inverse analysis method, and the horizontal axis provides the cracking strength results calculated with the calibrated empirical expression. It is evident that the inverse analysis method generally provides greater cracking strength values than the calibrated empirical expression regardless of casting methodology.

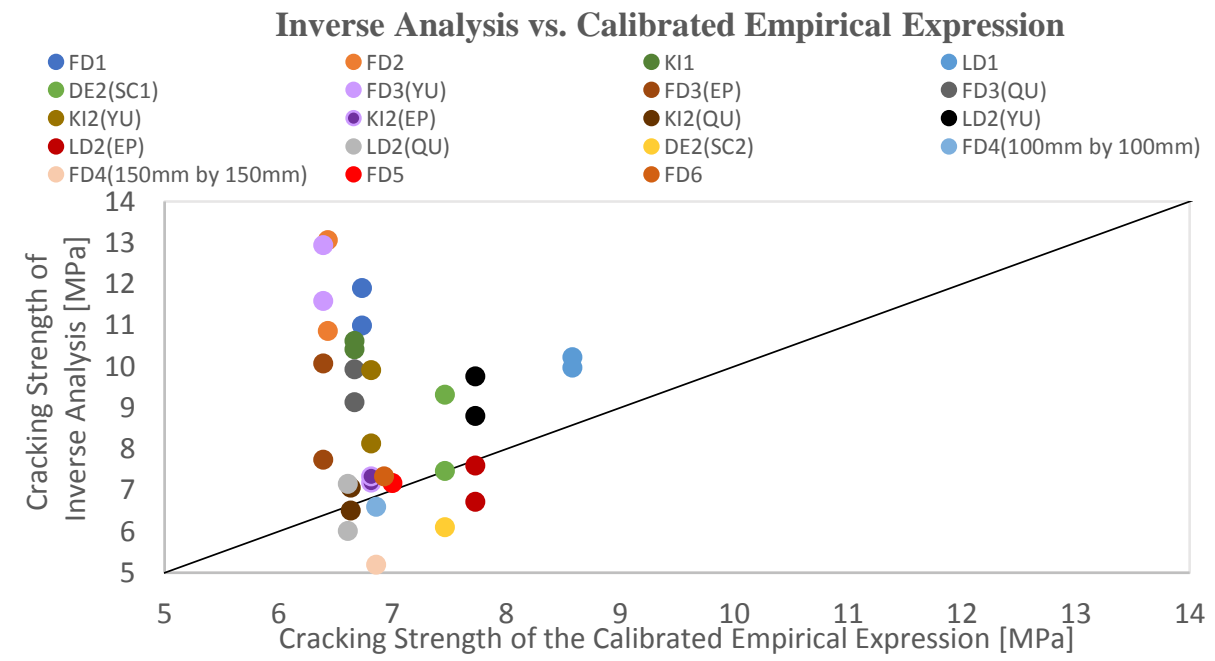

Figure 6- 42: Relationship between the inverse analysis method and the calibrated empirical expression (each point represents the average of a triplicate set of identical tests) 


\subsubsection{Inverse Analysis Method and Splitting Tensile Test}

Tensile strength results obtained from the inverse analysis method and splitting tensile test are illustrated in Figure 6-43, where the vertical axis provides the average cracking strength results of each set of prisms obtained from the inverse analysis, and the horizontal axis provides the true splitting tensile strength results. Two points were plotted for each design mix to compare the OW and R prism results with the true splitting tensile strength results, respectively. It is evident that the inverse analysis method provides greater cracking strength values than the splitting tensile test in general.

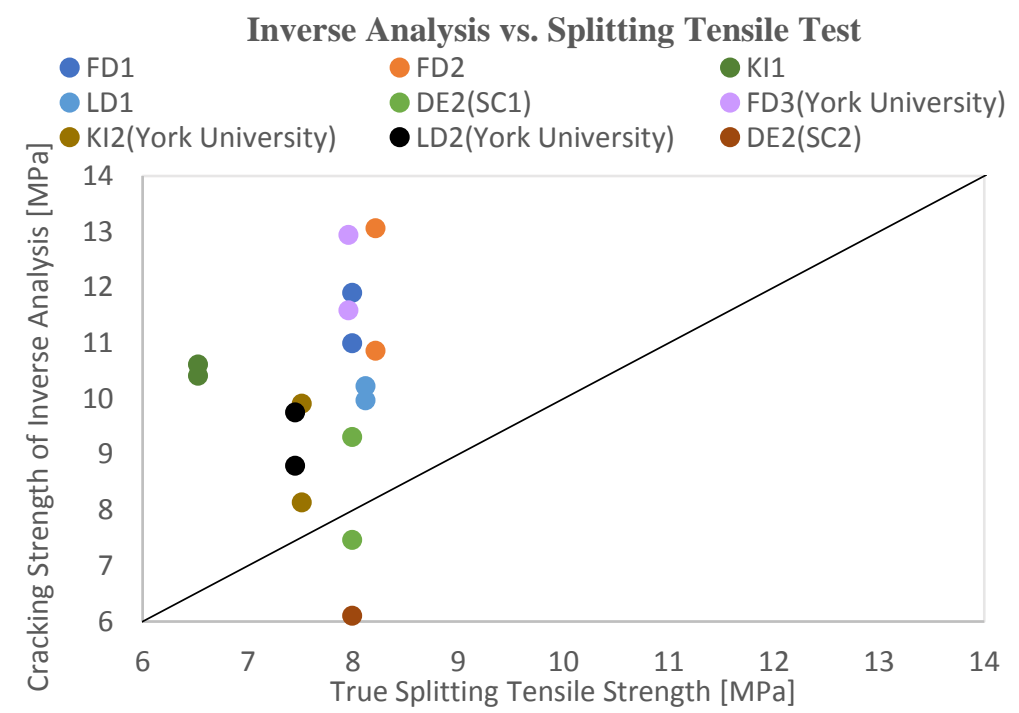

Figure 6- 43: Relationship between the inverse analysis method and splitting tensile test (each point represents the average of a triplicate set of identical tests)

\subsubsection{Inverse Analysis and Finite Element Analysis}

The relationship between inverse analysis and finite element analysis is depicted in Figure 6-44. Two points were plotted for each design mix to compare the results of OW and R prisms, and it is evident that inverse analysis generally provides greater values than finite element analysis in terms of both cracking strength and ultimate tensile strength. 
Inverse Analysis vs. Finite Element Analysis

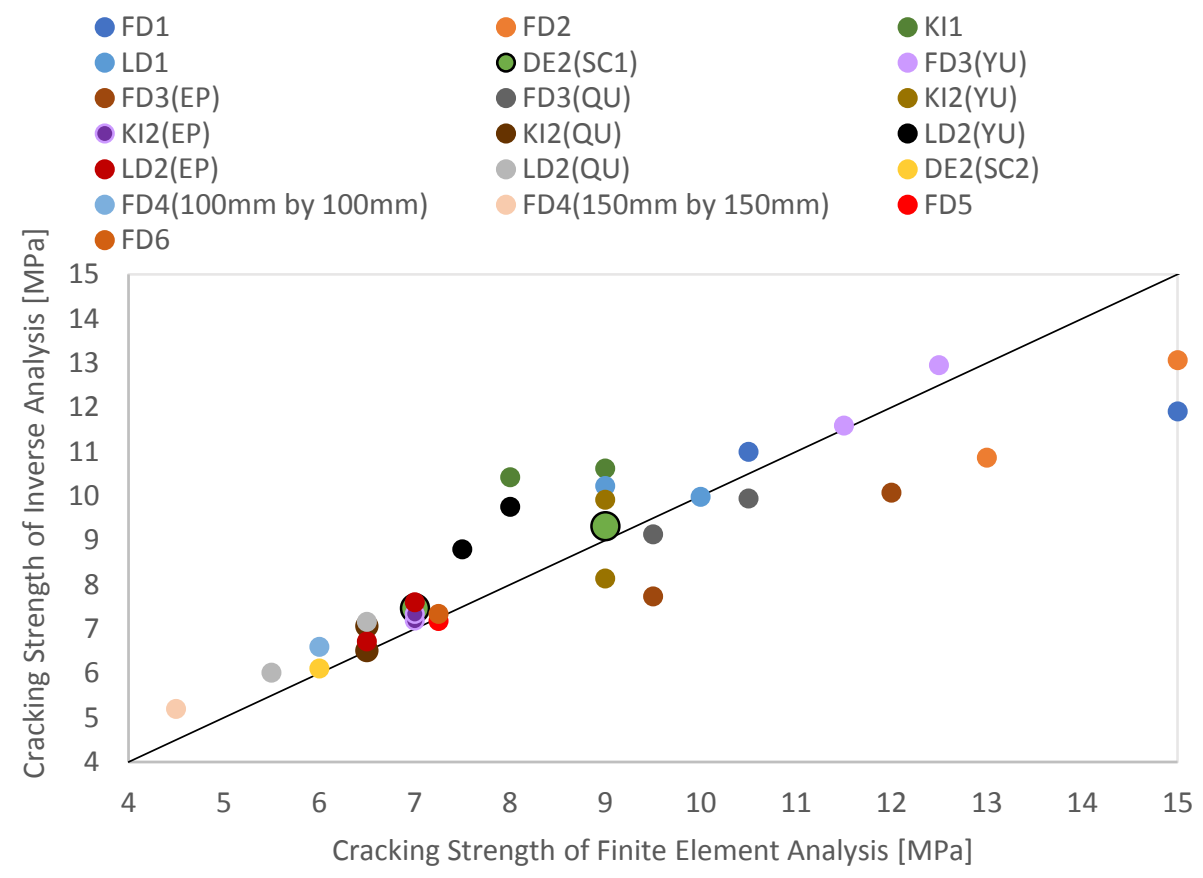

(a)

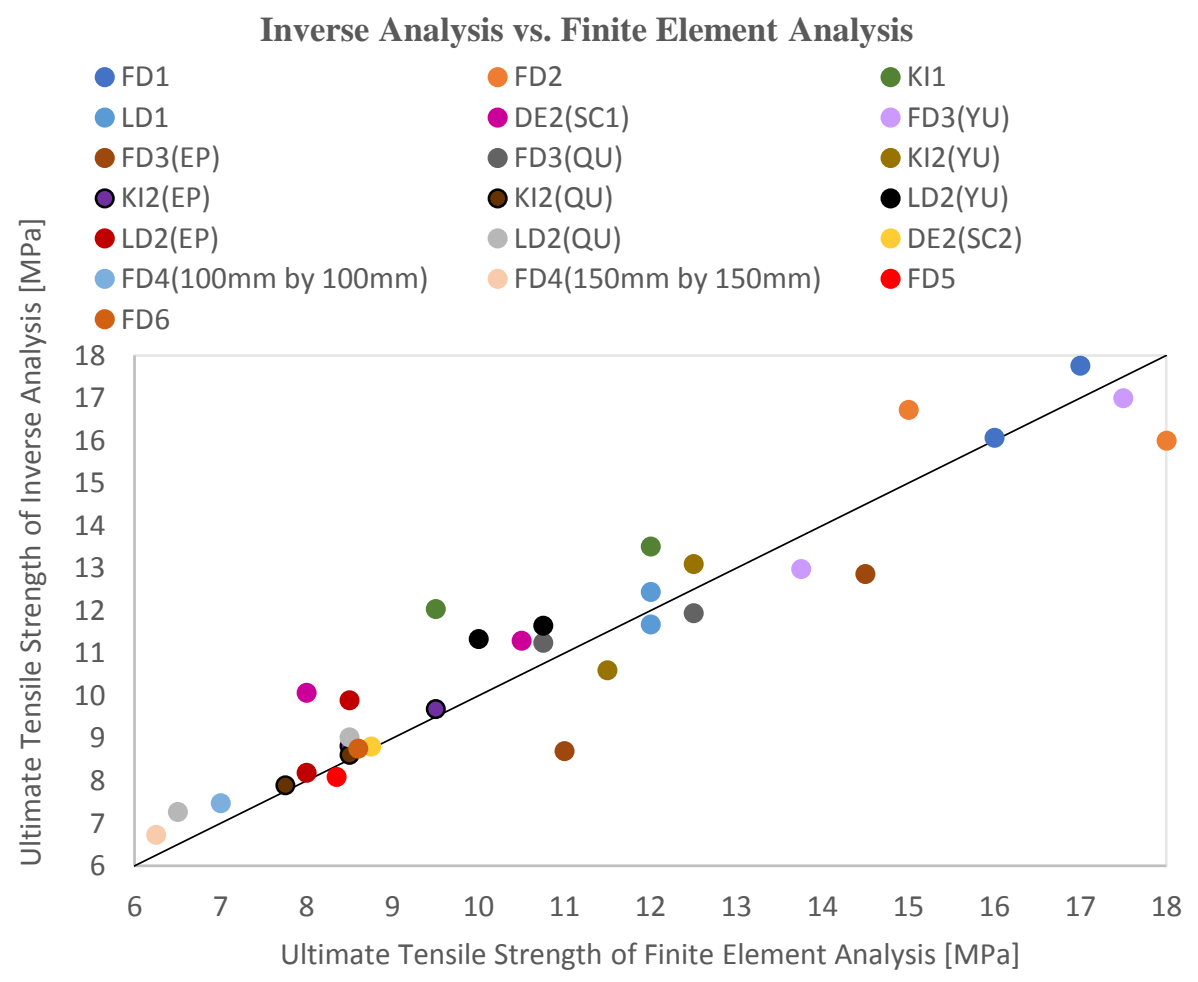

Figure 6- 44: Relationship between inverse analysis and finite element analysis: (a) Cracking strength; and (b) Ultimate tensile strength (each point represents the average of a triplicate set of identical tests) 


\subsubsection{Finite Element Analysis and the Calibrated Empirical Expression}

Figure 6-45 illustrates the relationship between finite element analysis and the calibrated empirical expression. Since the tensile stress-strain relationship utilized for modelling R short prisms from mixes FD1 and LD1 can not be verified by the response of long prisms (refer to Sections 6.3.4.1 and 6.3.4.3), three points were plotted for mixes FD1 and LD1, respectively, representing the finite element analysis results of OW prisms (both short and long prisms), R short prisms and R long prisms. In general, finite element models provide greater cracking strength values than the calibrated empirical expression.

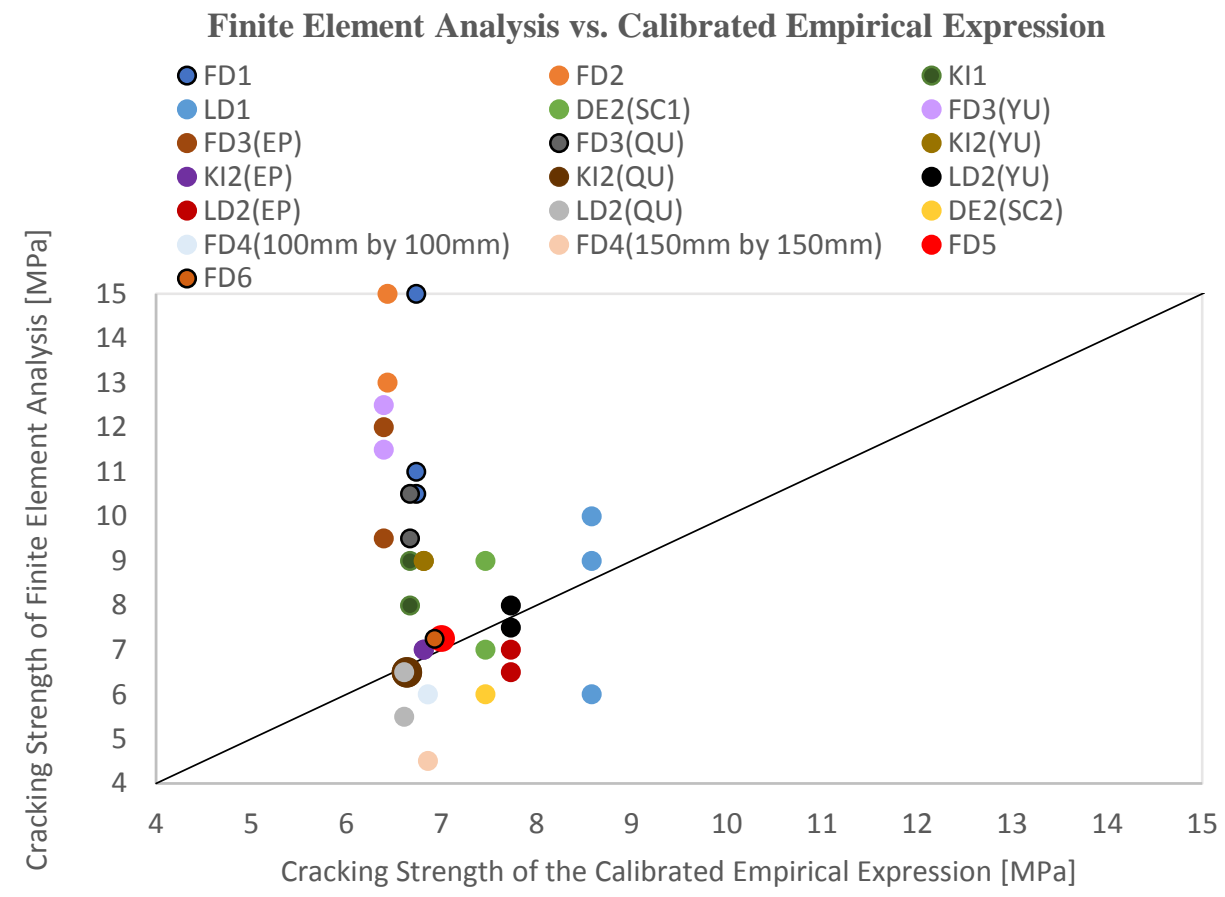

Figure 6- 45: Relationship between finite element analysis and the calibrated empirical expression (each point represents the average of a triplicate set of identical tests)

\subsubsection{Finite Element Analysis and Direct Tension Test}

Figure 6-46 illustrates the relationship between finite element analysis and DTT based on the experimental results of mixes FD1, FD2, KI1, LD1 and DE2 (SC1). The average tensile strength results of each type of direct-tension specimens were only compared with the cracking strength results of OW prisms obtained from VecTor2 because all direct-tension specimens were cast from one end of the mold with a single layer 
of fresh concrete. It is evident that finite element models provide greater cracking strength values than DTT in general.

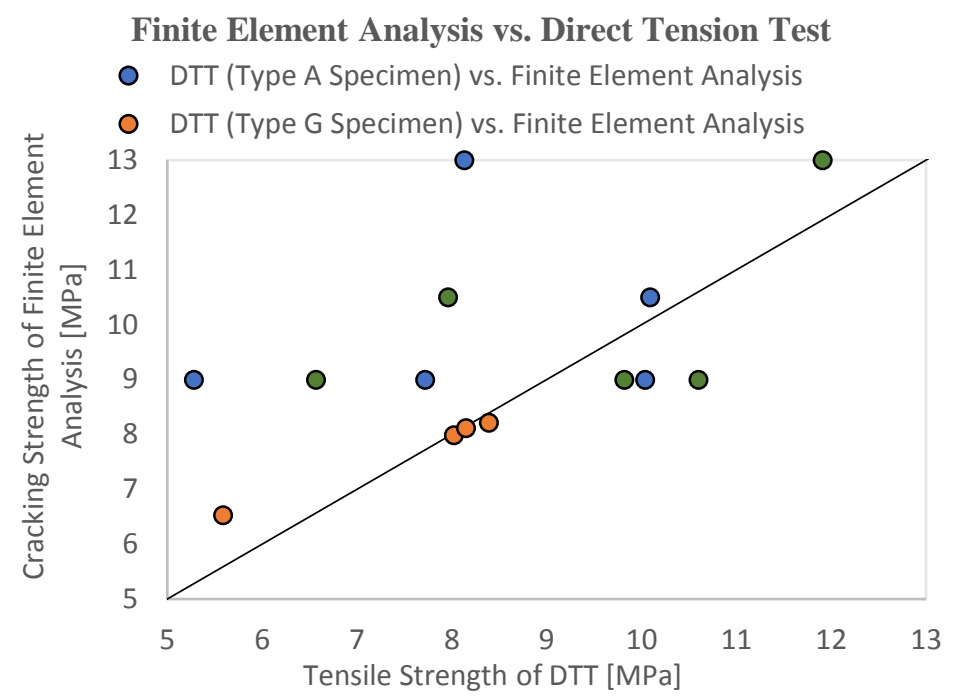

Figure 6- 46: Relationship between finite element analysis and direct tension test (each point represents the average of a triplicate set of identical tests)

\subsubsection{Finite Element Analysis and Splitting Tensile Test}

Figure 6-47 illustrates the relationship between finite element analysis and splitting tensile test, and it is evident that finite element models generally provide greater cracking strength values than splitting tensile test.

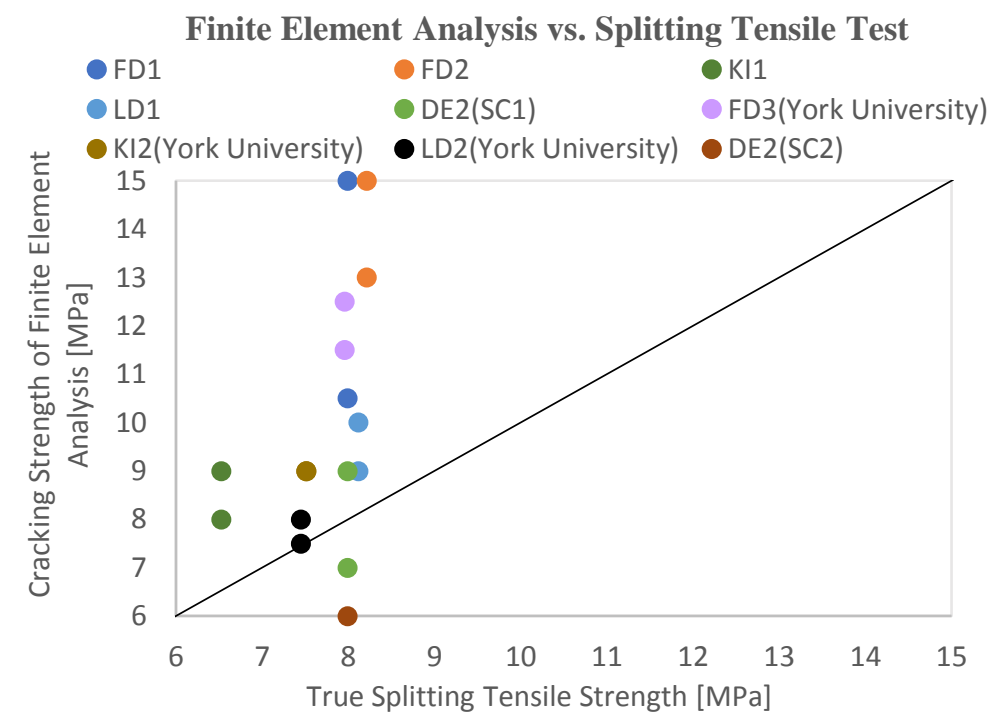

Figure 6- 47: Relationship between finite element analysis and splitting tensile test (each point represents the average of a triplicate set of identical tests) 


\subsubsection{Summary}

Figure 6-48 was plotted to summarize the relationship between the tensile strength results obtained from DTT, inverse analysis of FPBT, splitting tensile test, nonlinear finite element analysis and the calibrated empirical expression. It is evident that the inverse analysis method and finite element models generally provide relatively close values, and these values are generally greater than those obtained from other approaches. In addition, the calibrated empirical expression provides the lowest values in general, and the results obtained from DTT and splitting tensile test fall into the middle.

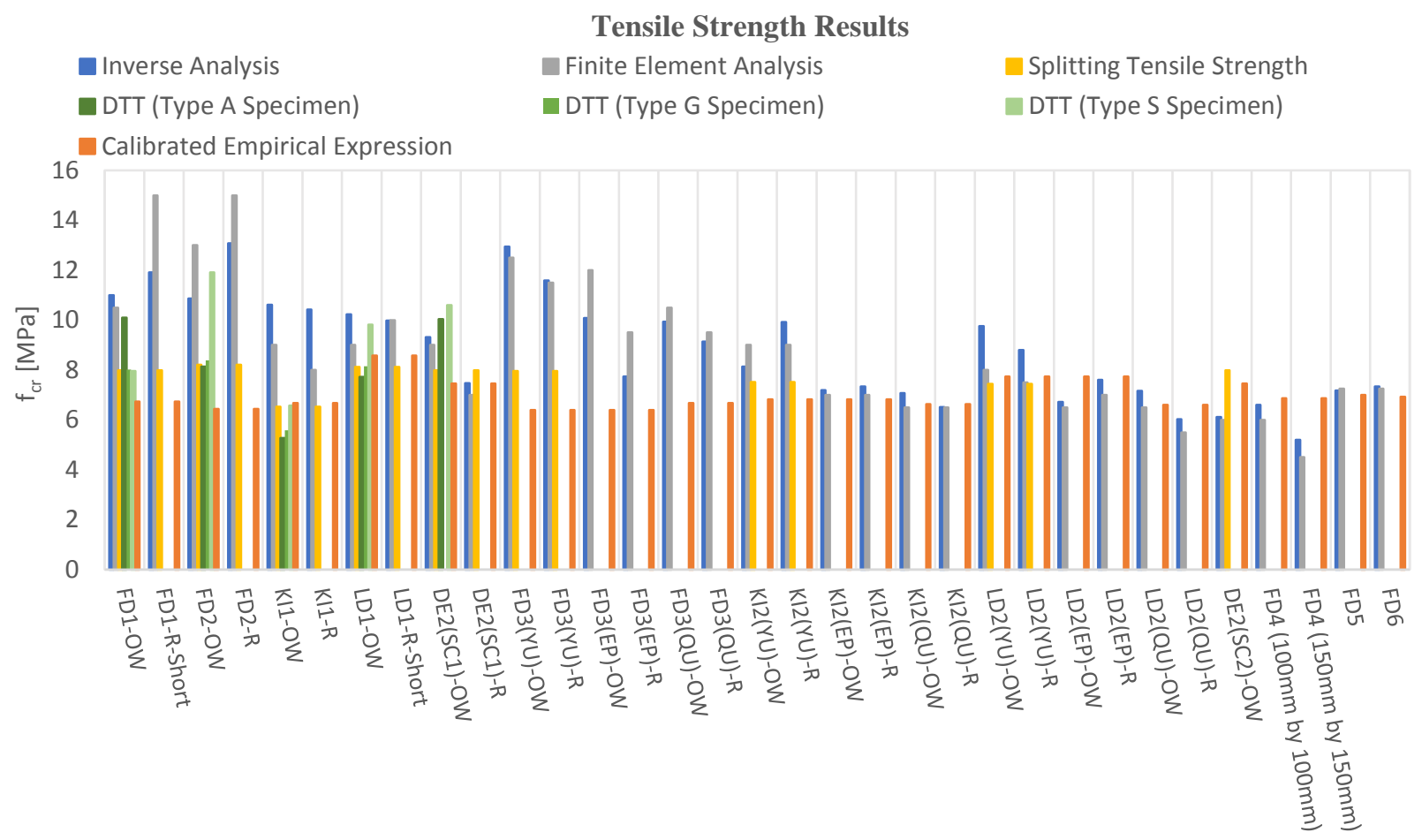

Figure 6- 48: Correlation of tensile strength results through direct tension test, the inverse analysis method, splitting tensile test, finite element analysis and the calibrated empirical expression (each bar represents the average of a triplicate set of identical tests)

\subsection{Effect of Flowability on Tensile Strength}

The results of average cracking strength and ultimate tensile strength of each set of prisms were plotted against the spread values measured immediately after concrete mixing. As illustrated in Figure 6-49, there is a downward trend in between the dashed lines, indicating that both cracking strength and ultimate tensile 
strength decrease when flowability of the design mix increases. For instance, segregation issue was observed through the cracks of bending prisms from mix FD4 due to the fact that mix FD4 was very flowable with a spread value of $247.5 \mathrm{~mm}$, leading to relatively low flexural and tensile strengths.

Cracking Strength vs. Flowability

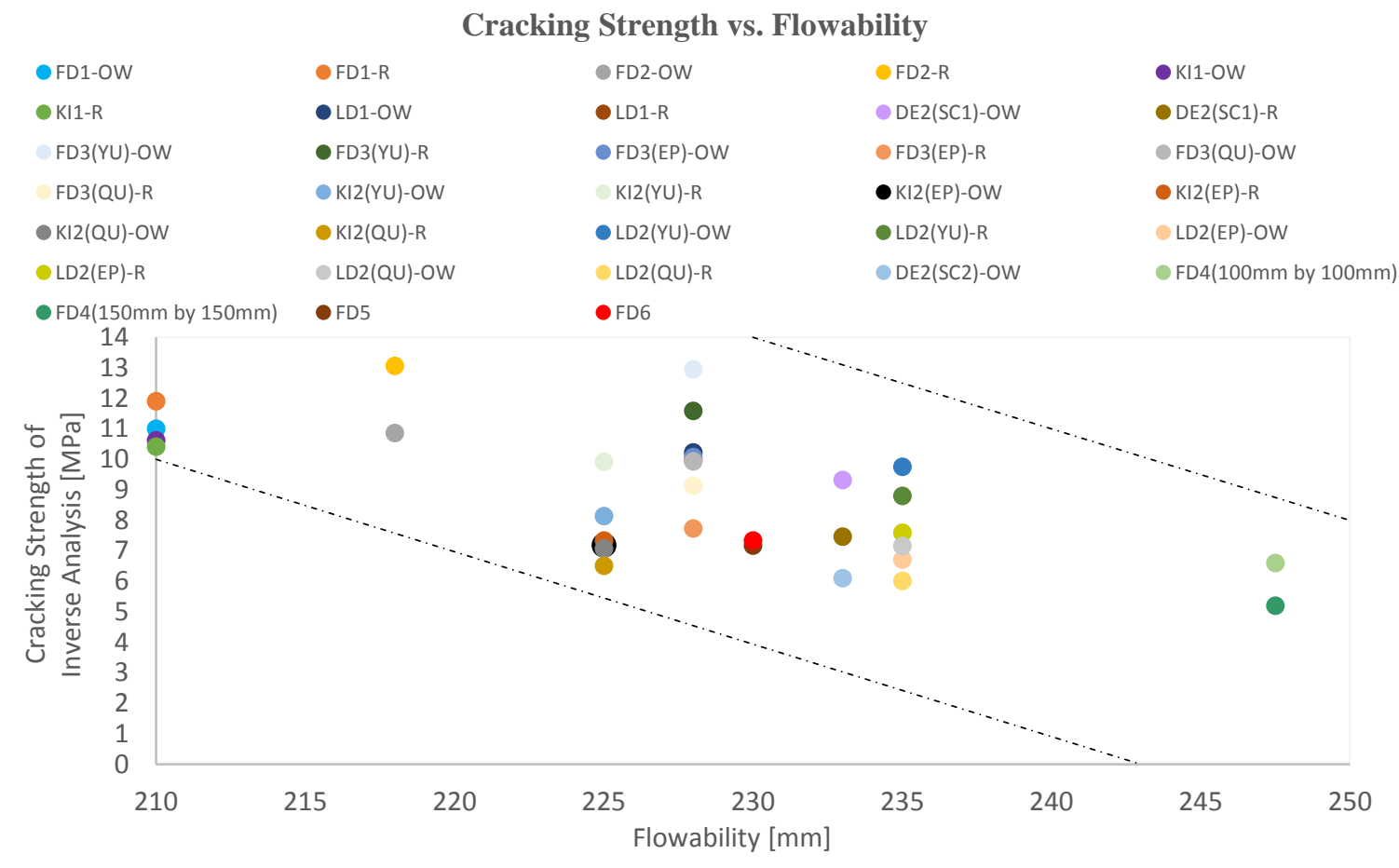

(a)

Ultimate Tensile Strength vs. Flowability

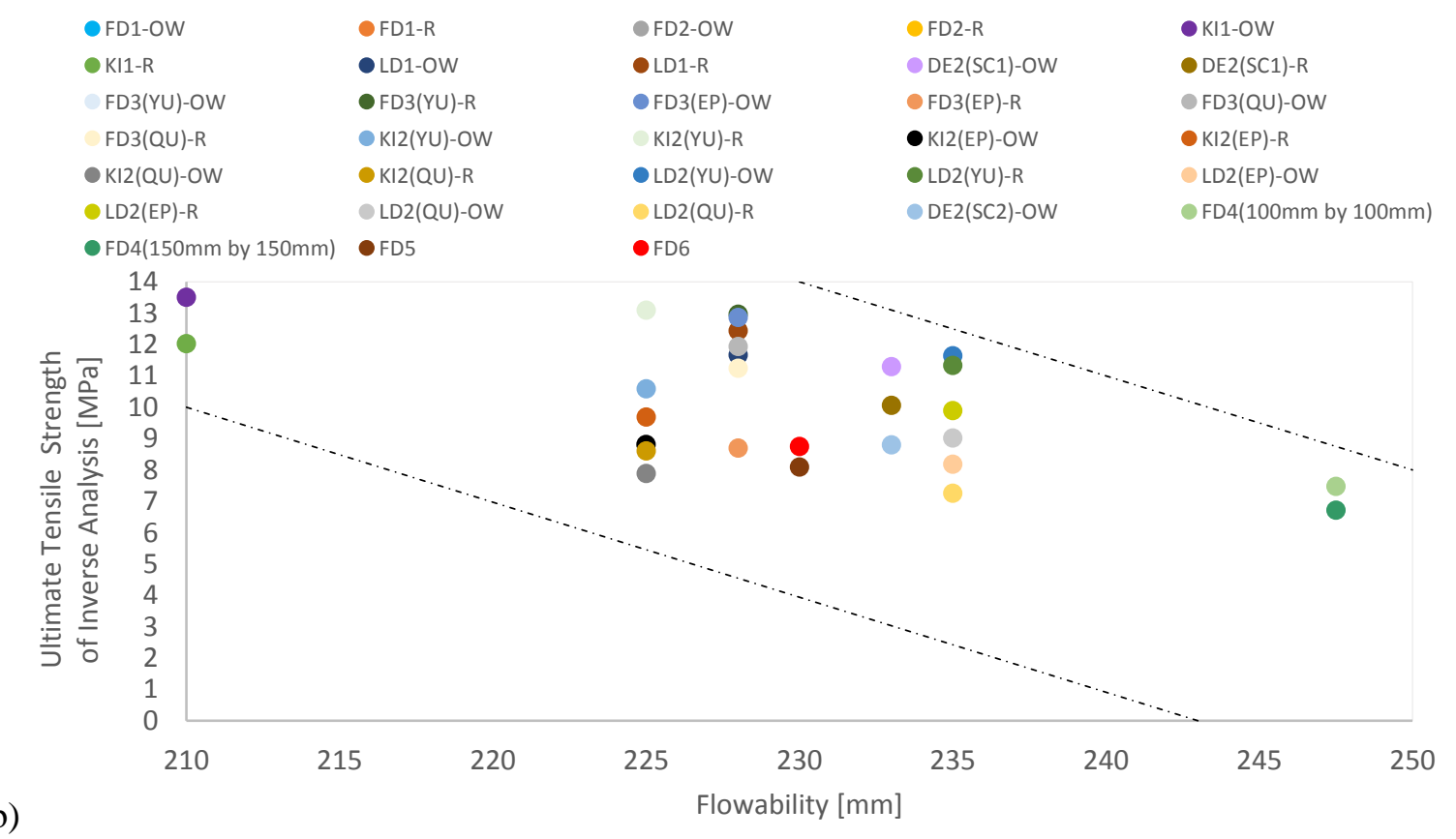

Figure 6- 49: Effect of flowability on tensile strength: (a) Cracking strength results of inverse analysis against spread values; and (b) Ultimate tensile strength results of inverse analysis against spread values 


\subsection{Important Parameters on Flexural Strength}

\subsubsection{Aspect Ratio of the Bending Prism}

Flexural strength result of each prism (both OW and R prisms) from mixes FD1, FD2, KI1, LD1 and DE2(SC1) tested during Phase 1 of the experimental program was plotted in Figure 6-50 to study the effect of aspect ratio of the bending prism on flexural strength, where the vertical axis provides the flexural strength results of long prisms ( $75 \mathrm{~mm}$ by $75 \mathrm{~mm}$ by $500 \mathrm{~mm}$ prisms), and the horizontal axis provides the flexural strength results of short prisms ( $75 \mathrm{~mm}$ by $75 \mathrm{~mm}$ by $280 \mathrm{~mm}$ prisms). Note that the results of the prisms which failed in torsion or shear were excluded. In general, the prisms with an aspect ratio of 1 exhibited greater flexural strength than those with an aspect ratio of 2.

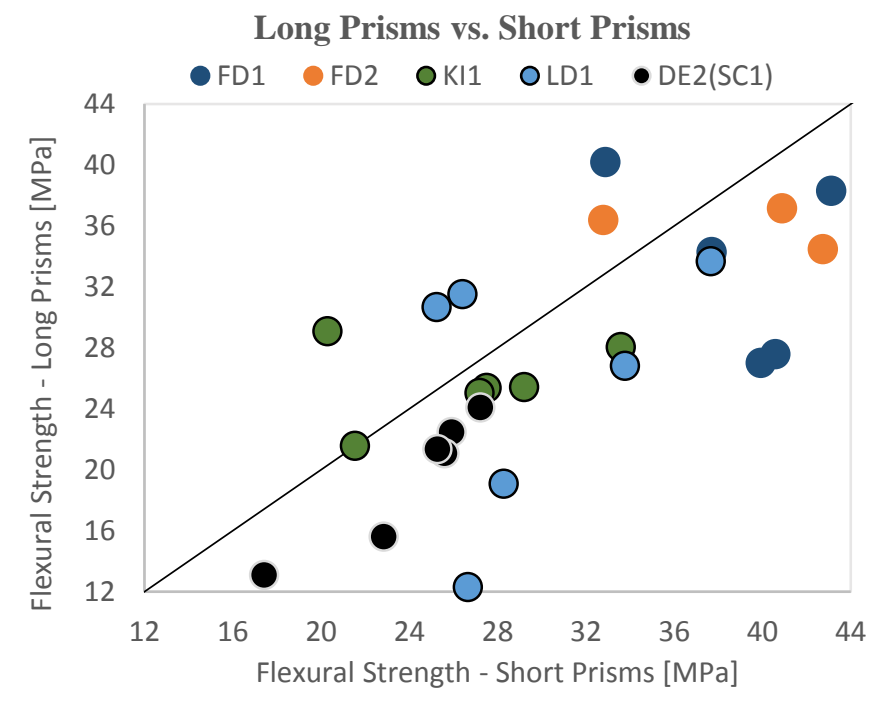

Figure 6- 50: Effect of aspect ratio of the bending prism on flexural strength

\subsubsection{Casting Methodology}

Flexural strength result of each prism tested during the experimental program was plotted in Figure 6-51, where the vertical axis provides the results of OW prisms, and the horizontal axis provides the results of $\mathrm{R}$ prisms. It is evident that OW prisms generally exhibited greater flexural strength than R prisms. 


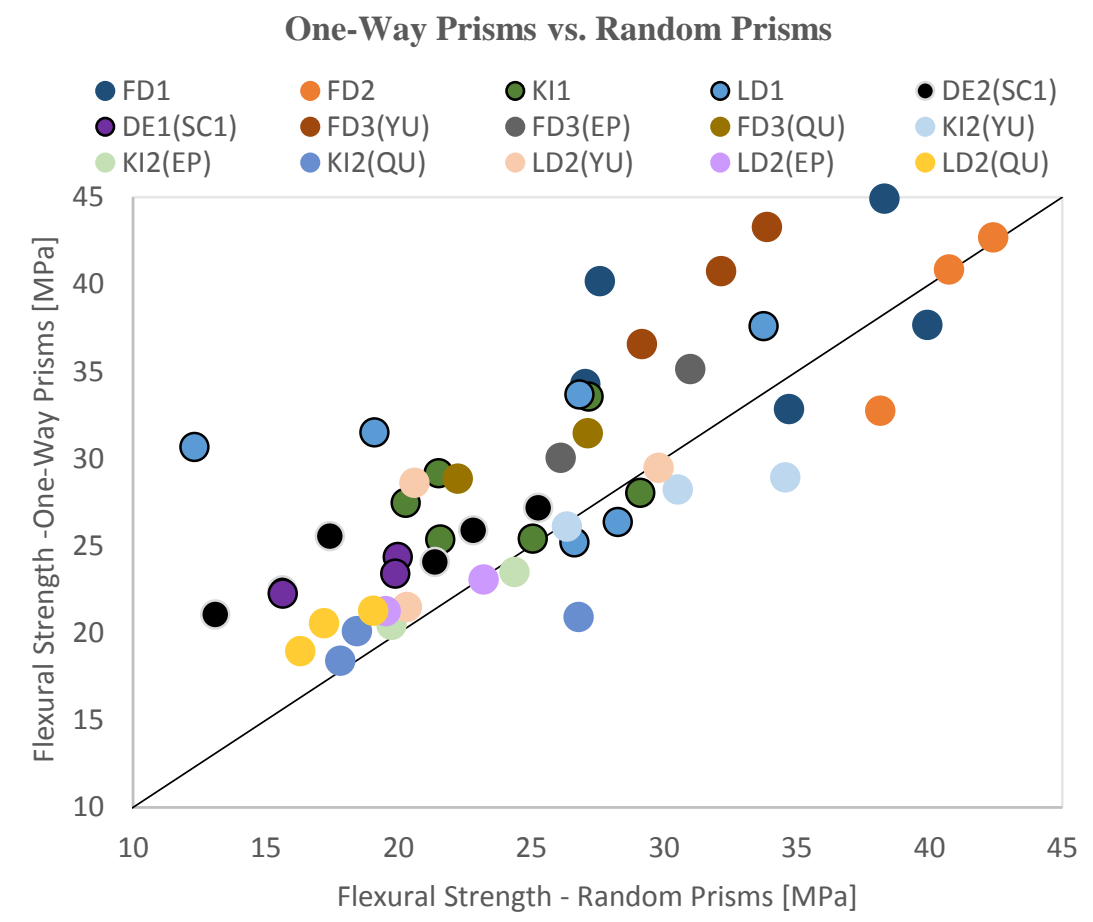

Figure 6- 51: Effect of casting methodology on flexural strength

In addition, as illustrated in Figure 6-52a, for the prisms from mixes FD1, FD2, KI1 and LD1, one of the prisms from each set whose equivalent flexural strength was close to the average strength of the same set was cut into two pieces near the failure plane, and the number of steel fibers within $1 \mathrm{~cm}^{2}$ area near the tension face of the prism was counted. As depicted in Figure 6-52b, the presence of a larger number of steel fibers near the tension face of the prism generally led to greater flexural strength regardless of the aspect ratio of the bending prism. For instance, the average flexural strength of OW long prisms from mix LD1 was $31.96 \mathrm{MPa}$ corresponding to the presence of 42 steel fibers within $1 \mathrm{~cm}^{2}$ area, and the average flexural strength of R long prisms of LD1 was 19.41 MPa corresponding to the presence of 22 steel fibers within 1 $\mathrm{cm}^{2}$ area. 


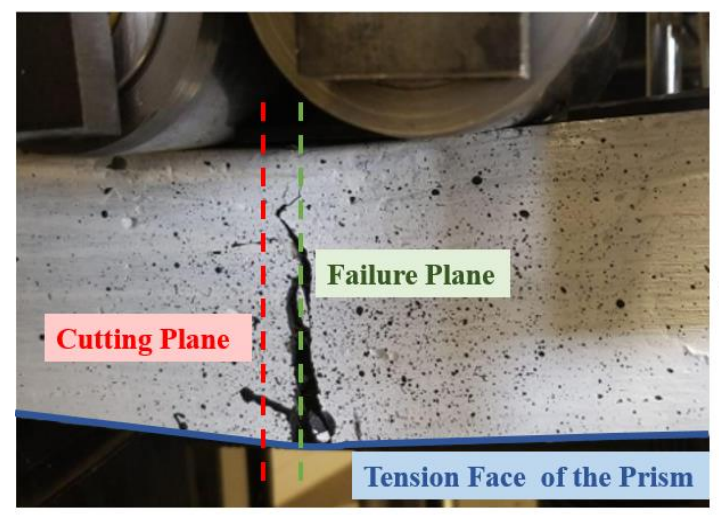

(a)

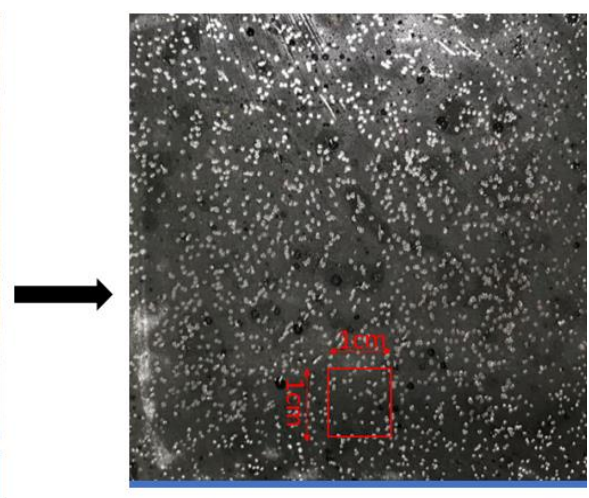

Tension Face of the Prism

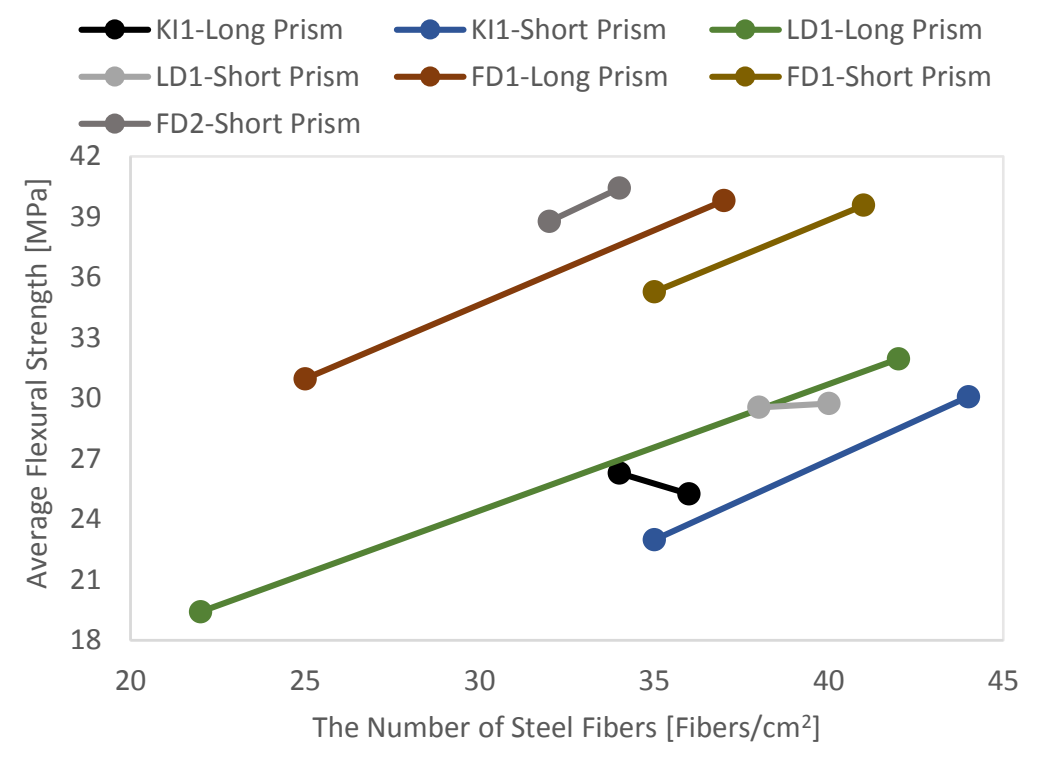

Figure 6- 52: The number of steel fibers present near the tension face of the prism: (a) Cutting plane and the area of interest; and (b) Relationship between the number of steel fibers present near the tension face of the prism and average flexural strength

\subsubsection{Volumetric Ratio of Steel Fibers}

Flexural strength result of each prism from mixes DE1 and DE2 tested with SC1 was plotted in Figure 653 , and it is evident that the prisms comprising $2 \%$ steel fibers exhibited greater flexural strength than those comprising $1 \%$ steel fibers regardless of casting methodology. 


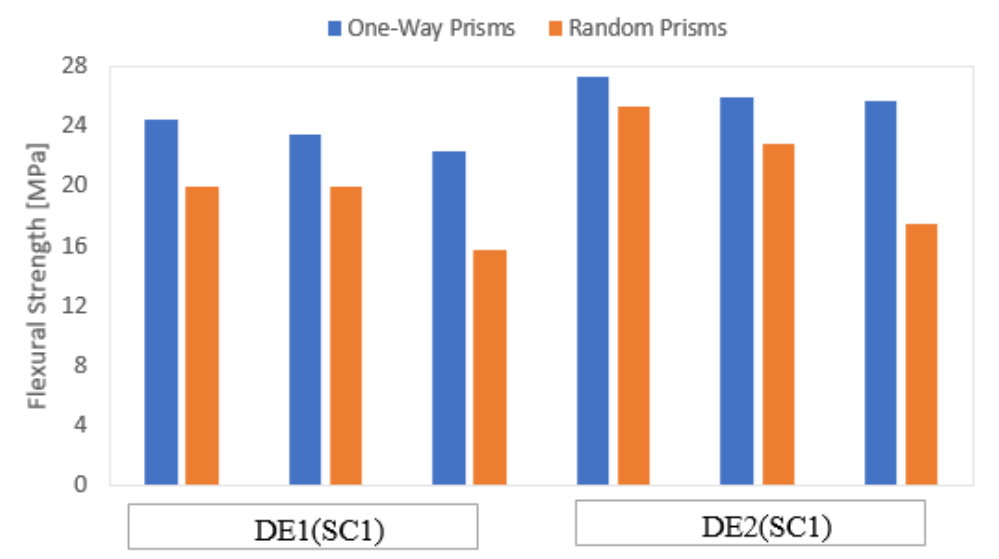

Figure 6- 53: Effect of volumetric ratio of steel fibers on flexural strength

\subsubsection{Degree of Preferential Fiber Alignment}

Figure 6-54 illustrates the average flexural strength results of each set of prisms from mixes FD4, FD5 and FD6. Note that mix FD4 comprises $13 \mathrm{~mm}$ long fibers, whereas mixes FD5 and FD6 comprise $20 \mathrm{~mm}$ long fibers. It is evident that the degree of preferential fiber alignment became more remarkable in the prisms containing relatively long steel fibers compared with the cross-sectional dimensions and resulted in higher flexural strength: the average flexural strength result of $100 \mathrm{~mm}$ by $100 \mathrm{~mm}$ prisms from mix FD4 is greater than that of $150 \mathrm{~mm}$ by $150 \mathrm{~mm}$ prisms from the same mix, and the average flexural strength results of 150 $\mathrm{mm}$ by $150 \mathrm{~mm}$ prisms from mixes FD5 and FD6 are greater than those with the same dimensions from mix FD4.

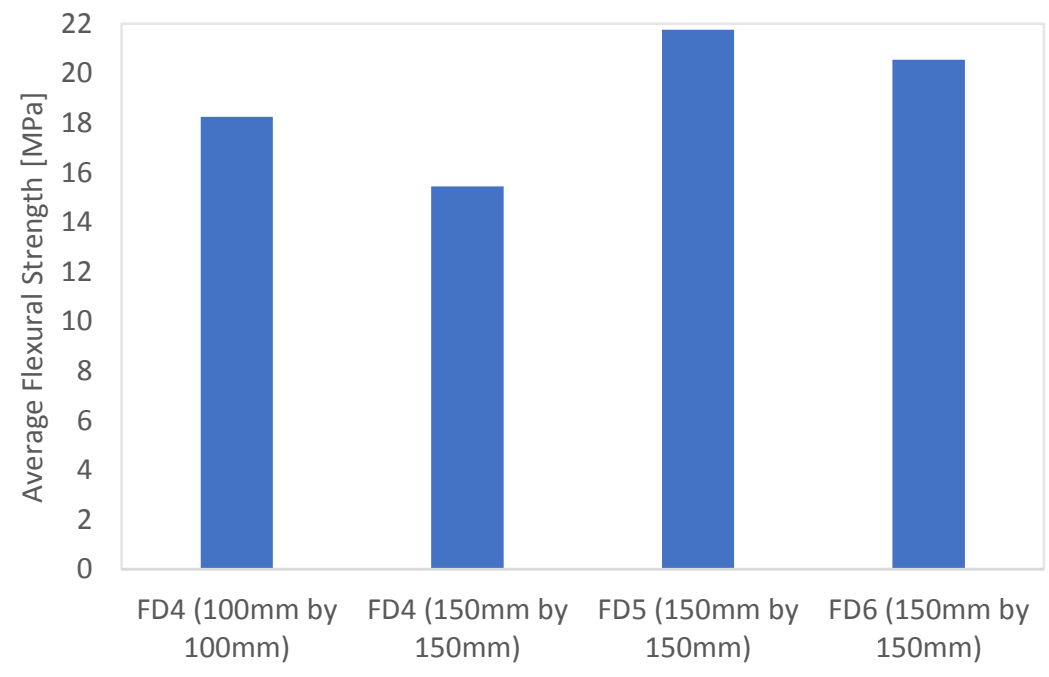

Figure 6- 54: Effect of degree of preferential fiber alignment on flexural strength 


\section{Chapter 7. Conclusions and Recommendations}

The work presented in this report concerned characterization of the mechanical and durability performance of ultra-high-performance steel fiber reinforced concrete (UHP-SFRC). The most critical of all the behavioral indices which makes this material attractive and promising for construction is its behavior in tension, particularly the strength at cracking and in the ultimate limit state, and the resilience it presents to tensile deformation. The distributed reinforcement in the form of steel fibers with specific volumetric ratio enables a resilient and ductile response in tension by delaying the occurrence of crack localization and sustaining large levels of deformation owing to the fact that UHP-SFRC fails under tension only when the steel fibers rupture or get pulled out of the cementitious matrix. In the present work, this behavior was experimentally and numerically confirmed and quantified. In addition, a detailed study was conducted in order to correlate the tensile strength results obtained from the direct tension test, splitting tensile test, inverse analysis of the four-point bending test and nonlinear finite element analysis. The relevance of a calibrated empirical expression which quantifies the tensile strength of UHP-SFRC in terms of the cylinder compressive strength of the material was correlated with the experimentally obtained values. Moreover, the effects of several important parameters on flexural strength including support and loading roller arrangements, casting methodology, volumetric ratio of steel fibers, aspect ratio of the bending prism and the degree of preferential fiber alignment were investigated. The conclusions and recommendations drawn from the study are summarized in the following sections.

\subsection{Conclusions}

\subsubsection{Cylinder Compression Test}

Ten proprietary and two non-proprietary design mixes were tested for the experimental program. The cylinder compressive strength results were greater than $120 \mathrm{MPa}$ for the majority of the mixes considered. Cylinders generally held their integrity beyond the attainment of peak load, and cracks formed parallel to the loading direction, revealing the bridging effect of steel fibers. In addition, compressive strength was insensitive to the volumetric ratio of steel fibers, and cylinders containing $2 \%$ steel fibers had a lower compressive strength than those comprising the same cementitious material but $1 \%$ steel fibers, due to fiber clotting. Moreover, compressive strength increased from $155 \mathrm{MPa}$ to $209 \mathrm{MPa}$ tested after 28-day and 156day curing periods, respectively. Segregation of fibers was observed in cylinders obtained from very 
flowable mixes (i.e., steel fibers accumulated towards the bottom of the mold and large voids appeared near the top with the cylinders failing prematurely in that region).

\subsubsection{Splitting Tensile Test}

Cylinders maintained their integrity after the longitudinal crack formed beyond failure. In addition, apparent splitting tensile strength increased from 25.1 MPa to 27.3 MPa tested after 28-day and 156-day curing periods, respectively. Moreover, owing to the biaxial stress state developed within the cylinder, apparent splitting tensile strength results were multiplied by a correction factor of $(1 / \pi)$ in order to compare with the tensile strength results obtained from other tension tests and the calibrated empirical expression.

\subsubsection{Direct Tension Test}

Setup conditions and tolerances in specimens' dimensions could have contributed to the development of initial eccentricities, and it was critical that bending moments inevitably developed when a crack initiated from one side of the specimen. An effective measure to encourage the formation of critical failure plane within the gauge length was by strengthening the end zones of the specimens with carbon fiber reinforced polymer (CFRP) strips with a high-shear-strength thixotropic epoxy. Tensile strength obtained were relatively consistent for all the UHP-FRC mixes considered, and for the three types of direct-tension specimens considered in the experimental program.

\subsubsection{Four-Point Bending Test}

Prisms containing $1 \%$ steel fibers by unit volume failed in a relatively brittle manner and exhibited less flexural strength compared with those comprising the same cementitious material but with $2 \%$ steel fibers. For the majority of the prisms tested, prisms with an aspect ratio of 1 developed greater flexural strength than those with an aspect ratio of 2 . In addition, the casting methodology had an influence on fiber distribution which was directly related to flexural strength: prisms cast by pouring fresh cementitious materials at different locations along the span of the prism with multiple layers (i.e., random prisms) generally exhibited lower flexural strength than those cast from one end of the mold in a single layer of material (i.e., one-way prisms). Moreover, the degree of preferential fiber alignment became more prominent in specimens that contained relatively long steel fibers as compared with the prims' crosssectional dimensions, leading to more favorable flexural strength results. Support rollers which were free 
to rotate about their own axes and did not translate horizontally caused a deviation between the prism's deflection curve and its natural beam elastica, producing spurious internal moments at the supports which partially counteracted the applied load and resulting in greater load carrying capacity and flexural strength values. Other standard testing details used routinely in the tests, such as the introduction of a compliant plaster layer between rollers and the prism introduced experimental errors which affected the resisted load and consequently the apparent tensile strength of UHP-SFRC. Additionally, flowable mixes were prone to segregation, effectively influencing the flexural strength results.

\subsubsection{Finite Element Modeling}

The nonlinear finite element models developed with VecTor2 were able to simulate the prisms subjected to four-point loading and to match the analytical resistance curve with that obtained experimentally by defining the tensile stress-strain curve. According to the finite element analysis results, all design mixes considered in the experimental program fell into the tension-hardening fiber reinforced concrete category.

\subsubsection{Tensile Behaviour of UHP-SFRC}

Tensile strength results were correlated through the direct tension test, splitting test, inverse analysis of four-point bending test, nonlinear finite element models, and the calibrated empirical expression. In general, inverse analysis procedures prescribed in Annex 8.1 of CSA-S6 (2018) and analyses conducted using finite element models provided relatively close values in terms of cracking strength and ultimate tensile strength. Besides, the tensile strength results obtained from direct tension tests were lower than the cracking strength results obtained from inverse analysis owing to the different stress fields developed in the direct tension test and the four-point bending test. In general, the true splitting tensile strength results were close to the tensile strength results obtained from direct tension tests. Additionally, the calibrated empirical expression proposed by Annex 8.1 of CSA-S6 (2018) provided the lowest cracking strength results among all of the approaches considered. According to the tensile properties acquired from inverse analysis and finite element analysis, all the design mixes considered in the experimental program had a cracking strength greater than $5 \mathrm{MPa}$, a hardening ratio greater than 1.1, and an ultimate tensile strain greater than $0.1 \%$, indicating that they were classified as tension-hardening fiber reinforced concrete. 


\subsubsection{Physical Characteristics of UHP-SFRC}

Durability tests, such as freeze-thaw exposure, did not produce any noticeable degree of concrete deterioration after subjecting the specimens to 300 cycles of temperature variation from $-18^{\circ} \mathrm{C}$ to $4^{\circ} \mathrm{C}$. Only slight reduction in the fundamental transverse frequency of UHP-SFRC was reported corresponding to a durability factor of 98.3. In another type of durability experiment, known as salt scaling test, the total mass of the flaked-off concrete from the test surface was $27.28 \mathrm{~g} / \mathrm{m}^{2}$ on average after exposing the specimen to freezing-thawing conditions for 50 cycles in the presence of the ponding of de-icing chemicals on the test surface. The average coefficient of thermal expansion of UHP-SFRC was $9.87 \mathrm{E}-06 \mathrm{~mm} / \mathrm{mm} /{ }^{\circ} \mathrm{C}$, which was slightly lower than that of conventional concrete. The physical characteristics were also studied experimentally; as an example, for one of the commercial mixes considered in the experimental program (i.e., mix FD4), the static modulus of elasticity was found to be $46.58 \mathrm{GPa}$, the Poisson's ratio was 0.204 , the water content was $2.76 \%$, and the void content was $4.3 \%$.

\subsection{Recommendations}

Recommendations drawn from the study are summarized as follows:

- An upper limit to the spread value of the two-minute flow test of $230 \mathrm{~mm}$ is proposed because flowable mixes are prone to fiber segregation.

- The gripping devices of the universal testing machine restrict the dimensions of direct-tension specimens, leading to preferential fiber orientation during concrete casting. Type A direct-tension specimens offer an alternative to the rather thin prisms used in accordance with the established procedures for testing in normal tension. Local failures at the grip locations can be eliminated by strengthening the specimens with CFRP strips near the ends: a scheme that functions more effectively than the established techniques according to which thin aluminum plates should be epoxy-glued in the ends where the grip pressures are applied.

- Support rollers of four-point bending test which rotate about their own axes and against horizontal translation tend to introduce internal moment and to spuriously increase the flexural strength. However, the test setup condition consisting of a fixed roller against rotation and horizontal translation on one side and a roller allowing horizontal translation on the other side develops an unsymmetric boundary condition. Therefore, it is proposed that the continued use of flexural tests for UHP-SFRC characterization should be amended with regards to the supports, so that both support rollers should be free to translate laterally. In addition, support rollers should be greased to minimize any potential frictional effects. 
- Regarding the execution of the four-point bending test, introducing compliant layers between the steel hardware and the specimen (e.g. plaster layers) should be avoided to minimize the risk of premature softening response.

- A good experimental practice is to cap the free surfaces of all freshly cast UHP-FRC mixes with glass plates to eliminate the roughness caused by steel fibers. Alternatively, it is proposed to rotate the bending prisms at 90 degrees with respect to the position at casting so that the surfaces in contact with the support and loading rollers are smooth.

- Prims with a shear-span aspect ratio of 2 are more adequate than those with an aspect ratio of 1 for the four-point bending test because they provide more conservative flexural strength results. In addition, it is proposed to increase the distance between loading rollers of the prisms with an aspect ratio of 2 to two times of prism depth so that loads are applied at the third points of the span of the prism, in order for the inverse analysis procedures prescribed in Annex 8.1 of CSA-S6 (2018) would be directly applicable; or otherwise a modified version of the inverse analysis should be included in the standard to enable inverse analysis of bending tests with different loading arrangements.

- Prisms cast from one end of the mold with a single layer of fresh cementitious materials (i.e., oneway prisms) tend to encourage fiber alignment, and their results are not representative of the onsite construction condition. To the contrary, prisms cast with layers (i.e., random prisms) generally provide more conservative flexural strength results, whereas their results scatter widely. Therefore, it is proposed to develop an empirical correlation factor between one-way and random prisms from further study.

- The cracking strength of UHP-SFRC calculated with the calibrated empirical expression is proposed to be set as the baseline on account of its simplicity and conservatism. 


\section{References}

AASHTO (2015) 'Coefficient of Thermal Expansion of Hydraulic Cement Concrete.'

AASHTO (2018) 'T132-87: Standard Method of Test for Tensile Strength of Hydraulic Cement Mortars.'

Abbas, S., Nehdi, M.L., and Saleem, M.A. (2016) 'Ultra-High-Performance Concrete: Mechanical performance, Durability, Sustainability and Implementation Challenges', International Journal of Concrete Structures and Materials, Vol.10, pp.271-295.

Abrishamabaf, A., Pimentel, M., and Nunes, S. (2017) 'Influence of Fiber Orientation on the Tensile Behaviour of Ultra-High-Performance Fiber Reinforced Cementitious Composites', Cement and Concrete Research, pp.28-40.

Addis and Bussell, M. (2002) 'Key Development in the History of Concrete Construction and the Implications for Remediation and Repair', Blackwell Science Serious, pp. 13-105.

AFGC-SETRA (Association Francaise de Genie Civil-Service d'etudes Techniques des Routes et Autoroutes) (2002) 'Ultra-High-Performance Fibre-Reinforced Concretes, Recommendations provisoires-interim recommendations', pp.98.

AFNOR NF EN 13670/CN (2013). 'Exécution des Structures en Béton-Complément National à la Norme NF EN 1367.'

Afroughsabet, V., Biolzi, L., and Ozbakkaloglu, T. (2016) 'High-Performance Fiber-Reinforced Concrete: A Review', Journal of Material Science, pp. 6517-6551.

Alkaysi, M., et al. (2016) 'Effects of Silica Powder and Cement Types on Durability of Ultra-HighPerformance Concrete', $I^{\text {st }}$ International Interactive Symposium on UHPC.

American Coal Ash Association (2015) 'American Coal Combustion Products Production and Use Statistics', ACAA.

American Concrete Institute (2011) 'C363.2R: Guide to Quality Control and Assurance of High-Strength Concrete', American Concrete Institute.

American Concrete Institute (2012) 'C1202: Standard Test Method for Electrical Indication of Concrete's Ability to Resist Chloride Ion Penetration, American Concrete Institute.

American Society for Testing and Materials (2002) 'C192/C192M: Standard Practice for Making and Curing Concrete Test Specimens in the Laboratory', The American Society for Testing and Materials.

American Society for Testing and Materials (2002) 'C215: Standard Test Method for Fundamental Transverse, Longitudinal, and Torsional Resonant Frequencies of Concrete Specimens', The American Society for Testing and Materials. 
American Society for Testing and Materials (2008) 'C666/C666 M: Standard Test Method for Concrete to Rapid Freezing and Thawing', The American Society for Testing and Materials.

American Society for Testing and Materials (2010) 'C469/C469M: Standard Test Method for Static Modulus of Elasticity and Poisson's Ratio of Concrete in Compression', The American Society for Testing and Materials.

American Society for Testing and Materials (2014) 'C11698: Standard Test Method for Autogenous Strain of Cement Paste and Mortar', The American Society for Testing and Materials.

American Society for Testing and Materials (2017) 'C157M: Standard Test Method for Length Change of Hardened Concrete', The American Society for Testing and Materials.

American Society for Testing and Materials (2017) 'C1856/C1856M: Fabricating and Testing Specimens of Ultra-High-Performance Concrete', The American Society for Testing and Materials.

American Society for Testing and Materials (2018) 'C596: Standard Test Method for Drying Shrinkage of Mortar Containing Hydraulic Cement', The American Society for Testing and Materials.

American Society for Testing and Materials (2005) 'C39: Standard Test Method for Compressive Strength.' American Society for Testing and Materials (2012) 'C1609: Standard Test Method for Flexural Strength.'

Amin, A., Foster, S., and Muttoni, A. (2015) 'Derivation of the $\sigma$-w Relationship for SFRC from Prism Bending Tests', Journal of Structural Concrete 16(1), pp.93-105.

Amin, A., Markic, T., and Kaufmann, W. (2019) 'Direct Tension Testing of SFRC-Some Peculiar Effects of the Ends Restraints', Conference Paper.

Annex 8.1 of CSA-S6 (2018) 'Canadian Highway Bridge Design Code - Annex 8.1 Fibre Reinforced Concrete (New Edition)', Canadian Standards Association.

Annex U of CSA-A23.1 (2019) 'Ultra-High-Performance Concrete', Canadian Standards Association.

Anson, M., Ko, J.M., and Lam, E.S.S. (2002) 'Advances in Building Technology.'

Astarlioglu, S., and Krauthammer, T. (2014) 'Response of Normal-Strength and Ultra-High-Performance Fiber-Reinforced Concrete Columns to Idealized Blast Loads', Engineering Structures, pp.1-12.

Baby, F., et al. (2013) 'UHPFRC Tensile Behaviour Characterization: Inverse Analysis of Four-Point Bending Test Results', Materials and Structures, pp.1337-1354.

Banthia, N. (2012) 'FRC: Milestone in International Research and Development', Proceedings of FIBCON2012, ICI, pp.48.

Bhalla, S., and Bhattacharjee, B. (2014) 'Reinforcement Corrosion in Concrete Structures and Service Life Predictions - A Review', $9^{\text {th }}$ International Symposium on Advanced Science and Technology in Experimental Mechanics.

Blais, P.Y., and Couture, M. (1999) 'Precast, Prestressed Pedestrian Bridge - World's First Reactive Powder Concrete Structure', PCI Journal 44(5), pp.60 -71. 
Bonneau, O. et al. (1996) 'Reactive Powder Concretes: From Theory to Practice', Concrete International 18(4), pp.47-49.

Bonneau, O., et al. (1997) 'Mechanical Properties and Durability of Two Industrial Reactive Powder Concretes', ACI Materials Journal 94(4), pp.286-290.

Bonneau, O., et al. (2000) 'Characterization of the Granular Packing and Percolation Threshold of Reactive Powder Concrete', Cement and Concrete Research 30(12), pp.1861-1867.

Brownyard, T.I., (1948) 'Studies on the Physical Properties of Hardened Portland Cement Paste', the Portland Cement Association.

Burrow, R.W. (1998) 'Visible and Invisible Cracking of Concrete', The American Concrete Institute, pp.78.

Canada West Foundation (2013) 'At the Intersection: The Case for Sustained and Strategic Public Infrastructure Investment', Canada West Foundation.

Canadian Center for Policy Alternatives (2013) 'Canada's Infrastructure Gap: Where It Came from and Why It Will Cost so much to Close', Canadian Center for Policy Alternatives.

Canadian Chamber of Commerce (2013) 'The Foundations of a Competitive Canada: The Need for Strategic Infrastructure Investment', Canadian Chamber of Commerce.

Canadian Standards Association (2014) 'A23.2: Concrete Materials and Methods of Concrete Construction', Canadian Standards Association.

Canadian Standards Association (2014) 'A23.2-9C: Compressive Strength of Concrete Specimens.'

Canadian Standards Association (2014) 'CAS A23.2-13C: Splitting Tensile Strength of Cylindrical Concrete Specimens', Canadian Standards Association.

Carmo, N.F., and Júlio, E. (2017) 'New Trends for Reinforced Concrete Structures: Some Results of Exploratory Studies', infrastructures.

Carmona, S., and Aguado, A. (2012) 'New Model for the Indirect Determination of the Tensile StressStrain Curve of Concrete', Materials and Structures, Volume 45, pp.1473-1475.

Casanova, P., and Rossi, P. (1996) 'Analysis of Metallic Fibre-Reinforced Concrete Beams Subjected to Bending', Materials and Structures, pp. 354-361.

Chan, Y. W., and Li, V.C. (1997) 'Effects of Transition Zone Densification on Fiber/Cement Paste Bond Strength Improvement', Advanced Cement Based Materials.

Chan, Y., and Chu, S. (2004) 'Effect of Silica Fume on Steel Fiber Bond Characteristic in Reactive Powder Concrete', Cement Concrete, pp.1167-1172.

Chanvillard, G. (2000) 'Characterisation of Fibre Reinforced Concrete Mechanical Properties: A Review', Proceedings of the fifth international RILEM symposium on fibre-reinforced concretes, pp. 29-50.

Chasioti, S. (2017) 'Hybrid Steel Fiber Reinforced Concrete in Shear: From the Material to the Structural Level', A Thesis Report. 
Cortes, D.D., et al. (2008) 'Rheological and Mechanical Properties of Mortars Prepared with Natural and Manufactured Sands', Cement Concrete, pp.1142-1147.

Cwirzen, A. (2007) 'The Effect of the Heat-Treatment Regime on the Properties of Reactive Powder Concrete', Advances in Cement Research 19(1), pp.25-33.

Darling, D. (2018) 'Ultra-High-Performance Concrete: Fundamentals and Applications', CorTuf UHPC.

Deeb, R., Ghanbari, A., and Karihalloo, B.L. (2012) 'Self-Compacting High and Ultra-High-Performance Concretes with and without Steel Fibers', Cement, Concrete and Composite, pp. 185-190.

Denarié, E., and Brühwiler, E. (2015) 'Cast-on site UHPFRC for Improvement of Existing Structures Achievements over the last 10 years in Practice and Research', Proceedings of the 7th International

Dhir, R.K. et al. (1988) 'Contribution of PFA to Concrete Workability and Strength Development', Cement and Concrete Research, pp,277-289.

Doiron, G. (2017) ‘UHPC Pire Repair/Retrofit Examples of Completed Projects in North America', AFGCACI-fib-RILEM International Symposium on UHPC.

Donatello, S., Tyrer, M., and Cheeseman, C.R. (2006) 'Recent Developments in Macro-Defect-Free Cements', Construction and Building Material.

Dunuweera, S., and Rajapakse, R.M.G. (2018) 'Cement Types, Composition, Uses and Advantages of Nanocement, Environmental Impact on Cement Production and Possible Solutions', Advances in Materials Science and Engineering.

EI-Tawil, S., Liu, Z., and Hansen, W. (2016) 'Effects of Silica Powder and Cement Type on Durability of Ultra-High-Performance Concrete.

El-Dieb, A.S. (2009) 'Mechanical, Durability and Microstructural Characteristics of Ultra-High-Strength Self-Compacting Concrete Incorporating Steel Fibers', Material Design, pp.4286-4292.

Eldin, H. et al. (2014) 'Mechanical Properties of Ultra-High-Performance Fiber-Reinforced Concrete', International Journal of Engineering and Innovative Technology, Vol.4, Issue 4, pp.4-10.

Federation of Canadian Municipalities (2012) 'Canadian Infrastructure Report Card', Federation of Canadian Municipalities.

FHWA-HRT-17-097 (2017) 'Ultra-High-Performance Concrete for Bridge Deck Overlays', U.S. Department of Transportation.

FHWA-HRT-18-036 (2018) 'Properties and Behaviour of UHPC-Class Materials', U.S. Department of Transportation.

fib Bulletin 62 (2012) 'Structural Concrete Textbook on Behaviour, Design and Performance (Second Edition)', fib National Member Groups, pp.1-35.

Gao, R. et al. (2006) 'Static Properties of Reactive Powder Concrete Beams', Engineering Materials, pp.521-527. 
Georgiou, A.V., and Pantazopoulou, S.J. (2016) 'Effect of Fiber Length and Surface Characteristics on the Mechanical Properties of Cementitious Composites', Construction and Building Materials, pp.1216-1228.

Ghafari, E., et al. (2012) 'Design of UHPC Using Artificial Neutral Networks', 10 ${ }^{\text {th }}$ International Symposium on Brittle Matrix Composites, pp.9.

Golaszewski, J., and Szwabowski, J. (2004) 'Influence of Superplasticizers on Rheological Behaviour of Fresh Cement Mortars', Cement and Concrete Research, pp. 235-248.

Gopalaratnam, V., and Gettu, R. (1995) 'On the Characterization of Flexural Toughness in Fiber Reinforced Concretes', Cement and Concrete Composites, pp.239-254.

Graybeal, B. (2007) 'Compressive Behaviour of Ultra-High-Performance Fiber Reinforced Concrete’, ACI Materials Journal, pp.146-152.

Graybeal, B., and Hartmann, J. (2003) 'Strength and Durability of UHPC', Concrete Bridge, pp.20.

Graybeal, B.A. (2006) 'Material Property Characterization of Ultra-High-Performance Concrete', U.S. Department of Transportation.

Graybeal, B.A., and Baby, F. (2013) 'Development of Direct Tension Test Method for Ultra-HighPerformance Fiber-Reinforced Concrete', ACI Materials Journal, pp,177-186.

Hassan, A., Jones, S., and Mahmud, G. (2012) 'Experimental Test Methods to Determine the Uniaxial Tensile and Compressive Behaviour of Ultra-High-Performance Fiber Reinforced Concrete', Construction and Building Materials, pp.374-882.

Hassani, H.M., Khalil, W.I., and Danha, L.S. (2014) 'Mechanical Properties of Reactive Powder Concrete with Various Steel Fibers and Silica Fume Contents', Acta Technica Corviniensis, pp.47-58.

Herold, G., and Muller, H. (2004) 'Measurement of Porosity of Ultra-High-Strength Fiber-Reinforced Concrete', Proceedings of the International Symposium on UHPC, pp.685-694.

Hooton, R.D. (1993) 'Influence of Silica Fume Replacement of Cement on Physical Properties and Resistance to Sulfate Attack, Freezing and Thawing, And Alkali-Silica Reactivity', Journal of Materials, pp.143-151.

Huang, W. et al. (2017) 'Effect of Cement Substitution on Hydration and Microstructural Development of Ultra-High-Performance Concrete', Cement, Concrete and Composites, pp.86 -101.

Huo, X., and Wong, L. (2000) ‘Early-Age Shrinkage of HPC Decks Under Different Curing Methods', The American Society of Civil Engineers.

Ingo, S., Jurgen, S., and Oliver, M. (2004) 'Effect of Mixing and Placement Methods on Fresh and Hardened Ultra-High-Performance Concrete', Proceedings of UHPC, pp,575-586.

Jiang, S.P., and Kim, B.G. (1999) 'Importance of Adequate Soluble Alkali Content to Ensure Cement Superplasticizer Compatibility', Cement and Concrete Research, pp. 71-78. 
Juanhong, L., Shaomin, S., and Lin, W. (2009) 'Durability and Micro-Structure of Reactive Powder Concrete', Journal of Wuhan University of Technology Material 24(3), pp.506-509.

Justs et al. (2011) 'UHPC Hardening under Pressure', $3^{\text {rd }}$ International Conference of Civil Engineering.

Kanakubo, T. (2006) 'Tensile Characteristics Evaluation Method for Ductile Fiber-Reinforced Cementitious Composites', Journal of Advanced Concrete Technology, 4(1), pp.3-17.

Kang, S.H. et al. (2017) 'Microstructural Investigation of Heated-Treated Ultra-High-Performance Concrete for Optimum Production', Journal of Materials.

Kazemi, S., and Lubell, A. (2012) 'Influence of Specimen Size and Fiber Content on Mechanical Properties of UHPFRC', ACI Materials Journal 109(6), pp.675-684.

Kim, D., Naaman, A., and El-Tawil, S. (2008) 'Comparative Flexural Behavior of Four Fiber Reinforced Cementitious Composites', Cement and Concrete Composites, 30, pp.917-928.

Kosmatka, S., and Panarese, W. (1988) 'Design and Control of Concrete Mixes', Portland Cement Association, pp.205.

Kumar, M.P., and Burrows, R.W. (2001) 'Durable Structures in the $21^{\text {st }}$ Century', Indian Concrete Journal.

Kusumawardaningsih et al. (2015) 'Tensile Strength Behaviour of UHPC', Procedia Engineering.

Kythe, P., Wei, D., and Okrouhlik, M. (2003) 'An Introduction to Linear and Nonlinear Finite Element Analysis; A Computational Approach.'

Lappa, E., Braam, C., and Walraven, J. (2004) 'Static and Fatigue Bending Tests Of UHPC', Proceedings of the International Symposium on UHPC, pp.449-458.

Larsen, I.L. et al. (2017) 'Determining the Environmental Benefits of Ultra-High-Performance Concrete as a Bridge Construction Material', Material Science and Engineering.

Lawrence, P., Cyr, M. and Ringot, E. (2003) 'Effect of Inert Materials on Short-Term Hydration', Cement and Concrete Research, pp. 1939-1947.

Lerrard, F., and Sedran, T. (1994) 'Optimization of Ultra-High-Performance Concrete by the Use of Packing Model', Cement and Concrete Research, pp.997-1009.

Li, Q. and Ansari, F. (2000) 'High-Strength Concrete in Tension', ACI Material Journal, pp. 49-57.

Li, V.C. (2002) 'Advanced in ECC Research', ACI Specimen Publication, pp,373-400.

Li, Z., et al. (2014) 'Development of Ultra-High-Performance Concrete for Shear-Keys in Precast Bridges

Using Locally Available Materials In South Carolina', $60^{\text {th }}$ Anniversary Convention and National Bridge Conference.

Li, Z.Q. (2015) 'Proportioning and Properties of Ultra-High-Performance Concrete Mixtures for Application in Shear Keys of Precast Concrete Bridges', A Thesis Report.

López, J., et al. (2016a) 'The UHPFRC Pedestrian Bridge Above The V-21 Highway in Puzol, Valencia: Design, Construction and Cost', Proceedings of the 4th International Symposium on UHPC. 
López, J., et al. (2016b) 'Characterisation of The Strain-Hardening Behaviour of UHFPRC Using the Third-

Point-Bending Test', Proceedings of the 4th HIPERMAT International Symposiun on UHPC.

Lopez-Flores, D.S. (1981) 'On the Distinction in Physical and Chemical Characteristics Between Lignitic And Bituminous Fly Ashes', Proceedings of the MRS Symposium, Materials Research Society.

Ma, J., and Orgass, M. (2004) 'Comparative Investigation on UHPC with and without Coarse Aggregates', Proceedings International Symposium on Ultra-High-Performance Concrete.

Ma, J., and Schneider, H. (2002) 'Properties of Ultra-High-Performance Concrete', Leipzig Annual Civil Engineering Report, pp.25-32.

Maca, P., Zatloukal, J., and Konvalinka, P. (2012) 'Development of Ultra-Higher-Performance FiberReinforced Concrete Mixture', IEEE Symposium, pp.861-866.

Magureanu, C. et al. (2012) 'Mechanical Properties and Durability of Ultra-High-Performance Concrete', ACI Materials Journal, pp.177-183.

Mahmud, G., Yang, Z., and Hassan, A. (2013) 'Experimental and Numerical Studies of Size Effects on Ultra-High-Performance Steel Fiber Reinforced Concrete Beams', Construction and Building Materials, pp.1027-1034.

Mansour, M., and Alkafaji, K. (2014) 'Performance of Reactive Powder Concrete Slabs with Different Curing Conditions', Journal of Engineering and Technology Research, pp.81-93.

Martínez, J.A.L. (2017) 'Characterization of The Tensile Behaviour of UHPFRC by Means of Four-Point Bending Tests', A Thesis Report.

Marzouk, H. and Chen, Z. W. (1995) 'Fracture Energy and Tension Properties of High-Strength Concrete', Materials Journal, pp. 108-116.

Matte, V., and Moranville, M. (1999) 'Durability of Reactive Powder Composites: Influence of Silica Fume on Leaching Properties of Very Low Water/Binder Pastes', Cement Concrete, pp.1-9.

Metha, K., and Monteiro, J. (2006) 'Concrete: Microstructure Properties, and Materials ( $4^{\text {th }}$ Edition).'

Millon, O., et al. (2012) 'Failure Mechanisms of UHPC Components Under Explosive Loading', Proceedings of the $3^{\text {rd }}$ International Symposium on UHPC and Nanotechnology for HighPerformance Construction Materials, pp.583-591.

Mindess, S., and Young, J.F. (1981) 'Concrete', Prentice Hall, Englewood Cliffs, New Jersey.

Mindess, S., Young, J.F., and Darwin, D. (2003) 'Concrete (Second Edition)', Pearson Education Inc.

Ministry of Transportation of Ontario (2015) 'Ontario Southern Highways Program 2015-2019' Ministry of Transportation of Ontario, pp. 49.

Ministry of Transportation, Ontario (2017) 'Method of Test for Scaling Resistance of Concrete Surfaces Exposed to Deicing Chemicals.'

Mowat Center (2014) ‘A New Framework for Renewing Canada’s Infrastructure', Mowat Center. 
Naaman, A., and Reinhardt, H. (2006) 'Proposed Classification of HPFRC Composites Based on Their Tensile Response', Materials and Structures, pp.547-555.

Nagataki, S., Sakai, E., and Tekeuchi, T. (1984) 'The Fluidity of Fly Ash Cement Paste with Superplasticizer', Cement and Concrete Research, pp,631-638.

Nehdi, M., Mindess, S., and Aitcin, P.C. (1998) 'Rheology of High-Performance Concrete: Effect of Ultrafine Particles', Cement Concrete, pp.687-697.

NF P18-470. (2016) 'Bétons - Bétons fibrés à Ultra Hautes Performances - Spécification, Performance, Production et Conformité'.

Nguyen, D., et al. (2014) 'Size and Geometry Dependent Tensile Behaviour of Ultra-High-Performance Fiber-Reinforced Concrete', Composites Part B: Engineering, pp.279-292.

Orgass, M., and Klug, Y. (2004) 'Fiber-Reinforced Ultra-High-Strength Concretes', Proceedings of the International Symposium on UHPC, pp,637-648.

Ostergaard, L.,Walter, R., and Olesen, J. (2005) 'Method for Determination of Tensile Properties of Engineered Cementitious Composites (ECC)', Proceedings of ConMar'05.

Painuly, P., and Uniyal, I. (2016) 'Literature Review of Self-Compacting Concrete', International Journal of Technical Research and Applications, pp.178-180.

Park, J.J. et al. (2008) 'Influence of The Ingredients on The Compressive Strength of UHPC As A Fundamental Study to Optimize the Mixing Proportion', Proceedings of the international symposium on UHPC, structural materials and engineering series, pp.105-112.

Perry, V. and Zakariasen, D. (2004) 'First Use of Ultra-High-Performance Concrete for An Innovative Train Station Canopy', Concrete Technology Today 25(2), pp.1-2.

Pierard, J., and Cauberg, N. (2009) 'Evaluation of Durability and Cracking Tendency Of UHPC', Creep, shrinkage and durability mechanics of concrete and concrete structures, pp. 695-700.

Piotrowaski, S. and Schmidt, M. (2012) 'Life Cycle Cost Analysis of a UHPC-Bridge on Example of Two Bridge Refurbishment Designs', Proceedings of Hipermat $20123^{\text {rd }}$ International Symposium on UHPC and Nanotechnology for High Performance Construction Materials, pp. 957-964.

Plank, J., Schrofl, C., and Gruber, M. (2009) 'Use of a Supplemental Agent to Improve Flowability of Ultra-High-Performance Concrete', $9^{\text {th }}$ ACI International Conference on Superplasticizers and Other Chemical Admixtures, pp. 1-16.

Portland Cement Association (2002) 'Causes of Concrete Deterioration', Portland Cement Association.

Qian, S., and Li, V. (2007) 'Simplified Inverse Method for Determining the Tensile Strain Capacity, Journal of Advanced Concrete Technology, 5(2), pp.235-246.

Rahman, M.A., and McQuaker, T. (2016) 'Application of Ultra-High-Performance Concrete in Expediting the Replacement and Rehabilitation of Highway Bridges', Ministry of Transportation of Ontario. 
Rangaraju, P.R. et al. (2013) 'Development of High-Performance Concrete for Application in Shear Keys in Precast Bridges, FHWA-SC-13-04a', FHWA, U.S. Department of Transportation.

Reda, M.m, Shrive, G., and Gillott, E. (1999) 'Microstructural Investigation of Innovative UHPC', Cement and Concrete Research, pp.323-329.

Reddy, M., Sekhar, V., and Krishna, M.N. (2012) 'Durability of Standard Concrete Incorporating Supplementary Cementing Materials Using Rapid Chloride Permeability Test', International Journal of Civil Engineering and Technology, Vol.3, Issue 2, pp. 373-379.

Reineck, H., and Greiner, S. (2004) 'Tests on Ultra-High-Performance Fiber-Reinforced Concrete Designing Hot-Water Tanks And UHPFRC-Shells', Proceedings of the International Symposium on UHPC, pp.361-374.

Reineck, K., and Frettlör, B. (2010) 'Test on scale effect of UHPFRC under bending and axial forces', $3 r d$ Fib International Congress, pp. 40.

Ricciotti, R. (2013) 'Technology: MuCEM in Marseille -Structural Applications of UHPC.'

Richard, P., and Cheyrezy, M. (1995) 'Composition of Reactive Powder Concretes', Cement and Concrete Research, pp.1501-1511.

RILEM TC 162-TDF (2002) 'Test and Design Methods for Steel Fibre Reinforced Concrete. Design of Steel Fibre Reinforced Concrete Using The $\varepsilon$-w Method: Principles and Applications', Materials and Structures, pp. 262-278.

RILEM TC 162-TDF (2003c) 'Test and Design Methods for Steel Fibre Reinforced Concrete: $\sigma-\varepsilon$ Design Method', Materials and Structures, pp.560-567.

Roux, N., Andrade, C., and Sanjuan, M. (1996) 'Experimental Study of Durability of Reactive Powder Concretes', Journal of Materials in Civil Engineering 8(1), pp.1-6.

Russell, H.G., and Graybeal, B.A. (2013) 'Ultra-High-Performance Concrete: A State-of-the-Art Report for the Bridge Community, FHWA-HRT-13-060', The Federal Highway Administration, McLean.

Schmidt, M. et al. (2003) 'Ultra-High-Performance Concrete: Perspective for The Precast Concrete Industry', Concrete Pre-casting Plant Technology, 69(3), pp.16-29.

Schmidt, M., and Fehling, E. (2005) 'Ultra-High-Performance Concrete: Research, Development and Application in Europe', $7^{\text {th }}$ International Symposium on Utilization of High Strength High Performance Concrete, pp.51-77.

Schmidt, M., Fehling, E., and Geisenhanslueke, C. (2004), International Symposium on Ultra-HighPerformance Concrete, pp.868.

Shaheen, E., and Shrive, N. (2006) 'Optimization of Mechanical Properties and Durability of Reactive Powder Concrete', ACI Material Journal 103(6), pp.444-451. 
Shehata, M.H., and Thomas, M.D.A. (2000) 'The Effect of Fly Ash Composition on The Expansion of Concrete Due to Alkali-Silica Reaction', Cement and Concrete Research 30(2000), pp.1063-1072. SIA 2052 (2014) 'Béton Fibré Ultra-Performant (BFUP): Matériaus, dimensionnement et exéctuion. Draft'.

Skazlic, M, Bjegovic, D., and Serdar, M. (2008) 'Influence of Test Specimens' Geometry on Compressive Strength of Ultra-High-Performance Concrete', Proceedings of the $2^{\text {nd }}$ International Symposium on UHPC, pp.295-301.

Sohail, M.G., et al. (2018) 'Advancements in Concrete Mix Designs: High-Performance and Ultra-HighPerformance Concretes from 1970 to 2016', Journal of Materials in Civil Engineering.

Soliman, A.M. (2011) 'Early-Shrinkage of Ultra-High-Performance Concrete: Mitigation and Compensating Mechanisms', The University of Western Ontario.

Soroka, I., and Stern, N. (1976) 'Calcareous Fillers and The Compressive Strength of Portland Cement', Cement and Concrete Research, pp.367-376.

Soutsos, M., Millard, S., and Karaiskos, K. (2005) 'Mix Design, Mechanical Properties, and Impact Resistance of RPC', International Workshop on High-Performance Fiber-Reinforced Cementitious Composites in Structural Applications, pp.549-560.

Srinivasa, C.H., and Dr.Venkatesh (2014) 'A Literature Review on Engineered Cementitious Composites for Structural Applications', International Journal of Engineering Research and Technology.

Sritharan, S., Bristow, B., and Perry, V. (2003) 'Characterizing an Ultra-High-Performance Material for Bridge Applications Under Extreme Loads', Proceedings of the $3^{\text {rd }}$ International Symposium on High-Performance Concrete.

Steil, Y., Karihaloo, B., and Fahling, E. (2004) 'Effect of Casting Direction on The Mechanical Properties of CARDIFRC', Proceedings of the International Symposium of UHPC, pp.481-493.

Sun, W., and Jiao, C. (2011) 'Experimental Study on Impact Tensile Behaviour of Reactive Powder Concrete', Journal of Guangzhou University, pp.42-47.

Suresh, D., and Nagaraju, K. (2015) 'GGBS in Concrete-A Review', IOSR Journal of Mechanical and Civil Engineering, Volume 12, Issue 4, pp.76-82.

Swar, S.K., and Sharma, S.K., and Sharma, H.K. (2015) 'Performance Characteristics of HPDSP Concrete: An Overview', High Performance Fiber Reinforced Cement Composites, pp.79 -86.

Tai, Y.S., EI-Tawail, S., and Chung, T.H. (2016) 'Performance of Deformed Steel Fibers Embedded in Ultra-High-Performance Concrete Subjected to Various Pullout Tests', Cement and Concrete Research, Volume 89, pp.1-13.

Taihan, J., Rossi, P., and Parant, E. (2012) 'Tensile and Bending Behaviour of a Strain Hardening CementBased Composite: Experimental and Numerical Analysis', Cement and Concrete Composites 34(2), pp.166-171. 
Teinchmann, T., and Schmidt, M. (2004) 'Influence of The Packing Density of Fine Particles on Structure, Strength and Durability Of UHPC', Proceedings of the $1^{\text {st }}$ International Symposium on UHPC, pp.313-323.

Thamilselvi, P. et al. (2017) 'State-Of-Art-Review of Slurry Infiltrated Concrete', International Journal of Technical Innovation in Modern Engineering and Science.

Thomas, M.D.A. et al. (1999) 'Use of Ternary Cementitious Systems Containing Silica Fume and Fly Ash in Concrete', Cement and Concrete Research, pp.1207-1214.

Thomason, J.C. (2009) 'Development of Higher Performance Structural Lightweight Portland Cement Concrete', Master's thesis.

Tuan, V.N. et al. (2011) 'The Study of Using Rice Husk Ash to Produce Ultra-High-Performance Concrete', Construction Building Materials, pp. 2030 -2035.

Tue, N., Orgass, M., and Ma, J. (2008) 'Influence of Addition Method of Plasticizer on The Properties of Fresh UHPC', Proceedings of the $2^{\text {nd }}$ International Symposium on Ultra-High-Performance Concrete, pp.93-100.

Vecchio, F. J. (2000) 'Disturbed Stress Field Model for Reinforced Concrete: Formulation', Journal of Structural Engineering.

Vecchio, F. J., and Collins, M. P. (1986) 'The Modified Compression Field Theory for reinforced concrete elements subjected to shear', ACI Structural Journal.

Vecchio, F. J., Wong, P., and Trommels, H. (2013) 'Vector2 User's Manual.', Second Edition.

Verma, M., and Sharma, S.K. (2019) 'Durability Properties of Densified Small Particles Based Concrete', International Journal for Research in Applied Science and Engineering Technology.

Vernet, P. (2004) 'Ultra-Durable Concretes: Structure at The Micro-and Nano-Scale', Cement and Concrete Research, pp.324-327.

Wang, D., et al. (2015) ‘A Review on Ultra-High-Performance Concrete: Part II. Hydration, Microstructure and Properties', Construction and Building Materials, pp.368-377.

Way, R., and Wille, K. (2012) 'Material Characterization of An Ultra-High-Performance Fiber-Reinforced Concrete Under Elevated Temperature', Proceedings of the $3^{\text {rd }}$ International Symposium on UHPC and Nanotechnology for High-Performance Construction Materials, pp.565-572.

Wille, K, and Cotulio, B.C. (2013) 'Development of Non-Proprietary Ultra-High-Performance Concrete for Use in the Highway Bridge Sector', FHWA-HRT-13-100.

Wille, K. and Cotulio, C.B. (2013) 'Development of Non-Proprietary Ultra-High-Performance Concrete for Use in the Highwat Bridge Sector', FHWA-HRT-13-100.

Wille, K. et al. (2012) 'Ultra-High-Performance Concrete and Fiber Reinforced Concrete: Achieving Strength and Ductility Without Heat Curing', Materials and Structures, pp.309-324. 
Wille, K., and Naaman, A.E. (2010) 'Bond Stress-Slip Behavior of Steel Fibers Embedded In UHPC', Proceedings of $18^{\text {th }}$ European Conference on Fracture and Damage of Advanced Fiber-Reinforced Cement-Based Materials.

Wille, K., and Naaman, A.E. (2013) 'Effect of Ultra-High-Performance Concrete on Pullout Behaviour of High-Strength Brass-Costed Straight Steel Fibers', ACI Materials Journal.

Wille, K., and Parra-Montesinos, G. (2012) 'Effect of Beam Size, Casting Method, And Support Conditions on Flexural Behaviour of UHPFRC', ACI Materials Journal 109(3), pp.379-388.

Wille, K., EI-Tawil, S., and Naaman, A. (2014) 'Properties of Strain Hardening UHPFRC Under Direct Tensile Loading', Cement and Concrete Composites, pp.53-66.

Wille, K., Kim, D.J., and Naaman, A.E. (2011) 'Strain-Hardening UHP-FRC With Low Fiber Contents', Materials and Structures, pp.583-598.

Wille, K., Naaman, A.E., and Parra-Montesinos, G.J. (2011) 'Ultra-High-Performance Concrete with Compressive Strength Exceeding 150MPa: A Simple Way', ACI, pp, 46-54.

Willey, J. (2013) 'Use of Ultra-High-Performance Concrete to Mitigate Impact and Explosive Threats', Master's thesis.

Winslow, D.N. et al. (1994) 'Pore Structure in Mortars and Concrete', Cement Concrete, pp.25-37.

Yang, I., Joh, C., and Kim, B. (2010) 'Structural Behaviour of Ultra-High-Performance Concrete Beams Subjected to Bending', Engineering Structures 32(11), pp.3478-3487.

Yau, G. (1998) 'Repair and Strengthening of Columns with Fiber Reinforced Composites', University of Toronto, pp.1-2.

Yazici, H. (2006) 'The Effect of Curing Conditions on Compressive Strength of Ultra-High-Strength Concrete with High Volume Mineral Admixtures’, Building and Environment, pp.2083-2089.

Ye, Y. et al. (2012) 'Mechanical Behaviour of Ultra-High-Performance Concrete Reinforced with Hybrid Different Shapes of Steel Fibers', In ASCE, CICTP, pp.3014-3028.

Yoo, D.Y., Lee, J.H., and Yoon, Y.S. (2013) 'Effect of Fiber Content on Mechanical and Fracture Properties of Ultra-High-Performance Fiber Reinforced Cementitious Composites', Composites and Structures, pp.742-753.

Yu, R., Spiesz, P., and Brouwers, H. (2015) 'Development of An Eco-Friendly Ultra-High-Performance Concrete with Efficient Cement and Mineral Admixtures Uses', Cement, Concrete and Composites, pp.383 -394.

Yu, R., Spiesz, P., and Brouwers, H.J.H. (2014) 'Mix Design and Properties Assessment of Ultra-HighPerformance Fiber Reinforced Concrete', Cement and Concrete Research, pp. 29-39.

Yun, L. (2010) 'Tension Stiffening Model for Reinforced Concrete Based on Bond Stress Slip Relation', Thesis Report. 


\section{Appendix A. Resistance Curves of Four-Point Bending Tests}

\section{A.1 Commercial FD2 Design Mix}
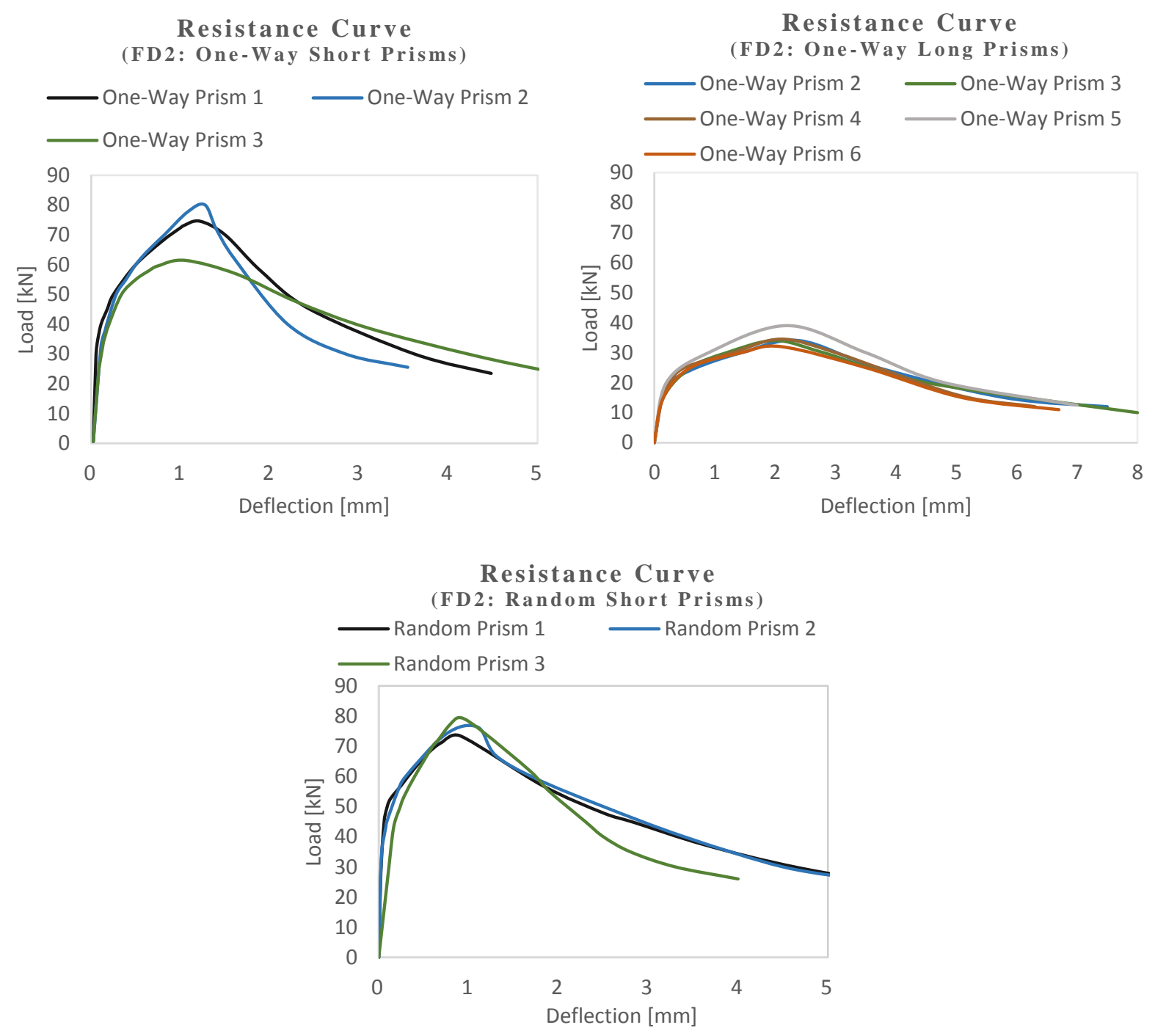

Figure A-1: Resistance curves of the prisms from mix FD2 


\section{A.2 Commercial KI1 Design Mix}
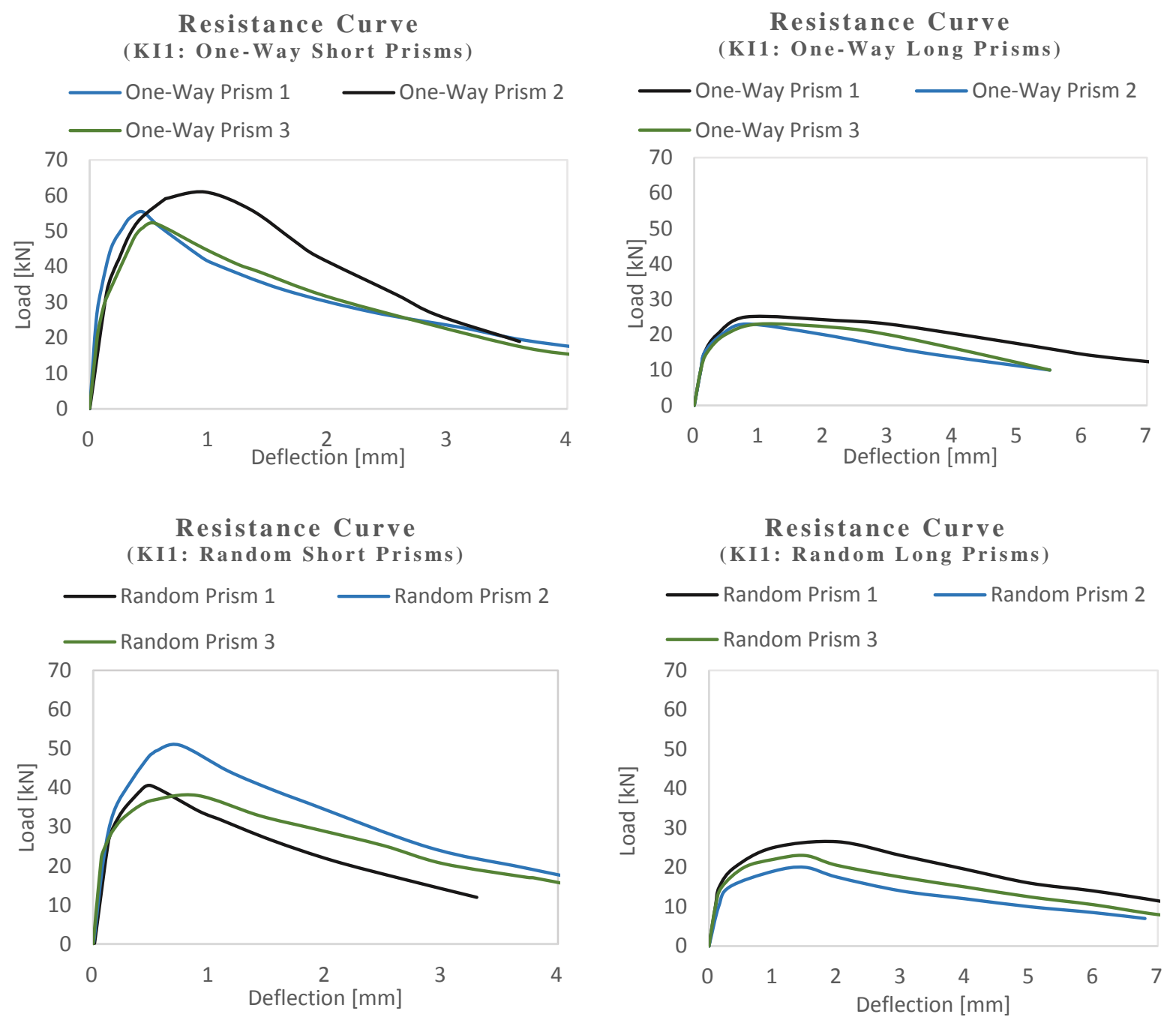

Figure A-2: Resistance curves of the prisms from mix KII 


\section{A.3 Commercial KI2 Design Mix}
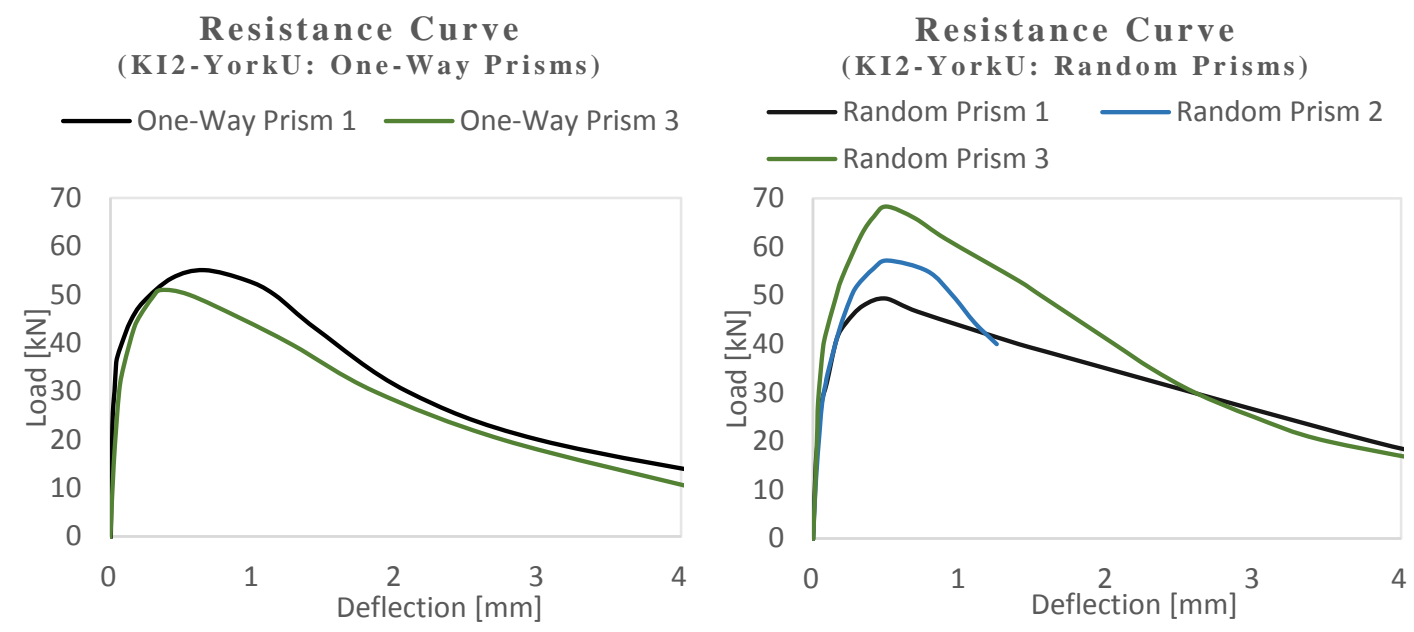

Figure A- 3: Resistance curves of the prisms from mix KI2 (York University)

Resistance Curve

(KI2-EP: One-Way Prisms)

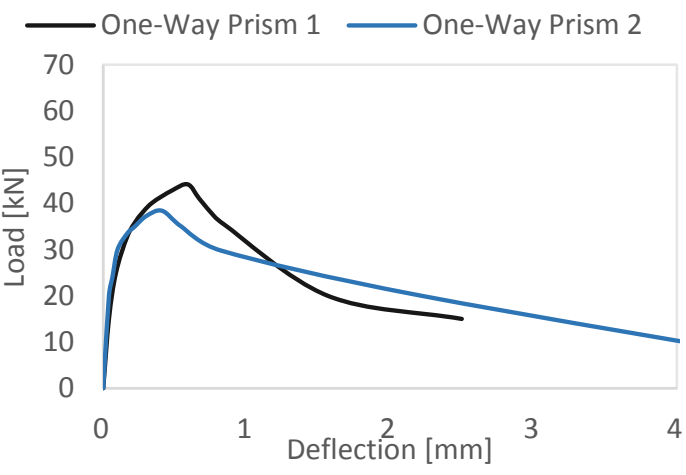

Resistance Curve (KI2-EP: Random Prisms)

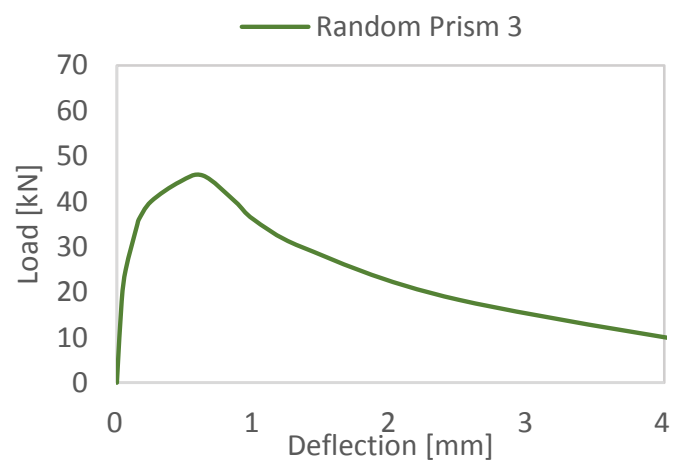

Figure A- 4: Resistance curves of the prisms from mix KI2 (École Polytechnique-Montréal)
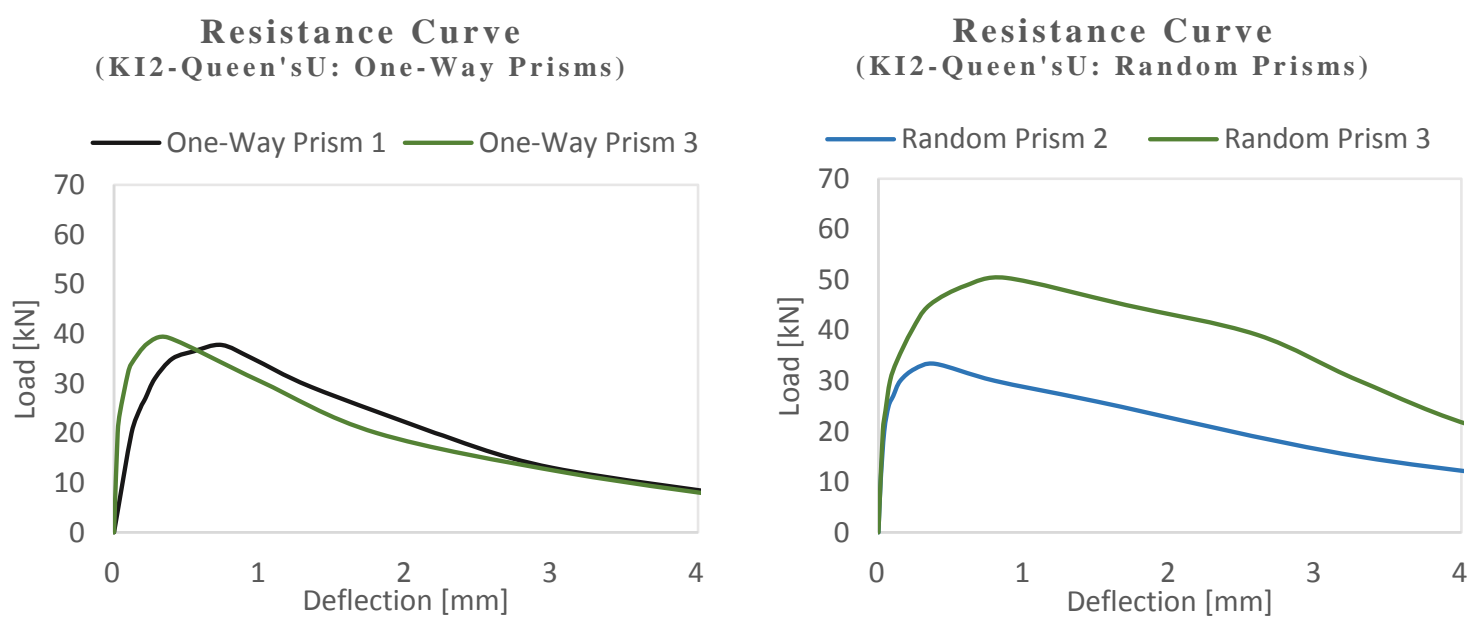

Figure A- 5: Resistance curves of the prisms from mix KI2 (Queen's University) 


\section{A.4 Commercial LD2 Design Mix}
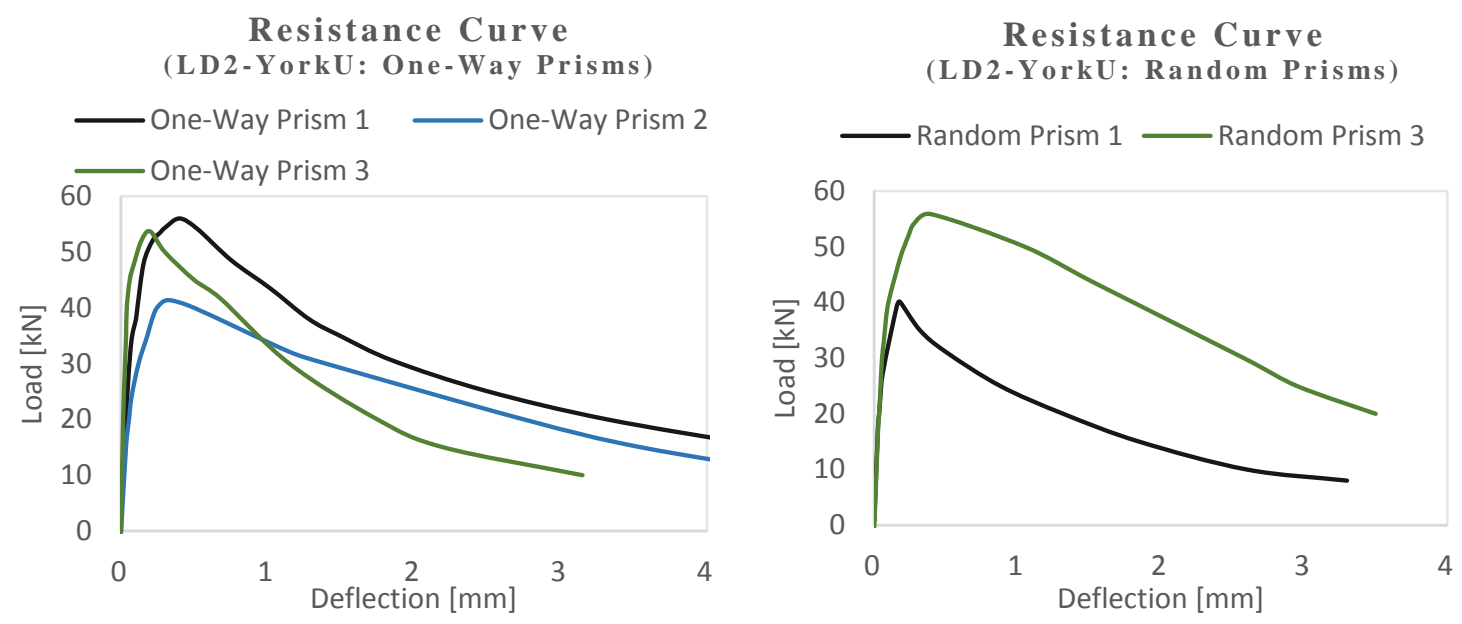

Figure A- 6: Resistance curves of the prisms from mix LD2 (York University)
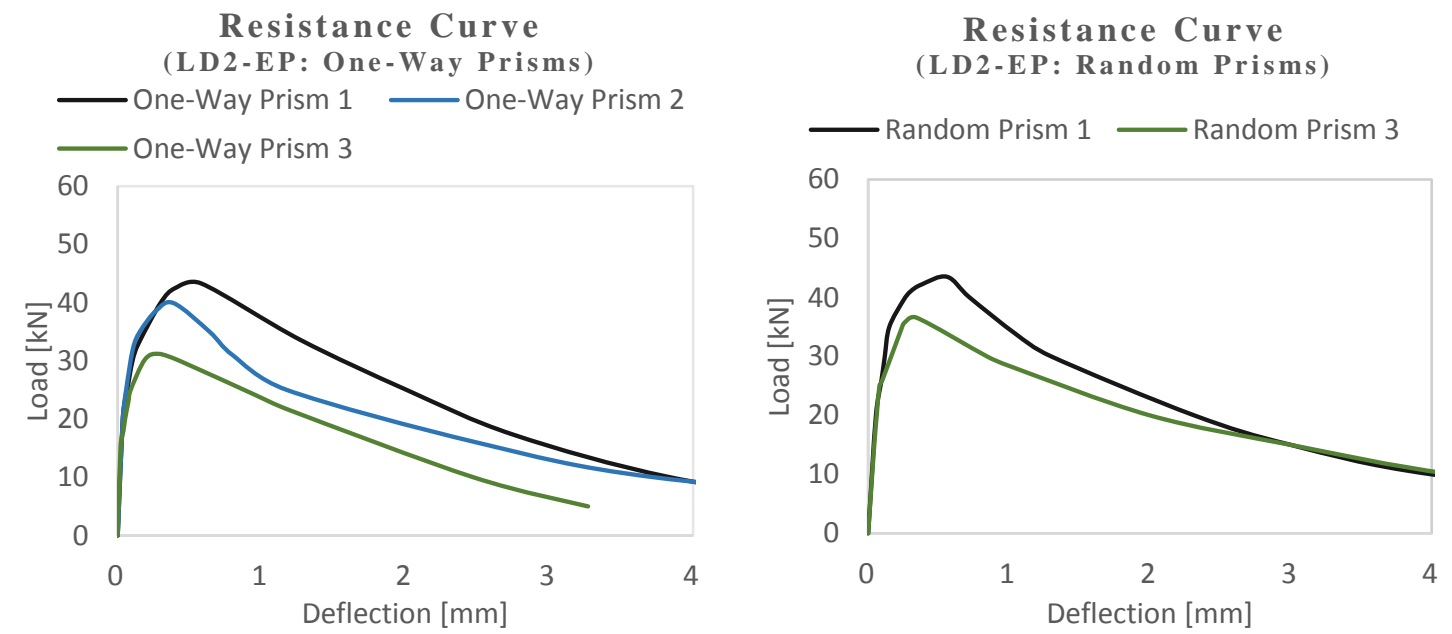

Figure A- 7: Resistance curves of the prisms from mix LD2 (École Polytechnique-Montréal)
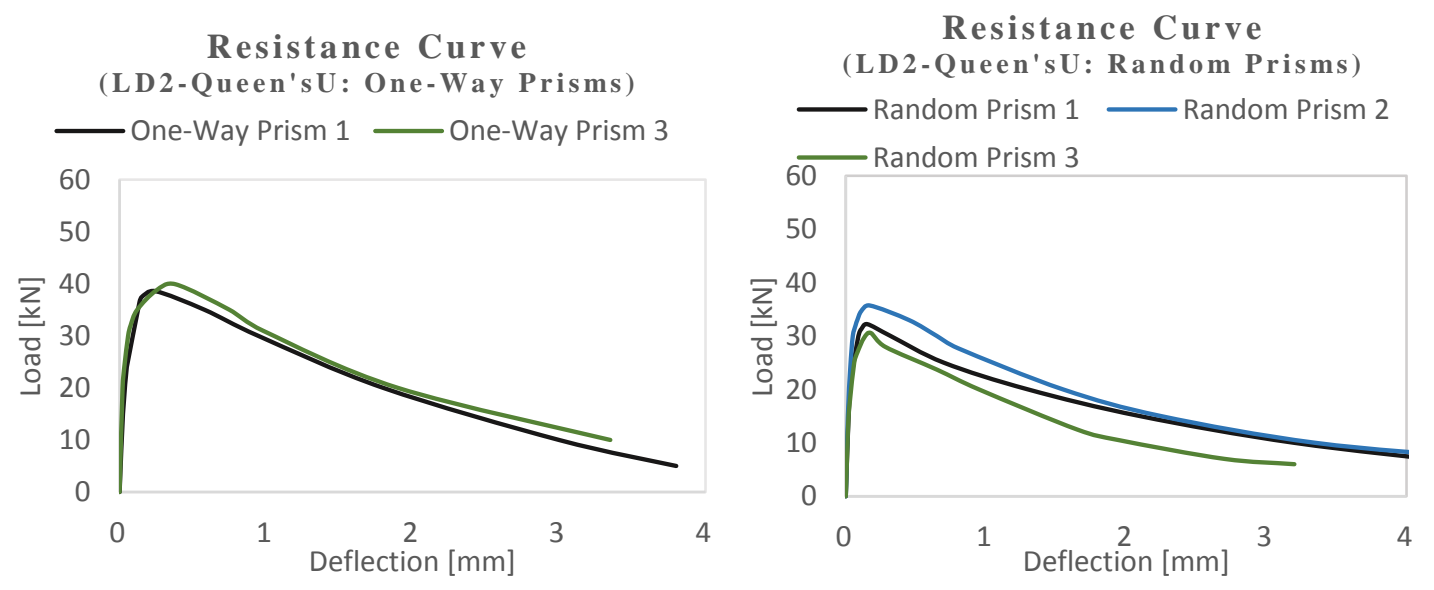

Figure A- 8: Resistance curves of the prisms from mix LD2 (Queen's University) 


\section{A.5 Commercial FD5 and FD6 Design Mixes}
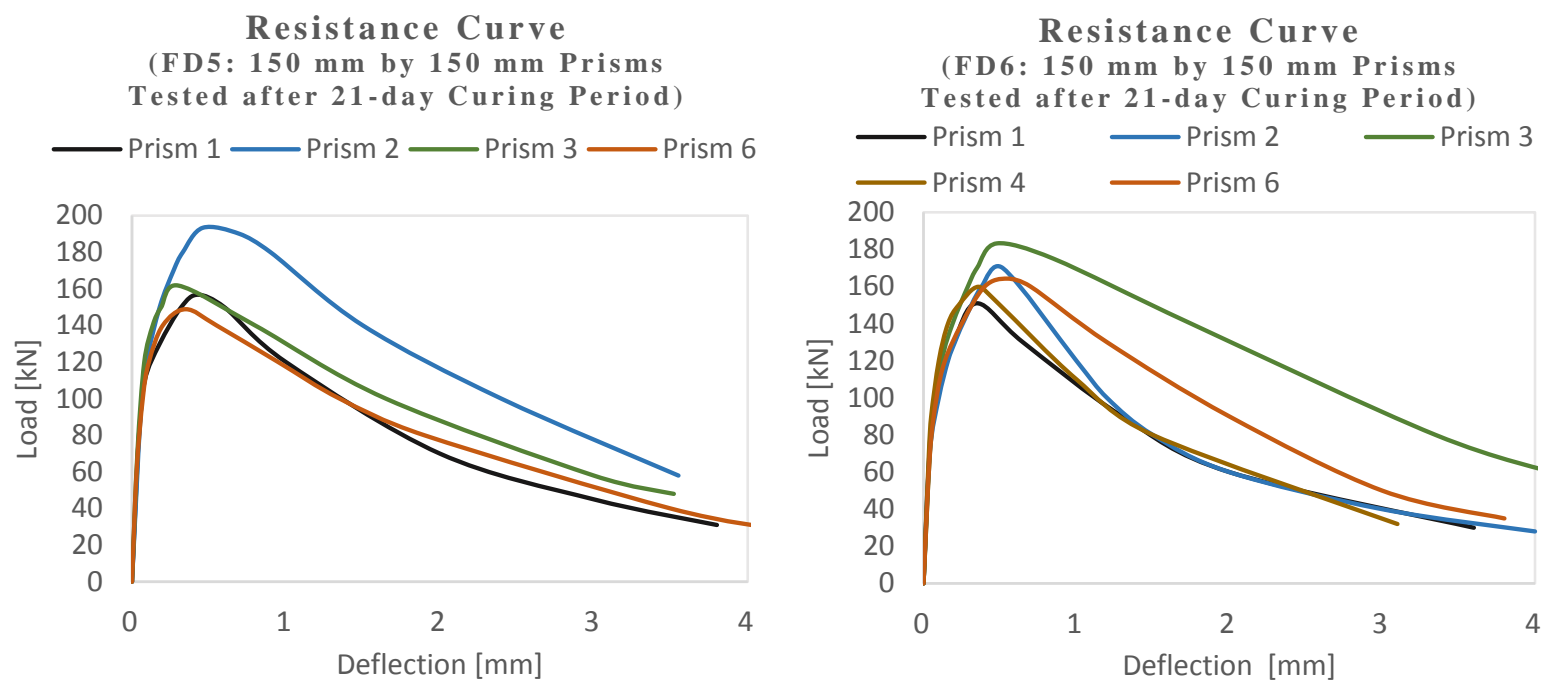

Figure A- 9: Resistance curves of the prisms from mixes FD5 and FD6 tested at the age of 21 days
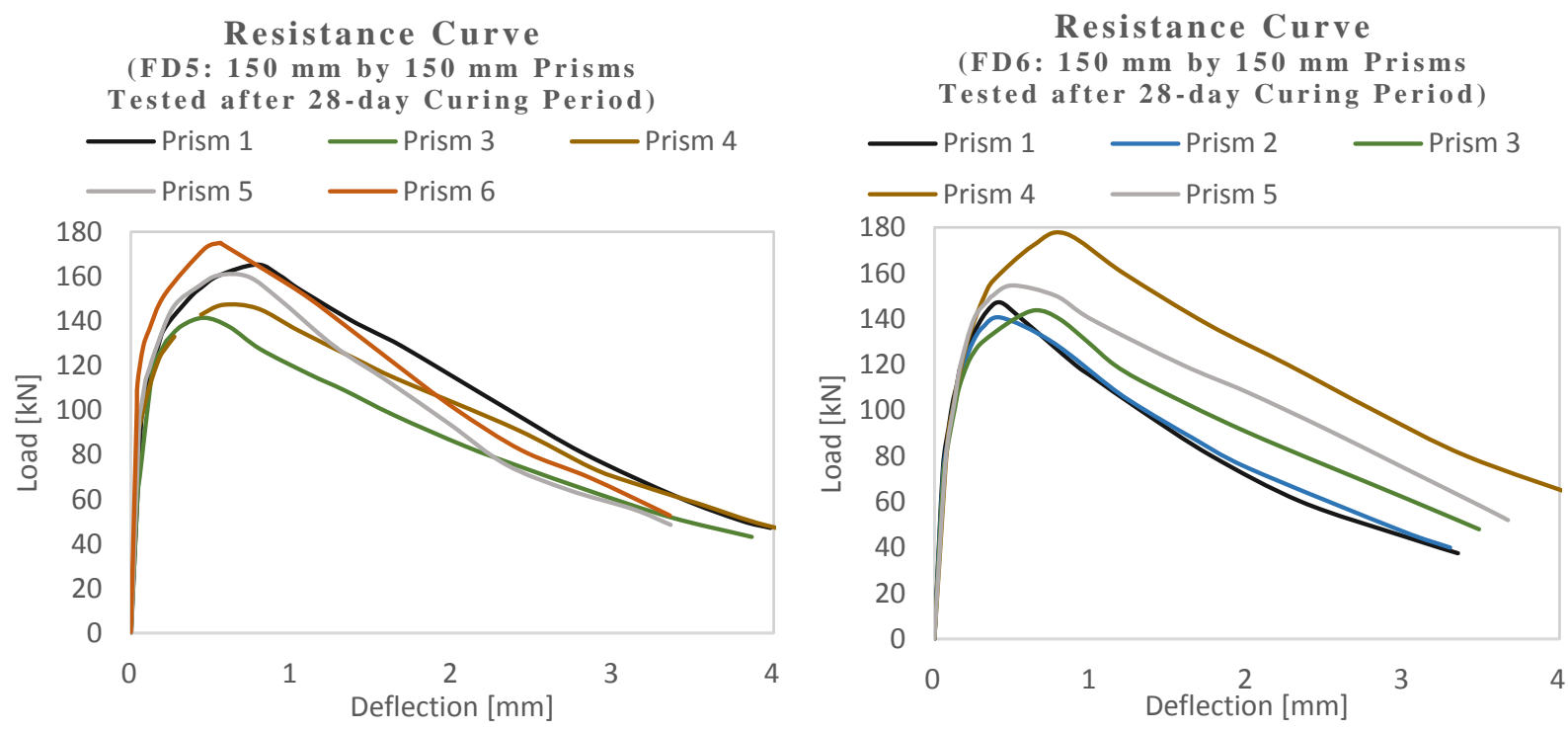

Figure A-10: Resistance curves of the prisms from mixes FD5 and FD6 tested at the age of 28 days 


\section{Appendix B. Inverse Analysis (The Four Characteristic Points)}

\section{B.1 Commercial FD1 and FD2 Design Mixes}

Table B-1: The four characteristic points (Mix FD1)

\begin{tabular}{|c|c|c|c|c|c|c|c|c|c|c|c|c|}
\hline \multirow{2}{*}{ Specimen } & $\mathbf{P}_{\mathbf{0}}$ & $\mathbf{P}_{1}$ & $\mathbf{P}_{2}$ & $\mathbf{P}_{3}$ & $\mathbf{P}_{4}$ & $\mathbf{P}_{\max }$ & $\delta_{0}$ & $\delta_{1}$ & $\boldsymbol{\delta}_{2}$ & $\delta_{3}$ & $\delta_{4}$ & $\delta_{\text {Pmax }}$ \\
\hline & {$[\mathrm{kN}]$} & {$[\mathrm{kN}]$} & {$[\mathrm{kN}]$} & {$[\mathrm{kN}]$} & {$[\mathrm{kN}]$} & {$[\mathrm{kN}]$} & [mm] & [mm] & [mm] & [mm] & [mm] & [mm] \\
\hline OW1 & 30.09 & 40.00 & 58.00 & 69.94 & 55.95 & 72.11 & 0.107 & 0.190 & 0.520 & 0.970 & 2.180 & 1.211 \\
\hline OW2 & 20.00 & 36.00 & 60.00 & 62.08 & 49.66 & 64.00 & 0.140 & 0.339 & 1.025 & 1.100 & 2.250 & 1.250 \\
\hline Average & 25.05 & 38.00 & 59.00 & 66.01 & 52.81 & 68.05 & 0.124 & 0.265 & 0.773 & 1.035 & 2.215 & 1.231 \\
\hline \multirow{2}{*}{ Specimen } & $\mathbf{P}_{\mathbf{0}}$ & $\mathbf{P}_{1}$ & $\mathbf{P}_{2}$ & $\mathbf{P}_{3}$ & $\mathbf{P}_{4}$ & $\mathbf{P}_{\max }$ & $\delta_{0}$ & $\delta_{1}$ & $\delta_{2}$ & $\delta_{3}$ & $\delta_{4}$ & \\
\hline & {$[\mathrm{kN}]$} & {$[\mathrm{kN}]$} & {$[\mathrm{kN}]$} & {$[\mathrm{kN}]$} & {$[\mathrm{kN}]$} & {$[\mathrm{kN}]$} & [mm] & {$[\mathrm{mm}]$} & [mm] & [mm] & [mm] & [mm] \\
\hline $\mathrm{R} 1$ & 30.00 & 48.00 & 70.00 & 76.28 & 61.03 & 78.64 & 0.180 & 0.385 & 1.050 & 1.310 & 3.400 & 1.700 \\
\hline R3 & 30.00 & 35.00 & 47.00 & 77.72 & 62.18 & 80.12 & 0.042 & 0.063 & 0.153 & 0.910 & 1.810 & 1.225 \\
\hline $\mathrm{R} 4$ & 30.00 & 35.00 & 45.00 & 74.69 & 59.75 & 77.00 & 0.080 & 0.121 & 0.280 & 1.300 & 2.700 & 1.700 \\
\hline Average & 30.00 & 39.33 & 54.00 & 76.23 & 60.99 & 78.59 & 0.101 & 0.190 & 0.494 & 1.173 & 2.637 & 1.542 \\
\hline
\end{tabular}

Table B- 2: The four characteristic points (Mix FD2)

\begin{tabular}{|c|c|c|c|c|c|c|c|c|c|c|c|c|}
\hline \multirow{2}{*}{ Specimen } & $\mathbf{P}_{\mathbf{0}}$ & $\mathbf{P}_{1}$ & $\mathbf{P}_{2}$ & $\mathbf{P}_{3}$ & $\mathbf{P}_{4}$ & $P_{\max }$ & $\delta_{0}$ & $\delta_{1}$ & $\delta_{2}$ & $\delta_{3}$ & $\delta_{4}$ & $\delta_{P \max }$ \\
\hline & {$[\mathrm{kN}]$} & {$[\mathrm{kN}]$} & & & & & & & & & & \\
\hline & & & & & & & & & & & & \\
\hline & & & & & & & & & & & & \\
\hline & & & & & & & & & & & & \\
\hline & & 34. & 48.33 & 69.88 & & & & & & & & \\
\hline \multirow{2}{*}{ Specimen } & & & & $\mathbf{P}_{3}$ & & & & $\delta_{1}$ & & $\delta_{3}$ & & \\
\hline & & & & & & & & & & & & \\
\hline & & & & & & & & & & & & .908 \\
\hline & & & 43. & & & & & & & & 50 & .100 \\
\hline & & 3 & 72.00 & 77.07 & & 79 & & 0.2 & & 0.7 & 90 & 0.915 \\
\hline Average & 26.99 & 42.33 & 55.50 & 74.12 & 59.30 & 76.41 & 0.052 & 0.113 & 0.280 & 0.747 & 1.753 & 0.974 \\
\hline
\end{tabular}

\section{B.2 Commercial KI1 Design Mix}

Table B- 3: The four characteristic points utilized for the inverse analysis of the short prisms from mix KII

\begin{tabular}{|c|c|c|c|c|c|c|c|c|c|c|c|c|}
\hline \multirow{2}{*}{ Specimen } & $\mathbf{P}_{0}$ & $\mathbf{P}_{1}$ & $\mathbf{P}_{2}$ & $\mathbf{P}_{3}$ & $\mathbf{P}_{4}$ & $\mathbf{P}_{\max }$ & $\delta_{0}$ & $\delta_{1}$ & $\delta_{2}$ & $\delta_{3}$ & $\delta_{4}$ & $\delta_{P \max }$ \\
\hline & {$[\mathrm{kN}]$} & {$[\mathrm{kN}]$} & {$[\mathrm{kN}]$} & {$[\mathrm{kN}]$} & {$[\mathrm{kN}]$} & {$[\mathrm{kN}]$} & {$[\mathrm{mm}]$} & {$[\mathbf{m m}]$} & {$[\mathrm{mm}]$} & {$[\mathrm{mm}]$} & {$[\mathbf{m m}]$} & {$[\mathrm{mm}]$} \\
\hline OW1 & 25.00 & 34.00 & 51.00 & 53.79 & 43.03 & 55.45 & 0.055 & 0.099 & 0.279 & 0.350 & 0.920 & 0.441 \\
\hline OW2 & 32.77 & 42.00 & 58.99 & 59.15 & 47.32 & 60.98 & 0.143 & 0.243 & 0.630 & 0.640 & 1.710 & 0.968 \\
\hline OW3 & 20.00 & 30.00 & 49.00 & 50.64 & 40.51 & 52.20 & 0.063 & 0.127 & 0.388 & 0.440 & 1.250 & 0.547 \\
\hline Average & 25.92 & 35.33 & 53.00 & 54.53 & 43.62 & 56.21 & 0.087 & 0.156 & 0.432 & 0.477 & 1.293 & 0.652 \\
\hline \multirow{2}{*}{ Specimen } & $\mathbf{P}_{\mathbf{0}}$ & $\mathbf{P}_{1}$ & $\mathbf{P}_{2}$ & $\mathbf{P}_{3}$ & $\mathbf{P}_{4}$ & $\mathbf{P}_{\max }$ & $\delta_{0}$ & $\delta_{1}$ & $\delta_{2}$ & $\delta_{3}$ & $\delta_{4}$ & $\delta_{P \max }$ \\
\hline & {$[\mathrm{kN}]$} & {$[\mathrm{kN}]$} & {$[\mathrm{kN}]$} & {$[\mathrm{kN}]$} & {$[\mathrm{kN}]$} & {$[\mathrm{kN}]$} & {$[\mathrm{mm}]$} & {$[\mathrm{mm}]$} & {$[\mathrm{mm}]$} & {$[\mathrm{mm}]$} & {$[\mathrm{mm}]$} & [mm] \\
\hline R2 & 22.01 & 34.00 & 48.00 & 49.37 & 39.49 & 50.89 & 0.091 & 0.180 & 0.480 & 0.535 & 1.540 & 0.744 \\
\hline
\end{tabular}



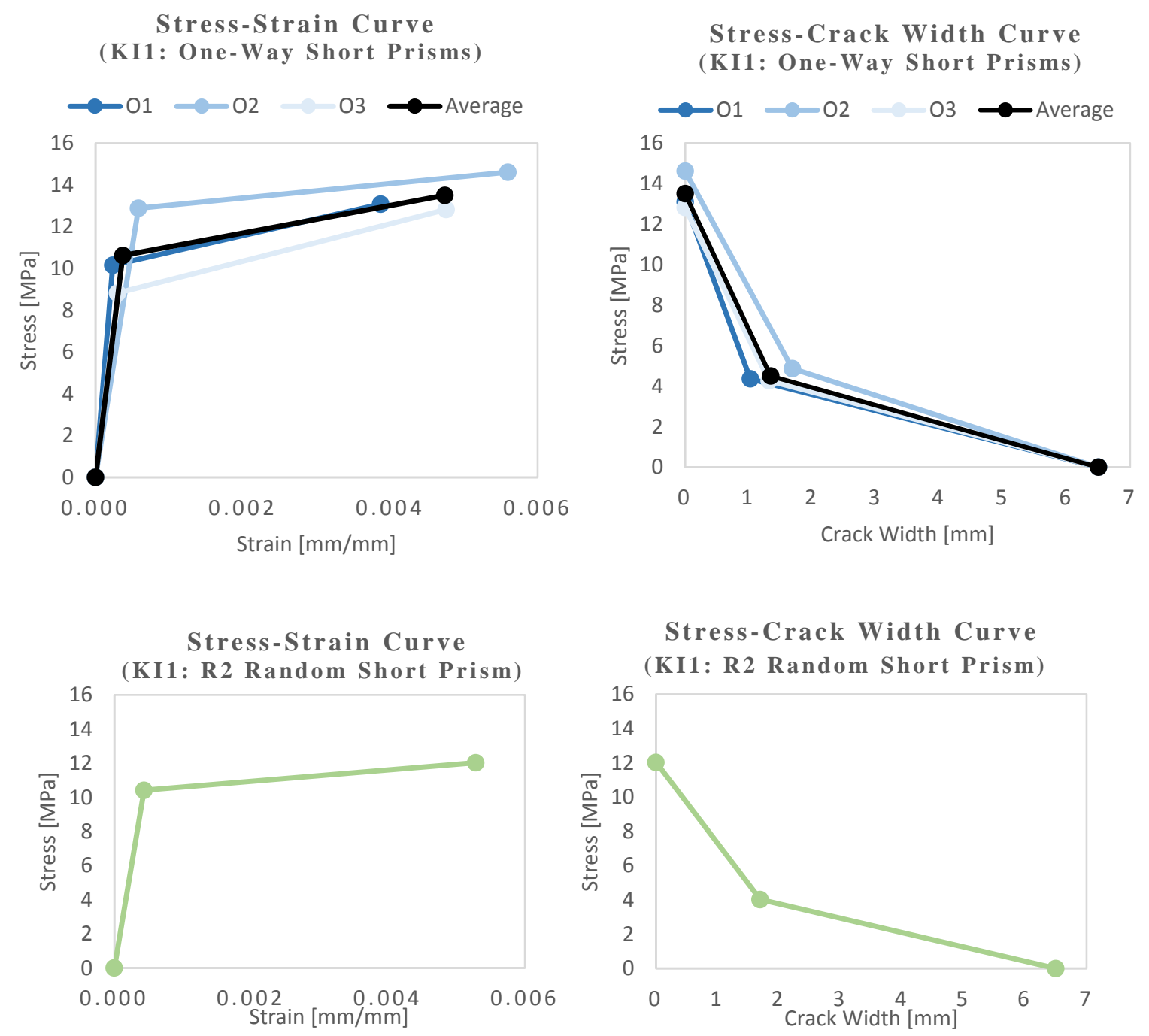

Figure B-1: Linearized tensile stress-strain-crack mouth opening relationships of the short prisms from mix KII

\section{B.3 Commercial LD1 Design Mix}

Table B-4: The four characteristic points utilized for the inverse analysis of the short prisms from mix LDI

\begin{tabular}{|c|c|c|c|c|c|c|c|c|c|c|c|c|}
\hline \multirow{2}{*}{ Specimen } & $\mathbf{P}_{0}$ & $\mathbf{P}_{1}$ & $\mathbf{P}_{2}$ & $\mathbf{P}_{3}$ & $\mathbf{P}_{4}$ & $\mathbf{P}_{\max }$ & $\boldsymbol{\delta}_{0}$ & $\delta_{1}$ & $\delta_{2}$ & $\delta_{3}$ & $\delta_{4}$ & $\delta_{\text {Pmax }}$ \\
\hline & [kN] & {$[\mathrm{kN}]$} & [kN] & {$[\mathrm{kN}]$} & {$[\mathrm{kN}]$} & {$[\mathrm{kN}]$} & {$[\mathrm{mm}]$} & {$[\mathrm{mm}]$} & {$[\mathrm{mm}]$} & {$[\mathrm{mm}]$} & [mm] & [mm] \\
\hline OW1 & 38.76 & 40.80 & 48.00 & 70.26 & 56.22 & 72.44 & 0.019 & 0.026 & 0.057 & 0.300 & 0.839 & 0.402 \\
\hline OW2 & 28.67 & 32.00 & 42.00 & 48.62 & 38.89 & 50.12 & 0.028 & 0.042 & 0.103 & 0.190 & 1.080 & 0.392 \\
\hline OW3 & 19.64 & 26.40 & 33.00 & 45.68 & 36.54 & 47.09 & 0.011 & 0.021 & 0.050 & 0.200 & 1.130 & 0.371 \\
\hline Average & 29.02 & 33.07 & 41.00 & 54.85 & 43.89 & 56.55 & 0.019 & 0.030 & 0.070 & 0.230 & 1.016 & 0.389 \\
\hline \multirow{2}{*}{ Specimen } & $\mathbf{P}_{\mathbf{0}}$ & $\mathbf{P}_{1}$ & $\mathbf{P}_{2}$ & $\mathbf{P}_{\mathbf{3}}$ & $\mathbf{P}_{4}$ & $\mathbf{P}_{\max }$ & $\delta_{0}$ & $\delta_{1}$ & $\delta_{2}$ & $\delta_{3}$ & $\delta_{4}$ & $\delta_{P \max }$ \\
\hline & {$[\mathrm{kN}]$} & {$[\mathrm{kN}]$} & {$[\mathrm{kN}]$} & {$[\mathrm{kN}]$} & {$[\mathrm{kN}]$} & {$[\mathrm{kN}]$} & {$[\mathrm{mm}]$} & [mm] & {$[\mathrm{mm}]$} & {$[\mathrm{mm}]$} & {$[\mathrm{mm}]$} & [mm] \\
\hline $\mathrm{R} 1$ & 27.37 & 36.00 & 52.00 & 61.38 & 49.10 & 63.28 & 0.052 & 0.093 & 0.255 & 0.510 & 1.550 & 0.709 \\
\hline R2 & 20.87 & 28.00 & 36.00 & 49.08 & 39.26 & 50.60 & 0.017 & 0.030 & 0.072 & 0.340 & 1.350 & 0.497 \\
\hline R3 & 22.00 & 35.00 & 52.64 & 53.35 & 42.68 & 55.00 & 0.042 & 0.143 & 0.365 & 0.400 & 1.350 & 0.630 \\
\hline Average & 23.41 & 33.00 & 46.88 & 54.60 & 43.68 & 56.29 & 0.037 & 0.089 & 0.231 & 0.417 & 1.417 & 0.612 \\
\hline
\end{tabular}




\section{B.4 Commercial DE2 Design Mix (SC1)}

Table B- 5: The four characteristic points utilized for the inverse analysis of the short prisms from mix DE2

\begin{tabular}{|c|c|c|c|c|c|c|c|c|c|c|c|c|}
\hline \multirow[b]{2}{*}{ Specimen } & $\mathbf{P}_{\mathbf{o}}$ & $\mathbf{P}_{1}$ & $\mathbf{P}_{2}$ & $\mathbf{P}_{3}$ & $\mathbf{P}_{4}$ & $P_{\max }$ & $\delta_{0}$ & $\delta_{1}$ & $\delta_{2}$ & $\delta_{3}$ & $\delta_{4}$ & $\delta_{P \max }$ \\
\hline & {$[\mathrm{kN}]$} & {$[\mathrm{kN}]$} & {$[\mathrm{kN}]$} & {$[\mathrm{kN}]$} & {$[\mathrm{kN}]$} & {$[\mathrm{kN}]$} & [mm] & [mm] & {$[\mathrm{mm}]$} & [mm] & [mm] & {$[\mathrm{mm}]$} \\
\hline OW1 & 20.00 & 30.00 & 39.00 & 45.59 & 36.47 & 47.00 & 0.035 & 0.070 & 0.171 & 0.350 & 1.410 & 0.600 \\
\hline OW2 & 20.00 & 30.00 & 43.00 & 48.81 & 39.05 & 50.32 & 0.020 & 0.039 & 0.104 & 0.200 & 1.250 & 0.538 \\
\hline OW3 & 21.86 & 30.00 & 45.00 & 46.80 & 37.44 & 48.25 & 0.120 & 0.221 & 0.620 & 0.680 & 1.310 & 0.807 \\
\hline Average & 20.62 & 30.00 & 42.33 & 47.07 & 37.65 & 48.52 & 0.058 & 0.110 & 0.298 & 0.410 & 1.323 & 0.648 \\
\hline \multirow[b]{2}{*}{ Specimen } & $\mathbf{P}_{\mathbf{0}}$ & $\mathbf{P}_{1}$ & $\mathbf{P}_{2}$ & $\mathbf{P}_{3}$ & $\mathbf{P}_{4}$ & $P_{\max }$ & $\delta_{0}$ & $\delta_{1}$ & $\delta_{2}$ & $\delta_{3}$ & $\delta_{4}$ & $\delta_{P \max }$ \\
\hline & {$[\mathbf{k N}]$} & {$[\mathbf{k N}]$} & {$[\mathbf{k N}]$} & {$[\mathbf{k N}]$} & {$[\mathrm{kN}]$} & {$[\mathbf{k N}]$} & [mm] & {$[\mathrm{mm}]$} & [mm] & [mm] & {$[\mathrm{mm}]$} & {$[\mathrm{mm}]$} \\
\hline R1 & 12.25 & 20.00 & 31.00 & 31.87 & 25.50 & 32.86 & 0.070 & 0.130 & 0.350 & 0.365 & 1.600 & 0.392 \\
\hline $\mathrm{R} 2$ & 15.00 & 24.00 & 38.00 & 41.49 & 33.19 & 42.78 & 0.065 & 0.137 & 0.400 & 0.520 & 1.250 & 0.600 \\
\hline R3 & 20.00 & 35.00 & 43.00 & 45.63 & 36.50 & 47.04 & 0.013 & 0.035 & 0.070 & 0.105 & 0.700 & 0.202 \\
\hline Average & 15.75 & 26.33 & 37.33 & 39.66 & 31.73 & 40.89 & 0.049 & 0.101 & 0.273 & 0.330 & 1.183 & 0.398 \\
\hline
\end{tabular}

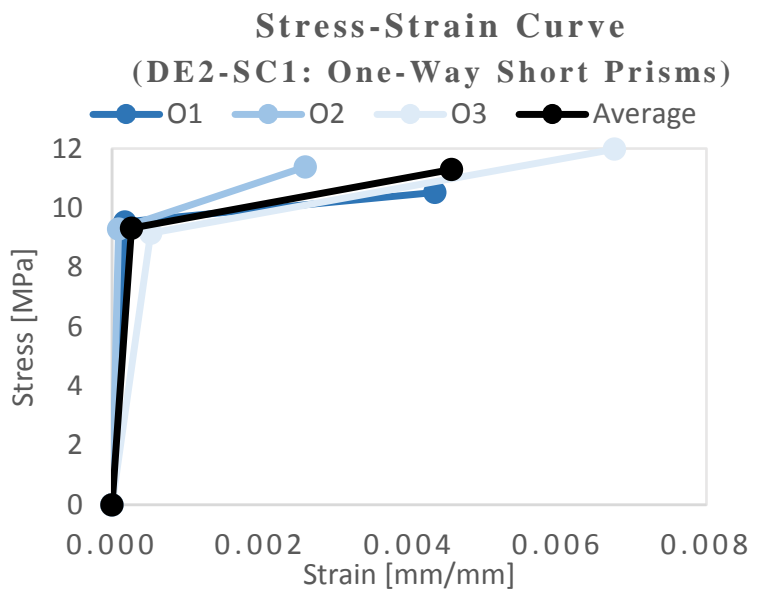

Stress-Strain Curve (DE2-SC1: Random Short Prisms)

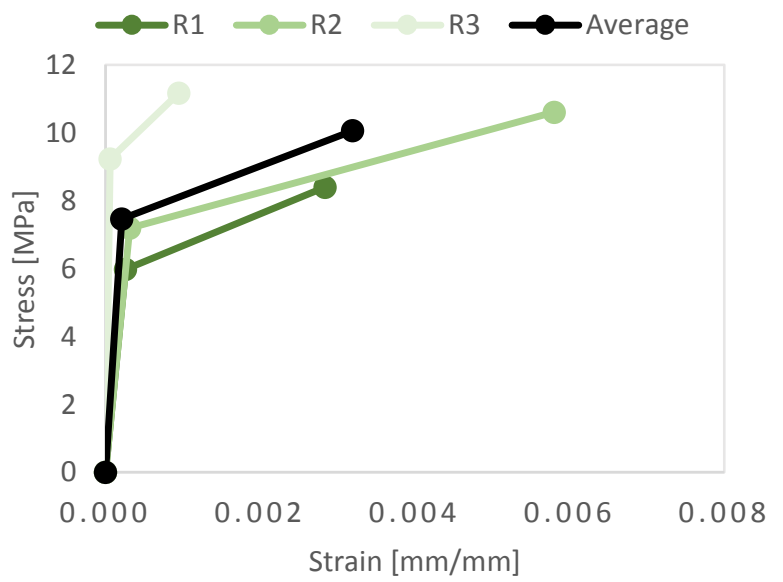

Stress-Crack Width Curve (DE2-SC1: One-Way Short Prisms)

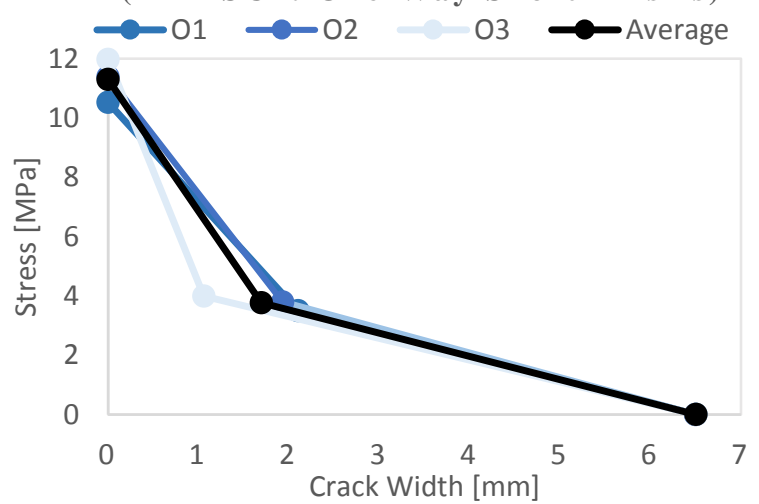

Stress-Crack Width Curve (DE2-SC1: Random Short Prisms)

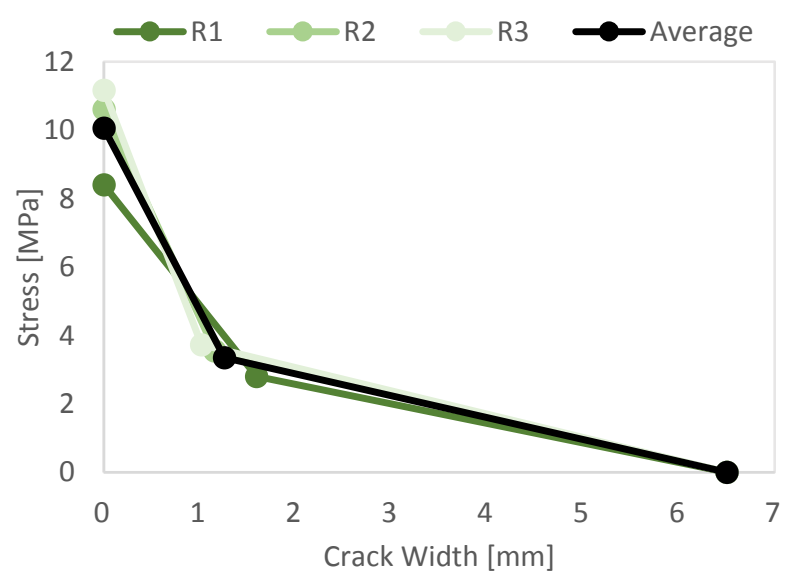

Figure B-2: Linearized tensile stress-strain-crack mouth opening relationships of the short prisms from mix DE2 tested with SC1 


\section{B.5 Commercial FD3 Design Mix}

Table B- 6: The four characteristic points utilized for the inverse analysis of the prisms from mix FD3 tested by York

University

\begin{tabular}{|c|c|c|c|c|c|c|c|c|c|c|c|c|}
\hline \multirow{2}{*}{ Specimen } & $\mathbf{P}_{\mathbf{0}}$ & $\mathbf{P}_{\mathbf{1}}$ & $\mathbf{P}_{\mathbf{2}}$ & $\mathbf{P}_{\mathbf{3}}$ & $\mathbf{P}_{\mathbf{4}}$ & $\mathbf{P}_{\max }$ & $\boldsymbol{\delta}_{\mathbf{o}}$ & $\boldsymbol{\delta}_{\mathbf{1}}$ & $\boldsymbol{\delta}_{\mathbf{2}}$ & $\boldsymbol{\delta}_{\mathbf{3}}$ & $\boldsymbol{\delta}_{\mathbf{4}}$ & $\boldsymbol{\delta}_{\mathbf{P m a x}}$ \\
\cline { 2 - 14 } & {$[\mathbf{k N}]$} & {$[\mathbf{k N}]$} & {$[\mathbf{k N}]$} & {$[\mathbf{k N}]$} & {$[\mathbf{k N}]$} & {$[\mathbf{k N}]$} & {$[\mathbf{m m}]$} & {$[\mathbf{m m}]$} & {$[\mathbf{m m}]$} & {$[\mathbf{m m}]$} & {$[\mathbf{m m}]$} & {$[\mathbf{m m}]$} \\
\hline OW2 & 65.00 & 78.00 & 100.00 & 141.44 & 113.15 & 145.81 & 0.065 & 0.104 & 0.252 & 0.870 & 1.800 & 1.035 \\
\hline OW3 & 55.00 & 70.00 & 88.00 & 118.29 & 94.63 & 121.95 & 0.080 & 0.134 & 0.310 & 0.700 & 2.140 & 0.783 \\
\hline Average & 60.00 & 74.00 & 94.00 & 129.87 & 103.89 & 133.88 & 0.073 & 0.119 & 0.281 & 0.785 & 1.970 & 0.909 \\
\hline \multirow{2}{*}{ Specimen } & $\mathbf{P}_{\mathbf{0}}$ & $\mathbf{P}_{\mathbf{1}}$ & $\mathbf{P}_{\mathbf{2}}$ & $\mathbf{P}_{\mathbf{3}}$ & $\mathbf{P}_{\mathbf{4}}$ & $\mathbf{P}_{\mathbf{m a x}}$ & $\boldsymbol{\delta}_{\mathbf{o}}$ & $\boldsymbol{\delta}_{\mathbf{1}}$ & $\boldsymbol{\delta}_{\mathbf{2}}$ & $\boldsymbol{\delta}_{\mathbf{3}}$ & $\boldsymbol{\delta}_{4}$ & $\boldsymbol{\delta}_{\mathbf{P m a x}}$ \\
\cline { 2 - 14 } & {$[\mathbf{k N}]$} & {$[\mathbf{k N}]$} & {$[\mathbf{k N}]$} & {$[\mathbf{k N}]$} & {$[\mathbf{k N}]$} & {$[\mathbf{k N}]$} & {$[\mathbf{m m}]$} & {$[\mathbf{m m}]$} & {$[\mathbf{m m}]$} & {$[\mathbf{m m}]$} & {$[\mathbf{m m}]$} & {$[\mathbf{m m}]$} \\
\hline R2 & 57.60 & 65.00 & 87.00 & 108.12 & 86.50 & 111.47 & 0.046 & 0.071 & 0.184 & 0.600 & 1.960 & 0.888 \\
\hline R3 & 49.87 & 71.00 & 84.00 & 112.82 & 90.25 & 116.31 & 0.054 & 0.102 & 0.225 & 0.680 & 1.600 & 0.887 \\
\hline Average & 53.74 & 68.00 & 85.5 & 110.47 & 88.38 & 113.89 & 0.050 & 0.087 & 0.205 & 0.640 & 1.780 & 0.888 \\
\hline
\end{tabular}

Table B- 7: The four characteristic points utilized for the inverse analysis of the prisms from mix FD3 tested by

École Polytechnique-Montréal

\begin{tabular}{|c|c|c|c|c|c|c|c|c|c|c|c|c|}
\hline \multirow{2}{*}{ Specimen } & $\mathbf{P}_{\mathbf{0}}$ & $\mathbf{P}_{\mathbf{1}}$ & $\mathbf{P}_{\mathbf{2}}$ & $\mathbf{P}_{\mathbf{3}}$ & $\mathbf{P}_{\mathbf{4}}$ & $\mathbf{P}_{\max }$ & $\boldsymbol{\delta}_{\mathbf{0}}$ & $\boldsymbol{\delta}_{\mathbf{1}}$ & $\boldsymbol{\delta}_{2}$ & $\boldsymbol{\delta}_{\mathbf{3}}$ & $\boldsymbol{\delta}_{4}$ & $\boldsymbol{\delta}_{\text {Pmax }}$ \\
\cline { 2 - 15 } & {$[\mathbf{k N}]$} & {$[\mathbf{k N}]$} & {$[\mathbf{k N}]$} & {$[\mathbf{k N}]$} & {$[\mathbf{k N}]$} & {$[\mathbf{k N}]$} & {$[\mathbf{m m}]$} & {$[\mathbf{m m}]$} & {$[\mathbf{m m}]$} & {$[\mathbf{m m}]$} & {$[\mathbf{m m}]$} & {$[\mathbf{m m}]$} \\
\hline OW1 & 45.00 & 55.00 & 78.00 & 97.19 & 77.76 & 100.20 & 0.060 & 0.100 & 0.260 & 0.820 & 1.700 & 0.960 \\
\hline OW2 & 52.00 & 62.50 & 76.00 & 113.69 & 90.95 & 117.21 & 0.052 & 0.083 & 0.190 & 0.820 & 1.550 & 1.050 \\
\hline Average & 48.50 & 58.75 & 77.00 & 105.44 & 84.36 & 108.71 & 0.056 & 0.092 & 0.225 & 0.820 & 1.625 & 1.005 \\
\hline \multirow{2}{*}{ Specimen } & $\mathbf{P}_{\mathbf{o}}$ & $\mathbf{P}_{\mathbf{1}}$ & $\mathbf{P}_{\mathbf{2}}$ & $\mathbf{P}_{\mathbf{3}}$ & $\mathbf{P}_{\mathbf{4}}$ & $\mathbf{P}_{\max }$ & $\boldsymbol{\delta}_{\mathbf{o}}$ & $\boldsymbol{\delta}_{\mathbf{1}}$ & $\boldsymbol{\delta}_{2}$ & $\boldsymbol{\delta}_{3}$ & $\boldsymbol{\delta}_{4}$ & $\boldsymbol{\delta}_{\text {Pmax }}$ \\
\cline { 2 - 13 } & {$[\mathbf{k N}]$} & {$[\mathbf{k N}]$} & {$[\mathbf{k N}]$} & {$[\mathbf{k N}]$} & {$[\mathbf{k N}]$} & {$[\mathbf{k N}]$} & {$[\mathbf{m m}]$} & {$[\mathbf{m m}]$} & {$[\mathbf{m m}]$} & {$[\mathbf{m m}]$} & {$[\mathbf{m m}]$} & {$[\mathbf{m m}]$} \\
\hline R3 & 40.00 & 45.00 & 58.00 & 75.95 & 60.76 & 78.30 & 0.035 & 0.053 & 0.127 & 0.530 & 2.100 & 0.690 \\
\hline
\end{tabular}

Table B- 8: The four characteristic points utilized for the inverse analysis of the prisms from mix FD3 tested by

Queen's University

\begin{tabular}{|c|c|c|c|c|c|c|c|c|c|c|c|c|}
\hline \multirow{2}{*}{ Specimen } & $\mathbf{P}_{\mathbf{0}}$ & $\mathbf{P}_{\mathbf{1}}$ & $\mathbf{P}_{\mathbf{2}}$ & $\mathbf{P}_{\mathbf{3}}$ & $\mathbf{P}_{\mathbf{4}}$ & $\mathbf{P}_{\max }$ & $\boldsymbol{\delta}_{\mathbf{0}}$ & $\boldsymbol{\delta}_{\mathbf{1}}$ & $\boldsymbol{\delta}_{\mathbf{2}}$ & $\boldsymbol{\delta}_{\mathbf{3}}$ & $\boldsymbol{\delta}_{\mathbf{4}}$ & $\boldsymbol{\delta}_{\text {Pmax }}$ \\
\cline { 2 - 14 } & {$[\mathbf{k N}]$} & {$[\mathbf{k N}]$} & {$[\mathbf{k N}]$} & {$[\mathbf{k N}]$} & {$[\mathbf{k N}]$} & {$[\mathbf{k N}]$} & {$[\mathbf{m m}]$} & {$[\mathbf{m m}]$} & {$[\mathbf{m m}]$} & {$[\mathbf{m m}]$} & {$[\mathbf{m m}]$} & {$[\mathbf{m m}]$} \\
\hline \multirow{2}{*}{ OW3 } & 40.00 & 75.00 & 80.00 & 93.38 & 74.71 & 96.27 & 0.040 & 0.150 & 0.200 & 0.400 & 0.850 & 0.451 \\
\hline \multirow{2}{*}{ Specimen } & $\mathbf{P}_{\mathbf{0}}$ & $\mathbf{P}_{\mathbf{1}}$ & $\mathbf{P}_{\mathbf{2}}$ & $\mathbf{P}_{\mathbf{3}}$ & $\mathbf{P}_{\mathbf{4}}$ & $\mathbf{P}_{\max }$ & $\boldsymbol{\delta}_{\mathbf{0}}$ & $\boldsymbol{\delta}_{\mathbf{1}}$ & $\boldsymbol{\delta}_{\mathbf{2}}$ & $\boldsymbol{\delta}_{\mathbf{3}}$ & $\boldsymbol{\delta}_{\mathbf{4}}$ & $\boldsymbol{\delta}_{\mathbf{P m a x}}$ \\
\cline { 2 - 13 } & {$[\mathbf{k N}]$} & {$[\mathbf{k N}]$} & {$[\mathbf{k N}]$} & {$[\mathbf{k N}]$} & {$[\mathbf{k N}]$} & {$[\mathbf{k N}]$} & {$[\mathbf{m m}]$} & {$[\mathbf{m m}]$} & {$[\mathbf{m m}]$} & {$[\mathbf{m m}]$} & {$[\mathbf{m m}]$} & {$[\mathbf{m m}]$} \\
\hline \multirow{2}{*}{ R } & 40.00 & 57.00 & 70.00 & 87.91 & 70.33 & 90.63 & 0.070 & 0.133 & 0.305 & 0.650 & 1.350 & 0.791 \\
\hline
\end{tabular}



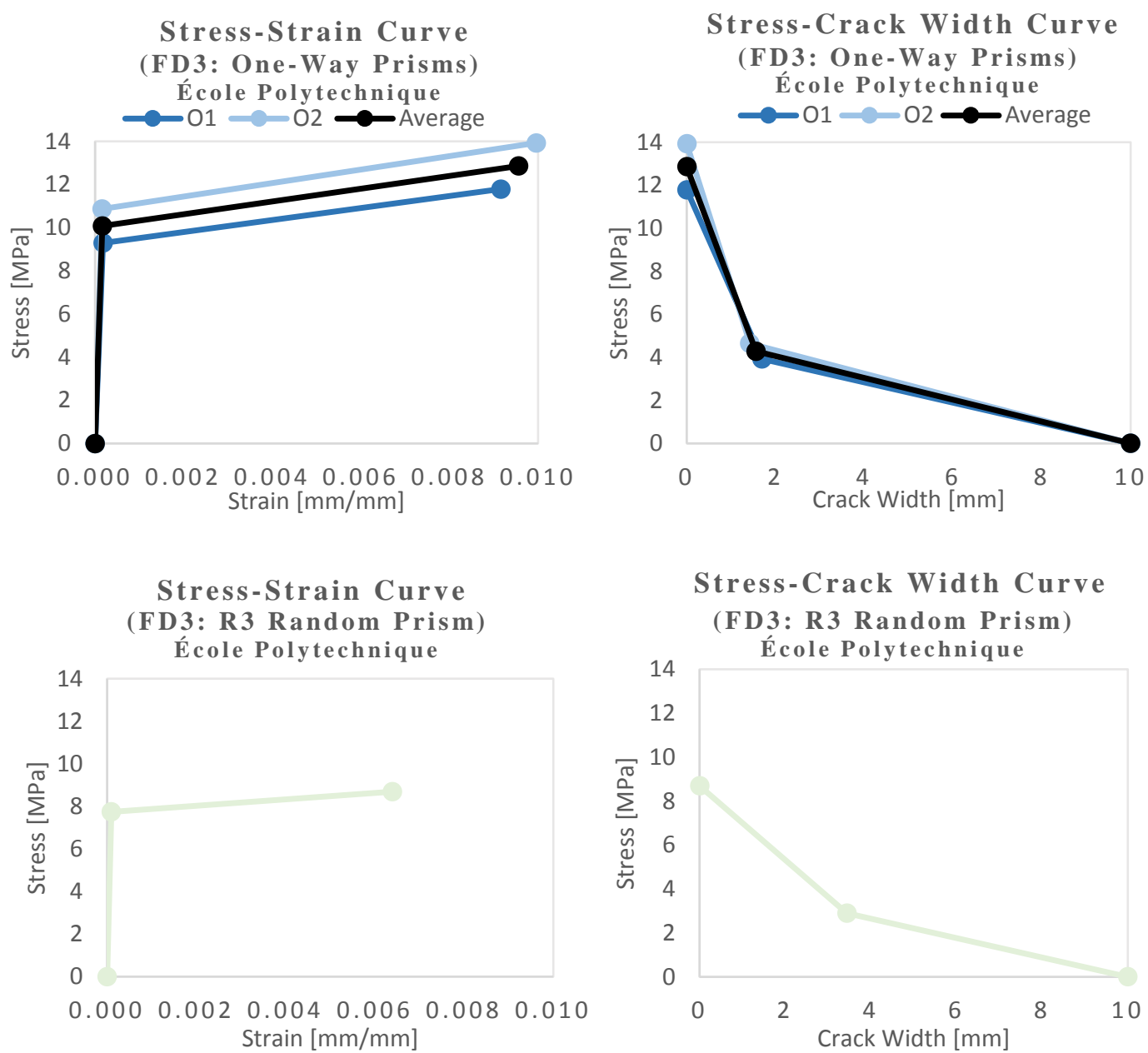

Figure B-3: Linearized tensile stress-strain-crack mouth opening relationships of the prisms from mix FD3 tested by École Polytechnique-Montréal
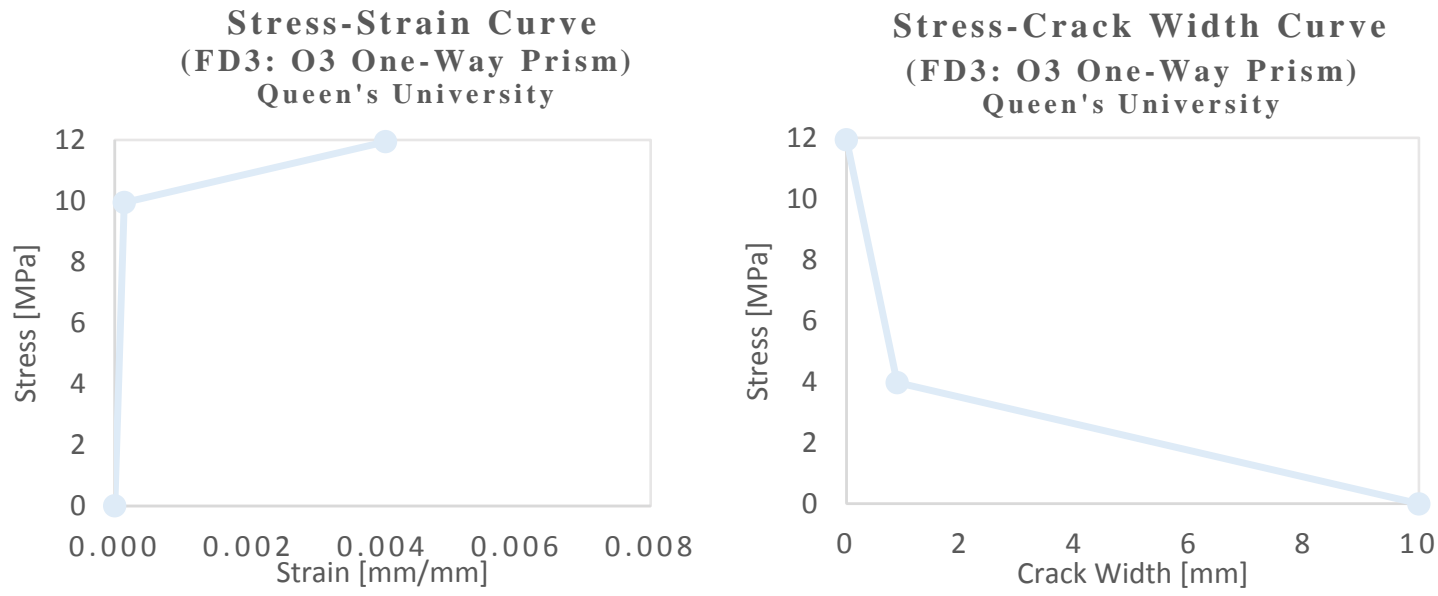

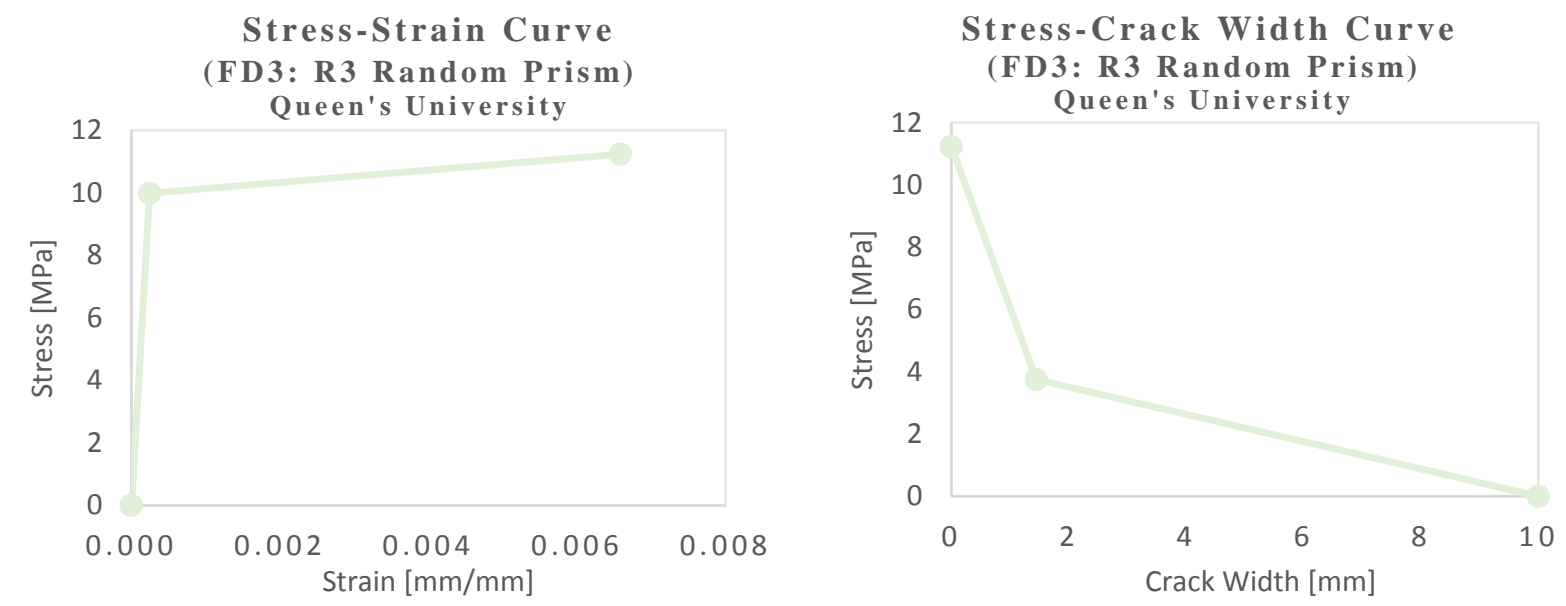

Figure B-4: Linearized tensile stress-strain-crack mouth opening relationships of the prisms from mix FD3 tested by Queen's University

\section{B.6 Commercial KI2 Design Mix}

Table B- 9: The four characteristic points utilized for the inverse analysis of the prisms from mix KI2 tested by York

University

\begin{tabular}{|c|c|c|c|c|c|c|c|c|c|c|c|c|}
\hline & $\mathbf{P}_{\mathbf{0}}$ & $\mathbf{P}_{1}$ & $\mathbf{P}_{2}$ & $\mathbf{P}_{3}$ & $\mathbf{P}_{4}$ & $P_{\max }$ & $\delta_{0}$ & $\delta_{1}$ & $\boldsymbol{\delta}_{2}$ & $\delta_{3}$ & $\delta_{4}$ & $\delta_{P \max }$ \\
\hline Specimen & {$[\mathrm{kN}]$} & {$[\mathrm{kN}]$} & {$[\mathrm{kN}]$} & {$[\mathrm{kN}]$} & {$[\mathbf{k N}]$} & [kN] & {$[\mathrm{mm}]$} & {$[\mathrm{mm}]$} & {$[\mathrm{mm}]$} & {$[\mathrm{mm}]$} & {$[\mathrm{mm}]$} & {$[\mathrm{mm}]$} \\
\hline OW1 & 22.84 & 27.00 & 37.00 & 53.43 & 42.74 & 55.08 & 0.010 & 0.020 & 0.050 & 0.430 & 1.450 & 0.660 \\
\hline OW3 & 14.98 & 30.00 & 43.00 & 49.45 & 39.56 & 50.98 & 0.020 & 0.061 & 0.160 & 0.295 & 1.280 & 0.360 \\
\hline Average & 18.91 & 28.50 & 40.00 & 51.44 & 41.15 & 53.03 & 0.015 & 0.040 & 0.105 & 0.363 & 1.365 & 0.510 \\
\hline & $\mathbf{P}_{\mathbf{o}}$ & $\mathbf{P}_{1}$ & $\mathbf{P}_{2}$ & $\mathbf{P}_{3}$ & $\mathbf{P}_{4}$ & $P_{\max }$ & $\delta_{0}$ & $\delta_{1}$ & $\delta_{2}$ & $\delta_{3}$ & $\delta_{4}$ & סPmax \\
\hline Specimen & {$[\mathrm{kN}]$} & {$[\mathrm{kN}]$} & {$[\mathrm{kN}]$} & {$[\mathrm{kN}]$} & {$[\mathrm{kN}]$} & {$[\mathrm{kN}]$} & {$[\mathrm{mm}]$} & {$[\mathrm{mm}]$} & {$[\mathrm{mm}]$} & {$[\mathrm{mm}]$} & {$[\mathrm{mm}]$} & {$[\mathrm{mm}]$} \\
\hline $\mathrm{R} 1$ & 20.00 & 28.00 & 41.00 & 47.90 & 38.32 & 49.38 & 0.032 & 0.059 & 0.161 & 0.340 & 1.600 & 0.496 \\
\hline R2 & 22.00 & 32.00 & 49.00 & 55.49 & 44.39 & 57.20 & 0.045 & 0.087 & 0.250 & 0.410 & 1.100 & 0.508 \\
\hline R3 & 20.16 & 39.00 & 49.00 & 66.26 & 53.01 & 68.31 & 0.027 & 0.068 & 0.157 & 0.415 & 1.400 & 0.501 \\
\hline Average & 20.72 & 33.00 & 46.33 & 56.55 & 45.24 & 58.30 & 0.035 & 0.071 & 0.189 & 0.388 & 1.367 & 0.502 \\
\hline
\end{tabular}

Table B-10: The four characteristic points utilized for the inverse analysis of the prisms from mix KI2 tested by

École Polytechnique-Montréal

\begin{tabular}{|c|c|c|c|c|c|c|c|c|c|c|c|c|}
\hline \multirow{2}{*}{ Specimen } & $\mathbf{P}_{\mathbf{o}}$ & $\mathbf{P}_{\mathbf{1}}$ & $\mathbf{P}_{\mathbf{2}}$ & $\mathbf{P}_{\mathbf{3}}$ & $\mathbf{P}_{\mathbf{4}}$ & $\mathbf{P}_{\max }$ & $\boldsymbol{\delta}_{\mathbf{o}}$ & $\boldsymbol{\delta}_{\mathbf{1}}$ & $\boldsymbol{\delta}_{\mathbf{2}}$ & $\boldsymbol{\delta}_{\mathbf{3}}$ & $\boldsymbol{\delta}_{\mathbf{4}}$ & $\boldsymbol{\delta}_{\text {Pmax }}$ \\
\cline { 2 - 15 } & {$[\mathbf{k N}]$} & {$[\mathbf{k N}]$} & {$[\mathbf{k N}]$} & {$[\mathbf{k N}]$} & {$[\mathbf{k N}]$} & {$[\mathbf{k N}]$} & {$[\mathbf{m m}]$} & {$[\mathbf{m m}]$} & {$[\mathbf{m m}]$} & {$[\mathbf{m m}]$} & {$[\mathbf{m m}]$} & {$[\mathbf{m m}]$} \\
\hline OW1 & 15.00 & 25.00 & 37.00 & 42.79 & 34.22 & 44.10 & 0.040 & 0.089 & 0.246 & 0.480 & 0.840 & 0.590 \\
\hline OW2 & 20.00 & 24.00 & 33.50 & 37.25 & 29.80 & 38.40 & 0.040 & 0.064 & 0.168 & 0.330 & 0.820 & 0.410 \\
\hline Average & 17.50 & 24.50 & 35.25 & 40.02 & 32.01 & 41.25 & 0.040 & 0.077 & 0.207 & 0.405 & 0.830 & 0.500 \\
\hline \multirow{2}{*}{ Specimen } & $\mathbf{P}_{\mathbf{o}}$ & $\mathbf{P}_{\mathbf{1}}$ & $\mathbf{P}_{\mathbf{2}}$ & $\mathbf{P}_{\mathbf{3}}$ & $\mathbf{P}_{\mathbf{4}}$ & $\mathbf{P}_{\max }$ & $\boldsymbol{\delta}_{\mathbf{o}}$ & $\boldsymbol{\delta}_{\mathbf{1}}$ & $\boldsymbol{\delta}_{\mathbf{2}}$ & $\boldsymbol{\delta}_{3}$ & $\boldsymbol{\delta}_{4}$ & $\boldsymbol{\delta}_{\mathbf{P} m a x}$ \\
\cline { 2 - 13 } & {$[\mathbf{k N}]$} & {$[\mathbf{k N}]$} & {$[\mathbf{k N}]$} & {$[\mathbf{k N}]$} & {$[\mathbf{k N}]$} & {$[\mathbf{k N}]$} & {$[\mathbf{m m}]$} & {$[\mathbf{m m}]$} & {$[\mathbf{m m}]$} & {$[\mathbf{m m}]$} & {$[\mathbf{m m}]$} & {$[\mathbf{m m}]$} \\
\hline R3 & 18.00 & 25.00 & 36.00 & 44.33 & 35.46 & 45.70 & 0.035 & 0.065 & 0.175 & 0.460 & 1.000 & 0.630 \\
\hline
\end{tabular}


Table B-11: The four characteristic points utilized for the inverse analysis of the prisms from mix KI2 tested by

Queen's University

\begin{tabular}{|c|c|c|c|c|c|c|c|c|c|c|c|c|}
\hline \multirow{2}{*}{ Specimen } & $P_{0}$ & $\mathbf{P}_{1}$ & $\mathbf{P}_{2}$ & $\mathbf{P}_{3}$ & $\mathbf{P}_{4}$ & $\mathbf{P}_{\max }$ & $\delta_{0}$ & $\delta_{1}$ & $\boldsymbol{\delta}_{2}$ & $\delta 3$ & $\delta_{4}$ & $\delta_{P \max }$ \\
\hline & {$[\mathbf{k N}]$} & {$[\mathbf{k N}]$} & {$[\mathbf{k N}]$} & {$[\mathbf{k N}]$} & {$[\mathbf{k N}]$} & {$[\mathbf{k N}]$} & {$[\mathrm{mm}]$} & {$[\mathrm{mm}]$} & {$[\mathrm{mm}]$} & {$[\mathrm{mm}]$} & {$[\mathrm{mm}]$} & {$[\mathrm{mm}]$} \\
\hline OW3 & 20.00 & 24.00 & 34.00 & 38.06 & 30.45 & 39.24 & 0.025 & 0.040 & 0.106 & 0.250 & 1.000 & 0.371 \\
\hline \multirow{2}{*}{ Specimen } & $\mathbf{P}_{\mathbf{o}}$ & $\mathbf{P}_{1}$ & $\mathbf{P}_{2}$ & $\mathbf{P}_{3}$ & $\mathbf{P}_{4}$ & $\mathbf{P}_{\max }$ & $\delta_{\mathbf{o}}$ & $\delta_{1}$ & $\delta_{2}$ & $\delta_{3}$ & $\delta_{4}$ & $\delta$ Pmax \\
\hline & {$[\mathbf{k N}]$} & {$[\mathbf{k N}]$} & {$[\mathbf{k N}]$} & {$[\mathbf{k N}]$} & {$[\mathbf{k N}]$} & {$[\mathbf{k N}]$} & {$[\mathrm{mm}]$} & {$[\mathrm{mm}]$} & {$[\mathrm{mm}]$} & {$[\mathrm{mm}]$} & {$[\mathrm{mm}]$} & {$[\mathrm{mm}]$} \\
\hline R2 & 10.00 & 20.00 & 27.00 & 32.37 & 25.89 & 33.37 & 0.015 & 0.040 & 0.102 & 0.250 & 1.500 & 0.390 \\
\hline R3 & 20.00 & 24.00 & 34.00 & 48.87 & 39.10 & 50.38 & 0.030 & 0.048 & 0.128 & 0.600 & 2.600 & 0.875 \\
\hline Average & 15.00 & 22.00 & 30.50 & 40.62 & 32.50 & 41.88 & 0.023 & 0.044 & 0.115 & 0.425 & 2.050 & 0.633 \\
\hline
\end{tabular}
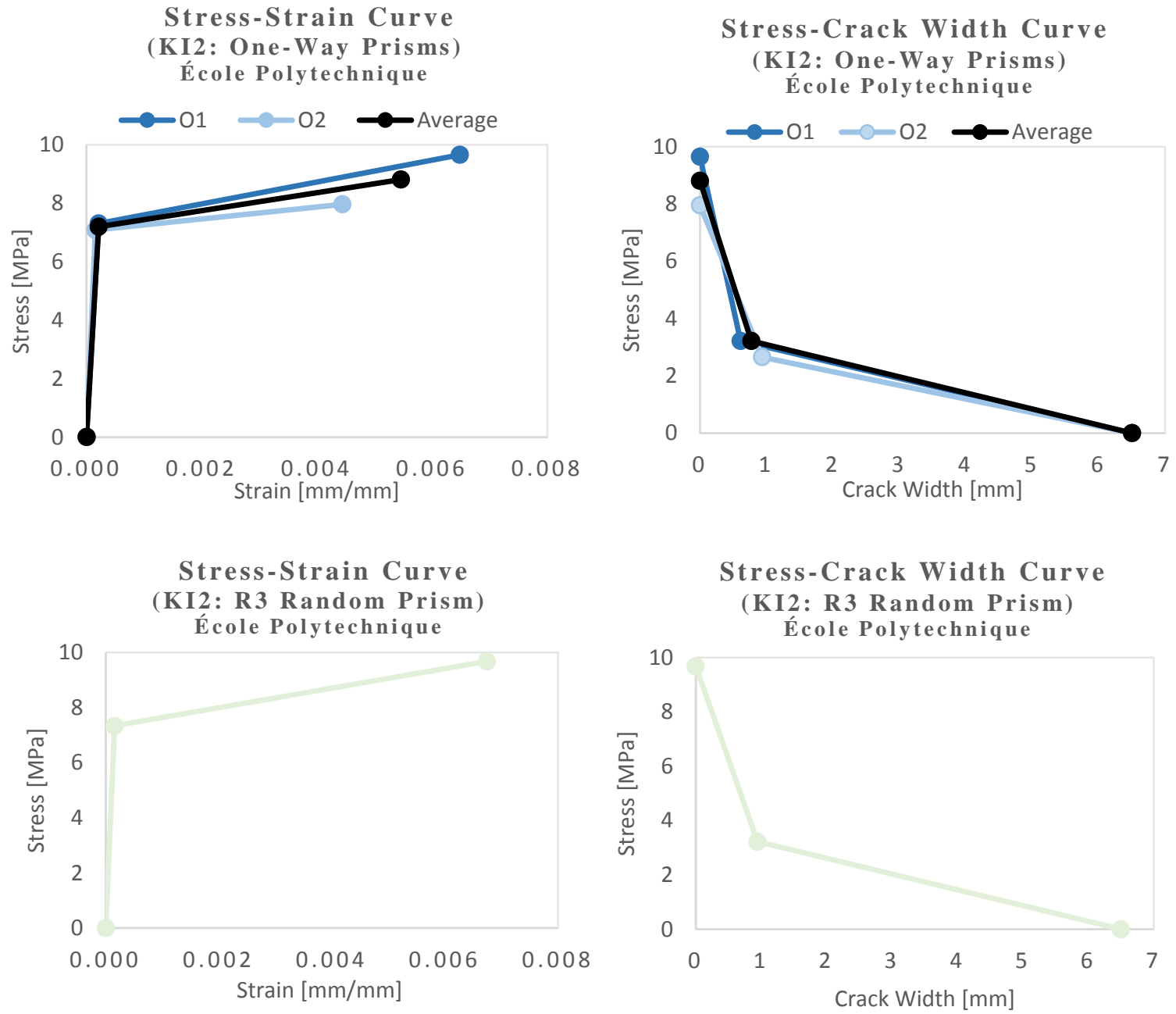

Figure B- 5: Linearized tensile stress-strain-crack mouth opening relationships of the prisms from mix KI2 tested by École Polytechnique-Montréal 

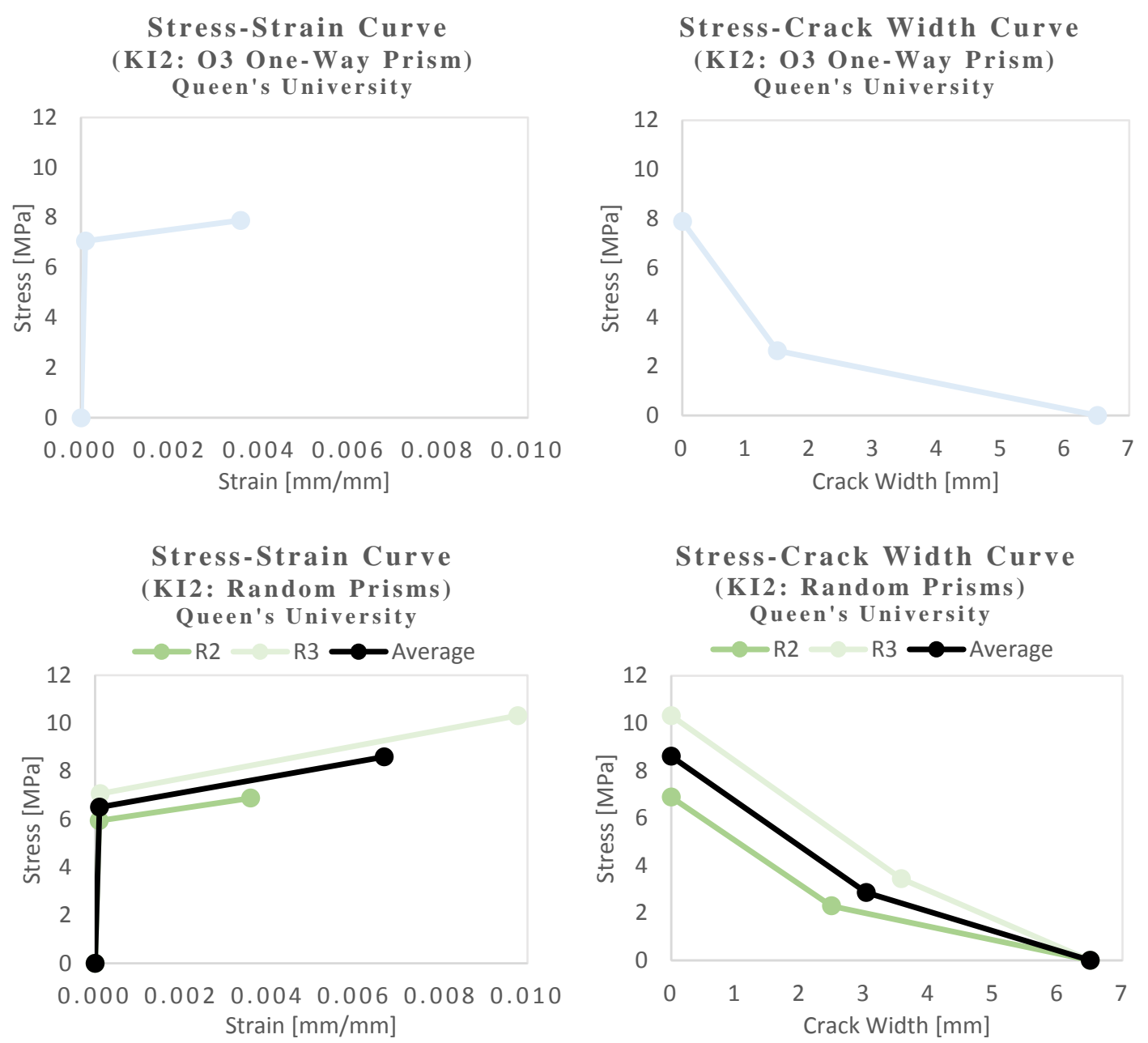

Figure B- 6: Linearized tensile stress-strain-crack mouth opening relationships of the prisms from mix KI2 tested by

Queen's University

\section{B.7 Commercial LD2 Design Mix}

Table B-12: The four characteristic points utilized for the inverse analysis of the prisms from mix LD2 tested by

York University

\begin{tabular}{|c|c|c|c|c|c|c|c|c|c|c|c|c|}
\hline \multirow{2}{*}{ Specimen } & $\mathbf{P}_{\mathbf{0}}$ & $\mathbf{P}_{1}$ & $\mathbf{P}_{2}$ & $\mathbf{P}_{3}$ & $\mathbf{P}_{4}$ & $\mathbf{P}_{\max }$ & $\delta_{0}$ & $\delta_{1}$ & $\delta_{2}$ & $\delta_{3}$ & $\delta_{4}$ & $\delta_{\text {Pmax }}$ \\
\hline & {$[\mathrm{kN}]$} & {$[\mathrm{kN}]$} & {$[\mathrm{kN}]$} & {$[\mathrm{kN}]$} & {$[\mathrm{kN}]$} & [kN] & [mm] & [mm] & [mm] & [mm] & [mm] & [mm] \\
\hline OW1 & 25.00 & 38.00 & 53.50 & 54.35 & 43.48 & 56.03 & 0.045 & 0.103 & 0.268 & 0.300 & 1.020 & 0.400 \\
\hline OW2 & 15.26 & 24.00 & 38.00 & 40.15 & 32.12 & 41.39 & 0.035 & 0.072 & 0.213 & 0.250 & 1.150 & 0.326 \\
\hline OW3 & 20.00 & 34.93 & 48.00 & 52.08 & 41.66 & 53.69 & 0.015 & 0.035 & 0.090 & 0.140 & 0.680 & 0.200 \\
\hline Average & 20.09 & 32.31 & 46.50 & 48.86 & 39.09 & 50.37 & 0.032 & 0.070 & 0.190 & 0.230 & 0.950 & 0.309 \\
\hline \multirow{2}{*}{ Specimen } & $\mathbf{P}_{\mathbf{0}}$ & $\mathbf{P}_{1}$ & $\mathbf{P}_{2}$ & $\mathbf{P}_{3}$ & $\mathbf{P}_{4}$ & $\mathbf{P}_{\max }$ & $\delta_{0}$ & $\boldsymbol{\delta}_{1}$ & $\boldsymbol{\delta}_{2}$ & $\boldsymbol{\delta}_{\mathbf{3}}$ & $\boldsymbol{\delta}_{4}$ & $\delta_{\text {Pmax }}$ \\
\hline & {$[\mathbf{k N}]$} & {$[\mathbf{k N}]$} & {$[\mathbf{k N}]$} & {$[\mathbf{k N}]$} & {$[\mathbf{k N}]$} & {$[\mathbf{k N}]$} & [mm] & {$[\mathrm{mm}]$} & {$[\mathrm{mm}]$} & [mm] & {$[\mathbf{m m}]$} & {$[\mathrm{mm}]$} \\
\hline R1 & 17.70 & 26.00 & 37.00 & 38.90 & 31.12 & 40.11 & 0.027 & 0.052 & 0.138 & 0.148 & 0.500 & 0.180 \\
\hline R3 & 17.29 & 33.00 & 40.00 & 48.00 & 54.19 & 50.00 & 0.028 & 0.068 & 0.100 & 0.182 & 0.280 & 0.200 \\
\hline Average & 17.50 & 29.50 & 38.50 & 43.45 & 42.66 & 45.05 & 0.027 & 0.060 & 0.119 & 0.165 & 0.390 & 0.190 \\
\hline
\end{tabular}


Table B-13: The four characteristic points utilized for the inverse analysis of the prisms from mix LD2 tested by École Polytechnique-Montréal

\begin{tabular}{|c|c|c|c|c|c|c|c|c|c|c|c|c|}
\hline \multirow{2}{*}{ Specimen } & $\mathbf{P}_{\mathbf{0}}$ & $\mathbf{P}_{\mathbf{1}}$ & $\mathbf{P}_{\mathbf{2}}$ & $\mathbf{P}_{\mathbf{3}}$ & $\mathbf{P}_{\mathbf{4}}$ & $\mathbf{P}_{\max }$ & $\boldsymbol{\delta}_{\mathbf{0}}$ & $\boldsymbol{\delta}_{\mathbf{1}}$ & $\boldsymbol{\delta}_{\mathbf{2}}$ & $\boldsymbol{\delta}_{\mathbf{3}}$ & $\boldsymbol{\delta}_{\mathbf{4}}$ & $\boldsymbol{\delta}_{\operatorname{Pmax}}$ \\
\cline { 2 - 13 } & {$[\mathbf{k N}]$} & {$[\mathbf{k N}]$} & {$[\mathbf{k N}]$} & {$[\mathbf{k N}]$} & {$[\mathbf{k N}]$} & {$[\mathbf{k N}]$} & {$[\mathbf{m m}]$} & {$[\mathbf{m m}]$} & {$[\mathbf{m m}]$} & {$[\mathbf{m m}]$} & {$[\mathbf{m m}]$} & {$[\mathbf{m m}]$} \\
\hline OW1 & 20.00 & 25.00 & 33.00 & 42.00 & 33.60 & 43.30 & 0.035 & 0.065 & 0.145 & 0.400 & 1.270 & 0.580 \\
\hline OW2 & 20.00 & 32.00 & 35.00 & 38.70 & 30.96 & 39.90 & 0.035 & 0.080 & 0.154 & 0.290 & 0.800 & 0.390 \\
\hline OW3 & 15.40 & 18.00 & 23.00 & 30.26 & 24.21 & 31.20 & 0.020 & 0.031 & 0.074 & 0.190 & 0.950 & 0.287 \\
\hline Average & 18.47 & 25.00 & 30.33 & 36.99 & 29.59 & 38.13 & 0.030 & 0.059 & 0.124 & 0.293 & 1.007 & 0.419 \\
\hline \multirow{2}{*}{ Specimen } & $\mathbf{P}_{\mathbf{0}}$ & $\mathbf{P}_{\mathbf{1}}$ & $\mathbf{P}_{\mathbf{2}}$ & $\mathbf{P}_{\mathbf{3}}$ & $\mathbf{P}_{\mathbf{4}}$ & $\mathbf{P}_{\max }$ & $\boldsymbol{\delta}_{\mathbf{0}}$ & $\boldsymbol{\delta}_{\mathbf{1}}$ & $\boldsymbol{\delta}_{\mathbf{2}}$ & $\boldsymbol{\delta}_{3}$ & $\boldsymbol{\delta}_{4}$ & $\boldsymbol{\delta}_{\operatorname{Pmax}}$ \\
\cline { 2 - 13 } & {$[\mathbf{k N}]$} & {$[\mathbf{k N}]$} & {$[\mathbf{k N}]$} & {$[\mathbf{k N}]$} & {$[\mathbf{k N}]$} & {$[\mathbf{k N}]$} & {$[\mathbf{m m}]$} & {$[\mathbf{m m}]$} & {$[\mathbf{m m}]$} & {$[\mathbf{m m}]$} & {$[\mathbf{m m}]$} & {$[\mathbf{m m}]$} \\
\hline R1 & 20.00 & 26.00 & 41.00 & 42.20 & 33.76 & 43.50 & 0.055 & 0.095 & 0.280 & 0.380 & 1.050 & 0.560 \\
\hline
\end{tabular}

Table B-14: The four characteristic points utilized for the inverse analysis of the prisms from mix LD2 tested by

Queen's University

\begin{tabular}{|c|c|c|c|c|c|c|c|c|c|c|c|c|}
\hline \multirow{2}{*}{ Specimen } & $\mathbf{P}_{\mathbf{0}}$ & $\mathbf{P}_{1}$ & $\mathbf{P}_{2}$ & $\mathbf{P}_{3}$ & $\mathbf{P}_{4}$ & $\mathbf{P}_{\max }$ & $\delta_{0}$ & $\delta_{1}$ & $\delta_{2}$ & $\delta_{3}$ & $\delta_{4}$ & $\delta_{P \max }$ \\
\hline & {$[\mathbf{k N}]$} & {$[\mathbf{k N}]$} & {$[\mathbf{k N}]$} & {$[\mathbf{k N}]$} & {$[\mathbf{k N}]$} & {$[\mathbf{k N}]$} & {$[\mathrm{mm}]$} & {$[\mathrm{mm}]$} & {$[\mathrm{mm}]$} & {$[\mathrm{mm}]$} & {$[\mathrm{mm}]$} & {$[\mathrm{mm}]$} \\
\hline OW1 & 15.00 & 24.00 & 36.50 & 37.43 & 29.95 & 38.59 & 0.023 & 0.049 & 0.140 & 0.170 & 0.950 & 0.243 \\
\hline \multirow{2}{*}{ Specimen } & $\mathbf{P}_{\mathbf{0}}$ & $\mathbf{P}_{1}$ & $\mathbf{P}_{2}$ & $\mathbf{P}_{3}$ & $\mathbf{P}_{4}$ & $\mathbf{P}_{\max }$ & $\delta_{0}$ & $\delta_{1}$ & $\delta_{2}$ & $\delta_{3}$ & $\delta_{4}$ & $\boldsymbol{\delta}_{\mathbf{P} \max }$ \\
\hline & {$[\mathbf{k N}]$} & {$[\mathbf{k N}]$} & {$[\mathbf{k N}]$} & {$[\mathbf{k N}]$} & {$[\mathbf{k N}]$} & {$[\mathbf{k N}]$} & {$[\mathrm{mm}]$} & {$[\mathrm{mm}]$} & {$[\mathrm{mm}]$} & {$[\mathrm{mm}]$} & {$[\mathrm{mm}]$} & {$[\mathrm{mm}]$} \\
\hline R1 & 15.00 & 22.00 & 31.00 & 31.26 & 25.00 & 32.22 & 0.020 & 0.039 & 0.103 & 0.110 & 0.700 & 0.153 \\
\hline R3 & 12.00 & 18.00 & 27.00 & 29.64 & 23.71 & 30.56 & 0.015 & 0.030 & 0.084 & 0.130 & 0.650 & 0.179 \\
\hline Average & 13.50 & 20.00 & 29.00 & 30.45 & 2436 & 31.39 & 0.018 & 0.035 & 0.094 & 0.120 & 0.675 & 0.166 \\
\hline
\end{tabular}
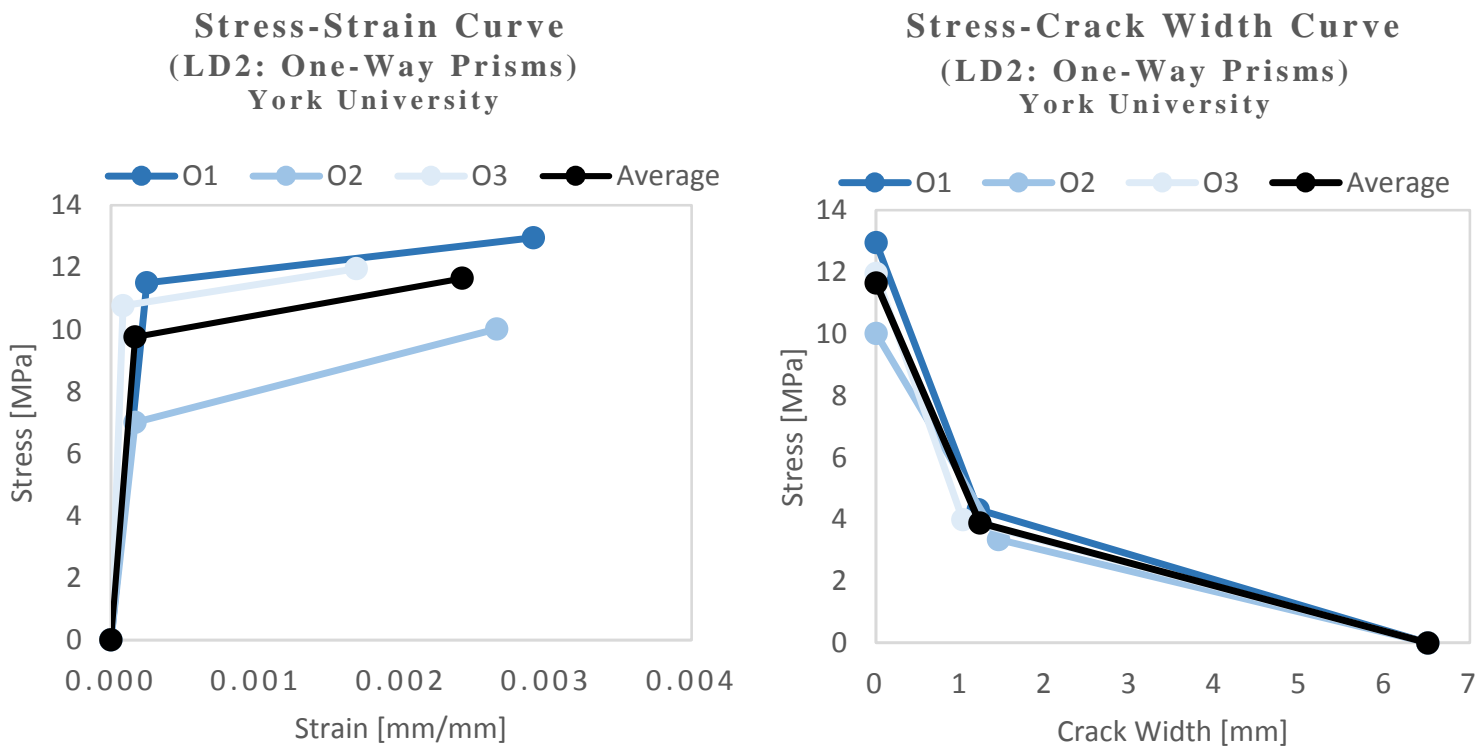

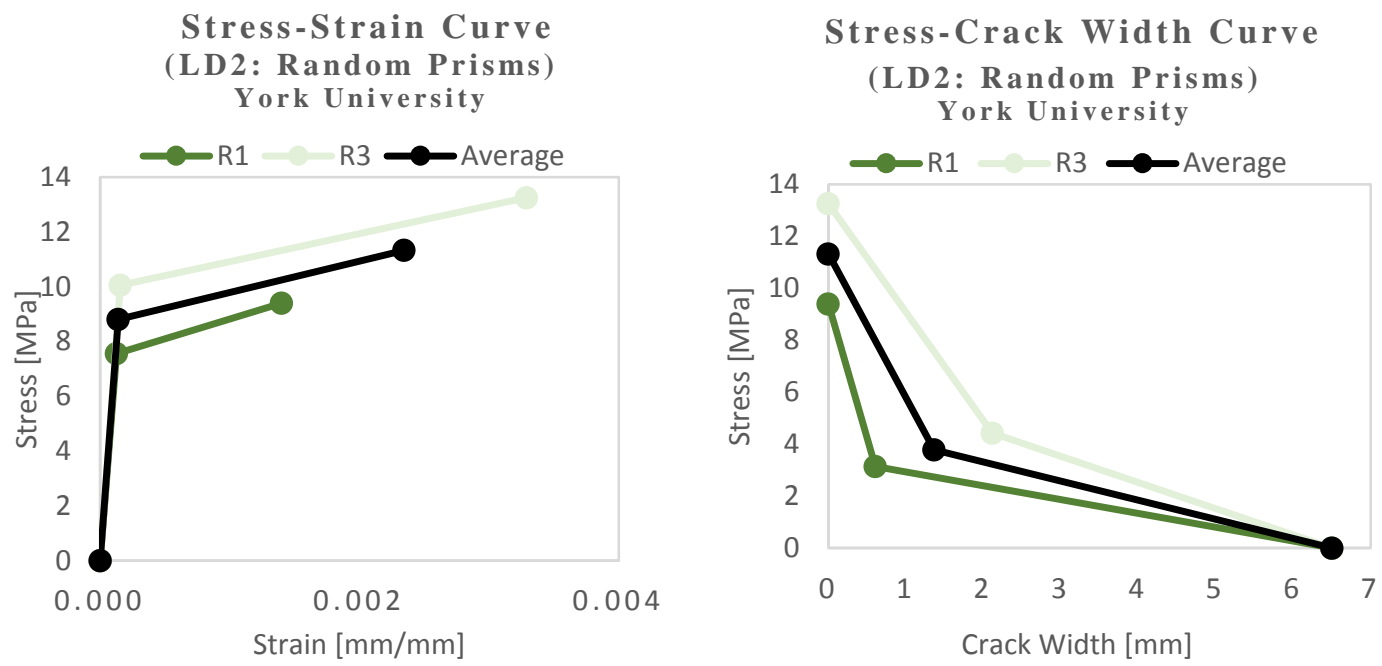

Figure B-7: Linearized tensile stress-strain-crack mouth opening relationships of the prisms from mix LD2 tested by York University
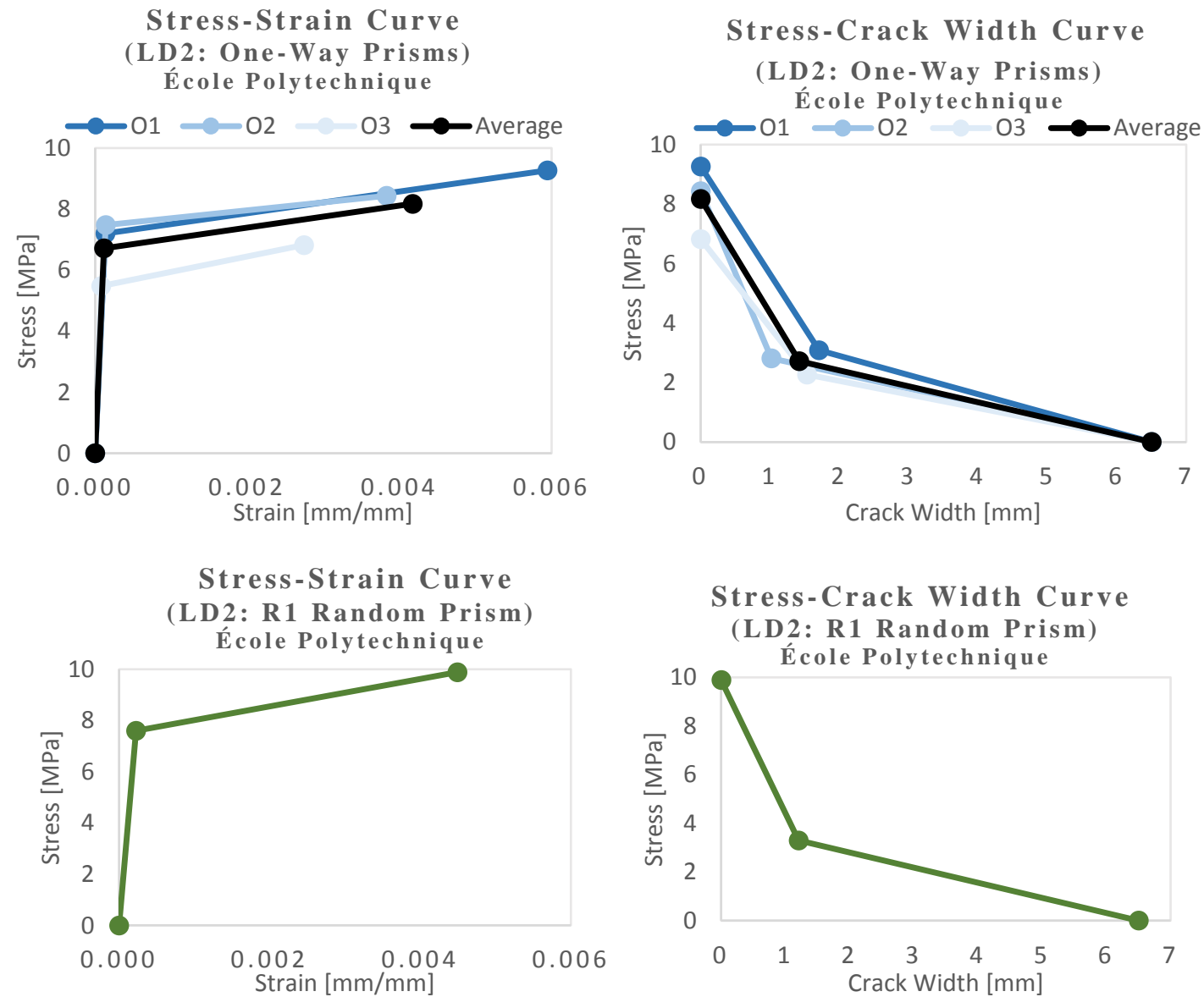

Figure B-8: Linearized tensile stress-strain-crack mouth opening relationships of the prisms from mix LD2 tested by École Polytechnique-Montréal 

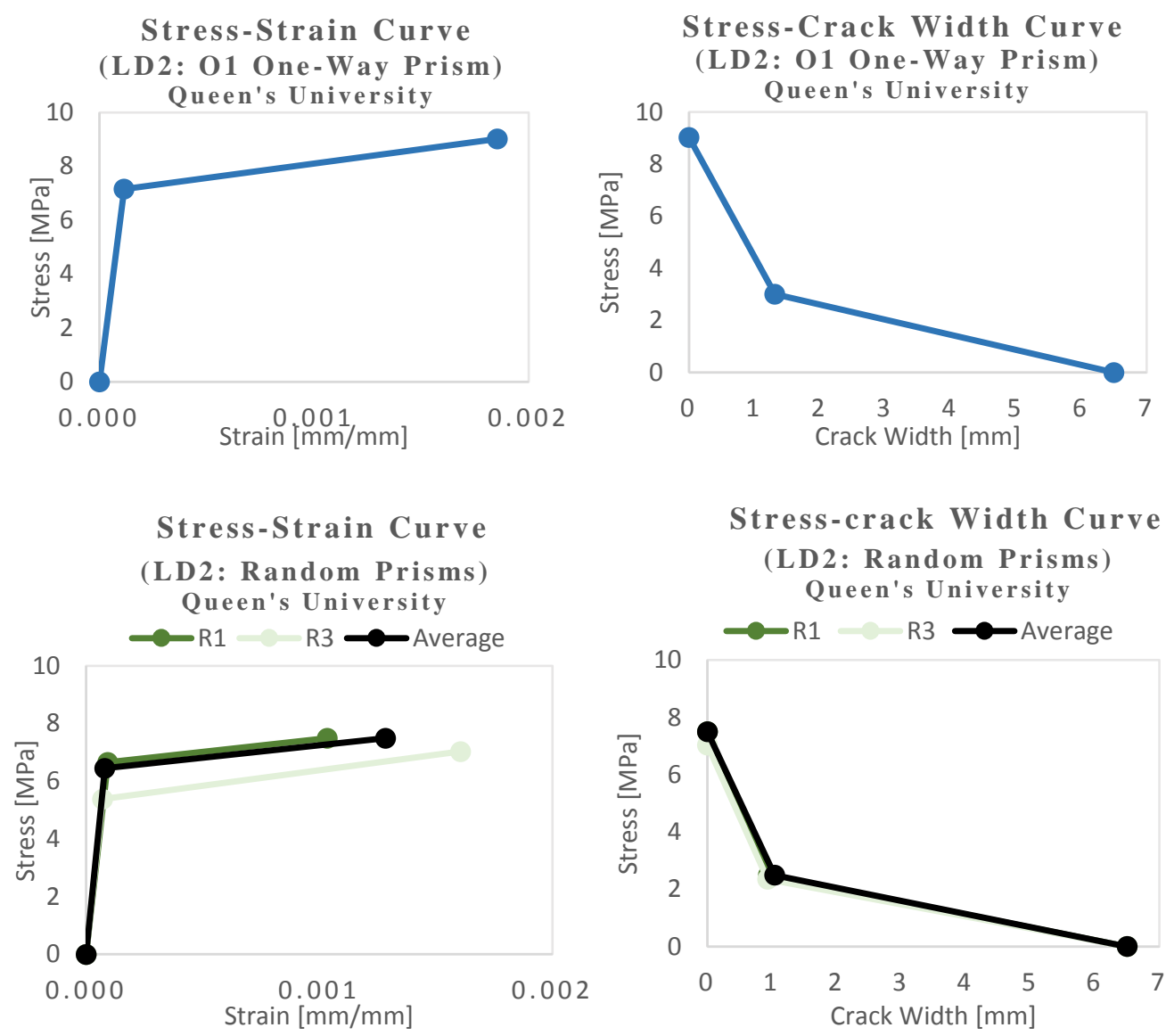

Figure B- 9: Linearized tensile stress-strain-crack mouth opening relationships of the prisms from mix LD2 tested by Queen's University

\section{B.8 Commercial DE2 Design Mix (SC2)}

Table B-15: The four characteristic points utilized for the inverse analysis of the OW prisms from mix DE2 tested with SC2

\begin{tabular}{|c|c|c|c|c|c|c|c|c|c|c|c|c|}
\hline & $\mathbf{P}_{\mathbf{0}}$ & $\mathbf{P}_{1}$ & $\mathbf{P}_{2}$ & $\mathbf{P}_{3}$ & $\mathbf{P}_{4}$ & $\mathbf{P}_{\max }$ & $\boldsymbol{\delta}_{0}$ & $\delta_{1}$ & $\boldsymbol{\delta}_{2}$ & $\delta_{3}$ & $\delta_{4}$ & $\delta_{P \max }$ \\
\hline Specimen & {$[\mathbf{k N}]$} & {$[\mathrm{kN}]$} & {$[\mathrm{kN}]$} & {$[\mathrm{kN}]$} & {$[\mathrm{kN}]$} & {$[\mathrm{kN}]$} & [mm] & [mm] & [mm] & [mm] & [mm] & [mm] \\
\hline OW1 & 15.00 & 22.00 & 34.00 & 35.34 & 28.28 & 36.44 & 0.075 & 0.147 & 0.420 & 0.460 & 0.950 & 0.520 \\
\hline OW2 & 11.00 & 17.50 & 28.00 & 32.50 & 26.00 & 33.50 & 0.060 & 0.109 & 0.290 & 0.400 & 1.550 & 0.470 \\
\hline OW3 & 15.00 & 21.00 & 31.50 & 38.43 & 30.74 & 39.62 & 0.020 & 0.038 & 0.107 & 0.350 & 0.750 & 0.496 \\
\hline Average & 13.67 & 20.17 & 31.17 & 35.42 & 28.34 & 36.52 & 0.052 & 0.098 & 0.272 & 0.403 & 1.083 & 0.495 \\
\hline
\end{tabular}




\section{B.9 Commercial FD4 Design Mix}

Table B-16: The four characteristic points utilized for the inverse analysis of the prisms from mix FD4

\begin{tabular}{|c|c|c|c|c|c|c|c|c|c|c|c|c|}
\hline \multicolumn{13}{|c|}{$100 \mathrm{~mm}$ by $100 \mathrm{~mm}$ Prisms } \\
\hline \multirow[b]{2}{*}{ Specimen } & $\mathbf{P}_{\mathbf{o}}$ & $\mathbf{P}_{1}$ & $\mathbf{P}_{2}$ & $\mathbf{P}_{3}$ & $\mathbf{P}_{4}$ & $\mathbf{P}_{\max }$ & $\delta_{0}$ & $\delta_{1}$ & $\delta_{2}$ & $\delta 3$ & $\delta_{4}$ & $\delta$ Pmax \\
\hline & {$[\mathrm{kN}]$} & {$[\mathrm{kN}]$} & {$[\mathrm{kN}]$} & {$[\mathrm{kN}]$} & {$[\mathrm{kN}]$} & {$[\mathrm{kN}]$} & [mm] & [mm] & {$[\mathrm{mm}]$} & [mm] & {$[\mathrm{mm}]$} & [mm] \\
\hline Prism 1 & 32.00 & 35.00 & 44.00 & 52.58 & 42.06 & 54.21 & 0.055 & 0.080 & 0.180 & 0.350 & 1.400 & 0.720 \\
\hline Prism 2 & 35.00 & 42.00 & 50.00 & 67.87 & 54.30 & 69.97 & 0.045 & 0.072 & 0.160 & 0.550 & 1.150 & 0.710 \\
\hline Prism 3 & 33.00 & 37.00 & 45.00 & 61.86 & 49.49 & 63.77 & 0.042 & 0.063 & 0.143 & 0.450 & 1.050 & 0.725 \\
\hline Average & 33.33 & 38.00 & 46.33 & 60.77 & 48.62 & 62.65 & 0.047 & 0.072 & 0.161 & 0.450 & 1.200 & 0.718 \\
\hline \multicolumn{13}{|c|}{$150 \mathrm{~mm}$ by $150 \mathrm{~mm}$ Prisms } \\
\hline \multirow[b]{2}{*}{ Specimen } & $\mathbf{P}_{\mathbf{0}}$ & $\mathbf{P}_{1}$ & $\mathbf{P}_{2}$ & $\mathbf{P}_{3}$ & $\mathbf{P}_{4}$ & $\mathbf{P}_{\max }$ & $\delta_{0}$ & $\begin{array}{lllll}\delta_{1} \\
\end{array}$ & $\boldsymbol{\delta}_{2}$ & $\delta_{3}$ & $\delta_{4}$ & $\delta$ Pmax \\
\hline & {$[\mathrm{kN}]$} & {$[\mathrm{kN}]$} & {$[\mathrm{kN}]$} & {$[\mathrm{kN}]$} & {$[\mathrm{kN}]$} & {$[\mathrm{kN}]$} & {$[\mathrm{mm}]$} & [mm] & [mm] & [mm] & {$[\mathrm{mm}]$} & [mm] \\
\hline Prism 1 & 62.00 & 72.00 & 88.00 & 120.35 & 96.28 & 124.07 & 0.080 & 0.124 & 0.284 & 0.700 & 1.230 & 0.820 \\
\hline Prism 2 & 59.00 & 71.00 & 92.00 & 118.53 & 94.83 & 122.20 & 0.050 & 0.080 & 0.193 & 0.405 & 0.950 & 0.520 \\
\hline Prism 3 & 55.00 & 65.00 & 84.00 & 106.00 & 84.80 & 109.28 & 0.052 & 0.082 & 0.200 & 0.400 & 0.810 & 0.520 \\
\hline $\mathrm{m} 4$ & 63.00 & 80.00 & 103.00 & 131.01 & 104.81 & 135.06 & 0.053 & 0.090 & 0.216 & 0.465 & 0.955 & 0.580 \\
\hline & 49.00 & 56.00 & 73.00 & 102.14 & 81.71 & 105.30 & 0.045 & 0.069 & 0.168 & 0.490 & 0.955 & 0.590 \\
\hline & 47.00 & 57.00 & 83.00 & 98.35 & 78.68 & 101. & & 0.078 & 0.212 & 0.354 & 1.002 & 0.520 \\
\hline Average & 55.83 & 66.83 & 87.17 & 112.73 & 90.19 & 116.22 & 0.055 & 0.087 & 0.212 & 0.469 & 0.984 & 0.592 \\
\hline
\end{tabular}

\section{B.10 Commercial FD5 and FD6 Design Mixes}

Table B-17: The four characteristic points utilized for the inverse analysis of the prisms from mix FD5 and FD6

\begin{tabular}{|c|c|c|c|c|c|c|c|c|c|c|c|c|}
\hline \multicolumn{13}{|c|}{ Mix FD5 } \\
\hline \multirow[b]{2}{*}{ Specimen } & $\mathbf{P}_{\mathbf{o}}$ & $\mathbf{P}_{1}$ & $\mathbf{P}_{2}$ & $\mathbf{P}_{3}$ & $\mathbf{P}_{4}$ & $\mathbf{P}_{\max }$ & $\delta_{0}$ & $\delta_{1}$ & $\delta_{2}$ & $\delta_{3}$ & $\delta_{4}$ & $\delta_{\mathbf{P m a x}}$ \\
\hline & {$[\mathrm{kN}]$} & {$[\mathrm{kN}]$} & {$[\mathrm{kN}]$} & {$[\mathbf{k N}]$} & {$[\mathrm{kN}]$} & {$[\mathrm{kN}]$} & [mm] & [mm] & [mm] & {$[\mathrm{mm}]$} & [mm] & [mm] \\
\hline Prism 1 & 90.13 & 96.98 & 133.52 & 160.34 & 128.28 & 165.30 & 0.050 & 0.071 & 0.188 & 0.545 & 1.696 & 0.784 \\
\hline Prism 3 & 61.26 & 77.40 & 131.03 & 137.82 & 109.04 & 141.43 & 0.040 & 0.066 & 0.205 & 0.316 & 1.325 & 0.456 \\
\hline Prism 4 & 90.03 & 93.20 & 125.82 & 142.81 & 114.27 & 147.23 & 0.047 & 0.065 & 0.166 & 0.436 & 1.647 & 0.574 \\
\hline Prism 5 & 96.59 & 102.29 & 124.88 & 155.73 & 124.58 & 160.54 & 0.050 & 0.070 & 0.159 & 0.482 & 1.347 & 0.548 \\
\hline Prism 6 & 80.00 & 100.00 & 136.03 & 172.50 & 138.09 & 177.94 & 0.029 & 0.037 & 0.123 & 0.459 & 1.331 & 0.558 \\
\hline Average & 83.60 & 93.97 & 130.26 & 153.84 & 122.85 & 158.49 & 0.043 & 0.062 & 0.168 & 0.448 & 1.520 & 0.584 \\
\hline \multicolumn{13}{|c|}{ Mix FD6 } \\
\hline \multirow[b]{2}{*}{ Specimen } & $\mathbf{P}_{\mathbf{o}}$ & $\mathbf{P}_{1}$ & $\mathbf{P}_{2}$ & $\mathbf{P}_{3}$ & $\mathbf{P}_{4}$ & $\mathbf{P}_{\max }$ & $\boldsymbol{\delta}_{\mathbf{o}}$ & $\delta_{1}$ & $\boldsymbol{\delta}_{2}$ & $\delta_{3}$ & $\delta_{4}$ & $\delta_{P \max }$ \\
\hline & {$[\mathrm{kN}]$} & {$[\mathrm{kN}]$} & {$[\mathrm{kN}]$} & {$[\mathrm{kN}]$} & {$[\mathrm{kN}]$} & {$[\mathrm{kN}]$} & {$[\mathrm{mm}]$} & [mm] & [mm] & {$[\mathrm{mm}]$} & {$[\mathrm{mm}]$} & {$[\mathrm{mm}]$} \\
\hline Prism 1 & 75.00 & 92.00 & 133.00 & 142.79 & 114.23 & 147.21 & 0.055 & 0.090 & 0.245 & 0.354 & 1.020 & 0.421 \\
\hline Prism 2 & 71.00 & 88.00 & 130.00 & 136.37 & 109.10 & 140.59 & 0.054 & 0.089 & 0.247 & 0.310 & 1.150 & 0.425 \\
\hline Prism 3 & 72.50 & 88.00 & 125.10 & 139.47 & 111.57 & 143.78 & 0.058 & 0.094 & 0.250 & 0.501 & 1.360 & 0.652 \\
\hline Prism 4 & 87.00 & 110.00 & 157.00 & 172.02 & 137.62 & 177.34 & 0.084 & 0.142 & 0.380 & 0.630 & 1.750 & 0.840 \\
\hline Prism 5 & 78.00 & 103.00 & 148.00 & 149.99 & 119.99 & 154.63 & 0.072 & 0.127 & 0.342 & 0.370 & 1.584 & 0.500 \\
\hline Average & 76.70 & 96.20 & 138.62 & 148.13 & 118.50 & 152.71 & 0.065 & 0.108 & 0.293 & 0.433 & 1.373 & 0.568 \\
\hline
\end{tabular}



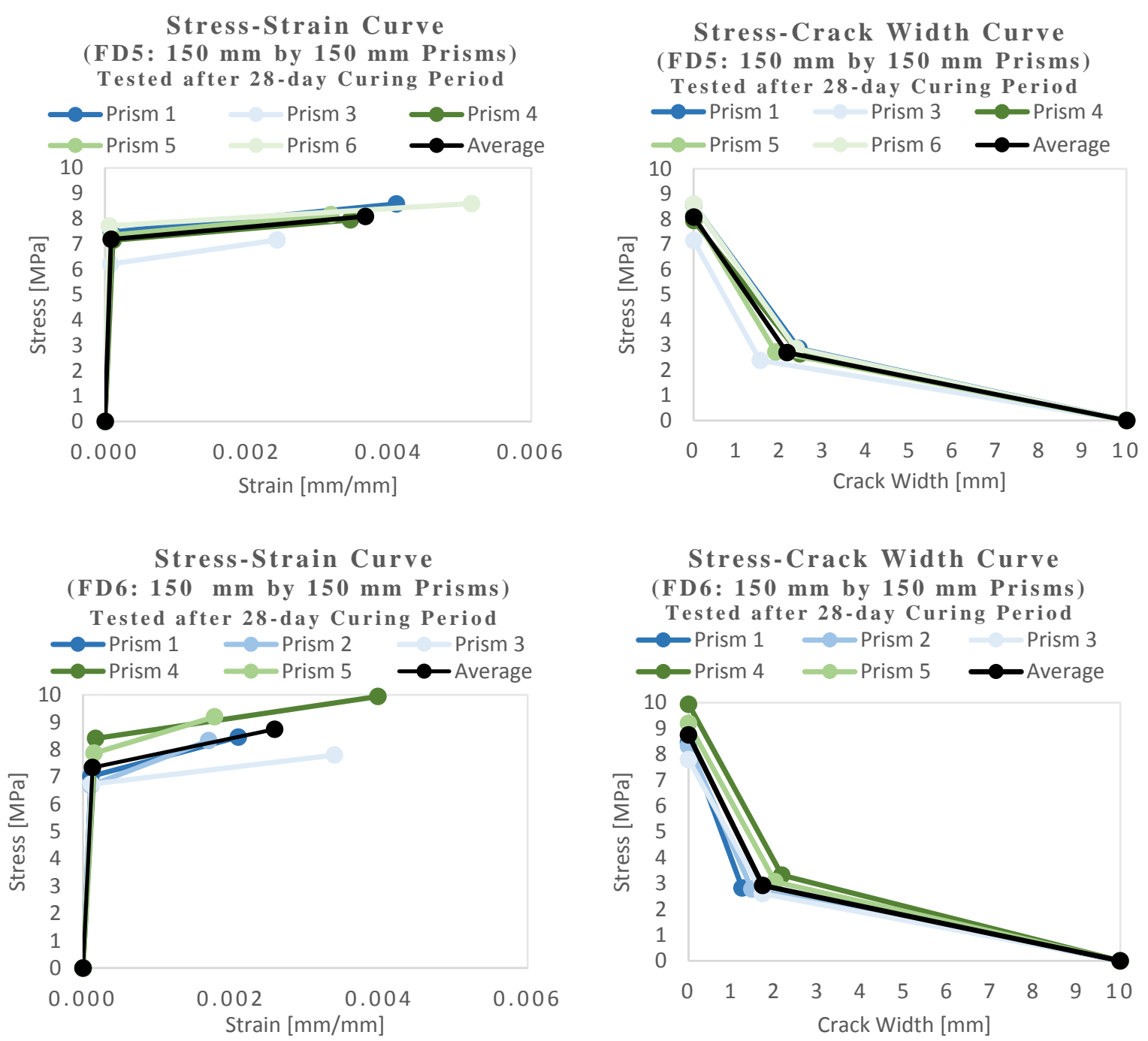

Figure B-10: Linearized tensile stress-strain-crack mouth opening relationships of the prisms from mixes FD5 and FD6 


\section{Appendix C. Analytical Resistance Curves}

\section{C.1 Commercial FD2 Design Mix}
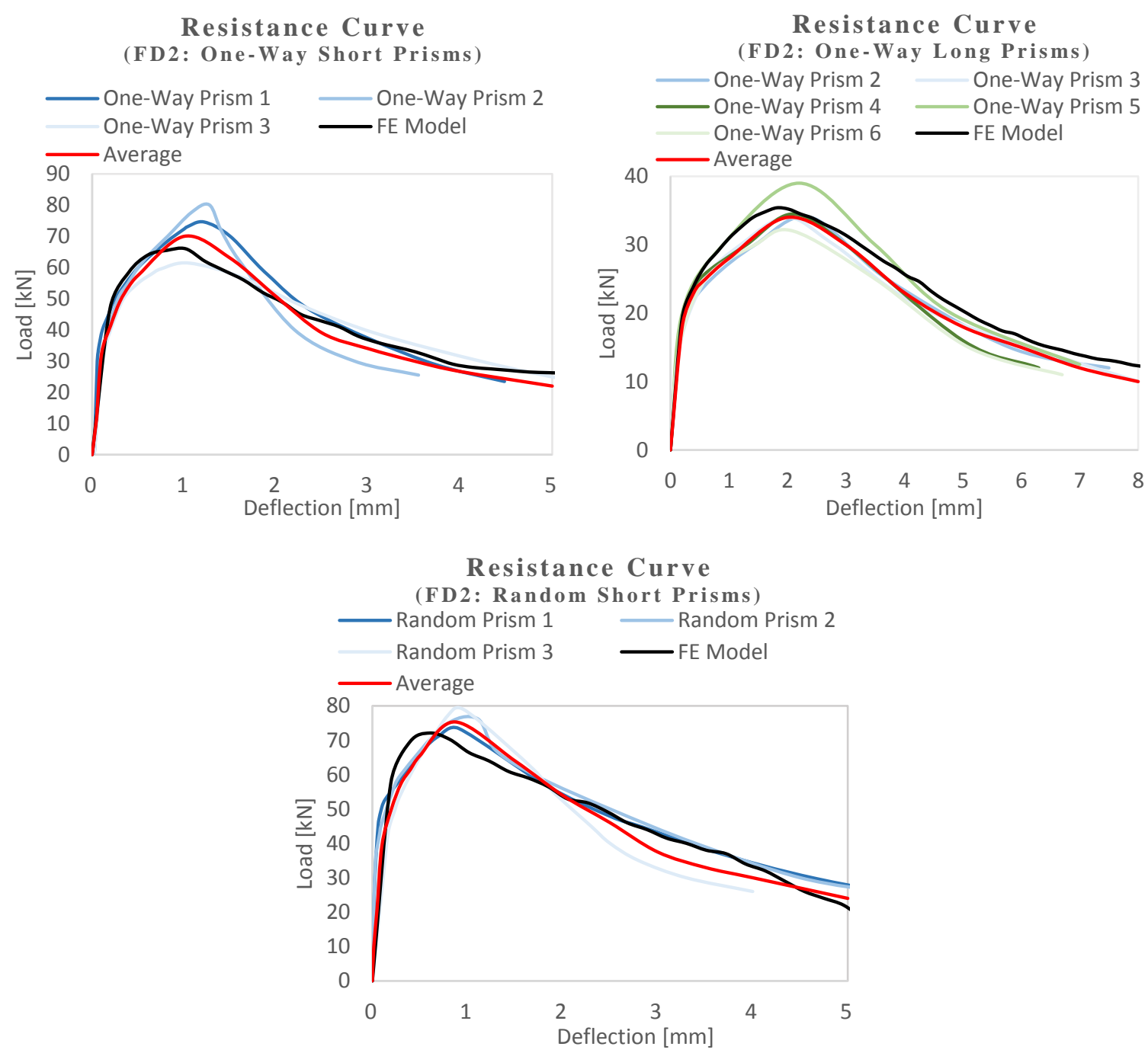

Figure C-1: Analytical resistance curves of mix FD2 


\section{C.2 Commercial LD1 Design Mix}
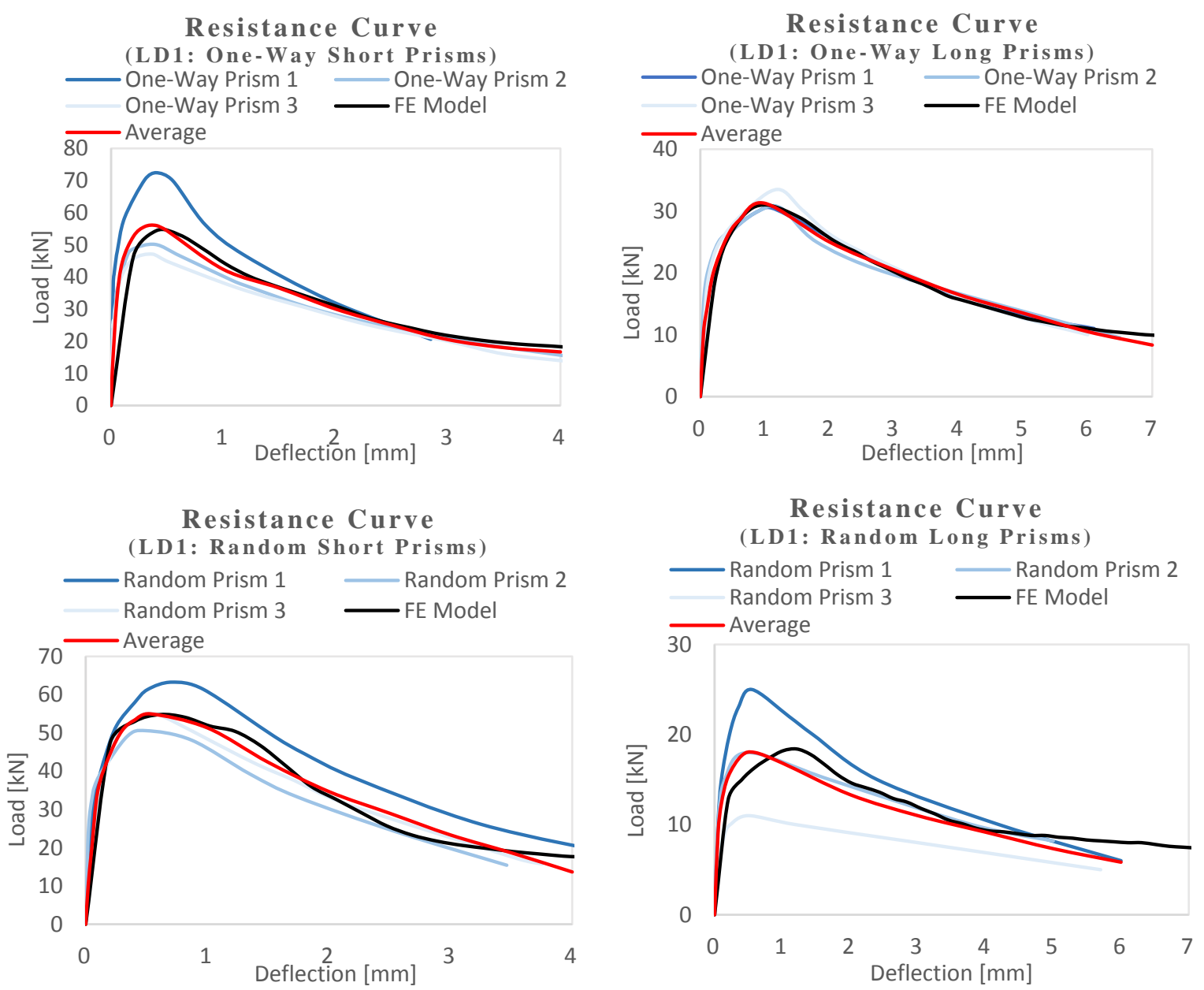

Figure C-2: Analytical resistance curves of mix $L D 1$

\section{C.3 Commercial DE2 Design Mix (SC1)}
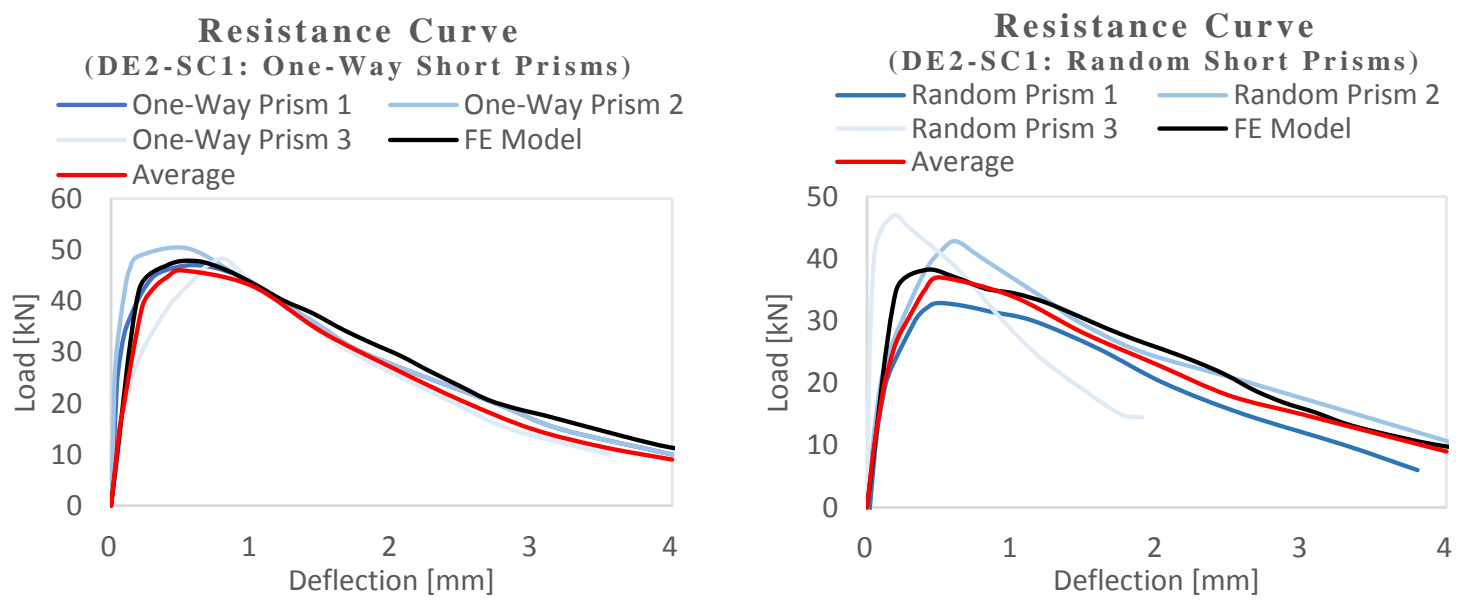

Figure C-3: Analytical resistance curves of mix DE2 


\section{C.4 Commercial FD3 Design Mix}
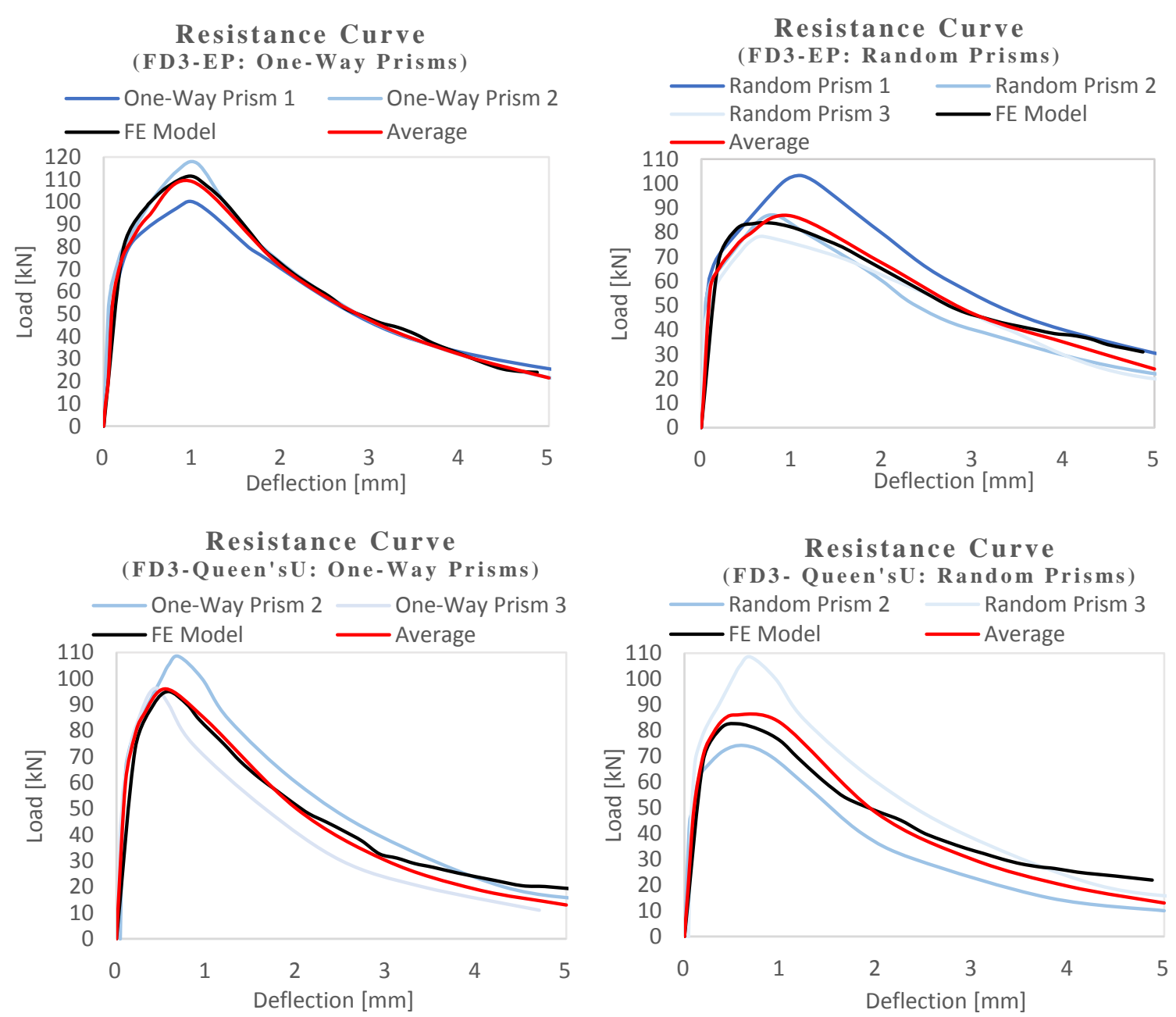

Figure C-4: Analytical resistance curves of mix FD3 


\section{C.5 Commercial KI2 Design Mix}
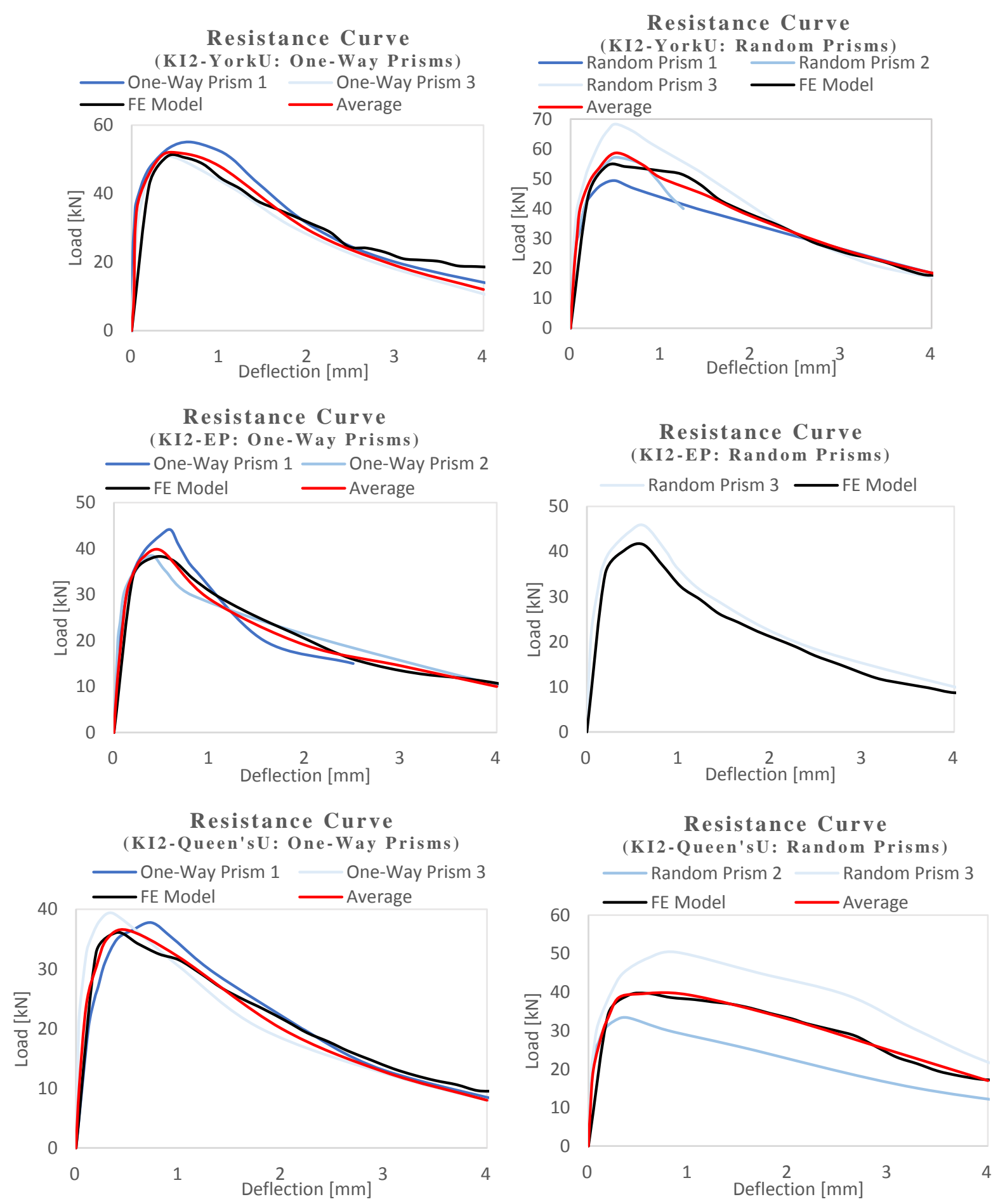

Figure C-5: Analytical resistance curves of mix KI2 


\section{C.6 Commercial LD2 Design Mix}
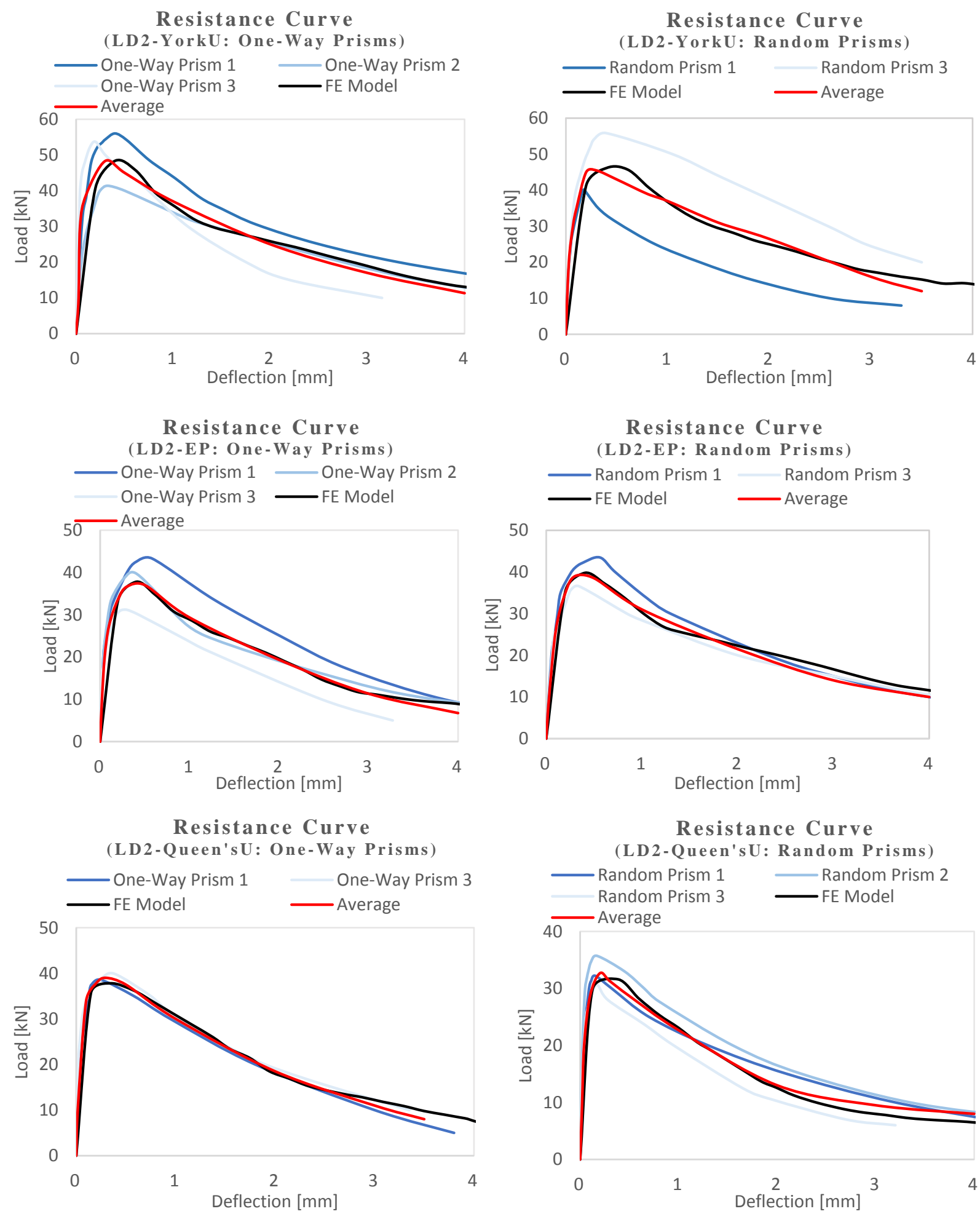

Figure C- 6: Analytical resistance curves of mix LD2 


\section{C.7 Commercial FD5 and FD6 Design Mixes}
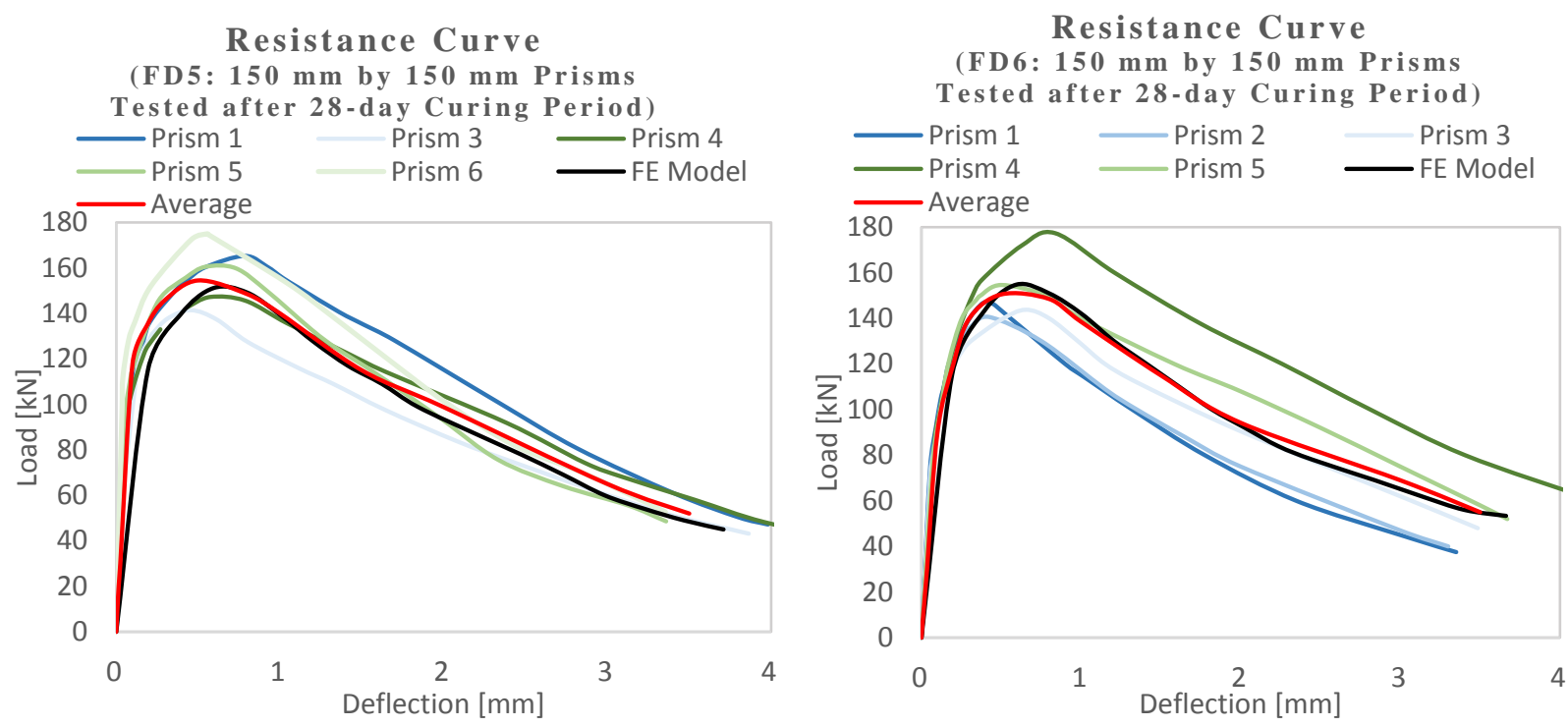

Figure C- 7: Analytical resistance curves of mixes FD5 and FD6 